Frühneuhochdeutsches Wörterbuch

Band 9.1 



\title{
Frühneuhochdeutsches Wörterbuch
}

Herausgegeben von

Ulrich Goebel • Anja Lobenstein-Reichmann

Oskar Reichmann

\author{
Begründet von \\ Robert R. Anderson · Ulrich Goebel \\ Oskar Reichmann
}

Band 9.1

l-maszeng

Bearbeitet von

Anja Lobenstein-Reichmann

und Oskar Reichmann

De Gruyter 
Das Frühneuhochdeutsche Wörterbuch ist auf einen Umfang von dreizehn Bänden zu jeweils rund 1000 Seiten berechnet. Die einzelnen Bände werden nebeneinander von je eigenen Bearbeitern oder kleinen Bearbeiterteams erstellt und in jeweils vier Lieferungen publiziert. Ein Registerband wird relevantes Datenmaterial des Wörterbuches zusammenstellen und damit für unterschiedliche lexikologische Untersuchungen erschließen.

Lieferung $1 \quad l$-leben, erschienen Dezember 1999

Lieferung 2 leben - leschen, erschienen September 2003

Lieferung 3 lescheur - machen, erschienen November 2011

Lieferung 4 machen-maszeng, erschienen Dezember 2012

bearb. von

Anja Lobenstein-Reichmann

bearb. von Oskar Reichmann

ISBN 978-3-11-031106-8

e-ISBN 978-3-11-031127-3

Bibliografische Information der Deutschen Nationalbibliothek

Die Deutsche Nationalbibliothek verzeichnet diese Publikation in der Deutschen Nationalbibliografie; detaillierte bibliografische Daten sind im Internet über http://dnb.dnb.de abrufbar.

(C) 1999/2003/2011/2013 Walter de Gruyter GmbH, Berlin/Boston

Satz: Meta Systems, Wustermark

Druck: Hubert \& Co. GmbH \& Co. KG, Göttingen

(0) Gedruckt auf säurefreiem Papier

Printed in Germany

www.degruyter.com 
anbrinne / vnnd alßdann nyemandt mehr sey / der jn loschen künde. Spechtler, Mönch v. Salzb. 36, 98 (oobd., 3. Dr. 14. Jh.): gelaubig sel tröst, herr, behend, | durch all dein güt lesch ab ir prend. Turmair 4, 81, 4 (moobd., 1522/ 33): Der gemein man [...] trank kain wein nit, lescht und püest den hunger, ersettiget die natur mit milich. Kummer, Erlauer Sp. 4, 459 (m/soobd., 1400/40): Lesche, herr lesche | disen man also vreche! | [...] | er prinnt recht als ein glit. - Luther, WA 34, 2, 403, 14; Reichmann, Dietrich. Schrr. 142, 12; SudHoff, Paracelsus 10, 273, 18 ; Andreae. Ber. Nachtmal 114v, 15; Pfeiffer, K. v. Megenberg. B. d. Nat. 391, 14; Alberus u v; Hulsius L iijr; Dict. Germ.-Gall.-Lat. 304.

3. >etw. (in der Regel: gebrannten Kalk) mit Wasser mischen<.

J. W. von Cube. Hortus 23, 3 (Mainz 1485): so faret eß von eynander glicher wyse als kalck der geleschet wirt mit wasser. BELKIN u. a., Rösslin. Kreutterb. 106, 16 (Frankf. 1535): Kalck. [...] Lebendiger kalck. Geleschter kalck.

4. >(Schulden) tilgen<. - Rwв 8, 1415 (a. 1354).

lescheur, s. leschen (V.) 2.

leschgrube, s. leschen (V.) 1.

leschhorn, das.

,Wassergefäß zum Löschen von Kerzen`; spöttisch auch mit Bezug auf die Nase.

FIscher, Folz. Reimp. 36, 81 (Nürnb., um 1520): Des dein harschober ungefer | Gar köstlich zu arswischen wer | [...] | Und dein nas gut zu eim leschorn. SACHS 14, 62, 10 (Nürnb. 1550): Hab ie auch ein schönes leschhorn, | Das benckt mir herab ubers maul. Ders. 20, 107, 9 (1559): Ich glaub, der nasen-küng dw seist, | Auß allen grossen nasn erkorn; | Du hast ie ein schönes leschborn!

leschkole, leschkrug, leschpandel, leschschaufel, leschspies, s. leschen (V.) 1.

leschtrog, der.

, Wassereimer zum Löschen von Feuer bzw. zum Abkühlen von heißen Gegenständen (in der Schmiede)<; auch obszön und abwertend für den Menschen sowie als Schimpfwort gebraucht; zu leschen (V.) 1.

Jungbluth, J. v. Saaz. Ackermann 24, 17 (Hs. 'omd., $\left.1465^{\urcorner}\right)$: ein mensche [ist] [...] ein leimen raubhaus, ein unsetig leschtrog [Var.: leschkrug], ein gemalte begrebnüß. FASTNACHTSP. 255, 11 (nürnb., v. 1486): Du leschtrog, harmkrug, lochrete tasch, | Du stinkender eimer, du kunige flasch. EвD. 733, 32 (15. Jh.): Das sie mir gern het ab gelescht mein prant | Unden in irem leschtrog (obszön). SACHs 17, 291, 16 (Nürnb. 1562): Nach dem der herr zu dem lesch- drock | Das glüend mennlein binein zug, | Daß das wasser ob ibm zsamb schlug | Und kühlet es fein sitlich ab. EBD. 292, 33: Der schmid dacht, kunst geht nit recht zu, | Und sie [weib] herauß der esse zog | Und warff sie nein in den leschtrog. MaAler 274v (Zürich 1561): Lóschtrog (der) Darin die schmid das loschwasser habend. - Bremer, Voc. opt. 14027; Voc. inc. Teut. o viijr; Dict. Germ.Gall.-Lat. 304.

\section{leschung, die.}

1. >Durstlöschung; Heilmethode, Heilmittel gegen Krankheiten, deren Bekämpfung man mit dem Löschen von Hitzezuständen in Vergleich setzte<; vgl. leschen (V.) 2. KeIL, Peter v. Ulm 35 (nobd., 1453/4): Ein ander gut leschung. [...] leg das über den presten. SudHoff, Paracelsus 5, 465, 32 (1527): die löschung [von Entzündungen] von inwendigen ist solatrum, nenufar, opium; von außen acetum, camphora. EBD. 10, 190, 34 (1536): als sich dan oftmals begibet in büchsenpulvers brant, das auch ein solche leschung muß fürgehen, vor dem und die hiz ausgezogen wird. diselbig leschung ist also: nim distillirten fröschleich, hauswurzen saft, [...] darnach so bind mit den andern brantsalben. MAALER 274v (Zürich 1561): Loschung deß Dursts. Restinctio sitis. - Sudhoff, a. a. O. 10, 186, 24.

2. >Löschung (einer Schuld)<. - Bdv.: vgl. ablöschung 1, ablösung 4, abtilgung 2, abwaschung 3, abwischung.

Mitzschke, UB Bürgel (thür., 1451): leschung etlicher anliegender notscbulde [...], damit der spital befallen ist. BINDEWALD, Texte schles. Kanzl. 131, 11, 24 (schles., n 1350): durch leschunge wille der scbult vñ ouch durch czunemyn der selbin stat.

leschwasser, leschwedel, s. leschen (V.) 1.

lese, die; $-\emptyset /-$.

1. >Weinernte<; als Metonymie: >Zeit der Weinlese (auch als Abgabetermin) ২. Rechts- und Wirtschaftstexte. - Bdv.: ernte, herbst 1; 3, weinernte; vgl. ärische, ${ }^{2}$ laube 3.

Buch Weinsb. 1, 278, 30 (rib., um 1560): dieweil es mit den kalten winen noch zu frohe und in der lasen allenthalben war, wolt ich nicht vergeblich binab nach Coln. RuDOLPH, Qu. Trier 186, 9 (mosfrk., 1593): welche [bürgermeister und rat] auf einen bestimmten tag die laß um die stadt herum zu besichtigen, nach befindung der sachen wiederum zurück referiren, ist es, daß der traub genug zeitig [...], sollen [...] die vorlaß angezeigt, die zeit der laß angeschlagen und publicirt werden. EBD. 320, 16 (1351): daz wir die lase setzen und daz wir zwen dage das vorlesen han, ee man gemeinlichen lese. Mell u. a., Steir. Taid. 244, 24 (m/soobd., 1638): des gotshaus sachen und diener seind des schifflons befreit, тие $\beta$ 
auch alle läre startin wie auch die möst in lösen bin und her fieren. Mell, Steir. Weinbergr. 119, 24 (smoobd., 1543): Zwai emmer most in gemainen lesen und 4 s $z u$ vogtrecht. - Bastian u. a., Regensb. UB 444, 3; Winter, Nöst. Weist. 1, 175, 2; Alberus Nn ivv; Öst. Wв. 1, $405 ; 4,89$.

2., s. lesen 4.

lesebuchstabe, s. lesen 3.

lesegeld, lesehafer, s. lese 2.

lesehaus, s. lesen 4.

leseheller, s. lesen 2.

lesekunst, s. lesen 3.

leselich, s. leslich.

leselon, s. lesen 2.

lesen, V., unr. abl.; zum bedeutungsgeschichtlichen Zusammenhang zwischen lesen 1 ; 2 > sammeln « und 3-6 >Geschriebenes lesen sowie zur Etymologie vgl. Dwв 6, 774; LeXer 1, 1888; KLuge/S., Et. Wb. 2002, 571.

1. setw. systematisch (vom Boden) auflesen, sammeln; etw. pflücken`; mit Verschiebung der Bezugsgröße: >etw. (einen Behälter) auflesend füllen`; speziell: >etw. aussortieren, das Gute, Wertvolle auswählen ‘; von Menschen: `jn. auswählen; jn. um sich scharen; sich mit jm. treffen, sich versammeln; jn. auserwählen`; von Vögeln: >etw. aufpicken`; offen zu 2. - Phras.: (jm. die) federn lesen >(jm.) schmeicheln〈; der jare an sich gelesen baben >älter geworden sein<; den kopf vol lesen > sich vollfressen<. - Bdv.: aufklauben 1, sammeln; vgl. abbrechen 7, auffaben 4, auflesen 1, aufraspen, colligieren, klauben 1. - Synt.: sich zusammen l. >sich versammeln<, etw. (z. B. parteken / brot / bolz. / kraut / wurz, eine blume, den weizen, die raden) l., etw. aus etw. l., etw. von etw. (z. B. von den bäumen, von der erde) l., jn. (belden) an sich l.; (verschoben:) den korb vol lesen.

Strehlke, Nic. Jerosch. Chron. 513, 1821 (preuß., um 1330/40): daz al dî pfafheit, | dî indirt dâ zu Akirs was, | mit vroidin sich zusamne las. LuTHER, WA 10, 1, 1, 666, 6 (1522): Szo wollen sie nur die feddern geleßen haben unnd mit fuchsschwentzen ubirweddellt seyn. EвD. 46, 441, 25 (1538): die lieben Christen, Die gelesen sind aus dem Judi- schen volck. Alberus, Barf. 188, 2 (Wittenb., 1542): Ein Briderlin war seer demutig / vnd bielt sich im Wald / vnd las Holtz zusamen. PeIL, Rollenhagen. Froschm. 100, 1787 (Magdeb. 1608): du must kein mangel leiden / | Die bröcklein nicht von bencken läsen. EвD. 123, 2484: Die [Hühner] lehrt er from vnd heußlich wesen / | Die körnlein von der Erden lesen. Thiele, Minner. II, 31, 12 (Hs. 'md./rhein., 1. V. 15. Jh. ') : sy hatte der jaer an sich gelesen, | sy hatte die jogent over strebet. KuRz, Waldis. Esopus 4, 7, 70 (Frankf. 1557): Jr seit am guten ort gewesen|Und habt ewrn kropff gar voll gelesen. BeCHSTEIn, M. v. Beheim. Evang. Mt. 13, 28 (osächs., 1343): Wiltu daz wir [...] lesin [LANG 1521: samlen; Luther 1545: aus getten] si zu samene? Und her sprach: Nein, daz ir lichte icht zi boufe lesinde di râten uz. roufit vorsament mit en ouch den weisze. Mon. BoICA, NF. 2, 1, 942, 23 (nobd., 1464): [Wilhelm Megerlein] hat auch recht, bolz zi lesen in afterslegen. KL ÄUI, Schweiz. Urbare 3, 193, 1 (halem., 137080): von den gutren ze Niderwile 31/2 pfennig lespfennig. Welti, Stadtr. Bern 503, 27 (halem., 15. Jh.): ₹ư welchen zitten er ein jegklich krut oder wurtz. [...] solle lesen und samnen. Dict. Germ.-Gall.-Lat. 304 (Genf 1636): lesen / Zusammen machen [...] Colligere. WintTerLin, Würt. Ländl. Rechtsqu. 2, 137, 11 (schwäb., 1605): Welcher biren, aichelin oder büechelin lißt, anderst dann es verkündet wirdt, soll [...]. Gilman, Agricola. Sprichw. 2, 81, 25 ([Augsb.] 1548): Federn klauben / Federn lesen / Heüchlen unnd schmaichlen / umb genieß willen. Primisser, Suchenwirt 3, 88 (oobd., 2. H. 14. Jh.): Di stoltzen held er an sich las, | Wie wol ez wer in fremdem lant. KLEIN, Oswald 123, 14 (oobd., 1415): Ain hand si mir im part vergass, die langen har si darawss las. EBD. 19, 161 (1416): Ain edler nam ward mir gelesen [rausgewählt]; wisskunte von Türkei. Munz, Füetrer. Persibein 26, 4 (moobd., 1478/ 84): da ich nächt hab manng schöne plúem gelesen. - HeLm, H. v. Hesler. Apok. 2976; Quint, Eckharts Pred. 1, 200, 11; Етtмüller, Heinr. v. Meißen 2, 15; SchotTENLOHER, Flugschrr. 64, 29; Hauber, UB Heiligkr. 2, 416, 32; Alberus gg iijr; Pfälz. Wв. 4, 940. - Vgl. ferner s. v. ätzen 1, aufklauben 1, parteke 1.

2. setw. (die Frucht, den Ernteertrag) einbringen〈; zumeist: >(Trauben im Weinberg) lesen`; mit mehrfacher Verschiebung der Bezugsgröße: den wein / weingarten, die reben lesen; im Unterschied zu 1 auf das von Menschenhand Gepflanzte bezogen; subst.: >Ernteertrag〈; metonymisch: ‘Zeit der Lese, Jahreszeit Herbst (auch als Abgabetermin relevant)<; als Spezialisierung zu 1 auffaßbar. - Gewisse Beleghäufung für Rechts- und Wirtschaftstexte. - Bdv.: $a b$ blatten 3, abbrechen 7, ausbrechen 3, herbsten, weinernen; vgl. abaugsten, abbringen 2, abfechsnen, bebalten $12,{ }^{2}$ berechen 2 , beimen 6. - Synt.: 
etw. (z. B. den weizen, die trauben, das obest von den bäumen) l.; verschoben: most / wein, reben, den weingarten l.; subst.: das lesen inbringen. Wbg.: lesassach >Traubenbottich ২ (vgl. zum Gw: assach), leselon >Bezahlung für die Erntehelfer`, lesenschaffer $>$ Aufseher über die Weinlese<, lesen(s)zeit, leset $>$ Herbst als Zeit der Weinlese<, leskernen >die nach der Hauptlese übrig gebliebenen Trauben lesen<(Gegenstand von Rechtsregelungen), leskerner, lestag >festgesetzter Tag für den Beginn der Weinlese`, leswagen > Wagen für die Weinlese<, 'lesegeld, lesehafer, leseheller, lesgarbe, lespfennig $\urcorner$ jeweils: >Abgabe (in Naturalien oder Geld) zur Erntezeit an eine bezugsberechtigte Person〈, lesstat 〉Ort, an den die Ernte eingebracht wird $<$.

Helm, H. v. Hesler. Apok. 20194 (nrddt., 14. Jh.): So werde wir gescheiden, | Die lieben von den leiden, IAls do man agaleize Leset den schonen weize. Grosse, Schwabensp. 177a, 9 (Hs. 'nd./md., um 1410?): Als dì din ouet lesest von den boimen. Hilliger, Urb. St. Pantaleon 353, 40 (rib., 2. H. 15. Jh.): wan man die wyne aldae lesen sall. STRUCK, Marienst. Wetzlar 570, 90 (hess., 1400): $28 \mathrm{tn}$. с₹и koste unde c₹u lone den wingarten in deme birbeste сұи lesen. Schmidt, Frankf. Zunfturk. 1, 11, 16 (hess., M. 15. Jh.): Frawwen [...] von irer arbeit mit miste tragen, brechen, hefften, reben lesen [...] zehen heller. ÖSTERLEY, Kirchhof. Wendunmuth 1, 248, 19 (Frankf. 1563): Ins Franckenland kam ein Baier im herbst, den wein lesen zu belffen. Schwartzenbach 2v (Frankf. 1564): Wir sagen Blumen / Opffel [...] abbrechen oder abnemmen / Aber nit Hopffen abbrechen / [...] / Also auch Wein oder Trauben lesen / vnd nicht abbrechen. ERmisch u. a., Haush. Vorw. 162, 10 (osächs., 1570/7): Im September lisset man wein in warmen landen. BurkhaRdT, UB Arnstadt 120, 29 (thür., 1369): daz. man dries in deme iare phliet c₹u sampne, an lesegelde unn an snetegelde. EBD. 216, 1 (1418): darnach sollen die genanten [...] leselon, tragelon, furlon, tretelon, butelon, kelterlon, adder was von dem wyn koift inzubrengen [...] und usgeben. Gille u. a., M. Beheim 222, 29 (nobd., 2. H. 15. Jh.): eur weingart ist volpracht furbas. | ist chain lesen darinne. KLÄUI, Schweiz. Urbare 3, 29, 26 (halem., 1324): Der kuster lat öch werden dem sigristen in dem Hove die lesgarbin, die ein ielicher buman [...] von alter gewonhet gen sol. Kocher, Rechn. Schönenwerd 177, 79 (halem., 1374): ze lesen die reben und den trottknechten XI. MAALER 261r (Zürich 1561): Der Läset. Der herpst. WinTterLin, Würt. Ländl. Rechtsqu. 2, 204, 34 (schwäb., 1624): so man anfacht zu leßen, soll ein jeder zu den keltern leßen. Drescher, Hartlieb. Caes. 279, 22 (moobd., 1456/ 67): ,Warumb babt ir nit gelesen ë̈ren weingarten bisher?" Der ritter sprach: ,Er ist langst gelesen.' Der prister sprach:
,Er ist in chain weis gelesen, wann ich hab in yecz gesehen steen voller weintrawben.' FUCHS, Kart. Aggsbach 387, 7 (moobd., 1469): davon man järleich gibt in dem lesen newin viertaill most. UhLIRz, Qu. Wien 2, 3, 4653, 12 (moobd., 1477): $[o b]$ das lesen destpas inbracht wurde. WinTER, Nöst. Weist. 1, 183, 26 (moobd., 1580): Eß soll auch ein ieder den pergrechtmost alsbalt er gelösen vom weingahrten zu meines gnedigen herrn etc. keller [...] füehren. EвD. 1, 530, 20 (um 1450): den [weingarten] sulln si nicht lesen dieweil ain weingarten in irer buet ze lesen ist; und darnach sulln si das ze wissen tun dem lesenschaffer im perghof ob er in well lesen lassen fur das perkerecht. EBD. 1, 531, 37: wellich leser oder leserin puttner [...] weinper verpergen, es sei in lesassach in puttn in furtucher [...7. EBD. 2, 75, 4 (1610): In verlesnen panthäding befindt sich das die gemain jährlichen lesenszeit ainem leswagen zue schicken schuldig. EвD. 2, 214, 22 (1437): Daz ist auch unser recht daz, wir kaufen most in dem lezen unz auf sand Merten tag. EBD. 2, 460, 15 (1506): so hat der richter mit der gemain zu setzen ain lesstag. EBD. 4, 110, 5 (1578): Die hieter sollen [...] auf die lößkörner ihr aufmerken haben und dieselben nit lößkörnen lassen. BIsCHOFF u. a., Steir. u. kärnt. Taid. 401, 24 (m/soobd., 16. Jh.): Vermerket die ruegatt die ain richter [...] pald nach dem lesen reiten, besitzen und den pauern den eid vorhalten soll. Mell u. a., Steir. Taid. 246, 31 (m/soobd., 1638): In diser weinzüerlei [...] haben zu lösenzeit die lösmaister [...] ir unterkomen und werden darin alle sachen zu dem lösen aufgehalten. Mollay, Ofener Stadtr. 217, 3 (ung. inseldt., 1. H. 15. Jh.): Den czehent sol man an aller ståt vonn den ofnern nemen auff der leß stat. - Hilliger, a. a. O. 278, 23; Herborn u. a., Rechn. Jülich 74, 17; Ermisch u. a., a. a. O. 161, 31; Rennefahrt, Zivilr. Bern 46, 31; BAstian, Runtingerb. 2, 335, 2; UhliRz, a. a. O. 2, 1, 736, 4; 2, 3, 4689, 9; 4994, 9; WINTER, a. a. O. 1, 250, 37; 3, 18, 2; Bretholz, Liechtenst. Herrsch. 220, 18; 339, 13; Mell, Steir. Weinbergr. 121, 2; 122, 24; 145, 16; Schmitt, Ordo rerum 635, 26; Dasypodius 371r; Dict. Germ.-Gall.-Lat. 304; Rwв 8 (hier eine Reihe weiterer Wortbildungen), 1233; PFÄLZ. Wв. 4, 941; Schweiz. ID. 3, 1419; Öst. Wв. 1, 405; Matzel u. a., Spmal. dt. Wortschatz. 1989, 190. - Vgl. ferner s. v. ackerbaunngschaft, afterlesen, ausbrechen 3, auslesen 2.

3. >(selbst) lesen, still, in Gedanken lesen; schriftlich fixierte Texte semantisch und kommunikativ erfassen (als individuelle kulturelle Fähigkeit)<; davon in den meisten Belegen nicht trennbar: >etw. (einen geschriebenen Text oder geschriebene Textteile, mit Bezugsgrößenverschiebung: ein Buch) rezipieren, etw. kognitiv auf- und wahrnehmen`; mit besonderer Betonung des Inhalts: >(einen Textinhalt) verstehen, erkennen; durch das Verstehen des Textes Einsicht in etw. bekommen, etw. erfahren; 
etw. erforschen, studieren ‘; oft ütr.; subst.: >Lektüre; gelesener Text. - Phras. (in Urkunden): etw. sehen, bören oder lesen. - Synt.: nicht l. können, nach dem buchstaben, nach dem sin l.; etw. (z. B. das buch / geheimnis / js. leiden, den brief / buchstaben, die copei / materie / tugend, schriften / verse, deutsch / latein, die evangelisten, St. Augustin) l., etw. in etw. (z. B. in dem ewangelio, in büchern / chroniken) l., die tugent an jm. l., etw. von jm. (z. B. von den märtyrern) l., jm. etw. für laster l., den glauben an im selber l. > sich den Glauben durch Lesen aneignen`, l., das [Objektsatz]; innerlich / inwendig / recht / übel / wol l.; subst.: des lesens fertig / läuftig sein, jn. auf lesen abrichten; das deutsche lesen, das lesen > die Lektüre < des ritters, der verstand des lesens. - Wbg.: lesbüchlein, lesebuchstabe, lesekunst, les(e)werk, lesewort.

Ingen, Zesen. Ros. 94, 5 (Hamb. 1646): Du / Liebe / lebrest jederman $|[. .]$.$| wie er die schriften lesen kan. PFEF-$ FERL, Weigel. Ges. 27, 11 (Hamburg 1646): gleich wie die Engel im Himmel das innere buch ibres Herzens lesen. EBD. 42, 12: ist kundt vndt offenbar an den Gelerten, daß sie ibnen selber nimmermehr mögen den Glauben lesen. LUTHER, WA 1, 274, 42 (1518): Erkennen, das Christus ist der rechte Brieff, [...], darinnen wir lesen, Lernen in sehen. EBD. 9, 548, 24 (1521): di Juden, die do dy schriefft lasßen und verstanden, sein dennoch nicht z $w$ im kumen. EBD. 28, 233, 11 (1528): Wer nu Christus leiden recht lesen wil. EBD. 31, 1, 67, 25 (1530): es sind doch ja nicht Lesewort, [...7, Sondern eitel Lebewort drinnen [jnn der ... schrifft]. EвD. 53, 598, 8 (1543): Da bórestu eine verstendliche rede, nach der Grammatica oder lesebuchstaben. EBD. 54, 30, 8 (1543): Wer aber des lesens leufftig und fertig ist, der leufft uber hin, fasset den synn, ungeacht, ob er etliche buchstaben oder wort nicht eigentlich ansibet, Ehe der ander ein wort buchstabet, hat er den gantzen brieff ausgelesen. PeIL, Rollenhagen. Froschm. 554, 1487 (Magdeb. 1608): Wie wir auch selbst bóren und lesen / | Das Alexander des sinns gewesen / | Als er wolt bezwingen die Welt. Toeppen, Ständetage Preußen 3, 500, 26 (preuß., 1452): Och zo weys ew. gn. wól, das ich nicht selben schreiben noch leszen kan. LOHMEYER, K. v. Nostitz 29, 21 (preuß., 1578): Thonius] und ich schigkten einmal in die cantzley nach dem registrant und lassen die copei. Chron. Köln 1, 50, 63 (rib., Hs. 1. H. 15. Jh.): Men leist, dat bie vurmals ein man | zein dusenden den sege ane gewan. Steer, W. v. Herrenb. Büchl. 132 (pfälz., 1436): Dauon wer geleret werden wil, der lese jnnerlichen jn dem obgenanten liecht. Froning, Alsf. Passionssp. 1172 (ohess., $1501 \mathrm{ff}$. ): ßo man lißet uberall, | das nymmandes vorsuchen sal | synen schepper! KüTHER, UB Frauensee 177, 36 (thür., 1390): Wir] bekennen offinlich an dysim brife alle den, dy in seben horin adir lesin, daz. [...]. KeHrein, Kath.
Gesangb. 1, 184, 6 (Bautzen 1567): Sólch Creutz billich zu preisen ist, | Doran man Gotts geheimnus list. M. CuNITIA. Ur. Prop. 147, 14 (Öls 1650): Nach dem ich von dem 5 ten Jahr an / in dem ich allbereit lesen kónnen / durch stete lesung so Geist $=$ so Weltlicher Historien [...] erk.and. Gille u. a., M. Beheim 82, 494 (nobd., 2. H. 15. Jh.): Man liset nit kainen puchstab, | das Kristus ye gelachet hab, [...] | $[\ldots] \mid$ der sin ist aber neuten $\mid Z u$ mainen noch zu lesen nach dem puchstaben dez gsanges. RupPrich, Dürer 1, 46, 46 (nobd., 1506): Lest den priff noch dem sin, ich hab geeilt. EBD. 1, 171, 82: Darumb sehe ein jeglicher, der do Doctor Martin Luthers bücher list, wie sein lehr so klar durchsichtig ist. Rieder, Gottesfr. 70, 10 (els., 1377): so ist die geschrift gar úbele $z$ ì lesende (২zu entziffern`). RolofF, Brant. Tsp. Widmung 50 (Straßb. 1554): Dieweil nun [...] auch die materi lustig ist zú lesen. Wickram 4, 9, 24 (Straßb. 1556): Dann ob sie gleich die kinder auff schreiben / rechnen und lesen wol abrichten [...7. Golius 153 (Straßb. 1579): Grammatica, die kunst recht und wol lesen / schreiben / reden oder außsprechen. BEHREND, Spangenb. Anbindbr. 1, 10 (Straßb. 1611): daß solche FangBrieffe / von vielen gelesen worden. BaCHMANN u. a., Volksb. 94, 4 (alem., 15. Jh.): Wie die mit einandren strittend, da₹ fint man in dem lesen des wirdigen ritters Sant Wilhelms ouch bernach an disem buoch. Rieder, St. Georg. Pred. 23, 28 (Hs. 「önalem., 1387`): dis drie tugend die wir an únserm herren sollent lesen, den antwúrtent dri ander tugent die an der sele sont sin. SСHмiDT, Rud. v. Biberach 73, 3 (whalem., 1345/60): want er heisset in latinscher zungen in tellectus, want er inwendig list vnd merket. Dict. Germ.-Gall.-Lat. 304 (Genf 1636): lesen / eine Schrifft vbersehen. KolLeR, Ref. Siegmunds 177, 17 (Hs. `Augsb., um 1440?) : etlich können die siben tagzeit nit petten noch singen noch lesen. SAPPler, H. Kaufringer 26, 136 (schwäb., Hs. 1472): gedultigkait in leiden ist $\mid$ grösser vil, als man da list. Kohler, Ickelsamer. Gram. 2, 5 (wohl `Augsb. 1. Dr. 16. Jh. ${ }^{7}$ ): das es die besten [...] stuck der Grammatic handelt. Námlich den verstand der Buchstaben, des lesens vnd der Teütschen wórter. EBD. 5, 8: Es ist [...] kaũ ain werck od. creatur auf erden, die zugleich zu Gottes ehr [...] mehr gebraucht würdt, dan die lesekunst. EBD. 5, 18: das mich Gott über dises sein ampt setzen wolt, das lesewerck zugebrauchen. EBD. 23, 2: will ich dauon auch ain wenig sage, vnd nit mehr dan souil zum teütschen lesen gehơrt. EBD. 42, 21: Damit dises buichlin auch ein schul oder leßbuichlin móg sein. SCHORER, Sprachposaun 43, 5 (о. O. 1648): wiewol er nicht lesen oder verstehen kunte / was dessen Jnhalt seyn móchte. KLEIN, Oswald 98, 3 (oobd., 1430): für alles, das ich hör, sich, lis, I mit gütem berzen freust du mich. Fichtner, Füetrer. Trojanerkr. 159, 7 (moobd., 1473/8): ich thät, was ir mich biesset - | und solt man mir's ymmer für laster lesen! TURMAIR 4, 329, 5 (moobd., 1522/33): Es ist nichts dan nur leswerk, g'swäz, träum, schuelertand. - Оовsснот, Spee/Seifert. Proc. 455, 15; Luther, WA 26, 237, 5; 578, 17; 610, 23; 54, 84, 34; Grosse, Schwabensp. 104a, 24; Quint, Eckharts Pred. 1, 127, 2; 2, 58, 2; Chron. Köln 1, 3279; Schützeichel, Mrhein. Passionssp. 
137; Kollnig, Weist. Schriesh. 276, 25; DüNNHAupt, Werder. Gottfr. v. Bullj. 18, 1; Oorschot, Spee/ Schmidt. Caut. Crim. 213, 3; Mathesius, Passionale 36r, 9; Roloff, Naogeorg/Tyrolff. Pamm. 7, 13; v. D. Lee, M. v. Weida. Spigell 19, 14; 79, 33; DoubeK u. a., Schöffenb. Krzemienica 728; Ingen, Zesen. Ged. 383, 11; Valli, Baldemann 20; v. Keller, Ayrer. Dramen 6, 3; 2876, 2; Bell, G. Hager 117, 2, 21; Thiele, Minner. II, 10, 28; Boos, UB Aarau 42, 4; Morrall, Mandev. Reiseb. 161, 16; Bauer, Geiler. Pred. 316, 24; 462, 11; Kohler, a. a. O. 5, 10; 7, 19; 16, 13; 19, 17; 20, 28; 29, 2; 37, 19; Schorer, Sprach-Verd. 18, 22; Bauer, Haller. Hieronymus-Br. 88, 11; DIEs., Imitatio Haller 89, 5; Pirrainen, Stadtr. Sillein 80r, 20. - Vgl. ferner s. v. abschreiben 1, altar 2, andacht 1, anleiten 1, arzetbuch, aussagen 3, balgen 3.

4. >etw. (einen Text) verlesen, (jm.) laut vorlesen, (jm.) etw. Geschriebenes vortragen, mitteilen`; speziell auf die Aussprache bezogen: >etw. laut artikulieren, prononzieren`; rechtssprachlich: >etw. öffentlich verkünden, etw. (z. B. ein Gesetz, eine Verordnung oder ein Urteil) durch die öffentliche Verkündigung rechtskräftig werden lassen`; in der christlichen Liturgie: >(Gottes Botschaft) verkünden, (die Messe) lesen, zelebrieren; gemeinschaftlich Kommunikation über / mit Gott halten〈; auch: >meditativ rezitieren (über einen wichtigen biblischen Text oder eine Textpassage)<. - Phras.: jm. den rechten, einen harten / scharfen text lesen >jn. scharf zur Rede stellen<; jm. den kalender lesen >jm. den Tod vorhersagen ‘; jm. sein legend lesen (Beleg s. v. legende 1), einer frauen die mette lesen (obszön); grillen und possen lesen. - Bdv.: vgl. auflesen 5 , belesen $1 ; 2$, jarzeiten, verlesen, vorlesen. - Synt.: etw. (z. B. den brief [vielfach], eid, die gerechtigkeit / klage / messe, das evangelium / herkommen / urteil, geschichten / verse / worte) l., etw. zu deutsch l., jm. etw. (z. B. die regel, das buch) l., etw. lesen hören, lesen hören, wie [...], etw. öffentlich l.; von jm. >über jn.८/ etw. l., von etw. l., wie [...]; subst.: das lesen der kirche >in der Kirche<, das öffentliche lesen; got mit lesen dienen. Wbg.: lesbank $1>$ Kanzel (dazu bdv.: letner, predigtstul), lese 2 (dazu bdv.: lection 1; a. 1561 f.), lesehaus, lesmesse >Totenmesse〈, lesschule 〉Schule, an der der Unterricht in Form von Vorlesungen erfolgt< (a. 1601).
Luther, WA 6, 239, 7 (1520): vil stifften, pfeyffen, lesen und singen [...]: dafur ist y hm [bose geist] nit leyde. EBD. 8, 496, 19 (1521): alle Christen haben gutt fug und recht, auß der heyligen schrifft tzu leßen und predigen. ЕвD. 30, 3, 461, 32 (1531): Jch wil dir, Juncker Meuchler, den recbten text lesen. EBD. 32, 398, 33 (1532): Stephanus [...] den hohen priestern ein harten scharffen text lieset. REISSENBERGER, Väterb. 163 (md., Hs. 14. Jh.): In das [bích] han ich den sin gewant | Und wil dar uz ze dute lesen, | Daz, mich nutze duncket wesen $\mid Z u$ horen der gemeinschaft. REDLICH, Jül.Berg. Kirchenp. 1, 249, 2 (Cleve 1532): das gein nuwerong widder die heilige sacramenten, widder die gesang und lesen der kirchen, [...] ingefurt sollen werden. BuCH WeINSB. 1, 106, 36 (rib., um 1560): ist wis sinen testament auch ain leismiss [...] fundeirt worden. Hilliger, Urb. St. Pantaleon 534, 17 (rib., 1655): nach ibrem absterben gleich lassen lesen per nostros conventuales 300 leessmessen. VOC. INC. TEUT. O viijv (Speyer um 1483/4): Lese hus stat'kamer lectoriũ é loc' legẽdi. Sievers, Oxf. Benedictinerr. 31, 26 (hess., 14. Jh.): so sal man ir [ins Kloster eintretende Schwester] dise regele lesen von anbeginne biz an daz ende. Schmidt, Frankf. Zunfturk. 2, 235, 31 (hess., 1490): sollen irs bantwercks meistere diß buche alle fronfasten den gesellen gemeynlich in eyme gebode tun lesen. KöBLER, Ref. Franckenfort 74, 21 (Mainz 1509): Wir wóllen auch das in allen sachen so in schrifften gehandelt werden / die ende vrteil in schrifften gegeben / vñ durch vnsern gerichtschryber gelesen sollen werden. KuRz, Waldis. Esopus 1, 79, 14 (Frankf. 1557): Fübrt sie [Vögel] mit jm ins hauß binein, | [...] | Da laß er jnen den Kalander, | Das jre keine wider kam. Karnein, Salm. u. Morolf 14, 1 (srhfrk., Hs. um 1470): Da man das ewangelium laß, | nu horent, was der frawwen opffer waß. Jungbluth, J. v. Saaz. Ackermann 31, 20 (Hs. ' omd., 1465ㄱ): Ich han von jugent auf gehöret lesen und gelernet, wie got alle ding beschaffen habe. LöscHER, Erzgeb. Bergr. 84, 21 (omd., 1559): So die mutzettel zweymal offentlich gelesen sein worden. OpITz. Poeterey 33, 29 (Breslau 1624): weil das ó von vnns als ein e [...] gelesen wirdt. Mon. BoicA, NF. 2, 1, 20, 29 (nobd., 1464): so man armen lewten die lantpucher list. v. Keller, Ayrer. Dramen 3013, 9 (Nürnb. 1610/8): Nun wil ich [...] [... | Der Frauen beut die Metten lesen (hier obszön). Moscherosch. Ges. Phil. v. Sittew. 15, 16 (Straßb. 1650): wenn sie [...] grillen und bossen lesen hören. Bremer, Voc. opt. 12016 (wobd. / oobd. 1328 ff.): lespankech [...] Canellus [...] in singulari est locus altus in templis, in quo stans prespiter indicit subditis agenda. SCHмIDT, Rud. v. Biberach 38, 19 (whalem., 1345/60): Wenne di bórest old lisest daz. ewig wort vnd die sel mit ein ander reden, so solt di nit liplich stim old liplich bilde bilden. MAaler 261r (Zürich 1561): Låsen vnd büchstaben außsprächen [...]. Das offentlich Lásen vnnd profitieren. Professio. Jerouscheк, Nürnb. Hexenh. 14r, 20 (obd., 1491): also le $\beta$ der richter od' laß lesen den proff. Bauer, Geiler. Pred. 464, 23 (Augsb. 1508): denn lobest / und dankest du got / mit werken. wenn du mit singen unnd lesen gott dienest. Barack, Zim. Chron. 3, 55, 15 (schwäb., M. 16. Jh.): Grave Friderrich lass den brief, das 
die umbstender das alles bören mögten. PRIMISSER, Suchenwirt 22, 54 (oobd., 2. H. 14. Jh.): Die weil ich pey der werlt waz, Ee ich der chlosen regel laz (hier wohl: >das Klostergelübde ablegen (). Mell u. a., Steir. Taid. 44, 24 ( $\mathrm{m} /$ soobd., 1523): [soll] in aines ieden freien willen steen was er nach seinem vermugen singen, lesen oder begenknus halten laßen well. Baptist-Hlawatsch, U. v. Pottenst. Vorr. 80 (moobd., A. 15. Jh.): Darumb pit ich in Christo Jhesu alle die, die es lesen oder hören lesen. - REDLICH, a. a. O. 1, 273, 35; Sievers, a. a. O. 21, 7; WeINGART u. a., Seelb. Rhodt 1, 15; KüTHer, UB Frauensee 114, 9; Opel, Spittendorf 141, 38; KISCH, Leipz. Schöffenspr. 411, 17; Mon. BolcA, NF. 2, 1, 25, 19; GiLman, Agricola. Sprichw. 1, 289, 18; Bihlmeyer, Seuse 414, 5; Vetter, Pred. Taulers 59, 11; 138, 13; 271, 2 ; 282, 28; Rieder, St. Georg. Pred. 98, 5; Turmair 5, 445, 20; Dirr, Münchner Stadtr. 173, 17; 404, 12; Moscouia D 2v, 38; Baptist-Hlawatsch, a. a. O. 28; 1470; Schmitt, Ordo rerum 133, 21; Voc. Teut.-Lat. dd vijv; z iixr; Rот 345; Rwв 8, 1234 f.; SCHweIz. ID. 3, 1420; 8, 614. - Vgl. ferner s. v. abdringen 3, abhören 1 , allegieren, altarstein 1, ampt 2, andacht 1, anfahen 1, aufmerken 2, auslecken 2, aussprechen 3.

5. setw. lehren; jn. unterrichten; jm. etw. vortragen, erklären, erläutern (zumeist vom lehrenden Vortrag bzw. einer akademischen Vorlesung)<. - Bdv.: vgl. anfüren 1, anrichten 17 , anzeigen 1 , beleren, belernen 2 , bereden 5 , bereiten 6 , informieren 2, instituieren, ${ }^{2}$ leren $1 ; 2$. - Wbg.: $\ulcorner$ lesbank 2, lesstul >Vorlesungskatheder $\urcorner$, leshaus >Hörsaalgebäudeく (a. 1593), lesrok >Amtskleidung des Diakons〈, lesstube.

Helm, H. v. Hesler. Apok. 578 (nrddt., 14. Jh.): Als sente Johannes drabe las: |, In dem beginne waz dar wort. Buch WeInsB. 2, 218, 19 (rib., 1571): die sint mit umbgegangen in der kirch lessrock und kappen. EBD. 3, 272, 4 (rib., 1585): Das kereichsfolk hat die chorkappen, gegere, lesrock, alfen angetain. SchüTZEICHeL, Mrhein. Passionssp. 491 (mrhein., um 1335): Die wisen arzede alle lesen, | daz. der siche si genesen, | so er slafen moge wol. GILlE u. a., M. Beheim 96, 74 (nobd., 2. H. 15. Jh.): dy [maister] salche ding fur geben | Mit lesen und mit tispotiern | und auch dar zu mit argubirn. MAaler 261r (Zürich 1561): Offentlich Lásen und leeren. Profiteri. Bremer, Voc. opt. 12016 (wobd., 14./15. Jh.): Pulpitum [...] lespanck [...] Pulpitum, analogium, ambo /-onis significant tabulam eleuatam, super quam libris alte positis quod scriptum est legitur clara voce. Dict. Germ.-Gall.-Lat. 304, 31 (Genf 1636): lesstube / f. Auditoire [...] Auditorium. [...] leßstul / m. Chaire de professeur, Cathedra. KLEIN, Oswald 24, 25 (oobd., 1423): All maister uns das lesen | aus der vil hailgen schrifft, | das kainer müg genesen, | wer inn der sünde gifft. SEEMÜLLER, Chron. 95 Herrsch. 12, 8 (oobd., Hs. 1. H. 15. Jh.): [Jupiter] macht die vrein künst, die darnach wurden ze Athenis gelesen. EBD. 82, 9: Ain weib ward pabst [...]. Darnach cham si gen Röm und lazz da manige grosse chunst. Ander maister, schuler und phaffen horten ir leczen, und was ze Röm die weil dhain maister, der al₹ maisterleich biet gelesen. Hohmann, H. v. Langenstein. Quästio 181, 4 (moobd., 1. H. 15. Jh.): so sind doch sunderleich syben [versüechung], [...] dye der hoch gelertt mayster Hainreich von Hässen bye cze Wienn hat gelesen. - Loose, Tuchers Haushaltb. 171; Bremer, Voc. opt. 6013; Schweiz. ID. 2, 1717.

6. >sich selbst etw. vortragen, etw. überlegen, im Geiste abwägen`; anschließbar an 2. - Bdv.: vgl. abwägen 4, aufwegen 5, auswegen 4, beschauen 6, besehen 7, besinnen 1, pensen, perpendieren.

Mayer, Folz. Meisterl. 71, 101 (nobd., 1517/8): Sie eillet ser $\mid$ Zum herczog [...] | [...] Die red sie eben fur sie las, | Wie sey dem fürsten mocht verkunden pas.

\section{lesenschaffer, lesen(s)zeit, s. lesen 2.}

$$
\text { leser, der; }-/-\emptyset \text {. }
$$

1. >Person, die Weintrauben pflückt; Erntehelfer ‘; zu lesen 2. - Meist Rechtsund Wirtschaftstexte. - Bdv.: herbster, samler; vgl. ableser, hauerpöbel. - Wbg.: leserin 1, leserlon.

Lamprecht, Dt. Wirtschaftsl. 3, 302, 23 (mosfrk., 1497): das sie [...] das dritteil gewas von den obgenanten wingarten geben sullen, ane einchen leser- ader dregerlone. ENGEL, Rats-Chron. Würzb. 190a, 20 (nobd., Hs. M. 17. Jh.): mußt man einem leser am montag post Martini geben zu lobn 18 s. EвD. 261, 3: da man anbub, zu lesen, gab man einem leser 4 schilling [...7; vndt man macht den lohn einem leser 12 ঐ. Kurrelmeyer, Dt. Bibel 9, 25, 4 Var. (Straßb. 1466): Bekere dein hant als der leser [Var. 14752-1518: weinleser] ₹ú dem kúbel. WeLti, Stadtr. Bern 566, 15 (halem., 15. Jh.): das des spitals volck, leser, herbst gesind, karrer, pfender vnd der meister ledig uber die brígg faren. MAALER 261r (Zürich 1561): Herpster, Läser. Vindemiator. WINTER, Nöst. Weist. 1, 531, 37 (moobd., um 1450): wellich leser oder leserin puttner tretter nachsteer etc., [...] weinper verpergen. - Aubin, Weist. Hülchrath 255, 36; Bastian u. a., Regensb. UB 446, 8; Dict. Germ.-Gall.-Lat. 304; PfÄLZ. WB. 4, 942.

2. >Rezipient eines Textes «; zumeist in der Anrede oder mit sonstiger Bezugnahme durch den Autor, häufig innerhalb einer captatio benevolentiae; vgl. lesen 3; 4. - Vorwiegend späteres Frnhd.; meist bildungssprachliche Texte.

SCHÖPpER Vorr. a IVr (Dortm. 1550): nachdem ein reguliertte unnd wolgesetzte Rede oder Schrifft [...] den zübórer oder Leser bewegt. Luther, WA 10, 1, 2, 211, 4 (1526): Dem 
Christlichen leser. Gnad und fryde [...]. EвD. 10, 2, 228, 8 (1522): ich [Luther] gebe keyn ablaß meynen leßern und bitte got, das [...]. EBD. 18, 136, 5 (1525): Und bitte dich, Christlicher leser, wöllest drauff sehen, Ich will dyr [...]. v. Keller, Amadis 5, 3 (Frankf. 1561): Vorrede des Teutschen Tranßlatoris, an den Läser. GVnstiger lieber Läser, Als [...7. Oоrschot, Spee/Schmidt. Caut. Crim. 380b, 34 (Frankf./M. 1649): Damit der Leser dieses verstehe / muß er wissen / daß [...]. OpITz. Poeterey 53, 16 (Breslau 1624): Jch hoffe aber / es wird mir der guethertzige Leser / in betrachtung der kurtzen zeit etwas vbersehen oder bedenkken / [...]. FrancK, Klagbr. 219, 5 ('wohl Nürnb. ${ }^{\top}$ 1529): SIbe durch Gott / mein Leser / wer zum theyl die bewschrecken sind. Trunz, Meyfart. Tub. Nov. Vorr. 4, 5 (Coburg 1626): Bitte aber den ginstigen Leser / er wolle es dem Drucker zu gut halten / wenn [...]. Wickram 4, 10, 19 (Straßb. 1556): Desshalben / lieber leser oder zübórer / euwer baider ungunst zu vermeiden / bab ich mich [...] entschuldigen wóllen. ANDrEAE. Ber. Nachtmal 27r, 6 ([Augsb.] 1557): Auß disem kan auch ain Christenlicher Leser wol vernemen / warum̃ [...]. KOHLER, Ickelsamer. Gram. 10, 4 (wohl 'Augsb. 1. Dr. 16. Jh. ${ }^{\top}$ ): so würdt doch solcher vnterschaid vom Teütschen leser nit vermerckt, vnd würdt das, $u,[\ldots]$ an solcher stat gebraucht. - Lemmer, Amman/Sachs. Ständeb. IX, 20; STOltzius, Chym. Lustg. 14, 3; Dünnhaupt, Werder. Gottfr. v. Bullj. 5, 1; 5, 4; 16, 8; Allg. Schau-Bühne 33, 29; 65, 57; M. CuNitia. Ur. Prop. 148, 1; Franck, a. a. O. 219, 1; v. Birken. Erzh. Österreich 77, 13; Jörg, Salat. Reformationschr. 746, 20; Heidegger. Mythoscopia 33, 21; 63, 14; VOC. INC. TEUT. o viijr. - Vgl. ferner s. v. äquinoktialisch, astronomisch, aufmerker, ausfürlich 2.

3. \Vorleser eines Textes «; im einzelnen: , Vorleser biblischer Schriften im Klosterrefektorium<; >Lektor im Dom<; >Erzähler`; >Lehrer`; > Archivar des Kammergerichtes ‘; > wiedertäuferischer Laienprediger〈; >Person, die Gesetzestexte oder Urteile öffentlich verkündet bzw. Leseunkundigen vorliest ‘; zu lesen 4. - Wbg.: leserin 2 >Vorleserin im Klosterrefektorium

Helm, H. v. Hesler. Apok. 3711 (nrddt., 14. Jh.): Des bit ich den lesere, | Swen her kumt in diz mere, | Daz ber mit schonen witzen $|[.]$.$| Antweder her entrumen | Oder$ ober var den lumen. LuTHER, WA 29, 687, 12 (1529): Ego sol ein prediger und leser sein. VeTter, Schw. zu Töß 48, 17 (Hs. 15. Jh.): und illt [sy] denn in den refentar und satzt sich nebent die leserin und loset begirlich dem Gotz, wort. LAUFs, Reichskammergo. 113, 5 (Mainz 1555): sechs redlich personen, deren zwo prothonotarii und zwo notarii und zwo leser des cammergerichts sein sollen. PALM, Veter Buoch 33, 2 (schles., Hs. E. 14./A. 15. Jh.): Er [Jesus] wart ein kint, dar nach ein lesere, do er das buch nam vnd las in der synagoga. Golius 158 (Straßb. 1597): Professor, ein offentlicher leser / oder Lebrer inn einer schïl. BACHMANN,
Morgant 5, 14 (halem., 1530): Er hatt alwegen lässer, die im die Biblen und kronicken lăssend. MAALER 261r (Zürich 1561): Láser / Der heyter und laut lißt. Recitator. Láser / ein knab oder diener der einem lißt. [...] Offentlicher Láser vnnd leerer. Professor. Chron. Augsb. 4, 237, 12 (schwäb., v. 1536): Die leser bie zu dem thom haben [...] etlich felden und ecker gehept. SeXauer, Schrr. in Kart. 240, 3 (nöst., v. 1450): Wann man in dem reuent izt so sol der kelnër besehen / daz chein tumult oder yemants anders stimm gehort werd / denn allain des leser. WINTER, Nöst. Weist. 2, 747, 37 (moobd., 1641): verpeüt unser gnediger herr gar ernstlich das man die stuck der rechten und seiner pot als die verschriben seint in ietlichem pantäding fürlegen sollen mit ainem lëser, oder ob man das nit haben mëcht mit ainem vorsprëcher. - LUTHER, WA 30, 2, 528, 28; KARNEIN, Salm. u. Morolf 451a, 5; LAufs, a. a. O. 272, 18; Euling, Kl. mhd. Erz. 789, 18; Chron. Augsb. 8, 360, 2; Wackernell, Adt. Passionssp. St. I, 97; DasyPODIUS 110r; SERRANus 117r; Rwb 8, 1236; SChweIz. ID. 3, 1419.

leserin $1 ; 2$, s. leser $1 ; 3$.

leserlich, s. leslich.

leserlon, s. leser 1.

leset, s. lesen 2.

lesewerk, lesewort, s. lesen 3.

lesgarbe, lespfennig, s. lesen 2.

leske, wohl der; aus tschech. dlesk (SuOLAHTI, Vogelnamen. 1909, 136).

>Kirschkernbeißer`. - SCHLES. WB. 2, 807 (a. 1603).

leskernen, leskerner, s. lesen 2.

leslich, auch lese- und leser-, Adj. >lesbar, entzifferbar; deutlich`; vgl. lesen 3. Reissenberger, Väterb. 35669 (md., Hs. 14. Jh.): er sach bi sich in den sant $\mid$ Mit ordelichen buchstaben $\mid$ Dise wort wol uz gegraben, | Die er sus leselichen las. Voc. INC. TEUT. oviijr (Speyer um 1483/4): Leslich legibilis. KöBler, Ref. Franckenfort 95, 11 (Mainz 1509): vff yedes halb blat vierundzwentzig zyle leserlicher schriffte / zymlicher wyße geschryben. MAaler 261r (Zürich 1561): Láßlich / Das man låsen mag. Legibilis.

lesmeister, Bw teils lese-, der; $-/-\emptyset$.

1. >Aufseher über die Weinlese`; vgl. le$\operatorname{sen} 2$.

Mell u. a., Steir. Taid 246, 31 (m/soobd., 1638): In diser weinqüerlei [...] haben zu lösen₹eit die lösmaister [...] ir unterkomen und werden darin alle sachen zu dem lösen aufgebalten. 
2. >ausgebildeter Mönch im Kloster, der die Novizen in den heiligen Schriften unterrichtet, Klosterlehrer, Theologe «; vgl. lesen 3; 4. - Beleghäufung für Texte der Sinnwelt ,Religion', auch Chroniken. Bdv.: ${ }^{2}$ lerer $1 ; 2$, professor.

Luther, WA 6, 291, 30 (1520): Solch ungeschickte ding (die Vernunft [...] dem göttlichen Gesetz angleichen wollen) solt ein lesemeyster vorbyn betracht haben, unnd gotliche werck [...] mit der schrifft [...] beweren. Fischer, Brun v. Schoneb. 12462 (md., Hs. um 1400): i₹ was Heinrich von Huxere, | ein barvuzbruder und predigere | und lesemeister zu Meideburg. Strauch, Par. anime int. 3, 4 (thür., 14. Jh.): in disir predigade lerit brudir Eckart Rube der lesemeister wi di inphabunge unsis herrin lichamin ist zveigirleige. VetTer, Pred. Taulers 196, 28 (els., 1359): die grossen pfaffen und die lesmeister die tispitierent weder bekentnisse merre und edeler si oder die minne. Kurrelmeyer, Dt. Bibel 6, 384, 15 (Straßb. 1466): was eúch schreibt essdras der briester der lesemeister der ee des herren. Warnock, Pred. Paulis 1, 1 (önalem., 1490/4): Dise nachgeschribnen, erlúchten lér hat uns geton der wirdig lesmaister herr Pauli, unser trúwer bichtvatter. SeEmüller, Chron. 95 Herrsch. 126, 25 (oobd., Hs. 1. H. 15. Jh.): Do auch der selb bischof dannoch was lesmaister des conventz ze Basel. Chron. Baier. StÄDte. Landsh. 296, 5 (moobd., 1450): wan es was ain Lesmaister zu den Predigern, der vast wider die Juden prediget. $\mathrm{UH}_{\mathrm{H}}$ LIRz, Qu. Wien 2, 3, 4337, 1 (moobd., 1470): Bruder Andre von Gaden, lesmaister der heiling Geschrifft, prior, und der convent gemain Prediger ordens [...]. - WYss, Limb. Chron. 4124, 56; Strauch, a. a. O. 6, 8; Sachs 6, 381, 1; Rieder, Gottesfr. 122, 35; Bolte, Pauli. Schimpf u. Ernst 1, 3; 181; Chron. Augsb. 6, 29, 4; Sсhmitt, Ordo rerum 97, 16; Alberus Yy iiv; Vorarlb. Wв. 2, 272. - Vgl. ferner s. v. barfusse, beischaft 2.

3. >Protokollant bei Gericht, Gerichtsschreiber $<$.

Barack, Zim. Chron. 3, 332, 1 (schwäb., M. 16. Jh.): wie er nun also diese wort gesagt, undersprücht im der ein lessmeister und protonotari, welche dann alle wort müssen protocolliren.

lesmesse, s. lesen 4.

lesrok, s. lesen 5.

lesschule, s. lesen 4.

lesstat, s. lesen 2.

lesstube, lesstul, s. lesen 5.

lestag, s. lesen 2.

lester, s. letzer.

lesung, die.

1. >Ernteertrag an Weintrauben; Weinernte<; zu lesen 2. - Bdv.: frucht, leset; vgl. 1 aust 2 , augst 2 , äher 2 .
Kurrelmeyer, Dt. Bibel 3, 445, 11 (Straßb. 1466): die weintrauben deiner erstlichen die samel nitt als ein lósung [Var. 1475²-1480: leset; 1483-1518: weynlesen; LUTHER 1545, 3. Mose 25, 5: soltu nit lesen]. - EbD. 6, 329, 7; Bremer, Voc. opt. 56038.

2. >interessiertes, andächtiges, prüfendes Rezipieren, stilles Lesen von Texten; genaues Studium eines Textes <; als Metonymie: >im Lesen erworbene Erkenntnis`; vgl. lesen 3; 4.

Bechstein, M. v. Beheim. Evang. 2, 19 (osächs., 1343): Abir di krigeschen bûchere und di alden bûchere di nu vil von gewonheit latînischer lesunge unglìch trûgen, die [...].

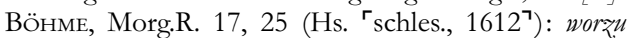
wir dir wie auch zu fleissiger lesung der Heiligen Schrifft treulich rathen. M. Cunitia. Ur. Prop. 147, 14 (Öls 1650): Nach dem ich von dem 5ten Jahr an / in dem ich allbereit lesen kónnen / durch stete lesung so Geist- so Weltlicher Historien [...] viel [...] erkand. WendeHORST, UB Marienkap. Würzb. 82, 17 (nobd., 1412): des alles zu einem gezugnisse unde warn urkunde diser besehung unde lesung. KuRRELMEYER, Dt. Bibel 6, 253, 11 Var. (Straßb. 1466): ich bit eúch hirumb: das ir eúch last genúgen an heimlicher letzigen [Var. Augsb. um 1475²-1518: lesunge] meins büchs. HEIDEGGER. Mythoscopia 43, 18 (Zürich 1698): da hab ich meine Fastnáchte mit Lesung deß Ciceronen zugebracht. RIEDER, St. Georg. Pred. 315, 20 (Hs. 「önalem., 1387`): daz. ist daz drit: lesung. daz. geschibt an drier hand buchen. Henisch 40 (Augsb. 1616): Alcoran / die lehre oder buch des Mabomets / Alcoranus / also genant von dem Arabischen Al / und Koran / lectio, lesung. Steer, K. v. Megenberg. Sel 435 (Hs. ' moobd., 1411`): intellectus, das ist gesprochen ein jnnrew lesung: jntelligere est intus legere, vnd ist die keraft, da sich die sel mit heft ïber alles, das ausser sinn begreiffen migen. - M. Cunitia. a. a. O. 148, 2; Hohmann, H. v. Langenstein. Untersch. 95, 68; Dasypodius 110r. - Vgl. ferner s. v. abschnit 4, atem 2, aufnemen 10, lesen 3.

leswagen, s. lesen 2.

leswerk, s. lesen 3.

letanei, die; zu mhd. letanîe (LeXer 1, 1890), dies über afrz. letanie letztlich aus griech. litaneia (KLuge/S., Et. Wb. 2002, 578).

>Litanei, mit dem Kyrie eleison beginnendes, aus der Anrufung von Heiligen und unterschiedlichen Bitten bestehendes, häufig in Prozessionen begegnendes Wechselgebet<. - Meist Wobd. / oobd. - Zur Sache: Lex. D. Mal. 5, 2010; RgG 1929, 3, 1670; Lthuк 6, 1075. - Bdv.: bitte 1, flehung, 
1 gebet 1, supplizierung. - Synt.: die l. singen (häufig) / anheben / beten; sich in den letaneien recht halten; mit der l. (um)gehen, bis zum ende bei der l. bleiben / verbarren; l. im kreuzgang; die schöne / wollautende l.

LUTHER, WA 2, 177, 37 (1519): Sollen wir [...] unß recht lalten yn [...] allen litanien. EвD. 30, 2, 250, 18 (1530): Cristlich Litaneien und gebet vor allerlei stennde vnnd not. EвD. 45, 321, 18 (1537): Da gehets in Capellen, Kirchen [...7: Salve Regina Und in der Litania: S. Maria, ora pro nobis. Chron. Strassb. 21, 14 (els., 1362): [Honorius] satte uf, daz, man alle sammestac gat mit der letanie zi sancte Peter. Krebs, Prot. Konst. Domkap. Protokoll 8319 (nalem., 1525): am dornstag, wann die sext gesungen ist, sollen die suben buspsalmen gesprochen vnnd daruff die letany gesungen werden. Roт 324 (Augsb. 1571): Letanei, Hertץliche / demuitige bit vnnd flebung / supplicierung / Jst ein Griechisch wort. Leidinger, V. Arnpeck 643, 33 (moobd., v. 1495): am montag sind sy umbgangen mit ainer process mit der lataney in irm kreuzgang. Moscouia D IIv, 33 (Wien 1557): So hebt der Diacon an die Lethaney erbarmb dich herr vnser. - Bernoulli, Basler Chron. 4, 4, 381, 30; Roder, Stadtr. Villingen 194, 16; Moscouia D IIIr, 24; A. À S. Clara. Deo Gratias 12, 25.

letare, Name.

>Lätare, Mittfasten, der vierte Sonntag der österlichen Bußzeit, benannt nach dem Introitusbeginn („Freue Dich“!) der Tagesmesse (relevant auch als Zinstermin)<.

WENDEHORST, UB Marienkap. Würzb. 53, 20 (nobd., 1390): die besitzere der selben miner zwey teile des hofs [...] sóllen alle jare îf den süntag zu mittervasten [...] antworten den vormuinden und pflegern der obgnant capellen dritthalb awelen lüters lynóls [...] und si sollen anbeben îf den süntag letare. Hauber, UB Heiligkr. 1, 639, 30 (schwäb., 1398): Dirr brieff ward geben in der vasten an dem sunnentag alz. man singet letare. - EBD. 2, 108, 6.

letfeige, s. lette.

lethargie, die; in beiden Belegen mit lat. Form; über lat. lethargia >Schlafsucht $<$ aus griech. lèthargia (KLuge/S., Et. Wb. 2002, 571; Schulz / Basler 2, 1542, 20).

>Lethargie, körperliche und seelische Erschlaffung, Untätigkeit als Folge von hauptkrankheiten

Sudhoff, Paracelsus 1, 83, 4 (um 1520): Der schlag hat in im etlich krankheiten, welche mit vil namen begriffen werden, als apoplexia, paralysis, lethargus. EвD. 9, 501, 1 (1531/ 5): das hauptwehe, hauptgeschwer, hauptgesucht und ander was bauptkrankheiten sind dermaßen auch entspringen, als paralysis, lethargia, tortura.

letner, s. letter. letsche, wohl die; formal wohl zu lattich. eine Pflanze, möglicherweise $>$ Klette . Alberus EE ijr (Frankf. 1540): Lappa personaria [...] est maxima, groß letschen / wañ sie stengel vnnd frücht bringt.

letschzaun, der; Bw Schreibform für latsche >Legföhre< (Dwв 6, 278).

,Zaun aus Kiefernholzı. - PFÄLz. WB. 4, 945 (a. 1565 f.)

letstein, s. lette.

lette, der; $-/-n$.

$>$ Lette, lehm-, tonartige Erde`, teils synonym mit ${ }^{2}$ leim und ton gebraucht, teils von diesen unterschieden; auch: >Schlamm, Dreck, Morastr. - Bdv.: drek, kot, ${ }^{2}$ leim, mergel, ton. - Synt.: den l. treten; ein l. sein; aus l. etw. brennen, etw. mit l. düngen / bedecken / pflästern, auf dem l. entwelen, in l. stehen; der blankiesige / weiche / weisse l. Wbg.: letfeige (Gw zu mhd. vige >Feige «, mit motivationeller Anspielung auf veige >furchtsam<; LEXER 3, 46; 345), lettechtig, let(ten)stein (wie leberstein).

Ziesemer, Proph. Cranc Jes. 64, 8 (preuß., M. 14. Jh.): unse vatir bistu, und wir syn ein let [WORMSER PrOPH. 1527: leym; LutHeR 1545: Thon] und du bist unse wirker. Lemmer, Amman/Sachs. Ständeb. 86, 3 (Frankf./M. 1568): Ein Ziegler thut man mich nennen / | Auß Láttn kan ich Ziegel brennen. HüBNER, Buch Daniel 1748 (omd., Hs. 14./A. 15. Jh.): Dir ertriche ist lette. ERmisch u. a., Haush. Vorw. 64, 31 (osächs., 1570/7): Wo nicht viel mist ist, alda tünge die sandichten ecker mit letten, die kalten mit leime, den lättigten gibe sand. EBD. 195, 39: Das die fische, [...] frischer werden dann zuvorn. Nim than und letten, thue gerstenmehl mit honig vormischet unter den leim. Wutke, Schles. Bergb., Cod. Sil. 21, 177, 13 (schles., 1625): Es sind auch sauerbrunnen und blaukiesige letten, die ein alaunbergwerck verkundschafften. KURRELMEYER, Dt. Bibel 7, 207, 13 (Straßb. 1466): Sy entwelten [...] in den bólern der erd oder auf den letten [LuTHER 1545, Hiob 30, 6: steinritzen]. EBD. 9, 9, 17: Ob du dich weschtest mit letten [Luther 1545, Jer. 2, 22: Laugen] [...7: du bist fleckhafftig vor mir. DAsYPODIUS 371v (Straßb. 1536): Lettechtig. Lutosus. [...] mit Letten odder Leymen bedecken. Delutare. die scheür mit Lett oder leymen pflástern. SudHOFF, Paracelsus 2, 293, 25 (1525/6): in den weg, wie obstêt, geschehen die resolution der lettsteinen, das ist der lebersteinen, der alabastern [...], so ein solchs genus an tag kompt und einfleußt, so entpfint es die art der erden. als dan hengt es sich an und macht ein misgewechs, gleich dem alabaster in etlicher gestalt, oder gleich den lebersteinen, und laßt das lauter wasser hindan fließen mit farben und form. wie dan die selbigen lettenstein sind an in 
selbs. MAALER 261r (Zürich 1561): Låttåchtig / Kaatig. Lutosus, Limosus. Argillatus. Láttåchtig oder Klipperåchtig ort. [...] Láttáchtiger grund. A. À S. Clara. Glori 31, 15 (Wien 1680): es muß unser lieber HErr den Feigen nicht wohl geneigt seyn / wenigst weiß ich / solche Feigen die Er hasset und verwirfft / diese seynd die Letfeigen / die jenige / so sich nicht trauen um Christi willen etwas zu leyden. - НüвNER, a. a. O. 1770; Löscher, Erzgeb. Bergr. 182, 23; Rohland, Schäden 465; Primisser, Suchenwirt 4, 478; A. À S. Clara. Glori 31, 17; Rechn. Kronstadt 2, 77, 44; Bremer, Voc. opt. 13025; Sсhмitt, Ordo rerum 30, 4; Voc. Teut.-Lat. t vir; Schöpper 77b; MaAler 261r; Rot 324; Golius 34; Pfälz. Wв. 4, 943; SchweIz. ID. 3, 1488/9. - Vgl. ferner s. v. anbeten 2, aufspalten 1, aufbrechen 12 .

lettechtig, lettenstein, s. lette.

\section{lettener, der.}

>auf dem ${ }^{1}$ letter Vortragender $\varsigma$.

Koller, Ref. Siegmunds 147, 40 (Hs. 'Basel, um $\left.1440^{\urcorner}\right)$: man sol alle accoliten, lettener, ewangelier und priester umbsust wiben.

${ }^{1}$ letter, letner (letzteres seltener), der; über ahd. / mhd. lector aus mlat. lectionarium >Buch mit liturgischen Texten, lectorium Lesepult ‘ (KLuge/S. 2002, 571; LeXer 1, 1851; Dжв 6, 794).

>Lettner, Lesepult zum Verlesen des Evangeliums; (zumeist kunstvoll errichtete) Trennwand zwischen Laien und Klerikern`, in frnhd. Zeit entwickelt zu >offene Querbühne in Stifts- und Klosterkirchen zwischen Schiff und Chor; Emporkirche<; dem Lettner oblag auch die Bekanntgabe weltlicher Nachrichten. - Gehäuft Rechtsund Wirtschaftstexte. - Zur Sache: LEx. D. Mal. 5, 1914; Hrg 2, 1855; Rwb 8, 1240. - Bdv.: vgl. lector.

Reduich, Jül.-Berg. Kirchenp. 1, 61, 27 (rib., 1487): als der priester up dem letter stainde ind soulde predigen. ÖsTERLEY, Kirchhof. Wendunmuth 1, 531, 28 (Frankf. 1563): etliche [thumbherren] von hoher geburt, welche auff dem lättner zugebört hatten. FolTz, UB Friedb. 1, 363, 47 (Friedberg 1387): wan si dan ende wollen gebin, so soln di scheffene daz. tuin virkunden obir den letter. BEYER, UB Erfurt 2, 342, 32 (thür., 1354): das her [pherrer] der vorgenanten gedenke. ober den lettir. Fuchs, Murner. 4 Ketzer 2746 ( ${ }^{2}$ wohl Straßb. ' 1509): Das sye jn an eim seil hinab |Vom lettner ab gelossen hant. FucHs, Kart. Aggsbach 23, 19 (moobd., 1371): sol ein ieglicher pharrer begen einen ewigen jartag meiner hawsfrawn [...] und mit gedáchtnuzz auf dem letter. - Aubin, Weist. Köln/Brühl 114, 12; Wyss, UB
Deutschord. Hessen 192, 20; Bolte, Pauli. Schimpf u. Ernst 1, 204; Martin, H. v. Sachsenh. Tempel 703; Qu. Wien 2, 2, 3608, 25, UB ob DER ENNs 10, 240, 30. - Vgl. ferner s. v. accolit.

${ }^{2}$ letter, letterwechsel, s. litter.

lettich, wohl der.

wie lette. - Wbg.: lettichberg, lettichleim.

Kurz, Waldis. Esopus 4, 38, 52 (Frankf. 1557): So man euch [...] | Mit lättich leym die Lippen schmiert, | Bleibt drinn ligen derselbig dreck. Wutke, Schles. Bergb., Cod. Sil. 20, 85, 26 (schles., um 1450): do ist ein lettich bergk, do findistu och yn deme lettich sulch bley gedegin. - SCHMIDT, Hist. Wb. Elsaß 219.

lettichberg, lettichleim, s. lettich.

$$
\text { letticht, s. lettig. }
$$

lettig, letticht, Adj.

>aus Letten; lettenhaltig; wie Letter. Bdv.: kötig, schleimerig.

Ermisch u. a., Haush. Vorw. 64, 32 (osächs., 1570/7): Wo nicht viel mist ist, alda tünge die sandichten ecker mit letten, [...], den lättigten gibe sand. EBD. 99, 28: Ist das feld zehe oder lätticht, so muß man daßelbige mit sandichter erden vormischen. EBD. 161, 7: wo der bodem salzig, sumpficht, lettig oder bitter ist. SudHOFF, Paracelsus 13, 274, 10 (1530): welche stern in im haben die lettich art vom schwefel. Sснмiтt, Ordo rerum 26, 43 (oobd., 2. H. 15. Jh.): chötig oder lettig erd. - Kurrelmeyer, Dt. Bibel 3, 223, 9; Sudhoff, a. a. O. 4, 559, 2. - Vgl. ferner s. v. lette, lettich.

${ }^{1}$ letze, die; $-\emptyset /-\emptyset$; zu mhd. letze >Hinderung< (LeXer 1, 1891; vgl. auch Dwв 6, 797 f. und mit Zweifeln an der Einheit des Wortes: SchweIz. ID. 3, 1562); Bed. 7 semantisch auf lezt 1 , lezte bezogen.

1. >Befestigungsanlage generell<; im einzelnen: >Erdaufwurf; mit Mauerwerk verstärkter Wall; befestigte Stelle innerhalb einer Befestigungsanlage<; auch: >Tor im Gemeindehag‘; differenziertere Beschreibungen von Befestigungsanlagen im SchweIz. ID. 3, 1558, dort auch zusätzliche (metonymische) Bedeutungen verbucht: >Gebietsgrenze<; >von der Grenze umschlossenes Gebiet<; in 1 Beleg (s. u. BARACK) Tendenz zu > Wacht, Lauer $\measuredangle$ - Dichte Belegung für das Wmd. / Nobd. / Wobd.; gehäuft Rechts- und Wirtschaftstexte, auch berichtende Texte. - Bdv.: bastei, bolwerk, 
festung, (land)wer, mauer, turn, wache, were, zaun. - Synt.: die / eine l. aufwerfen / bauen / besetzen / bessern / brechen / gewinnen / machen, jm. die l. angewinnen; auf die l. kommen / ziehen, ausserhalb der l. liegen, inner der l. sitzen, in die l. brechen, an der l. schalmutzen, sich auf die l. verfügen, jn. auf die l. (ver)ordnen, in einer l. gesessen sein, zu den l. senden, in die l. zu den waffen treten; die l. zu Appenzel; die alte / gemauerte / gute / starke / wüste l. Wbg.: letzelen seine Verschanzung anbringen (a. 1468), lezgeselle.

Meisen, Wierstr. Hist. Nuys 911 (Köln 1476): In allen letzen dorch dye stat $\mid$ Al malsch dayr zo den wapen trat. Rudolph, Qu. Trier 100, 12 (mosfrk., 1593/94): Es sollen alle letzgesellen einem scbützenmeister [...] nacbkommen, sie fleißig anmabnen, ibre letzen oder orther, [...] besuchen. SchмidT, Frankf. Zunfturk. 1, 45, 25 (hess., 1595): Wurde [...] ein ufflauff in oder vor der statt, [...] so soll ein ider alßdann uf die lets, dabin er verordnet, sich alßbaldt verfügen. ЕвD. 1, 321, 35 (1478): die jhenen, die an die porten uff thorne und letze z $u$ kommen bescheyden sint. GILLE u. a., M. Beheim 453, 842 (nobd., 2. H. 15. Jh.): Dar nach fürt er sy auff die türn | und die maür die zeschossen würn, | Und zaigt in all die leczen, $\mid$ wie er die waz beseczen. BaumanN, Bauernkr. Rotenb. 491, 26 (nobd., 1525): damit die burger gemustert, und alle pastey und letz. werden besetżt. SCHotTenloher, Flugschrr. 82, 15 ([Würzb.], 1523): Eyn fuß volgke er auch mit im nam, | Mit welchem uberzog er Met\%, | Dem ließ er vast eyn wuste letz. BARACK, Teufels Netz 3408 (Bodenseegeb., 1. H. 15. Jh.): ich [Teufel] lig allzit an der letz. | Und warten mit minem net: Roder, Hugs Vill. Chron. 20, 10 (önalem., 1503): Und zugend fir ain letze, da lagend etlich Frantzoßen an. CHRON. StRAsSB. 692, 14 (els., A. 15. Jh.): so zogetent alle antwerg für das münster geveffent und ein teil uf die muren und an die letzen. Argovia 4, 104, 29 (halem., 1590): dasz man nun binfüro von der trotten by der gmeind reben, ein lezi oder thor mache. Graf-Fuchs, Ämter Interl./Unterseen 60, 15 (halem., 1349): daz aber wir wider die Waltlúte súllen werinen und letzinen machen. RenNeFahrT, Stadtr. Bern 248, 23 (halem., 1387): und sullent öch die selben stett [...] inrent iren slossen und letzinen ane allen dienst beliben. HaLtaus, Liederb. Hätzlerin 1, 14, 12 (schwäb., 1471): Gang [wacbter] pald binwider an die let?. $\mid$ Vnd hab die rie dein. Chron. Augsi. 5, 79, Anm. 1 (schwäb., 1523/7): man spilt nun offenlich auff der gassen, auf den bencklin vor den heussern oder in den lezen. Baumann, Bauernkr. Oberschw. 38, 26 (schwäb., v. 1542): Es was ach ain groß, mercklich folck versamlet, burguß und Etzlent an der letzin bey Mals im tal, [...], hetten sy die burguschen daselbst an der letzin ein leger gemacbt. Fichtner, Füetrer. Trojanerkr. 532, 5 (moobd., 1473/8): Hiemit dy Chriechen fueren aus der letze | für Thenadon bindter den wald. - MeIsen, a. a. O. 150; V. Anshelm. Berner Chron. 1, 373, 18; 4, 72, 4; GeIer, Stadtr. Überl. 79, 31; MülLER, Alte Landsch. St. Gallen 264, 34; Dierauer, Chron. Zürich 66, 12; Tobler, Schilling. Bern. Chron. 1, 33, 30; LuginbüHL, Brennwalds Schweizer Chron. 1, 284, 5; 286, 1; Diehl, Dreytw. Essl. Chron. 44, 14; Roder, a. а. O. 9, 10; Rwв 8, 1241; PғÄLz. Wв. 4, 947; VorARLb. Wв. 2, 273; SChWeIZ. ID. 3, 1556; 1558; Sсншёв. Wв. 4, 1195.

2. >Verletzung, Zufügung von (meist:) körperlichem oder (seltener:) psychischem Schmerz<; teils mit gen. explicativus; vgl. letzen 1; 2. - Verstexte. - Bdv.: vgl. beinschrot, beschädigung 2, beleidigung 2, beschämnis, geserde, injurie, lämde 1, läme, loch 8, wunde.

Bergmann, Ambr. Liederb. 36, 14 (Frankf. 1582): fürwar mit nicht, $\mid$ ich von dir sets, mit keiner lets, $\mid$ freundlich dich sicher alles leids erget:. Gille u. a., M. Beheim 140, 133 (nobd., 2. H. 15. Jh.): tut in we, | dar gat hat solcher lecze | Gewalt, daz er sy allso slebt. EBD. 447, 58: wanne | er [Nero] must leiden des tades lec₹: Primisser, Suchenwirt 7, 155 (oobd., 2. H. 14. Jh.): Dem hat der tót versniten Sein hertz mit swinder letze. KLEIN, Oswald 36, 7 (oobd., 1426): So hat ir [weib] lets. mit scharpfer wetz. und sneller betz. I mein hail auf erd erloffen.

3. >Abschiedsmahl, -essen, -trinken<; metonymisch: >Speise, Getränke eines Mahles «, bei Luther mit Bezug auf das sakramentstiftende Abendmahl Christi mit seinen Jüngern; vgl. letzen 3. - Bdv.: vgl. ausschenk. - Wbg.: letzemal (verdeutlichend; a. 1650), leapredig, leaschiessete (a. 1616).

LutHer, WA 28, 510, 1 (1529): non est aliud quam ein letzpredige, damit er gesegnet hat [...]. ЕвD. 41, 41, 2 (1535): audivimus die libliche, schone rede in caena, da er die letze cum suis discipulis bat gehalten. EвD. 46, 268, 22 (1538): Also stehet nu Christus unnd ißet die letze. BucH WeInsB. 3, 290, 23 (rib., 1585): Den mittag drunken sie die letz im Kleinen Pallast. EBD. 5, 480, 18 (rib., um 1560): So emantz verreist, drinkt oder schenket der nit die letz? BOLTE, Pauli. Schimpf u. Ernst 1, 13, 7 (Straßb. 1522): da wolt er die Letz, mit ir essen, und lind sie. ScHIB, H. Stockar 108, 10 (halem., 1520/9): Wier beschürmtend im sin scblesslin und den win und bielt uns erlich mit sim volk. mit der letzsin. - Sachs 17, 247, 32; Goedeke, Fischart. Schiff 975; Dict. Germ.-Gall.-Lat. 304, 40; 395, 1; Schweiz. ID. 4,$160 ; 8,1447$.

4. >Abschied 〈; dazu metonymisch mit offenem Übergang: >Abschiedsgeschenk, Gabe; Trinkgeld; gütige Hinterlassenschaft (dies letztere mehrfach auf heilsgeschichtliche Tatbestände, wie den Hl. Geist, die 
Sakramente, bezogen)<; auch: >Einkommen e. P.«. - Bdv.: vgl. abdank, abdankgeld, abdankung 1; 2, abscheid 1, abschied 1, liebnis 1; 3, liebtat. - Synt.: die / eine l. (aus)teilen / nemen / baben / lassen / verzeren, jm. eine l. (binter)lassen / geben; etw. zur l. tun, jm. etw. zu(r) l. aufsetzen / geben / beiligen (sein blut) / lassen / schenken / senden; die erliche / gemeine l. Wbg.: letregeld >Trinkgeld beim Abschied<, letzepfennig (a. 1374), letzepredig (a. 1637), letzeschrift (um 1640), letzesteuer (a. 1651; zum Gw vgl. steuer 4; 5; a. 1651), letzetrab, wohl >letzter Weg, Gang`(a. 1593; Gw nicht völlig klar; s. SCHweIz. ID. 14, 52).

Luther, WA 2, 685, 12 (1519): diß [todt] ist eyn [...] eußerlicher abschided von dißer welt, und wirt urlaub und letze geben dem gut. EBD. 30, 3, 279, 25 (1531): Und wóllen also zur letze ein walfertlin mit einander thun. EBD. 45, 467, 17 (1537): als der [Heiland] von jnen [Jüngern] scheidet, zur letze gibt, der gleichen nimer kein Mensch [...] gegen seinen liebsten und besten freunden tun und redenkan. $\mathrm{BuCH}$ WeINSB. 1, 302, 32 (rib., um 1560): hat schon ein doctor etwas mehe [als der licentiat] gerechtigkeit, das er ein let?. haben mag, das solte $z$ dur stain. SсHмiDT, Frankf. Zunfturk. 1, 264, 22 (hess., 1407): die [maß] sal der knecht von dem letzegelt betzalen, ließe der geselle aber keyne letzegelt, so sollen iß die gesellen gemeynlich betzalen. BERGmanN, Ambr. Liederb. 118, 60 (Frankf. 1582): Aus schwererpein, das hertze mein, | der liebsten allein, | schenk ich das liedlein zur letzen. Schмiтz, Schiltb. 143, 23 (Frankf. 1597): Nach dem nun der Keyser hinweg gewesen / und den Schildischen ein gute Letze binderlassen hette. GOLDAMmER, Paracelsus. B. d. Erk. 42, 7 (obd., Hs. n. 1570): darvf er [Cristus] jnen zu ainer letzen geben hat denn hailigen gaist. MAYER, Folz. Meisterl. 5, 25 (nobd., v. 1496): hat nit Cristus ym abscheiden $\mid$ Unß geben zu der lecz die siben sacrament? ЕвD. 85, 40 (1517/8): die pschneidung, dar in der zart | Iesüs gab die lecze | Der alten ee. Rupprich, Dürer 1, 156, 114 (nobd., 1520): Ein stüber hab ich des wirths knecht zu lecz geben. Rieder, Gottesfr. 97, 15 (els., 1380): ich hatte dir me zu letze gesant, wenne daz mich dise vart gar vaste entblosset het. Pfeiffer-BeLli, Murner im Glaubensk. I, 2, 16, 5 (Luzern 1526): do gab ich kein letz. vnd scheiß ins bet. Sснів, H. Stockar 129, 21 (halem., 1520/9): kost mich der ritt 3 gl, verzert und lietzgelt gen, als mian mir schank. JöRG, Salat. Reformationschr. 866, 17 (halem., 1534/5): ling noch eynmal zu letzj (herr vergib uns unser sünd) unserm herr gott jns füdloch. Dict. GERM.Gall.-Lat. 304 (Genf 1636): letz. / f. abscheydt. Drescher, Hartlieb. Caes. 205, 20 (moobd., 1456/ 67): das an Gots leichnam tag er [...] sein heyligs plìt uns aufseczt und beyligt zu einer lecz. und seiner gedächtnüsz: KLEIN, Oswald 85, 8 (oobd., 1423): banzer und armbrost, darzu die eisenbüt, | die liessens uns zu letze. - Lu-
THER, WA 21, 372, 31; 47, 311, 36; Wyss, Limb. Chron. 58, 5; v. Liliencron, Dür. Chron. Rothe 449, 3; Thiele, Minner. II, 4, 55; Lindqvist, K. v. Helmsd. 432; Bernoulli, Basler Chron. 4, 97, 13; 6, 407, 7; Schade, Sat. u. Pasqu. 3, 4, 23; LuginbüHl, Brennwalds Schweizer Chron. 2, 302, 5; 423, 31; 306, Var.; Drescher, a. a. O. 148, 8; Niewöhner, Teichner 476, 29; Rwi 8, 1241; Martin/Lienhart 1, 216; Schmidt, Hist. Wb. Elsaß 220; Schwёв. Wв. 4, 1196; SchweIz. ID. 3, $1560 \mathrm{f}$; 5, 403; 1125; 9, 1589; 11, 1338.

5. >übles Andenken, üble Hinterlassenschaft, Schaden «. - Obd.

Roloff, Brant. Tsp. 2156 (Straßb. 1554): Dein [Salomol ebre hastu wuist thün vermosen $\mid$ Deim somen ein bóß letze gelossen. Adomatis u. a., J. Murer. Nab. 1263 (Mühlh. 1556): So wil ich dir [...] Gwüßlich ein thüre letze bringen. Goedeke, Fischart Flöh Haz, 1679 (Straßb. 1594): Dem liß aus rachgir ich ain letr, | Und gab im in die seit ain pfetz. SAPPLER, H. Kaufringer 1, 112 (schwäb., Hs. 1464): du hast ain böse letz. [mord] gelaun. Chron. Augsi. 5, 272, 1 (schwäb., 1495): allweg ließ es [kranckbait] im ain letzi. Barack, Zim. Chron. 4, 82, 33 (schwäb., M. 16. Jh.): Es haben die spannischen rauden im ein soliche letzin gelasen, das er dessen doch letstlichen sterben müesen. SpILlER, Füetrer. Bay. Chron. 178, 25 (moobd., 1478/81): umb alle die eer, [...7, was sy geflissen, das sy im zu letz. wolt lassen ain getranck des grymmigen tods. - SACHS 13, 437, 11; 17, 242, 1; Adomatis u. a., J. Murer. Abs. 1748; Sсншёв. Wв. 4, 1196.

\section{6. >Zeichen, Andenken .}

Karsten, Md. Paraphr. Hiob 3, 163, 103 (omd., 1338): dienyl ich Thomas] dich [Maria] doch nit gsehen verscheiden, so bitt ich dich doch vmb ein letzin so wirfft sy ime iren gürtel berab.

7. \Abschluß, Schluß, Ende<; auch: >Überbleibsel, letzter Restく. - Phras. : auf die letze, zur letze >zuletzt, zu guter Letzt<. Bdv.: vgl. ausgang 13, beschlies 2, grund 13.

Luther, WA 22, 193, 7 (1544): Des hat er dieses tages, [...], das vorspiel angefangen, eben zur letze vor seinem abscheid. EBD. 22, 194, 8: da er [...] in Tempel gehet und zur letze noch leret bis auff den tag seines leidens. EBD. 30, 2, 144, 2 (1529): hatten die Juden schier nichts mehr von yhrem Kónigreich, Herodes war die letze. Chron. Nürnb. 2, 311, 15 (nobd., 1449): auf die letz. kauft der rat als vihe. $\mathrm{zU}$ DoHNA u.a., Staupitz/Scheurl 185 (Nürnb. 1517): wann sie sich befleisen [...] fern zu sein von den zuneigungen zu sünden, das ist von der letze [reliquiae] der sünde. SACHS 21, 548, 24 (Nürnb. 1552): Auch möcht mir an der lez. abgon | An profant und municion. BeLL, G. Hager 7, 2, 15 (nobd., 1592): auf das er solcher mas $\mid$ Die in dem necz. | waren im gsecz | er lösete da an der lecæ. Chron. AugsB. 7, 425, 2 (schwäb., zu 1564): well er [Sathan] zur letz. wunder anrichten und das größt übel stiften. - SACHS 15, 
352, 34; 18, 486, 21; 20, 291, 21; Sсншёв. Wв. 4, 1195.

${ }^{2}$ letze, letzie, letzige, lezge, die, $-n /-$; zu mhd. lëcze > Vorlesung <, dies aus lat. lectio (LEXER 1, 1852).

1. >Bibelabschnitt〈; dazu als Metonymie: >Lesung oder gesungener Vortrag eines Bibeltextes im Gottesdienst, bei der Totenmesse, der Vigil, im Klosterleben, bei der Mahlzeit (jeweils innerhalb christlicher Riten)<; oft im Orientierungsfeld von ${ }^{1}$ bet 1 , epistel, evangelium, gesang 1 , lere 5 , predigat 1; 2. - Meist Texte der Sinnwelt ,Religion / Didaxe. - Synt.: die l. anheben / begehen / balten / lesen / singen (mehrfach) / bören, jm. die l. sagen; die l. weitschweif sein, die geschrift inhalten; der letzen nicht verstehen; grosses volk bei der l. haben, in der l. geschrieben stehen, an die l. gehen; die l. des evangeliums, der alten e; die heilige / tägliche l.; fleis der letzen; vigilie mit 9 letzen.

Quint, Eckharts Pred. 2, 6, 2 (E. 13./A. 14. Jh.): [ein wörtelin] daz stât geschriben in der leczien, die man biute liset. Sievers, Oxf. Benedictinerr. 21, 4; 12 (hess., 14. Jh.): Diz ist die letze von ezzene. [...] Dan sal da grozze stille sin zu dische, daz [...] ockert die letze gehort werde. Neumann, Rothe. Keuschh. 2187 (thür., 1. H. 15. Jh.): di letzie unnd di predigate ist in swer, $\mid$ si horten vil liber ander mer. Mon. Bolca, NF. 1, 574, 36 (nobd., 1414): ein vigilie singen mit newn leczen mit einem erberen leichczeichen und vier kerczen. Kurrelmeyer, Dt. Bibel 2, 116, 11 (Straßb. 1466): das selb decksal in der lece (Var. 1470; $1475^{1}-1478$ : lere] der alten ee beleibt es vngeoffenbart. CHron. AugsB. 4 , 177, 7 (schwäb., v. 1536): Man bat zü den Carmeliten [...] offenlich wie auff der hochen schul die evangelia und epistel Pauli gelesen, und sind vil frauen und mann an dise letzgen gangen. RuH, Bonaventura 324, 16 (oschwäb., 2. V. 15. Jh.): Die letzig ist weitswaiff, [...], wann die letzig des dryuachen wegs die halt inn die gantz, hailgen geschrifft. Drescher, Hartlieb. Caes. 68, 26 (moobd., 1456/67): ward er von seinem preial gefragt, [...] wes er ob dem tisch gedacht, die weil [...] er der leczen nicht verstind. EBD. 134, 23: do die leczen des heyligen ewangely angehaben was, do flog die selbe taube aber von dem chreücz, auff seinen pfeyler. EBD. 247, 30: des geleichen sagt er in von den vigilien, welch münch und was leczen oder was responsz. sy gesungen hetten. BuissSEN, Dur. Rat. 10, 12 (moobd., 1384): der wort sind drey underschayden: pet, gesang, lezczen. - CHRON. KöLN 2, 180, 2; Wendehorst, UB Marienkap. Würzb. 326, 35; Kurrelmeyer, a. a. O. 3, 8, 1; Adrian, Saelden Hort 7252; Jaspers, St. v. Landskron 52r, 6; Hauber, UB Heiligkr. 1, 568, 20; Sexauer, Schrr. in Kart. 265, 5;
Hohmann, H. v. Langenstein. Untersch. 95, 72 Vogel, Urk. Heiliggeistsp. 1, 379, 25; Reithmeier, B. v. Chiemsee 94, 9; Voc. Inc. TeUt. o viijv; SChweIz. ID. 3, 1572.

2. >Lehre, belehrende Aussage; Lektion ‘; auch personifiziert; mit negativen Nuancen Teil von Phrasemen; ansatzweise Lösung aus christlichem Kontext; als Metonymie zu 1 auffaßbar. - Gehäuft Texte der Sinnwelt ,Religion', auch der Didaxe. Phras.: jm. die letze (auch: leviten) lesen a) >jm. Unangenehmes sagen $<$; b) >in. abrichten $<$. Bdv.: vgl. beischaft 2, beispiel 3, bericht 2, berichtung 1 , confession, ${ }^{1}$ glaube $1 ; 5 ; 6,{ }^{2}$ lere 4 ; 5, (-)predigt. - Synt.: die l. lesen / kennen / hören, jm. eine l. geben; l. geheissen sein, die l. etw. bezeichnen; jm. in der l. etw. sagen; l. der otmutigkeit; die böcbste / nützeste l.

LutHer, WA 34, 1, 118, 3 (1531): da [am rbimen ... Christum Jesum) bette einer ein letzen dran. QuINT, Eckharts Pred. 1, 241, 1 (E. 13./A. 14. Jh.): Dat wir van jhms xp̃s de letze der oitmodycheit leren. Altmann, Wind. Denkw. 280, 26 (wmd., um 1440): die Swebeschen meigede die sint einfaltig gewesen, | nü hat man also die letzen in wol gelesen, | das sie die kunste triben recht. THIELE, Minner. II, 31, 514 (Hs. ' ${ }^{\mathrm{md}}$./rhein., 1. V. 15. Jh. ${ }^{\top}$ ): spade und vru | moessent si in erbeide wesen | und ir lectze over lesen. MEIJвоом, Pilgerf. träum. Mönch 969 (rib., 1444): So sagen ich uch in deser letzen: $\mid$ Mit der werelde en soelt ir uch neit ergetzen. BöMER, Pilgerf. träum. Mönch 13170 (rhfrk., um 1405): Sij erfullet das hertze und nit den buch | Mit yrer guder süßer spisen genuch. | Sij ist geheissen die Letze | Und studieret die gesetze | Und dar zu auch die heilige schrifft. Williams u. a., Els. Leg. Aurea 303, 6 (els., 1362): vnd ist bute mine lecze, daz ich sol globen an got. RIEDER, Gottesfr. 21, 17 (els., 1382/91): eine letze, [...] wie er leren solte alle untugent úber winden. Ders., St. Georg. Pred. 252, 9 (Hs. 'Önalem., 1387?): Dú fúnfte letage ist gútú zubt. Strauch, Schürebrand 48, 13 (els., E. 14. Jh.): ich bitte úch, brudere, daz ir dise letze [...] mit wackerbeit der sinne alle zit bórent. BARACK, Teufels Netz 10476 (Bodenseegeb., 1. H. 15. Jh.): Da wil ich dir denn ain letzgen [Var. C: leviten] lesen, | Das si niemer me mag genesen. BaChmann, Haimonsk. 144, 13 (halem., 1530): Her münch, könnend ir üwere lätžgen wol ins tǔffels nammen? BAuER, Imitatio Haller 71, 22 (tir., 1466): das ist die böchist und nüczist lëczen, da der mensch sich selbs recht erkchent vnd sich diemuetigen ist. - ThIELE, a. a. O. II, 31, 279; 31, 578; Bömer, a. a. O. 13188; BARAck, a. a. O. 3182; Rieder, a. a. O. 9, 16.

3. `akademische Vorlesung «; als Metonymien: >Stoff der Vorlesung $<;>$ Lehrstuhl $<$; >Unterricht «; >Schulklasse «. - Bdv.: vgl. colle- 
gium 2, lection 1, lectur. - Synt.: die l. erlangen / balten / bören / lesen / repetieren / schreiben / disputieren / einziehen; in die l. gehen, um die l. sollicitieren, jn. in die l. setzen; das erklären / läutern der letzen; die deutsche l. Wbg.: letzenzeit.

Buch WeinsB. 1, 66, 4 (rib., um 1560): [ich] wolt nit mehe under der letzenzit spaceren gain. ЕвD. 4, 266, 40 (1596): als d. Reck, ein raitzman zur zit, und lic. Hulss, syndicus eins raits, umb die ordinari(e) lectur und letze in iure, so [...] vaceirte, zu beiden seiten beftich solliciteirt [...], sie ₹̌ erlangen. EвD. 5, 277, 12 (rib., 1586): Disse gab uns die oberste camer an der straissen zu, da mir unse letz, repiteirten, laissen, schriben, disputeirten. GERHARD, Hist. alde e 4787 (omd., um 1340): $Z u$ der zit Aristotiles, $|[. .]$.$| Horte$ PLatonis letzen. SudHoff, Paracelsus 4, 144, 10 (1527): sie baben dadurch sie, die meinen so in mein lezgen gont zu doctoriren binder triben. Bolte, Pauli. Schimpf u. Ernst 1, 191, 31 (Straßb. 1522): ein Nobilist, ,... ., der hort Letzgen von demselben Doctor. MAaler 269r (Zürich 1561): Der glertisch sein in der Letzgen. Ducere classem. Ein yetzliches kind oder schuiler in sein Letzigen setzen. ЕвD. 364r: Disputation oder gespräch und letzgen die man in der scbúlen baltet. Müller, Nördl. Stadtr. 513, 9 (schwäb., Hs. 14./ A. 15. Jh.): in der andern letzigen soll er haben ain baccularium oder sunst ain verstendtlichen gesellen, desgelichen in den andern letggen, die die schüler tugentlich und vleisslich underweisen. - Chron. Köln 3, 876, 15; Buch Weinsb. 5, 180, 16; Bernoulli, Basler Chron. 5, 466, 20; SChweIz. ID. 3, 1572/3.

4. >Schulklasseく. - SchweIz. ID. 3, 1573 (a. 1561).

${ }^{3}$ letze, s. lez 2.

letzegeld, s. ${ }^{1}$ letze 4.

letzelen, s. ${ }^{1}$ letze 1.

letzeltiat, der.

Spielform zu lizentiat.

SCHade, Sat. u. Pasqu. 2, 145, 30 (els. 1521): es stat manicher ölgötz auf die kanzel [...] und wellent dannocht etlich Lezeltiat haißen.

letzemal, s. ${ }^{1}$ letze 3.

letzen, $\mathrm{V}$.

1. >die Ausführung von etw. behindern, hemmen; js. Handlungen oder Gefühle beeinträchtigen; jn. an etw. hindern, stören, sich jm. entgegenstellen; jn. täuschen; sich e. S. (z. B. einem gebot) entgegenstellen; etw. zu Ende bringen`; speziell: >jn. gefangen nehmen<; refl.: >sich trüben (von der
Freude)<; sich heben (von einer Stimmung); offen zu 2. - Gewisse Beleghäufung für Texte der Sinnwelt ,Religion / Didaxe', auch Chroniken. - Bdv.: abhalten 1, bedrangen 1; 2, irren 2, bekümmern 2, leidigen 2; vgl. abbindern, stören 2. Ggs.: ergetzen. - Synt.: die freude sich l.; etw. (z. B. den hof, die liebe / trene, das gebot / gesez. / trauren) l., jn. (z. B. mit worten, an treuen, an dem bund, an seinem sitze) l., jn. l., das [...], der sin, die (ehafte) not jn. l., die werke die engel l., die furcht gottes die hochfart l.; subst.: seinen mut sonder letzen volbringen. Wbg.: letzenis >Hinderung<(dazu bdv.: vgl. letzung), letzer.

KOCHENDÖRFFER, Tilo v. Kulm 5757 (preuß., 1331): Di vorchtte Gotes leczet | Di hochvart. Leman, Kulm. Recht 2, 5, 43 (Thorn 1584): Kumet yener der noch des is do ist und beret das yn eheaste [sic für ehaft] not abe gelettrit der richter sal ym syn teil wedir geben (Belegschreibung auch als abletzen lemmatisierbar). Fischer, Brun v. Schoneb. 7528 (md., Hs. um 1400): ab mich min sin nicht letzet (〉täuscht, im Stich läßt ‘), | so vloz u乏, sime libe saf. Quint, Eckharts Pred. 1, 200, 6 (E. 13./A. 14. Jh.): ob sie [engel] ibt geletzet werden von den werken, daz sie unser büetent. Меijвоом, Pilgerf. träum. Mönch 5151 (rib., 1444): So dat en wenich letzenisse | Mir brecbte groisse hyndernisse. SChüTZeichel, Mrhein. Passionssp. 157 (mrhein., um 1335): Icb hade ez allen minen sin $\mid$ mit flize dar vf geseczet, | wie ich hede geleczet | bit hoffart und mit frazheit $\mid$ daz. [...]. HüBner, Buch Daniel 7005 (omd., Hs. 14./A. 15. Jh.): Er [norden kung] volbrenget sinen mut | Volleclich sundir letzen, | Sich da widir setzen | Niemant tar. Goedeke u. a., Liederb. 333, 104 (о. O. o. J.): der ar begrifs; do wart sich ir frend lezen. GILle u. a., M. Beheim 234, 139 (nobd., 2. H. 15. Jh.): davon sol ein iglicher crist $\mid$ dis petrieger und checzer $\mid$ Und die verkerten leczer $\mid$ vermeiden und in wider stann. v. KelLer, Ayrer. Dramen 19, 26 (Nürnb. 1610/8): [Eur gnad] Soll keinen zweiffel an uns setzn, $\mid$ Daß eur gebot wir solten letzn. MatTHAEI, Minner. I, 12, 273 (Hs. ' wobd., 15. Jh. ${ }^{7}$ ): do man mit unstátem müt | lieb und trúw wolt letzen. THIELE, Minner. II, 6, 153 (Hs. 'ndalem./sfrk., 1470/90?): wan ich mynen bulen an sich $|[\ldots]|$ so wirt myn drurn geleczt $\mid$ zu band er mich ergeczt $\mid$ unmutes. Chron. Strassb. 38, 7 (els., 1362): der wolt den bof zu Frankefurt letzen und irren. LEMMER, Brant. Narrensch. 110a, 18 (Basel 1494): die sich zu disch dint setzen | Vnd andere an dem sytzen letzen $\mid$ Die vor jn soltten syn gesessen. АDомatis u. a., J. Murer. Bab. 406 (Zürich 1560): Vil minder uns der Cyrus letzt $\mid$ der gágen uns ein flieg wirt gschátzt. HaLtaus, Liederb. Hätzlerin 2, 35, 29 (schwäb., 1471): Mein trauren tuist du letzen IVnd pringst mir fräden. BARACK, Teufels Netz 36 (Bodenseegeb., 1. H. 15. Jh.): der tiifel die welt mit striken hat überlait. [...], Wer mag den striken allen usgan?" $\mid[.$. 
,Ain recht diemütig man; | Der zeit im durch sin netz, | Da ain andra inn wirt geletzt. EBD. 42: wir hangend in sinem netr. Und werdend allsament geletst. EвD. 7413: Die rechten ler und gesetżt $\mid$ Werdent ietz von küngen geletzt. CHRON. Augsi. 3, 366, 7 (schwäb., E. 15./A. 16. Jh.): wer der rapp nit worden geletst, | die vogthey het er auch besetzt. Leman, a. a. O. 2, 5, 63; Haltaus, a. a. O. 1, 47, 38; Rwв 8, 1242; Schles. Wв. 2, 808.

2. >jn. physisch oder psychisch verletzen, jm. körperlichen oder finanziellen Schaden zufügen; jn. e. S. berauben, an der Ehre kränken; (ein Tier) verletzen; jn. töten`; speziell (mit Bezug auf Sachen): retw. schädigen, zerbrechen, vernichten «. Phras.: jm. das leben letzen. - Bdv.: s. u. SCHÖPPER, ferner: bestricken 1, kränken, kratzen, leichen 2, reissen, schädigen, schlagen, schmähen, schmälen, verderben, verseren, verwunden; vgl. angreifen $10 ; 11 ; 14 ; 15$, bekrenken $1 ; 3$, bekümmern $4,{ }^{1}$ beschweren $1 ; 3-4 ; 7$, betrüben $2 ; 4$, diemütigen, freidigen, leidigen 1 , lützen, uneren, verkleinen. - Synt.: etw. (z. B. ein gut / geschïr, eine bürgschaft, js. ere, den leumund / stam, ein pferd) l., eine grobheit jn. l., jn. (z. B. den begaber, das weib, die frommen, den verzagten) l., jm. ein werk. l., jn. e. S. (Gen., z. B. der zeit) l., jn. an das baupt, am mute, an dem leibe l., den schalk mit schalk l. > einen schalk schalk nennen (Verbalinjurie)<; am gemächt geletzet sein; das falsche letzen (subst.).

Schöpper 20b (Dortm. 1550): Nocere. Letzen verletzen verunrechten beleidigen beschedigen vernachteiligen 1 krencken schwächen. Helm, H. v. Hesler. Apok. 3858 (nrddt., 14. Jh.): wer Gote widerstrebit, | Sine zit her halb nicht enlebit, | Die Got im hat gesetzet | Her enwerde ir [zit] geletzet, | Daz sie der tot undervat. EвD. 16282: Die sich dir wider setzen | Und nu din ere letzen | Mit valschlicher lere. LuTHER, WA 2, 89, 23 (1519): Welche seyn aber die frucht? [...], Kratzen, Reyssen, Lettzen, und kein guth worth. Chron. Magdeb. 2, 176, 3 (nrddt., Hs. 1601): do haben der Doctor und sein Vicarius wiederumb heraber geworffen und ein Schmedeknecht hart ans Heupt geletzt. STREHLKE, Nic. Jerosch. Chron. 8398 (preuß., um 1330/40): [si] letatin | mit den spern der cristinen pfert. ReIssenberger, Väterb. 18888 (md., Hs. 14. Jh.): Der mac vil wol in [stam] letzen, | Da₹ er joch gar virtirbet. Мегјвоом, Pilgerf. träum. Mönch 655 (rib., 1444): De groffheit mochte sij wale me letz̧en | Dan de salve kunde ergetzen. BELKIN u. a., Rösslin. Kreutterb. 150, 16 (Frankf. 1535): Wer geletzt were an dem gemecht / der neme diß puluers. KuRz, Waldis. Esopus 4, 2, 195 (Frankf. 1557): Wer einen schalck mit schalck wil letzen, | Der muß ein auff die schiltwacht setzen. BoLte,
Pauli. Schimpf u. Ernst 2, 95 (Frankf. 1560): die unversehen Geschutz letzen mehr. Bergmann, Ambr. Liederb. 225, 48 (Frankf. 1582): kein ritter lebt im lande, | der jbn [Hector] mocht letzen auff seinem pferd. NeumanN, Rothe. Keuschh. 2150 (thür., 1. H. 15. Jh.): [di trunkenheit] da man den lümund letzet mete. Bechstein, M. v. Beheim. Evang. Lk. 6, 28 (osächs., 1343): Bittet vor di, di üch letzin [Mentel 1466 / Froschauer 1530: laidigent; LuTHER 1545: beleidigen]. LuTz, Buch Alfadol 31ra, 5 (obd., n. 1478): [Dein feindt] gedenken nymer so groslich dich zu letzen alß sy es vor gewölt han. PyrITZ, Minneburg 1261 (nobd., Hs. um 1400): Netze, kalter regen risel! | Lety, arger winter sur und kealt! Neubauer, Kriegsb. Seldeneck 88, 25 (nobd., 2. H. 15. Jh.): Die knecht sollenn sich verbuten einander selbst zu schlagen oder zu leczenn. BAUMANN, Bauernkr. Rotenb. 412, 14 (nobd., n. 1525): Ir vil wurden [...] mit dem geschutz, an iren leyben und glidern schwerlich verwundt und geletzt. SACHs 16, 482, 12 (Nürnb. 1562): Mit käuffen in den stich ibn setzen, | Mit borgen oder bürgschafft letzen. EBD. 16, 505, 6: Der spötter, die mit irn bonwortn | Fromb leut fatzen [...] | [...] | An glimpff und ebren sie zu letzen. EBD. 20, 175, 11 (1560): last uns alle wehr besetzen, | Auff daß die Römer uns nit letzen! MAYER, Folz. Meisterl. 34, 375 (nobd., v. 1496): als der sunnen scheine | Dring durch die fenster eine | Und sie nit lecz. BELL, G. Hager 620, 5, 8 (nobd., 1591): das hercz, das schlecht oft den man $\mid$ Dut ein ver zagten leczen. MatTHAei, Minner. I, 12, 704 (Hs. ' wobd., 15. Jh. ${ }^{7}$ ): das er mit valschem wencken | die frowen haut in laid gesetat | das sie an hohem mut letzt. Fuchs, Murner. 4 Ketzer 4413 (rwohl Straßb. ${ }^{7}$ 1509): Hab ich die frummen drinn geletat / | [...] / | Das es mir ist von hertzen leidt. Bihlmeyer, Seuse 397, 20 (alem., 14. Jh.): bòse zungen hant mich geletzet. JörG, Salat. Reformationschr. 332, 2 (halem., 1534/5): fand da comun / da schryer / da sunder personen / [...] / geletzt von disemm gifftt / und bestrickett jn diser tüfelspündtnus. RenNefahrT, Wirtsch. Bern 739, 4 (halem., 1539): Wellicher stuben gsell dem andern sin werck letst, [...], derselbig soll für ein straff [...] vervallen sin. KöBLER, Stattr. Fryburg 103, 3 (Basel 1520): weñ die begabt person / den begaber leţte oder schmechte / an sinẽ eren / lyb oder güt / mit worten oder wercken. FuCHS, Murner. Geuchmat 374 (Basel 1519): Kein wyb vff dise erd ye kam, | Die ich geletzet hab, frow scham. MaAler 269r (Zürich 1561): Edelgestein / Letzen oder bråchen. Haltaus, Liederb. Hätzlerin 1, 39, 8 (schwäb., 1471): Fraw, ich furcht ser der claffer mund | Vnd auch ir valsches letzen. Hör, Urk. St. Veit 180, 13 (moobd., 1414): Wir süllen awch das egenantt güet stiftleych [...] inhaben noch in chainerley weiz.. smeln noch letczen. Gierach, Märterb. 3134 (Hs. rmoobd., A. 15. Jh. $\left.{ }^{\urcorner}\right)$: der chaiser enpot in herwider do ineinen gluenden ofen sey seczen | und ir leben also leczenn. BAUER, Haller. Hieronymus-Br. 11, 1 (tir., 1464): si verschrenkhten seine wëg, vnd die strik richtetten si zu läczen seinen füessen. STrehlKe, a. a. O. 495, 16612; 567, 22828; KurZ, a. a. O. 1, 9, 63; 2, 48, 17; Gerhard, Hist. alde e 1889; Thiele, Chron. Stolle 133, 19; 402, 28; Grosch u. a., 
Schöffenspr. Pössneck 291, 29; KIsCH, Leipz. Schöffenspr. 167, 9; Chron. Nürnb. 1, 158, 15; Reichert, Gesamtausl. Messe 4, 39; SACHS 13, 26, 26; 111, 30; $15,541,10 ; 17,464,18 ; 494,9 ; 18,45,37 ; 18,149$, 6; 18, 481, 23; 20, 429, 34; Chron. Strassb. 690, 9; LuginbüHL, Brennwalds Schweizer Chron. 2, 285, 4; Bihlmeyer, a. a. O. 85, 3; 109, 6; Bernoulli, Basler Chron. 4, 4, 219, 15; Koppitz, Trojanerkr. 5909; 11508; Rennefahrt, Stadtr. Bern 416, 23; Müller, Alte Landsch. St. Gallen 64, 22; V. Anshelm. Berner Chron. 2, 81, 25; JörG, Salat. Reformationschr. 453, 11; 493, 27; 769, 5; 924, 9; Pfeiffer-Belli, Murner im Glaubensk. I, 3, 19; Adomatis u.a., J. Murer. Bab. 4094; Gehring, Würt. Ländl. Rechtsqu. 3, 195, 24. Österley, Steinhöwels Äsop 1358; Chron. Augsb. 5, 180, 8; Primisser, Suchenwirt 8, 167; NiewÖhner, Teichner 306, 3; Gierach, Märterb. 1258; 12174; LeIDinger, V. Arnpeck 558, 2; Bauer, Haller. Hieronymus-Br. 11, 1; Voc. Teut.-Lat. k viv; Hulsius L iijr; Schmidt, Hist. Wb. Elsaß 220; Baumann-Zwirner, Augsb. Volksb. 1991, 273; RaABe, Wortsch. Murner 2, 437. - Vgl. ferner s. v. ablegung 1, ${ }^{4}$ bas 4.

3. >sich von jm. / etw. verabschieden; mit jm. (den Zurückbleibenden) vor dem gehen, reiten, scheiden einen Trunk, ein letztes Essen einnehmen und auf diese Weise den Abschied zelebrieren, dadurch Lebewohl sagen, eine Abschiedsfeier vollziehen<; offen zu 4. - Vorwiegend Texte der Sinnwelt ,Religion / Didaxe', auch berichtende Texte. - Bdv.: vgl. abdanken 3; abgnaden, abletzen, valete geben. - Synt.: jn. l.; sich (z. B. freundlich / lieblich) l., sich etliche tage l., sich e. S. (z. B. der ere, des kindes / weibes) l., sich mit jm. (z. B. mit den freunden) / von jm. (z. B. von dem könig, von der schar) l.; jn. l. Wbg.: letzetrank, leztrunk (auch letze-), letzewort.

Helm, H. v. Hesler. Apok. 22146 (nrddt., 14. Jh.): Wie dan der sich bie letzet | Der eren, gutes, libes, | Des kindes und des wibes | Und alles daz her ie gewan. Luther, WA 26, 457, 33 (1528): [Christus] fehet nu nach dem letzetrunck und abendmal ein newes an Und spricht, es sey sein leib, [...]. EвD. 27, 103, 7 (1528): , das ist mein leib', da sthet das newe abentmal, ubi den letztrunck geben hat. EBD. 41, 46, $10(1525 / 35)$ : et dat eis letz, Et auff den letz trunk dispertit. ЕвD. 46, 96, 33 (1537): wie sich gute freunde unternander pflegen zu letzen. Ders. Hl. Schrifft. Offb. Vorr. 2471, 24 (Wittenb. 1545): Kompt im XX. Cap. auch her zu der Letzetranck. / Gog und Magog (hier ütr.). Bolte, Pauli. Schimpf u. Ernst 2, 113 (Frankf. 1583): Also letzten sich diese zwey [Liebenden] wol mit einander. Wie es nun Zeit war, das sie von einander solten scheiden, | zeigt ihm die Fraw an, [...7. SACHS 2, 236, 1 (Nürnb. 1540): Endtlich [...] | Letzten sie sich noch etlich tag. | Darnach sie [...] urlaub namen. Warnock, Pred. Paulis 11, 134 (önalem., 1490/4): [do er] solt schayden, er sich mit inen [frúnden] fruntlichen waz letzen und dar jungst nachtmal mit inen begieng. B̈̈CHTOLD, N. Manuel. 410, 611 (1523/6): wo die söltind werden gletzt, | Die lib und güt hand zu uns gsetzt. Chron. Augsi. 4, 150, 6 (schwäb., v. 1536): Pfaltrgraff Oth Hainrich hat sich am 5. tag maii hie mit hertzog Wilhalm von Bayern geletzt und ist [...] gem hailigen grab zogen. BAUMANN, Bauernkr. Oberschw. 45, 29 (schwäb., v. 1542): An dem tag, alß sy binweg mußten faren, gab ich inen allen ze morgen zu essen, letzet mich mit inen. BäumKer, Geistl. Liederb. 51, 11, 1 (oobd., 2. H. 15. Jh.): Leczt dich noch bie ain weyl von mir! | wer ways, wan mich mer sehen wirst! SeEmüller, Chron. 95 Herrsch. 140, 6 (oobd., Hs. 1. H. 15. Jh.): Herczog Albrecht von Österreich und graf Meinhart von Tyrol [...] sich ze Augspurg liepleich leczten von chünig Rudolffen und riten von danne. SPECHTLER, Mönch v. Salzb. 37, 34 (oobd., 3. Dr. 14. Jh.): got letzt sich vor mit seiner schar (auf den Abschied beim letzten Abendmahl bezogen). Sснмітт, Ordo rerum 672, 10 (oobd., 2. H. 15. Jh.): Valefacere [...] gesegen [...] vrlaub nemmen [...] leczrzen oder gesegen. Weber, Füetrer. Poyt. 107, 5 (moobd., 1478/84): Dy müed sich hett dy nacht von im geletzet, | er ging. Turmair 4, 63, 4 (moobd., 1522/33): [drei herren] wolten sich vor von einander letzen, und in ein ewige gedechtnus machen. - LuTHER, WA 26, 461, 37; Kehrein, Kath. Gesangb. 3, 102, 5; Kopp, Volks- u. Gesellschaftsl. 56, 59; GOEDEKE, Fischart Schiff 1076; Spechtler, a. a. O. 41, 12.

4. >jn. / sich an e. S. (an Speise, Trank, Geselligkeit, an der Natur) erfreuen, sich laben, sich erfrischen; sich mit jm. (einem Mann / einer Frau, auch erotisch) vergnügen «. - Bdv.: ergötzen, ${ }^{1}$ laben 1, lerzen 3, weiden; vgl. gäuchen 2, jubilieren. Ggs.: kränken. - Synt.: jn. (auch: sich) l., sich erlich l., sich mit etw. (z. B. mit gesange) / jm. (z. B. mit einem fräulein / weib), an etw. (z. B. am tanze), zu etw. (z. B. zum -essen) l., die augen / sinnen l.; subst.: jm. letzen gescheben.

Ingen, Zesen. Rosenw. 10, 9 (Hamb. 1642): Du Außbund aller Zier [...] | Wo ich mit úberfluß die Augen letz' und weide. EBD. 10, 16: Ich wolte tag fur tag die matten Sinnen letzen $\mid$ und mich mit diesem Volck [...7 stets ergotzen. EBD. 15, 2: WOl dem / der sich [...] | letzen kann und laben hier. Еттмüller, Heinr. v. Meißen 386, 15 (md., Hss. 14./15. Jh.): den wolte ich vürbaz, mit gesange letzen. OpITz. Poeterey 24, 5 (Breslau 1624): Jch wil weil ich kan mich letzen.| Bitte meine guete Brúder | Auff die music vnd ein glaß. Gille u. a., M. Beheim 354, 160 (nobd., 2. H. 15. Jh.): daz yeder hete | ain fraulin an dem pete, | [... $\mid$ Da welt wir uns genug $\mid$ mit in leczen und lerczen. Chron. Nürnb. 5, 723, 4 (nobd., 1490): [die erbarn frawen] baten sein küniglich wirde ze bleiben und sich mit in 
zu letzen am tant\%: SACHS 14, 84, 15 (Nürnb. 1550): Derbalb ich noch ein monat bleib, $\mid Z u$ letzen mich mit diesem weib. Haltaus, Liederb. Hätzlerin 1, 25, 88 (schwäb., 1471): [du stoltzer Jüngeling] Ich will mich mit dir letzen. EвD. 2, 5, 66: Geschach dir ye chain letzen | Von mir in langer zeitt. BARACK, Zim. Chron. 4, 82, 9 (schwäb., M. 16. Jh.): do wolt er sich mit dem grafen zum nachtessen letzen. - Ingen, Zesen. Ged. 385, 4; Chron. Baier. StÄdte. Regensb. 97, 24

5. > freundlich gegen jn. sein, sich mit jm. gutstellen

SACHs 17, 246, 22 (Nürnb. 1562): ich muß mich mit ibm letzen; Dann er wird ietzt gen himel fahrn. | Wenn ich auch komb binauff nach jarn, | So wird er mir auch wider schenkken.

6. >etw. abgrenzen, eine Grenze ziehen`. - Bdv.: begrenzen, ${ }^{2}$ bereinen, besteinen, grenzen 3, vermarken. - Wbg. (als Ütr. hier anschließbar): lę̧eit.

Schöpper 77a (Dortm. 1550): Conterminare. Grentzen letəinen. Diefenbach, Mlat.-hd.-böhm. Wb. 118: Extremitas $i$. finis vel finalitas leç̧ait.

letzenis, s. letzen 1.

letzenzeit, s. ${ }^{2}$ letze 3.

letzepfennig, letzepredig, s. ${ }^{1}$ letze 4.

letzer (der), s. letzen 1.

letzer, lester, Adj.; als Komparativ zu lezt rückgebildet.

in der Reihe erer / erst / letzer: >später, später stattgefunden, später vollzogen; später in der Zeit liegend; letztgenannt<; teils mit Tendenz zur Abwertung, dann: >minder, eingeschränktく. - Phras.: unser frawen tag der letzere > Fest der Geburt Mariae (8. Sept.) \& gegenüber dem früher (am 15. August) liegenden Fest der Himmelfahrt Mariae. - Wbg.: letzern >zurücksetzen, abwerten, schmälern`.

Helm, H. v. Hesler. Apok. 676 (nrddt., 14. Jh.): Daz wir der namen nicheinen $\mid$ Weder grozeren noch cleynen, $\mid$ Noch minneren noch meren, | Noch letzeren noch eren. EBD. 3570: dine lezzeren werk | Schinet vor mir herren | Maniger den erren. EBD. 11174: ir kein der drier| Ist mechtiger noch vrier | Noch gewaldiger noch herer, | Noch letzerer noch erer, | Wen die drie sint allentsamen | Ein war got. CHRON. KöLN 2, 25, 6 (rib., 1374): in deme selven jaire quamen de denzer zu unser vrawwen dage der lester (Mariä Geburt) zu Collen. Bindewald, Texte schles. Kanzl. 128, 24, 2 (schles., 1362): An dem Montage vor vnsir vrowen tag der Leczczirn
Sprach Muschil Jode Tilkin roten an vm vumfczig marc schult. Chron. Nürnb. 5, 636, 1 (nobd., E. 15. / A. 16. Jh.): hernach die letzern 59 wochen gab man das gemelt prot iede wochen neur einen tag auß. v. Keller, Ayrer. Dramen 2020, 26 (Nürnb. 1610/8): Die let乏ter kinst ist schier die best. | Lernt mich die letzter kunst allein! - HeLm, a. a. O. 8928; 16251; v. Keller, a. a. O. 2441, 10.

letzern, s. letzer.

letzeschrift, letzesteuer, letzetrab, s. ${ }^{1}$ letre 4.

letzetrank, letzetrunk, s. letzen 3.

letzewort, das; -/auch $-\emptyset$.

>Abschiedswort<, in der Regel mit Bezug auf das sakramentstiftende Abendmahl Christi mit seinen Jüngern; vgl. letzen 3.

Kuther, WA 45, 623, 18 (1537): Das ist nu ein seer tröstlich [...] Letzewort, das er jnen lesst nicht stete und schlösser [...], sondern den frieden als den böhesten schatzjn Himel. EBD. 45, 623, 5: Das sind die letze wort als des, der da wil binweg scheiden. EBD. 45, 627, 11; 46, 98, 11; 46, 111, 3.

letzie, s. ${ }^{2}$ letze.

letzig, Adj.

>verleumderisch; schadhaft; verletzt<; vgl. letzen 1; 2. - Bdv.: vgl. ausrichterisch, franenwirtisch, giftig 3, hönlich, kläpperig, lästerlich 2, schandbar, schmäblich. - Wbg.: letrigen >jn. beleidigen; zum Zorn reizen<.

Chron. Köln 2, 451, 30 (Köln 1499): den willen wir strenglichen straifen mit dem swert als einen der unser overschaft ind majestait geletziget hait. VOC. Ex QUO, O 155 (Eltville 1467): Offendere [...] est aliquem facere irasci, letzigen und erzornen. Franz u. a., Qu. hess. Ref. 4, 188, 7 (hess., 1538): als er nit abstund mit letaiger zungen und schrecklichem angesichte zu poltern. LÖFFLER, Columella/Österreicher 2, 162, 17 (schwäb., 1491): das nit die gantz̧bait unsers angefangnen wercks, als ob im ain gelid angeschnitten wer, letzig oder gesechen wurd. GEHRING, Würt. Ländl. Rechtsqu. 3, 590, 9 (schwäb., 1588): welcher den andern lemig, letzig, painschröt oder lidtief schlegt. - Rwв 8, 1244.

$$
\begin{aligned}
& \text { letzige, s. }{ }^{2} \text { letze. } \\
& \text { letzigen, s. letzig. } \\
& \text { letzung, die; - } \emptyset /- \text {. }
\end{aligned}
$$

$>$ Benachteiligung, Beeinträchtigung, Beleidigung, Schädigung, Verletzung e. P., Angriff auf jn. (auch: auf eine Gruppe); Herabwürdigung einer Institution`; vgl. letzen 1; 2. - Rechts- und Wirtschaftstexte, Chro- 
niken. - Bdv.: s. u. Beleg SCHÖpper, ferner: abbruch 3, betrübung 2, frefel, hindernis, lämung, malefiz, mishandel, schmach, verwüstung; vgl. letzenis. Ggs.: beschirmung 2.

SchÖpper 15b (Dortm. 1550): Offendiculum. Ergernus ansto $\beta$ strauchstein fall letzung. LOESCH, Kölner Zunfturk. 11, 214, 8 (rib., 1375): dat de vurs. Johan van engein van diesen vurs. punten letzunge of hindernisse an ieme hait. HeRTEL, UB Magdeb. 3, 442, 39 (1492): ungerechts unde swarliker lettinge balven an obme irgangen. HAAs u. a., Erasmus/Jud. Klag 4v, 3 (Zürich 1521): [ob] schon etwas letzung zu zyten jnfiel / da durch die früntschafft etlicher maß betribt [...] wurd / das [...]. MülleR, Alte Landsch. St. Gallen 161, 32 (halem., 1525): es sig wider die er Gottes oder zu letzung und betrübung des nechsten mentschen. V. Anshelm. Berner Chron. 1, 379, 7 (halem., n. 1529): sóllend si im schuldig sin, bilf ze tün zu beschirmung und zu látzung, wie die notturft wurd erbeischen. JÖRG, Salat. Reformationschr. 82, 28 (halem., 1534/5): [Lutrer] stallt gar grusam unerbört artickell [...] alles mit verwustung und let乏ung jn r(omischen) hof. GeHRING, Würt. Ländl. Rechtsqu. 3, 675 (schwäb., 1530): wer den anderen ain schlechte fliesende wunden schlöcht [...], darauß kein lözung oder lamung volgt, der [...]. - SснмidT, St. Kastorst. 2, 471, 15; Kehrein, Kath. Gesangb. 3, 8, 2; DasypoDius 155r; Schweiz. ID. 3, 1563. - Vgl. ferner s. v. anfängen 1.

$$
\text { leu, s. }{ }^{1} \text { lewe. }
$$

leuch, Genus und Etymologie unsicher. eine Farbe eher ein Stoff.

SKÁLA, Egerer Urgichtenb. 257, 9 (nwböhm., 1579): ein weiber Rock von Praun leuch.

leuchse, die; zu mhd. liuhse >Stemmleister (LeXer 1. 1941); Vielfalt der Schreibformen: $-e i-$, $-s s-$ statt -chs- (dazu: FRNHD. Gr. \L 53, 4; 56, 3; SchweIz. ID. 3, 1047; 1344).

1. >Nagel, der durch die Achskappe und die Achse verläuft‘; er sichert die Stabilität der Radspur und hält gleichzeitig die Stützstange für die Wagenrunge; insofern auch (als Metonymie): >Stemmleiste für die Rungen . - Obd.; Rechts- und Wirtschaftstexte, Wörterbücher. - Bdv.: lünse. - Wbg.: leuchsenloch >durch Achse und Radkappe gehendes Loch für die leuchse«, leuchsenring > eiserner Ring, der die Leuchse hältı, leuchsenstift, leuchshole (wie leuchsenloch).

Voc. Teut.-Lat. s iv (Nürnb. 1482): Lan. [...] od' runagel od' kypff oder leuchse. EBD. mm viv: Wagennagel od' runnagel od' leuch $\beta$. EBD. bb vr: Runnagel od' spannágel od' leuchßhol. Gehring, Würt. Ländl. Rechtsqu. 3, 151, 9 (schwäb., 1536): von den leißelringen von dem größten 6 s und von den andern zwaien ringen von jedem 3 s. ze machen. EBD. 30: von ainer lumen oder leisselensteff 1 \$, von ainer bichs oder leisselenring 1 \& [...] geben. BREMER, Voc. opt. 27033 (schwäb., 1. V. 15. Jh.): Limo [...] nabennagel [...] wagennagel [...] lone [...] laim [...] liuchsen [...] est clavis axi in anteriori parte infixus et inpediens cantum elabi; uel est baculus an anteriori parte [axis] sursum protensus, vulgariter liuchsen [...]. Et est luno nomen tractum a vulgari theutonico. EBD. 27035 (wobd. / oobd., 15. Jh. f.): Columbar [...] nabenlún [...] línenloch [...] láwchsenloch [...]. Et in proposito tanssumitur pro foramine in axe, per quod transfigitur luno. Sснмiтt, Ordo rerum 247, 16 (oobd., 2. H. 15. Jh.): Trabale lunse lewchsen. Winter, Nöst. Weist. 2, 527, 18 (moobd., 15. Jh.): prëst ïr ainem ein wid oder ein leichsten, so sol er gen an ewr holz. [...] und sol nimant darumb nichts phlichtig sein. - VOC. Ex QUO, Index 457; WVOC. INC. teut. o vijr; Matzel u.a., Spmal. dt. Wortschatz. 1989, 194 (mit Diskussion der Etymologie).

2. >Fangarme des Polypen<; als Ütragung zu 1 (i. S. von >Stemmleiste `) auffaßbar. - SchweIz. ID. 3, 1047 (a. 1563).

leuchsenloch, leuchsenring, leuchsenstift, leuchshole, s. leuchse.

leuchte, die; $-\emptyset /-n$. - Gehäuft nrddt. / md. / nobd. Belege.

1. >Lichtquelle (z. B. eine Wachskerze)<; zumeist als pars pro toto für: >Lampe, Laterne «. - Phras.: die leuchte aleine tragen >die Verantwortung allein übernehmen «. - Bdv. : s. u. SсHÖPPER, vgl.: ampel 1, lampe, liecht 1; 2. - Synt.: die l. bereiten / bessern / finden / machen / nemen, zu stücken schlagen; die l. (Subj.) [wo] bängen / scheinen; des hochmeisters l.; die brennende / grosse / hangende / verbrante l.; das liecht aller leuchten (bezogen auf Christus). Wbg.: leuchtenmacher (auch - $\ddot{a}$ - im Gw). SchÖpper 81b (Dortm. 1550): Lucerna. Liechtstart leuchter lucern latern leuchte. Helm, H. v. Hesler. Apok. 1794 (nrddt., 14. Jh.): Unser herre Jhesu Crist, | Der liecht aller luchten ist. Ders, Maccabäer 7134 (omd./nrddt., Hs. A. 15. Jh.): [han wir] die lubten burnende bereit. LoescH, Kölner Zunfturk. 1, 58, 9 (rib., 1344): ever solen deselve meister kerzen genuch geven up ire kost in de [zwa] lichtin under den gedemen. Chron. KöLn 3, 672, 29 (Köln 1499): so bait he ouch [...] gesatrt dat Marienbilde ind ein hangende luchte. Frantzen u. a., Kölner Schwankb. 3, 1104 (Köln um 1490): Szo eyne luchte up eyneme gulden luchtere schynende is. Buch WeINSB. 1, 47 (rib., um 1560): Tilman Weitmesser hat die lucht allein getragen, war ein from menschs. 
EBD. 85, 7: vur jeder deur bink ein lucht mit brennenden kerzen. EBD. 4, 183, 22 (1594): das jeder burger [...] ein brennende lucht vur dem haus hangen gehat. KuRz, Waldis. Esopus 4, 93, 54 (Frankf. 1557): Ein alter Müller one Korn, | Ein Leuchtenmacher obne Horn. Lexer, Tucher. Baumeisterb. 145, 25 (nürnb., 1464/75): hat man ettwan vill leuchten machen lassen, die man auch auß geben wolt in ettlich eckhäuser. - JоАснім, Marienb. Tresslerb. 459, 25; Ziesemer, Marienb. Ämterb. 149, 31; Ders., Gr. Ämterb. 186, 10; 713, 22; Buch WeInsb. 1, 354, 11; 356, 23; 5, 40, 9; Struck, Joh. Pfannstiel 183, 52; Hertel, Hall. Schöffenb. 2, 333, 23; Dict. Germ.-Gall.-Lat. 305, 17; BüCHER, Berufe Frankf. 1914, 79.

2. >Leuchten, Glanz, Leuchtkraft; von einer Lichtquelle ausgehende Helligkeit‘; vielfach tropisch im Anschluß an 1 gebraucht, dann z. B.: >abschreckendes Beispiel, Orientierungshilfe`; >lichthaft gedachte Seinsform (Christi) <. - Phras.: jn. für eine leuchte in der nacht vorstellen >jn. als Warnung, als abschreckendes Beispiel hinstellen $<$ - Bdv.: vgl. geleuchte, glanst 1 , glänster, glan₹ 1, glänze 1, heitere, kläre, klarbeit 1.

Helm, H. v. Hesler. Apok. 2155 (nrddt., 14. Jh.): Also irouget her ouch sich, | Als ber wir thuen danne, | Deme guten Johanne | Mit also liechter luchten | Daz her sunnen duchten | Sin von ebenglicher mugent. EBD. 22310: Daz ist aber Got Jhesus Crist, | Der luchten alles liechtes ist. Perez, Dietzin 2, 392, 16 (Frankf. 1627): Jhr kónnet ewern Freunden billich für ein Leuchte in der Nacht vorgestellet werden / mit ewerm bosen unnd verrucbten Leben / daß sie nemblich eweren bósen Außgang betracbten. HüBNER, Buch Daniel 2014 (omd., Hs. 14./A. 15. Jh.): Lylien sten in vuchte |In drierbande luchte, |Wiץ, gel, bla, der varwen fin. SACHs 19, 248, 14 (Nürnb. 1563): Dann das gebot ist in der nech | Ein leucht, und liecht ist das gesetz. KLEIN, Oswald 106, 45 (oobd., 1411?): Auffrüstigkliche wunne, sunne, | brunne, meines herzen feuchte, | leuchte deiner öglin klar | gar mich verzucket.

\section{leuchtefas, das.}

>Leuchte, Lichtquelle‘; in allen Belegen in religiöser Ütr.; vgl. leuchten 2; 5. - Bdv.: vgl. lampe.

Helm, H. v. Hesler. Apok. 20917 (nrddt., 14. Jh.): Gotes schin; | Der ist daz ware luchtevaz. EвD. 22313: Daz ist aber Got Jhesus Crist, | Der lucbten alles liechtes ist. | Bi dem wanderet die diet, $|[. .]$.$| Wen daz lamb ist ir luchte vaz:$ EgGers, Psalter 36, 2 (thür., 1378): Wan du derluchtes, herre, min luchtevas. ReIssenberger, Väterb. 6173 (md., 14. Jh.): Apollonius was sin name, | Dem lande gar ein lubtevaz: - Helm, a. a. O. 22257.

leuchtekerze, s. leuchten 2.

\section{leuchten, V.}

1. >eine Lichtquelle benutzen, um einen Weg (auch ütr.) durch Beleuchtung anzuzeigen . - Wbg.: leuchthof >(durch Tageslicht) beleuchteter Hof`, leuchtrahe >Stange, an der die Leuchte befestigt wird`( $\mathrm{Gw} z \mathrm{zu}$ mhd. rahe >Stange<, LeXer 2, 335).

SchmidT, Frankf. Zunfturk. 1, 195, 2 (hess., 1489): man soll auch des nachts keyn garn setzen, auch nit leuchten. SKÁLA, ENGEL, Rats-Chron. Würzb. 309, 122 (nobd., Hs. M. 17. Jh.): thür, die da gehet auf den leuchthof. Dirr, Münchner Stadtr. 420, 23 (moobd., um 1365): Swer wagenlàwt [...] herberget, der sol in mit únsliecht láwchten. KLEIN, Oswald 6, 54 (oobd., um 1425?): got, schepfer, leucht mir Wolkensteiner klar! - SAPPLER, H. Kaufringer 5 , 142; Matzel u. a., Spmal. dt. Wortschatz. 1989, 195.

2. >leuchten, glänzen, strahlen, Licht werfen (von Bezugsgrößen, denen das Leuchten als wesenhaft zugeschrieben wird) <; vielfach tropisch. - Bdv.: brehen, scheinen; vgl. glänzen 1; 2, glasten 1. - Synt.: der bliz. / comet / funke / bimmel / mond / scheiterbaufe / stern,

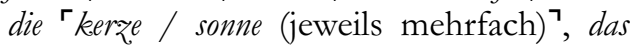
liecht (häufig) l.; die leuchtende fackel / lucerne, das leuchtende liecht. Wbg.: leuchtekerze, leuchtepfanne, leuchtkrone, 「leuchtschirbel gefäßartiger Leuchtkörper?, leuchtsel (a. 1545), leuchtung 1. Jодснім, Marienb. Tresslerb. 362, 36 (preuß., 1405): $5 \mathrm{~m}$. an $1 \mathrm{frd}$. vor 5 messinges luchtkeronen. Ziesemer, Gr. Ämterb. 308, 31 (preuß., 1420): 6 holczynne luchtekerczen, 1 czenynne lampekanne. EBD. 591, 11: 3 beslagene eymer, 1 luchtschirbil. QuiNT, Eckharts Pred. 1, 326, 9 (E. 13./A. 14. Jh.): Sô diu sunne ûfbrichet, daz ist des morgens lieht; dar nâch sô liubtet si ie baz. EBD. 2, 30, 6: daz. [oberste der sêle] in dem liget bedecket als ein ursprunc alles guotes und als ein liubtende lieht, daz alle zit liubtet, und als ein brinnender brant. CHRON. KöLn 2, 2v, 7 (Köln 1499): dye historien synt gelych als luchtende fackelen vnd reytzung off stuppung zo den doegenden. EBD. 606, 35: die [kertze] luchte so schoin ind so clair. STEER, W. v. Herrenb. Büchl. 153 (pfälz., 1436): mit hilffe jres [sele] liechts, das da lüchtet der vernonfft. VOC. INC. TEUT. p ir (Speyer um 1483/4): Liecht das võ im selbs scheint vnd leucht. JürgEs u. a., Waldecker Chron. 350, 15 (wmd., 1546): 2 luchte pfannen. Oоrschот, Spee/Schmidt. Caut. Crim. 367b, 13 (Frankf./M. 1649): wie in vnserm lieben Teutschlandt [...] die Scheiter hauffen / Leuchten. FEudeL, Evangelistar 5, 27 (omd., M. 14. Jh.): daz licht luchtete in dem vynsternysse. Bechstein, M. v. Beheim. Evang. Mt. 5, 15 (osächs., 1343): nimant intzundet eine lucerne und setzit si undir eine mầ, abir ûf einen lûchter, ûf daz, si lûcbte [MeNTEL $1475^{2} \mathrm{f}$ : 
kertzstal] alle den die [...]. v. D. Broek, Suevus. Spieg. 151r, 39 (Leipzig 1588): nach absterben Keyser Ludwigs / hat auch ein Comet 3. Monat lang geleuchtet. BöHme,

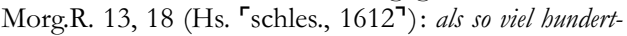
tausend Sonnen leucbten werden. v. Keller, Ayrer. Dramen 915, 4 (Nürnb. 1610/8): O grechter Richter [...] | laß leuchten die Sonn der Grechtigkeit! VetTer, Pred. Taulers 378, 22 (els., 1359): Ze gelicher wis als die klare sunne schinet, so verblendet si alle die lúchtunge der sterne. KurRELMEYER, Dt. Bibel 7, 37, 17 (Straßb. 1466): diser auslegung ditz buichs hab ich gegeben ein kurtze wach zeit bey dem leuchtscherben. BRÉvarT, K. v. Megenberg. Sphaera 7, 18 (noobd., 1347/50): der [bimel] haizzet der feurein bimel, davon, daz er an im selber zemal leubtend und prehend ist. BAuER, Haller. Hieronymus-Br. 1, 12 (tir., 1464): Als das materleich liecht nücz ist, das da leüchten ist den menschen in den vinstern [...] wëgen. - Ziesemer, Marienb. Ämterb. 10, 1; Luther, WA 22, 369, 10; Peil, Rollenhagen. Froschm. 86, 1352; Quint, a. a. O. 240, 4; JAHR, H. v. Mügeln 1026; 2118; 2343; Vetter, a. a. O. 333, 9; SteEr, Schol. Gnadenl. 5, 75; SAPpler, H. Kaufringer 17, 192; Brévart, a. a. O. 34, 7; Volkmar 238; Pfälz. Wв. 4, 958. - Vgl. ferner s. v. asterion.

3. >leuchten, glänzen, angenehmen hellen Schein geben (von Bezugsgrößen, denen das Leuchten als auszeichnende Eigenschaft zukommt oder zugeschrieben wird)<. - Bdv.: fünkeln, glänzen 1, glästen 1, ${ }^{2}$ gleissen, scheinen, schimmern. - Synt.: der carfunkel / palast / stein, die krone / lasur, das antliz / gold / gut l., etw. sam ein liecht, als das feuer l.; der leuchtende stein, das leuchtende har. Wbg.: leuchtig 2, leuchtnis >das Strahlen

Helm, H. v. Hesler. Apok. 21986 (nrddt., 14. Jh.): Ir [der stein Krisoparus] luchtnisse sulchen schin treit | Daz sie luchten in dem vinstern. Wunderlich, Fierrabr. 51, 15 (Simmern 1533): Diese Jungkefraw [...] het ein leuchtend har / als das schón gold. KarneIN, Salm. u. Morolf 9, 2 (srhfrk., Hs. um 1470): Ein kron trug die kunigin, | die luchte recht als der sonnen schin. BeLKIN u. a., Rösslin. Kreutterb. 168, 2 (Frankf. 1535): Peridonius ist ein steyn der leuchtet ann der farb als das feur. STRAUCH, Par. anime int. 138, 21 (thür., 14. Jh.): wan Got ein spigil ist in deme alliz gut lûcbtit. v. Tscharner, Md. Marco Polo 58, 33 (osächs., 2. H. 14. Jh.): wen her [rubyn] luchtit sam eyn licht unde burnit glich eyme vugir. PyriTz, Minneburg 369 (nobd., Hs. um 1400): Irs namen luchtig gymme Its durch sußet als ein zimme. Gille u.a., M. Beheim 267, 18 (nobd., 2. H. 15. Jh.): ir antlut leuchtet alz ein schmit vor tag. Pfeiffer, K. v. Megenberg. B. d. Nat. 465, 17 (oobd., 1349/50): Vertillus ist ain läubtend stain genuog klâr. KLein, Oswald 34, 1 (oobd., 1416): Es leucht durch graw die vein lasur. - BELKIN u. a., a. a. O. 64, 10; LAUChert, Merswin 9, 26; Weber, Füetrer. Poyt. 323, 2;
Alberus Kk iijr. - Vgl. ferner s. v. abnemen 18, abwischen 1 , baldekin 1 .

4. >an-, hereinbrechen (vom Tag) <. Bdv.: vgl. anblicken 2, anfallen 1, anstossen 8; 12, 2 aufbrechen, aufgeben 15, bereinfallen 2, berfürbrechen 1, berfürkommen 3. - Wbg.: leuchtig.

Bechstein, M. v. Beheim. Evang. Lk. 23, 54 (osächs., 1343): der tag was der vorbereite tac, und der sunâbint lûchtite in. Pyritz, Minneburg 4793 (nobd., Hs. um 1400): Ez sy luchteg oder tymber, | So get vor uz von ir [wip] ein schimber. - Bechstein, a. a. O., Mt 28, 1.

5. in einer hohen Anzahl von Belegen ütr. von Gott, Gottes Allmacht, von religiös durch Herrschaft oder sonstige Qualitäten ausgezeichneten Persönlichkeiten, auch von der Minnedame, der Seele u. ä. gesagt; >in hellem Licht erstrahlen, leuchten, glänzen, in etw. / jm. aufleuchten «. Gehäuft Texte der Sinnwelt ,Religion / Didaxe'. - Phras.: als ein drek / eine rostige pfanne leuchten. - Bdv.: brehen, brennen 16, glinstern 1, glühen 1, scheinen; vgl. glorieren 2. - Synt.: ein liecht (Akk.) l.; got, Christus, das kindlein, Darius, der herre, der beilige, der mensch, der beichter, Maria, die fraue, die magd, das weib, der frommen leib, das angesicht gottes, die sele, die almächtigkeit / einigkeit / keuschheit / liebe, das evangelium / liecht / wort, js. lob, die werke l.; die leuchtende anweisung / warbeit. Wbg.: leuchterin, leuchtig 2 > hellı, leuchtung 2.

Helm, H. v. Hesler. Apok. 3487 (nrddt., 14. Jh.): Des [Crist] lucbtene sulche craft truc| Daz sie den tuveln wider sluc. Luther, WA 10, 1, 1, 628, 18 (1522): blindheyt, die das Euangelium nit sihet, obs wol leuchtet. EBD. 33, 52, 23 (1530): die Veter leuchten gegen jme [Moses] als ein Dreck. in einer Latern. ReIssENBERgER, Väterb. 15359 (md., Hs. 14. Jh.): So kumt die luterinne, | Die sunne rebter minne, | Die bringet vreude vollenkumen. Froning, Alsf. Passionssp. 174 (ohess., $1501 \mathrm{ff}$.): ich [Lucifer] werde widder als ee | viel schoner dan die sonne | und luchte als eyn rostrige phanne! Jostes, Eckhart 2, 3 (14. Jh.): Cristus ist mir alle ding. Da geschiecht ein leuchen und ein widerleuchen sin selbes natur. EBD. 45, 30: Di bildreich form got\%, [...] dar an leubtet daz pild aller ding ungeformet in einvalticheit. Di selb form di leubt einvalticlich ein libt in allen geisten. STRAUCH, Par. anime int. 46, 28 (thür., 14. Jh.): ich were noch seliger ob ich volgite der lere und geordinit were zu der luchtunge der engle. Neumann, Rothe. Keuschh. 2402 (thür., 1. H. 15. Jh.): thud der schonen kuscheit ere, | di luchtid vor andern togunden sere. Pyritz, Minneburg 3411 (nobd., Hs. um 
1400): luchticlichen git er [munt] glitzen | Recht sam ein uber heller blitzen. GiLle u. a., M. Beheim 161, 543 (nobd., 2. H. 15. Jh.): das ein menschen pleüd |also chreftigen tute | Und freuet seinen müte $\mid$ und sein leiden cæu leuchtung wendt. Kehrein, Kath. Gesangb. 1, 5, 6 (Nürnb. 1631): Der Frommen Leib wird leuchten schon. BIHLMEYER, Seuse 244, 2 (alem., 14. Jh.): die bibtere lúbtent in grünender schonbeit. Chron. Strassb. 306, 5 (els., A. 15. Jh.): Daryus [...], der do lübtet mit den götten von Persa, enbüte mime diner fröude. Sснмidt, Rud. v. Biberach 170, 13 (whalem., 1345/60): daz dv gotlich wúrkvnge lúchtet in dem menschen. PÄPKE, Marienl. Wernher 14225 (halem., v. 1382): Sú ist dú werde maget rain $\mid$ An súnde und äne main $\mid$ Dú inder welt lúcht allain. Gilman, Agricola. Sprichw. 2, 214, 7 ([Augsb.] 1548): Ain Herr on Ehre / leüchtet also wol | Als ain schón Saal / finsternuß vol. Kummer, Erlauer Sp. 3, 946 (m/soobd., 1400/40): [jamer] umb den vil lieben berren mein, | der da leuchtet für der engl schein! BAUER, Imitatio Haller 78, 26 (tir., 1466): es geschicht etwenn, das der vnpekechant mensch leüchten ist durch das guet lob. - LuTHER, WA 21, 457; 21, 494, 16; 22, 414, 6; QUINT, Eckharts Pred. 1, 31, 7; 2, 277, 4; DERs., Eckharts Trakt. 111, 21; 210, 15; HüBner, Buch Daniel 6598; Jostes, a. a. O. 75, 33; Pyritz, a. a. O. 4788; Kehrein, a. a. O. 1, 20, 1; 2, 385, 6; BeLl, G. Hager 41, 3, 2; 46, 3, 22; Bihlmeyer, Seuse 186, 12; 299, 21; WiLliams u. a., Els. Leg. Aurea 466, 19; 549, 20; Gilman, a. a. O. 1, 85, 20; Schmidt, a. a. O. 37, 16; SAppler, H. Kaufringer 1, 89; SpILler, Füetrer. Bay. Chron. 119, 29. - Vgl. ferner s. v. anbaftung, anweisung $2,{ }^{2}$ aufbrechen, ausbund, auswendig 9.

6. >in den Sinnen als Bild erscheinen, als Bild aufleuchten`; ütr.: >etw. besagen`. Texte der Sinnwelt ,Religion'. - Wbg.: leuchtung 3.

Helm, H. v. Hesler. Apok. 18337 (nrddt., 14. Jh.): zu der vesperzit $\mid$ Ein lucbtenunge schinet $\mid$ Die nimmer mer vorswinet. HüBnER, Buch Daniel 760 (omd., Hs. 14./A. 15. Jh.): Kunic, sich, dich beduchte, $\mid$ Als dir din sinne luchte, | Wie eine sule stu ${ }^{\mathrm{i}}$ de | Vor dir. VetTer, Pred. Taulers 22, 14 (els., E. 14. Jh.): Er sol ufston, surge, sprichet dis wort: stant uf'; dis lúbtet iemer als ob der mensche darzu tü súlle, er müs ufston von allem dem daz Got nút enist. - НüвNER, a. a. O. 3572.

leuchtenmacher, s. leuchte 1.

leuchtepfanne, s. leuchten 2.

leuchter, der; $-s /-\emptyset$, auch $-e$.

>großer Kerzenhalter, Kerzenständer, Kandelaber; Menora‘; metonymisch dazu: >Licht; Lichtquelle, die auf den Kerzenhalter aufgesetzt wird (z. B. eine Kerze)<; ütr.: >Person, die eine Leuchte trägtı. - Bdv.: s. u. SCHÖPPER, ferner: kerzenstok, lampe, liechtstok. - Synt.: den l. waschen, dem kloster einen l. schenken; der l. [wo] stehen, in die kirche gehoren; das liecht auf dem l. brennen, etw. (z. B. die luzerne) auf den l. einsetzen, die lampe fürwärts dem l. scheinen, jn. (got) in dem l. beschauen; der alte / aufgebängte / eisene / guldene / irdene / kupferne / messingene / überzinkte / vierfache / weisse / zinnene l.; 3 par leuchter; das liecht des leuchters. Wbg.: leuchterwurf $>$ Wurf mit einer Leuchte (als Straftat)<. SCHÖPPER 81b (Dortm. 1550): Lucerna. Liechtstart leuchter lucern latern leuchte. Helm, H. v. Hesler. Apok. 1614 (nrddt., 14. Jh.): Do sach ich siben luchtere, | Die waren von golde. EBD. 1664: Daz Got in siben luchteren|Johannen sich beschouwen biez. $|[\ldots]|$ Daz ist ein stark gedute. | [... | Der luchter ist des liechtes stadel, | Alz ist daz liecht des libes adel. EвD. 7768: Da vor sach der gute Johan |Crist in den luchteren stan | Glich menschlichen antlitzen. LuTHER, WA 8, 378, 31 (1521): Alsqo mocht er [Gott] it₹t tzu den stifftern, reuchern, sengern, klengern und leuchtern sagen: Meynet ybr [...]. EвD. 10, 1, 1, 677, 21 (1522): da ist keyn tzill noch masse, [...] monstrant\%, silbern bild unnd kleynod, leuchter, kertzen, liechte, weyrauch. EBD. WA 23, 558, 11 (1527): Er [Prophet] habe ein gesicht gesehen, wie ein gülden leuchter stehe zwisschen zweyen ólebewmen. DERs. Hl. Schrifft. 4. Mose 8, 2 (Wittenb. 1545): Wenn du die Lampen auffsetzest / soltu sie also setzen / das sie alle sieben fürwerts dem Leuchter [MENTEL $1475^{2} f$.: Körtzstal] scheinen. KollNig, Weist. Schriesh. 144, 30 (rhfrk., 1620/1710): blutrunst, item kanten-, kraußen-, gläßer-, leuchter-, teller- und dergleichen wür. FEUDEL, Evangelistar 143, 4 (omd., M. 14. Jh.): Noch sy inpurnen dy lucernen unde inseczen sy nicht under den scheffel, sundir uf den luchter uf daz. sy lucbten. HüBnER, Buch Daniel 4537 (omd., Hs. 14./A. 15. Jh.): Blicke warf er [kung] stetlich dar | [...] |An die gelit me und me $\mid$ Die da schriben kein im e | Und des luchters lichtis schin. RöHrich u. a., Cod. Dipl. Warm. 4, 554, 2 (omd., 1434): VII czenen kannen und 1 eren luchter. Gille u.a., M. Beheim 441, 85 (nobd., 2. H. 15. Jh.): du pist der guldin leuchter, der $\mid$ im thempel Salamane erlaucht mit siben lampen kler. BARACK, Zim. Chron. 1, 92, 33 (schwäb., M. 16. Jh.): das vor vilen jaren ain freiberr von Zimbern dem closter ain guldinen leuchter [...] arbait, so im latein genennt würt candelabrum miri operis, zu eewiger gedechtnus geschenkt hab. CHRON. AugsB. 8, 360, 20 (schwäb., zu 1548): auf jedem altar ist gestanden ain guldin creutz, [...], sechs schön silberin leichter, darauf sechs kertzen. Bauer, Haller. Hieronymus-Br. 4, 10 (tir., 1464): er hat geleücht als das liecht, das da prinnt auf dem leüchter mitten in dem tempel gottes. - LuTHER, WA 47, 686, 29; 49, 732, 13; Thielen, Gr. Zinsb. Dt. Ord. 48, 5; 119, 39; Jürges u. a., Waldecker Chron. 352, 10; Bechstein, M. v. Beheim. Evang. Mk. 4, 21; Küther, UB Frauensee 410, 4; KIsCH, Leipz. Schöf- 
fenspr. 411, 10; Lippert, UB Lübben 2, Einl. 54, 17; Chron. Augsb. 2, 385, 14; Müller, Welthandelsbr. 289, 5; Bauer u. a., Kunstk. Rud. 1605; Uhlirz, Qu. Wien 2, 3, 5481, 17; ZingerLe, Inventare 8, 2, 15; 27, 2, 18; 98, 2, 30; Dasypodius 23r; 371v; MaAler 269r; Golius 321; Hulsius L iijr; Dict. Germ.-Gall.-Lat. 305, 20; PfäLz. WB. 4, 957. - Vgl. ferner s. v. ampulle 2.

leuchterin, s. leuchten 5 .

leuchterwurf, s. leuchter.

leuchthof, s. leuchten 1.

leuchtig 1;2, s. leuchten 3; 4.

leuchtkrone, s. leuchten 2.

leuchtnis, s. leuchten 3.

leuchtrahe, s. leuchten 1.

leuchtscherbe, leuchtschirbel, leuchtsel, s. leuchten 2.

leuchtung $1 ; 2 ; 3$, s. leuchten $2 ; 5 ; 6$.

leucophlegmatica, die; aus dem Griech. über lat. leucophlegmatica >Bleichsucht «(GEORGES 2, 624).

eine Krankheit, wohl > Wassersucht .

Scholz, Lanfrank. Chir. Parva 235r, 1 (md./oobd., 1446/8): mercke: ist das sy ist vber den ganczen leyp, so macht leucoflematicam, / das ist waßerkeyt.

leuerachtig, Adj.; $\mathrm{zu}{ }^{1}$ lauer.

>schlaftrunken «.

MAALER 269 (Zürich 1561): Leürachtige augen / Von grossem schlaaf der einen ankompt. - SCHWEIz. ID. 3, 1377.

leug, Genus? Aus franz. lienes, so das Glossar zur u. a. Ausgabe.

ein Entfernungsmaß (laut Umfeld des ersten Belegs etwa eine halbe teutsche meile). Morrall, Mandev. Reiseb. 5, 4 (schwäb., E. 14. Jh.): das wasser der Tonaw [...] hatt als ain ungestumen gang in das mere das es sü $\beta$ ist in dem mer bin in zwaintzig loüg. Rauwolf. Raiß 14, 12 ([Lauingen] 1582): fart biß in Cypern, (dabin noch 1300 [meiln] seind / deren 3 ein Frantzosische leucam oder meiln machen).

leuge, wohl der; Lemmaansatz unklar.

Monatsname, möglicherweise >Juli<.

Chron. Augsb. 4, 120, Anm. 1 (schwäb., v. 1536): Des jar ward auch der rerkast gemacht bei unser Frawenbrieder und gesetrt im lewge 1508. - Sсншёв. Wв. 4, 1989. leugnen (+ Wortbildungen), s. läugnen (+ Wortbildungen).

leumd(e), s. leumund.

leumden, $\mathrm{V}$.

> ehrend oder verleumderisch über jn. sprechen〈; als part. Adj. (ge)leumdet 〉von gutem bzw. üblem Ruf «, wohl auch mit Tendenz zu >beschuldigt<; zu leumund 1, zum part. Adj. vgl. auch leumund 5. - Bdv.: loben 1, ${ }^{1}$ preisen 1; vgl. (negativ): abbrechen 11, ausmären, beklaffen, beschämen 3, schänden, schelten, schmähen, unleumden.

Voc. Ex Quo, D 329 (rhfrk., 1418): Diffamare beschemen [...] / schmehen uel eyn bose gerecht machen. VOC. INC. TEUT. o viijv (Speyer um 1483/4): Leumt mẽsch Famosus. BIschoff u. a., Steir. u. kärnt. Taid. 60, 26 (m/soobd., 1537): mit wol geloumbten männern. - SCHweIz. ID. 3, 1273; Vorarlb. Wв. 2, 276; SChWÄв. Wв. 4, 1205.

\section{leumdig, Adj.}

>von gutem Ruf, gut beleumundet ‘; zu leumund 1b. - Oobd.; Rechtstexte. - Bdv.: vgl. geleumt, gerüchtig. Ggs.: schädlich, unleumdig.

Siegel u. a., Salzb. Taid. 84, 33 (smoobd., 1654/68): ist er aim pflëger tang, nuz und gefölig, ist guet, ob nit, so sëzen sie ain gueten, frumen, leibumbtigen (Var. C: leumbtigen) gerichtsman al 14 tag. EBD. 287, 25 (1494): es soll auch ain ieder hauswirt oder angesezzner man aufnemen leuntig poten, knecht und diern, [...], die sich allenthalben gegen den nachtpawrn mit beschaidenhait [...] baltent. - WinTer, Nöst. Weist. 4, 425, 23 (hier Var.: leutig); Rwв 8, 1257.

leumdung, die.

>Gerücht, Kunde, Gerede`; zu leumund 3. - Bdv.: vgl. gerücht 4, leumund 3.

Bernoulli, Basler Chron. 4, 373, 4 (alem., 1348): es stünd ein gross lumdung uff, das die Juden die Cristenheit dilken woltten mit vergift.

leumlos, Adj.; zur Sippe von leumund.

>böse, ruchlos`. - Bdv.: erlos, verbannen; vgl. anrüchtig 1 , ruchlos.

Kurrelmeyer, Dt. Bibel 8, 8, 10 Var. (Straßb. 1466): Der weg seint verkert vnd ir geng seint verpannen [Var. 1475²-1480: lewmloß; 1483-1518: erloß.

leumund, leumd(e), leumut, lümut, der; -s oder -en/-; aufgrund etymologischer Undurchsichtigkeit starke Formvariation: Kontraktionen, Entrundungen (-ei-), Zer- 
dehnung der ersten Silbe (zu leine-), unterschiedlicher Stand der Diphtongierung sowie der Umlautkennzeichnung (bezogen auf die erste Silbe), volksetymologische Sinngebung(sversuche) der ersten Silbe (z. B. lein-, ${ }^{3}$ langen-, leut-) sowie der zweiten Silbe (-mund, -mut); teilweise völlige Deformation der Schreibung (z. B. zu land).

1. >Ruf, öffentliche Einschätzung, Leumund, Urteil der sozialen Umwelt über eine Person oder Personengruppe mit Bezug auf ihr sittliches Verhalten`; a) teils neutral ohne Attribut; b) teils trotz fehlenden Attributs aus dem Textzusammenhang als positiv oder negativ gemeint erschließbar; c) teils mit Wertadjektiven, meist gut oder böse, attribuiert und dann ansatzweise phrasematisch; offen zu 2-5. - Gehäuft 'Rechtstexte, Texte der Sinnwelt ,Religion / Didaxe', berichtende Texte $\urcorner$. - Bdv. (alle Nuancen zusammenfassend): ${ }^{1}$ achtung 4, argwan 1, besagung, erbarkeit, ere, gerücht 5, geschrei 9, glimpf 1; 3, leutlob, lob 1; 2, name, ruf, sage, schmähe, wort. - Synt.: einen (guten / bösen) l. haben, den l. abschneiden / ärgern, kränken / letzen / schwächen / vermeiligen, js. l. rauben, einen guten l. bören, einen bösen l. gewinnen; der l. (Subj.) umgehen / wachsen, jm. lieb sein, sich weit ergiessen, einem apfelschal gleich sein; eines guten leumundes sein; den verdacht durch l. anzeigen, an js. l. rüren, jn. am l. töten, jn. mit dem l. bemorseln, mit dem l. betragen werden, in einen bösen l. kommen, in bösem l. stehen, jn. in einen l. werfen, von bösem l. sein, jn. von dem guten l. bringen; der l. des vaters; der argwönige / böse / dunkle / erliche / gemeine / grosse / gute / offenbare / offene / übele l.; abstrickung / verderben / verkleinerung / zier des leumunds.

$\mathrm{Zu}$ a): Laufs, Reichskammergo. 187, 22 (Mainz 1555): soferr dann die klagendt parthei den verdacht durch gnugsam anzeyg oder eyn gerücht, leumuth oder aber durch eynen zeugen [...] anzeygt. Hertel, Hall. Schöffenb. 2, 223, 21 (osächs., zu 1424): daz her sy geschulden hat mit reden vnde worten, dy on ere lip vnde lumund ruren. JEROUsCHEK, Nürnb. Hexenh. (obd., 1491): dar die erste anzeugüg sol seinb der lumüt mit dem argkwon das iist das geschrey das gemainẽ volcks. BACHMANN, Morgant 290, 36 (halem., 1530): dienyl er [Gannellon] den lümbden hat, das er der gröst verretter. WedLeR, W. Burley. Liber 55r (moobd., v. 1452): du macht / ettwann wol betrogenn werden mit dem lüntden, aber mit der gewissenn nymer. BAPTISTHlawatsch, U. v. Pottenst. 244 (moobd., A. 15. Jh.): Aines menschen leïndt warumb der ainem appffelschal geleich ist. - KÖBler, Ref. Wormbs 341, 6; Chron. AugsB. 8, 431, 29. - Zu b): Strehlke, Nic. Jerosch. Chron. 323 (preuß., um 1330/40): daz sich [...] | sin limmunt und sîn name $|[\ldots]|$ an gütem rüche wîte irgốz. LUTHER, WA 10, 1, 1, 652, 12 (1522): das sie [diaboli] dieselben $z u$ schanden machen, yhren leuttmund offentlich rawben. EBD. 30, 1, 169, 7 (1529): Darümb wil Gott des nehisten leumund, glimpff und gerechtickeit so wenig als gelt [...] verkürtzt haben. Neumann, Rothe. Keuschh. 4229 (thür., 1. H. 15. Jh.): so wirt si [maget] zu der stunde| bemorselt mit dem limunde. EBD. 5615: ab si dich vordencken| unnd dinen lumund dar umme kerencken. KöBLER, Ref. Nürnberg 85, 2 (Nürnb. 1484): das der Clager dem oder denselben der person oder habe vnd gut auf sein anpringen verpotten wordê wer vngüttlich gethan. vñ vnpillich in sóllichen Rüff. schmehe vnd lewmüt geworffen hette. JörG, Salat. Reformationschr. 417, 3 (halem., 1534/5): wider die xij ort der Eygnoschaft zu abstrickung und vercleynerung jrs glimpfs, lümdens und eeren. Bauer, Geiler. Pred. 467, 32 (Augsb. 1508): da aines dem andren mit seinen worten sein eer abschneydet / und ym seinen laümden schwóchet. Müller, Grafsch. Hohenb. 2, 294, 23 (schwäb., 1535/6): der Brun der mayer waz. in ainen lümden, er het ain ku angangen. Rudolf, Peuntner. Sterbek. 150va, 32 (moobd., n. 1434): Vergibst du [...] allen den, die dich gelaidigt habent an deinem leichnam, an deiner seel, an deinem gut, an deinem lanndt und an deinem lob? Karnein, de amore dt. 165, 368 (moobd., v. 1440): so ain junckfraw in der lieb vnd mynn wol gelert ist vnd macht damit ir güt wort vnd lewnt wachssen, so mag ir man [...] kayn recht haben wider sie zu sprechen. - FRANCK, Decl. 344, 23; Bihlmeyer, Seuse 8, 7; Wagner, Erk. Ps.-J. v. Kastl 8, 33; UB OB Der EnNs 10, 296, 3; Pfeiffer-Belli, Murner im Glaubensk. I, 2, 23, 27; HaAs u. a., Erasmus/Jud. Klag 7r, 1; Leidinger, A. v. Regensb. 605, 7; Türk, Wortsch. Dietr. v. Gotha. 1926, 73; MaAler 275v; Schmidt, Hist. Wb. Elsaß 229. - Zu c): Luther, WA 30, 1, 360, 3 (1529): das wir unsern nebisten nicht felschlich beliegen [...] odder bösen leumund machen. KoHLer u. a., Peinl. GO Karls V. 20, 20 (о. O. 1532): ob der verdacht ein [...] leychtfertige person vonn bosem leymadt vnnd geruch sey. OORSCHOT, Spee/ Schmidt. Caut. Crim. 287a, 14 (Frankf./M. 1649): Wann nun diese angezeigte Persohnen [... wegen solcher eintzigen Besagung / vnd gegen sie entstandenen bösen Leumuths / zur Hafft und Folter hingerissen wird. ENDERs, Eberlin 3, 13, 7 (Erfurt 1523): was ist erberkyt vnnd gutter lewmbd nut:, so man sich da durch erhebt vber und widder Gottis wort. Chron. Nürnb. 1, 345, 4 (nobd., 1420/41): da bracht hertzog Rudolf von Bayrn sein muetter in einen großen leinmuet mit einem ritter. MAYer, Folz. Meisterl. 100, 289 (o. O. o. J.): schand die auß dem ubeln leumant des vaters kümpt. Franck, Decl. 346, 32 (Nürnb. 1531): die sauffsücht / 
durch welche die gesundtheit des gemuths zerkeruppelt / [...] die vetterliche erbgutter verzert vnd zerstreut / der gü leumbd außgemustert werden. Kurrelmeyer, Dt. Bibel 8, 43, 8 (Straßb. 1466): der gut leumunt derfaißt die bain [Var. 1475²/ 1576: laymung; 1480: leümung; 1483-1518: lewmut; Luther 1545, Spr. 15, 30: Gerücht. B̈̈сHTOLD, H. Salat 139, 4 (Freiburg 1537): So dann gar nach in ganzer tütscher nation etwas dunklen lumbdens und sag krücht und umbgat von dem [...] fründ Gottes, brider Clausen. ReNNEFAHRT, Recht Laupen 207, 8 (halem., 1545/6): wo die unseren von Loupen einen an das gricht welten wetzen, der ein bósen lümbden hette. Österley, Steinhöwels Äsop 310, 77 (Ulm 1474/82): so ist diser arme man ains guoten lümden alle syt gewesen. VOC. Ex QUO F 48 (oobd., 1458): Fama ein geruchte vel luumont [...] nomen bonarum et malarum rerum. NiewÖHNER, Teichner 656, 23 (Hs. $\Gamma_{\text {moobd., } 1469\urcorner)}$ ): wer ein frawn tugenthaft $\mid$ pringt von irm gueten lewnt, $\mid$ der ist ir veint. - KOHLER u. a., Bamb. Halsger. 32, 11; SACHS 17, 220, 25; Geier, Stadtr. Überl. 46, 11; Köbler, Stattr. Fryburg 214, 3; Welti, Stadtr. Bern 52, 14; B̈̈сhtold, a. a. O. 189, 255; Dreckmann, H. Mair. Troja 23, 12; Müller, Stadtr. Ravensb. 130, 4; Dirr, Münchner Stadtr. 567, 24; Drescher, Hartlieb. Caes. 65, 20; Schwartzenbach 40v; Rwв 8, 1257 f.

2. >Verleumdung, üble Nachrede, Ehrverletzung; Beschuldigung, im Unterschied zu 1 deutlicher negativ und eher als Handlung gedacht. - Bdv.: bedassung, erverletzung; vgl. achterkosung, argwan 1, calumnie, erabschneidung, gerïcht 5, geschelle 1, binterrede, klaffe 1, klafferei, klapper 2, klapperei, laster 3, lästerung 2, lästerwort, liegezunge, nachrede, teiding 7, unglimpf.

Toeppen, Ständetage Preußen 2, 546, 14 (preuß., 1443): das manicherley rede, bedassunge und lunemund, [...], abgetan werden. Leman, Kulm. Recht 2, 5, 23 (Thorn 1584): So sal man [...] ym slan vyrayk slege. vnd sal deme vatir busse geben vor bosen logen munt [Var.: lumunt] den ber von der tachtir hat gemacht. Bihlmeyer, Seuse 66, 23 (alem., 14. Jh.): daz der bose limde also dur die stat wart gend úber den brider. RODER, Stadtr. Villingen 180, 8 (önalem., 1582): leimmuot und erverlötzung mag einer, [...], woll appellieren. Sснів, Urk. Laufenb. 138 (halem., 1450): Als denn leider Ulrich Vógtlin [...] von eins bósen liumden wegen [...] in den thurn komen. BONER, Urk. Aarau 663, 3 (halem., 1502): die von des hertten schweren limden wegen der hexery, [...], gefangengesetzt worden war, schwört nach der Freilassung Urfehde. Karnein, de amore dt. 239, 6 (moobd., v. 1440): er wirt vnder dem volck versmecht vnd mit pösen leuntten vermayligt. - BerNoulli, Basler Chron. 5, 266, 19.

3. `Gerücht, Kunde, unbestätigtes umlaufendes Gerede über einen Sachverhalt«.
- Mehrfach berichtende Texte. - Bdv.: beruf 1, gerücht 4, geschrei 10, märe, ruf; vgl. leumdung. - Synt.: den l. bören; der / ein l. sein / werden / bleiben / ausgehen, jn. betrüben, aufs land kommen, auf jn. gehen, das [...]; der gemeine (mehrfach) / offene / offenbare / starke l.

Schöpper 59b (Dortm. 1550): Fama. Lümbd leumbd leymat geruicht ruff geschrey. BeCHSTEIN, M. v. Beheim. Evang. Lk 4, 14 (osächs., 1343): Jhêsus ist wider gegangen in der craft des geistes in Galilêam, und der limunt [MENTEL 1466: mer; $1475^{2}$ : geschray; LuTHER 1545: das gerüchte] ginc $\hat{u} z$ durch daz kinicriche allesament von ime. KURRELMEYer, Dt. Bibel 5, 17, 6 (Straßb. 1466): es ist nit ein gíter leumund [LuTHER 1545, 1. Kön. 2, 24: geschrey] den ich hór: das ir macht zu vbergeen das volck des herren. Diст. Germ.-Gall.-Lat. 305 (Genf 1636): Leumb / Beruff / m. Geruchte [...7, Rumor. Vetter, Schw. zu Töß 22, 23 (Hs. 15. Jh.): davon kam sy in sólichen lúnden das man wond das sy ainen schweren siechtagen het, und hie von ward sy vil verschmáhet. Buck, U. v. Richent. Chron. Conz. 144, 18 (alem., um 1430): ward zwüschen inn zwain ain täding angefangen und ward gemainer lün, sy wärind mit ainander gericht. Barack, Zim. Chron. 1, 9, 38 (schwäb., M. 16. Jh.): als noch ain gemeiner leumbd, auch wol glaublich, in die erst christenlich kirchen derselben enden verwendet. EBD. 1, 20, 31: das bei unsern vorfarn ain gemainer leumbedt gewesen, es hab [...]. - Bechstein, a. a. O. Mt. 9, 26; KurRelmeyer, a. a. O. 9, 508, 6; Rennefahrt, Staat/Kirche Bern 385, 25; V. Anshelm. Berner Chron. 3, 104, 10; Jörg, Salat. Reformationschr. 740, 15; 741, 9; Chron. Augsb. 8, 430, 2; Rwв 8, 1261.

4. >öffentliche Einschätzung eines Sachverhaltes $<$.

Rieder, St. Georg. Pred. 86, 15 (Hs. 「önalem., 1387`): ir sont nit gedenken won das war si [...] und daz ewig si und daz, gütes lúmden si. Chron. Strassb. 434, 2 (els., A. 15. Jh.): die heilge kirche [...] ist vil geswechet und beswert und die wandelunge het einen bósen lymüt. LöFFLER, Columella/ Österreicher 1, 175, 2 (schwäb., 1491): das kain gemain gestalt oder geschlecht der winreben zi setzen sy dann us $\beta$ dem laimden, und kains lang qu buwen dann das durch erfarung bewert ist.

5. >Beschuldigung, Vorwurf, Anzeige vor Gericht, Anklage «. - Bdv. : in₹icht, klägde 2; vgl. anklage, antastung 3, anzieg, auflag 3, beklagung 2, besagung 3, beschuldigung, bezicht 1, berieg, gerieg, incusation, insimulation.

Pfeiffer, Frk.-bay. Landfr. 86, 4 (o/nobd., 1379): Da warde erteilt, daz er sich desselben lewmunts und inzibt entslaben schölt mit sein ainshant mit dem ayde. DinkLage, Frk. Bauernweist. 75, 41 (nobd., 1523): was mit leumund und warer rüge reyn fur gericht kompt, das wey $\beta$ man nymands. Rennefahrt, Stadtr. Bern 307, 36 (halem., 1403): so 
sóllen wir ze beiden teilen fü den ... schultheissen und rete ze Berne komen und den lumden und klegde der gevangnen person da selbs furlegen.

\section{leumut, s. leumund.}

leun, s. leunen.

leunen, V.; zu mhd. liunen (LeXer 1, 1942).

>auftauen; Tauwetter eintreten $\varsigma$. Bdv. auftauen, ${ }^{2}$ tauen. - Wbg.: leun >das Auftauen<.

Lexer, Tucher. Baumeisterb. 253, 24 (nürnb., 1464/ 75): wo jebling ein leunen oder weichs wetter an viell. EBD. 29: der [snee] dann dick ist und sich richt in leun und abgeen will. Chron. Nürnb. 5, 562, 1 (nobd., E. 15./A. 16. Jh.): erst Kungundis da ließ die kelt erst ab und leunet es. EвD. 695, 32: an sant Katarina tag da regentz, und leunet den gantzen tag.

leustel, die; etymologische Zuordnung? >äußere grüne Schale der Nußく. - Wbg.: leustelen >Nüsse schälen<. - ScHмIDT, Hist. Wb. Elsaß 221 (a. 1494 für beide Bildungen).

\section{leustelen, s. leustel.}

leut, der / das; -es/-. - Im mittleren Frnhd. auslaufend.

1. >geringe Anzahl bis größere Menge von Menschen; Volk; Menschheit generell; einem Herrscher zugehörige Menschen, Untertanen, Leute ‘. - Vielfach Texte der Sinnwelt ,Religion'. - Wbg.: leutlos >menschenleer< (um 1476), leutsdrek, leutskot, leutsstein (zur Erklärung s. den Beleg), leutswurm wohl eine Laus oder ein Darmwurm. Strehlke, Nic. Jerosch. Chron. 4145 (preuß., um 1330/40): An cleiderin richeite | und ubirvluzzikeite | nicht achtit noch daz, selbe lût. Grosse, Schwabensp. 104a, 34 (Hs. ' $\mathrm{nd} . / \mathrm{md}$., um 1410?): Mich süchet vil lites nach miner vfirstende. Helm, H. v. Hesler. Nicod. 4390 (nrddt., 14. Jh.): du [Crist] bist war heil des lutes. Ders., H. v. Hesler. Apok. 5323 (nrddt., 14. Jh.): Lut und vie wirt selic dort. Leman, Kulm. Recht 2, 23 (Thorn 1584): Begrift eyn man eyne iuncorouwe vf eyme ackir, do nicht lute en ist. Fischer, Folz. Reimp. 9b, 100 (Nürnb., um 1485/86): alls es [worber >eine Wahrsagerbeerex] ein richen hot >riecht<, | So iß nicht anders dan leütskot. SACHS 17, 203, 20 (Nürnb. 1557): Der feyhel schmeckt gleich wie leutsdreck! Hulsius L iijr (Nürnb. 1596): Leut / gens. Williams u. a., Els. Leg. Aurea 564, 14 (els., 1362): Von disem zeichen wart vil lutes glǒbig. SCHADE, Sat. u.
Pasqu. 2, 150, 26 (els. 1521): an feirtag, so vil leut züm opfer kumpt in allen dörfern. MERzDORF, Historienb. 673, 3 (alem., Hs. 2. H. 14./A. 15. Jh.): so wart er rich und gewan in demselben zil gar vil lútes und gütes. Adrian, Saelden Hort 5212 (alem., Hss. E. 14./15. Jh.): swaz lútes in der gegene $\mid$ man da sach kainen presten han. SCHMIDT, Rud. v. Biberach 73, 1 (whalem., 1345/60): Verstentnissi ist ein kraft der sel [...], zi ervarne gotlich heimlichi, vnd dar ziv k.vmt wenig luttes'. PÄPKE, Marienl. Wernher 141 (halem., v. 1382): Den ainen tail si măssen | Mit trúwen dem armen lút. Pfeiffer, K. v. Megenberg. B. d. Nat. 32, 25 (oobd., 1349/50): die veigen machent nibt guot pluot und dar umb machent si dem menschen vil leutswürm. EBD. 305, 16: pediculus haizt aigencleich ain füezling. daz ist ain leuteswürml oder ein kintpeiæ, und haizt dar umb ain füezling [...], daz. ez vil füez hât. EBD. 456, 8: Piropholos mag leutsstain haizen. wan [...], der stain ist gar edel und wirt auz ains menschen hery, daz mit vergift ist getatt, wan daz herz mag in feur nibt verprinnen. - Helm, H. v. Hesler. Apok. 18302; Pyritz, Minneburg 1926; Williams u. a., a. a. O. Aurea 632, 24; 671, 29; 750, 16; Baumann-Zwirner, Augsb. Volksb. 1991, 112.

2. >Kriegsvolk; Kriegsknechte; militärisches Aufgebot<. - Bdv.: vgl. kriegvolk.

PäPke, Marienl. Wernher 10004 (halem., v. 1382): Das si [Beine Christi] do von ainander nút $\mid$ Bringen mocht das vaige lút. Bernoulli, Basler Chron. 5, 5, 175, 7 (alem., 1421): die kurfúrsten [...] schribent iederman sine summ lút?, mit wie vil er solte kommen. Chron. STRAssb. 856, 12 (els., A. 15. Jh.): do wart ouch menig schif so vol lütes, das dar. schif underging.

\section{leutbet, leutbrief, s. leute 5 .}

leutbescheisser, s. leute 2.

leutbeschreiber, s. leute 1.

leutding, s. leute 5 .

leute, rhein. vereinzelt: lieden, die; pl. tantum. - Vgl. auch Dwв 6, 873 ff.; Rwв 8, 1262 f.; Verwijs/Verdam 4, 521 ff.; speziell zum Problem der Bedeutungsbeschreibung von nhd. Leute: E. LANG, in U. Kramer, Lexikol.-lexikogr. Aspekte [...]. 2000, 1-40. Die Wortbildungen sind teilweise auch zu leut stellbar, so vor allem bei fehlendem finalem $-e$ des Bw.

1. >Menschen, menschliche Wesen; Bevölkerung eines Gebietes〈; auch: >Menschenfigur<. - Wbg.: leutbeschreiber >Ethnologe $<$, leuteharn, leuteschar, leutefras (ein Spitzname), leutmörder, leutschädig, leutscheu, leutschreiig >ruchbar, bekannt<, leutsterben, leut- 
stube >Badestube, Gemeinbad`, leuttrieger (a. 1532), leutverblender (dazu bdv.: zäuberer), leutverfürer, leutverfürung > Verschleppung von Menschen<, leutverkëufer.

Luther, WA 17, 2, 125, 23 (1525): Darumb sóllt dieser spruch billich die ketzer meyster und leut mórder erschrecken. EBD. 30, 2, 43, 11 (1529): Es sind ihenseit des bergs auch leute. Quint, Eckharts Trakt. 43, 12 (E. 13./A. 14. Jh.): daz. unmügelich ist bî den liuten, da₹ ist mügelich bî gote. Chron. Köln 2, 57, 12 (rib., 1416): volk mit wunderlichen namen: Ludevrais, Kickpot, Rodehunt. BÖMER, Pilgerf. träum. Mönch 4925 (rhfrk., um 1405): Ich han einen stein, der ist dar zu geachtet $\mid$ Das er die lude, [...], unsichtlich machet. Tiemann, E. v. Nassau-S. Kgn. Sibille 149, 10 (rhfrk., um 1435): ich sitzen bie ferre von den lüden / vnd han ouch wedder bette noch stro. VOC. INC. TEUT. o viijv (Speyer um 1483/4): Leut Hoĩes in plurali nũero. Froning, Alsf. Passionssp. 1979 (ohess., 1501 ff.): wan uch die ludeschare | begynnet hassen bie dorch mich. JungBluth, J. v. Saaz. Ackermann 24, 6 (Hs. romd., 1465?): Dein kurze vernunft, [...] wil aus leuten mer machen dann sie gewesen mügen. Sснмітт, Ordo rerum 130, 17 (omd., 1466): Pigmeus clein lutte. Bechstein, M. v. Beheim. Evang. Mt 4, 19 (osächs., 1343): Kümit nâch mir, und ich mache ûch werden fischêre der lûte [LUTHER 1545: menschen Fischer]. Thür. Chron. 1r, 18 (Mühlh. 1599): In der andern [Kammer] waren fleischliche Thier / In der dritten / waren die Leute / vnd jbre Speise [...] / vñ in der bóhe / [...] / woneten / die Vógel. MYLius H 2v (Görlitz 1577): Prastigator Zeuberer / Leuth verblender. CHRON. STRASSB. 51, 24 (els., 1362): und verberjete dar gantze welsche lant an güte, an lüten und an vihe. Kurrelmeyer, Dt. Bibel 2, 211, 32 Var. (Straßb. 1466): und den gemein vnkeuschern der mann beyliger vnd den pflagern den lugnern vnd den mainaidern [Var. 1475-1508: den schlagern oder leút verfúrern]. DAsYPODIUs 135r (Straßb. 1536): Misanthropos, Ein leütscheüch / einer der mit niemants gemeinschafft. BREMER, Voc. opt. 41085 (halem., 1329 f.): Vango lútverkóffer. Plant u. a., Main. Naturl. 296v, 13 (ohalem., Hs. E. 14. Jh.): arabia. dez. lande liute vahẽt, dc iar an in dem sumer. PÄpke, Marienl. Wernher 4555 (halem., v. 1382): Und machtond lúte, als man saget, | Nach dem kinde und nach der maget | Bilde schóne. RoHLAND, Schäden 468 (nalem./schwäb., 1400/33): leg es [berginschmer] ... in lütte harn. EBD.: das die [siechen] j̈e zu wilen badetten in schweiß bädern oder in bütten, nit in den grossen lütt stuben. Morrall, Mandev. Reiseb. 118, 6 (schwäb., E. 14. Jh.): in der ynsel fint man als clain lut als die zwerge sind. Chron. Augsb. 3, 78, 22 (schwäb., E. 15. Jh.): Des jars was ain grosser leutsterben bie. GILman, Agricola. Sprichw. 1, 382, 22 (Hagenau 1534): die verruchten kriegßleutte / und leütmórder haben sich darauff verlassen. Pfeiffer, K. v. Megenberg. B. d. Nat. 7, 34 (oobd., 1349/50): daz die läut und diu tier in den kalten landen babent gestracktez hâr. EBD. 491, 1: diu läutel kindelnt in dem dritten jâr und altent in dem abten. BRÉvarT, K. v.
Megenberg. Sphaera 42, 16 (noobd., 1347/50): daz ist nach der leut angesibt in dem satzie dez augenenders. GROssMANN, Unrest. Öst. Chron. 44, 9 (oobd., 3. Dr. 15. Jh.): [dye Turckhen] tetten da grossen wuest mit pranndt, mordt und lewdtverfuerung. BRUnNer, Rechtsqu. Krems u. Stein 136, 1 (moobd., 1476): und solchs alles in stille handlet, damit das nicht leuthschrayig werde. Munz, Füetrer. Persibein 442, 7 (moobd., 1478/84): vor vinster er lüczel wesste, | wo er zer nacht aw lewten sollte kumen. TuRMAIR 4, 209, 32 (moobd., 1522/33): Die alten geschicht-, lenderund leutbeschreiber, [nennen] all nation [...] gegen mittentag [...] swarz, Moren. Bischoff u. a., Steir. u. kärnt. Taid. 490, 2 (m/soobd., 1608): alle diejhenige so grosse riden oder andere vieb- und sonderlich leutschödige bunt haben. Schützeichel, Mrhein. Passionssp. 184; Sievers, Oxf. Benedictinerr. 31, 13; SснÖNBaCH, Adt. Pred. 19, 25; Moscherosch. Ges. Phil. v. Sittew. 7, 15; Gilman, Agricola. Sprichw. 1, 187, 12; Hulsius O ivv, Schwerz. ID. 14, 627. - Vgl. ferner s. v. abscheide, abseben 3, alum 1, angehen 17, auszälen 6.

2. >Leute, Menschen, Personen im Nähebreich, die als pragmatisch bekannt vorausgesetzt werden`; sie werden demzufolge nicht eigens durch syntaktische oder textliche Bestimmungen charakterisiert. Phras.: (nicht gerne) bei den leuten sein $>$ (nicht gerne) unter Leuten sein ‘; $j n$. in die leute tragen >jn. verleumden`. - Wbg.: leutbescheisser >Betrüger`, leutgeheier (dasselbe; Gw zu geheien >betrügen< Dwв 4, 1, 1, 2, 2346 f.), leutliebig >beliebt<, leutlob >guter Leumund<, leutschinder 1, leutverräter.

SCHÖPPER 63a (Dortm. 1550): außrúffen |angeben |jn die leut in bilden $\mid$ jn die leut tragen. LuTHER, WA 10, 1, 2, 365, 20 (1526): das die leüt sind in sünden gelegen biß über die oren. EBD. 30, 2, 448, 21 (1530): Meinet yhr denn [...], ybr vertzweiffelten leüt verrether vnd Gotts lesterer, das [... . EBD. 30, 3, 419, 2 (1531): der Luther thut dem Kaiser gewallt und unrecht, sonst heisst mans felschlich und widerset:lich angelogen, das er yn dermassen in die leuthe tregt. EBD. 47, 300, 38 (1537): ehr [Gott] saget, ich sols bej den leuthen suchen, bej meinem bruder, der mit mir eine Tauffe [...] hatt. PeIL, Rollenhagen. Froschm. 549, 1346 (Magdeb. 1608): Der Wolff solt bey nacht schleichen gehen / I Jns Haus borchen / grindlich erfabren / | Was jbre Feind für Leute waren. Thiele, Minner. II, 27, 435 (Hs. ' ${ }^{\text {md./rhein., }} 1$. V. 15. Jh. $\left.{ }^{\top}\right)$ : et syn vele lieden nocbtien $\mid$ der $y m$ der nyter cleyn ontsien. KuRz, Waldis. Esopus 4, 50, 90 (Frankf. 1557): Sie nams und gab jm funff Mattheier. I Da lacht derselbig Leutgeheier. v. Keller, Amadis 386, 31 (Frankf. 1561): Denn er die seine für solche Leut hielt, daß er für gewiß achtet, daß Olinas gar nicht zween so gute bekommen köndte. Bolte, Pauli. Schimpf u. Ernst 1, 103 (Straßb. 1522): ein erfarner Schüler [...] ein Lütbescheisser. MAALER 
269r (Zürich 1561): Wenn er nit gern bey den Leüten was. Dict. Germ.-Gall.-Lat. 305 (Genf 1636): Leutliebig / e. Affable, doux, gracieux, courtou. Chron. Augsi. 9, 368, 2 (schwäb., 1532): er fragt die leit am selben ort, |Wo ist die frau, die sas am weg? Barack, Zim. Chron. 3, 211, 25 (schwäb., M. 16. Jh.): Und muess man die leut reden lasen, die gens köndens nit. BAuer, Geiler. Pred. 318, 15 (Augsb. 1508): So vil du mer mit den leüten redest so vil du unsynniger würst. KLEIN, Oswald 112, 96 (oobd., 1438): hat ainer neur ain urtailer | und da bei leute nach der swër, | si volgen al dem selben nach. Voc. Ex QUO F 48 (oobd., 1468): Fama ein geruchte [...] luumont [...] lewt lob. - PYritz, Minneburg 57, 4; Bernoulli, Basler Chron. 6, 210, 28; Kummer, Erlauer Sp. 2, 9; Dict. Germ.Gall.-LAt. 305, 34; Schweiz. ID. 8, 916. - Vgl. ferner s. v. abreden 7, almei, angeben 3 .

3. >Erwachseneく.

Gilman, Agricola. Sprichw. 1, 432, 16 (Hagenau 1534): Auß kindern werden auch leut. - Rwв 8, 1271.

4. >Leute, Menschen, Personen, die durch ein Adjektivattribut so charakterisiert werden, daß der gesamte Ausdruck semantisch weitgehend durch das Adjektiv besetzt wird ‘ die fremden / geistlichen / wilden (usw.) leute können dann als >Fremde / Geistliche / Wilde verstanden werden. Der häufiger (als in der nhd. Schriftsprache) begegnende bestimmte Artikel situiert die Bezugspersonen tendenziell als pragmatisch (meist situationell, auch textbedingt) bekannt. - Synt. (in Auswahl, ansatzweise nach sachlichen Kontiguitäten): die alten / augschwerenden / aussetzigen / blinden / gebresthaften / bitzigen / kranken / lamen / sinlosen / toten / geistlichen / weltlichen / erbaren / erlichen / biderben / freien / grossen / guten [auch: >Aussätzige<; vgl. gut, Adj., 16] / geschworenen / schepfenbaren / volkommenen / gefangenen / frommen / anbetenden / zunemenden / armen [vgl. arm, Adj., 4] / reichen / elenden / heidnischen / israelischen / gebrochenen / losen / bergezogenen / fremden / unnützen / schädlichen / schändlichen / übeltätigen / abenteuerlichen / behenden / bösen / gelerten / schwachen / verfluchten / verfürten / einfältigen / geistlosen / törechten / weisen / jungen / ebelichen / ledigen / wunderlichen / wilden / gedachten l.; alle l. >jedermann〈, andere l. >andere «. Wbg.: leutesel (als Kennzeichnung von buler).
Helm, H. v. Hesler. Apok. 7094 (nrddt., 14. Jh.): Daz. wir unse ougen salben mite $\mid$ Noch der ougswernder lute site. Mieder, Lehmann. Flor. 150, 4 (Lübeck 1639): Wenn Mann vnd Weib einander schlagen / so ist das der Gewinn / daß ander Leut zu lachen haben. Quint, Eckharts Pred. 1, 8, 6 (E. 13./A. 14. Jh.): Diz sint harte tôrebte liute, die alsô koufen wellent mit unserm herren. EBD. 1, 342, 4: ze der âbentwirtschaft ladet man grôze liute und liebe liute. LoEsCH, Kölner Zunfturk. 11, 257, 8 (rib., 1487): umbdat desselven Jacops vader rinsnider gebrochenre lude geweist, [...], deshalven unbequeme were. BECKERs, Bauernpr. 51, 14 (Köln 1515/18): Die frome luyd steruen gern. Gropper. Gegenw. 17r, 973 (Köln 1556): Die armen verfürten leuth / die in jetzberurtem groben Jrthumb stechen. LAMPRECHT, Dt. Wirtschaftsl. 3, 265, 20 (mosfrk., 1418-30): betwang, als dan unsere armen lude undersessen und burgere in den vier dorferen [tegelich lident]. EBD. 3, 300, 30 (1447): und moegen [...] sich auch daselbs bin als andere frie Triesche lude bestaden. KöBlER, Ref. Wormbs 313, 7 (Worms 1499): das sie ime offenlich in bysyn erbarer lute vnd nit heimlich vertrut bett. EBD. 343, 21: võ denen die einen gehessig widerwertig vnwillig oder sust lychtfertig oder klapperich lute syn. FRONING, Alsf. Passionssp. 871 (ohess., 1501 ff.): das der toden viel uffstehen, | und die lamen lude viel gehen. v. D. LEE, M. v. Weida. Spigell 22, 10 (omd., 1487): Zcwischen eblichen lewten. das ist manne und weÿbe, ein naturliche liebe vnd frúntschafft sej. KüTHER, UB Frauensee 275, 40 (thür., 1495): sollenn dy gedachtenn lyge [...] die macht habenn, dennselbenn [sehe] uffzubrechen. Jungbluth, J. v. Saaz. Ackermann 4, 16 (Hs. ' ${ }^{\circ}$ omd., 1465ㄱ): Guter gewissen, freuntholt, getreu, gewere und zumale gütig was sie gen allen leuten. EBD. 10, 11: wie die behenden, ebenteuerlichen, hochgelerten und allerlei meisterschaft wol vermügenden leute, [...], müßen zu nichte werden. SchöNBACH, Adt. Pred. 7, 28 (osächs., 1. H. 14. Jh.): Liebe lite, waz mach erger sin wan daz der mensche hoffe nach dem gite und nibt nach gote? Bindewald, Texte schles. Kanzl. 26, 3 (schles., 1349): daz vor vns [... kumen sint di erlichen luyte Wiske vnde Niclaus peczsch gebrude'. Dinklage, Frk. Bauernweist. 43, 18 (nobd., 1451): das die herschaft haben die recht, auf aller herren gut schadber leut anzugreufen. Fischer, Folz. Reimp. 28, 43 (Nürnb., 1488): So spricht der ein: „sich, der statfarr!" | Der ander: ,jo, ydermans narr." | „Der lewtesel“, heist yn der dritt. Dietrich. Summaria 20r, 24 (Nürnb. 1578): das dise gnadenpredig den weysen vnd verstendigen verborgen / vnd den armen und elenden leutlein offenbart werde. Williams u. a., Els. Leg. Aurea 53, 3 (els., 1362): das blimen vnd dich die vf dem altar sant Stephans worent gelegen, gobent vil gebresthaften lúten gesuntheit. EBD. 163, 3 (els., 1362): Daz den weltlichen lúten ist eine kleine sunde daz ist eime geistlichen menschen eine uil swere súnde. Moscherosch. Ges. Phil. v. Sittew. 36, 32 (Straßb. 1650): Jhr Alte seit wunderliche Leute, sprach ich, vnd in gemein könnet ibr nicht wobl sehen oder leiden, daß Junge Leute auch etwas Freude [haben]. BERNoulu, Basler Chron. 6, 129, 17 (alem., 1526): [die Widertoiffer] baben [...] vil armer fromer einfaltiger lutten verfurt. Welti, Stadtr. Bern 94, 22 (halem., 1401): Als ietr. 
langzit gros rede vnd kelegde gewesen ist von den unsern und ouch von frómden liten. EBD. 106, 18 (1419): gebresten, den biderb lüt habent in großen gechen tóden. EBD. 111, 18 (1343): wie man von kranken alten liuten, [...] kein sunder telle noch güt nemen sol. EBD. 432, 20 (E. 15./A. 16. Jh.): die, so dieselben vnnutzen lut busenn, hoffenn. KLÄUI, Schweiz. Urbare 3, 220, 30 (halem., 1400): die beissent stülsessen, $d z$ waren frije lüt, die bebuben dem gotzhuß sin recht. Stammler, Berner Weltger. 134 (ohalem., 1465): stand vff, jr totte lüttel | Ze gericht mussent jr büttel. EBD. 484: dise verfluichten lüte Sönd wir uerurteilen büte. KÖBLER, Stattr. Fryburg 60, 9 (Basel 1520): Wyber vnd iung lüt under zwentzig iaren / mogen umb lyb und leben nit kuntschafft sagen. EBD. 119, 4: Gebrechhafftig / siñlosz lüt sollen Vogt haben. Gilman, Agricola. Sprichw. 2, 133, 22 ([Augsb.] 1548): Thut doch die Bawren / unnd meine arme leüte auß dem hausse. Morrall, Mandev. Reiseb. 156, 1 (schwäb., E. 14. Jh.): da sind vil vilder lút, [...], die grinent als ain ber. Spechtler, Mönch v. Salzb. 39, 35 (oobd., 3. Dr. 14. Jh.): Ave, volle freud und wunn, | der saligen leben, | armer lewt tröstlicher prun. KLEIN, Oswald 39, 34 (oobd., um 1426?): die armen leut beswär ich ser. BRUNNER, Rechtsqu. Krems u. Stein 53, 21 (moobd., 1393): zu gleicher weis, als die anderr herren gaistlicher und weltlicher leute in unserm land raichent. Grothausmann, Stadtb. Karpfen 16, 27 (mslow. inseldt., 1512): feśt gePetten, den bemelten Armen Leütten śeinen Vnterśaśsen [...], gnad Zue bewaisen. EBD. 25, 2 (1501): Iśt er durch gePett guetter Leüt abgePetten worden. EBD. 34, 26 (1604): vnd herr Rośenman es mit guten leuten widerumb verglichen. - Grosse, Schwabensp. 226a, 3; Koller, Reichsreg. Albr. II. 168, 7; Sievers, Oxf. Benedictinerr. 33, 11; BuchDA, Schöffenspr. Pössneck 4, 217; Gille u. a., M. Beheim 194, 4; Thiele, Minner. II, 14, 179; Gilman, Agricola. Sprichw. 1, 4, 25; 1, 463, 21; 1, 523, 6; SudHofF, Paracelsus 2, 254, 25; 14, 154, 11; GRAF-Fuchs, Ämter Interl./Unterseen 302, 14; 307, 7; Welti, Stadtr. Bern 103, 32; Ott-Voigtländer, Rezeptar 216r, 14; SAPPler, H. Kaufringer 27, 19; BAumAnN, Bauernkr. Oberschw. 111, 5; Klein, a. a. O. 111, 172; Pfeiffer, K. v. Megenberg. B. d. Nat. 7, 28; Piirainen, Stadtr. Sillein 41a, 14; 56a, 27; 70a, 8; DERs., Igl. Bergr. \32; Cirullies, Rechtsterm. Anh. 1981, 245; PFälz. Wв. 4, 964. - Vgl. ferner s. v. abenteuer 6, abtrünnig 1, affengespenst, anbeben 5, ${ }^{2}$ alber, bedunken 3, israelisch.

5. >einem Herrscher, einem mit Herrschaftsaufgaben Beauftragten, einer Grundherrschaft, auch z.B. dem Satan, rechtlich / tatsächlich in unterschiedlicher Form der Abhängigkeit Zugeordnete, Untergebene`; mit Tendenz zu >Untertanen`; auch: >einem Geistlichen anvertraute Gemeindemitgliederく. - Synt.: die l. betreffen, js. l. um schuld aufhalten, die l. als eigene leute versprechen, $j m$. die l. absetzen (>Anspruch auf sie erhe- ben (), dem Sathan die l. abfangen; jm. l. werden, die l. zins geben, zu recht stehen, culmisches recht haben; den leuten schwer sein / tun; reich an leuten (sein), glauben auf die l. setzen, anspruch bin zu den leuten gewinnen, etw. mit den leuten beweisen, über die l. richten, segnung über die l. sein, die kunst jn. um die l. bringen; l. des herren / mannes, der herschaft; leben / recht / sitte der l.; die eigenen l.; in Formeln: land und leute; leute und gut / habe; leute und untersassen; diener, bürger und leute; leute und grund und boden; land, leute und untertanen; leute, land und (andere) rechte; leute, gerichte und dingstätte; leute, land, stand und eren. Wbg.: leutbet, leutbrief >Verzeichnis der Hörigen` (a. 1554), leutding $>$ meist ungebotenes, mit landsässigen Bauern besetztes Landgericht< (Rwв 8, 1274), leutehof $>$ Pfarrhof $<$, leutmarkt $>$ Sklavenmarkt $<$, leutschinder 2.

Thielen, Gr. Zinsb. Dt. Ord. 124, 22 (preuß., 1437/ 8): her hot polnisches recht, seine leute haben col[misch] recht. Luther, WA 10, 2, 114, 2 (1522): Wilche sind die [Bepstische Bischoff]? [...] bürle haben, [...], bübsch bengst reytten, fürsten böff hallten, officiales, das ist leut schinder, neeren. EвD. 37, 39, 35 (1533): da regire die ochsen, kue [...], gesind, stad, land und leut. EвD. 41, 62, 18 (1535): Rex habet geld, gut, land, leut, schlosser und sted. Grosse, Schwabensp. 89a, 14 (Hs. 「 $\mathrm{nd} . / \mathrm{md}$., um 1410?): Swer den liten icht tüt, der roubet daz, gottes bis vnde ouch den heren, des len se sin. Holland, H. J. v. Braunschw. V. e. vngerat. Sohn 338, 23 (Wolfenb. 1594): wil ich meinem eltesten Sohn Probo die Regierung, vnd Land vnd Leute nach meinem Tod aufftragen. Schade, Sat. u. Pasqu. 1, 9, 74 (o. O und J.): Daß in [bauren] bleibt weder haut noch har, | Daß sie ir leutbet möchten geben. LAMPRECHT, Dt. Wirtschaftsl. 1230, 6 (mosfrk., 14. Jh.): quod omnes homines, [...7, quilibet eorumdem tenetur unum d. in ultimo termino videlicet feria secunda post Iohannis, qui dicitur luitdink, et ex illis denariis dantur cellerario domini Treverensis 3 s. et 6 d. VAlLI, Baldemann 243 (rhfrk./nobd., um 1350): Sus wart mir cron, lant, lute. Eggers, Psalter 4, 7 (thür., 1378): Gotis ist daz heil, vñ vbir dine lute [Luther 1545, Ps. 3, 9: Volck] ist din segenunge. Thiele, Chron. Stolle 517, 15 (thür., 3. Dr. 15. Jh.): also solches unser person selbest, unser eygen land, luthe unnd underthan betreffe. ERmiscH, Freib. Stadtr. 240, 10 (osächs., 1335): Eines iklichen herren [...] lute, [...], der da schuldic ist bi in der stat, di heldet man wol uf vor in umme di schult mit rechte. DinkLage, Frk. Bauernweist. 56, 12 (nobd., 1370): Es hat auch die herschaft die recht, das sie alle die leut, die uff dem Eigin sietzen, sollen versprechen als ir eigin leut. [...] und sollen die leut niergent zu recht steen dann vor der vorgeschrieben herschaft. [...] denselben $\operatorname{zin} \beta$ sollen die leut [...] geben der vorgeschrieben herschaft. 
ReichmanN, Dietrich. Schrr. 234, 7 (Nürnb. 1548): Deñ damit [wort] fehet man jhm [Sathan] die leut ab. Schade, Sat. u. Pasqu. 3, 181, 3 (obd. 1525): ein bischof beißt ein ufseher, der halb im gebürt, daß er acht hab und sorg auf das leben und sitten seiner leuten. CHRON. STRAssB. 754, 14 (els., A. 15. Jh.): do ging ein für uf in unser frowen lütehof zu Grienecke. MAALER 269r (Zürich 1561): Leütmarckt / ort da man leybeigen leüt feil hat vnd sy verkaufft. Venalitium. Gilman, Agricola. Sprichw. 2, 172, 32 ([Augsb.] 1548): Es ist auch der Tirannen art / das sy auff ire leütte kainen glauben setzen / Sondern auff frembde leüte bawen sy. KLEIN, Oswald 112, 157 (oobd., 1438): wo gaistlich berschen leut und lant, | da wirt mer ungeleichs erkant, | wann fürsten. Thiel u. a., Urk. Münchsm. 226, 37 (moobd., 1478): das kain werntlicher richter [...] nichts zu richten sullen haben in vnnserm gotzhaws [...] weder vber leutt noch vber guet dan vmb dreyerlai sach, das ist dewb, totslag vnnd notnuft. MeLL, Steir. Weinbergr. 110, 20 (smoobd., 1543): ander herren leut, die auch perkgnossen sein. BAPTIST-HLAwATsch, U. v. Pottenst. 176 (moobd., A. 15. Jh.): Da werden die posen berren gestraffet die iren lewten swër sind. - Grosse, Schwabensp. 107a, 21; PeIL, Rollenhagen. Froschm. 585, 2516; Merk, Stadtr. Neuenb. 27, 11; Hör, Urk. St. Veit 97, 8; Turmair 4, 37, 14; Bischoff u. a., Steir. u. kärnt. Taid. 243, 38; DoubeK u. a., Schöffenb. Krzemienica 295; Rwв 8, 1273. - Vgl. ferner s. v. absetzen 2 , anrede 1, anschlag 7, arbeiten 9, aufbeben 30 .

6. > einem Herrscher, einer kriegführenden Partei (o. ä.) zugehöriges bewaffnetes Volk, Soldaten, Heeresangehörige<; als Spezialisierung zu 5 auffaßbar. - Synt.: die l. erschlagen / fangen, so viel l. bringen, als [...]; die l. (Subj.) jn. anbegreifen, ab den kielen stehen; den leuten die zeitung sagen; das tor mit leuten besetzen, jm. etw. mit leuten bestellen; die gemeinen l. >Kriegsknechte<, die erlichen / guten l. >vornehme Kriegerı; die l. von der landschaft.

PeIL, Rollenhagen. Froschm. 590, 2653 (Magdeb. 1608): Die Rómer / so auff kundschafft giengen / | Sagen die zeitung jbren Leuten. Tiemann, E. v. Nassau-S. Kgn. Sibille 155, 26 (rhfrk., um 1435): Der konnig von Coine kame zu erste yme dar / vnd brachte so viel lude / als er gehaben konde. KarneIn, Salm. u. Morolf 380, 3 (srhfrk., Hs. um 1470): roß und lüte bieß er abe den kielen stan. v. Tscharner, Md. Marco Polo 25, 1 (osächs., 2. H. 14. Jh.): do sint me wen $X$ tusint geczelt uf geslagin siner lute. Neubauer, Kriegsb. Seldeneck 73, 13 (nobd., 2. H. 15. Jh.): an den eussersten zweyen zeilnn soln liegen die gemeinen guten leut vonn der landschafft. KLEIN, Oswald 26, 46 (oobd., 1427): zwar oben, niden, binten, vor | was mir die büt mit leuten wolbestellet. Moscouia Cijr, 27 (Wien 1557): der óberste Haubtman vnnd vil gueter leüt waren gefangen. - Neubauer, a. a. O., S. 174; Chron. Augsb. 1, 65,$18 ; 248,2 ; 5,345,27$; Primisser, Suchenwirt 28, 243. - Vgl. ferner s. v. anbegreifen.

leuteharn, s. leute 1.

\section{leutehof, s. leute 5 .}

leutenant, leutener, der; -s/ -leute, auch - $\emptyset$ (für erstere Form); aus frz. lieutenant, dies letztlich aus locum tenens (KLUGE/S., 2002, 572), insbesondere bei der zweiten Form volksetymologische Anlehnung an leute 6.

>Offizier unterhalb des Hauptmannes`, mehrfach in seiner Funktion als Vertreter eines Vorgesetzten angesprochen. - Vielfach berichtende Texte. - Bdv.: befelshaber, befelsman, fäner, fänrich, feldweibel, hauptman 2, obrist, schütze, söldner, stathalter, verweser. Wbg.: leutenantschaft (a. 1617).

LutHer, WA 49, 735, 32 (1545): Der oberste Leutenant oder Heubtman, dem der Kriegsfurrst das Feld befoblen hat. Chron. Magdeb. 2, 50, 28 (nrddt., 1565/6): ist Hans von Cöln, des obersten in Magdb. Leutenantt, [...] mit 24 kanen zu wasser ausgefallen. HАMPE, Nürnb. Ratsverl. 1, 498, 27 (nobd., 1554): sol man ine anbaims vordern unnd schreyben, seinem leutenampt bevelch zu thun, ine mittler zeit zu vertretten. BeLl, G. Hager 411, 2, 1 (nobd., 1595): Der leiden ambt von papa $\mid$ war ein hussar vnd ein christ. Golius 169 (Straßb. 1597): Legatus ducis, des Hauptmans / Statthalter / Leutenant. EвD. 185: Legatus classis, des óbersten der Armada Leutenant. Bernoulli, Basler Chron. 6, 44, 2 (alem., 1513): was herr Hans Stoltz houptman, Ulrich Falckner lütener. V. Anshelm. Berner Chron. 3, 13, 25 (halem., n. 1529): so also allenthalb boptlút, lútiner, venner, und derglich doppelsóldner gesezt. DieHL, Dreytw. Essl. Chron. 54, 25 (schwäb., 1549): Also seind die bemelttenn 5 baupttleutt nebenn einander geryttenn, auff sey ire 5 leuttenamptt, auff sie ire 5 fenderich. CHron. Augsb. 8, 418, 1 (schwäb., zu 1556): welche seine bauptlent, leutenambt, fendrich, feldwaibel gewesen, [...] wird alles nacheinander verzaichnet. - PEIL, Rollenhagen. Froschm. 635, 4040; Sснів, H. Stockar 69, 20; Ukena, Zuger Trag. 720; Welti, Stadtr. Bern 664, 33 f.; Chron. Augsb. 8, 420, 9; Maaler 275v; Hulsius L iijr; Dict. Germ. Gall.-Lat. 305, 32; Eckel, Fremdw. Murners. 1978, 127; Jones, French Borrowings 393; 397; SchweIz. ID. 3, 1527.

leutenantschaft, leutener, s. leutenant.

leutern, s. läutern.

leuteschar, s. leute 1.

leutesel, s. leute 4.

leutfras, s. leute 1.

leutfresser, der; $-/-\emptyset$.

>Menschenfresser, Kannibale<; meist ütr., dann: >Gewalt ausübender Kraftkerl; 
Großmaul, rücksichtsloser Machtmensch`; vgl. leute 1. - Bdv.: vgl. Kannibale, menschenfresser.

SCHÖPPER 67b (Dortm. 1550): Tyrann witerich. blutbundt vnderdrucker lewtfresser. LuTHER, WA 10, 1, 1, 620, 17 (1522): Ditterich von Bern oddr Hildebrantt odder Roland, odder wie man sonst dieselben grossen morder unnd leuttfresser nennen will. EBD. 621, 12 (1522): Bapst, Cardinal, Bischoff, pfaffen, [...] sind die letzten und mechtigisten kerle, ryßen, leuttfresser und Herodes. EвD. 15, 413, 6 (1524): Herodes tentonice, riß', [...] Saxonice, kerl', die grossen leutfresser, ut Dittrich von Bern, qui multa fecerunt. EBD. 16, 208, 31 (1524): das er [Herrgott] den Scharrbansen und Leutfressern den mut nimet. EBD. 17, 1, 329, 31 (1525):

1. Co. 15. mors', du leutsfresser, ubi nunc aculeus' mortis, quo yderman perimitur, ubi est? DAsYPODIUs 323v (Straßb. 1536): leutfresser, $m$. anthropophagus. - LuTHER, WA 10, 1, 1, 630, 3; Hulsius E ivv.

leutgebe, s. leitgebe.

leutgeben, s. leitgeben.

leutgeheier, s. leute 2.

leuthaus, s. leithaus.

leutkauf, s. leikauf.

leutkilche, die; auch -kirche.

>Pfarrkirche (im Unterschied zur Klosterkirche)<; vgl. leute 5 (letztgenannte Nuance), ${ }^{1}$ kilche 1; 2. - Alem.; Rechts- und Wirtschaftstexte, auch Chroniken.

Boos, UB Aarau 73, 2 (halem., 1351): daz si dar inne gotes dienst begen und haben, aslo daz ez der lewtkylichen daselbs ze Arow [unschedlich sei]. Welti, Stadtr. Bern 89, 29 (halem., 1370): waz der liden sol, der deheinen freuel oder einung begat in dem kilchof der litkilchen. MERz, Urk. Bremgarten 350, 3 (halem., 1447): als wir vnsser lutkilchen langczitt ettwas kernengelcz schuldig sind. MüLLER, Stadtr. Ravensb. 166, 8 (oschwäb., 1373): Wenn ain lihe ist oder mer bie ze der lutkirchen, so ez verschlagen ist und man die lut an daz velt let, daz denn niement sol der lich messen noch opfrent. - Chron. STrassb. 730, 13; MERK, Stadtr. Neuenb. 150, 22; 151, 28; ToBler, Schilling. Bern. Chron. 1, 60, 9; Rennefahrt, Stadtr. Bern 283, 28; KLÄUi, Urk. Hermetschwil 46, 3; 68, 9; Dierauer, Chron. Zürich 215, 17; Rwв 8, 1278. - Vgl. ferner s. v. bare 2 .

leutliebig, s. leute 2.

leutlob, s. lente 4.

leutlos, s. leut 1.

leutmarkt, s. leute 5 .

leutmörder, s. leute 1. leutpriester, der; $-s /-\emptyset$.

>Pfarrer, Weltgeistlicher (im Unterschied zum Ordensgeistlichen), der in Vertretung des Kirchherren die Seelsorge gegen Entgelt ausübte<; vgl. lent 1 , priester 2. - $\mathrm{Zu}$ Wort und Sache: K. Kunze, in P. Kesting, Würzb. Prosat. 2, 1975, 35-76; Rwв 8, $1282 \mathrm{f}$. - Starke Beleghäufung für das Alem. - Bdv.: s. u. SCHÖPpeR, ferner: kaplan, priester 2. - Synt.: den l. laden / erfordern / setžen / wälen / präsentieren; der l. jn. bannen, sünde erkennen, jm. das sacrament versagen; dem l. etw. gebieten / befelen / geben; die kilche mit einem l. besetzen, sich an den l. keren, jn. zu l. machen; der l. des gotteshauses / ordens; der gute l.; das haus, der rat des leutpriesters. Wbg.: leutpriesterei.

Schöpper 64b (Dortm. 1550): Pastor. Pfarrherr pastor pfärner seelsórger leutpriester kirchberr templierer. LEMAN, Kulm. Recht 2, 5, 46 (Thorn 1584): vyndet eyn man gut vffe der vryben strasse boben der erden. das sal her deme nesten lutpryster geben [...] vnd her sal also sprechen. Das gut beuele ich vch vf vwir truwe [...]. So sal der lutprister alle syn tage in syner prediget [...] kundigen daz do gut gefunden sy. Williams u. a., Els. Leg. Aurea 582, 20 (els., 1362): Es was ein lútpriester, der enkunde keine ander messe denne alleine von vnser frowen. ILling, Albert. Sup. miss. 2244 (els., n. 1380): ein lü[t]prister sólte offenliche eime súnder das sacremente nút versagen, so er zu dem alter offenliche keme vnder andern lüten, vmbe beimeliche súnde, die der lú $t$ tpriester erkante in der bibte. Merk, Stadtr. Neuenb. 172, 27 (nalem., 1478): zu welcher zeit ain luprister oder caplon, er sige elich oder nit, [...] mit tod abschaiden wirt. SCHADE, Sat. u. Pasqu. 3, 281, 1 (Straßb. um 1545): der pfarrherr [...] schicket sie [weib] züm dechan seines capitels, der dechan zim leutpriester des stifts seines bistumbs. [...] darnach schickt sie der priester zum erzpriester: der spricht ir dann die lang absoluz: VetTer, Schw. zu Töß 87, 37 (Hs. 15. Jh.): hat mir der gut lüppriester von Bichlense gelert sprechen, wenn ich nit gnaden het, und das was also: „Her, [...]“. Rennefahrt, Staat/Kirche Bern 13, 29 (halem., 1324): die selben [buch] sol ein lupriester da selbentz an kettenen machen in einer librari. Ders., Stadtr. Bern 352, 26 (halem., 1401): daz si und ir nachkomen [...] die ... kilchen ze Grenchen [...] mógent frilichen besetzzen mit einem kilchherren oder lüppriester. MAag u. a., Habsb. Urbar 2, 1, 745, 4 (alem., 1394): Die burger wellent ain schultheizzen; si wellent ouch ainen litpriester. Welti, Stadtr. Bern 247, 19 (halem., n. 1437): die sol und mag vnser lupriester und vnser ertapriester darumb bannen vnd mit geistlichem gerichte wisen, zu lassen von dem vnrechten [Ehebruch]. BONER, Urk. Aarburg 87, 70 (halem., 1484): das járlichen [...] uss unnserm korncasten zi Arburg vier malter dinckell [...] einem pfarrer oder 
lütpriester werden geben. HaAs u. a., Erasmus/Jud. Klag 1r, 7 (Zürich 1521): durch meister Leo Jnd lütprister des gots huß Einsydlen vertütscht. BREMER, Voc. opt. 36039 (halem., 1328 ff.): Plebanus lutpriester [...] Plebanus est, qui habet curam animarum plebis in aliqua parrochia constituta. Et dicitur [plebanus] a plebe. Merz, Urk. Lenzb. 23, 3 (halem., 1413): von eins lütpriesters wágen, den sy ze erwelen und einem kilchberren ze presentieren haben, das im der liche vnd in denn bischoff inuestier, vnd ouch das ein lütpriester keinen sigristen nit setz, one der von gmeinen burgeren erwelt werde. Chron. Augsb. 2, 214, Anm. 1 (schwäb., Hs. 16. Jh.): die maur bey der thumbrobstey und lewpriesterey niederzewerffen. v. Maren, Marquard. Ausgabe 40, 5 (Venedig 1483): ist man gebunden in sechserlày weise den egistlichen vater zu eren als den babst und den bischof und den lewtbrister und den beichtiger. - Chron. STRAssB. 566, 20; Leisi, Thurg. UB 8, 222, 16; Bernoulli, Basler Chron. 6, 457, 1; KLÄUI, Schweiz. Urbare 3, 224, 21; Schib, Urk. Laufenb. 84, 5; 169, 21; Rennefahrt, Recht Laupen 145, 28; Nyberg, Birgittenkl. 1, 272, 13; Chron. Augsb. 2, 214, 4; Müller, Stadtr. Ravensb. 81, 23; Voc. Teut.-Lat. y viiv; Rwв 8, 1287; SCHWEIZ. ID. 856

\section{leutpriesterei, s. leutpriester.}

\section{leutschädig, leutscheu, s. leute 1.}

leutschinder $1 ; 2$, s. leute $2 ; 5$.

leutschreiig, s. leute 1.

leutsdrek, s. leut 1.

\section{leutselig, Adj.}

sgegen andere zuvorkommend, offen und freundlich; höflich und dadurch in die Gesellschaft passend (von Menschen, menschlichen Handlungen und Haltungen); schön, anmutig (von Sachen) ‘; vgl. leut 1 , leute 1 ; 2. - Gehäuft Texte der Sinnwelt ,Religion'. - Bdv.: s. u. ScHöPper, ferner: behegelich 1, edel, geschlacht, holdselig, lind (Adj.) 2, ordlich, sanft, schön, sitlich, zü̈htig. Ggs.: menschenfeind. - Synt.: l. sein; l. antworten / reden; der leutselige anblik, die leutselige gebärde / ordnung / stat, das leutselige kind / weib, leutselige füsse.

Schöpper 11b (Dortm. 1550): Comis. Lieblich freundtlich grüßbar gesprách leutselig gútig. LuTHER, WA 10, 1, 1, 98, 6 (1522): Alßo nennen die naturlich meyster etlich thierer menschenlieber odder leuttselig, alß da sind die hund, pferd. EBD. 22, 391, 17 (1544): wie er [Herr] gemenget ist mitten unter das Volck als ein freundlicher, leutseliger Man. VolKMAR 201 (Danzig 1596): leutselig / holdselig. PeIL, Rollenhagen. Froschm. 226, 5606 (Magdeb. 1608): weil ich so leutselig war / | Dem Wandersman kroch aus dem Weg / | Das er sicher gieng seine steg. МЕјјвоOм, Pilgerf. träum. Mönch 6760 (rib., 1444): Ich leiden sij [lude] ouch ind setzen | Up luytzliche stede sich zo ergetzen. STRAUCH, Par. anime int. 72, 36 (thür., 14. Jh.): daz Got sine irweltin mit also luitseligir ordenunge der vollincumminheit brengit zu sime glichnisse. SACHS 19, 324, 6 (Nürnb. 1563): Daß er [knabe] wird zü̈htig und geschlacht, | Ihn sittlich und leutselig macht. Thiele, Minner. II, 24, 107 (Hs. rwobd., 15. Jh. ${ }^{\urcorner}$): eyn lutzelich wyf kan winder werken. CHron. Strassi. 298, 22 (els., A. 15. Jh.): Troeye was die witeste stat an begriffe, die lütseligeste an gezierde. BIHLMEYER, Seuse 216, 24 (alem., 14. Jh.): O du [Herr] lútseliger anblik aller beiligen. EBD. 311, 16: so út lútzeliges, út vróliches oder búgliches in dinem müte oder libe uf stet, so hab einen geswinden ker mit einem uftragenne in got. ADRIAN, Saelden Hort 10407 (alem., Hss. E. 14./15. Jh.): so lútesalig gebarde | gewan nie wib uf erde. Pfeiffer, K. v. Megenberg. B. d. Nat. 48, 27 (oobd., 1349/50): kurz, und leutsalig füez. bedäutent ainen unkäuschen menschen. TurmaIR 4, 864, 24 (moobd., 1522/33): Hadrianus Antoninus Pius ist [...] under allen fürsten der güetigist und lindist senftest leutsäligest gewesen. - LuTHER, WA 10, 1, 1, 97, 3; 26, 89, 29; Strauch, a. a. O. 120, 27; SAchs 14, 37, 5; 136, 36; Pyritz, Minneburg 72, 36; 120, 27; Bihlmeyer, a. a. O. 22, 7; Adrian, Saelden Hort 4410; Alberus kk ijr; Dict. Germ.-Gall.-Lat. 305, 38. - Vgl. ferner s. v. behegelich 1 , behende 2 , behüten 9 .

\section{leutseligkeit, die.}

>von Gott, einer Person oder ihrer Haltung (u. ä.) ausgehende, auf den Mitmenschen gerichtete Freundlichkeit, gnädige Höflichkeit, liebevolle Zuwendung`. Bdv.: s. u. SCHÖPPER, ferner: clemen₹, demut, gezierde 1, gnade 9, holdseligkeit, schönheit. Synt.: der l. nachdenken; zu l. geneigt sein, mit l. in js. gunst kommen, von der l. gelokt [sein]; die l. dei; die zarte l.

SCHÖPPER 11b (Dortm. 1550): Comitas. Lieblichkeit freundtlichkeit leutseligkeit gútigkeit. LuTHER, WA 49, 639, 5 (1544): Ista est, die freundlickeit und leutseligkeit Dei nostri Salvatoris', sic appellans freundlickeit. Est vir ausdermassen gútig, kan die leute wol umb sich leiden. [...] qui den leuten nachleufft und wil umb sie sein. Non ut mendici, qui den leuten stelen. Sed sic ,leutselig', das er inen múge helffen. Alberus $\mathrm{r}$ ijv (Frankf. 1540): Philanthropus, leutselig / der die leut lieb hat. Philanthropia. leutseligkeyt. OоRsснот, Spee/ Schmidt. Caut. Crim. 230a, 38 (Frankf./M. 1649): Herren seind gemeinlich zur Clemenz / Gnade / vnd Leuthseeligkeit insonderbeit geneigt. Thiele, Minner. II, 31, 680 (Hs. ' ${ }^{\mathrm{md}}$./rhein., 1. V. 15. Jh. ${ }^{\top}$ ): ein l ich dir gloesen kan | das mit lutselkeit ein man | sal komen in der wibe gonst. Bihlmeyer, Seuse 378, 9 (alem., 14. Jh.): so man aller lútselikeit, gezierde, schonheit ie grundlosklicher na gedenken 
kan, so man es ie úberswenklicher in dir [Herr], zartes lieb, vindet. - PeIL, Rollenhagen. Froschm. 19, 23; Luther, WA 24, 333, 31; v. BIRKEN. Erzh. Österreich 74, 10; Bihlmeyer, a. a. O. 14, 29; 452, 18; Dict. Germ.Gall.-Lat. 305, 40. - Vgl. ferner s. v. ausgesamnen, leutselig.

leutskot, s. leut 1 .

leutsstein, s. leut 1.

leutsterben, leutstube, s. leute 1.

leutswurm, s. leut 1.

leuttrieger, leutverblender, leutverfürer, leutverfürung, leutverkäufer, s. leute 1.

leutverräter, s. leute 2.

levanda, s. levieren.

levant, Name; aus ital. levante >Osten< (Pfeifer, Et. Wb. 2000, 796).

1. >Osten (als Richtungsangabe)<.

Haszler, Kiechels Reisen 310, 33 (schwäb., n. 1589): Von gemelter sacristey bey dreyssig paß zur linckhen hanndt gögen Levante vom chor ein cleine capell. KLEIN, Oswald 17, 20 (oobd., 1410): Die brüff (ital. provo >Bug〈) ze hant ker in levant, $\mid$ und nim ze hilf an allen tant $\mid$ den wint ponant.

2. >Ostwind

KLeIN, Oswald 20, 2 (oobd., 1415): Es seusst dort her von orient $\mid$ der wind, levant ist er genent. - Vgl. ferner s. v. alabanda.

\section{leviathan, der.}

Name eines biblischen Meeresungeheuers, das in unterschiedlicher Gestalt auftritt: als Walfisch (s u. LuTHER, Hiob 41, 1; vgl. auch Ps. 104, 26), oder als Schlange (Jes. 27, 1) ; ütr. gebraucht zur Kennzeichnung des Chaos, der Gottesferne, des Bösen und Teuflischen. - Meist Texte der Sinnwelt ,Religion'.

Helm, H. v. Hesler. Apok. 4882 (nrddt., 14. Jh.): Daz her sich nicht liez betoren | Den argen Leviathan. LuTHER, WA 32, 41, 14 (1530): Meinestu auch, [...], das du den Leviathan wolst auferziehen mit einem hammen und sein zunge mit stricken binden? Ders. Hl. Schrifft. Hiob 3, 8 (Wittenb. 1545): Es verfluchen sie die Verflucher des tages / und die da bereit sind zu erwecken den Lewiathan. EBD. Hiob 40, 20 (Marg.): [Behemoth] Heisst alle grosse vngehewre Thier. Wie Leuiathan alle grosse vngehewre Fische. Aber dar vnter beschreibet er die gewalt und macht des Teufels und seines Gesinds / des gottlosen Hauffens in der Welt. EBD. Hiob
41, 20: KAnstu den Leuiathan ziehen mit dem hamen / vnd seine Zungen mit einem strick fassen? EBD. Hiob 41, 20 (Marg.): Leviathan nennet er die grossen Walfisch im meer / Doch darunter beschreibt er der welt Fürsten / den Teufel mit seinem Anhang. EBD. Jes. 27, 1: $Z V$ der zeit wird der HERR beimsuchen mit seim harten / grossen und starcken Schwert / beide den Leviathan / der eine schlechte Schlange / und den Leviathan / der eine krumme Schlange ist / vnd wird die Drachen im Meer erwürgen. Fastnachtsp. 12, 24 (nürnb., v. 1486): Die dritten drei [... | Got speist auß seinen wirden hoch | Vom eingehurn biß auf den floch; | Die vierden drei got unbefilt $\mid$ Stet mit dem leviassan spilt. SuDHOFF, Paracelsus 8, 43, 29 (1530): Jr seind aus der schlangen art und lassen euer vergiften nicht; wo irs anderst mögen auslassen, so muß es sein. darumb aus der leviathan art seint ir mir gehaß. - EBD. 12, 214, 3. - Vgl. ferner s. v. anhangen 7.

levieren, V.; letztlich aus lat. levare $>$ heben`(GEORGES 2, 628).

>aufziehen, aufkommen, sich erheben (von einem Unwetter) <. - Bdv.: vgl. aufwelgen, berkommen 6. - Wbg.: levanda.

Mathesius, Passionale 43v, 23 (Leipzig 1587): Mein trewer Knecht wird es wol außrichten / Aber er wird mussen eine leuanda zunor / oder Hebe haben / vnd eine Thruma sein / welche den Namen vom Erbóben oder Erheben hat. MülleR, Welthandelsbr. 231, 2 (schwäb., 1508): daß durch fortuna, levieren des sturmwetters oder [...] daß guetter da an landt komen.

levit, der; -/en.

1. >Angehöriger eines der zwölf Stämme Israels, benannt nach dessen Stammvater Levi, dem dritten Sohn Jacobs (Gen. 29, 34) <. Die Leviten wurden in 5. Mose 1, $47 \mathrm{f}$. dazu bestimmt, die ,Priester' im Volk Israel zu stellen; folglich: >jüdischer Priester; Tempeldiener $\triangleleft$ - Texte der Sinnwelt ,Religion', oft Bibeltexte. - Bdv.: ambächter, pfaffe 1, pförtner, priester, epistler 1. - Wbg.: levitenstand.

LuTHER, WA 8, 516, 5 (1521): Es nabmen wol die Leviten vom volck J srael yhre opffer, aber sie assen nichts von dem, das gott sollt geopffert werden. EBD. 10, 1, 2, 364 (1526): der Samariter [...], der kumpt her und thit mehre den der Priester und Levit. EBD. 10, 3, 341, 13 (1522): Nun haben die allerbailigisten leüt, als priester und leviten, dem gesatz nit gni̊g mügen thin. EBD. 31, 1, 399, 28 (1530): als da sind Vaterund Mutterstand, Priester-stand, Leviten-stand nach dem gesetz. Mosi. EBD. 46, 728, 14 (1537/8): das sie [Kinder von Jsrael] den Leviten jre narung und unterhaltung geben solten. EвD. 47, 377, 29 (1538): Es muste alles den Priestern und Leviten beimfallen, damit sie unterbalten [...] werhen. EBD. 
52, 566, 32 (1544): Daher denn solche kleider [prieserliche Amtstracht] zum teil noch den namen haben und Leviten röck heissen. Ders, Hl. Schrifft. 4. Mose 1, $47 \mathrm{f}$. (Wittenb. 1545): Aber die Leuiten nach jrer Veter stam wurden nicht mit unter gezelet. Vnd der HERR redet mit Mose / und sprach. Den stam Leui soltu nicht zelen [...] wenn man reisen sol / So sollen die Leuiten die Wonung abnemen. EBD. 5. Mose 18, 1: DJE Priester / die Leuiten [MenteL 1466-1470: ambechter] des gantzen stams Leui sollen nicht Teil noch Erbe haben mit Jsrael / Die opffer des HERRN und sein erbteil sollen sie essen. EBD. Ri. 17, $11 \mathrm{ff}$.: DER Leuit trat an zu bleiben bey dem Man / vnd er bielt den Knaben gleich wie einen Son. Vnd Micha füllet dem Leviten die hand / das er sein Priester ward [...] Nu weis ich das mir der HERR wird wolthun / weil ich einen Leuiten zum Priester habe. EвD. Apg. 4, 36: JOses aber [...] vom geschlecht ein Leuit aus Cypern. Feudel, Evangelistar 4, 2 (omd., M. 14. Jh.): santen dy Juden von Jherusalem pristere unde leviten cqu Johanni. Bechstein, M. v. Beheim. Evang. 71, 8 (osächs., 1343): Marcus êwangelista gotis irwelte und Pêtri sun in der toufe [...], ein Lêvîte nâ dem vleische und ist bekart zu dem gloubin Christi. Fischer, Folz. Reimp. 9a, 128 (Nürnb. 1479): In dem do kam der böchst levit | In gancz. levitischem gewant. EBD. 132: Nun der levit het vor im [Rabbi] ston | Ein napf. Reichmann, Dietrich. Schrr. 225, 8 (Nürnb. 1548): Denn Hohepriester / Leniten / Phariseer / Herodes / Pilatus / alle waren sie wider jn. Kurrelmeyer, Dt. Bibel 6, 239, 11 (Straßb. 1466): das do gesament hetten die leuiten und die portner von manasse. ADrian, Saelden Hort 2432 (alem., Hss. E. 14./ 15. Jh.): die juden in den ziten | priester und leviten | santen aber zu Johanne. - Luther, WA 10, 1, 1, 262, 2; Ders. Hl. Schrifft 5. Mose 14, 27; 16, 4; 18, 6; 26, 12; Ri. 17, 7; 9; Lk. 10, 32; Bechstein, a. a. O. Lk. 10, 32; DAsypodius 112r. - Vgl. ferner s. v. benennung 2, gottesdienst 2 .

2. im Christentum: >Diakon, Hilfspriester, der bei kirchlichen Zeremonien assistiert, Kirchendiener, häufig die Person, die aus dem Evangelium vorliest «. - Bdv: vgl. geselle 10, geselpriester, glokner, kirchendiener, ${ }^{1}$ letter, lezner. - Wbg.: levitisch 2.

Goldammer, Paracelsus. B. d. Erk. 35, 1 (obd., Hs. n. 1570): wo rue ist / do ist Cristus / do durffen sie kains priesters / appostells / leuit / predicant. CHRON. BAIER. STÄDTE. Regensb. 42, 26 (noobd., 1521): es hetten dy fronambter all sambt den leviten piß auff zwen verschlaffen. EBD. 169, 1 (1541): darnach gingen 6 persevanten in iren levitischen habitn. Uhurz, Qu. Wien 2, 2, 3732 (moobd., 1457): Michel Wülffing, levit dacz sand Stephan. - TürK, Wortsch. Dietr. v. Gotha. 1926, 70.

3. im Phrasem jm. die leviten lesen >jn. tadeln, zurechtweisen, mit jm. schimpfen, jm. eine Strafpredigt halten «. - Bdv. : jm. den golter fegen / lausen / zausen, jm. eine scharfe lection / seine legende / die letze lesen.

Roloff, Brant. Tsp. 1647 (Straßb. 1554): Ich will wol selber zu in gon | Und ine die Leviten der mossen lesen |Das [...]. - Vorarlb. WB. 2, 277; Schweiz. ID. 3, 1150.

levitenrok, der; - /auch $-\emptyset+\mathrm{Uml}$.

>Messgewand des Kirchendieners bzw. Diakons <; zu levit 2. - Bdv.: mesgewand.

Bolte, Pauli. Schimpf u. Ernst 1, 70 (Straßb. 1522): ist Gewonheit, das man dem letst Evangelier oder Epistler, dem laßt man den Levitenrock uff dem Halß ligen. CHron. Augsb. 4, 299, 1 (schwäb., v. 1536): Der kaiser hat angelegt ain alb, handfanen und stol und ain levitenrock wie ein evangelier. - Struck, Joh. Pfannstiel 55, 46; Boner, Urk. Brugg 373, 16; UhLirz, Qu. Wien 2, 3, 5293, 39; Zingerle, Inventare 47, 1, 1; SChWeiz. ID. 6, 832.

levitenstand, s. levit 1.

levitisch, Adj.

1. 'zum Stamm der Leviten, zu den jüdischen Priestern bzw. Tempeldienern gehörend bzw. diese Zugehörigkeit symbolisierend $<$; zu levit 1 .

Luther, WA 8, 555, 8 (1521): das alle recht Christglewbige menschen rechte [...] priester sind [...], wie die Levitischen priester waren ynn der Synagogen. EBD. 15, 49, 29 (1524): wie Gott das Levitsche Priesterthum [...] verordenet hat das sie der bücher bütten [...] sollten. EBD. 23, 585, 28 (1527): das Christus priestertum [...] solte ein anders denn das Levitische priesertum sein. EBD. 33, 644, 39 (1532): Gottes Son ist hoeber denn alle Stifft, Kloester [...], denn der Levitische Gottesdienst, der Tempel zu Jerusalem oder die Leviten [...]. ВЕCHSTEIN, M. v. Beheim. Evang. 71, 8 (osächs., 1343): Wan her [Marcus êwangêlista] bewîsende di ordenunge lêvitischer irwelunge. Kurrelmeyer, Dt. Bibel 6, 183, 5 (Straßb. 1466): ir habt vor eúch leuittisch meister. LuTHER, WA 46, 569, 15. - Vgl. ferner s. v. levit 1.

2., s. levit 2.

${ }^{1}$ lewe, löwe, lebe, leu, der; $-n /-n$; teils lat. Form: leo.

1. >Löwe`; der Löwe gilt als König der Tiere, als Symbol von Kraft, Tapferkeit, Mut und Stärke; er erscheint demzufolge vielfach in heraldischen oder vergleichbaren herrschafts- und ordnungsdokumentierenden Zusammenhängen (als Wappentier, in der Münzprägung, als Tuchzeichen; vgl. auch 5); in theologischen Kontexten steht er als Symbol für Christus wie für den Teufel; das Bild des Löwen begegnet vereinzelt 
auch $\mathrm{zu}$ dekorativen Zwecken (etwa in Form einer löwengestaltigen Kanne); häufiger Gebrauch des Wortes in Vergleichen und in Verbindung mit anderen mächtigen Tieren (bär 1, leopard, tieger, wolf). - Phras.: einen lewen giessen > sich erbrechen ‘; den lewen bei den klauen urteilen >von äußeren Kleinigkeiten auf das Ganze schließen. - Bdv.: s. u. Schöpper. - Synt.: den l. bändigen / füren / (er)schlagen / sehen, jm. einen l. bringen; jn. einen l. nennen; der l. erseufzen, jn. bebüten / erquicken, dem anderen das haupt abreissen; dem l. etw. (z. B. feuer) geben, den mund aufreissen; jn. vor die l. werfen, von den l. erlöst werden; als ein lewe streiten, etw. nemen / regieren, gleich dem lewen etw. werben; antliz. / zäne / mund / har / baut / gestalt / beine / mut / stimme / welf / haus des lewen; der adelliche / alte / aufgerekte / brüllende / edle / grimme / bellische / küne / lebendige / lügende (s. lägen) / reissende / schlafende / schreiende / starke / unverzagte / wilde / zornige lewe, die blauen / vergoldeten lewen; die fabel, das gleichnis vom lewen. Wbg.: lewenbein, lewenbirne, lewenblat, lewenfarben, lewenfleisch, lewenfus, 「lewengarten, lewengatter, jeweils $>$ LÖwengrube $\urcorner$, lewengeschrei, lewengewalt, lewengrif >Muttermal< (a. 1474), lewenhar, lewenhaupt, lewenjeide, lewenkind, lewenklaue, lewenknochen, lewenkeraut, lewenlunze (zum Gw s. ${ }^{1}$ lunze), lewenmilch (eine besonders wirksame Medizin), lewenpelz (wie lewenhaut), lewenplappert >Plappert mit dem Bilde eines Löwen (= 22 Angster; a. 1504), lewenskraft, lewentappe, lewentier, lewenwelf >Löwenwelpe, catulus leonis〈, lewen₹winger $>$ Löwenbändiger〈, lewig > verwegen, unerschrocken〈, lewin.

SchÖpper 4a (Dortm. 1550): Diabolus. Teuffel sathan Faland alte schlang vatter der ligen beelzebub versucher menschenfeindt starcker Behemot brüllender lew. InGEN, Zesen. Ros. 70, 23 (Hamb. 1646): hat man gelebret / wi die leuen und tiger=tibre ándlich zu bándigen sein. Ziesemer, Proph. Cranc Jes. 31, 4 (preuß. M. 14 Jh.): als der lewe brymmet und daz. lewenwelf [ЕсK 1537: gwálf] ubir synem aze, wen [...]. EBD. Jer. 51, 38: si werdin brummin mit einander als dye lewen und werdin schuttin ire mane als di lewenwelf. EBD. Dan. 14, 30: Den [Daniel] stiesen si in den lewengatir, [...]. ouch waren in dem gatir siben lewin. EBD. Os. 5, 14 (preuß., M. 14. Jh.): wen ich bin als eine lewineluncze LutHeR 1545: Lewe] Effraym und als eine lewinwelf [LUTHER 1545:
Jungerlewe] dem buse Juda. EBD. Mi. 5, 8: als ein lewe undir den tyren des waldes und als ein lewenwelf in der herten des vyes. EвD. Na. 2, 13: dine lewelin wirt das swert vorzeren. Ders.\#, Gr. Ämterb. 250, 24 (preuß., 1508): 1 furhang mit einem weissen leben. PeIL, Rollenhagen. Froschm. 21, 4 (Magdeb. 1608): nennet den Kónig zu Sichem einen Ochsen / Judam einen Lewen. EBD. 531, 788: besser sey der Hirschen Heer / | Welcher Hertzog ein Lewe wer / | Denn ein Kriegsvolck von eiteln Lewen / | Die ein Hirsch führt mit furcht und schewen. EBD. 555, 1534: Schawt / da kompt her vnser Cuman / | Hat ein Lewenpeltz, angethan. EвD. 573, 2134: Das wir daselbst die Meus vmbracht / | Mit Lewen Muth / und Lewens kerafft. Helm, H. v. Hesler. Apok. 8408 (nrddt., 14. Jh.): [herre Jhesus Crist] ist her lewe genant, | Wen her warb in den ewen | Glich einem kunen lewen $\mid Z u$ der werlde sins vater wort, $|[\ldots]|$ Der lewe dutet ouch Marken, | Wen her kundete den starken. Ders., Maccabäer 1974 (omd./nrddt., Hs. A. 15. Jh.): Daniel in siner einvalt | wart irlost u₹ lewengewalt. REISSENBERGER, Väterb. 38065 (md., 14. Jh.): Eime lewen tiere|Wart da geruofen schiere. Fischer, Brun v. Schoneb. 9399 (md., Hs. um 1400): der lebe bezeichent, so man seit, | uns di obirmutigen kundikeit, | [..] der lebarde bezeichent di sundere. EBD. 10125: zwuschen lewenkinder, her seit, | slif ich mit grozer trubheit. | welchez, waren die lewenkint sprich? | iz, waren di schecher. Helbig, Qu. Wirtsch. 5, 44, 38 (md., 1562): Dann sollen die zihen [...] in die vass eingeschlagen und das rechte Altenbergische zeichen, den leuen, darauf brennen lassen. Chron. Köln 1, 1093 (rib., Hs. 1. H. 15. Jh.): men saich sy alse lewen stryden. Buch WeInsB. 1, 227, 9 (rib., um 1560): [Keppel] hat vur sich [...] ein wapen vom keiser erworben, ein guldin leben mit einem guldin peil in den klawwen in eim swarzen schilde, uff dem helm ein halben leben. KARNEIN, Salm. u. Morolf 438, 1 (srhfrk., Hs. um 1470): Salmon gewan eins lewen mubt. BelKin u.a., Rösslin. Kreutterb. 56, 6 (Frankf. 1535): Agathes oder Agapis der Steyn hat grosse tugent / besonder der ein farbe hat gleich eins lowen haut oder lowen har. Alberus EE ijv (Frankf. 1540): cauda porcina, meerbirsen / lewenkraut / meergries / meerhanff. [...] pardalium, cornutum papauer, lewenblat. [...] dori petron, lewenfü / synaw / vnser frawen mantel. HaRms u. a., Alberus. Fabeln 170, 8 (Frankf./M. 1550): Dachs / Biber / Marder / Wisel / Mauß / | Die kamen auch furr Lowen hauß. Stambaugh, Milichius. Zaubert. 11, 28 (Frankf./M. 1563): denn ewer widersacher der teuffel geht umbher wie ein brullender Lón. GeRHARD, Hist. alde e 908 (omd., um 1340): Du solt ruen in rechtem sinne, Sam ein lew und ein lewinne| Man vorchtet ummuozen sere. EBD. 4382: Van dem engel. der en troste | Und uz dem lewengarten loste. HüBner, Buch Daniel 73 (omd., Hs. 14./A. 15. Jh.): Danyelem den reinen, | Den Got von der lewen munt $\mid$ Machte zeichenlich gesunt. JAHR, H. v. Mügeln 95 (omd., Hs. 1463): der leu bedütet Bemerlant. Eggers, Psalter 9, 13 (thür., 1378): Das nimant neme mine sele als eyn leywe. EBD. 30, 8: als ein lawwin welf, daz da wonet in deme vorborgen. v. Tscharner, Md. Marco Polo 33, 19 (osächs., 2. H. 14. Jh.): Dy [slangin] han czwey kny bi dem 
houbte unde lewin vuze. Lippert, UB Lübben 2, 281b, 25 (osächs., 1564): Ein messerner lebe, da man waßer innenn tregett. KeHrein, Kath. Gesangb. 1, 230, 3 (Bautzen 1567): Todt und leben tratten in kampff, | Ein starcker Lew und schwaches Lamb. Opitz. Poeterey 27, 7 (Breslau 1624): [Namen für den Weingott:] Lówen=zwinger / Hertz=fänger / Hertzen=dieb. ReICHERT, Gesamtausl. Messe 95, 19 (Nürnb. um 1480): als dy sel des hochfertigen wuerd eingan in einen loewen und dy sel der unkewschen wuerd eingan in ein hund. Voc. Teut.-Lat. g viiir (Nürnb. 1482): Pferdthar od' iubar. od' lewenhar. Franck, Klagbr. 234, 19 ('wohl Nürnb.' 1529): mag dein K. M. den leowen bey den klaben vrteiln / wie doch der gantz leow sey. Hulsius L ivr (Nürnb. 1596): Lówin / vne Lyonne. [...7. Lowen geschrey / rugiment de Lyon. SACHS 3, 494, 6 (Nürnb. 1527): Das grewlich thier [...]. | Das het ein löwenhaubt. Ders. 20, 270, 4 (1563): Daß man in füren must zu beth, | Da er ein löwen giessen thet (hier wohl: > sich erbrechen ). BELL, G. Hager 37, 1, 30 (nobd., 1593): der teüfel, ist kein schwacher. | der ge het vmb her runde | [... | wie ein lew. Thiele, Minner. II, 13, 439 (Hs. ' ndalem.) sfrk., 1470/90?): ein kruc₹, des starcken leuwen styme, | alsus er unns erlost $\mid$ mit hertter, bitter marter. Bolte, Pauli. Schimpf u. Ernst 1, 63 (Straßb. 1522): Wan wir Lewenmilch betten und man sie damit salbt, so wird sie genesen. Dasypodius 374r (Straßb. 1536): Lówen geschrey. Rugitus, [...]. Lowen tapen. Trifolium, uel Planta leonis. SuDHOFF, Paracelsus 14, 545, 2 (um 1567): sol eben zu der zeit ein löwe von mitternacht [Bezug auf Christus] komen, welcher dem adler nachfolgen [wird]. VeTtER, Pred. Taulers 321, 16 (els., 1359): der túfel der lóffet umbe als ein brimmender liggender lówe. NiewÖHNER, Teichner 566, 1 (Hs. 「önalem., um 1433?): Aller tier fürst der loow. WeLti, Stadtr. Bern 447, 15 (halem., 1451): Der zeichen sind III, das erst ist ein ochs, gilt Viii halr. ze schowen, ein leo XVI halr (der Löwe fungiert hier als Tuchzeichen). LAUATER. Gespånste 17r, 4 (Zürich 1578): Von lóuwen schrybt man / daß sy sich durch kein Bogen oder butzẽwerch lassind erschreckẽ. Dañ der lơuw ist ghertæt / dapffer / vnerschrocken / vnd ein künig vnder den vierfússigẽ thieren. BARACK, Teufels Netz 5528 (Bodenseegeb., 1. H. 15. Jh.): An der kanzel ist er ain lowen | Und getar kïnig und berren drowen. Haltaus, Liederb. Hätzlerin 2, 85, 140 (schwäb., 1471): libe dochter, nỹ̃ dich an | Leïig und betring yederman! DieHL, Dreytw. Essl. Chron. 55, 4 (schwäb., 1549): Alda [...] zwu gar gross seüllen auff zwen grosse loüwenn gesetzt. EBD. 94, 14: Darnach hand sich zwen lewenn herfyrgethan und auch gegenn einander gezogenn und anfachenn zu kempffenn und der lew auff der lencken seitten hatt dem ander das hauptt abgerissenn. HeNisch 391 (Augsb. 1616): Hessische birn / Lówenbirn / scherersbirn / Helsiacum. Spechtler, Mönch v. Salzb. 30, 2 (oobd., 3. Dr. 14. Jh.): Christus erstuend mit siges van, | do wart aus lamb ein leb getan | mit hochzeitleicher sigeskeraft. EBD. 1, 131: Ruff uns recht als der leo tüt, | speis uns mit pelikanes plít. Pfeiffer, K. v. Megenberg. B. d. Nat. 22, 34 (oobd., 1349/50): dar umb hat der leb nibt marks, wann ez wirt verzert von der übrigen nâtürleichen bits, die in des lewen painen ist. EBD. 143, 3: sô diu lewinn gepirt, sô slâfen die lewel drei tag unz der vater kümt. EBD. 144, 13: Plinius spricht, da , lewenflaisch und allermaist sein herz, den läuten guot sei. EBD. 156, 18: dâ sint die lebinne, die unkäuschent mit mangerlei tiern [...], dâ von koment die parden. BAUER u. a., Kunstk. Rud. 1157 (oobd., 1607/11): der fuß von metal mit lewenklawen. EBD. 1424: Ein geschirr von jaspis, lewenfarb mit 2 handhebin. Fichtner, Füetrer. Trojanerkr. 73, 3 (moobd., 1473/8): Auss pern- und lewenpainen - | daraus sy [helden] assen allreit nur das marck. | Die lewin muesten die tapen helden trencken. EBD. 302, 5: Achilles vacht in lewens weyse. SPILler, Füetrer. Bay. Chron. 18, 8 (moobd., 1478/81): Ignatius ward für lewen und peren geworfen. Turmair 5, 136, 7 (moobd., 1522/33): da zogen si mit den bunden an das lewenjaid. - Holland, H. J. v. Braunschw. V. e. vngerat. Sohn 374, 33; Luther, WA 41, 38, 17; 41, 464, 14; JoAchim, Marienb. Tresslerb. 475, 23; Fischer, Brun v. Schoneb. 2588; BucH Weinsb. 1, 187, 7; Meisen, Wierstr. Hist. Nuys 2115; Wyss, Limb. Chron. 30, 18; Tiemann, E. v. NassauS. Kgn. Sibille 169, 15; Fellmann, Denck. Schrr. 2, 95, 5; Froning, Alsf. Passionssp. 6726; Österley, Kirchhof. Wendunmuth 4, 259, 17; 260, 1; OoRsснот, Spee/Schmidt. Caut. Crim. 403a, 21; Hübner, Buch Daniel 4512; 5731; Thiele, Chron. Stolle 87, 12; Thür. Chron. 19v, 11; Bechstein, M. v. Beheim. Evang. 6, 6; v. Tscharner, Md. Marco Polo 23, 19; 70, 14; 72, 7; Gille u. a., M. Beheim 26, 1; 238, 249; Mayer, Folz. Meisterl. 14, 33; Kurrelmeyer, Dt. Bibel 2, 501, 14; 5, 288, 57; Bihlmeyer, Seuse 260, 16; Bernoulli, Basler Chron. 6, 473, 24; BachmanN, Morgant 248, 23; Morrall, Mandev. Reiseb. 123, 13; Barack, Zim. Chron. 1, 320, 28; Gilman, Agricola. Sprichw. 2, 135, 30; 216, 19; KLeIN, Oswald 67, 21; 81, 30; Bauer u. a., Kunstk. Rud. 323; Bauer, Haller. Hieronymus-Br. 10, 34; 20, 4; BaUmanN-Zwirner, Augsb. Volksb. 211; Dasypodius 31r; Schweiz. ID. 3, 950; 5, 133. - Vgl. ferner s. v. abwerfen 12, ${ }^{1}$ als 2 , anlegen 5 , antependium, antragen 2, ${ }^{1}$ ar 2 , arenflügel, aufarbeiten 4, aufrecken 1, aufsperren 2, aufwischen 2, ausrotten 3, ${ }^{1}$ backe 1, pantel, ${ }^{1}$ bär 1, pard, bärin 1, barreweis, bebrunzen, ${ }^{1}$ leffel, leopard, lewenzan 1.

2. `Sternbild Löwe<; dazu metonymisch: >Monat August als Sichtzeit des Sternbildes $<$.

Stoltzius, Chym. Lustg. 78, 1 (Frankf./M. 1624): Der grine Low. Was bdeut der Lów / mit seinen Stern? |Was bdeut die Sonn / welche gar gern |Vom Lowen wird gehalten fest? EвD. 106, 3: Africa gibt / obn allen schertzn / | Warme Lowen mit rothem Hertzn. Stackmann u.a., Frauenlob 3, 16, 3 (Hs. ' schles., 14. Jh. `): Noch süzer dan ins lewen hitzic sunne ein schate schine. SuDHOFF, Paracelsus 14, 411, 35 (um 1567): luna hat von sol und vom löwen und der jungfrau die lautere reinikeit und große bestendikeit wider die macht des feurs sol, löwe jungfrau. Menge, 
Laufenb. Reg. 319 (Hs. ' nalem., um 1470?): He ist die sunne Im löwen | Mit siner hitze Im trowen | Glich als der löwe in zorne tüt. BRÉvART, K. v. Megenberg. Sphaera 23, 3 (noobd., 1347/50): die zal der bimelzaichen die sint also: wider, obs, zwinlein, krebs, leo, juncfreulein, [...]. KLEIN, Oswald 22, 35 (oobd., 1422): welcher von der Sunne | orient geboren ist, | dem geit der Leo die wunne. - JAHR, H. v. Mügeln 507; Sudhoff, Paracelsus 8, 288, 15; 10, 144, 28; Martin, H. v. Sachsenh. Tempel 386; BrÉVART, a. a. O. 23, 17; 37, 19; 50, 7; Golius 18.

3. in dem Phrasem: roter lewe: >Metall (aufgrund der Farbe möglicherweise Gold; alchimische Kontexte).

Sudhoff, Paracelsus 14, 14, 394, 1 (1568): Die materi der tinctur ist ein ding, so du mich recht auf spagirisch verstehest, welches von dreien ein éin wesen durch die vulcanische kunst ausgehen [...] mag. und das ich dirs mit seinem namen nach altem brauch nenne so ists der rote löw, vilen genant, wenigen bekant. - BARKE, Spr. d. Chymie. 1991, 287.

4. >Gehilfe des Scharfrichters, Henkersknecht, Büttel (der Stadt Nürnberg)<. Nobd. - Bdv.: vgl. büttel, haher, peiniger 2, kästiger, richter, scherge. - Wbg.: lewenknecht.

Chron. Nürnb. 1, 273, 12 (nobd., 1388): It. ded. Albrecht, leben, 11/2 \& hl. vom streichampt. EBD. 4, 180, 2 (15. Jh.): auf der maur der stat [...] pei der plaich und des lebin maur, da gieng man auf über. LEXER, Tucher. Baumeisterb. 112, 1 (nürnb., 1464/75): man lest auf der stat kost niemant sein schlöt fegen dann [...] dem nachrichter, leben, buntschlaber, den zweien statbirten. EBD. 259, 5: soll der stat paumeister mit dem pfenter und in sunderbeit mit dem leben bestellen, das man den schön prunnen [...] mit kremen nit versetzen loß. EBD. 267, 33: hat der leb dorpei ein sweinstall steen, [...] dorein er die swein spert, die er auf der gassen vindet. Hampe, Nürnb. Ratsverl. 1, 82, 8 (nobd., 1498): Dem Gareysen, leben, ist vergonndt, ein tafelein ein $\beta$ gemelds [...] verneuen zu lassen. Euling, Kl. mhd. Erz. 411, 1 (nobd., E. 15. Jh.): Von dingen die geben vil poser cristen. Richters knecht, verreter, leben und schergen. RuPPRICH, Dürer 1, 168, 80 (nobd., 1521): Jch hab zu trinckgeld geben dem meßner und löwen knechten 3 stüber. - Chron. Nürnb. 5, 690, 5; Lexer, a. a. O. 202, 13; Pfeiffer, Frk.-bay. Landfr. 160, 31 : Rwв 8, 1482. - Vgl. ferner s. v. aufzukken.

5. der Löwe als Identifikationszeichen von Gesellschaften, Bünden oder Zünften; metonymisch: Name von deren Räumlichkeiten. - Obd. - Wbg.: lewler, lewlerbund.

Chron. Nürnb. 3, 290, 24 (nobd., 1450/80): etliche sant Wilhelmes geselleschaft, etliche die geselleschaft der panthier oder der lowen geselleschaft. GEIER, Stadtr. Überl. 523, 15 (nalem., 1560): so gehn beede erwelt new und alt burgermaister, stattaman, die räth $u \beta$ dem lewen, $[.$.$] in die hindern$ newen stüben. ReNnEFAHRT, Wirtsch. Bern 290, 8 (halem., 1576): uß wöllichen [...] ursachen dann gesagte fürtrager vom Löuwen ... uns ... gepätten haben, wir wöltend unsere burger [...] by loblichem altem harkhommen [...] belyben lassen. Turmair 5, 518, 31 (moobd., 1522/33): Der fürsten punt nent man den ,lewlerpunt darumb, das die fürsten von Baiern ainen lewen füerten. LeIDINGER, V. Arnpeck 681, 7 (moobd., v. 1495): di ritterschaft in und vor dem wald betten ainen punt gemacht wider herzog Albrecht und nennten sich die lebler. - GEIER, a. a. O. 552, 19; LEIDINGER a. a. O. 683,$34 ; 705,13$.

6. ein Geschütz.

Schottenloher, Flugschrr. 97, 6 (Würzb. 1523): Da wardt gschossen syben tag $|[\ldots]|$ Die abgestummelt scharpffe metz. | Het darzu auch das best getan. | Der leb thet guten nachtruck han | In vorgeborte locher kleyn | Die rays er gewaltig all zu eyn.

${ }^{2}$ lewe (auch mit Hebung des Wurzelvokals: $-i-)$, die; zu mhd. lêwe >Fischhecke (Lexer 1, 1894); vgl. auch läuwe.

>Fischhecke«. - Wbg.: leiweide >Fischfanggebiet, das mit Fischhecken befischt wird « (Schreibung -ei- des Bw undurchsichtig).

Grimm, Weisth. 4, 514, 19 (nalem., 1442): wer do wil lewen befohen. MErk, Stadtr. Neuenb. 139, 21 (nalem., 1681): sovil die leiweid anlangen tuet, es solle jehrlichen [...] die leiweid angehalten werden, dan man die leiweid teilet. Das bolz sollen sie auf den osenden hawen und, wan sie dis liben baben, sollen sie das holz wider binder sich auf die böhe legen und wan, einer ein lihen hat und ongevohr einer auf- oder ab mit einem garn abzeücht, so solle er bei dem wenigsten die lihen ongeirrt nicht darein stecken oder storen bei der straf ein pfund fünf schilling stebler [...]. Und wan einer ein lihen in einem rinnenden waßer hat und daßelbige von einem andern abgekert und gewendt wurde, so hat derselbe seinen bahn in disem waßer, der jenige, so dises waßer abkehren will, der solle demselben, deme die liben ist, anzeigen.

lewenbein, lewenbirne, lewenblat, lewenfarben, lewenfleisch, lewenfus, lewengatter, lewengarten, lewengeschrei, lewengewalt, lewengrif, s. ${ }^{1}$ lewe 1 .

\section{lewengrube, die.}

>Löwengrubeく, ein als Loch, Mulde, Vertiefung gedachter Ort, in dem Löwen gefangen gehalten werden.

MAYer, Folz. Meisterl. 45, 22 (nobd., v. 1496): Got yn bin furet pey dem hare $\mid Z u$ Daniel ind lebengruben zware. Gille u. a., M. Beheim 172, 41 (nobd., 2. H. 15. Jh.): Durch neid man auch Danieln erbub | und warff in in die leben grub. - Sснмітт, Ordo rerum 35, 15.

lewenhar, lewenhaupt, s. ${ }^{1}$ lewe 1. 
lewenhaut, die.

>Fell eines Löwen (oft als Attribut der Königlichkeit)<. - Bdv.: lewenpelz.

PeiL, Rollenhagen. Froschm. 554, 1508 (Magdeb. 1608): [Der Esel] fand am weg auß ungeschicht / | Ein Lewenhaut wol zugericht / | So einem Junckherrn in dem Jagen / | Entfallen war. v. Tscharner, Md. Marco Polo 24, 28 (osächs., 2. H. 14. Jh.): hus, das ist gemacht von rore und ist bedact mit lewin butin. EBD. 25, 6: dy cameren und der sal sint bedact mit den lewin butin. Koppiтz, Trojanerkr. 20209 (Hs. 'noschweiz., 15. Jh. ') : Sy trïgend alle claider an | Gemachett uss löwen bütten. EBD. 20232: uff töttlicher vartt, | Menig löwen butt verschrotten ward (bildlich für: > fielen im Kampf $)$ ). Turmair 4, 123, 23 (moobd., 1522/33): das er [Hercules] ein lewenhaut an bat gebabt, wie unser fürsten ietzo wolpel\%. BAuer u. a., Kunstk. Rud. 1326 (oobd., 1607/11): Ein geschirrl von topazio, wie ein lewenhautt. - PeIL, a. a. O. 555, 1537; BAUER u. a., a. a. O. 1935; Dict. Germ.-Gall.-Lat. 310, 29.

lewenherz, das.

>einem Löwen verglichener Mut, unerschrockene Tapferkeit

Thür. Chron. 8r, 22 (Mühlh. 1599): [Scipio] empfing ein Lowen hertze / unnd Trat für die Rómer [...] vnd sprach. Ukena, Luz. Sp. 2636 (halem., 1575): Wölff / halb Tüfel er an inen findt | Mitt löwenhertz, sy [Soldaten] bgabet sind.

lewenjeide, lewenkind, lewenklaue, s. ${ }^{1}$ lewe 1.

lewenknecht, s. ${ }^{1}$ lewe 4.

lewenknochen, s. ${ }^{1}$ lewe 1.

lewenkopf, der; -/auch $-\emptyset+\mathrm{Uml}$.

bildliche Darstellung eines Löwenkopfes in unterschiedlicher Ausformung und Funktion (als Dekoration an Geschirren, an der Kleidung, als Hausschild o. ä., als Symbol von Macht, Stärke, Tapferkeit, Reichtum); vgl. ${ }^{1}$ lewe 1 ; auch 5.

Chron. Magdeb. 2, 235, 24 (nrddt., 1505): sollen auf allen kleyderen, an Roßenkereutzen, lowenkopffen heften dergleichen uber zwey marck silbers gewichte nicht haben. CHrON. Nürnb. 5, 667, 22 (nobd., E. 15. / A. 16. Jh.): der kaufman bieß [...] Paner an sant Gilgen gassen in dem haus zu den lebenköpfen. Koller, Ref. Siegmunds 340, 12 (Hs. ${ }^{\ulcorner}$Basel, um 1440`): das dirte zeichen, ₹u der lincken siten gefürt soll werden, ist [...] geteylt enmitten mit einem güldenen strich, in dem ober teil zwen güldin lowenkopf, [...] jegelicher lowenkopf hat drige füren flammen zü dem munde usschiessen. Bauer u. a., Kunstk. Rud. 115 (oobd., 1607/11): anathomirter lewenkopf, allein die gebain und seine zähnt. EBD. 1032: 7 lewenkö̈f von weissem augstein. - Koller, a. a. O. 341, 24; 30; Bauer, u. a., a. a. O. 1596. lewenkraut, lewenlunze, lewenmilch, lewenpelz, s. ${ }^{1}$ lewe 1.

lewenpfennig, der.

>Löwenpfennig, im meißnischen Münzsystem übliche Münzeく; vgl. ${ }^{1}$ lewve 1 .

Luther. Hl. Schrifft. Offb. 6, 6 Marg. (Wittenb. 1545): drey mass Gersten vmb einen grosschen [Marg.: ein Grosche belt dreissig lawen pfennig] / und dem Ole und dem Wein thu kein leid. THiele, Chron. Stolle 462, 26 (thür., 3. Dr. 15. Jh.): gemeyne wine eyn fertel umme dry lawen phenge. - Hertel, UB Magdeb. 3, 769, 8; Rwв 8, 1484.

lewenplappert, lewenskraft, lewensweise, lewentappe, lewentier, lewenwelf, s. ${ }^{1}$ lewe 1.

lewenzan, der.

1. \Zahn eines Löwen`, zu ${ }^{1}$ lewe 1.

Heцm, H. v. Hesler. Apok. 14150 (nrddt., 14. Jh.): Als sie dan daz volk zu strazen | Irre irrekeit wol gewenen, | So bizen sie iz mit lewen zenen. ZIESEMER, Proph. Cranc. Joel 1, 6 (preuß., M. 14. Jh.): sine zebene sint als lewenzebene und sine baczehene sin als lewwenwelfis. BAUER u. a., Kunstk. Rud. 107 (oobd., 1607): 19 löwen, bern unnd wolfsszän. inn einer gemalten langlicbten scattel.

2. eine Blütenpflanze; zu den botanischen Bezugsmöglichkeiten s. Marzell 5, 339.

Alberus EE ivv (Frankf. 1540): lewenzan / wissen lattich / [...] augen wurtzel / eyer blum / dotter blum. MAALER 275r (Zürich 1561): Lóuwenæan ein kraut. Alcen.

lewenzwinger, s. ${ }^{1}$ lewe 1.

lewer, le, der; zu mhd. lê, lêwer > Hügel (LEXER 1, 1845; 1895).

>Hügel, Erdaufwurf (als Grenzzeichen)<. - Bdv.: vgl. anschütte 1, aufwurf 1. - Wbg.: lewerhanfe.

Kochendörffer, Tilo v. Kulm 64 (preuß., 1331): plan | Da ich di blumen vinde | Vol craft der suzen winde, | Di sich czu der nwen e | Scbrenken uz dem alden le (hierher als dichtungssprachliche Ütr.?). STACKMANN u. a., Frauenlob 5, 38, 14 (Hs. ' $\mathrm{md}$. auf nd. Grundlage, v. M. 14. Jh. '): hochvart ist uf der tugende le | ein blünder kle. Seemüller, Chron. 95 Herrsch. 120, 8 (oobd., Hs. 1. H. 15. Jh.): Chünig Bela [... trat an ain leber und wolt da von verren des streites zu sehen. WinTER, Nöst. Weist. 1, 103, 12 (moobd., 15. Jh.): sol der galgen steen auf dem lebar bei den Graslussen. EвD. 2, 55, 34 (1697): binweggackern der rain, abstoßung der marchstain und lewer. EBD. 2, 74, 26 (moobd., 1610): Die gemärk sollen alßbalten nach verlëßener panthäding [...] mit vleiß ubergangen, erbebt, die 
aufgerichten leberhaufen beschiettet werden. EBD. 3, 373, 30 (um 1545): von der obrn gassn gend die grünt unz auf den leber, vom leber unz in den Khatzngrabn. - EтTMüLLER, Heinr. v. Meißen 367, 12; Rwв 8, 1287; PfäLz. Wв. 4, 878; Sснміdт, Hist. Wb. Elsaß 216; Rwв 8, 1287; SCHWEIZ. ID. 3, 1544.

lewerhaufe, s. lewer.

lewig, lewin, s. ${ }^{1}$ lewe 1.

lewler, lewlerbund, s. ${ }^{1}$ lewe 5.

lez, Adj.; zu mhd. letze, lez > verkehrtı (Lexer 1, 1890), nhd. letr (Dwi 6, 794).

1. >im Unterschied zu dem stehend, was recht, richtig ist oder als recht, richtig gilt, unrecht, falsch, verkehrt‘; im einzelnen: >links〈; > umgekehrt (von Kleidungsstükken)<; >rückseitig (von Papier)<; >vom Glauben abgefallen<; offen zu 2-4. Beleghäufung für das Wobd. - Phras.: der letze pelz, > falscher Pelz` (spöttisch für die Bekleidung ärmerer Leute); lez stehen >im Unrecht sein <; lez, dran sein >irren<; es geht lez. $z u$ >es geht nicht mit rechten Dingen zu<; lez oder recht haben $>$ recht oder unrecht haben<; jn. lez stellen >jn. ins Unrecht setzen<; das lez, herumber keren o. ä. >das Innere nach außen kehren`; im Beleg ütr.: >sich schamlos zur Schau stellen<; dem rok das lez auskeren >alles auf den Kopf stellen`; jm. gilt (etw.) lez oder recht >jm. ist etw. gleich ; es get lez und überzwerch >es geht drunter und drüber<; es geht jm. lez, >es geht jm. schlechtr. - Bdv.: s. u. SCHÖPPER, ferner: übel, umgewendet, überzwerch, verkeret. Ggs.: recht (oft). - Synt.: j. / etw. l. sein; retw. (z. B. das rechte) l. machen, jn. / etw. l. stellen (präd. Attr.), jn. / etw. l. verstehen, etw. l. angreifen / ausrichten, jm. l. oder recht gleich gelten; den letzen (>Verkehrten «) umbringen; der letze glaube / bändschub / verstand / weg, die letze hand / meinung. Wbg.: lezgläubig >abergläubisch〈, leqverständig > widersprüchlich .

SCHÖpper 90a (Dortm. 1550): Contrarius Widerstendig widerwertig entgegen lătz, widersins widerspilisch. SсHÖNBACH, Adt. Pred. 10, 5 (osächs., 1. H. 14. Jh.): zo mustu sten [...] von gote gesundert und gewizet zu der lezen hant (>zur Linken `) mit den stinkenden ciegen. GiLle u. a., M. Beheim 82, 450 (nobd., 2. H. 15. Jh.): qu laichen [...] die
| glaubigen menschen, nur das sy | werden ringer und leczer. Spanier, Murner. Narrenb. 26, 31 (Straßb. 1512): So louffens [frouwen] mit den puren knaben |Vnd gilt in glych letz, oder recht. EвD. 40, 61: so ist es buiben werck |Vnd gat offt letz und überzwerg. EBD. 41, 60: So ists [dyn tocbter] wol selber so gelert, | Das sy das letz herumbher kert. EBD. 42, 92: Die schencken machens alles schlecht, | Wer es let\%, so würd es recht. DAsYPODIUS 371v (Straßb. 1536): letzuerstendig red / widerspilische red. Antiphrasis. GoedeKe, Fischart Flöh Haz 1387 (Straßburg 1594): Dieselb [weib] hett nach alten gebreuchen, | Die her von Eve belz solln raichen, | Ain letzen belz um, sah daraus $\mid$ Wie ain schildkrott aus irem haus. SudHoff, Paracelsus 2, 383, 14 (1525/6): das die rechte meinung verstanden werde, und nit die leze. B̈̈снтоLD, N. Manuel. Papst 85, 1427 (Zürich 1525): Gott geb, ir habind denn glich ja letz. oder recht, | So wil's trüwlich mit üch han. EBD. 156, 641 (1526): Wenn] das ross sitzt uf dem man, | So müss es alles letz zügan! V. Anshelm. Berner Chron. 4, 145, 25 (halem., n. 1529): wie wol einer Eidgnoschaft nie wirs, nit so úbel und låz gangen. MAALER 106r (Zürich 1561): Sich nit Entsetzen oder sich nit látr, gestellen ab menschlichen zúfálen / dultigklich leyden. EBD. 261r: An das Látz theil des papeyrß schreiben oder auf das rauch theil, [...]. Latze rádt / auff das wiederspil des das man thün solt / gegáben. [...] Látzer glaub. Superstitio. Látzglóubig. Superstitiosus. Látz, machen / Vmbkeeren / Das inner außhin keeren. [...] EBD. 261r; Látz stellen die zeügen. Eleuare testimonia. Einsi fráfenheit Látz stellen. Audaciam alicuius confutare. [...] Einen seiner ziuersicht Látz. stellen. Rennefahrt, Zivilr. Bern 756, 7 (halem., 1615): das er sölchen kauff [...] obn einichen betrug, falschen schyn und allen anderen látzen verstandt züzüchen begere. BARACK, Zim. Chron. 3, 574, 9 (schwäb., M. 16. Jh.): schlecht in der könig user kereften mit der letzen handt unversehenlich ins angesicht. DieHL, Dreytw. Essl. Chron. 115, 3 (schwäb., 1552): Es haist dem rock das letz herausskertt, darum mus sich gottes wortt mitt vill seltzamer brattick [...] besudlen lan. KLEIN, Oswald 112, 238 (oobd., 1438): man well dann felschen gots gesetz. | und das gerechte machen let\%: - LuGINвüHL, Brennwalds Schweizer Chron. 2, 289, 7; 313, 25; Qu. Schweiz. Gesch. 1, 48, 1; Adomatis u. a., J. Murer. Abs. 447; Bab. 4615; UkenA, Luz. Sp. 2780; Hulsius Q iijr; Sснмidt, Hist. Wb. Elsaß 219; SchweIz. ID. 3, 1550; Schwёв. Wв. 4, $1193 \mathrm{f}$.

2. >verdreht, linkisch, ungeschickt, der normalen Erwartung entgegengesetzt, unangemessen (von Menschen, Verhaltensweisen u. ä.)<; auch: >merkwürdig〈. - Phras.: der letze meier >schräger Vogel<. - Bdv.: gäuchisch, link 2, tölpisch, übel, ungeschikt; vgl. lurtsch, lurz 2, täppisch. - Synt.: l. wirken, sich l. stellen / verkeren, etw. l. [tun], zur hand nemen; der letze kopf, das letze wort. - Wbg.: ${ }^{3}$ letze, leakopf 1. 
MiedER, Lehmann. Flor. 650, 29 (Lübeck 1639): Let\%kopff und Narren machens noch al. EBD. 838, 3: Mancher schleifft / hebt und trägt sein Creutz so vngeschickt / letz und tólpisch / das sich zu verwundern. LuTHER, WA 21, 468, 10 (1544): DAS sind nu eitel letze wort, so Christus seinen Jingern gibt. Bolte, Pauli. Schimpf u. Ernst 1, 94 (Straßb. 1522): er was also ein letzer Meyer, das er nichtz. für gut wolt haben. TitTmann, Schausp. 16. Jh. Funk 180, 228 (Bern 1551): Wie kanst du dich gar lätz. stellen! FUCHs, Murner. Geuchmat 3137 (Basel 1519): Welcher tüffel sy doch leret, | Das sy sich alß letz verkóret. ADOMATIs u. a., J. Murer. Ufferst. 577 (Basel 1567): ir klagend all on underscheid | Mit láren worten unütr. geschwátz. | ich wólt mich ouch gern stellen làtz: MeISEN u. a., J. Eck 55, 22 (Ingolst. 1527): dan yetlicher letzer kopff in bewegung seins boffertigen bertzen [...] im firgenommen hat, etwas në̈ß in der beyligen kirchen [ein₹üfieren]. MaAler 261v (Zürich 1561): Làtz. / Vngeschick.t. Ineptus. Látz. / Gouchisch. [...] Látze. Peruersitas. - Unena, Zuger Trag. 2460; Schmidt, Hist. Wb. Elsaß 219; SchweIz. ID. 3, 1552; Vorarlb. WB. 4, 1194.

3. >einen erwarteten Qualitätszustand, eine Funktion nicht oder nur eingeschränkt erfüllend, schlecht, minderwertig (von Sachen unterschiedlichster Art) <. - Wobd. / oobd. - Bdv.: vgl. anbrüchig, arg (Adj.) 1, gebrestlich, lästerig 2.

Bolte, Pauli. Schimpf u. Ernst 1, 332 (Straßb. 1522): Es kam uf einmal ein alt Weib mit einem letzen Schleier. WARnock, Pred. Paulis 7, 40 (önalem., 1490/4): dar er kainen gebresten hab, [...], nit ain letz. og hab, nit schilche oder krume nasen. NiewÖHNER, Teichner 686, 27 (Hs. 'moobd., 1469?7): als der scher (>Wühlmaus`) wult im pacht $\mid$ und macht wisen und áckher letzzen (flekt. präd. Attr.). Winter, Nöst. Weist. 3, 327, 33 (moobd., A. 16. Jh.): wolten [si] das letzer [vleis] bie verkaufen, wiert ainer des uberfaren, ze wandl 6 th. Bischoff u. a., Steir. u. kärnt. Taid. 510, 35 (m/soobd., 1515): das guet heuslich [...] innen ze haben, damit es nur pesser und nit letzer werde. Siegel u. a., Salzb. Taid. 217, 34 (smoobd., 1624): die heusser sind wandlpär und mugen bësser und lëtzer werden. Müller, Handel Paumgartner 271, 29 (Tirol 1545): 41 ster des letzern Imbster pro 4 fl. - JASPERs, St. v. Landskron 137v, 8; MüLLER, Welthandelsbr. 183, 20; WINTER, a. a. O. 2, 168, 28; VORARLb. WB. 4, 1194. - Vgl. ferner s. v. abessend.

4. >eigenwillig, zielstrebig auf etw. gerichtet, begierig auf etw.; feindselig; störrisch, widerspenstig «. - Bdv.: eigenrichtig, feindselig, greulich 1; vgl. abscheu (Adj.) 1, köpfisch, starhälsig, starkhalsig, starrig. - Wbg.: lezkopf 2, lezköpfig >starrköpfigく.

Gille u. a., M. Beheim 52b, 31 (nobd., 2. H. 15. Jh.): Sy sein gar lecz | auf pos auf secz. Bolte, Pauli. Schimpf u. Ernst 1, 194 (Straßb. 1522): Letzkospff thin nit güt. Ukena, Luz. Sp. 2842 (halem., 1575): Man muß sich in den poßen stellen / | Ob ettlich sich letz böggen wellen / | So nim ich sy mitt gwallt darvon. MAALER 261r (Zürich 1561): Låtzkeópffig. Duricapitones. Látzkopff Eigenrichtig. Peruicax. - Martin/Lienhart 460; Schweiz. Id. 3, 1553 f.

lezelte, lezelter, lezelterin, lezeltlach, s. lebzelte.

$$
\begin{aligned}
& \text { lezge, s. }{ }^{2} \text { letze. } \\
& \text { lezger, s. lezner. } \\
& \text { lezgeselle, s. }{ }^{1} \text { letze } 1 . \\
& \text { lezgläubig, s. lez } 1 . \\
& \text { lezgrabe, der. }
\end{aligned}
$$

> Grenzfurche, -graben (zwischen Grundstücken); Wehrgraben<; zu letzen 6, vgl. grabe 2 ; 3. - Halem. - Bdv.: vgl. anfurche.

Argovia 4, 267, 11 (halem., 1400/1450): [Der vmbkreis] gat von demselben markstein an den Pilgerweg und in den Lezgraben: und den Leagraben of an den markstein. LuginbüHL, Brennwalds Schweizer Chron. 1, 376, 17 (halem., 1508/16): [si] leitend sich an die Spanweid, enhalb den usseren lezgraben. BachmanN u. a., Volksb. 30, 33 (alem., 15. Jh.): [sy] bliessend ir strithorn und kamend herus uber den leczgraben an das feld. - LugINBüHL, a. a. O. 1, 357, 12; Welti, Urk. Rheinfelden 114, 43, 4; Schweiz. ID. 2, 682 .

lezheit, s. letzen 6 .

lezkopf $1 ; 2$, s. lez $2 ; 4$.

lezköpfig, s. lez 4.

lezner, lezger, der; -/- $\emptyset$; starke Schreibvariation infolge morphologischer Undurchsichtigkeit.

$>$ Geistlicher niederen Ranges; Subdiakon<; zu ${ }^{2}$ letze 1. - Wobd. / oobd. - Bdv.: epistler, evangelier, priester 2; vgl. levit 2. Wbg. : leznerskleid.

Barack, Teufels Netz 4590 (Bodenseegeb., 1. H. 15. Jh.): Letzner und och kolner | Lernend och die selben mer | Und tuond sich der wibi übergeben | Und hand och gar ain wilds leben. Bremer, Voc. opt. 19004 (halem., 1329 f.): Substile lerznerscleit [...] leccierskleit [...] epistler rokech [...] est vestis subdyaconi solempnis super alban induta in diebus festivis. EBD. 36048: Subdyaconus lechzger [...] letaner [...] epistler [...] vir ab episcopo consecratus ad infundendum aquam et vinum in calicem et ad apponendum hostiam. Buck, U. v. Richent. Chron. Conz. 105, 1 (alem., um 1430): [hertrog Ludwig] was beklaidet mit ainem rock als ein letzger. EвD. 139, 30: und bott der letzgner dem ewangelier die schüß- 
len mit dem brott. Dierauer, Chron. Zürich 23, 8 (halem., 1415/20): Keiser Karlus stifte die Probsty Zurich und bewidmet die mit einem propst und mit vier und zwenzig korberren, das sint acht prietser, acht ewangelier und acht lechziner. Boot, Cassiodor. Hist. Eccl. 153, 16 (moobd., um 1385): dy daucht daz guet sein, [...] daz dy pischof und dy priester, dy ewangelier und dy leczner, nach der weich nimer mit iren weiben scholten slaffen, dy si vor der weich bieten genomen. - Boner, Urk. Aarau 191, 5; SchweIz. ID. 3, 1563.

leznerskleid, s. lezner.

lezpredig, s. ${ }^{1}$ letze 3.

lezschiessete, s. ${ }^{1}$ letze 3.

lezt, lest (etwas seltener als erstere Form), Adj., in 3 nur adv. Gebrauch.

1. >letzt, zeitlich gesehen nach allem anderen (z. B. nach dem ersten); schließlich ‘; in adv. Gebrauch: ‘zuletzt`. - Phras. (gehäuft; teils mit Substantivierung, dann von lezte keine klare Trennung möglich): am lezten o. ä., auf das lezte, fürs lezte, in dem lezten, zum lezten o. ä., zu lezt, jeweils >zuletzt, schließlich«; das lezte mal. - Wbg.: leztlebend >jn. überlebend «, leztlich.

Quint, Eckharts Pred. 1, 389, 3 (E. 13./A. 14. Jh.): der êrste begin ist durch des lesten endes willen. Jâ, got der rouwet selbe nibt dâ, dâ er ist der êrste begin; er rouwet dâ, dâ er ist ein ende und ein raste alles wesens. PEIL, Rollenhagen. Froschm. 521, 448 (Magdeb. 1608): Weib halt ein / | Odr es sol dein letzter trunck sein. BECKERs, Bauernpr. 55, 14 (Köln 1515/18): in den lesten trweyen frydagen in dem mertzen. KöBlER, Ref. Franckenfort 54, 7 (Mainz 1509): dann darnach das letstlebende sich widderumb in die zweyte ehe verandert. Opes, Spittendorf 128, 41 (osächs., um 1480): Derhalben were yn letzst zu santh Mauritz vorbalt gesehen. Grosch u. a., Schöffenspr. Pössneck 235, 36 (thür., 1474): $Z c u$ deme letçtin uffgebote ist Jhaen von Thumpelingen vor gerichte [komen]. SERMON THAulerI IIIra, 10 (Leipzig 1498): Nun haben wir gesprochen von der erstenn und letzsten geburt, wie wir yn der letzsten an der ersten sollen lere nemẽ. B̈̈CHTOLD, N. Manuel. 277, 537 (Basel 1530): Sie ist weder die erst noch letst, | Die mit dem

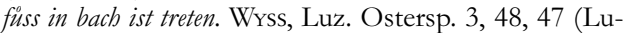
zern 1616): Das ist deß Herren letste wunder, I so er mit andacht thatt besunder $\mid$ Vor sinem todt. Chron. Augsi. 5, 104, 24 (schwäb., 1523/7): das schlos zů Hochenaschperg, das was das lötzst, das der pundt eingenomen hat. CHRON. Augsi. 8, 405, 11 (schwäb., zu 1552): auf den lesten tag des monats augusti. Winter, Nöst. Weist. 3, 729, 4 (moobd., A. 16. Jh.): welicher pawman der löst ist umb die fruemößzeit dem erstn ain metzn habern geben. PIIRAINEN, Stadtr. Sillein 96a, 34 (sslow. inseldt., 1378): Der svntag waz der erste tag der ye wart und der auch wirt der leczte. $\mathrm{Zu}$ den Phrasemen: SCHÖPPER 47b (Dortm. 1550): Postremo. Lăst / letətlich zum letzsten endtlich. Меijвоом, Pilgerf. träum. Mönch 6289 (rib., 1444): Du en kans up dat leste geyn guyt gehain, | He en wille deilachtich syn da an. Loesch, Kölner Zunfturk. 2, 396, 3 (rib., 1489): ind $u$. g. die beide ampte int lest vereiniget haven. CHrON. KöLN 2, 11v, 6 (Köln 1499): zo dem lesten so sullen sy verkriegen die krone der merteler. v. D. LeE, M. v. Weida. Spigell 77, 18 (omd., 1487): Am letzsten er vor dem babst vorclaget [worden]. Roloff, Naogeorg/Tyrolff. Pamm. 105, 878 (Zwickau um 1540): Noch eins fürs letæt. v. Keller, Ayrer. Dramen 2204, 6 (Nürnb. 1610/8): wenn er mich bie finden thet. I Ich euch das letzt mal gsehen hett. VETTER, Pred. Taulers 66, 28 (els., E. 14. Jh.): wie sint sú in daz. urteil Gottes in dem lesten gevallen. Wiessner, Wittenw. Ring 4607 (ohalem., 1400/08): ker dich auf das best | Und auf daz. gnädigost ze lest! CHron. AugsB. 2, 68, 26 (schwäb., Hs. 16. Jh.): daß er gottes leichnam begert hett an dem letsten. Pirrainen, Stadtr. Kremnitz 84 (mslow. inseldt., 1537): Zum Letzten, Wollen wier Iederman treulich gewarnet haben. - BAUMANN, Bauernkr. Rotenb. 104, 21; Wyss, Limb. Chron. 68, 26; KöBler, Ref. Frankkenfort 39, 10; 43, 17; Chron. Augsi. 8, 157, 2. Zu den Phras.: Kurz, Waldis. Esopus 3, 26, 3; v. Liliencron, Dür. Chron. Rothe 689, 17; Williams u. a., Els. Leg. Aurea 346, 7; Chron. Augsb. 1, 99, 9; 303, 25; Roth, E. v. Wildenberg 124, 20; Kummer, Erlauer Sp. 6, 56; Rwв 8, 1244 f. - Vgl. ferner s. v. absenz 2, ausrecken 5, ${ }^{2}$ auf $9,{ }^{1}$ base 4 .

2. an 1 anschließbar eine Fülle von Phrasemen um den Tod des Menschen, das Jüngste Gericht; teilweise elliptischer Gebrauch (Auslassung des Subst.). Ordnung so weit wie möglich und sinnvoll nach dem Alphabet: das lezte (abend)essen >letztes, sakramentstiftendes Abendessen Jesu mit seinen Jüngern<; der lezte abschied, das lezte abscheid / scheiden (o. ä.) jeweils euphemistisch für das Sterben, den Tod, die Todesnot; der leate atem; die leate auferstende >Auferstehung des Menschen`; das lezte ende >letzte Zeit eines Lebens; Todeszeit, Tod «; die lezte fart / hinfart; der lezte gang; das lezte gericht >das jüngste Gericht<; das lezte keichen; die leate lässe >letzter Stich beim Kartenspiel` (vgl. Dжв 6, 213; 772); der lezte lon; das lezte nachtmal wie das lezte (abend)essen; die lezte reitung $>$ die letzte Abrechnung «; die lezte not $>$ Todesnot «; die lezte stunde >die Todesstunde`; das lezte urteil 〉Jüngstes Gericht<; der lezte wille; der lezte tag, die lezten tage >Tag(e) vor dem 
Tod, Todestag〈; das lezte testament; die lezten zeichen; die lezte(n) zeit(en) oft in präp. Verbindung an / zu den lezten zeiten (o. ä.) für den Tod des einzelnen Menschen, für die Endzeit und die Zeit des jüngsten Gerichts (im einzelnen kaum trennbar); der lezte zorn; die lezten züge.

Belege in der Reihenfolge der Nennungen (mit semantisch bedingten Abweichungen): FronING, Alsf. Passionssp. 3069 (ohess., 1501 ff.): dijt sal das leste essen synn, | das ich mit uch essen sail! ReICHERT, Gesamtausl. Messe 11, 10 (Nürnb. um 1480): da er sprach an dem letsten abentessen nach dem als er den jungernn het das heylig sacrament gegeben. BAuer, Haller. Hieronymus-Br. 21, 7 (tir., 1464): Das ewenpild hat ins lassen Christus an dem lësten abent ëssen, da er wüsch die füesse seiner junger. ScHÖрPER 113a (Dortm. 1550): Mors. Todt sterben I abgang letzstes abscheid. Kenrein, Kath. Gesangb. 1, 269, 2 (Nürnb. 1631): Glorwuirdig Er zum Oelberg kam, |Da Er den letzten abschiedt nam. KLeIN, Oswald 35, 36 (oobd., 1409/10): göttlich geburd durch magt mensch rain, | hilf an dem letzten schaiden! V. Anshelm. Berner Chron. 2, 134, 6 (halem., n. 1529): dass si biss an letsten aten wie die kálber bláreten. SACHS 15, 342, 9 (Nürnb. 1562): Und hat sein unart gar ein end $\mid$ Biß zu der letżten aufferstebnd. SсHÖNBACH, Adt. Pred. 14, 14 (osächs., 1. H. 14. Jh.): min lip mize sterben als die gerechten und min leste ende muze sich gelichen den selben. Bӧнме, Morg.R. 13, 7 (Hs. ${ }^{\ulcorner}$schles., 1612 7 ): Mensch [...] der du an diesem letzten ende der zeit in so grosser sicherbeit lebest. Langen, Myst. Leben 156, 6 (nobd., 1463): da mit er [poser geist] / anficht d) krancken an dem leczten ende. WYss, Luz. Ostersp. 3179 (Luzern 1571): So sich thit scheiden die seele min $\mid A m$ lettsten end von minem lyb. OpITz. Poeterey 23, 23 (Breslau 1624): Worzue dienet das studieren / | [...] ?| Vnter dessen laufft die Bach $\mid$ Vnsers lebens [...] | [...] Auff jbr letztes ende bin. Primisser, Suchenwirt 32, 33 (oobd., 2. H. 14. Jh.): Nu let er auf der lesten vart $\mid$ Sein grozzes guet auf erden. SсHÖNBACH, Adt. Pred. 7, 16 (osächs., 1. H. 14. Jh.): 요 ffe daz $d u$ behalden werdes an diner leste hinevart. Fastnachtsp. 1451, 20 (nobd., 15. Jh.): Das lest oder gemein gericht wirt sein an dem end der wellt. LutHer, WA 34, 2, 282, 25 (1531): Also [...] gewiß soll ich auch sein, wenn ich den letzten gang gehe unter die Erden. Rотн, E. v. Wildenberg 4, 29 (moobd., v. 1493): ir müst warlich darumb antwort geben vor dem letəten gericbt. KLeIN, Oswald 29, 24 (oobd., 1409, 1426?): Maria, hab, bilf an dem letzten keichen. SACHS 20, 77, 34 (Nürnb. 1559): Treib auch das spil der letzten le $\beta$, [möglicherweise Umdeutung von lässe auf les >lectioব| Das mir offt war gar herb und reß. GILLE u. a., M. Beheim 81, 33 (nobd., 2. H. 15. Jh.): das ist ein gross czaichen vermelt, | das in solch creitlich glike $\mid$ Czu einem lesten lon sey gebm, $\mid$ als den grechten ir ewigs lebm | auch ist ir lester lone. WYss, Luz. Ostersp. 6677 (Luzern 1545): wisch in ir füs, irs werden gsen, $|[\ldots]|$ nam ouch mit in sin letst nachtmal $\mid$ zu Hierusa- lem in einem saal. SpeChTLer, Mönch v. Salzb. 33, 47 (oobd., 3. Dr. 14. Jh.): die letżten raitung für uns gilt, wann wir bie müssen sterben. Primisser, Suchenwirt 41, 700 (oobd., 2. H. 14. Jh.): daz uns in der lesten not Chains pósen geistes ang anscbilh. LuTHER, WA 53, 276, 2 (1542): Das sind die rechten letzten schrecklichen [...] plagen. EBD. 46, 62, 18 (1537): von dem letzten Regiment des Endchrists, nemlich des Bapstumbs. Wyss, Luz. Ostersp. 9284 (Luzern 1545): verlass in nit in der letsten stund! SCHÖNвасH, Adt. Pred. 32, 41 (osächs., 1. H. 14. Jh.): erlose mich von dem ewigen tote an dem vorchsamen tage des lesten urteiles. SCHÖPPER 106a (Dortm. 1550): Testamentum Testament erb gemächt seel gerecht letzter will. v. D. LEE, M. v. Weida. Spigell 83, 2 (omd., 1487): Der letzte wille des vorstorben menschen sall Jn allen dingen [gehaldenn werden]. Moscherosch. Ges. Phil. v. Sittew. 47, 37 (Straßb. 1650): Anstatt daß sie sich [...] der Sterblichkeit vnnd ibres Endes erinnern [...], so fangen sie an von der verstorbenen Letztem Willen oder Testament vnd der verlassenschaft zu erzeblen. Grothausmann, Stadtb. Karpfen 70, 22 (mslow. inseldt., 1602): 1602, Ortnet die Tugendtsame fraw Catharina [...] bej guter vernunfft mit vornemlicher teutlicher rete, ibren letzten willen wie volget. HeNsChel u. a., Heidin 952 (nobd., um 1300): Din ich nibt vergezze [...] $\mid$ Biz an minen letsten tak. Vetter, Pred. Taulers 34, 32 (els., E. 14. Jh.): in disen lesten tagen, das do sint die tage des heiles, so enmag enkein mensche niemerme genesen [...] dan úbermitz da minnekliche wasser des tiches, daz ist daz blit unsers herren. BAUER, Haller. Hieronymus-Br. 12, 30 (tir., 1464): er ist pesammen sein schaff in sein scboff an den lesten tägen. Grothausmann, Stadtb. Karpfen 68, 2 (mslow. inseldt., 1586): haben wir vns dem lezten teśtament nach gebalten. LUTHER, WA 29, 619, 5 (1529): es sollen die letzten zeichen nicht ferne sein. EвD. 54, 472, 13 (1548): Was wunder ists, das auch wir, so jtzt, in dieser letzten schrecklichen zeit, Christum predigen. Dubizmay, kurß zu Teutze 16, 13 (hess., 1463): küm mir zu bilff an | meynnem letzten zeytten. Reichert, Gesamtausl. Messe 92, 33 (Nürnb. um 1480): das Got der herre [...] richten und urteylen wirt eyn yegklichen menschen an seynen letzten zeyten. Wyss, Luz. Ostersp. 10083 (Luzern 1545): es würt zu den letsten sytten kon $\mid$ sim glouben ein widerspeniger trut: BAUER, Haller. Hieronymus-Br. 13, 3 (tir., 1464): Da in nu sein leste zeit wart nöten au₹ zugen von dem leib. Luther, WA 19, 193, 15 (1526): Also hat er auch Christum [...] ynn die welt gesand, ehe denn der letzte zorn des jungsten gerichtes kompt. EBD. 28, 670, 23 (1529): Guiter kónnen nicht helffen in den letżten zigigen. SAPPLER, $\mathrm{H}$. Kaufringer 16, 555 (schwäb., Hs. 1464): wie gern ir [tiefel] den menschen beraubt $\mid$ an den lețsten zügen sein $\mid$ der tugent rain. - Elliptisch: LOHMEYeR, K. v. Nostitz 159, 26 (preuß., 1578): wil ich alles, das ich weiß, auff meine letzte binder mir verlassen. LOESCH, Kölner Zunfturk. 4, 387, 9 (rib., 1397-1409): sal man in ermanen, wat he deme beilgencruir, wil geven [...] ind an sime lesten, dat be sin gunst ouch deme heilgencruiz bewise. BuCH WeINsB. 1, 262, 6 (rib., um 1560): in sinem lesten bat er sinen einigen son 
Eduardum zum koning ingesatzt. Moscouia CIv, 40 (Wien 1557): So aber der Großfürst Hans an seinem letaten gelegen / und die Geistliche jne der gewissen vermant / hat [...]. - Bauer, Imitatio Haller 97, 16; Golius 116. Vgl. ferner s. v. abschied 2, augenede, ausbleiben 3, auslassen 12 , ausrichtung 6.

3. von einem später als das Bezugsgeschehen liegenden Zeitpunkt aus gesehen auf den zuletzt stattgefundenen oder einen kürzlich abgelaufenen Vorgang bezogen: $>$ neulich, letzthin, vor kurzem .

Loesch, Kölner Zunfturk. 11, 548, 14 (rib., 1454): darup begeren ich guetlich wire eirsambeit ind wissheit zo wissen, dat ich lest uiren frunden, die bij mir wairen, der gelegenheit en deil gesacht hain. Kurz, Waldis. Esopus 4, 65, 1 (Frankf. 1557): ZV Maintz am Rhein ich lesten war. OpeL, Spittendorf 135, 2 (osächs., um 1480): seyne gnade und der rath hetten letzst zu sant Mauritz yre antwort gehort.

4. >letzt, hinterst, entlegenst, nach allem anderen (unter dominant räumlichem Aspekt, teils auf die Linearität von Texten bezogen) $<$.

Grosse, Schwabensp. 47a, 37 (Hs. rnd./md., um $\left.1410^{\urcorner}\right)$: de vinfte sibbe stet an dem ersten lede des vengers. [...] de sebede an dem lesten lede vor deme nagele. STREHLKE, Nic. Jerosch. Chron. 27734 (preuß., um 1330/40): dî êrsten und dî lesten [dorfir] | dî branten sî̀ gar abe. ROT 303 (Augsb. 1571): Declinirn, [...] die letzt silben eines worts in ein andre lencken. Chron. Augsb. 4, 410, 1 (schwäb., zu 1490): da die ersten nachet bei Jnningen wassend, da wassend die lesten noch bei Gegingertor. Hohmann, H. v. Langenstein. Quästio 203, 52 (moobd., 1. H. 15. Jh.): als sand Paul spricht ad Ephesios am lesten. - Gerhard, Hist. alde e 2869; Rohland, Schäden 465; Brévart, K. v. Megenberg. Sphaera 47, 14.

5. >zuletzt genannt<; in einer Reihe von Bezugsgrößen mit Absetzung von erst, ehe gesprochen, dann den Zeitverlauf des Textinhalts betonend. - Wbg. : leztgenant.

Quint, Eckharts Pred. 2, 433, 5 (E. 13./A. 14. Jh.): $N \hat{u}$ lâze ich die lesten zwei und rede von dem êrsten stücke. Chron. Augsb. 1, 62, 17 (schwäb., zu 1378): uff dem egesprochen letasten ros rait ain ersamer ritter. TURMAIR 4, 345, 1 (moobd., 1522/33): lezgenanter künig Schirm verfertiget herwider sein rät ab zue dem grossen Alexander. OоRsсhот, Spee/Schmidt. Caut. Crim. 335, 21.

6. dient der Charakterisierung des Endgliedes einer eher logisch reihend als zeitlich oder räumlich gedachten Reihe.

SсHÖNBach, Adt. Pred. 24, 31 (osächs., 1. H. 14. Jh.): daz [virr] sol der priester viten und sol drier hande bolz dar uf werfen tegiliches [...]. daz erste holz ist daz, gotes wort der beiligen lere, daz ander ist gut bilde beiliges lebenes, daz leste ist daz ammacht des beiligen gotes dinest. Turmair 4, 426, 7 (moobd., 1522/33): 'Dialectica' ist die dritt und lest kunst, so auch wort und red antrift. CHRON. BAIER. STÄDTE. Regensb. 98, 12 (noobd., 1529): Sambstag Bricci hab ich den lesten zehet von Tegerbeim außtailt.

7. dient der Kennzeichnung der äußersten Seinsmöglichkeit einer Bezugsgröße, darunter endzeitlicher Projektionen: ‘äuBerst, höchst; definitiv $\iota$, zum Teil auch zu 2 stellbar. - Texe der Sinnwelt ,Religion“.

Helm, H. v. Hesler. Apok. 19007 (nrddt., 14. Jh.): Die [Degen] zeigent dan ir leste tugent $\mid$ Mit degentlicher beldes mugent. EBD. 22913 (nrddt., 14. Jh.): Alpha und O ich bin, | Erst und let:t, ende und begin. Luther, WA 46, 453, 8 (1538): Johannes ist der letzte und gibt den letze trunck. EвD. 49, 741, 14 (1545): Die Donnerschlege [... sind ein [...] vortraber des letzten donnerschlags. [...]. das wird die letzte Posanne. Strauch, Par. anime int. 71, 11 (thür., 14. Jh.): wan alse liplich frucht das leiste unde daz suriste daz da ist in dem menschin. Mathesius, Passionale 50v, 7 (Leipzig 1587): Der seine besten Tage gelebet hat / das ist / der aller letate / geringste / vnterste vnnd außwurfling / vnter vnnd von allen Menschen Kindern. Kurrelmeyer, Dt. Bibel 3, 89, 12 (Straßb. 1466): on zwifel bie hab ich gesehen die letzsten ding dez sehenden mich. Chron. Augsi. 7, 78, 8 (schwäb., zu 1548): so möcht daraus anders nichts dann diser stat endtlicher, letster undergang und eusserist verderben zü gewarten.

lezte, die; möglicherweise formale und semantische Beeinflussung durch ${ }^{1}$ letze 7.

>Ende, Schluß<. - Phras.: an der lezt; auf die lezte. - Bdv.: ende; vgl. ausgang 13, beschlies 2 , grund 13.

Fischer, Brun v. Schoneb. 2575 (md., Hs. um 1400): doch ist unse letzte ie der tot. Scholz, Lanfrank. Chir. Parva 28v, 10 (md./oobd., 1446/8): auf dy leczte leg auf dy wunden werg. SACHs 18, 287, 4 (Nürnb. 1562): Stürtzst sie zu boden an der letż. Rupprich, Dürer 1, 153, 196 (nobd., 1520): Auff die lecit kam ein groser trach. ReIcHMANN, Dietrich. Schrr. 147, 14 (Nürnb. 1548): Der gute wein ist behalten biß auff die letot. Chron. Augsb. 2, 286, 10 (schwäb., Hs. 16. Jh.): das was die letzt, damit gieng der frid an des morgens am freitag. Chron. Augsb. 5, 217, 2 (schwäb., 1523/7): auff die lötzst ist er gäcblingen erschwartzt und auf der kantzel gestorben. - DOUBEK u. a., Schöffenb. Krzemienica 566.

leztens, s. lezt 3.

leztgenant, s. lezt 5.

leztlebend, leztlich, leztmals, s. lezt 1 .

leztrunk, s. letzen 3.

lezverständig, s. lez 1. 


\section{leztwerf, s. lezt 3.}

lib, s. libra.

liban, der; Bergname.

>Libanon (in einem geographisch kaum festlegbaren Sinne)<; auch bildlich und ütr., in letzterem Falle: >Weihrauchく. - Wbg.: libanisch.

LuTHER, WA 19, 421, 12 (1526): Dann Libanon auch dasselbige gebirge Hermon ist. Das dis sey der synn: Du hast ym Libanischen lande, das ist ym Judischen lande, grosse frevel geubt. Bihlmeyer, Seuse 13, 28 (alem., 14. Jh.): als der hobe liban unverschniten smaket [...T, also bin ich ein blindes [...] lieb. Kurrelmeyer, Dt. Bibel 6, 125, 12 (Straßb. 1466): Wann sende mir auch zedrine bóltzer [...] von dem liban [Var. W, 15. Jh.: aus dem libanischen walde]. RAUwolf. Raiß 23, 27 ([Lauingen] 1582): Tripolis [...] ligt [...] an den vorbergen des hohen Gebürges Libani. - BIHLMEYER, a. a. O. 310, 12.

libanisch, s. liban.

libel, das; -s/-; zu mhd. libel (LeXer 1, 1895), dies aus lat. libellus (GEOrges 2, 560).

>kleines Büchlein, schriftliche Darlegung rechtsrelevanten Inhalts ‘; bei Bezug auf ein verbales Vergehen z. B. ’Schmähschrift〈, bei Bezug auf die Klageerhebung (häufiger) bzw. den Vortrag einer Beschwerde im einzelnen z. B. >Klageerhebung, -schrift ‘; >Bitte, Beschwerder. - Bdv.: anforderung 3, anklagezettel, bitte 1, brief $1-5$, büchlein (s. buch 1; 3), pasport 1; 2, fürtrag, geschrift 1, (schriftliche) klage 2, rechtshandel, schrift, supplication, zuspruch. - Synt.: l. tun >Klage erheben<, ein l. artikulieren / bedürfen, verfertigen / stellen / vorbringen, [wie] verstehen, klar ausdrucken > formulieren<, jm. ein l. geben, überantworten, ein l. in schriften einlegen, zu den-krankheiten ergründen; kraft des libels etw. ordnen / setzen; dem richter etw. ane l. erbieten, in einem l. etw. stellen > festlegen<, begriffen sein, etw. in ein l. zusammenziehen, jm. in einem l. etw. (z. B. böse worte) anschlagen; das eingelegte / grosse / neue l., gleich lautende libelle; das l. wieder dem gegenteil; in libels weise. Wbg.: libellieren >jn. publizistisch schmähen< (zur erstgenannten Variante).

SCHÖPpER 93a (Dortm. 1550): LIBELLVS. Libell clag zuspruch anfurderung. KӧвLER, Ref. Franckenfort 96, 15 (Mainz 1509): ein jglicher clager / er thu sein forderung durch sein fürsprechen müntlich / oder lege sein clage oder libell jn schriffte jn. LAUFs, Reichskammergo. 256, 32 (Mainz 1555): In der sachen zwischen $A$. und B. gib ich diese geschrift, nemlich libell, exceptiones, articulos, replicas, duplicas. KoHLer u. a., Bamb. Halsger. 134, 2 (Bamb. 1507): schmehschrifft, zu latein libel famoss genant. FucHs, Murner. 4 Ketzer 383 ('wohl Straßb. ${ }^{\top}$ 1509): Darumb Wygand in seim libell | Doctor Branten vil manche schnell | Mit bosen worten aneschlig. KuRz, Murner. Luth. Narr 1986 (Straßb. 1522): Wa iemans thet vnß widerstant, | So riefft im vß so thür den wein, [...] | Vnd facht in an zu libillieren, | Vil bosser stück doch nit probieren. GRAF-FuCHS, Ämter Interl./Unterseen 437, 7 (halem., 1605): das sölliche verbesserete [...] artickel zusampt den alten, [...], in ein nüw lybell züsamengezogen [...] werde. Rот 325 (Augsb. 1571): Libell, Buchle. Jtem ein schrifftliche bit und furtrag / die man sonst ein supplication nennt. Jtem ein rechts handel in gschrifft gestelt. Jtem gschrifftliche verkindung zum rechten. Jtem ein Boßpart / oder zettel von eim Zolner. GeHRING, Würt. Ländl. Rechtsqu. 3, 456, 13 (schwäb., 1574): soll biemit und in allweg, was in diesem libel von puncten zu puncten underschidlich begrifen, unwiderrieflich, trewlich, [...] gehalten [...] werden. - Laufs, a. a. O. 260, 35; 262, 22; FAstnachtsp. 991, 11; V. Anshelm. Berner Chron. 4, 269, 12; PfeifferBelli, Murner im Glaubensk. II, 13, 28; DERs., Murner. Kl. Schrr. 6, 84, 30; Chron. Augsb. 4, 289, 7; 6, 74, 6; 7, 135, 3; Gehring, a. a. O. 3, 436, 2; SсHib, Urk. Laufenb. 363, 114; Eschenloher. Medicus 45, 12; Wopfner, Bauernkr. Tirol 6, 9; MaAler 270v; Rwb 8, 1288; Vorarlb. Wв. 2, 277; Eckel, Fremdw. Murners. 1978, 127. - Vgl. ferner s. v. antworter 2.

libellieren, s. libel.

liberal, Adj.

> freigiebig, großzügig, wohltätig ‘. - Bdv. s. u. SchÖPPER, vgl. ferner giftig 1. - Wbg.: liberalität.

SCHÖPPER 11b (Dortm. 1550): Benignus. Gútig freundtlich 1 milt freymilt liberal gastfrey gabreich freygebig gutthätig. CHRON. Köln 2, 519, 28 (Köln 1499): der vurß buschof was ein weidelich liberail ind wise furst. BARACK, Zim. Chron. 2, 331, 26 (schwäb., M. 16. Jh.): das er sich entlichen entschloss, er wellt auch ainmal s. Niclaus sein und in seinem dorf under den paurendöchtern umbher terminirn und sein liberalitett erzaigen. Rот 324 (Augsb. 1571): Liberalitet, Trewe, und milte hand oder freygebigkeit.

liberalität, s. liberal.

liberanzbrief, der; $\mathrm{Bw}$ aus mlat. liberare >bürgen` (NiermeYer 1, 1002, 794).

$>$ Garantieurkunde über eine Geldschuld r. - Rwв 8, 1290 (a. 1532).

liberation, s. ${ }^{1}$ liberieren.

${ }^{1}$ liberei, die; zu mhd. liberîe >Bibliothek (LeXer 1, 1896), dies über das Rom. aus lat. liber(arium). 
>Bibliothek«; vereinzelt mit Tendenz zu $>$ Buchladen, Buchhandlung $`$; tropisch auch im Sinne von >geistliches Rüstzeug‘; >einzelne Bücher; Schriften`; positive und negative Bewertung der Bibliothek (letzteres in Richtung auf >Scheinwissen`) ausgewogen. - Bdv.: bibliotheca, bücherbehaltnis, bücherstat, buchgaden, buchkammer, buchladen. Synt.: 'die / eine l. angeben / an-, aufricbten, bauen, zu wege bringen?, jeweils >gründen`, die l. baben / beschauen / erreichen / weofüren; die l. (Subj.) die apoteke / arzenei der sele sein; in die l. kommen, in der l. sitzen, etw. (z. B. bücher) in der l. behalten, etw. in der l. aufputzen, ütr.: den harnisch in der l. schlagen, sich auf die $l$. verlassen, auf die l. geneigt sein; die l. des domstiftes, der gelerten; die alte / falsche / gelerte / grosse / köstliche / überflüssige > reichhaltige $<$ l. LUTHER, WA 15, 49, 12 (1524): das man fleys und kosten nicht spare, gutte librareyen odder bücher-heuser [...] zuverschaffen. Goedeke u. a., Liederb. 259, 70 (Marb., 1549): zun waffen wöllen wir greifen, | den harnisch legen an, | den Paulus hat geschlagen | in seiner liberei. Gerhard, Hist. alde e 4554 (omd., um 1340): Erdras hatte wol besachet | Di librarie und gemachet. Lemmer, Brant. Narrensch. 1, 4 (Basel 1494): Vff myn libry ich mych verlan |Von buchern hab ich grossen hort | Verstand doch drynn gar wenig wort. Goldammer, Paracelsus 6, 194, 12 (1530): also werden verschwinden die großen falschen libereien, die großen sinagogen, die großen stift, kloster. SPANIER, Murner. Schelmenz. 34, 13 (Straßb. 1512/3): Das ist biß har die libery [vom Hrsg. als liberei 2 interpretiert]: | bòse werck - ein bóß geschrey! MaAler 270v (Zürich 1561): Liberey (die) Ein ort da man bucher behaltet. Bibliotheca, Libraria. Haltaus, Liederb. Hätzlerin 1, 28, 169 (schwäb., 1471): Vnkeüsch, wucher und Symoney, | Das kartenspil vnd quatter drey | Ist der gelerten librey, | Da sy studieren ynn. Chron. AugsB. 9, 122, 15 (schwäb., 1544/5): daß er [...] überflissige libereien mit allerlai biechern, göttliche kunst lerende, auffgerichtet hat. Roт 325 (Augsb. 1571): Librei, Buicher bebaltnus / Buicher stat / Büchladen oder gaden. HeNISCH 92 (Augsb. 1616): Die Liberey ist der Seelen apoteck / artzney. - RuppRICH, Dürer 173, 23; SACHS 4, 41, 19; 16, 390, 8; 20, 110, 17; 243, 1; Kurrelmeyer, Dt. Bibel 10, 274, 22; Morrall, Mandev. Reiseb. 161, 19; Chron. Augsb. 3, 457, 1; Barack, Zim. Chron. 1, 423, 20; Baumann, Bauernkr. Oberschw. 81, 12; Turmair 1, 114, 24; 4, 309, 18; 5, 164, 13; Qu. Brassó 4, 182, 1; Voc. INC. TeUt. o viijv; MaAler 82r; SCHMidt, Hist. Wb. Elsaß 221; Eckel, Fremdw. Murners. 1978, 128. - Vgl. ferner s. v. angeben 8.

${ }^{2}$ liberei, die; zu mhd. liberîe >Abzeichen (Lexer 1, 1896), dies über das Rom. aus mlat. liberata >Geliefertes ‘; s. Niermeyer 1, 2002, 794; Eckel, Fremdw. Murners. 1978, 128; Jones, French Borrowings 399.

>Abzeichen (von Herrschafts- und Standesrängen) auf der Kleidung ‘. - Vielfach berichtende Texte. - Synt.: (die) l. tragen / erstiegeln, aus den ärmeln abtrennen, jm. die l. geben; mit l. geburt sein, etw. zu l. brauchen; die l. des königs / bischofs / herzogen / berren / knaben / lakeien; die l. mit dem kreur. Wbg.: ${ }^{2}$ liberieren >jn. auszeichnen<.

Altmann, Wind. Denkw. 381, 14 (wmd., um 1440): do hette er [keiser] den zwein geben sin liberige. Österley, Kirchhof. Wendunmuth 2, 60, 10 (Frankf. 1602): alles volck zu roß und fuß fast wol gebutzt mit federbuschen und andern liberey, berpaucken, trummeten. EuLING, Kl. mhd. Erz. 281, 5 (nobd., E. 15. Jh.): Wer schone weip bit umb ir myn | und dar aüff legt sein witz. und syn | mit boffiren gen und sten und cleyd, $|[\ldots]|$ mit lieberey, rossen, federspil. V. Anshelm. Berner Chron. 4, 179, 26 (halem., n. 1529): do wurdend etlich Eidgnossen vom keiser ouch zü mötwilliger bracht gelifret. Chron. Augsb. 2, 27, 27 (schwäb., Hs. 16. Jh.): der trueg hertzog Hansen von Münichen librei und büchs und sprach, er wer bertzog Hansen pot. DieHL, Dreytw. Essl. Chron. (schwäb., 1549): die hernn der landtschaft [...], habentt auch ire sunder lyfryenn gebebbt. EBD. 54, 10: Her Frantzenn lyfrey und kleydung ist gewessenn gelb und schwartz samett. KLEIN, Oswald 26, 8 (oobd., 1427): nach ainem plümlin was mir we, $\mid$ ob ich die Liberein da möcht erstiglen, | von ainer edlen künigin | in mein gewalt verriglen. - Fischer, Folz. Reimp. 25, 441; Diehl, a. a. O. 52, 6; Chron. Augsb. 7, 186, 8; 8, 363, 20; Vorarlb. Wb. 2, 290 .

\section{${ }^{1}$ liberieren, V.}

>jn. befreien «. - Wbg.: liberation.

LutHer, WA 16, 254, 37 (1524): auff das man der herrlichen Liberation der Kinder Jsrael aus Egypten nicht vergesse. Rот 325 (Augsb. 1571): Liberirn. Erlosen / erretten. Liberation, Erlosung / freymachung. - SCHwEIz. ID. 3, 983.

${ }^{2}$ liberieren, s. ${ }^{2}$ liberei.

libertas, lat. Flexion, libertät, die.

>Privileg, Befreiung von sozialen, rechtlichen Verpflichtungen`; mehrfach mit Bezug auf die ,alten Freiheiten' gebraucht. Bdv.: s. u. Beleg Luther (lat.), ferner freiheit, gnade 10. - Synt.: l. haben / recuperieren; sich der l. gebrauchen; lust / willen zu der l. tragen; die l. der Teutschen; die alte / hergebrachte / keiserliche / bäpstliche l. 
Luther, WA 10, 1, 1, 648, 8 (1522): hatt der Bapst und geystlich recht ybr priuilegia, libertates, immunitates, indulta, gratias und eyttel außtinge. Chron. AugsB. 7, 262, 2 (schwäb., zu 1522): zu der alten, wol hergebrachten libertet und freihait der Teutschen. EBD. 16: damit Gottes ehre und wort gefürdert, auch die alte libertet und freibait der Teutschen recuperirt und in den vorigen [...] stand gestellet und gebracht werde. EвD. 9, 79, 4 (1544/5): dieweil Augspurg [...] hoch löblich befreit und sich der libertas des reichs gebraucht. RoT 324 (Augsb. 1571): Libertet, Freiheyt. - Eckel, Fremdw. Murners. 1978, 127.

libertät, s. libertas.

libertiner, auch libianer, der; $-/-\emptyset$; aus lat. lïbertinus (GeORges 2, 640).

eine religiöse Glaubensgemeinschaft in Jerusalem (vgl. Apg. 6, 9). - Zur Sache: LThK 6, 1019; vgl. auch RGg 3, 1929, 1630 mit einer weiteren Bedeutung: >Vertreter einer quietistischen Mystik

BeLL, G. Hager 64, 1, 20 (nobd., 1594): Edlich stunden auch auff dar bej;, $|[. .]$.$| aus der schul hisen die libianer.$ Kurrelmeyer, Dt. Bibel 2, 303, 18 (Straßb. 1466): etlich stunden auf von der synagogen die do ist geheissen der libertiner. - Vgl. ferner s. v. asia 1.

libeter, Adj. zum südslov. / oberung. Ortnamen libethen.

>libether, in Libethen abgebaut (von Schwarzkupfer) <; es wurde in den Hütten Sohl (Oberungarn) oder St. Georgenthal (Thüringen) versaigert (so das Glossar zu u. a. Ausgabe).

Helbig, Qu. Wirtsch. 5, 92, 13 (md., 1503): was gemeine rotte oder libetter Kupffer im Sol auch gemacht werden, sollen in gemein zu gewin vnd verlust verkaufft werden.

\section{libianer, s. libertiner.}

libra, abgekürzt $l b$. und lib., teils lat. Flexion.

>Pfund ८, Gewichtseinheit für Waren und Münzen; ütr. auch für das Sternzeichen >Waage gebraucht. - Wbg.: librament, librieren > wiegen

Löscher, Erzgeb. Bergr. 192, 18 (omd., 1554/1633): Zwo wogen annabergisch eißen baben gewogen 84 lb. MoN. BoICA, NF. 2, 1, 51, 23 (nobd., 1464): so ist bestymmet in den buichern die món₹, [...], ye dreissig pfenn. für ein lib. werung genant. Plant u. a., Main. Naturl. 301a, 7 (ohalem., Hs. E. 14. Jh.): Ein ander zeichen libra. so sint tac $v \bar{n}$ naht als gelich lanc als der ein ding wege mit einer wagen. Broszinski, Minner. Chir. Parva 76r, 6 (halem., 2. H.
15. Jh.): tu das saft daruon und mit ij lib. schwini schmer, das alt sye. Müller, Nördl. Stadtr. 71, 17 (schwäb., 1421): Welche partie sich des satzte, als oft das von ir vor rate oder gerichte geclagt wirt, als oft git sie ie ein lib. Rот 325 (Augsb. 1571): Librament, gwicht / pfächt / abmessung. EBD.: Librirn, Wegen / oder mit der bleywag vnnd richtschnur abmessen. - Weingart u. a., Seelb. Rhodt 297, 1; Mon. Boica, NF. 2, 1, 303, Anm. 2; Müller, Alte Landsch. St. Gallen 8, 7; 15, 15; Broszinski, a. a. O. S. 319.

librament, librieren, s. libra.

\section{licon tripon, Genus?}

>Licon Tripon<, ein Arzneimittel gegen Harn- und Blasensteine aus dem Antidotarium Nicolai (so KeIL, s. u.).

KeIL, Peter v. Ulm 238 (nobd., 1453/4): Zu dem stein. Nym plut von einem fuchs; [...], vnd mach verbena-wurtz. zu puluer vnd thus in einen wein [...] oder nym electuarium ducis oder licon tripon mit warmem wein: pricht den stain.

\section{lidig, s. ledig.}

lieb, das (meist) / der (seltener); es/ -.

1. >Geliebte, Liebchen, Geliebter`; meist auf die Frau bezogen; vgl. lieb (Adj.) 6; auch diminutiv gebraucht. - Gewisse Beleghäufung für poetische Texte.

Luther, WA 5, 655, 304 (um 1535): Ein lieb sucht das ander. Karnein, Salm. u. Morolf 388, 2 (srhfrk., Hs. um 1470): Die liebe ist gar verlorn, | sie hat den heiden zu einem andern liebe erkorn. Schaer, Pyr.-Thisbe-Sp. III, 197, 1047 (osächs., 1607): die haben [...] | Einander gehalst vnd geküst, | wie zweyer liebichen gebrauch i(e)st. OpITz. Poeterey 31, 18 (Breslau 1624): wer nicht kan noch mag | Sein lieb sebn wann er wil / wird alt auff einen tag. v. Keller, Ayrer. Dramen 2126, 6 (Nürnb. 1610/8): Mein voriges Lieb thut todt liegen, | Für die ich in die Höll wehr gstiegen. Pfeiffer, K. v. Megenberg. B. d. Nat. 249, 25 (oobd., 1349/50): sie [-snecken] begernt des bimeltawes rebt als ain fraw irs liebes begert. Primisser, Suchenwirt 28, 110 (oobd., 2. H. 14. Jh.): Mein lieb der hat wol bundert | Tzu lieb im ausgesundert. KLEIN, Oswald 34, 46 (oobd., 1416): wenn sich mein houpt wirt senken | gen deinem veinen mündlein rot, | so tü mich, lieb, bedencken. Kummer, Erlauer Sp. IV, 652 (m/soobd., 1400/40): wann ich dir fleißleich gedienet han | recht als ein rechter chanman, | der nimer lieb hat dan ains. - Wyss, Limb. Chron. 65, 25; Moscherosch. Ges. Phil. v. Sittew. 48, 24; Klein, a. a. O. 20, 39. - Vgl. ferner s. v. ankallen.

2. >in der mystischen Liebesbeziehung zwischen got / Christus einerseits und der sele / dem Menschen andererseits sowohl für Gott / Christus wie für die Seele / den Menschen als Ziel der Liebe gebraucht $<$; Ütr. 
zu 1; erotische Bildlichkeit der Belege. Meist Texte der Sinnwelt ,Religion', speziell der Mystik; älteres und mittleres Frnhd.

Quint, Eckharts Pred. 604, 8 (E. 13./A. 14. Jh.): Dâ von sprichet diu sêle in der minne buoche: , min liep hât mich anegesehen durch einen schranz'. HüBNER, Buch Daniel 3500 (omd., Hs. 14./A. 15. Jh.): ,Min lieb, du [3493: sele] bist suberlich, | Lustic mir [3498: Cristus] an allen schrantr. BiHLmeYer, Seuse 480, 15 (alem., 14. Jh.): so wir daz gótlich liep [...] ie dicker an blicken. Vetter, Pred. Taulers 383, 16 (els., 1359): Ach aller liepstes einiges liep [bezogen auf unseren herren], were ich des wirdig das [...]. Lauchert, Merswin 4, 25 (els., 1352/70): das dv [got] wellest ane sehen dine grúndelose irbermede, und wellest min liep und min gespunze sin. Pfeiffer, K. v. Megenberg. B. d. Nat. 278, 26 (oobd., 1349/50): got abt die sêl nibt klain, er schätzet si als sein schatzpar liep, nâch im selber gepildet. - Vgl. ferner s. v. ausgesamnen.

3. `Geliebtes, Geschätztes, Ersehntes, Verehrtes, Gegenstand existentiellen Bemühens; friedliches, freundliches Verhalten, das zu dem Ersehnten führt` (dies letztere im SchweIz. ID. 3, 987, mit reicher Belegung); vgl. lieb (Adj.) 5.

Jostes, Eckhart 67, 7 (14. Jh.): Daz ein ist, waz der mensch liebes [dies als Subst. zu lieb (Adj.) 5] hat in dirre werlt, also schier als er dez enphindet, daz im daz ein bindernuzz ist an iens lieb, so sol er diz lieb durch iens lieb lazzen. Vetter, Pred. Taulers 38, 29 (els., E. 14. Jh.): die ime denne in diseme vinsternisse truwe hant geleistet, die furt er on alles mittel in sin unsprechenliches ewiges liep. SAPPLER, $\mathrm{H}$. Kaufringer 26, 52 (schwäb., Hs. 1472): leiden ist ain kurzes lait $\mid$ und ain langs lieb.

4. 'Zuwendung, Trinkgeld, kleine Vergütung «. - Bdv.: vgl. liebe 3, liebnis 2 .

Argovia 9, 118, 1 (halem., 1530): das dem aman alls lieb beschech, soll er den kouf verkünden in der kilchen. SCHWEIZ. ID. 3, 986.

\section{5., s. liebde 5 .}

lieb, Adj.

1. >angenehm, lieb, das Gefühl der Freude, des Glückes, Wohlbefindens vermittelnd; eine Bezugsgröße, einen Sachverhalt als angenehm empfindend, so beurteilend, als ob er angenehm sei<; von Bezugsgegebenheiten unterschiedlichster Art gesagt; vgl. lieben 1, auch 2. - Phras.: es / etw. ist jm. lieb oder leid; jm. etw. zu lieb tun; der teufel möge jm. lieber wesen! > es wäre jm. lieber, dass der Teufel ihn holte, als [...] «. - Bdv.: angenem 1; 2, genäme, geneigt, günstig 1, hold, wert, wolgefällig. Ggs.: leid (Adj.) 1; 4, wieder. - Synt.: etw. / j. l. sein, j. [wo] l. >gern gesehen< sein, jm. etw. (z. B. das leben) l. sein, jm. j. (z. B. das kind) / etw. (z. B. die ere, das federspiel) l. sein, jm. etw. l. sein, das [...] / ob [...] / zu [...], jm. etw. lieber sein, ehe [...], jm. j. lieber sein, denne [...], jm. j. lieber werden, wan [...], jm. l. tun, jn. l. halten / gewinnen / meinen, etw. l. achten, sich etw. l. sein lassen (z. B. geld), jm. etw. zu l. machen, jn. jm. (z. B. got) l. machen; der liebe brief / gast / rebensaft, das liebe ungemach / volk / vaterland. Wbg.: lieblächeln, lieblellen >schmeicheln< (a. 1518), liebleller (a. 1518), liebsam, liebschlek >Leckerbissen<, liebspeise (dasselbe), liebsprecher >Schönredner, Schmeichler (insofern hierher; andere Deutung im Dwв 6, 976/9; das Stichwort liedsprecher spricht für die Deutung im Dwв), liebtraben (Beleg s. v. liebkosen 2).

SchÖpper 10b (Dortm. 1550): Charus. Lieb holdt werdt freundtlich gunstig geneigt zuthätig holdselig. LuTHER, WA 30, 2, 279, 24 (1530): Und war Luther das liebe kind, und fegete die stifft und pfarren von solchem treudel marck.t. PEIL, Rollenhagen. Froschm. 547, 1288 (Magdeb. 1608): Die Geste blieben in dem Nest / | Das war jhnen das liebst vnd best. Lappenberg, Fleming. Ged. 79, 3 (1635): Es ist mir besonders lieb, mein Polus, daß ich dich itat bei mir befinde. Grosse, Schwabensp. 201a, 8 (Hs. ' nd./md., um $\left.1410^{7}\right)$ : wu lip im sin veder spil was; halp also vil sal her ime geben. Quint, Eckharts Trakt. 24, 9 (E. 13./A. 14. Jh.): daz im wirt sin vater, muoter, bruoder und swester hundert wis lieber wan sie ieze sint. FronING, Alsf. Passionssp. 2736 (ohess., 1501 ff.): Byß wilkom, Davidis sone! | du host mer nu liebe gethayn! VOC. INC. TEUT. $\mathrm{p}$ ir (Speyer um 1483/4): Liebsprech' Gannio. i. adulator. Mathesius, Passionale 33v, 26 (Leipzig 1587): das jhrs ja euch auch als ewer Symbolum vnd Bekentnis / lieb vnd werth achtet. Bindewald, Texte schles. Kanzl. 28, 10 (schles., 1350): daz si nur von firdebalbir bufen daselbist geschos heischen vnde nemin sullin [...], alz lip in vnser bulde сұи behaldin sy. Opitz. Poeterey 42, 5 (Breslau 1624): So windtsch' ich daz mein feind dich moge lieb gewinnen. Henschel u.a., Heidin 1127 (nobd., um 1300): Im mobte liber sin der tot $\mid E$ denne er so groze not $\mid$ Lide durch eines keisers wip. FISCHER, Folz. Reimp. 25, 167 (Nürnb. 1497): [Unkeusch] Lieblechelt, bendkratzt, schmucat und gelimpft. Reichmann, Dietrich. Schrr. 146, 17 (Nürnb. 1548): Kinder sind den Eltern nimmer lieber / [...] / denn so sie kranck sind. v. KeLler, Ayrer. Dramen 3232, 29 (nobd., um 1600): Nun hat Er Ein schwester, genandt | Apollonia, die In gwan lieb |Vnordenlicher Wey/3. SCHADE, Sat. u. Pasqu. 3, 1, 4 (orhein. 1520): under wölchen [ge- 
schöpf] er [schöpfer] zwo in sonderbeit lieb gemeint, begabet mit unußsprechlichem kleinet der verstentnus. Gilman, Agricola. Sprichw. 1, 17, 13 (Hagenau 1534): Er ist lieb unnd werdt gehalten. BeHrend, Spangenb. Anbindbr. 4, 46 (Straßb. 1611): David den Nabmen hett bequem / | Weil er lieb war und angenehm | Bei Gott. DAsypodius C, 412r (Straßb. 1536): lieb Schleck oder speiß. Philtrum. Roloff, Brant. Tsp. 497 (Straßb. 1554): Da gab ein güt frë̈ndt mir den rath $\mid$ Das ich doch etwas liebs doch werffen soth $\mid$ Ins mer. BaCHMANN, Haimonsk. 55, 33 (halem., 1530): Wir dörffend zuo Dordonna niemmand fürchten, wann wir sind da lieb. Ders., Morgant 34, 8 (halem., 1530): ich will nŭt mer an dissem hăf beliben, da die verrether lieber sind dann die frommen. MAaler 270v (Zürich 1561): Das Lieb vnd angenám vatterland. Patria antiqua. [...]. Einem abwásenden etwas zeLieb vnnd zu gefallen thin. EBD. 271r: Gott Lieb vnnd angenám. [...]. Jch begár das von dir so es dir Lieb ist [...7. EBD. 509r: laß dir dz. schnód gált nit so lieb sein. STEER, Schol. Gnadenl. 3, A2, 213 (schwäb., 1447): die gnad haizzet man also das sy den menschen got liep machet. SAPpler, H. Kaufringer 1, 360 (schwäb., Hs. 1464): nichtz, liebers was in irem waun | dann das kindelein verwassen. EBD. 15, 44: wär es dir lieb oder laid, | ob ain man läg bei mir hinn | hie an dem pett. Barack, Zim. Chron. 4, 234, 36 (schwäb., M. 16. Jh.): Ee ein liebs, kumend bundert laid. | Recht wie der schat ist unser leben. CHron. Augsi. 3, 262, Anm. 7 (schwäb., E. 15. Jh.): ain junger kardinal [...] der waß dem bapst fast lieb auff die welsch artt. KLEIN, Oswald 25, 20 (oobd., 1414): mit süssen worten leise | wirt mir vil liebs beschert. ЕвD. 88, 7: so wer ain laid ... vertuscht, | das hail drung mich zu liebem ungemach. Hohmann, H. v. Langenstein. Quästio 195, 230 (moobd., 1. H. 15. Jh.): der ritter ist seinem herren vil lieber, der mändleich in dem streyt dy veint hat ïberbunden, den der in den streytt nye cham. Kummer, Erlauer Sp. 5, 142 (m/soobd., 1400/40): ich hach im soleich sleg an, | im möcht der teufel lieber wesen. v. Maren, Marquard. Ausgabe 89, 2 (Venedig 1483): Jr [ráine megdt] rede ist messig ir stym ist senftmutig ir geper sind liebsam. - KARNEIN, Salm. u. Morolf 412, 2; v. Tscharner, Md. Marco Polo 2, 26; Fastnachtsp. 8, 26; 1344, 8; Rupprich, Dürer 1, 151, 18; v. Keller, a. a. O. 1166, 13; 1285, 11; 2885, 16; Strauch, Schürebrand 44, 5; Argovia 9, 118, 1; Bachmann, a. a. O. 7, 26; SAppler, a. a. O. 17, 40; Klein, a. a. O. 64, 10; Uhlirz, Qu. Wien 2, 3, 4257, 9; Sснмidt, Hist. Wb. Elsaß 223. - Vgl. ferner s. v. ${ }^{1}$ als 1 , anfallen 5 , angesicht 8.

2. dient attributiv in der Anrede, in titelähnlichen Formeln und in formelhaft referierenden Substantivgruppen der Herstellung und Einforderung von Vertrautheit, Verbindlichkeit, Kooperativität von Personen, die einer bestimmten herrschaftssozialen, teils konstitutionellen (sowohl niedrigeren, abhängigen, untergebenen als - sel- tener - höheren Ranges) zu der Person stehen, die als Textautorität fungiert; vielfach im Umfeld mit andächtig 3, edel, erenfest, ersam, erwirdig, getreue, beimlich 4, streng, weise; vgl. am ehesten lieben 3; 7. - Synt.: unsere lieben bürger / untertanen / getreuen / andächtigen; in Augsb. Chroniken auch: sonder lieben, lieben besonder. Wbg.: liebgeld >Hilfssteuer, Karitativgeld` (a. 1581).

Loesch, Kölner Zunfturk. 2, 292, 2 (rib., 1495): So als sich irrunge [...] tuschen unsen lieven, getruwen ind samenburgeren den meisteren ind amptzgenoissen der vilzhuedemechere [...] erbaven hatten. KüTHER, UB Frauensee 226, 12 (thür., 1450): Unßern gunst tquvor, probist eptischin [... unßis closters czu dem Sebe liebin andechigin. Roloff, Naogeorg/Tyrolff. Pamm. 15, 95 (Zwickau um 1540): das ich E. G. und Ernvehsten vor allen andern meinen gantz. lieben Herrn und Freunden dise meine arbeit [...] / zugeschriebn. v. D. BROEK, Suevus. Spieg. 144, 29 (Leipzig 1588): die ander Tugendt ist / Wenn sie sich jrer lieben Vnterthanen trewlich annemen. BINDEWALD, Texte schles. Kanzl. 132, 2, 4 (schles., 1373): wir karl, [...], empiten Johel Rotlewin, [...] vnsirm leibin getrwen, vnsir gnade. RuppRICH, Dürer 1, 46, 44 (nobd., 1506): vnd last mich wissen, ob vch libs gestorben sey. Chron. Augsi. 3, 299, 27 (schwäb., 1467): Den ersamen weysen unser lieben besunder burgermaister und ratte der stat Augspurg. DiRR, Münchner Stadtr. 598, 15 (moobd., 1377): Lieben herren, ewch bitt die gemain, daz ir mit ewer weisheit [...]. - Grosch u. a., Schöffenspr. Pössneck 308, 12; Bindewald, a. a. O. 150, 1 (4); Fastnachtsp. 1000, 16; Chron. Augsb. 3 , 287, 27; 289, 5; Rwв 8, 1311. - Vgl. ferner s. v. andächtig 3, innebalten 1 .

3. dient attributiv (auch in der Superlativform) in der Anrede oder (etwas seltener) in referierenden Substantivgruppen der Herstellung oder Einforderung von Höflichkeit, Verbindlichkeit, Vertrautheit bzw. der Charakterisierung einer Person des Nähebereichs (der Familie, der Dienerschaft, der engeren Lebensgruppe), dann: >geliebt, lieb, wert ( oder dies formelhaft abschwächend); vgl. lieben 5. - Synt.: $j$. (z. B. die frau) jm. l. sein; lieber bruder / man / son / herre / knecht / leser / nachbar, lieber Peter, liebe fran / mume, liebes kind, liebe diener / freunde / gesellen / kinder (Klosterangehörige); der liebe gemal / nachbar / vater / vetter, die liebe hausfrau / base. Wbg.: lieberman >dem Lehensherren angenehmer und bevorzugter Mann، (a. 1434 f.), liebvater. 
PeIL, Rollenhagen. Froschm. 635, 4044 (Magdeb. 1608): [DEr Kónig] Sprach auch die Krieger tapffer an / | Frisch auff sprach er / Jbr lieben Mann. Tiemann, E. v. Nassau-S. Kgn. Sibille 118, 8 (rhfrk., um 1435): Lieben fründe / jch wil es zü mal gerrne dün. Sievers, Oxf. Benedictinerr. 11, 6 (hess., 14. Jh.): durch daz, liebe sustere, ist uns vor zu warnene, [...] daz uns got an heiner zit nit insi abe kerende zu deme ubel. v. Keller, Amadis 9, 20 (Frankf. 1561): Vnnd thun mich also biemit, freundtlicher lieber läser, [...], zu deinen günsten befehlen. ULNER 82 (Frankf. 1577): Der tódlich Abgang deines lieben Gemabls ist mir von Hertzen leid. DoubeK u. a., Schöffenb. Krzemienica 107 (schles. inseldt., 1456): do sprach Maczko: liber Peter, ich gabe dir kejune scholt eyn dan sachen. EBD. 684 (1480): wÿ das ir mutter hot gesprochen off dem tot bette: liben kinder, ir sollet wessen, wÿ das [...]. Henschel u. a., Heidin 872 (nobd., um 1300): Vnd rvmet sich nibt da bi | Wie libe im sin vrowe si. Rupprich, Dürer 1, 45, 4 (nobd., 1506): Mein willing dinst zip vor, lÿeber her Pirykeamer. WICKRAM 4, 14, 23 (Straßb. 1556): Lieber nachbaur nit also / wir wóllend gute liebe freundt mit einander sein. STEER, Schol. Gnadenl. 1, 8 (noschweiz, 15. Jh.): dar vmb, min liebes kint, daz din menschlich krankheit in der gnäd, [...] gesterket [...] werde. Adrian, Saelden Hort 2886 (alem., Hss. E. 14./15. Jh.): liebvatter [Bezug auf Herodes] ich [tohter, Salome], bedarf, | so disu red stat sin, I daz du daz, tust mit aiden schin. B̈̈CHTOLD, N. Manuel Krankh. 229, 8 (1528): man brent den lieben selen weder öle, anken noch unschlit und tüt inen nüt gìts nach. WYss, Luz. Ostersp. 10119 (Luzern 1545): lieben diener, varend hin | vnnd teylend ïch in kreyss der wellt. SpechtLer, Mönch v. Salzb. 23, 24 (oobd., 3. Dr. 14. Jh.): do er [Jesus] vil geng bin tet $\mid$ mit seinen lieben jungern zart. RINTELEN, B. Walther 180, 30 (moobd., 1552/8): Zu Außrichtung und Vollziehung diß meines lezten Willens [...] ordne ich vielgedachte mein liebe Haußfran [...]. - SCHüTzEICHEL, Mrhein. Passionssp. 478; Perez, Dietzin 1 203, 14; 205, 14; v. Keller, Ayrer. Dramen 1208, 19; Vetter, Pred. Taulers 321, 13; Roloff, Brant. Tsp. 78; Wyss, a. a. O. 549; SAppler, H. Kaufringer 14, 600; DreckmanN, H. Mair. Troja 18, 16; SchweIz. ID. 4, 266. Vgl. ferner s. v. äne, aufbauen 2, aufbereiten 4, ${ }^{1}$ base 3 , pate 1.

4. als relativ festes, ansatzweise phrasematisiertes Attribut gebraucht für die Personen der Dreifaltigkeit, die Mutter Gottes und weitere gottnahe Personen, ütr. auch für Gottes Haltung (seinen willen), eine religiöse Institution (z. B. die Kirche) u. ä.; vielfach im Superlativ; oft in der Anrede; mehrfach mixtura verborum; vgl. liebe 11, lieben 11. - Oft Texte der Sinnwelt ,Religion', auch ,Recht'. - Phras.: unsere liebe frau, dazu weitere Bildungen: unser lieben frauen bruderschaft / distelwasser (eine Arznei) / bim- melfart / tag. - Synt. (oft ansatzweise phrasematisiert): der liebe got / berre / vater; der liebe son, das liebe haus / kind (für Jesus Christus), die liebe kirche / mutter, der liebe schaz. (für Maria), die lieben altväter / beiligen / patriarchen / propheten / stifter, der liebe Alexius / Johannes; mit mixtura verborum z.B.: der liebe wille gottes (mehrfach). Wbg.: liebergöttig >jämmerlich`(a. 1651), liebsehen (subst.) >liebendes Sehen

Alberus, Barf. 7, 2 (Wittenb., 1542): Franciscus bat die Mutter Gottes umb ein trost / da erschien sie jm mit jrem lieben Kinde. Grosse, Schwabensp. 40, 1, 11 (Hs. rnd./ md., um $\left.1410^{\urcorner}\right)$: vil libe here vnde god, hemelesche vater, sint daz, dů vns zů diner hoen gotheit [...] getelet hast, des sistů iumber geloùed. QuINT, Eckharts Pred. 1, 26, 1 (E. 13./

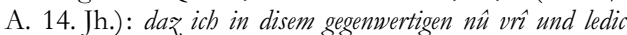
stüende nâch dem liebesten willen gotes. LAU, Qu. Neuß 173, 11 (rib., 1496): die kertze, die sij zo den Minrebroederen in de broderschaft Unser liever Frouwen [...] geordeniert. BEKKERs, Bauernpr. 56, 6 (Köln 1515/18): ITem regenet es an vnser lieuen frauwen dach als sy ouer dat geberch ginck. EBD. 59, 30: sal he kriegẽ dat ewich loin | Mit allen lieuen beil'gen in dẽ óuerstẽ throin. Rosenthal. Bedencken 35, 26 (Köln 1653): Wann deine liebe Kirch / in jhrer wolgemeinter Andacht verspottet wirdt. KeHreIn, Kath. Gesangb. 3, 156, 7 (Köln 1582): Ach thu dich mein erbarmen, | Du lieber trewer Got. EвD. 277, 44: Daß ich gepflantzt im lieben haus des Herren | Fein grinen mag, [...] Von fruchten reich. SснмiDT, Frankf. Zunfturk. 1, 13, 24 (hess., 1439): das kayn burger [...] uff die hochgetzijde, Unsrer Lieben Frauwen tage, sondage, der aposteln tage und anderer heilge tage [...] keynerlei werglich arbeid tun sulle. Dubizmay, kurß zu Teutze 2, 2 (hess., 1463): Ditz ist der Kurß zu Teutze von vnser lieben frawen. Bechstein, M. v. Beheim. Evang. Mt. 3, 17 (osächs., 1343): Dirre ist mîn lîber sun in dem ich mir habe behagit [LuTHER 1545: wolgefallen habe]. Reichmann, Dietrich. Schrr. 81, 1 (Nürnb. 1548): Lieber Vater / laß deinen gnedigen willen vber uns gehen. Bihlmeyer, Seuse 378, 2 (alem., 14. Jh.): daz du, truter herr, ein sunderliches minnekliches liebsehen uf mich betist. STRAuch, Schürebrand 39, 10 (els., E. 14. Jh.): der liebe sant Alexius ein arm verworfen lidig abgescheiden versmebet leben furte. EBD. 41, 19: unsere lieben stiftere, die heiligen großen gottes frúnde, uns vil [...] alle eigenschaft widerroten [...] hant. Warnock, Pred. Paulis 12, 85 (önalem., 1490/ 4): wie hailgklich [...] die lieben altvätter, patriachen und propheten lebtend. Bauer, Geiler. Pred. 102, 19 (Augsb. 1508): daz haisset kain vermessenhait / wann er ist gehorsam, dem liebsten willen gottes. BRÉvarT, K. v. Megenberg. Sphaera 7, 20 (noobd., 1347/50): der [feurein bimel] hat kainen lauf, sunder got rit mit seinen lieben darinne. SPECHTLER, Mönch v. Salzb. 1, 51 (oobd., 3. Dr. 14. Jh.): [Got] Hat ie bievor der minne pfeil | drei ganzperson so gar subteil | geloket zu der liben eil. MeLl u. a., Steir. Taid. 241, 39 
(m/soobd., 1638): der fürnembste patrocinium und kürchtag ist am suntag nach Unser lieben frauen himelfart. EIs u. a., G. v. Lebenstein 44, 5 (oobd., 1. V. 15. Jh.): Vnser lieben frawen distel wasser Item das wasser ist gut, welcherlaj prechen der mensch in im hat. - Tiemann, E. v. NassauS. Kgn. Sibille 125, 26; Dubizmay, a. a. O. 77, 17; Rupprich, Dürer 1, 176, 154; VetTer, Pred. Taulers 155, 29; Eichler, Ruusbr. steen 453; Piirainen, Stadtr. Sillein 36a, 5; SchweIz. ID. 2, 523. - Vgl. ferner s. v. auserwälen 1 , antichrist.

5. >jm. wichtig, angelegen, teuer; wertvoll (von Vorhandenem); innig erwünscht, ersehnt (von Unsicherem oder nicht Vorhandenem)<; vgl. lieben 5; 6. - Synt.: jm. etw. (z. B. die sele) lieber sein, den [...], jm. l. zu lernen sein; jn. l. halten; der liebe friede / wille, die liebe freibeit, das liebe Asia / teutschland / getreide.

v. D. LeE, M. v. Weida. Spigell 22, 20 (omd., 1487): Dem nach laß dj̈r dein ßele ljeber sein, den lejppp adder leben eins andern. OORschot, Spee/Schmidt. Caut. Crim. 241b, 36 (Frankf./M. 1649): Vnser liebes Teutschlandt hat diese Exempel gesehen / vnd kans niemãd wieder sprechen. Logau. Abdank. 163, 21 (Liegnitz 1651): [haben] nichts so inniglich und bertalich erseufftzet / [..] / alß den lieben FRJEDE. Lauchert, Merswin 7, 31 (els., 1352/70): daz du [herre] mir gebest zi tinde was din aller liebester wille si. MaAler 272r (Zürich 1561): Ein diener der seinen herren Lieb halt [...]. Pfeiffer, K. v. Megenberg. B. d. Nat. 119, 45 (oobd., 1349/50): die sinnereichen schuoler, den haiz und lieb ze lernen ist. Bischoff u. a., Steir. u. kärnt. Taid. 292, 13 (m/soobd., 17. Jh.): damit daß liebe getrait desto sicherer obne schaden möge stehn. - PeIL, Rollenhagen. Froschm. 537, 979; BeLKin u.a., Rösslin. Kreutterb. 118, 3; Roloff, Naogeorg/Tyrolff. Pamm. 381, 3737; Henschel u. a., Heidin 887.

6. >geliebt, erotisch begehrt (mit Bezug auf die geliebte Frau gesagt); verliebt (von der Haltung des Mannes)<; vgl. liebe 4, lieben 8; 9. - Synt.: jm. l. sein / werden; jn. l. gewinnen; der liebe blik / gedanke / grus / lust, die liebe braut / frau / sonne, das liebste ein, das liebe Mäzlein / weib, der liebste mein. Wbg.: liebängeln >liebevoll auf jn. blicken; jn. erotisch begehrend anschauen

Luther, WA 21, 415, 36 (1544): Das es nicht mus geschielet, sondern liebengelt heissen. EBD. 45, 404, 32 (1537): wenn man spricht: Sybe dein Sone schilet, So spricht der Vater: Eß lieb ewgelt. Pyritz, Minneburg 1761 (nobd., Hs. um 1400): Die frawen rant ich undervorcht $\mid$ Do an mit lieben blicken. Gille u.a., M. Beheim 68, 1 (nobd., 2. H. 15. Jh.): Die liebste mein $\mid$ macht mir gross pein $\mid$ und sendlich leiden. Wiessner, Wittenw. Ring 5208 (ohalem., 1400/ 08): Triefnass andacht die was gross / Gen seines lieben Mät\%- leins schoss. KleIN, Oswald 18, 76 (oobd., 1416): ich brunne, wenn mich hitzt die liebe sunne [bezogen auf: künigin, frowe, weib]; |Won ich ir bei, so ist unfrei mein mitt und mass. EBD. 47, 43 (v. 1408?): zart liebstes weib, | den jamer hie vertreib. Wackernell, Adt. Passionssp. Pf. II, 1164 (tir., 1486): Ich [ein diabulus] haysz dy lewt springen an dem tancz. Und die pueben lieb augeln auf dy frawen. - KARnein, Salm. u. Morolf 4, 5; Henschel u. a., Heidin 1173; KleIN, a. a. O. 1, 24; 20, 18; 20, 53; 22, 128 ; 42, 1;92, 38; 97, 12; 121, 27.

7. dient der nachsichtig liebevollen und zugleich distanzierenden Charakterisierung von Bezugsgrößen, die in einem gewissen Gegensatz zu lieb stehen.

GAJEK, Seidelius. Tych. 26, 27 (Breslau 1613): Jst das nicht eine Antichristische rede, das die liebe einfalt geweiset wirdt, auf die schwachen Element der Welt. Kopp, Volks- u. Gesellschaftsl. 148, 12 (Hs. 'pfälz., M. 16. Jh. ') : das jar ist mir worden so lang, | das liebe >schwere lange jar ist mir worden lang genueg. WICKRAM 4, 10, 5 (Straßb. 1556): Wann sie dann ausgelert haben / lauffen sie dabin wie das liebe > unverschuldet unvernünftige vieh / meinen sie habend ibre jar der lehr schon überkummen.

liebäugeln, s. lieb (Adj.) 6.

liebbehaft, Adj.; hier zu (be)haft gestellt; vgl. Dwв 6, 964 mit dem Ansatz liebhaft, dies auch bei A. À S. Clara. Glori 13, 25; 30, 22.

> fest und ohne Eigennutz auf die Liebe zu Gott und mittels dieser auf die Liebe, Fürsorge gegenüber den Menschen gerichtet<; vgl. liebe 5; 10.

Gille u. a., M. Beheim 129, 138 (nobd., 2. H. 15. Jh.): darumb ist ain ander | liebhaben oder liebbehafft | haisst ain lieb babende freuntschafft, | die nit hat gerens wander.

liebbrief, s. liebe 11.

liebde, die.

1.; 2.; 3., s. liebe 6; 10; 11.

4. >Liebe, erotische Zuneigung zwischen Mann und Frau<; vgl. liebe 9. - Wmd. Bdv.: bulschaft 1, minne.

Thiele, Minner. II, 28, 33 (Hs. 'rmd./rhein., 1. V. 15. Jh. $\left.{ }^{7}\right)$ : myn leyt eme lieft, myn liefde eme leydt $\mid$ wat mich ervrauwet, dat is eme leyt. EBD. 30, 373: bricht dan des geluckes brucke, | so ist sin leifd scheir en wech. CHRON. KöLN 2, 473, 17 (Köln 1499): ein geil unkuisch wif, die gesan einen mechigen greven an leifden ind boilschaf. $\mathrm{BuCH}$ WeInsB. 5, 495, 22 (rib., 2. H. 16. Jh.): Leiff haven und leffde belen | Doit leiff und leven quelen. Tiemann, E. v. Nassau-S. Kgn. Sibille 124, 6 (rhfrk., um 1435): jch 
wolte ee sterben / dan ich eyme solichem duffel von liebde ein gert (>im geringsten $`$ ) wart $z u$ rete. - FRANTZEN u. a., Kölner Schwankb. 1, 45, 5; 55, 1.

5. Anrede zwischen hoch gestellten Verwandten oder Standespersonen; vgl. lieb (Adj.) 2, liebe 1. - Wbg.: lieb (das/der) 5.

Buch Weinsb. 2, 305, 11 (rib., 1575): hab ich an den edlen und erentfesten jonker Wilhem Goltstein, [...], geschriben und siner leibden zu verstain geben, das [...]. KüTHER, UB Frauensee 389, 1 (thür., 1530): Ferners haben wir seiner liebden begeren nach personlich unnd mitt andern umb die brieff uber unser closter See balten suochung gethan. CHRON. Nürnb. 3, 381, Anm. 3 (nobd., 1440/4): so nü die leüffte aller lannde, [...], fremde und wilde sein, bedeüchte es dann ewrer liebde geratten sein, daz. [...]. v. Keller, Ayrer. Dramen 726, 4 (Nürnb. 1610/8): So hat sie [Keiserin] hoch kein Vrlaub gnommen | Von eur Liebt jbren Herrn Brüdern. Chron. BAIER. StÄDte. Regensb. 77, 21 (noobd., 1525): dieweill sein lieb kaiserlicher majestät im beiligen reich teuzscher nacion oberester stathalter sey.

liebe, die; $-\emptyset /-$.

1. >natürliche (d.h. ,nicht aus einer Rechtspflicht entspringende“; Rwв 8, 1305) soziale Achtung, Wertschätzung des anderen, soziale Verantwortung, Gemeinschaftssinn, Verläßlichkeit stiftende innere Freiheit`; von hier aus Tendenz zu >Begünstigung` (in den Belegen als Warnung / Verbot o. ä. angesprochen); in 1 Beleg (s. u. Rennefahrt, Stadtr. Bern): >vertraglich geregeltes Miteinander`; offen $\mathrm{zu} 2$; 4. Texte der Sinnwelten ,Recht / Wirtschaft sowie ,Religion / Didaxe'. - Phras.: liebe und leid leiden > etw. gemeinschaftlich tragen - Bdv. (bzw. bereichszugehörig): dienst, einigkeit, freundschaft, ${ }^{1}$ gunst 5 , buld, minne, steuer 3; 4, treue, wolgefallen, zuneigung. Ggs.: leid 1, ungerechtigkeit, zwietracht. - Synt.: l. haben / beweisen / fürgeben, die l. erhalten / erneuern; die l. (Subj.) ungefärbt sein, verkälten serkalten<; etw. durch l. tun, durch / zu liebe oder leid (ansatzweise phrasematisiert) (etw. tun); die beidnische / natürliche / lautere / reine / rechte / (ge)ware / sondere l.; friede mit l. Wbg.: liebezorn >in liebe 1 gründender gerechter Zorn〈, liebgefallen, lieblos 1 >nicht liebenswürdig〈, lieblïge >Lüge, um jm. nicht weh zu tun, Lüge aus Verantwortung, zur Abwendung von Schaden<, liebreich 1 >vertrauenswürdig, zuverlässigく, liebschaft 1.
ScHÖPpER 10b (Dortm. 1550): CHARITAS: Liebe büld freundtschafft minne gunst zuneigung. LUTHER, WA 8, 283, 16 (1521): Weyl dem menschen, der die liebe hatt, keyn gepott nott ist. EBD. 27, 12, 22 (1528): buiusmodi officiosa mendatia, lieblugen, da ich eim zu gut lieg, non incommodat, sed ist im gut. Luther, WA 17, 2 44, 26 (1525): Also ist auch die reyne liebe eyn seltzam ding auff erden. Nicht das Liebe an sich selbs unreyne sey, Sondern das sie liebe fur geben. PFEFFERL, Weigel. Ges. 8, 8 (Hamb. 1646): Der Apostel saget, dem Gerechten ist kein Gesetz, gegeben sondern er thut das gesetze ohn zwang und beschwernis aus lauter Liebe. Grosse, Schwabensp. 86a, 31 (Hs. 「nd./md., um 1410?): her [man, der kempen sal] muz eynen vor sich setzen, der iz tu dirch liebe oder durch pfennige. EBD. 140a, 13: Also strak. ist iz vmme diq gerichte, dar man noch wedder lip noch dirch leit noch dorch gü noch dorch vrinde willen nicht richten sal wen dorch rechticheyt. BÖMER, Pilgerf. träum. Mönch 2276 (rhfrk., um 1405): Des andern ubels machen ich myn, | Und allen muß myn gut gemeyne sin. | Minen name ob ir den wissen wollet, | Geware Liebe [personifiziert] ir mich nennen sollet! LAMPRECHT, Dt. Wirtschaftsl. 327, Anm. 8 (mosfrk., 1598): Ein jeder inwohner zu Fell ist das jahr durch [...] zwên tage zu graben [...] durch sich selbst oder einen anderen lieberichen arbeiter zu erscheinen schuldigh. SCHMIDT, Frankf. Zunfturk. 1, 218, 11 (hess., 1355): so sulden wir, die scheffen und der rat, mit den vorgen. bantwerken und sie mit uns, und wir, die vorgen. hantwerke, mit den scheffen und dem rade und sie mit uns getruweliche lyp und leyd lyden. KöBLER, Ref. Franckenfort 27, 4 (Mainz 1509): Jch. N. schwere das ich in diser sachen niemandt zu lieb oder zu leid / noch umb miedt oder gab [...] / sunder allein die warbeit [...] / offenbarẽ [...] wól. Schwartzenbach E vjr (Frankf. 1564): Dienst [...] Dienstbarkeit. Handreichung. Wolgefallen, Liebgefallen. Behegligkeit. Bechstein, M. v. Beheim. Evang. Mt. 24, 12 (osächs., 1343): wan di ungerechtikeit sal ubirfluzzic werden, sô vorkeldet di lîbe in manigen heržcen. Jungbluth, J. v. Saaz. Ackermann 24, 1 (Hs. romd., 1465): Liebe nicht allzu lieb, leit nicht allzu leit sol umb gewin und verlust bei weisem manne wesen. FASTNACHTSP. 1152, 39 (nobd., 15. Jh.): Noch hat er [mussigener] weder lieb noch gunst $\mid Z$ u den die im sein brot gewynnen. SudHOFF, Paracelsus 7, 377, 23 (1529): nun zwingt die christliche liebe [...], ja auch die heitnische natürliche liebe, wie ir wolten, das euch beschehe in nöten, also solt ir auch dem notürftigen bergegen erstatten. WIESSNER, Wittenw. Ring 3599 (ohalem., 1400/08): Got mach euch sälden reich! | Ewer liebschaft gfiel mir wol. RenNEFAHRT, Stadtr. Bern 272, 11 (halem., 1388): Und sulllen öch bi den selben eyden dis vorgenant burgrecht und luter liebu und trüwe von dishin ewenklich [... mit unsern eyden ernuwern. Ders., Recht Laupen 56, 28 (halem., 1471): ob inen vor ziten etwas gónnen und erloubt sie in dem selben holtr, das sie von liebe und keins rechten wegen beschechen. EвD. 143, 19 (1513): die selben all sóllen landträcht kouffen und mit den unsern im landtgricht lieb und leid tragen. Ders., Zivilr. Bern 763, 26 (halem., 1615): Welcher [...] mit den ubrigen stubengsellen in dem einen und anderen kein lieb und leyd hat, derselbig soll [...]. MAALER 
271r (Zürich 1561): Råchte Liebe die ein mensch gegen dem anderen hat. Charitas, Communis fides. EBD. 272r: Liebloser leyb / Vngeraatsamet. Incultum corpus. SAPpler, H. Kaufringer 29, 4 (schwäb., Hs. 1472): weliches under den zwaien wär | baß ze loben und ze krönen, | die lieb oder die schöne. Gilman, Agricola. Sprichw. 2, 137, 8 ([Augsb.] 1548): alß haben sy [Hunde] dem armen Lazaro mehr diensts und liebe beweiset / dann der Reiche Wanst. Primisser, Suchenwirt 46, 86 (oobd., 2. H. 14. Jh.): „So bin ich die Schón genannt", | [...] |,So bin ich die Lieb". - KöBleR, Ref. Wormbs 37, 24; DoubeK u. a., Schöffenb. Krzemienica 264; 503; Uhlirz, Qu. Wien 2, 3, 4473, 5; Grossmann, Unrest. Öst. Chron. 88, 36; Bischoff u. a., Steir. u. kärnt. Taid. 48, 32; GrothausmanN, Stadtb. Karpfen 113, 12.

2. >Gunst, Gunsterweisung eines sozial Überlegenen gegenüber einem Unterlegenen; als Gunst erklärte Bereitschaft eines in der Seelsorgehierachie Befugten zur Lesung einer Messe o. ä.〈; in allen Belegen rechtsformelhaft oder Teil eines Rituals. Meist rwobd./oobd.; Rechts- und Wirtschaftstexte?. - Bdv.: freundschaft, wolmeinung; vgl. anst, bene, benevolenz, glimpf 6, gnade 9, gratia $1,{ }^{1}$ gunst 4 .

Bindewald, Texte schles. Kanzl. 25, 3 (schles., 1349): das wir [wenczlaw] mit gutem willen [...] baben gegeben durch rechtir libe vnd fruntschaft willen Der Edeln furstynne vnde vrowen katherynen, [...], eyne mark groschen. V. ANSHELm. Berner Chron. 4, 228, 27 (halem., n. 1529): dass uwer grosmáchtikeit in semlicher achthabung, andacht, frintschaft und liebe wólle verharren. Rennefahrt, Statut. Saanen 166, 39 (halem., 1533): haben wir von sonderbarer lyeby und früntschaft, ouch uß sondern gnaden und von keins recbten wegen, gantz früntlicher wolmeynung [...]. HöR, Urk. St. Veit 71, 26 (moobd., 1352): Ich pruder Philipp prior [...], verjeben offenleich an disem brief, daz wir durch besunder lieb und trew vnd umb gelt [...] bintz. nacht ein vigilii vnd dez. morgens ein selmezze singen. EBD. 138, 30 (1390): Wir graf Hainreich [...] bechennen offenleichen an dem brief, daz. wir Petern dem Griesteter, [...] die lieb und genad getan haben umb datz. gut zu Räut, [...] haben wir im [...] ledig lazzen. - Leisi, a. a. O. 8, 374, 21; Hör, a. a. O. 185, 28.

3. >kleinere Zuwendung, Gabe, Geschenk<; zu lieb (das/die) 4. - Phras.: sant Johannis liebe ein in St. Johannis Namen gesegneter Wein; jn. einer liebe gewären >jm. einen Gefallen tun`(ironisch).

Helbig, Qu. Wirtsch. 3, 95, 33 (md., 1493): die phenner sollen den knechten fortmeher kein lon adder libe gebin. Chron. Nürnb. 3, 149, 26 (nobd., 1488): do sprach der ritter: ,reich mir vor sant Johannes lieb'. do trug er ein groß glas mit wein her. GoedeKe, P. Gengenb. 126, 332 (o. O.
1516): Der lieby wil ich sie [pfaffen, leyen] geweren, | Vnd inen auch die seckel leren [ironisch].

4. >liebevolle, natürliche, nicht zweckgerichtete und nicht erotische Zuneigung eines Menschen zu einem oder mehreren anderen`; bezogen auf das Verhältnis von Familienangehörigen untereinander (Eltern und Kinder, auch Ehegatten), generell auf das als Familienbindung gedachte Verhältnis von Personen, die Umgang miteinander haben, darunter auch z. B. zwischen Angehörigen verschiedener sozialer Schichten (etwa Bürgern und König); vgl. lieben 5, lieb (Adj.) 2; 3. - Bdv.: einigkeit, gefallen, treue; vgl. andacht 4, geneigigkeit, geneigung. - Synt.: (keine) l. zu jm. haben (z. B. christen zu heiden, die bürger / Franzosen zu einem könig), seine l. zu jm. wenden, l. in der ehe halten, jm. (z. B. dem vater) l. erzeigen; die l. ein werk des willen sein; der affe seine junge für l. zu tode drücken; etw. mit l. sehen, jm. (z. B. dem keiser) zu l. [etw. tun]; die l. des vaters gegen den kindern, die l. der eltern / untertanen (zu jm.); die sundere / väterliche l.; der friede der liebe. Wbg.: liebesstrafe, liebgenies, liebwirdig, liebzorn.

Luther, WA 21, 307, 20: Das [Kinder mit ruten straffen] sind amptschlege und Liebestreiche, nutzlich [...]. EBD. 37, 384, 18 (1534): ira, quae venit ex amore, [...], Das ist der gmeine lieb zorn. Helbig, Qu. Wirtsch. 1,. 91, 27 (md., 1423): Ouch sal eyn iclicher wergkmeister in unser hern phlege nach nyrgen in der stad von keyme gesellen heymlichen liebgeniss neme nach geschenck.te dar umme, das [...]. SCHWARTZENвАСН 55r (Frankf. 1564): Holdtselig. Lieblich. Schmaichlich. Liebwirdig. Allg. Schau-BüHne 49, 18 (Frankf. 1699): Dieser Herr hatte keinen ebligen Sohn / wandte derowegen sein Gemuth und Liebe zu diesem seinen unächten / $\operatorname{der}[\ldots$... v. D. LeE, M. v. Weida. Spigell 22, 32 (omd., 1487): saltu dï liebe deines eblichen gemahels vorsetzen vor dy lïbe deiner eldern. Logau. Abdank. 168, 28 (Liegnitz 1651): Der FRJEDE der Liebe / der Einigkeit / Aufrichtigkeit und Trew / welchen Sie / geliebter Herr Obme / Zeit jhres Ehstandes / so lieblich / so lóblich mit einander gepflogen. Reichmann, Dietrich. Schrr. 128, 33 (Nürnb. 1548): wie er [feind] die bertzen von einander reysse / Das gleich / wie jr yetzundt lust vnd lieb gegen einander habt / er vnlust / vnd feindschafft móge anrichten. EBD. 130, 1: dazu belffen / das eynigkeyt vin liebe in der ehe gehaltē werde. Das geschicht aber also / dz er [man] mit vernunfft / vñ nit mit gewalt faren / [...] soll. Bachmann, Morgant 64, 18 (halem., 1530): Du magst wol verstăn, daz sy kein liebe zuo dir hand und werden dir nüt halten waz sy dir verheissen hand: wann sy sind Kristen und du ein heid. MaAler 271r (Zürich 
1561): Liebe deß vatters vnd der muter gegen den kinden. Henisch 29 (Augsb. 1616): Der Aff trucket für libe sein Junge zu todt. Grossmann, Unrest. Öst. Chron. 126, 2 (oobd., 3. Dr. 15. Jh.): das der bischolff dem kayser zu lieb und gevallen dem von Gran, [...], das pistumb soldt ubergeben. - Gilman, Agricola. Sprichw. 1, 84, 20; Warnock, Pred. Paulis 23, 295; PÄPKE, Marienl. Wernher 11029; Eschenloher. Medicus 56, 22; Chron. Augsb. 3, 129, 20; 312, 27; Dict. Germ.-Gall.-Lat. 306, 19.

5. >christlich begründete Liebe des Menschen zu anderen, verständnisvolle, liebende, nachsichtige Haltung, fürsorgliches Bemühen um den anderen'; besonders auf protestantischer Seite Betonung des glaubens als des theologischen Grundes der liebe sowie der christlichen Freiheit, aus der sich die werke, mit ihnen die caritas im Sinne sozialer Verpflichtung herleiten; dem gegenüber auch Begründung des guten Werkes aus der Hoffnung auf Rechtfertigung, Sündenvergebung. - Texte der Sinnwelt ,Religion', auch der Didaxe. - Bdv. (bzw. bereichszugehörig): andacht 4, demütigkeit, geduld, geduldigkeit, freundschaft, glaube 4, minne. Ggs.: arglistigkeit 1; 2. - Synt.: l. untereinander haben, in die berzen setzen, der prediger l. haben (sollen), jm. l. erzeigen; l. (Subj.) freundlich / langmïtig sein, alles glauben / boffen / dulden / vertragen, nicht das ire suchen, sich nicht erbittern lassen, keine secten machen, das gesez, meistern, fordern, das [...], eine -waffe wieder die arglistigkeit (des teufels) sein; ane l. nichts gut sein, aus l. zu jm. reden, ${ }^{\circ}$ aus der l. ein werk machen, durch l. sünde tilgen $\urcorner$ (beides als Vorwurf gegen die ,Werkgerechtigkeit ${ }^{\top}$ ), durch l. die sünde (anderer leute) vergeben, in l. leben, in reiner l. minnen, nach l. stellen, das gesez, nach der l. bengen / lenken, jn. zu l. ziehen, der glaube jn. zu l. (des nächsten) neigen; die l. des nächsten, gegen dem nächsten / menschen, zu dem orden; die auferbauliche / brüderliche / schwesterliche / brünstige / cbristenliche / emsige / heisse / bohe / stete l.; der lon, die frucbt, regel, das mas der l. Wbg.: liebheit (semantisch hierher, morphologisch eher zu lieb, Adj.), liebreich 2.

LUTHER, WA 10, 1, 2, 398, 33 (1526): Liebe und not maistern alle gesatze, und kaine gesetz sol sein, es sol nach der liebe gebeügt und gelencket werden. EBD. 10, 3, 342, 17 (1522): wen die werck nit aus der lieb gescheen, so sein sie gar lautter nichts, dan an die lieb ist nichs gut. EBD. 11, 249, 20 (1523): liebe unnd glawbe machen keyne secten noch unterscheyd eußerlich. EвD. 17, 2, 159, 31 (1525): Dieser frucht [Euangelion] nachfolgen denn die fruchte des geysts, die werck, als gedult, liebe, trewe. EвD. 21, 411, 20 (1544): ,Fur allen dingen aber habt unternander eine brinstige Liebe, Denn die Liebe decket auch der sinden menge.' [...], Hie sagt er nu, wie sie auch sich gegen andern Leuten sollen halten, Und fasset biemit alle guten werck [...], so wir unserm Nehesten schuildig sind, in ein starck wacker wórtlin, das er nennet Brúnstige liebe. EвD. 21, 415, 16 f. (1544): die Papisten [...] Machen aus der Liebe des Nehesten ein werck oder tugent gegen Gott, Wollen darnach daraus schliessen, Das durch unsere Liebe unser sunde [...] getilget werden, [...] unser sünde zu decken fur Gott, da gehoret ein ander Liebe zu, nemlich, des Sons Gottes, welcher [...] durch solche Liebe auch uns ein Exempel gegeben, das wir auch (durch die Liebe) anderer Leute sunde, [...] vergeben sollen. Ders. HI. Schrifft. 1. Kor. 13, 1 (Wittenb. 1545): WEnn ich mit Menschen vnd mit Engel zungen redet / vnd bette der liebe nicht / so were ich ein donend Ert\%. EвD. 4: DJe Liebe ist langmütig und freundlich / die liebe einert nicht / die liebe treibt nicht mutwillen / sie blehet sich nicht. Quint, Eckharts Pred. 1, 79, 12 (E. 13./A. 14. Jh.): als S. Augustinus spricht: waz der mensch liebhat, daz. wirt er in der liebe. STRUCK, Cist. Marienst. 1348 (mosfrk., 1492): einträchtig in göttlicher Liebe, [...], Liebe (leyffhayt), Reinigkeit des Leibes und Armut des Geistes leben. SсHÖNBACH, Adt. Pred. 11, 12 (osächs., 1. H. 14. Jh.): Petrus und Andreas. bi den zwein soltu merken zweir hande minne: minne got und minne din ebencristen. di minne und die libe sol haben ein igelicher pretigere. v. D. Broek, Suevus. Spieg. 178v, 23 (Leipzig 1588): Darumb treibet [...] jhn sein liebreiches Hertze / den Nebesten vmb seiner mengel oder vbertretung willen / trewlich zu straffen. Williams u. a., Els. Leg. Aurea 738, 22 (els., 1362): Jst es aber daz du in reiner liebe minnest so gewinnet er [engel] dich so liep also mich und erzoget dir sine gnode. KOLLER, Ref. Siegmunds 289, 48 (Hs. `Augsb., um 1440?7): auch mag er [prister] plab tragen in rechter emsiger und steter lieb zu leben. Anderson u. a., Flugschrr. 7, 7, 27 (Straßb. 1524): DEr glaub an Christũ macht vnß frum̃ vor got / vnd neigt uns zü lieb des nåchsten. SudhofF, Paracelsus 14, 133, 33 (1529/32): also stellen sie [Wesen ohne Seele] nach solcher liebe gegen dem menschen, auf das sie mit den menschen in der selbigen büntnus seind. NYBERG, Birgittenkl. 1, 335, 38 (oobd., 1461): daz der selb Herr, [... w wöll uns raiczen und enczunden in seiner vnd auch in pruderlicher vnd swesterlicher lieb vnd wercken der güttikeit. EBD. 2, 271, 10 (schwäb., um 1543): [bischoff Friderich] het besundere lieb vnd andacht zi dem heiligen orden. BAUER, Geiler. Pred. 9, 10 (Augsb. 1508): aynem ainfaltigen christen menschen ist genig daz er hab cristenliche liebe. Rudolf, Peuntner. Sterbek. 148rb, 18 (moobd., n. 1434): vil grósleicher vodernt und begernt von uns got und die lieb, das wir vmb das geistleichen leben und hail vnsers nachsten sarig und fleizz tragen. Reithmeier, B. v. Chiemsee 2, 3 (München 1528): Awsserhalb der lieb ist der glawb nichts wie Paulus erzellt, 
wann jch gleich allen glawb hiet [...], und daneben khain lieb so bin jch doch nichts. BAuer, Zist.-Pred. Haller 59, 332 (tir., 1466): Der lon der pruederleichen lieb das ist die lieb Kchristi. - Luther, WA 10, 1, 2, 64, 31; 17, 2, 91, 4; 21, 415, 19 ff.; 32, 385, 10; ANDERson u. a., a. a. O. 5, 5, 24; 5, 6, 21; Reichmann, Dietrich. Schrr. 235, 29; Dietrich. Summaria 20v, 22; Bauer, Geiler. Pred. 473, 21; Baptist-Hlawatsch, U. v. Pottenst., S. 222. Vgl. ferner s. v. arglistigkeit 2, auferbaulich 2, parteïg 1, becker.

6. >tiefe Neigung, Verlangen, Streben, Begehren der menschlichen Natur generell oder des einzelnen Menschen zu etw. (meist:) negativ, (seltener:) positiv Bewertetem hin<; dieses wird in der Regel als Besitz, in Einzelfällen als erstrebte Tätigkeit gedacht; vgl. lieben 6. - Texte der Sinnwelt ,Religion', auch ,Didaxe'. - Bdv.: affect 1, begerung 6, beweglichkeit 2, eigenschaft, fleis, ${ }^{1}$ lust 1 , neigung, verblendung, wolgefallen. - Synt.: $l$. an etw. legen, zu etw. haben, für etw. (z. B. für warbeit) annemen, die l. büssen >erfüllen`, jm. die l. fremd machen; die l. (Subj.) ein ausbruch der herzigung sein, jn. zu etw. bewegen / zieben; mit l. ungefangen, zu etw. geneigt sein, etw. mit $l$. ausricbten; $\ulcorner$ die l. der geschrift / kunst / warheit / weisheit / welt, der zeitlichen dinge, des vaterlandes', jeweils >zu [...]<; die l. zum geld / gejägde, zu dem leibe; die grosse / lautere / tolle / weltliche l. Wbg.: liebde 1.

LUTHER, WA 32, 448, 33 (1532): sondern trittest aus der pan [>irrst ab<] und denckest nur wie du deine lust und liebe zum geld buissesst. Buch WeInsB. 3, 241, 28 (rib., 1584): er hetas (einen Mord) aus sich selbst getain ausser eifer gegen die catholisce religion und leifden des fridens. DEDEKIND/ Scheidt. Grob. 87, 38 (Worms 1551): daß sie [menschlich natur] zu allem / das verbotten ist / ein lieb / lust / vnd wolgefallen hat. VetTer, Pred. Taulers 37, 5 (els., E. 14. Jh.): ein fri [...] gemute, das ungevangen ist von allen dingen, noch mit luste noch mit liebe. Williams u. a., Els. Leg. Aurea 201, 6 (els., 1362): Du [Herre] hast weltliche liebe mir frómde gemaht, du hast mir keraft geben daz ich alle pin han úberwunden. MaAler 50r (Zürich 1561): Der ein lust und liebe zum gjägt [...] hat. HeYdn. MAISTER 3r, 16 (Augsb. 1490): von grosser liebe wegen der weißheit / mocht er [Tales] zeit nit haben dem gelt nach zestellen. BAUER, Geiler. Pred. 322, 12 (Augsb. 1508): welche tier den flaischlichen gedenken nachfolgen / das sind die außbrüch deiner hertrigungen Die erst / ist. liebe Die ander glüst. KOHLER, Ikkelsamer. Gram. 7, 14 (wohl `Augsb. 1. Dr. 16. Jh. `): vn bewegt mich darzu nichts anders dañ die liebe vnd lust diser seynen subtilen kunst. HeNisch 29 (Augsb. 1616): Affect / bewegligkeit zu oder von dem jenigen das vns gut vnd bóß dunckt zu sein / beweg des gmuits / affectus, perturbatio [...] derer seind viererley / lieb / haß / hoffnung / forcht. STEER, K. v. Megenberg. Sel 164 (Hs. 'moobd., 1411ㄱ): ein andrew aigenschaft der sel, das ist, das sy natürleich genaigt ist von notdurft der natur mit grosser lieb zü dem leib vnd erk ükchung ds leichnames. BAUER, Imitatio Haller 75, 25 (tir., 1466): die lauter lieb der warhait die sol dich cziehen cұue dem lesen. Dies., Zist.-Pred. Haller 47, 128 (tir., 1466): Die lieb der welt die pegert der ganczen welt vnd würt nimmer ersatt. - Alberus, Barf. Vorr. Alb. 7, 2; Quint, Eckharts Pred. 1, 215, 8; M. Cunitia. Ur. Prop. 154, 7; Reichmann, Dietrich. Schrr. 76, 23; Rennefahrt, Zivilr. Bern 103, 21; DERs., Staat/Kirche Bern 418, 5; Eichler, Ruusbr. obd. Brul. 2, 736; Gilman, Agricola. Sprichw. 1, 18, 24; Bauer, Geiler. Pred. 318, 32; Hohmann, H. v. Langenstein. Quästio 209, 61; VOC. INC. TEUT. p ir; MAAler 271r; HarsdoerfFer. Trichter 3, 318, 14

7. >als unrecht, unordenlich, ungeordnet, unredlich, unrein, eigen o. ä. charakterisierter Hang zur Sexualität, zu sich selbst, zum Besitz, zum Hochmut, zum Zweifel, zu Verwegenheit ‘; erhöhte Belegdichte für den Bereich der Sexualität (insbesondere zur außerehelichen Liebe, dann z. B.:) >sexuelle Beziehung zwischen Heiratsverwandten`, andeutungsweise >verwegene Neigung zur Teufelsbuhlschaft, auch zur Sodomie<; im einzelnen: \Unzucht; Habgier; Ehrsucht usw. ; im Hintergrund stehen die 7 Hauptsünden; die genauen Ausprägungen von liebe im Sinne dieses Bedeutungsansatzes werden teils verschleiert; vgl. den Stellenkommentar mit Lit. zum Beleg Wagner. - Texte der Sinnwelt ,Religion/ Didaxe'. - Bdv.: begerung 1, brunst 5; 6, senung, wollust; vgl. belangen 1, geilheit 2, gelüstung, beimliche 5. - Wbg.: liebkind (auch liebes-) > unehelich geborenes Kind beider lebender Elternteiles (14. Jh.f.).

SteER, W. v. Herrenb. Büchl. 520 (pfälz., 1436): Welicher nü das gewiecht hat der vnördenlichen liebe jn siner sele, $\operatorname{der}$ [...]. Jostes, Eckhart 65, 21 (14. Jh.): Sunderlich lieb on bute die ist ein valsch minnerin und ist ein ubergreifferin dez rebten gemaheln und ist ein zustorerinne dez frids (es folgen weitere Nennungen). WAGNER, Erk. Ps.-J. v. Kastl 9, 21 (nürnb., 1. H. 15. Jh.): wie ubel alle sein innern kereft [...] vergift sein mit [...] unredlicher, ungeordenter lieb, baß und poß senung, boffnung und verczweiffelung, forcht und kinheit. ЕвD. 9, 37: unkewsch, wollust, inprunst des fleysch, bewegung des fleyschs, unlawterheit, vermayligung, snodikeit, 
unschamheit, unvernunft, aygene lieb, haß gotes, begerung der werlt. SACHS 15, 373, 17 (Nürnb. 1558): Deß menschen leib, fleisch unde blut, | Welches von natur ist nicht gut, | Sonder vol begierd und affect $\mid$ Und eigner lieb beimlich vol steckt. v. Keller, Ayrer. Dramen 1285, 26 (Nürnb. 1610/18): Die Königin vnd Stiefmutter mein | Ist voll in brunst und Lieb vnrein. EICHLER, Ruusbr. steen 59 (els., sp. 14. Jh.): do mitte wurt vertriben alle verbildunge vnd alle ungeordente liebe zi den creaturen. Warnock, Pred. Paulis 16, 128 (önalem., 1490/4): So denn der mensch wendt, er hab ain gaistliche liebe zü sinem hailgen, so ist er bös vigind bie mit sinen tusigfaltigen listen und enzúndt den menschen mit liplicher unrainer liebe, also daz er etwenn begert, liplich mit im ze fallen und ze súnden. MAALER 271r (Zürich 1561): Hurische Liebe. SAPPLER, H. Kaufringer 16, 289 (schwäb., Hs. 1464): die ander lang, als man list, | ist unrecht lieb, [...]. | an den zwain hangen alle sünd. EBD. 338: lieb des flaisch; lieb der ern; lieb des guotz: Chron. AugsB. 9, 107, 16 (schwäb., 1544/5): ain grober, harter mann, der was in unordenlicher liebin gegen ainer junckfrauen, [...], entzündet. Turmair 4, 1025, 9 (moobd., 1522/33): Sein obgenanten sun Crispum [...] redt die stiefmueter Fausta umb unordenliche lieb an. - Neumann, Rothe. Keuschh. 1077; Bauer, Imitatio Haller 100, 13; Rwв 8, 1312. Vgl. ferner s. v. angemuten.

8. >Liebes, Angenehmes, Freude, was jm. im Unterschied zu leid zuteil wird`; zu lieben 1. - Vorwiegend Texte der Sinnwelt ,Religion / Didaxe'. - Phras.: las mich mit lieb! \laß mich mit Frieden / in Ruhe «! (hierher?).

QuiNT, Eckharts Pred. 1, 136, 1 (E. 13./A. 14. Jh.): Waz ist widersatzunge? Liep und leit, wî und swarz das hat widersatzunge, und diu enblîbet in wesene nibt. Ders., Eckharts Trakt. 406, 5 (E. 13./A. 14. Jh.): [Volkomeniu abegescheidenheit] wil alsô stân von ir selber, niemanne ze liebe noch ze leide. VetTer, Pred. Taulers 86, 7 (els., E. 14. Jh.): sú [junger] nament fride in unfriede und in liebe leit, und in dem tode nament sú daz leben. Kurz, Murner. Luth. Narr 4298 (Straßb. 1522): Laß mich mit lieb, das rat ich dir, | Vnd halt mir nit dein dochter für, $\mid$ Jch würd sunst sein dein ewig findt. SAPpler, H. Kaufringer 1, 196 (schwäb., Hs. 1464): si funden vor der statt stan | ain haus mit bösem obedach; | den gesten lutzel lieb geschach. KLEIN, Oswald 115, 78 (oobd., n. 1438): Wer ain andächtigs herze trait, | den kümbert weder lieb noch laid. - ScHмIDT, Frankf. Zunfturk. 1, 91, 35; v. Keller, Ayrer. Dramen 3397, 34; Schade, Sat. u. Pasqu. 2, 23, 2.

9. >Liebe zwischen Mann und Frau, körperliche und psychische Zuneigung zwischen Erwachsenen beider Geschlechter «; vereinzelt offen zu: >zwischenmenschliche, sozial geprägte Zuneigung` (vgl. 1) sowie zu: sungezügelte sexuelle Leidenschaftı (vgl. 7); vgl. lieben 9. - Phras.: der liebe buch / getöne >Hohes Lied〈; einander für liebe fressen > heiß verliebt sein «. - Bdv.: brunst 5 , bulschaft 1, ${ }^{1}$ lust 1 ; 2, minne, neigung; vgl. liebschaft 2. Synt.: die l. beschreiben, l. zu jm. baben / tragen, $j$ s. l. begeren, jm. (die) l. öfnen / anzeigen / abschlagen / versagen, die l. einem handel vergleichen; die l. (Subj.) alweg wären, sich verlieren, pein bringen, nicht ane leid ergehen, sich bis in das mark setzen, $j$ s. herz verstricken, giftig als ein atter sein, zu wegen bringen, das [...], l. sich mit l. vereinen, die l. arzenei, der herzen band sein; der l. (Gen.obj.) pflegen; in l. behaften, verhaftet / entbrant sein, bewegt / entzündet werden, jm. in l. gefangen sein, sich in l. gegen jn. annemen (z. B. gegen hadermetzen), sich mit l. gegen einander halten, bei jm. mit l. wonen, jn. (z. B. die jungfrau) um l. ansuchen, von l. reden, jn. von l. küssen; frau liebe (personif.); die l. des bräutigames / mannes, eines töchterleins (jeweils gen. subjectivus), die l. der fran (gen. objectivus), die l. zu jm.; die blinde / grosse / innigliche / rechte / stäte / unzerbrochene / qüchtige l.; der weg / strik, die ordnung / göttin, das band / schwert / zeichen der l.; durch l. und treue willen, die anreizung zu der l. Wbg.: liebebrunst, liebesarm >liebender Arm〈, liebeschmery, liebesfalke, liebeskraft, liebeskus, liebessache, liebhalb > aus Lieber, lieblos 2 >ungeliebt‘, liebmal >Kußfleck ‘, liebsenend, liebtrank 1, liebtrunk, liebung 4.

LuTHER, WA 32, 374, 9 (1532): Inn der erst gehets wol so an, das sie [eheleut] ein ander [...] fur liebe fressen wollen. Volkmar (Danzig 1596): Philtrum, ein liebtrunk, nach-

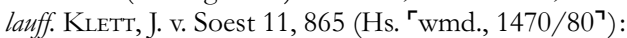
by by klerlichen wurt erkant, $\mid$ das lyb ist tzweyer hertzer bant. EвD. 11, 1106: dy lyb dy wyl bescheyden syn, | und ist das nyt, so bryngt sy pyn. EвD. 11, 1390: merck, wy von Amazonia $\mid$ lybhalp wass schyr geworden gra $\mid$ des selben landes kong. VOC. INC. TEUT. p ir (Speyer um 1483/4): Liebũg Dilectõ. i. Amor. Kopp, Volks- u. Gesellschaftsl. 156, 21 (Hs. 'pfälz., M. 16. Jh. `): amm suessen schlaff, den sie do thet $\mid$ in rechter lieb mit liebesarmen umbschrenket. EBD. 157, 13: ich bin behafft mit liebes kraft, | mit Adams ripp durchschoßen. Dedekind/Scheidt. Grob. 125, 2 (Worms 1551): Küß an ein backen daß es schmatzt / | Das beißt das liebmal angesatzt. HüBNER, Buch Daniel 2079 (omd., Hs. 14./A. 15. Jh.): In der liebe buche dort | Hore Salomonis wort. EвD. 3500: In der liebe gedone | Sprichet er gar wunneclich. BöHмE, Morg.R. 149, 2 (Hs. rschles., 1612 ${ }^{7}$ ): wann dieselbe [zwey junge menschen] an einander 
erbitzen in liebe brunst / so [...7. INGEN, Zesen. Ged. 384, 35 (Breslau 1641): weil alle die anmuthigen Liebes=Küsse / [...] / von der Góttin der Liebe Venus sollen [...] an den schönen Himmels=Saal geháfftet werden. ЕвD. 391, 27: Des Liedes Inhalt war ja von lauter Liebes $=$ Sachen / das muß sie alles gestehen. Pyritz, Minneburg 1503 (nobd., Hs. um 1400): Min trost, min wunne, min liebes valk, In din suße minne du mich walk! zu Dohna u. a., Staupitz/Scheurl 129 (Nürnb. 1517): Nun ist aber des geists antastung ein anrürn der lieb des breutigams zu der liebe der breut, des geists zum geist [hier bildlich]. SACHS 15, 200, 10 (Nürnb. 1562): Daß er vor lieb-senendem schmertzen | Ward kranck von seim gemüt. Ders. 21, 477, 7 (1548): Da mir mein berz $\mid$ In liebe-schmery $|[\ldots]|$ So yniclich entzündet wart. Thiele, Minner. II, 2, 31 (Hs. rndalem./sfrk., 1470/ $\left.90^{7}\right)$ : so muß got ummer erbarmen | das ich liebloses wib | den sinen mynngernden lib $\mid$ in keinen kumber ye gewarff. Williams u. a., Els. Leg. Aurea 598, 23 (els., 1362): Der waz so stolcz von libe vnd von ingent das dise frowe sterklich in sine liebe wart beweget, [...7. Do von begúnde sú mit flisze betrabten wie su in úberkeme daz er zu liplichem valle mit ir keme. Matthaei, Minner. I, 11, 179 (Hs. rwobd., 15. Jh. $\left.{ }^{7}\right)$ : 'ich haiß frow Lieb und hon den sin $\mid$ das ich da all zitt gern bin | wa sich lieb mit lieb ver aint. EBD. 15, 75: die funfft [frowe] nem ich uch schier: | das was frow Lieb, die mengem man | lib und gü gewund an. RolofF, Brant. Tsp. 938 (Straßb. 1554): Under eim baum als ich euch sag | Da sie mit im der liebe pflag. MaAler 271r (Zürich 1561): Liebe die sich biß in das margk binein gesetzt hat. EBD. 271v: Einem die Liebe offnen / anzeigen vnnd nit verhálen. [...]. Eines frommen eegemahels Liebe wäret allwág. LAUATER. Gespånste 33r, 11 (Zürich 1578): der ist in liebe eines schónẽ züchtigen tóchterlins eines burgers daselbst / entzündt worden. Dict. Germ.-GAlL.-LAt. 307, 18 (Genf 1636): Liebtränck / m. Poison pour fairs aimer. BRANDSTETTER, Wigoleis 193, 28 (Augsb. 1493): wonet herr gabon bey seiner frawen mit [...] jnniklicher liebe biß si schwanger ward. KLEIN, Oswald 56, 22 (oobd., 1417/8?): Mein hery, das prüfft vil offt und dick, | das seltram blick, pringt freundlich schrick, | in der lieben strick. EBD. 64, 2 (1416/ 7?): Gar wunniklich hat si mein hers, besessen, | in lieb ich ir gevangen bin mit stëtikait. KARNEIN, de amore dt. 182 (moobd., v. 1440): ir werd gesehen sam ain grosser vberfarer der geliebten lieb, das ir so vnbesinlich [...] tuet aischen vnd piten der lieb und minn. Uhlirz, Qu. Wien 2, 3, 3959, 11 (moobd., 1460): seiner Frau Margrethen durch sundere lieb und treun willen, so sie ihm in seiner krankhait bewaist bat. - KLetT, a. a. O. 11, 788; Tiemann, E. v. NassauS. Kgn. Sibille 121, 4; v. Keller, Amadis 402, 16; Ders., Ayrer. Dramen 3006, 17; 3099, 4; Oesterley, Kirchhof. Wendunmuth 2, 496, 15; 3, 181, 5; НüвNER, a. a. O. 5563; 7466; OpITZ. Poeterey 11, 25; 21, 21; Reichmann, Dietrich. Schrr. 129, 20; Goedeke, P. Gengenb. 128, 414; Roloff, a. a. O. 541; 856; SAPpLER, H. Kaufringer 11, 74; BrandstetTER, a. a. O. 226, 11; Klein, a. a. O. 1, 18; 1, 39; 20, 70; KARNEIN, a. a. O. 180; Dasypodius 155v; Crecelius 1, 560. -
Vgl. ferner s. v. abflüchtig, abschlagen 22, anbringen 5, anfesseln, antastung 2, atter, augenblicken, begeren 1.

10. >dem Menschen aus gnade von Gott gegebene, ihn gotförmig machende, durch asketische übung, den glauben, demut u. ä. Haltungen wieder auf Gott zurückführende Liebe, inbrünstige Ausrichtung des Menschen auf Gott, Liebe zu Gott<; in 1 Beleg (s. u. v. Tscharner): >Liebe, Verehrung eines heidnischen Gottes`; vgl. lieben 10. Texte der Sinnwelt ,Religion', oft der Mystik und Scholastik, auch der Didaxe. Bdv.: andacht 1; 2, brand 10, einbrunst, minne, verlangen; vgl. geminne, gierde 3, ${ }^{1}$ lust 5 . Synt.: l. zu got baben, zu Christo tragen / bringen, die übung mit bilfe der gnade l. wirken; die l. (Subj.) zu got wachsen, jn. gotformen, zu got aufiiehen, im menschen die gestalt der tugend gebären, die l. alle dinge verschmaben, [etw.] erkriegen / minnen; der l. durchbrünstig sein; in der $l$. alle gesaz begriffen sein, die furcht in die liebe geben, sich in l. zu got erbeben, in gottes l. steigen > hinaufsteigen`, Christi mit l. geren, mit l. den zugang zu got zieren, von l. innigkeit zu got kommen; die l. gottes, einer lere, der beiligen schrift (jeweils: >zu [...]<); die befindliche / geistliche / innige / inbrünstige / ware l., die götliche l. >Liebe zu Gottı; die inbrunst, das feuer, die gebote der l. Wbg.: liebde 2, liebespfeil >Liebe (des Menschen) zu Gottı, liebung 5.

Helm, H. v. Hesler. Apok. 17408 (nrddt., 14. Jh.): Wen sie [Gots irwelte] nimmer werden kul| Der waren Gotes minnen, | Der sie vor liebe brinnen. Мејјвоом, Pilgerf. träum. Mönch 2685 (rib., 1444): Yederman hain ich vrede gegeven; $\mid N u$ hoede den mallich gantz ind even $\mid N a$ der liefden de zo mir drait $\mid$ Eyn eicklicher. KeHREIN, Kath. Gesangb. 2, 640, 11 (Köln 1619): O Herr gib mir der liebten brandt, | Mein schwachbeit ist dir wolbekandt, | Leßt du mich auff mir selber stahn. Dubizmay, kurß zu Teutze 48, 7 (hess., 1463): beyliger geyste erfülle deyner gleubigen bertzen und eÿnzunde dar In das fewer deyner libe. DünNHAupt, Werder. Gottfr. v. Bullj. 24, 12 (Frankf./M. 1626): Vnd auff mein Andtlitz mich zu seinen Füssen neig / | Mit Demuth | Glauben / Lieb Furcht / Hoffnung angethan. v. TscharNER, Md. Marco Polo 14, 1 (osächs., 2. H. 14. Jh.): Von den dy ir wip lygin den markt lutin durch di libe irer gote. STEER, Schol. Gnadenl. 6, 79 (moobd., 15. Jh.): disen frid wurcket vnd sacht ein ïbernaturleich tugent, das ist dy genämmachent lieb, dy dann gotformet den menschen vnd gepirt yn ym alle gestalt der tugent, wann yn zwain gepoten der lieb sind begriffen alle gesaczt. KeHREIN, Kath. Gesangb. 
2, 497, 10 (Nürnb. 1631): Der Heiligen gsell mócht er Ignatius von Loyola] sein, | Mit Verlangen, | Liebspfeil sein Hertz durchdrangen. Vetter, Pred. Taulers 381, 4 (els., 1359): des bitte ich úch umbe alle die liebi und minne die ir zu Gotte hant. Eichler, Ruusbr. obd. Brul. 2, 243 (els., E. 14. Jh.): Beuintliche minne vnd liebe, das ist ein begirlicher smackender gelust, den man het zi gotte alse ziv einem ewigen gite [...]. Benintliche liebe git vrlop allen creaturen nach der gelust [...]. Innige liebe benindet sich begerende von innen mit ewiger minnen [...]. Innige liebe verzicht vnd versmahet libtekliche alle ding, vf das sv́ erkeriegen moge, daz sú minnet. Vs diser beuintlichen liebe kumet innekeit ziv gotte. MICHELs, Murner. Badenf. 19, 26 (Straßb. 1514): Frow, all sünd verzich ich [Cristus] dir Vmb liebin die du dreyst zu mir. Morgan u. a., MHG. Transl. Summa 230, 15 (schwäb., 14. Jh.): do brabt der mensche sin liebi zuo der minnen gottis alse zuo dem ende, unde also des gliches zuo allen den andern dingen. BAUER, Geiler. Pred. 462, 12 (Augsb. 1508): wann seine [des Cristophorus] reden flackrent von einbrunst der liebe. PRIMISSER, Suchenwirt 10, 220 (oobd., 2. H. 14. Jh.): Gotleicher lieb inhitaig vrisch|Was er durchprinstig als ein glüt. Buijssen, Dur. Rat. 2, 4 (moobd., 1384): ir bewarent ewren sin mit liebe der heiligen schrifte und ander sitigen lere. KLEIN, Oswald 4, 7 (oobd., 1421): Wer liebe trait | ze got, von dem si [liebe] kompt, daran si hafftet, | so wirt der wille pald gescbick.t, | das er tëglichen trachtet, | wie er die liebe darzu fickt [> fügt 1], | das si nicht werd geferret gotes prust. Baptist-Hlawatsch, U. v. Pottenst. 1108 (moobd., A. 15. Jh.): die weil wir in der werlt sein so müssen wir in den creaturn als an ainer laytter in gotes lieb vnd sein erchantnüss steygen. Höver, Bonaventura. Itin. 3, $589 \mathrm{f}$. (moobd., 1450/60): das ist Jhesum mit liebender andacht ansicht auff gehenckten am kreúcz. durch andacht, glauben, hoffnung vnd liebe, zu verwundrung zi erfrówung, zi lobung, zu liebung, zu jubilierung. BAuER, Zist.-Pred. Haller 47, 130 (tir., 1466): die lieb gottes die ist nicht anders pegern denn got alain. Die götleich lieb die ist gar edel: sÿ hat alle bübsche ding lieb. - Quint, a. a. O. 264, 3; Steer, W. v. Herrenb. Büchl. 495; Dubizmay, a. a. O. 92, 17; SChÖNBACH, Adt. Pred. 22, 31; zu Dohna u. a., Staupitz/Scheurl 130; FrancK, Klagbr. 226, 1; VetTer, a. a. O. 36, 32; Eichler, Ruusbr. steen 72; 586; 878; Warnock, Pred. Paulis 5, 46; 27, 83; Hohmann, H. v. Langenstein. Quästio 191, 147; BAPTist-Hlawatsch, a. a. O. 33 .

11. >Liebe als Qualität, Seins-, Existenzform Gottes, der er den Menschen in einem als -giessen, an-, en-, eræünden gedachten Prozeß teilhaftig werden läßt und aus und in der er seine Erlösungstat vollzogen hat<; auch für >Gottく gebraucht; zu lieben 11. Texte der Sinnwelt ,Religion / Didaxe'. Bdv.: brunst 5, güte 4, milde, minne; vgl. gnade 1; 3, gottesliebe, 1 gunst 3, gütlichkeit 1. Ggs.: has 1, neid, zorn. - Synt.: got die l. ausgiessen; die l. (Subj.) ewig / fest / stark / unwandelig sein, von got kommen, gottes $l$. in das her: gegossen sein, die liebe der weg gottes [...] sein, got die l. sein; die welt der l. (gottes) überzengen; jn. der götlichen l. > mit Gottes Lieber enzünden; aus l. für jn. leiden, durch die l. gottes vergottet werden, in der $l$. die tugend erzünden, sünde wieder die $l$. [Gottes] begehen; die l. gottes (gegen uns), des sones, Christi, des herren; die götliche l. > Gottes Liebe`, die beimliche / rechte / süsse l.; die gabe / süssigkeit der l., die brunst der l. - Wbg.: liebbrief >Brief der / über die Liebe Gottes`, liebde 3, liebestrafe (im Ggs. zu zornstrafe), liebezeichen, liebmildreich, liebreich 3, liebung 6, liebtrank 2.

LuTHER, WA 1, 162, 16 (1517): das die libe gottis starck ist wie der todt. EBD. 9, 128, 4 (1518): Wye hoch got antreugt die lieb, die er selbs ist. EвD. 28, 734, 22 (1529): wir haben im newen Testament viel ein gróssers Liebe zeichen Gottes gegen uns denn jene im alten Testament. EвD. 35, 448, 6 (1524): du susse lieb schenck. uns deyne gunst, | las uns empfinden der liebe brunst. EBD. 52, 286, 2 (1544): das die straff, die Gott auff uns legt, ein liebe srtraff und nicht ein zorm straff sey. Pfefferr, Weigel. Ges. 12, 35 (Hamb. 1466): er leidet, stirbet, [...], alles vmb vnsernt willen, das ist die Welt zu vberzengen der versönung vnd liebe Gottes kegen vn $\beta$ vnd alle Menschen zuuersichern. WAGNER, Erk. Ps.-J. v. Kastl 16, 36 (nürnb., 1. H. 15. Jh.): AUGUSTINUS spricht: Die lieb ist der weg gots zu der sel und der weg des menschen czu got. Gille u. a., M. Beheim 69, 80 (nobd., 2. H. 15. Jh.): mit dem geist du [heilger geist] mich enczund | deiner gotlichen liebe. Fellmann, Denck. Schrr. 2, 25, 18 (nürnb., Hs. 1525): der [...] wirt durch die liebe Gottes gantz, vergottet und Gott in im vermenscht. KeHreIn, Kath. Gesangb. 1, 41, 1 (Nürnb. 1631): Ein Engelischer Bot, |Vom Himmel gesendet wardt, | Von dem liebreichen GOtt, Gabriel. EвD. 1, 326, 5: Dein Blut im streit ein Krafftwehr ist, | Dein Blut der Seelen Liebtranck ist. EвD. 372, 50: Du Liebmiltreicher HERR und GOTT, | Mach mir beyn Außerweblten Platr: Koller, Ref. Siegmunds 230, 15 (Hs. um 1474): daz. wir [...] dancken syner [gottes] liebden und gnaden, die er uns in alle dyngen bewyst hat, sunderlich in synem beiligen lyden gantz unßer heyl instet. BiHLMEYeR, Seuse 484, 11 (alem., 14. Jh.): gute tútsche búchlin sint dines gottlichen liebes liebbrieff. Gilman, Agricola. Sprichw. 1, 285, 26 (Hagenau 1534): Gott ist die liebe / [...] / darumb hatt er auch sein milte gutte unnd liebe außgegossen / unnd in der ersten schepffung / [...] / schaffte er voradt. MoRGAN u.a., MHG. Transl. Summa 408, 22 (schwäb., 14. Jh.): die gotliche liebi, alse vil si von teile gotlicher getat ist, alse vil ist si enig unde unwandelich. KLEIN, Oswald 4, 16 (oobd., 1421): lug, hoffart, spot, hass, zoren, neid | götliche liebe nicht melt. - Quint, Eckharts Pred. 2, 388, 97; Gille u. a., a. a. O. 69, 188; zu Dohna u. a., Staupitz/ 
Scheurl 103; Spechtler, Mönch v. Salzb. 12, 11; 37, 124; Rudolf, Peuntner. Sterbek. 150rb, 40; 153ra, 5; Baptist-Hlawatsch, U. v. Pottenst. 1888; Bauer, Zist.-Pred. Haller 83, 80. - Vgl. ferner s. v. ausdrucken 6.

liebebrunst, s. liebe 9.

\section{liebeln, V.}

> sich liebedienerisch, scherzend, spielend verhalten ‘; vgl. lieben 1 ; 3. - Bdv.: vgl. augendienen, hofieren, liebkosen 2.

SChÖpper 61a (Dortm. 1550): \qürtlen lieblen beyen täntzlen. Mieder, Lehmann. Flor. 397, 27 (Lübeck 1639): Zu Hof gehet man mit Leuten vmb wie Kinder mit Poppen / die sie bald schmucken vnd liebeln / bald schlagen. Schade, Sat. u. Pasqu. 2, 2, 27 (md. 1521): oft wann ich das [pfertlin] uß dem stall gezogen, hab ich es gestreicht und geliebelt, auch etwa uf sein köpflin gekë̈sset. Alberus Y IVv (Frankf. 1540): Mulceo [...], palpo, ich streich / streichel / liebel. SACHS 17, 57, 36 (Nürnb. 1553): Wechelt und thut an mir auffspringen [ein Hund] | Und liebelt sich mit allen dingen?

\section{lieben, V.}

1. >jm. lieb, angenehm sein, gefallen, Freude, Vergnügen bereiten`; prototypisch mit Subj. d. S. und Dat. d. P. - Gehäuft Texte der Sinnwelt ,Religion / Didaxe'. Bdv.: gefallen. Ggs.: ${ }^{1}$ leiden (V.) 3. - Synt.: rgerste (Subj.) l., das beten nicht l.?, jeweils absolut; jm. etw. (Subj., z. B. ere / gut / treue / tugend / zucht, js. scherzen, der krieg / reisdienst >Kriegsdienst<, die freiheit / wahrheit, der meistergesang, das böse, die bulschaft / sünde / tyrannei / welt) l., jm. etw. (z. B. müssigkeit, irnis seiner gnaden) nicht l., im einzelnen z. B. der zorn dem teufel, einfaltigkeit dem weisen, das kebsweib js. gesicht (Dat.), (von einen Tier:) der geis die berge, dem esel futter l., jm. etw. (z. B. genäsch) für etw. (>mehr als « Anderes, z. B. für ritterschaft) l., (jm.) l. [+ Infinitiv(satz) mit oder ohne zu], z. B. (jm.) zu lieben / leben / sterben, [wo] zu bleiben l., dem esel l., im stal zu liegen >dem Esel gefällt es, wenn [...] <, jm. l., was [...]; die got liebende gerechtigkeit > die Gott wohlgefällige Gerechtigkeit .

Luther, WA 32, 444, 29 (1532): Was dem menschen liebet, das ist sein Gott, denn da tregt jn sein bertz zu. HeLm, H. v. Hesler. Apok. 23132 (nrddt., 14. Jh.): allen den da lugene | Libit unde trugene. BergmanN, Ambr. Liederb. 55, 24 (Frankf. 1582): mir liebt jhr zucht und wandel, |jbr weis und geberd. HüBNER, Buch Daniel 2318 (omd., Hs. 14./A. 15. Jh.): Gerste liebet, spru leidet. Jungbluth, J. v. Saaz. Ackermann 14, 11 (Hs. 「omd., 1465`): das haben begert die philosophen, wann sie sprechen, am besten zu sterben, wann am besten liebet zu leben. Opes, Spittendorf 90, 33 (osächs., um 1480): seyne gnade, [...], dem irrenisse der seynen billich nicht liebete. GILLE u. a., M. Beheim 176, 105 (nobd., 2. H. 15. Jh.): Der czorn liebt auch dem teufel, wan | er wirt des menschen her daran. SACHS 16, 174, 9 (Nürnb. 1561): Noch liebt das kebsweib mehr deim gsicht [poetisch für: >Dird. LAuchert, Merswin 3, 8 (els., 1352/70): in diseme lebende waz ich ettewie vil iare, also daz. mir die welt ie me und ie me liebende wart. Goldammer, Paracelsus 6, 190, 4 (1530): das ist sanft und milt vor got, daß dein frucht auf erden also gruenet und got liebet und gefällt, daß [...]. Lemmer, Brant. Narrensch. 18, 30 (Basel 1494): Dem wisen liebt eynfaltikeyt. Bolte, Pauli. Schimpf u. Ernst 1, 196 (Straßb. 1522): Also verbot einer seiner Frawen, sie solt nit in die Mistlachen gon, da geliebt es ir erst. Bauer, Geiler. Pred. 316, 8 (Augsb. 1508): gleych als ainem esel / dem liebt futer und bew und im stal zu ligen. Barack, Zim. Chron. 4, 231, 6 (schwäb., M. 16. Jh.): Was ich hab usserlesen, | Das liept aim andern auch, I Sie war mein buol und ich ir gauch. PRIMISSER, Suchenwirt 31, 185 (oobd., 2. H. 14. Jh.): Jm liept daz genesch fur ritterschafft. Pfeiffer, K. v. Megenberg. B. d. Nat. 128, 3 (oobd., 1349/50): Diu wild gaiz. ist gar ain weis tier, dem liebent hôch perge. KLEIN, Oswald 68, 23 (oobd., 1417): Für all diss werlt liept mir dein er | und wil der vil bas wesen undertan. Rudolf, H. v. Langenstein. Erch. 12, 4 (moobd., 1393): ez ist chain tegleich svnd, oder sy werd todleich, wenn si dem menschen mit gewonhait liebt. EBD. 38, 74: Der psalm ist mir vngesmach ze lesen, daz peten liebt nicht. Turmair 1, 430, 11 (Augsb. 1517): iam faxo scies ,wil dir thuen was dir liebt', dii faxint, got gebs'. KeHreIN, Kath. Gesangb. 2, 587, 5 (München 1586): Deins nechsten Weib solst nit begern, | Sein Gut laß dir nit lieben. WINTER, Nöst. Weist. 1, 1008, 10 (moobd., 17. Jh.): dero weegen diß orths auch fehrner mit nothwendigen beambten versehen, die gott liebende gerechtigkeit administriret. - MAYER, Folz. Meisterl. 7, 19; SACHS 17, 278, 21; 20, 290, 3; ReichmanN, Dietrich. Schrr. 143, 11; Bell, G. Hager 32, 3, 18; 465, 1, 6; 476, 1, 14; Chron. Strassb. 118, 28; Kurrelmeyer, Dt. Bibel 5, 8, 9; Fuchs, Murner. Geuchmat 1619 f.; Haszler, Kiechels Reisen 318, 27; Primisser, a. a. O. 1, 158; 22, 125; Spechtler, Mönch v. Salzb. 33, 92; Klein, a. a. O. 71, 12; 115, 82; Grossmann, Unrest. Öst. Chron. 117, $26 ; 138,7$.

2. >jm. belieben, jm. gefallen, etwas zu tun, Gefallen an etw. finden`, von Machtbefugten (oft von Gott) gesagt; auch: >etw. wollen, planen`; im Unterschied zu 1 mit stärkerer Betonung der Handlungsabsicht. - Phras.: so es dir (ge)liebt. - Synt.: got etw. l.; jn. (z. B. got) lieben, zu [...]. 
Loesch, Kölner Zunfturk. 11, 548, 17 (rib., 1454): Hedden wire liefden dairenbinnen gelieft, darbizoschicken, so weulde ich gerne darbij sijn komen. BoberTAG, Schwänke 317, 26 (Frankf. 1563): in ansäbung deines allhie einsamen, armutsäligen läbens, [...], so dirs liebt, begib dich [...]. SERMON Thauleri 11vb, 13 (Leipzig 1498): es mag nymmer Bo klein leidẽ auff dich gefallen got hab es vor ewiglich an gesehenn ṽ̃ das geliebet vnd das gemeinet. BACHMANN, Morgant 117, 6 (halem., 1530): das der Krysten got der almechtig oberst got were, [...], und gantz güettig ist und erbermklich, das in geliept hat den friden zemachen zwüschend den zwey besten rittern in aller Kristenheyt.

3. >sich jm. angenehm, beliebt machen<; mit negativer Wertung: >sich (bei jm.) einschmeicheln<; auch: >jm. / sich jn. / etw. angenehm, gefällig machen ren Frnhd. auslaufend; oft Texte der Sinnwelt ,Religion / Didaxe'. - Bdv.: beglimpfen 1 , bebagen 1, benedeien 3; 4, loben 1, rümen, schönen, vereren. - Synt.: der bund, die minne sich l., die vögel sich jm. l., j. sich jm. l., z. B. j. sich dem abgot, got, dem könige, den engeln / menschen / leuten, der lieben frauen, der diete l., j. sich der welt l., j. sich e. S. (z. B. der minne) l., j. sich zu jm. (z. B. zu Neronem) l.; nicht reflexiv: j. got (Dat.), einem menschen l., j. jm. etw. l., etw. (z. B. arme / lefzen) jn.jm. l., j.js. namen l. > angenehm machen $<$.

Ziesemer, Proph. Cranc Jes. 66, 3 (preuß., M. 14. Jh.): wer do gedenket des wyrouchs, der ist als der sich lyebet [worms. Proph. 1527: rumet; Dietenberger 1534: verehret; EcK 1537: benedeit; LuTHER 1545: lobet deme abgote. HüBNER, Buch Daniel 3474 (omd., Hs. 14./A. 15. Jh.): Die [crone] Got den gelobet hat $\mid$ Swelch im lieben mit der tat. PALm, Veter Buoch 16, 26 (schles., Hs. E. 14./A. 15. Jh.): da von wirt der man tugenhaft und liebet sich den engelen und gote. STACKMANN u. a., Frauenlob 8, 14, 10 (Hs. 'nobd., 3. V. 15. Jh. `): Solt er davon nicht riche $\sin |[. .]$.$| und lieben sich dem künig Constantin. BIHL-$ MEYER, Seuse 120, 19 (alem., 14. Jh.): herr, nu han ich alle min tag dinen wirdigen namen geeret und vil wit und breit mengem menschen geliept. EBD. 456, 1: dú süsse minne vahet an underwilen mit leide und wirt aber lieb und liebet sich zu allen ziten. EBD. 550, 24: si [bleiche lefzen, blitige arme] liebent dich mir minnenclich und eignent mich dir gentzlich. Williams u. a., Els. Leg. Aurea 401, 5 (els., 1362): Hie zwischent hette sich Symon der zöberer zu Nerone geliebet. Bobertag, Schwänke 29, 5 (Straßb. 1522): wan sie lieben sich wie ein bund der heffen bricht. Rieder, St. Georg. Pred. 182, 1 (Hs. 「önalem., 1387`): dar nach beginnet er es in ze liebenn und raitzet sú uff daz. sunder ding. VETTER, Schw. zu Töß 111, 15 (Hs. 15. Jh.): so was sy doch noch fil edler von manigfaltigen tugenten, mit den sy sich liebt Got und den lútten. Österley, Steinhöwels Äsop 53, 2 (Ulm 1474/ 82): so schmukt er [bund] synen schwancz, und kryset uff der erden wider zuo dir und liebet sich. Primisser, Suchenwirt 13, 51 (oobd., 2. H. 14. Jh.): Er was, der sich der mynne | Chund lieben mit gelimpfe. - Helm, H. v. Hesler. Apok. 4401; Quint, Eckharts Pred. 2, 290, 8; v. GroOte, Muskatblut 33, 37; Bihlmeyer, a. a. O. 422, 12; Kurz, Murner. Luth. Narr 1247; JöRG, Salat. Reformationschr. 281, 21; SpIller, Füetrer. Bay. Chron. 133, 13; Schmidt, Hist. Wb. Elsaß 223.

4. >jm. liebevoll, nachgiebig, schonend entgegenkommen

Leisi, Thurg. UB 6, 858, 34 (Konstanz, 1333): wáre, daz lantgebreste von hagel wurde, so sont si úns liben an dem vorgenanten zins.

5. >jm. lieb, wert, teuer sein, Ziel von js. Zuneigung, Liebe sein, jm. gefallen`; meist in der Richtung vom Mann (als dem Liebenden) auf die Frau gesehen, oft mit erotischer Komponente. - Bdv.: wolgefallen. Ggs.: ${ }^{1}$ leiden (V.) 1. - Synt. (prototypisch): j. (z. B. das weib) jm. (dem Mann) l., j. js. sinne / gier, inniglich, ob allen >mehr als alle‘, für die ganze welt l., j. jm. zu einem manne l.

LutHeR. Hl. Schrifft. Sir. 7, 28 (Wittenb. 1545): HAstu ein Weib / das dir liebet / So las dich nicht von jr wenden. Bergmann, Ambr. Liederb. 179, 24 (Frankf. 1582): der mir liebt, den las ich mir nicht leiden. Pyritz, Minneburg 4708 (nobd., Hs. um 1400): Wie kan sie halt derweren mir, | Sie lieb dem sin und miner gir | Immer, immer von tag zu tagen? v. GroOte, Muskatblut 37, 69 (nobd., 1. H. 15. Jh.): mir liebt ein knabe, ist freüden rich $\mid$ in mynes hertzen grunde. FastNaChTSP. 634, 5 (nobd., 15. Jh.): Sie liept mir für har auß raifen [zu ranfen] | Und liept mir für die haut ab straifen. EBD. 1404, 13: Er liebt mir sicherlich allein $\mid$ Fur alles das auf erden mag geleben. TrtTMAnN, Schausp. 16. Jh. Ayrer. Phaenicia 195, 230 (Nürnb. 1618): sie [jungfrau] mir auch liebt für die ganz welt. GolDAMMER, Paracelsus 7, 172, 22 (1530): und hat ein andere gesehen, die ibm geliebt hat, so hat er sie auch zur ebe genomben. Adrian, Saelden Hort 1695 (alem., Hss. E. 14./ 15. Jh.): din liep, daz liep, ain kindellin | sol lieben dir. BARACK, Zim. Chron. 4, 221, 27 (schwäb., M. 16. Jh.): Ir [Fraw] liept mir ob allen, | Die weibs nammen gewunnen. KLEIN, Oswald 26, 100 (oobd., 1427): wër ich ain weib, umb alles güt $\mid$ so möcht er mir nicht lieben. ЕвD. 74, 10 (v. 1408?): Ach raines töckel, traute, schöne tocke, $\mid$ du liebst mir mit dem zipfel an dem rocke. LeIDINGER, A. v. Regensb. 595, 32 (oobd., um 1430): Welcher ir liebät zu einem mann, das sy den näm, und doch ein sölichen, der das könikereich auszerichten wirdig wär. - FRANTZEN u. a., Kölner Schwankb. 1, 54, 7; BergmanN, a. a. O. 18, 34; 180, 7; 182, 5; Kehrein, Kath. Gesangb. 1, 49, 3; Goedeke u.a., Liederb. 25, 5; SAchs 16, 414, 6; 
Bolte, Pauli. Schimpf u. Ernst 1, 45, 35; Wiessner, Wittenw. Ring 1552; KLeIN, a. a. O. 87, 34.

6. >etw. (das in der Regel positiv oder negativ bewertet wird) lieben, mögen; sich e. S. mit überduchschnittlicher bis existentieller Intensität widmen, zu etw. stehen<; mit Tendenz zu: >etw. anpreisen $<$ - Oft Texte der Sinnwelt ,Religion'. - Bdv.: gefallen 6. Ggs.: hassen 1; 2, ${ }^{1}$ leiden (V.) 2; 3. Synt.: den fras > Gefräßigkeit‘, die einigkeit / gerechtigkeit (mehrfach) / keuschbeit / reue / sasse >den Müßiggang< / sünde, die zunftliche regierung, das vaterland / sakrament, gottes wort, das böse / laster l., eine kunst, kinderdinge, die seiten (eines Instrumentes) l.; der die einsamkeit liebende stand, die geliebte kürze. Wbg.: ${ }^{2}$ liebern >etw. höher veranschlagen, als es in der göttlichen Ordnung ist ‘ (dazu Ggs.: leidern).

Helm, H. v. Hesler. Apok. 2039 (nrddt., 14. Jh.): Man sal di ruwe lieben, | Daz Gotes wort in schieben | Den harten, die vorsteinet sint. Quint, Eckharts Pred. 388, 91 (E. 13./A. 14. Jh.): Liebest du die gerechtigkeit / nach dem sy gerechtigkeit ist in dyr oder über dich / so (l)iebest du die gerechtig(k)eitt nicht als sy ann jr selber ist [...] / Aber du nemmest sy geteiltt. RosentHaL. Bedencken 8, 3 (Köln 1653): Nun aber [...] haben die jenigen Gottes Lieb nit / welche die Eynigkeit der Kirchen nit lieben. Gropper. Gegenw. 18v, 5 (Köln 1556): das Sacrament aber seins Leibs vnd Blìts / [...] solt / sonder heiliglich gelibt / angericht vnd gnossen werden. HüBNER, Buch Daniel 8191 (omd., Hs. 14./A. 15. Jh.): Beten, wachen ist zu swer $\mid$ Den, die die zwei [vras, saze] bie lieben. Roloff, Naogeorg/Tyrolff. Pamm. 9, 37 (Zwickau um 1540): das der Jugnt solch irthumb offenbart | In ursach geb gots wort dest mebr zu liebn. Ingen, Zesen. Ged. 389, 6 (Breslau 1641): MEin liebster Opitius rúbrte die Seiten / | Die jederman liebte. M. Cunitia. Ur. Prop. 147, 34 (Öls 1650): als in meinem einsamkeit liebend $=$ und úbendem Stande / [...] nutzbares z. wirken. Dünnhaupt, Werder. Gottfr. v. Bullj. 25, 30 (Frankf./M. 1626): Wie wider dein Gesetz / [... / Jch stets die Laster liebt. Pyritz, Minneburg 4102 (nobd., Hs. um 1400): Was ir but liebt, daz leit ir morn. KurreLMEYER, Dt. Bibel 10, 72, 19 (Straßb. 1466): ir do habt in haß das gut und liebt das bóse. Pfeiffer-Belli, Murner. Kl. Schrr. 6, 78, 5 (Straßb. 1520): das wir lieber ein menschen erkennen für ein oberkeit den got dar zu sag ich das wir das weder zu lieberen noch zu leideren haben / sunder so es got also verordenet hatt. Goldammer, Paracelsus 3, 282, 10 (1530/5): daß wir die bilder nit ehren, fubren, nebren die sich an gottes statt setzen, malen gott ein rock an, den er nie geliebt hat [Anspielung auf den päpstlichen Ornat?]. Chron. Augsb. 9, 87, 11 (schwäb., 1544/5): domit die gemaine zunftliche regierung, [...], für andren [...] regimenten geliept und gelopt werden sollte. RudOLF, H. v. Langenstein. Erch. 24, 10 (moobd., 1393): wann dï sunt dem tewfel besunder geuelt vnd liebt zu des menschen vnhail. - M. CunITIA. a. a. O. 177, 17; zu Dohna u. a., Staupitz/Scheurl 178; 181; Mannack, Rist. Pers. 121, 15; v. Keller, Ayrer. Dramen 2130, 3.

7. >etw. (Geschäftliches, Rechtsrelevantes) akzeptieren; jn. als etw. anerkennen«. Bdv.: verjaworten.

v. Bunge, Livl. UB 817, 41 (nrddt., 1412): Das liebeten die boten und riten als am Sonnabende nest dirgangen von binnen. Peil, Rollenhagen. Froschm. 6654 (Magdeb. 1608): Es wollen auch die Fürsten Sieben / | Jhn als jbres Reichß Kónig lieben. DoubeK u. a., Schöffenb. Krzemienica 102 (schles. inseldt., 1455): als das dï erber leute han gemacht das wil ich liben vnd czu steen. EвD. 170: sy haben Hannus Welczels eewirtÿnne cleÿder geschaczt vor czw marg vnd vor IIII groschen, vnd dy entschechtunge hot Hannus Welczel gelipt vnd vorj̈owart.

8. >jn. lieben, gerne haben, mögen; jm. mit der moralisch und / oder religiös gebotenen Zuneigung begegnen und ihn entsprechend behandeln «. - Texte der Sinnwelt ,Religion / Didaxe'. - Bdv.: vgl. ${ }^{1}$ leiden (V., unr. abl.) 4. Ggs.: durchächten, hassen 1. - Synt., teils absolut gebraucht: j., die sele (Subj.) l.; mit dem herzen / gemüt l.; prototypisch: j. jn. l., z. B. j. den feind / nächsten, der könig Daniel, die mutter den erben l., der affe ein kind vor dem anderen l.; der bote geliebt werden, dichter geliebt werden wollen.

Luther, WA 10, 1, 2, 393, 36 (1522): so ich meinen nechsten liebe, so belffe ich im, bschütze in. EBD. 16, 22, 32 (1524): und Pharao Tocbter wird offt mit ime [Moises] gespielet, in getentzelt, auch seer geliebet haben. EBD. 17, 2, 149 , 9 (1525): Hie [im ampt] heysst es lieben und nicht geniessen, und doch nicht lassen verdriessen. EвD. 21, 308, 2 (1544): so man, die da sundigen [...] mit stilschweigen stercken solt zum bosen, das were nicht geliebt. PeIL, Rollenhagen. Froschm. 515, 280 (Magdeb. 1608): Den [Erben] Jch / sein Mutter / das elend Weib / | Mebr liebten / denn das Hertz im Leib. Kurz, Waldis. Esopus 2, 16, 3 (Frankf. 1557): wenn die Aff gebert | Bey paren, sie jr Kinder nert, Der thut sie eins vorm andern lieben. HüBNER, Buch Daniel 5073 (omd., Hs. 14./A. 15. Jh.): Der kunic begonde in [Danyel] Lieben vor den andern vil. SERMON THAuleRI 4vb, 8 (Leipzig 1498): Sal sy [sele] liebẽ das thut sie mit dem willen. Logau. Abdank. 169, 15 (Liegnitz 1651): Sonst giengen die Boten deß FRJEDENS daselbst jmmer auß und ein / Assen und Truncken / das ist / worden geliebt geehrt und befódert. zu DoHNA u. a., Staupitz/Scheurl 180 (Nürnb. 1517): Niemant mag zweien herrn dienen: antweder wirdet er einen hassen und den andern lieben. GOLD- 
AMmer, Paracelsus 2, 52, 8 (1531/4): folgt also ein ordnung der gaben eines ieglichen gegen dem nechsten. dann der den nechsten liebet, der liebet auch gott. Dict. Germ.-Gall.Lat. 306, 22 (Genf 1636): Lieben / lieb baben / eine Neigung zu einem haben. Wolf, Norm im sp. Ma. 60, 74 (oobd., 1486): yr sult lieben eur veint vnd sult pitten fur dy, die euch durchechten. - LuTHER, WA 10, 1, 2, 376, 31; Pfefferl, Weigel. Ges. 5, 16; Schorer, Sprachposaun 51, 12; MaAler 271v. - Vgl. ferner s. v. anschieben 2.

9. >jn. erotisch lieben, eine Zuneigung zu einer Person des anderen Geschlechtes haben`, mit etwa gleicher Häufigkeit auf den Mann wie auf die Frau als Liebende bezogen. - Bdv.: vgl. bulen 2. - Synt.: mehrfach absolut (ohne Obj.): inniglich / beimlich / stil / unerlich / unsinnig / verderblich l.; prototypisch: j.jn. l., z. B. die frau den bulen l., die magd die töchter l., als ob [...]; das zuckersüsse lieben (subst.).

Luther, WA 32, 382, 32 (1530/32): Du Richter solt [... straffen und ein jglicher sein gemalh haben und lieben. INGEN, Zesen. Ros. 80, 12 (Hamb. 1646): laß uns lieben / ehe wier | ganz vergráusen. EBD. 91, 22: daß ich sie liebe / welches doch auch nicht meine / sondern ibrer schónheit schuld ist. v. Keller, Amadis 402, 11 (Frankf. 1561): vnd hat jn [Ritter] so inbrünsliglich lieb, daß vnmüglich, daß sie jn böher lieben köndt. OpITz. Poeterey 49, 13 (Breslau 1624): Liebe nun wer nur zue lieben | Rechten fug und mittel hat. ZU DoHna u.a., Staupitz/Scheurl 128 (Nürnb. 1517): Das alles ist fürwar fast beschwerlich, bis so lang der geliebet sein handt heimlich einlest und das schwechst an der breut leib berürt. PANZER, Merlin Füetrers 215, 1 (moobd., 1473/8): Des dy geliebtdenn [>die Liebenden`] pflagen, | ist mir vil lutzel kund. FucHs, Murner. Geuchmat 1672 (Basel 1519): Ein yede frouw irn bülen triebt, | Ob sy jn schon recht wider liebt. BARAcK, Zim. Chron. 2, 433, 12 (schwäb., M. 16. Jh.): Die [dienstmagdt] hat die jungen döchter geliept, [...] auch alle geperden [...], als ob sie ain mannlichen affect het, gebraucht. - PeIL, Rollenhagen. Froschm. 126, 2586; Ingen, a. a. O. 70, 33; 78, 7; v. Birken. Erzh. Österreich 83, 24; v. Keller, Ayrer. Dramen 2796, 31; MAaler 165v.

10. >(Gott oder eine gottnahe Person: Maria) mit religiöser Inbrunst, mit der Aussicht auf Behütung, aufgrund gängiger Moralvorschriften, aufgrund des Glaubens lieben, verehren<; auch absolut gebraucht, teils in Verbindung mit eren, ${ }^{1}$ glauben (V.) 1 , loben 2. - Texte der Sinnwelt ,Religion / Didaxe'. - Synt.: auch mit Gen. d. P.

Luther, WA 10, 2, 497, 11 (1522): dich [got], es gee uns wol oder ubel, zсu loben, zсu lieben, unnd zсu eren. EвD. 10,
3, 101, 11 (1522): daß ein solch Hertz GOtt und Christum lieben musse. ЕвD 11, 249, 20 f. (1523): wer mehr glewbt unnd liebt, der ist volkomen. Dubizmay, kurß zu Teutze 18, 16 (hess., 1463): SAncta Maria [...] deyn geret meyn hertz, zu lieben [...] das du meyn voittin seyst vor dem teuffel. zu Dohna u. a., Staupitz/Scheurl 117 (Nürnb. 1517): Die breut des ersten grads lieben und werden geliebt, nemen aber nit sunderliche zeichen der lieb. ReICHMANn, Dietrich. Schrr. 250, 21 (Nürnb. 1548): Gott will barmhertzig sein in das tausent geschlecht / denen / so jn lieben. RolofF, Brant. Tsp. 655 (Straßb. 1554): sie [frawen] liebten Gott den Herren | Lebten in tugendt zucht und eren. GiLman, Agricola. Sprichw. 2, 5, 7 ([Augsb.] 1548): da in der Herr fragt / Petre amas me, Petre / liebest du mich? Höver, Bonaventura. Itin. 2, 587 (moobd., 1450/60): Wellicher [...] Jhesum mit liebender andacht ansicht auff gebenckten am kereúcz: - v. Keller, Ayrer. Dramen 2129, 10, Trunz, Meyfart. Tub. Nov. 84, 6. - Vgl. ferner s. v. arbeiter 1, arm (Adj.) 1.

11. >jn. (auch: die welt) lieben<, von Gott als dem Liebenden / Erlösenden gesagt; schwache Belegung.

LutHeR, WA 10, 2, 497, 30 (1522): unser got und Vatter, der uns geliebt hat. ANDERson u. a., Flugschrr. 5, 2, 3 ([Zwickau] 1524): Das ist mein gebot / dz jr ein ander lieb habt / wie ich eüch geliebt hab. BELL, G. Hager 75, 3, 17 (nobd., 1594): also bat got glibt die welt, | das [...]. v. Keller, Ayrer. Dramen 2131, 20 (Nürnb. 1610/8): Gott ist ein keuscher treuer Gott, | Libt all, die jhn lieb baben.

lieber, Adv. (1), Interj. (2).

1. >lieber, eher das Eine als das genannte oder vorausgesetzte Andere<; vgl. lieb (Adj.) 1. - Phras.: lieber sterben, dan [...]; lieber beim teufel wonen, dan [...]; lieber wäre ich nie geboren. - Bdv.: ehe, mer. - Synt.: lieber etw. geben / wagen / tun / wollen, dan / den [...] / als [...], ehe [...], lieber etw. sein (z. B. tot) / werden, als $[\ldots]$.

PeIL, Rollenhagen. Froschm. 581, 2388 (Magdeb. 1608): Es wer auch rathsam / nute, und gut / | Das man lieber Geld geb denn Blut. Quint, Eckharts Pred. 290, 11 (E. 13./A. 14. Jh.): ich entrüege daz base kleit lieber dan debein ander kleit. CHRON. KöLn 1, 1021 (rib., Hs. 1. H. 15. Jh.): ire alre hertze dachte al ein, | dat sij lieuer alda sturuen $\mid$ dan [...]. Tiemann, E. v. Nassau-S. Kgn. Sibille 141, 10 (rhfrk., um 1435): Darvmb wil ich mich viel liebir wappenen / und wil mit myner glenen zü yme in den plane ryden. Froning, Alsf. Passionssp. 4875 (ohess., $1501 \mathrm{ff}$ ): ich wolde lieber uber bundert myle gan, | dan das ich dyt vornumen han! Оовsснот, Spee/Schmidt. Caut. Crim. 254b, 38 (Frankf./M. 1649): diß die wir viel lieber unserẽ Eyffer raum geben / alß vnsers Gesetzgebers Christi Gebott folgen wollen. Henschel u.a., Heidin 1822 
(nobd., um 1300): Lieber wer ich nie geborn. zU DoHNA u. a., Staupitz/Scheurl 252 (Nürnb. 1517): das wir lieber wölten ze nicht werden dann leben. FrancK, Decl. 346, 5 (Nürnb. 1531): das schier gar kein mensch ist / der nicht lieber ein büler [...] wól genant werden / dann ein volle ki. v. Keller, Ayrer. Dramen 3004, 4 (Nürnb. 1610/8): Ich wolt warlich lieber sein todt, | Als mit eim solchen Mann behencken. Gilman, Agricola. Sprichw. 2, 141, 2 ([Augsb.] 1548): so solt der Man / die Kinder / das Gesinde / unnd gantze Nachbaurschafft / lieber beim Teüfel wonen / dann bey ainem solchen Weibe. - Lemmer, Schernb. Frau Jutte 903; v. D. LeE, M. v. Weida. Spigell 22, 1; Vetter, Pred. Taulers 59, 12; Morgan u.a., MHG. Transl. Summa 392, 11. - Vgl. ferner s. v. absein 1 , annemen 16 , auch 7.

2. dient der Redeeröffnung bzw. dem Fortgang des Gespräches, dann: >nun, ach, und, mit Verlaub, quaeso $<$.

Luther. Hl. Schrifft. 1. Mose 12, 13 (Wittenb. 1545): Lieber [Mentel 1466: ich bit dich; ähnlich ECK und Dietenberger] so sage doch / Du seist meine Schwester. Ders., WA 48, 179, 4 (1547): LJeber, Wer gleubet doch das, das vnser Gebet so angeneme sey? SACHs 13, 202, 26 (Nürnb. 1556): Ey, lieber, der narr ist abentewrisch, | Ist gar einfeltig und sebr beurisch. v. Keller, Ayrer. Dramen 2368, 21 (Nürnb. 1610/18): M. Simon sagt: So seit jbr Mabler? Ey lieber, lieber! MAaler 272r (Zürich 1561): Lieber ist es ein wunder? In spottswey\}. Mirum nerò? Lieber ja / à ja binder sich / Spottwort. [...]. Lieber ja / als ob er nitt dainnen seye / å ja / áben ja. Eia uerò quasi von sit intus. Lieber meine / ich bitt dich darumb / Ein wort lieb zekosen oder zeermanen. Sodes. Lieber sag wilt du ácht. Dic Sodes. [...]. Lieber meine sag mir / ist ers? Jch bitt dich darumb / ist ers? Obsecro an is est? Ukena, Luz. Sp. 658 (halem., 1575): Lieber was manglet an Jesu Christ | Der durch ïch ziom Tod kon ist. - Holland, H. J. v. Braunschw. V. e. vngerat. Sohn 337, 23; TrtTmanN, Schausp. 16. Jh. Ayr. 164, 1360.

lieberman, s. lieb (Adj.) 3.

liebergöttig, s. lieb (Adj. 4).

${ }^{1}$ liebern, V.; zu mhd. liberen sgerinnen< (LeXer 1, 1896).

sgerinnen, steif werden (vom Blut)<. Bdv.: beliebern, berinnen (V., unr., abl.) 3, coagulieren, gerinnen, grieseln, ${ }^{2}$ kallen; vgl. ${ }^{2}$ lippen.

Strehlke, Nic. Jerosch. Chron. 17561 (preuß., um 1330/40): daz blût im $\hat{u}$ dem labe twanc, $\mid$ daz iz $\hat{u}$ den wundin dranc| bî stuckin, want ì itzunt was | gelibbrit. Luther, WA 50, 526, 34 (1539): das [Bezug auf blut wurst ist nicht allein dünne blut, sonndern auch gelievert. $\mathrm{J}$. W. von Cube. Hortus 114, 22 (Mainz 1485): Wenn das blut am lybe gelibbert were von slegen. ERmisch u. a., Haush. Vorw. 74, 30 (osächs., 1570/7): Dis ist auch einem men- schen gutt vors gelieferte blutt im leibe. - STEDTFELD, RogerGlosse 84.

${ }^{2}$ liebern, s. lieben 6 .

liebesarm, liebeschmerz, liebesfalke, liebeskraft, liebeskus, s. liebe 9.

liebespfeil, s. liebe 10 .

liebessache, s. liebe 9.

liebesstrafe, s. liebe 11.

liebestreiche, s. liebe 4.

liebezeichen, s. liebe 11.

liebezorn, liebgefallen, s. liebe 1.

liebgeld, s. lieb (Adj.) 2.

liebgenies, s. liebe 4.

liebgezal, s. liebæälig 1.

\section{liebhaben, V.}

1. >sich zu etw. (positiv oder negativ Bewertetem) hingezogen, von etw. angezogen fühlen, e. S. (einer Tugend, einem Laster) zuneigen ‘; vgl. lieb (Adj.) 1; 5, lieben 6. Beleghäufung für Texte der Sinnwelt ,Religion / Didaxe'. - Synt.: etw. (z. B. den frieden, die ere / kirche / finstere nacht / ritterschaft / tugend / welt, das geld / haus / hausgeräte / recht / unrechte / liecht / leben / wildbrät, die winked) l.; der finstere (Gen.obj.) l. Wbg.: liebbabung 1.

KochendörfFer, Tilo v. Kulm 3750 (preuß., 1331): Er [Judas] was ein dieb | Und hatte vil winkel lieb. LUTHER, WA 9, 602, 31 (1521): wer die erbe oder das geldt lib gat, der [...]. EBD. 10, 1, 2, 361, 11 (1526): das ein weib [... das bauß und hausgeräth, vich und anders liebe babe. EвD. 52, 304, 6 (1544): Wer nun die Christliche Kirch lieb hat unnd gern jbr wolfart sábe, der [...7. Buch WeinsB. 5, 492, 27 (rib., 2. H. 16. Jh.): Hab leiff, was nit mag vergain, | So sal din hertz in freuden stain. WYss, Limb. Chron. 44, 18 (mfrk., Hs. 2. H. 16. Jh.): darzu hatte he [herzog] ritterschaf lip unde schonete darane keine koste. EgGers, Psalter 20, 18 (thür., 1378): der aber daz vnrechte lip hat, der hassit sine sele. СснӧмвасH, Adt. Pred. 6, 36 (osächs., 1. H. 14. Jh.): si [werld] wiset dir maniger bande zirait, durch da: tu sie lieb babest und vorgezzest der libe dines schepheres. REICHERT, Gesamtausl. Messe 27, 22 (Nürnb. um 1480): Dar bey sollen wir lernen und die tugent der keuscheyt lieb zu haben. Kurrelmeyer, Dt. Bibel 2, 432, 17 (Straßb. 1466): der so wil liebhaben das leben: vnd gesechen die gutten tag: der tzping sein zungen von dem vbelen. HaAs u. a., Eras- 
mus/Jud. Klag 6v, 8 (Zürich 1521): kunst macht ein menschen / liebhabung vnd durchgründung der wy/ßheit macht meer dann menschen. Gilman, Agricola. Sprichw. 2, 134, 31 ([Augsb.] 1548): wann er [Got] uns ain frummen Herren gibt / der Frid und Recht lieb hat. - Jungbluth, J. v. Saaz. Ackermann 31, 18; Gille u. a., M. Beheim 81, 223; Moscherosch. Ges. Phil. v. Sittew. 38, 11; Wagner, Erk. Ps.-J. v. Kastl 4, 40; Sappler, H. Kaufringer 14, 150. - Vgl. ferner s. v. ${ }^{1}$ an 16.

2. >jn. (auch: Tiere) lieb haben; jn. lieben; jn. hoch achten, hoch schätzen; jn. im Sinne des Caritas- oder des unio-Gedankens der Mystik liebevoll behandeln, Sorge für jn. tragen`; vgl. lieben 8. - Gehäuft Texte der Sinnwelt ,Religion / Didaxe'. Synt.: die mutter (Subj.) l. (absolut); etw. (Akk.obj.: ein Tier, z. B. den wind, pferde) / jn. (z. B. die menschen, den bösen / nächsten / feind, das kind) l., jn. mit dienst, zu hofe l., die laus den betler, das tier die jungen $l$.

LUTHER, WA 12, 465, 34 (1523): nun fort soll ewer leben dabin gerichtet sein, das ir ander lewt lieb habt und dienet. EBD. 16, 494, 35 (1525): wenn zween einander lieb haben, acht sich ein yglicher nicht bóher denn der ander. EBD. 24, 409, 27 (1527): Naturlich ists, das ein vater sein kind, das weib den man lieb hat und frólich sey, wens yhm wol gehet. EвD. 33, 40, 20 (1530): Sie haben Gott lieb nicht anders denn, wie die Leuse den Betteler lieb haben. Quint, Eckharts Pred. 1, 79, 7 (E. 13./A. 14. Jh.): Du solt alle menschen dir gelich liebhaben und gelich achten. Tiemann, E. v. Nassau-S. Kgn. Sibille 127, 21 (rhfrk., um 1435): Der selbe Abrye von Mondidyre / der hat eynen wint erzogen den hat er gar liep. Bechstein, M. v. Beheim. Evang. Mt. 5, 43 (osächs., 1343): gesprochen ist: habe lîb [LuTHER 1545: lieben] dînen nêbstin und hazze dînen vîent. Abir ich sage ûch: habit lîb ûwere vîende. v. TsCHARnER, Md. Marco Polo 7, 25 (osächs., 2. H. 14. Jh.): do werdin si [pfert] vorkouft und sint lib gehat. v. KELLER, Ayrer. Dramen 3401, 6 (nobd., um 1600): Es wern Verdiende leudt darauß, | Die man Zu boff am liebsten hat. RuH, Bonaventura 357, 16 (orhein., um 1480): so mustu, durch betrachttung des bildes vnd glichniß der götlichen majestet in allen mönschen, sy also mit inniger begirde siner minsamkeit liep han vnd vnder in allen besunder die durfftigen vnd krancken mönschen sorg tragen und baben, [...], glich alß ein getruwe mütter liep hat vnd sorgt fir iren aller liebsten sin. GiLman, Agricola. Sprichw. 1, 72, 29 (Hagenau 1534): Wer sein kind lieb hat / der sparet der ruthen nicht. EBD. 491, 18: Si hatt yhn lieb / ja auff der seitten da die tasche hangt. EBD. 2, 207, 4 ([Augsb.] 1548): Ain frumb man hat ain andern lieb | So liebet ain dieb den andern dieb. WArNOcK, Pred. Paulis 6, 134 (önalem., 1490/4): Aristotiles sprach: ,Omne animal diligit etc., ain jegkelich ding, daz hát lieb sin gelich'. StrehlKe, Nic. Jerosch. Chron. 20400; Quint, a. a. O.
1, 37, 8; Feudel, Evangelistar 67, 7; Roloff, Brant. Tsp. 709; SAPpler, H. Kaufringer 24, 101. - Vgl. ferner s. v. angeboren.

3. 'jn. lieben, eine erotische, tendenziell: eine sexuelle Beziehung zu jm. haben ; vgl. lieb (Adj.) 6, lieben 9. - Wbg.: liebhabenlich $>$ liebreizend <, liebhabung 2.

Luther, WA 9, 542, 14 (1521): wenn es keme, das zoweie zсu eynander lib betten. Frantzen u.a., Kölner Schwankb. 3, 88b (mnl./Köln um 1490): eyne eerlike vrouwe is leefhebbelick, eerwerdich unde begherlyck. WYss, Limb. Chron. 26, 1 (mfrk., 3. Dr. 14. Jh., Hs. 17./ 18. Jh.): darnach hatte der selbe konig ander wibe liver dan si. VOC. INC. TEUT. p ir (Speyer um 1483/4): Liebhabũg Adamatio [...] Dilectio. Kopp, Volks- u. Gesellschaftsl. 82, 20 (Hs. 'pfälz., M. 16. Jh. ${ }^{7}$ ): mit der [schöne] du hast mich hart und vast $\mid$ lieb habenlich besessen. v. TSCHARNER, Md. Marco Polo 61, 18 (osächs., 2. H. 14. Jh.): so ist eczliche husvrowe di do spricht, si babe yn allir libist gehat, di legit sich williclichin cæu dem manne in das vugir. FASTNACHTSP. 876, 8 (Straßb. o. J.): Mich dunckt, es thet mir gnienen so wee, | Als sölt ich einen andern mann, | Dann dich, min Uoly, lieben lan. Bachmann, Morgant 150, 35 (halem., 1530): Sy fuortend guot läben mit ein andren als zwey lieb habende. FisCHER, Eunuchus d. Terenz 80, 19 (Ulm 1486): das drit haist suavium und ist der unkeüschen untzimlichen liebhabenden küssen. KLEIN, Oswald 69, 69 (oobd., 1417): got waiss wol, wie | ich dich lieb hab. DAsYPODIUS 300v. - Vgl. ferner s. v. ausbrechen 13.

4. >(Gott) lieben<, in spiritueller Einheit mit bören / sehen / anbeten / fürchten / erkennen / loben / erwirdigen / böhen / verzeiben, wolsprechen >alles Selbstbezügliche aufgeben sowie (vereinzelt:) >(Gott) in Erlösungssehnsucht achten, ehren, lobpreisen`; vgl. lieben 10. - Texte der Sinnwelt ,Religion / Didaxe'. - Bdv.: minnen. - Synt. (abs.): hitziglich / volkommenlich l.; got (meist) / Christum / Jesum, den schöpfer, bräutgeben, den götlichen namen l., got geistlich, in seinem mute l.; der got liebhabende mensch.

Luther, WA 8, 293, 24 (1521): alle, die Christum lieb haben. Helm, H. v. Hesler. Apok. 11130 (nrddt., 14. Jh.): Hete wir den Got nicht lieb | Der uns nach im gebildet hat. BöMER, Pilgerf. träum. Mönch 6016 (rhfrk., um 1405): Sij [sele] gesebe und erkente nach geschichte | Yren schepper und hette yn liep. WAGNer, Erk. Ps.-J. v. Kastl 16, 44 (nürnb., 1. H. 15. Jh.): wie wir c₹u im [got] gen und in im pleiben, nicht mit den fußtriten des leibs, sunder mit der begerung der sele, nicht leiplich geent, sunder geistlich liebhabent. Und darumb wer volkomlicher und bycziglicher lieb hat, der laufft sneller und kompt pelder. GILlE u. a., M. Beheim 75, 23 (nobd., 2. H. 15. Jh.): Got uns darumb schaffen pegund, 
| das wir in bie erkanten und | in lieb heten und lobten. Eichler, Ruusbr. steen 1198 (els., sp. 14. Jh.): der müß got also liep han, daz er mit frýgem mute vnd umb die ere gottes sich alles des verzihen mag, daz er vnordelichen vebet. Höver, Bonaventura. Itin. A, 157 (moobd., 2. H. 15. Jh.): das du in allen creaturen deinen got, sehest, börest, khennest, lobest, anpettest, lyebhabest, fürchtest vnd cayrest, grozmachest vnd auch erwirdigest vnd höchest. EBD. 557: dy [...] nicht wellent got in disen allen erkhennen, wolsprechben und liebhaben, das ,sy vnentschulldigt seinn', wenn si nicht wellend üwergefüert werden, von den vinnsternüssen in das ïberwunnderleich lyecht gotes'. HoHmanN, H. v. Langenstein. Quästio 185, 53 (moobd., 1. H. 15. Jh.): Wir wissen, das den got liebhabunden menschen allew ding gedeichen zu güet. Bauer, Imitatio Haller 43, 19 (tir., 1466): als er denn selbs spricht: ,Wer [getrene sel] mich [prentgebe] ist lieb haben, der haltet auch mein rëd. - LuTHER, WA 9, 602, 32; GILLE u. a., a. a. O. 71, 145; 125, 7; BAuer, a. a. O. 102, 21. - Vgl. ferner s. v. ${ }^{2}$ anliegen 2.

5. >(den / einen Menschen, auch: eine dem Menschen mögliche Qualität) lieben (von Gott als dem Liebenden gesagt)<; vgl. lieben 11.

Ziesemer, Proph. Cranc Jes. 61, 8 (preuß., M. 14. Jh.): ja byn ich eyn herre lyephabende [LuTHER 1545: lieben] daz. gerichte und hassende den roub zu opphir. Grosse, Schwabensp. 41a, 23 (Hs. ' $\mathrm{nd}$./md., um 1410?): wir sollen mit vrede vnde sine mid eyn ander lebon; wan werdichlich leben had vnser here vil liep. Roloff, Brant. Tsp. 144 (Straßb. 1554): Gott hat von ewigkeit mich [Tugent] eracht | Und bei im gehaben lieb und wert. WARNOCK, Pred. Paulis 6, 137 (önalem., 1490/4): welcher mensch, der tugentrichlich lept und gerecht ist, der gelichet sich gott nach sinem vermugen, und darumb so muss er von gott lieb gehept werden. EBD. 139: Der herr ist gerecht und hát lieb die gerechtikait; und sin antlút sicht die gelichait (zum spirituellen Hintergrund s. gleichbeit 7). Spechtler, Mönch v. Salzb. 1, 37 (oobd., 3. Dr. 14. Jh.): got hat dein [bezogen auf Maria] ainikhait | lib für gemainikhait. - WARNOCK, a. a. O. 6, 131.

liebhabenlich, s. liebhaben 3.

liebhaber, in 1 Beleg mit Uml.: -bäber, der; $-s /-\emptyset$.

1. >Person, die sich zu einer (meist positiv, seltener negativ bewerteten) Sache / Handlung / Bezugsgröße hingezogen fühlt, Liebhaber, Gönner, Freund von etw.<; auch: >Kenner e. S.<; die bevorzugten Zielbereiche des liebhabers sind moralische Werte, Tugenden, Wissenschaften, Künste (jeweils positiv), Gegenstände der Wissenschaft, Kenntnis (neutral), weltimmanente Haltungen und Ziele, Sünden; vgl. liebhaben
1. - Bdv.: freund, minner. - Synt.: den liebhabern erscheinen; der l. des friedens / geldes / gesanges / getrankes / lustes / (gemeinen) nutzes / rechtes / reiches / vaterlandes / wortes, der erbarkeit / gerechtigkeit / musik / muttersprache / poesie / vernunft / warbeit / weisheit / welt (mehrfach), der antiquitäten / bergwerke / eren / geschichten / (zeitlicher) güter / künste / kräuter, der neuen mären; der rechte l. Wbg.: liebhaberin 1.

LutHeR, WA 17, 1, 227, 24 (1525): Weil nun unser Kurfurrst / seliger ein sonderlicher Liebhaber des beiligen worts gewesen ist. Chron. Magdeb. 2, 136, 16 (nrddt., Hs. E. 16. Jh.): Herr Georgius Butze [...], welcher ein besonder Liebhaber des worts [...] was. SCHEIN, NA 2/1 11b, 12 (Leipz. 1627): auf vieler Cantoren freundliches Zuschrei-

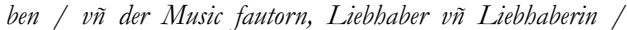
instendiges suchen. Wutke, Schles. Bergb., Cod. Sil. 21, 125 (schles., 1560): Der Fürst von Baiern auf Glatz sei bergverständig und ein Liebhaber der Bergwerke. CHRON. Nürnb. 3, 170, 7 (nobd., 1488): was Wenzeslaus gantz. ungeleich dem vater, Carolo, unertig, ein liebhaber des lusts und floch aller arbeit. WicKRAM 4, 12, 9 (Straßb. 1556): sie was ein weib der ehren ein liebhaberin. SAPPLER, H. Kaufringer 19, 32 (schwäb., Hs. 1472): o ir liebhaber der welt, | ir suocht das leben in dem lant, | das des todes ist genannt. Heydn. maister 28r, 14 (Augsb. 1490): fleißlich wachẽ die nature d' ding zuerjnnern die wir philosophos das ist liebhaber d' weißeit nennen. EBD. 19r, 22: wie sich einer verbuten solt $d z$ er nit würd ein liebhaber des getrãckes. RAUwOLF. Raiß 9, 14 ([Lauingen] 1582): mit Jacobo Renaudo, einem Hocherfarnen Mann / vnd liebhaber der Kreutter / welcher [...7. Höver, Bonaventura. Itin. A, 26 (moobd., 2. H. 15. Jh.): wir süllen auch sein lyebhawer der weishayt, dye vns rüefftt. Grossmann, Unrest. Öst. Chron. 216, 30 (oobd., 3. Dr. 15. Jh.): der [cardinal] was ein prechenhaffter herr, sunder hochgelert und liebhaber der gerechtikait. BAUER, Zist.-Pred. Haller 40, 59 (tir., 1466): Kchristus der hat nur alain erschinen [...] den gueten getreuen menschen vnd nicht den [...] liebhabern der welt. - Luther, WA 30, 3, 473, 35; Stoltzius, Chym. Lustg. Dedic. 3; Opitz. Poeterey 33, 2; 57, 23; Harms u. a., Alberus. Fabeln 31, 15; Vetter, Pred. Taulers 258, 20; Roloff, Brant. Tsp. Widm. 51; Memminger Chron. Beschr. 1, 3; 28, 6; VOC. INC. TEUT. p ir; MAaler 272r; Rwb 8, 1311. Vgl. ferner s. v. antiquität.

2. >Person, die eine andere aus sozialer oder christlicher Motivation heraus schätzt, ehrt, achtet, Begünstigter, Freund, Helfer, Unterstützer, Förderer; Günstling` (letzteres unter kritischem Aspekt); vgl. liebhaben 2. - Wbg.: liebhaberin 2.

Ziesemer, Proph. Cranc Jer. 30, 14 (preuß., M. 14. Jh.): alle dine [Jsrael, Juda] libhaber [Worms. Proph. 
1527: güten günner] habin din vorgezzen und suchin dich nicht. WARnOCK, Pred. Paulis 13, 8 (önalem., 1490/4): Johanes Pauli [...] ain rechter liebhaber únser selen, hát uns disse materi also gebredget. Chron. Augsi. 5, 31, 29 (schwäb., 1523/7): [hertzog Ulrich] behielt nun 1 edelman bei im, der hies Hans von Hutten, der was sein liebhaber ainer. EвD. 234, 16: doch so hett er [hertrog] ain liebhaber, der was ain Spaniol, [...], der regniert den hoff allen. Pfeiffer, K. v. Megenberg. B. d. Nat. 73, 17 (oobd., 1349/50): als lang wert diu lieb gegen got und gegen den menschen, als lang daz. wert daz man lieb hât, ez sei dann daz der liebhaber sein liep verlies. Nyberg, Birgittenkl. 2, 284, 11 (oobd., 1538/40): ein guter prediger vnd groser liebhaber vnd wolthatter des ordens. BAuER, Haller. Hieronymus-Br. 8, 25 (tir., 1464): Er [Jeronimus] ist ain liebhaber der prüeder.

3. >liebende männliche Person, Liebhaber, Verlobter<; vereinzelt mit positiver, mehrfach mit neutraler, oft mit negativer Konnotation; vgl. liebhaben 3. - Bdv.: buler; vgl. amant, amase, amoureux, beigenosse, beischläfer, bule 1, buler, heirat (der) 4, ${ }^{2}$ leffel, lescheur. - Synt.: etw. (kleine afterbelle) einen l. bedeuten; der l. blind, mit fleischlicher l. befangen sein, die länge der zeit nicht vertragen; ein l. werden; dem l. behagen / genahen; mit einem l. handeln; der l. der frauen / buren / weiber; der arme / heftige l.; die gegenwärtigkeit des liebhabers; das jungfräulein ane l. Wbg.: liebhaberfasnacht, liebhaberin 3 (dazu bdv.: vgl. beschliesserin, betgespiele, bule 2).

Ingen, Zesen. Ros. 72, 13 (Hamb. 1646): daß ich eher ein Libhaber ward / eh ich verstund / was Libe war. ÖsTERLEY, Kirchhof. Wendunmuth 2, 496, 20 (Frankf. 1602): alle die jenigen, so sie [jungfrau] in gegenwertigkeit ibres liebhabers gesehen. Neumann, Rothe. Keuschh. 1082 (thür., 1. H. 15. Jh.): di liebheber sint alle blint $\mid$ di mit fleislicher liebe befangen sint. EBD. 4566: mit der hoffard, | das si sich uss smocken zard $\mid$ unnd iren liebheber behage. FASTNACHTSP. 632, 1 (nürnb., 15. Jh.): DI GROSZ LIEBHABERVASNACHT [es folgt das Fastnachtspiel]. KurreLMEYER, Dt. Bibel 10, 5, 3 (Straßb. 1466): Ich genachen meinen liebhabern [Luther 1545, Hos. 2, 5: bulen] die mir gebent mein brot. BaCHMANN, Haimonsk. 10, 1 (halem., 1530): des vyl frowen umm ire man kămmend [...] und sovyl junckfröwlin on liebhaber. MAALER 272r (Zürich 1561): Ein háfftiger Liebhaber der weyberen. BARACK, Zim. Chron. 4, 188, 14 (schwäb., M. 16. Jh.): der het sich in abwesen des bischofs zu seiner beschliesere oder liebhabere gelegt und het ir ein kindt bevolchen. KARNEIN, de amore dt. 185, 9 (moobd., v. 1440): Wär aber ain weib so geittig, das sy ir lieb und mynn durch gellt oder guet geben wolt, daz. wär nit ain rechte liebhaberjnn. Pfeiffer, K. v. Megenberg. B. d. Nat. 49, 6 (oobd., 1349/50): wem der afterpell pain klain sint, daz bedäut ainen liebhaber der frawen. - INGEN, a. a. O. 70, 25; FrANCK, Decl. 334, 29; DAsYPODIUS $303 r$.

4. >den christlichen Gott (meist: Christus) Liebender, Verehrender`; vgl. liebhaben 4. - Texte der Sinnwelt ,Religion / Didaxe'. - Wbg.: liebhaberin 4.

Luther, WA 41, 134, 19 (1534): das sie [Kliglinge] unter dem schein [...] des Euangelij und Christi freunde und liebhaber konnen Christum und seine Christen verfolgen. GERHARDT, Meister v. Prag 65, 15 (Hs. ' nobd., 1477 ) : der gotes liebhaber ist und seinen willen tut den erbort er. GILLE u. a., M. Beheim 125, 70 (nobd., 2. H. 15. Jh.): wir sollen wellen, das all dy $\mid$ menschen mit uns auff erden bie $\mid$ seien gotes liebhaber. MAYER, Folz. Meisterl. 11, 109 (nobd., v. 1496): Der heilig geist: , die freye | Hab ich gesucht und ir zu kunfft | Mir außervelet gare $\mid Z u$ eyner liphabrine $\mid G e^{-}$ spons, praut und gemael mir. Warnock, Pred. Paulis 3, 212 (önalem., 1490/4): wer es niemant gesin denn der minrich liebhaber des gecrútzgoten Jhesu, der engelschlich doctor Sant Bonaventura, [...], wir söltind [...]. BAUER, Zist.-Pred. Haller 93, 231 (tir., 1466): die frend Kchristi, sÿ seÿ würchent oder leidleichen [...] oder si së̈ des liebhabenten menschen oder des liebhabers. - LuTHER, WA 54, 262, 14; WARNOCK, a. a. O. 3, 283; BAuer, Imitatio Haller 45, 23; 91, 24; Dies., Zist.-Pred. Haller 85, 126; Sснмітт, Ordo rerum $125,3$.

5. > Gott in seiner Seinsqualität als Liebender des Menschen und aller Werte‘; im Beleg KurRelmeyer: > eifernder Gott (des Alten Testamentes)<; vgl. liebhaben 5. Texte der Sinnwelt ,Religion / Didaxe'.

Jungbluth, J. v. Saaz. Ackermann 34, 27 (Hs. romd., $\left.1465^{\urcorner}\right)$: aller reinigkeit liebhaber, hasser aller unfletigkeit; [...]; einiger, aus des anfang alle sach ewiglich nimmer weichen mag; alte warheit - erhöre mich! MAYER, Folz. Meisterl. 11, 59 (nobd., v. 1496): Hie gibet zu verstente | Got vater wie | Sie sey die unzuprochen | Und keusch irem liphaber rein | Sich ewig hat gezweiget. KurrelmeYer, Dt. Bibel 3, 286, 11 Var. (Straßb. 1466): Wann ich bins der herr dein got starker recher (Var. 1475²-1518: ein ewferender liebhaber): heimsüchent die vngankeit der vetter in die sune. WARNOCK, Pred. Paulis 11, 19 f. (önalem., 1490/4): Der vil sälig [...] Sanctus Johannes hát sich selber [...] verainget in die liebe sins liebhabers [lat.: dilectoris] Jhesu. SPECHTLER, Mönch v. Salzb. 13, 1 (oobd., 3. Dr. 14. Jh.): Des menschen liebhaber $\mid$ sand zu der maide ber $|[\ldots]|$ nur ainen engel klar.

liebhaberfasnacht, s. liebhaber 3.

liebhaberin $1 ; 2 ; 3 ; 4$, s. liebhaber $1 ; 2$; $3 ; 4$.

\section{liebhabung, die.}

1.; 2., s. liebhaben 1; 3. 
3. >das Lieben, die caritative, brüderliche, fürsorgliche Liebe zu e. P.^; vgl. liebe 5, liebhaben 2.

Kurrelmeyer, Dt. Bibel 1, 427, 7 (Straßb. 1466): wie christus dy acht seligkeyt erzelet. Vnd darnach vil schóner lere. gepot vnd verbot tett. [...]. vom ayd. von gedult. vnd liebhabung der veynd. Roder, Hugs Vill. Chron. 117, 34 (önalem., 1525): Wo ir das thind, beschiecht daran der will gottes in berfillung sines gebotts von briderlicher liebhabing. Höver, Bonaventura. Itin. B, 415 (moobd., 1450/60): ob du gesechen macht des begynne liepleich liebhabung mit begnadender liebe.

4. >das Lieben, die Liebe des Menschen zu Gott<; vgl. liebhaben 4. - Bdv.: vgl. geminne, gierde 3.

Gille u. a., M. Beheim 125, 82 (nobd., 2. H. 15. Jh.): han ich mich pedabt diser liet, | etwaz auss czu erreüten | Und mit getibten czu erklern | von der liephabung unsers hern. EBD. 138, 49: dy selb liebhabung und | der gut will allermerste, | Den wir haben czu got dem frann. EвD. 142, 98: so werden fürbaz mere |All seine [des menschen] werk, mit aine | auss der liebhabung gacz gesacht. Hohmann, H. v. Langenstein. Untersch. 117, 17 (moobd., 1. H. 15. Jh.): Noch es ist sich ze wundern, wenn, sam Reichardus spricht, „an allen zweifel, chain ding gevelt got als, sam die liebhabung der sel". - Vgl. ferner s. v. behalten 4, inbrunst 2.

liebhalb, s. liebe 9.

\section{liebhart, der.}

>Vertrauter, Nahestehender; Liebling; Günstling ; vgl. lieb (Adj.) 2; 3. - Bdv.: vgl. bule 3, confident, ${ }^{2}$ gespan, geteling, günstling, liebhaber 2. - Synt.: den l. zälen >verschonen</ benken; j. js. (auch: gottes) l. sein; seinem l. etw. (z. B. ein schlos) geben; der l. des gubernators/ königs. Wbg.: liebhartin.

Tobler, Schilling. Bern. Chron. 2, 75 Anm. (whalem., 1484): Die herzogin mocht nit sovil an im haben, wie wol si sin liephartin miteinandern gewesen was. V. ANSHELM. Berner Chron. 2, 268, 27 (halem., n. 1529): das ungwinglich gacht schloss zu Meyland, [...] gab er in sinem liebhart, Bernhardin de Curt, welchen er uss nut vil gemacht hat. - EBD. 1, 245, 7; SCHмidt, Hist. Wb. Elsaß 223; Schwerz. ID. 2, 1644; 3, 991

liebhartin, s. liebhart.

liebheit, s. liebe 6.

\section{liebkallen, V.}

>schönreden, schmeicheln, schleimen «. Bdv.: vgl. adulieren, aufheben 33, bestreicheln, blandieren, glattieren, bälen, heucheln, klemenzen
2, klünzeln, liebern, liebkosen 2, liebreden. Wbg.: liebkaller (dazu bdv.: hoflecker, schlauch, zutütler).

SACHs 20, 12, 21 (Nürnb. 1564): Also kan ich in auch in allen|Auß falschem bertzen süß liebkallen. ÖsterLey, Steinhöwels Äsop 78, 26 (Ulm 1474/82): Er lert die nidern demütikait, sich allweg vor schmaichern und liebkallern büten. Fischer, Eunuchus d. Terenz 45, 6 (Ulm 1486): diser scen wirt Gnato in getzoggen der ain zütutler was oder liebkaller. EBD. 45, 17: Diser lieb kallender sitten die nun [...] in allem stät gaistlich und weltlich den fürgang hond. EBD. 86, 2.

liebkaller, s. liebkallen.

liebkind, s. liebe 7.

liebkosel, liebköseln, s. liebkosen 2.

\section{liebkosen, V.}

1. >jm. / e. S. liebevoll, freundlich entgegentreten, liebevoll mit jm. umgehen, zu jm. sprechen`, auch: ‘jm. schmeicheln«. Meist Texte der Sinnwelt ,Religion / Didaxe'. - Wbg.: liebkosung 1 >das Preisen, Lob<.

Ziesemer, Proph. Cranc Jes. 66, 12 (preuß., M. 14. Jh.): uf den knien werden sy uch lyebekosen [Worms. Proph.: kurtzweilen; Froschauer 1530: werdend erfrówet; Eск 1537: schmaichlen; LUTHER 1545: freundlich halten] rechte als eine mutir lyebekoset irem kynde. EBD. Jer. 5 , 31: dy propheten haben gewyssaget logene und di pristir haben gelibekoset [ECK 1537: klopften; LuTHER 1545: berrschen] dar₹u mit iren benden. LUTHER, WA 21, 527, 8 (1544): das er sich solche gute meinung kutzeln liesse, jm widerumb zu beucheln und liebkosen. Quint, Eckharts Pred. 34, 7 (E. 13./A. 14. Jh.): alsus liepkôset uns got, alsus vlêhet uns got, und got enmac nibt erbeiten, biz sich diu sêle gesmucket und geschelet von der crêatûre. v. Tscharner, Md. Marco Polo 34, 22 (osächs., 2. H. 14. Jh.): Des suchin yn [man] sine mok unde vrunde glich eyner di do lyt in deme kindbette, unde libekosin mit ym unde vroygin sich mit ym. JunGBLUTH, J. v. Saaz. Ackermann 26, 9 (Hs. 'omd., 1465ㄱ): Rhetorica, blüender grunt der liebkosung, hilfet da nicht mit iren blüenden und wol geferbten reden. GILLE u. a., M. Beheim 15 Überschrift (nobd., 2. H. 15. Jh.): von der lieb kosung Sand Augustin (es folgt ein Gotteslob). Kurrelmeyer, Dt. Bibel 3, 157, 20 (Straßb. 1466): sein sele was zugehaft mit ir: vnd er trost die trawrigen lieb kosende [LuTHER 1545, 1. Mose 34, 3: redete freundlich mit jr.

2. >jm. / sich selbst schmeicheln, schönreden, schöntun, sich schönrednerisch bei jm. einschmeicheln, jm. nach dem Mund reden, sich anbiedern; mit betrügerischer Absicht seinen Vorteil suchen 
ane liebkosen >ohne zu schmeicheln`. - Gewisse Beleghäufung für Texte der Sinnwelt ,Religion / Didaxe'. - Bdv. (und Orientierungsfeld): s. u. SCHÖPPER, ferner: bescheissen 3, gleichsen 1, bändschmieren, binterreden, hofieren, kleispern, listen, triegen, zussern, fuchsschwänze verkaufen, (jm.) den fuchsschwanz streichen, die federn abklauben, spek durchs maul ziehen, das maul schmieren; vgl. liebkallen. Ggs.: droben. - Synt.: etw. (Subj., z. B. der geiz, die zunge) l.; e. P. (z. B. dem babst, sich / im selbst) / e.S. (z. B. dem adel / volk, der welt) l.; mit jm. l. (z. B. mit herren / frauen), subst.: $j$ s. liebkosen verbüten, mit liebkosen reden, etw. ane liebkosen ausfüren / beschreiben, jn. durch liebkosen [wo] einflicken, jn. mit liebkosen von etw. weisen; das falsche liebkosen. Wbg.: liebkosel, liebköseln.

SchÖpper 61a (Dortm. 1550): Adulari. Schmeicheln liebkosen obrenkitzeln zu dutteln schmieren augendienen liebtraben küntalen obrenkrawen federklauben streicheln I zártlen lieblen beyen tantzlen auffheben 9 beucheln gleißnen. JuNGBLuth, J. v. Saaz. Ackermann 28, 15 (Hs. 'omd., $\left.1465^{\urcorner}\right)$: Triegen, listen, smeichen, spinnen, liebkosen, widerburren, lachen, weinen kan sie [weib] wol in einem augenblick; angeboren ist es sie. EвD. 29, 22: On liebkosen mit kurzer rede: aller werlt aufhaltung, [...], sint die werden frauen! WAGNER, Erk. Ps.-J. v. Kastl 2, 26 (nürnb., 1. H. 15. Jh.): wenn wir uns also inwendig in unser aigen gewissen bekennen, wir wollen uns dann selbs liebkoßen. FISCHER, Folz. Reimp. 25, 151 (Nürnb. 1479): [Geicz] Hendschmirt, libkost, dreugt arm und reich, | Pleibt bie ungesettet ewicleich. FrancK, Decl. 336, 16 (Nürnb. 1531): das wir das zussern / kleispern / liebkosen und schmaichlend federklauben der schnóden hüren verbuitten. BIHLMEYER, Seuse 364, 28 (alem., 14. Jh.): des [gottes] minneklich zúrnen besser ist, denn falsches liepkosen. RuH, Bonaventura 358, 6 (orhein., um 1480): daz. du niemer weder in wortten, wercken oder wissen etwaz vnwillens, [...] noch figenlichen gebrechttes, [...], binderredens, ergerniß, liebkossen [...] durch dich andern ein vrsach sigest. Moscherosch. Ges. Phil. v. Sittew. 9, 22 (Straßb. 1650): Es ist heucheley, es ist schmeichelei, Liebkosen, heimliche Boßheit. EBD. 44, 22: bei den geringsten Handwercken, die alle sich in ibrem Wesen selbst schmeichlen vnd Liebkosen. MAALER 272r (Zürich 1561): Liebkosen / Eigentlich wie die hund mitt dem schwantz. Adulari. Zäntzlen. [...]. Dem volck Liebkosen / zántzlen / vnd spáckle durchs maul ziehen. [...7. Sich durch Liebkosen in einsi gmeinschafft eynflicken. EвD.: Das Liebkóßlen. Assentatiuncula. Dict. Germ.-Gall.-Lat. 306, 37 (Genf 1636): Liebkosen / denn Fuchßschwantz. einem streichen / Schmeichlen. EBD. 41: Das (liebkosen) n. Fuchßschwántzerey. EBD. 307, 1: Liebkosel / Flattner / Fuchßschwaintzer. - PFefFerL,
Weigel. Gn. S. 134, 5; Kehrein, Kath. Gesangb. 3, 203, 21; Wagner, a. a. O. 10, 39; Bihlmeyer, a. a. O. 461, 11; Ruh, a. a. O. 354, 30, Pfeiffer-Belli, Murner. Kl. Schrr. 7, 9, 6; 9, 68, 32; Roloff, Brant. Tsp. 457; Adrian, Saelden Hort 2536; Chron. Augsb. 9, 42, 7; 84, 29; SpIller, Füetrer. Bay. Chron. 214, 16; Schmitt, Ordo rerum 687, 12; SchwartzenBach 74v; Ulner 253. - Vgl. ferner s. v. beissen 7.

liebkoser, der.

`Schönredner, Schmeichler, Person, die jm. zum eigenen Vorteil nach dem Munde redet und entsprechend handelt «; zu liebkosen 2. - Bdv. s. u. Schöpper und Rot; vgl. assenzial. - Wbg.: liebkoserin.

SCHÖPpER 60b (Dortm. 1550): Adulator. Schmeichler liebkoser obrenkitzler zu duttler suppen fresser obrenmelcker obrenkräwer wortschleiffer zungenträscher athem verkeuffer federklauber jaherr maulberer hollwanger zungentrager kautzenstreicher tellerschlecker bauchdiener fuchsschwäntzer augendiener. bypocrit heuchler gleißner. ToEPPEN, Ständetage Preußen 5, 740, 30 (preuß., 1523): Wie manigfeltige gewaltige eingriffe den steten und gerichten wider alte herkomende gewonbeit irer privilegien und gerechtigkeitten durch anleytung werntlicher rete und liebkosern geschen. Gille u. a., M. Beheim 443, 190 (nobd., 2. H. 15. Jh.): but dich vor den lieb kasern, | den man zu haf gar wal gehorcht. Hulsius P ijv (Nürnb. 1596): Schmeylerin / liebkoserin. BaumanN, Bauernkr. Oberschw. 61, 4 (schwäb., v. 1542): möchtest ach gedencken, ich were ain henselin, ain suppenesser oder liebkoser. Roт 287 (Augsb. 1571): Adulator, zuschmeichler / liebkoser / obrnplaser / federklauber / bellstreicher / suppenfresser / Gnaden diener. Turmair 4, 433, 18 (moobd., 1522/33): Es ist gar kain belonung der êrberkait [...], nur aigennützig geltnarren und liebkoser sein überal die pesten. Voc. inc. Teut. p ir; Dasypodius $117 \mathrm{v}$; Hulsius A ivv.

liebkoserin, s. liebkoser.

liebkosung, die; $-\emptyset /-e n$.

1., s. liebkosen 1.

2. >Schmeichelei, eitle Selbstdarstellung, schönrednerische Selbsterhebung; Reden mit betrügerischer Absicht`; als Metonymie: >Bestechung, Bestechungsgeschenk «; $\mathrm{zu}$ liebkosen 2. - Bdv.: vgl. augendienst, heuchelei 1, gleichsnerei, heuchlerei, pfauenstrich; zur Metonymie: vgl. liebnis 3.

Wagner, Erk. Ps.-J. v. Kastl 12, 44 (nürnb., 1. H. 15. Jh.): Wann die werlt treibt und jagt den menschen c₹u [...] ergeytigen, gluckpern dingen als reichtum, er, liebkoßßung, aygene lieb sein. MAYer, Folz. Meisterl. 9, 50 (nobd., v. 1496): Der das gespey | Der libkosung | Und spotters zung 
| Ym so lest pringen pey. Kurrelmeyer, Dt. Bibel 8, 77, 19 (Straßb. 1466): darnach vint er gnad bei im. merr denn der do betreugt durch die liebkosung der zungen [LUTHER 1545, Spr. 28, 23: der da heuchelt]. MaAler 272r (Zürich 1561): Liebkosung. Assentatio, Adulatio, Blandimentum. Vngeschickte Liebkosungen oder schmeichlungen / die weder form nach gestalt habend. Rотн, E. v. Wildenberg 15, 33 (moobd., v. 1493): do er [Julius Cesar] kam in Allexandria, schickt im der konig von Egipten entgegen zu liebkosung das haubt Pompeius und seinen harnasch. - DAsypodius 19 r.

lieblächeln, lieblellen, liebleller, s. lieb (Adj.) 1.

\section{lieblich, Adj.}

1. >liebevoll, freundlich; jm. Liebe, Freundlichkeit, Zuneigung spendend und dadurch Behagen, Trost gewährleistend`; das gesamte Spektrum von Liebe als der Existenzform Gottes (und diese auch in weltlichen Kontexten mitmeinend) bis hin zu erotischen, sozialen, rechtlichen Haltungen und Handlungen des Menschen umfassend; vereinzelt mit Tendenz $\mathrm{zu}>$ falsch, Freundlichkeit vortäuschend, schmeichlerisch gebraucht; vgl. lieb (Adj.) 1; 2; 6. Gehäuft Texte der Sinnwelt ,Religion / Didaxe'. - Bdv.: brüderlich, freundlich (mehrfach), gespräche, gnadenreich, grüsbar, gütig 2; 4, holdselig, leutselig, minniglich, sanftmütig, schmeichlich, süs, tröstlich, wollüstig. Ggs.: freislich, neidig. - Synt.: j. (z. B. Jesus) / etw. (z. B. die beiwonung) l. sein; sich l. erzeigen, etw. (z. B. den wein) l. ansehen / geren, etw. l. tun, den gottesschein l. beschliessen, jn. l. aufnemen / anlachen / empfangen / eriiehen / küssen / minnen / umfangen, von im > sich lassen, jm. l. zusprechen, den mund darbieten, l. bei jm. sitzen, mit $j m$. kosen / umgehen, miteinander vereinbart / verteidigt / vereint sein, miteinander leben, etw. l. mit jm. teilen, sich l. mit jm. vereinen; der liebliche blik / beiland / klos (ütr. für >Mensch`) / verkündiger, die liebliche geselschaft / predigt / sonne / vereinung, das liebliche angesicht, liebliche gebärden / worte. Wbg.: liebliche (bdv.: leutseligkeit, lieblichkeit 1, minne).

SCHÖPPER 11b (Dortm. 1550): Comis. Lieblich freundtlich grúsbar gespräch lentselig gútig. LUTHER, WA 1, 275, 16 (1518): wie guttlich und lieblich Christus mit den leuten gehan- delt hat. EBD. 48, 135, 6 (1546): wo fur man yhnen halten sol, nemlich nicht fur einen Richter [... Sondern fur einen lieblichen Heiland und trostli(ch)en freund. EBD. Bibel 7, 32, 20 Marg. (1522): Guttickeyt ist die liebliche geselschafft vnternander vnd freuntlichs wesen. Grosse, Schwabensp. 153a, 39 (Hs. ' nd./md., um 1410?): wir heyzen ouch daz mordere, swer mit deme anderen izzet unde trinket vnde liepliche in grozet vnde sleit on ane sine schuilde. STACKMANN u. a., Frauenlob 6, 10, 2 (Hs. 'md. auf nd. Grundl., v. M. 14. Jh. $\left.{ }^{\urcorner}\right)$: ich wunschte, swa zwei lieblich gern | der minnen stern, $\mid$ ir beider berze offen wern. JUNGBLUTH, J. v. Saaz. Ackermann 9, 10 (Hs. 'omd., 1465?): Wie liebe sach ich mir, wann sie so züchtigliches ganges pflag und alle ere bedenken kunde, und sie menschliches geslecbte da lieblich schaute. EвD. 25, 40: der mensche ist [...] allein der lieblich kloß, dem geleich niemant dann got gewürken kan. PALM, Veter Buoch 20, 9 (schles., Hs. E. 14./A. 15. Jh.): Der appet enpfienc sie gutlichen vnd liez sie liplichen von im. FRANCK, Decl. 345, 25 (Nürnb. 1531): seiteinmal er [der truncken] den wein mebr dann goldt acht / ya den lieblicher dan die Sonne ansibet. Reichmann, Dietrich. Schrr. 132, 35 (Nürnb. 1548): So wirdt ewer beywonung freuntlich vnd lieblich sein. VetTer, Pred. Taulers 100, 17 (els., E. 14. Jh.): Der nücbterliche mensche der tüt sin werg lieplichen. EBD. 372, 28 (1359): als die lipliche sunne hat ein stetes unzellich wurken und influs in das ertrich. BinlmeYer, Seuse 14, 30 (alem., 14. Jh.): Wannen kumpt ellú zartheit, schonheit, herzlust und lieplichi? DAsYPODIUs 373r (Straßb. 1536): Lieblich / schmeichlich. KoppITz, Trojanerkr. 15623 (Hs. ' noschweiz., 15. Jh. `): Lipplichen er sy dicke | Mitt mangem senften blike | Herlichen anne sach. STAMmLER, Berner Weltger. 461 (ohalem., 1465): Denn wil min uatter tusent stund | Lieplich kiüssen an sinen mund. UB ZUG 2434, 9 (halem., 1399): Hand sie vor mihr geoffnet, daß sie do lieplich und eigenlich mit einanderen vereinbert und bericht sind, das [...]. BRAndstetter, Wigoleis 228, 7 (Augsb. 1493): die mynnigklich larie dancket jm gar züchtigklich mit lieplichen worten. Spechtler, Mönch v. Salzb. 6, 11 (oobd., 3. Dr. 14. Jh.): du [gotes porte] bracht uns den waren gotesschein, | den hat dein käwscher leib | liebleich beslossen. Baptist-Hlawatsch, U. v. Pottenst. Vorr. 85 (moobd., A. 15. Jh.): daz sie daz icht vnwirdichleich und neydichleich peyssen und fraisleich reissen, sunder liepleich ond prüderleich straffen. Wolf, Norm im sp. Ma. 58, 70 (oobd., 1486): dy ministri sullen sy liebplich auf nemen vnd guetigklich. BAuER, Zist.-Pred. Haller 83, 86 (tir., 1466): O Jesu [...], du pist aller suess, $d u$ pist aller liepleichen, $d u$ pist gancz. und gar wolustig allen den, die dich da lieb haben. - Luther, WA 10, 3, 239, 3; 244, 2; 48, 205, 5; Helm, H. v. Hesler. Apok. 18486; Quint, Eckharts Pred. 2, 387, 43 f.; Buch Weinsb. 1, 97, 29; Wunderlich, Fierrabr. 125, 16; Froning, Alsf. Passionssp. 6422; Mone, Adt. Schausp. 1, 2317; Pyritz, Minneburg 4037; Reichmann, a. a. O. 251, 22; Roloff, Brant. Tsp. 1923; V. Anshelm. Berner Chron. 5, 222, 28 ; Wyss, Luz. Ostersp. 2876; SAPpler, H. Kaufringer 6, 195; 12, 224; 15, 33; 95; Chron. Augsb. 1, 64, 8; 
Klein, Oswald 12, 83; 114, 11; Uhlirz, Qu. Wien 2, 3, 4010, 9; Kummer, Erlauer Sp. 3, 1046. - Vgl. ferner s. v. angesicht 6, anheben 4, anlachen 1, arbeit 3, auftun 4 .

2. >beliebt<.

Chron. Köln 2, 581, 18 (Köln 1499): do versoinden si sich ind maechten sich overmitz ir gelt so liefelich, dat si der buschof wederumb zo sime hove lies komen.

3. >den Sinnen, dem Empfinden, dem Urteil des Menschen angenehm wahrzunehmen, als angenehm aufzufassen, zu beurteilen ‘; im einzelnen: a) >dem Auge angenehm wahrzunehmen, schön «. - Bdv.: angenem 2, annemlich 2, gleissecht, lustlich, schön, wunderlich. Ggs.: bäslich 1. - Synt.: der liebliche augenblik / kupferstich / ort, das liebliche angesicht / knäblein / silbergeschir, die liebliche gestalt / sonne / stätte / ursacherin; von ansehen l. - b) > angenehm zu hören, zu vernehmen, weich, wohlklingend. - Bdv.: angenem 2, hel 2, rein, sanft. - Synt.: die stimme l. werden; etw. (z. B. bucbstaben) l. lauten / singen (mehrfach); der liebliche gesang (mehrfach), die liebliche aussprechung / melodie, das liebliche deutsch / gerausche. - c) >angenehm im Geruch oder Geschmack, wohlschmeckend, wohlriechend, gut, fein «. - Bdv.: anmütig 1, süs, wolgeschmak. Ggs.: bitter 1. - Synt.: l. schmecken / riechen; der wein, das kraut l. schmecken; obs, l. zu essen, wein, l. zu trinken, keäuter, l. zur speise; der liebliche geruch / geschmak, wein. - d) >angenehm zu spüren, lau (vom Wind), warm (vom Sommer, von der Sonne); zart (von der Berührung) ( jeweils auf den Tastsinn beziehbar). - e) >als tröstlich empfunden (hinsichlich Gottes); als unschätzbar zu betrachten (vom Frieden); spannend (von Geschichten)<. - Bdv.: anmütig 1, belustigend; lustig; bübsch, schön. - Synt.: l. ₹u lesen; das liebliche gut / befinden (gottes), die liebliche behausung.

$\mathrm{Zu}$ a): Kehrein, Kath. Gesangb. 3, 49, 1 (Köln 1583): vns ist geboren heut, $\mid$ [... | Ein Kindlein so wuderlich, | Von ansebn sebr liebelich, | Jn seiner Menschbeide. HoffMEISTer, Kuffstein. Gef. A viijv, 2 (Leipzig 1625): grosse Straff und schmertzen / wegen dessen schönen und lieblichen Vrsacherin / [...] gedultig zu leiden. - Dünnhaupt, Werder. Gottfr. v. Bullj. 3, 16; v. Keller, Amadis 378, 10; Ders., Ayrer. Dramen 1313, 25; Thür. Chron. 6r, 12; Sermon Thauleri 11ra, 31; Gille u.a., M. Be- heim 70, 201; Rupprich, Dürer 2, 409, 35; EICHLER, Ruusbr. obd. Brul. 2, 480; Bihlmeyer, Seuse 103, 18. - Vgl. ferner s. v. ${ }^{1}$ abkeren 5, annemlich 2. - Zu b): Kohler, Ickelsamer. Gram. 30, 31 (wohl rAugsb. 1. Dr. 16. Jh. $\left.{ }^{\urcorner}\right)$: Buchstaben die [...] nit ₹u hart, sonder feyn lieblich vnd senfft lauten. Lemmer, Amman/Sachs. Ständeb. 108, 2 (Frankf./M. 1568): Mit gar lieblicher Melodey | So pfeiffen wir bie alle drey. OpITz. Poeterey 29, 3 (Breslau 1624): Der klare brunnen quilt mitt lieblichem gerausche. Goldammer, Paracelsus 5, 179, 11 (1530): spotweis redt David zu den abtgottern, die auf erden so lieblich gesungen haben. - Opitz. a. a. O. 31, 24; Gille u. a., M. Beheim 161, 427; zu Dohna u.a., Staupitz/Scheurl 44; Trunz, Meyfart. Rhet. 59, 7; Schorer, Sprachposaun 51, 13. - Zu c): PeIL, Rollenhagen. Froschm. 139, 2978 (Magdeb. 1608): Welchs Kreutlin jr so lieblich schmecket / | Das sie für tod ernider lag. MAALER 272v (Zürich 1561): Lieblichen schmócken vnd riechen / Ein lieblichen vnnd angenámen geschmack gáben. LAUATER. Gespånste 18v, 24 (Zürich 1578): einer der das feber hat / trinckt ein lieblichẽ suissen wyn / der bedunckt jn bitterer dañ ein gall. - Ralegh. America 22, 44; Haszler, Kiechels Reisen 324, 19; 330, 15; Dict. Germ.-Gall.-Lat. 307, 11. Vgl. ferner s. v. angelica, immenkraut. - Zu d): SCHÖPPER 75b (Dortm. 1550): Aura. Sanffte lufft lieblicher lustiger suisser windt. LUTHER, WA 4, 656, 13 (1517?): und scheint die Sonne lieblich. MAALER 272v (Zürich 1561): Lieblich / tugendtlich anrüren / streichlê. [...7. Lieblicher oder angenámer summer. - Zu e): Pfeffere, Weigel. Ges. 39, 1 (Hamb. 1646): daher wust Adam von keinem geseze, [...], vnd war nichts des Menschen, alß das lieblichste süße befinden, des einwonenden Gottes, aus Gnade, Friede, Ruhe, freüde, volle genüge in seinem Herzen. Logau. Abdank. 167, 6 (Liegnitz 1651): Der Zeitliche FRJEDE / ist ein liebliches und erfreuliches Gutt. MaAler 272v (Zürich 1561): Liebliche lustige erzellung. [...]. Liebliche schrifften oder úbungen / die einen vast belustigend. HeNisch 183 (Augsb. 1616): Kinder sind das lieblichste pfand vnd band der ehe. - v. Keller, Ayrer. Dramen 6, 4. - Vgl. ferner s. v. aufwachsen 1.

liebliche, s. lieblich 1.

lieblichkeit, der; $-\emptyset /-$.

1. >Liebe, Freundlichkeit, Zuneigung (in der unter lieblich 1 angegebenen semantischen Weite); Liebesbezeugung`; zu lieblich 1. - Bdv.: s. u. SCHÖPPER, ferner: liebe $1 ; 4$; sänfte.

SCHÖPPER 11b (Dortm. 1550): COMITAS. Lieblichkeit freundtlichkeit leutseligkeit guitigkeit. INGEN, Zesen. Ros. 82, 20 (Hamb. 1646): Du hast zu allerehrst den Adel der liebe verunehret / $d u$ hast seine fróliche liebligkeiten versáuret. EBD. 116, 4: Ich [...] wundsche / daß [...] die zubereitung unserer liebligkeiten nicht so harte schmerzen sein mógen. BöMER, Pilgerf. träum. Mönch 2277 (rhfrk., um 1405): Dann liebe heldet die in lieplicheit $\mid$ Die die ander halden in 
snodikeit. Thiele, Minner. II, 12, 173 (Hs. 'ndalem./ sfrk., 1470/907): din liplichkeit uff allen orten secze, | das er in keyner wise | dins willens unnd dins gebets ye icht verlecze. EвD. 30, 263 (Hs. 'md./rhein., 1. V. 15. Jh. ${ }^{7}$ ): want scamde und sceimde hant dicke geleynt | tuischen leiven leiflicheit. MAaler 230v (Zürich 1561): Holdsáligkeit / lieblichkeit / und gnadreiche zereden. - KeHrein, Kath. Gesangb. 1, 311, 37; MAALER 272v.

2. >angenehmes Aussehen; angenehmer Geschmack; angenehme Lautung (z. B. der Sprache)<; vgl. lieblich 2.

Ingen, Zesen. Ros. 88, 24 (Hamb. 1646): wie sehr mißbrauchet doch ein woblgestaltes gesichte seiner liebligkeit. PEREZ, Dietzin 1 41, 5 (Frankf. 1626): Die Bienen oder Jmmen locken den Menschen mit der süß-vnd lieblichkeit deß Honigs an sich. EBD. 321, 29: jüngling / der solchen Wollust vnd Lieblichkeit des Weins nicht geachtet. TRunz, Meyfart. Rhet. 1, 63, 13 (Coburg 1634): Weil jhm [Redner] obliget erstlich zubeweisen [...]: Zum andern zubelustigen / und das ist die suisse Liebligkeit. KoHLER, Ickelsamer. Gram. 34, 31 (wohl 'Augsb. 1. Dr. 16. Jh. `): Vmb solcher grosser nutzbarkeit, vnnd seiner lieblichait wille, solten ye die teüschen jre sprach bas verstehn lerne. EBD. 41, 21: dz die nachgende silben vm senftigkait und lieblichait des lauts wille auch aines der duplierten buchstabē bedórffe. - Göz. Leichabd. 273, 1; Opitz. Poeterey 16, 34.

lieblos, Adj.

1.; 2., s. liebe 1; 9.

3. >ohne js. Liebe lebend, jeder Liebe eines anderen entbehrend, verlassen elend, trostlos. - Wbg.: lieblose >Verlassenheit als Nicht-geliebt-werden des mystischen Menschen .

Bihlmeyer, Seuse 10, 7 (alem., 14. Jh.): Dar umbe gie er ellend und lieblos und enzoh sich mit groser gezwungenheit. EBD. 261, 21: Swenne du dich denne aller menschen liebes, nutzes und trostes verzibest, denn so vil es din bärú notdurft ist: so verwiset din lieblosi alle, die mich do ze der stunde liessen. EBD. 276, 7: kint mins, owe min kint, wie bin ich [Maria] nu so lieblos! Wie ist min hery so gar trostlos worden! - BIHLMEYer, a. a. O. 446, 1; SCHMidt, Hist. Wb. Elsaß 222.

lieblose, s. lieblos 3 .

lieblüge, s. liebe 1.

liebmal, s. liebe 9.

liebmildreich, s. liebe 11.

liebnis, das/die. - Nrddt. / md.; gehäuft Rechts- und Wirtschaftstexte.

1. >Freundschaftsgabe, freundschaftliches Geschenk; Ehrenbezeugung`. -
Phras.: zu liebnisse >zuliebe «. - Bdv.: gabe 1, geschenk 1; vgl. liebung 2, liebtat, schenkung.

v. Bunge, Livl. UB 543, 32 (nrddt., 1406): und redt dozu das beste um unsern willen, das sie [Stadt Lübeck] uns in eime sulchen zu lipnisse werden, of das her Jacop sinen fromen domete schaffe. Toeppen, Ständetage Preußen 3, 67, 32 (preuß., 1448): do welde ewir gnode in nicht so vil zcu lipnicz thun, das [...]. Reduich, Jül.-Berg. Kirchenp. 1, 298, 25 (rib., 1536): das dan s. churf. g. us dem ein liefnis dede, nit us plicht. Chron. Köln 3, 837, 11 (Köln 1499): die zokumst keiser Frederichs geschach niet sonder groisse treffeliche liefnis der stat van Coellen an den keiser. BUCH WEINSB. 1, 138, 9 (rib., um 1560): und ist folgens noch fil und fil daruff ergangen, on das geschenk, schade und leifnis. v. LiLIENCRON, Dür. Chron. Rothe 268, 17 (thür., 1421): die keiserynne die bilt das reich mit dem ssone gar irbarlichen [...], die irbarn grossen berren unde fursten mit guticheit unde fruntschaft, die andern dornechst mit gabe unde lipnisse. v. Tscharner, Md. Marco Polo 23, 1 (osächs., 2. H. 14. Jh.): und alle dy Tartirn, [...], dy gebin ym [chaam] czu lipnisse groze gobe von golde [...] - ReDLich, Jül.-Berg. Kirchenp. 2, 2, 106, 38; STruck, Cist. Marienst. 1140; Rwв 8, 1313.

2. `Zuwendung, Vergütung, die über den Lohn oder eine ausgehandelte Abgabe hinausgeht<; vgl. liebe 3; 5. - Bdv.: genies 2, pacht 1; 2, trankegeld, vorteil; vgl. lieb (das) 4, liebung 2.

Helbig, Qu. Wirtsch. 1, 90, 6 (md., 1408): Welch smid seynen knechten sichilin gebit zcu lipnisse [...] der is vorfallen sechs pfund wachs. Hilliger, Urb. St. Pantaleon 582, 43 (rib., 1681): sollen die gewohnlige pfacht jahrligs zablen [... und vor trugenweink auf einmabl geben 30 rblr. neben liebnus. ERMisch, Sächs. Bergr. 110, 13 (osächs., 1492): man soll binfur kein liepnus auf zechen oder butten nyemants geben. LÖsCHER, Erzgeb. Bergr. 187, 1 (omd., 1554/1633): Lipnis oder tranckgelt giebet man albier nicht obne sonderliche zuelaßunge des bergkmeisters. WutKe, Schles. Bergb., Cod. Sil. 20, 168, 28 (schles., 1509): Es soll auch keinem arbeiter mehr lohnes gegeben werden, denn wie itzund bishero der gebrauch gewesen, [...] auch keinerlei libnis obne zulassung des bergmeisters. WeIzsäckeR, Graupn. Bergb. 184, 36 (osächs., 1532): Der mollmeister lipnis halben sal es gehalten werden noch vormug der ordnung. - Hilliger, a. a. O. 544, 41; Froning, Alsf. Passionssp. 2, 63, 20; Wutke, Schles. Bergb., Cod. Sil. 20, 254, 29; Rwв 8, 1314.

3. >kleinere Gabe, Geschenk in Geld oder Naturalien, das man jm. des eigenen Vorteils willen zukommen läßt<; mit Tendenz zu >Bestechungsgeschenk $<$. - Bdv. fürdernis, gabe 2, geschenk 2, genies 2, genus 1; vgl. liebtat, liebkosung 2. - Synt.: l. nemen / unterlassen, jm. l. bieten; dem l. fluchen / folgen; 
jn. durch l. verschonen, mit l. anreizen, etw. zu l. geben.

Ziesemer, Proph. Cranc 262, 22 (preuß., M. 14. Jh.): alle habin sy [vurstin] gabe lieb und volgin liebnisse. HeLBIG. Qu. Wirtsch. 2, 40, 21 (md., 1379): tet ir daz nicht und meindit hirunder yemandis durch fruntschaft, gabe adir lipnizs willen zu vorschonen, so [...7. LoEscH, Kölner Zunfturk. 1, 120, 26 (rib., 1421): were sache, dat einich meister dem anderen [...] einich liefnis boede of dede bieden, daromb sulchen kneicht of gesinde sime meistere afbendich gemacht wurde. LAMPrECHT, Dt. Wirtschaftsl. 3, 313, 14 (mosfrk., 1509): auch sal kein kelner bi sinen plichten und geloebden, so ein iglicher unsem genedichten herrn gedaen haed, van pechten [...] buiseren aeder anderen guteren einich geschenk gaeb leibnis aeder genosse nemen und kein furtel broechen, das im zu nutz. [...] reichen maech. Wolf, Rothe. Ratsged. F 560 (thür., 3. Dr. 14. Jh.): Nicht gabe nemen | Die da mochten brengen daz schemen, | Noch liepnisse. EBD. B 363: Fluch auch lipnisse, | Ringe nicht sere nach genisse! - Loesch, a. a. O. 2, 10,8 .

\section{liebpflegung, die.}

$>$ Liebe zwischen ehegenossen, braut und bräutigam als Entsprechung der Liebe zwischen Christus und der Seele<; vgl. liebe 10; 11.

zU Dohna u. a., Staupitz/Scheurl 120 (Nürnb. 1517): inmasen auch der nackent Christus sich nit mag verneinen den nackenden. Wiewol in abgestolner liebpflegung izuzeiten die bitzig erzeigung fürdringt, nichtsdestweniger haben dise ungezweifelt gewies, das sie zu vergleichung leiplicher reichtumb und wollust bundertseliglich nemen [...] das ewig leben.

liebrede, s. liebreden.

\section{liebreden, V.}

>schmeicheln, schönreden`. - Bdv.: vgl. liebkallen, liebkosen 2. - Wbg.: liebrede, liebreder.

Luther WA 2, 64, 1 (1519): Das man liebgeredt und geschmeichelt hat. Schade, Sat. u. Pasqu. 1, 5, 164 (o O. o. J.): die orden des bettelstabs | Heucheln schmeicheln und liebereden dem bapst, | Daß sie durchs bapsts privilegien bleiben frei ungestraft. VOC. INC. TEUT. $\mathrm{p}$ ir (Speyer um 1483/4): Liebkoser Adulator [...] lieb reder. KeHrein, Kath. Gesangb. 2, 486, 6 (Nürnb. 1631): Acht kein Liebred noch schmeicheln, |Auch kein Marter noch schmert?.

liebreder, s. liebreden.

liebreich $1 ; 2 ; 3$, s. liebe $1 ; 5 ; 11$.

liebsam, s. lieb (Adj.) 1.

liebschaft, die.

1., s. liebe 1.
2. >Liebe zwischen Mann und Frau, erotische Zuneigung ‘; zu liebe 9.

WIESSNER, Wittenw. Ring 1680 (ohalem., 1400/08): Won die rebte liebeschaft $\mid$ Zwïschent zwaien hat ir cbraft. EвD. 1793: Daz ich da her so mangen tag, | Taugen liebschaft gen euch [puol] trag. EвD. 3486: Von rebter liebschaft sich die leut $\mid$ Nemen scbüllen, nit umb gelt.

liebschlek, s. lieb (Adj.) 1.

liebschwinderin, die.

$>$ Nachtigall .

Pfeiffer, K. v. Megenberg. B. d. Nat. 221, 9 (oobd., 1349/50): dar umb haizt si [nabtigal] ze kriecbischer sprâch phylomena, daz ist sô vil gesprochen sam ain liebswinderinne, wan si swindet und nimt ab von rebter lieb irs gesanges un₹ in den tôt.

liebsehen, s. lieb (Adj.) 4.

liebsenend, s. liebe 9.

liebspeise, liebsprecher, s. lieb (Adj.) 1.

liebstöckel, der/das; aus lat. levisticum officinale; aufgrund etymologischer Undurchsichtligkeit hohe Schreib- und Formenvarianz, teils mit volksetymologischer Umdeutung: laub-, leb-, le-, leib-, lied- in Kombination mit -stein, -stek, -stich, -stok, -stöckel, -stuk, -stuckel.

>Liebstöckel, Badekraut`, eine als Küchengewürz, als Wildköder sowie zu Heilzwecken verwendete Pflanze; vgl. Marzell 2, 1264 f. mit umfänglicher Dokumentation der Bezeichnungen und kulturgeschichtlicher Information. - Vielfach Pharmazeutische und medizinische Fachtexte. - Wbg.: liebstöckelblat, liebstöckelrörlein, liebstöckelsaft, liebstöckelsame, liebstöckelwasser, liestöckelwura(el).

Follan, Ortolf. Arzneib. 132, 13 (rib., 1398): mache er dyt plaster: nym leuestoc, [...], stoz se eyn wenich an waszere vnde leg se nederbalf dez nauels. EскHARDT, Ohess. Klöster 2, 447, 42 (hess., 1460): Contra strangwineam [...7 Nempt dilßensamen, [...], lebestuckelsamen. Alberus CC ijv (Frankf. 1540): Angelica [...] Herculeum, deß heyligen geyst wurt? / brustwurtzel / wechst vff den bergen / Vnd ist der liebenstóckel schwester / vertreibt gifft. ERMISCH u.a., Haush. Vorw. 79, 16 (osächs., 1570/7): Ein andere vihearzney, [...] 2 lot. baldrain, 2 lot. meisterwurzel, 3 lot. liebstökkel. EвD. 80, 23 (17. Jh.): Vor das vibe ein bewertes pulfer. Rp. [...] 2 lot baldrianwurzel, 2 lot libsteinwurzel, 6 lot lorbeern. ЕвD. 229, 14 (E. 16./A. 17. Jh.): Wildpret an einem ort zusammen bringen. Gesamblet ein fessel voll menschenharn, 
leim von backöfen, haringlack. liebstockelsaft, aspensaft. EвD. 229, 26: Die pfädt und genge derselbigen refir, da das geschleck. und sulzen bingeschlagen werden, sollen mit wasser, darein liebstöckel, birschwurzel, birkenschos, haselnschos ingesotten worden. KeIL, Peter v. Ulm 47 (nobd., 1453/4): Wiltu ein wunt-pflaster machen, Nim altes smer und meyen-schmaltz [...] und new wachs vnd lobstuckel vnd patonig. OTт-VOIGTLÄNDER, Rezeptar 211v, 14 (Hs. ' nalem., um 1400?): So du din antlit wellest schón machen: Nim / eselmilch [...] vnd nim mornendes lubstikel wurc\%. EBD. 216v, 13: Nim ruten / und des venchels wurczen vnd lubstichels / bletter. Gleinser, Anna v. Diesb. Arzneib. 1989, 183 (1658): so soll man laubstickel rörlin nemmen und eim dardurch binden in mund daz. waßer blaßen. HaAge, Hesel. Arzneib. 15v, 10 (Hs. rnoobd./md., E. 15. Jh. $\left.{ }^{\urcorner}\right)$: Wiltu machen ein gutt wasser fur die gesbulst, so nym vollich plud [...], dar zu nim nachtschaden und fenchel und laub stuck. Eis u. a., G. v. Lebenstein 75, 14 (oobd., 15. Jh.): Lusteckwasser Ist guet fur die geschwulst. Deinhardt, Ross Artzney 222, 368 (oobd., 1598): Für die khelsucht Nimb ludtsteckhen, khraut und wurzen. - Alberus DD IVv; Hajek, Güte spise 54; ERMisch u. a., a. a. O. 212, 10; 227, 11; KeIL, a. a. O. 161; 205; S. 419; Broszinski, Minner. Chir. Parva 78v, 14; Bremer, Voc. opt. 50076; Voc. Ex QuO, Index 459; MaAler 272v; 277r; SChweiz. Id. 10, 1664; VorARLB. WB. 2, 257.

liebstöckelblat, liebstöckelrörlein, liebstöckelsaft, liebstöckelsame, liebstöckelwasser, liebstöckelwurz(el), s. liebstöckel.

\section{liebtat, die.}

$>$ Geschenk, Liebesgabe`, mit Tendenz $\mathrm{zu}$ : >Bestechungsgeschenk<; vgl. liebe 3. Bdv.: vgl. gabe 1, liebnis 3. - Synt.: eine l. nemen, jm. eine l. beweisen / tun; etw. (einen handed in l. anstehen lassen; die grosse / gefällige l. - Wbg.: liebtätig (hier Bezug auf liebe 1; 5; 10 möglich).

Kehrein, Kath. Gesangb. 1, 254, 2 (Bautzen 1567): So jemand bawt auff diesen grund, Im lieb thetigen glauben, | Den mag der feint zu keiner stund, | Der seligkeit berauben. EBD. 2, 549, 4 (1584): In allgemeinem glauben stet\%, |Gehorsamlich wilfaren, | Vnd im liebtátigem gesetz, | Góttliches wort bewaren. Lemmer, Brant. Narrensch. 104, 31 (Basel 1494): Daniel keyn liebdat nemen wolt. - SCHwEIz. ID. 13, 2028.

\section{liebtätig, s. liebtat.}

liebte, der/die; $-/-n,-\emptyset$.

>der / die Liebende<; zu lieben 9.

Lappenberg, Fleming. Ged. 65, 268 (1631): Das ganz. Hartenstein erschallt von dem Geschrei | und jauchret mitte drein: Glück zu, ihr Liebten zwei! EBD. 66, 304: Geht, Liebte, gebt nur fort | und gebet gute Nacht! SCHAER, Pyr.Thisbe-Sp. III, 202, 1196 (osächs., 1607): Ach, das zween Liebten auff Erden. | Müssen so verfolget werden.

liebtraben, s. lieb (Adj.) 1.

liebtrank $1 ; 2$, s. liebe $9 ; 11$.

liebtrunk, s. liebe 9.

liebung, die; $-\emptyset /-$.

1. >Entgelt, Lohn, Bezahlung als angemessene Gegenleistung für erbrachte Mühen, Arbeit, Tätigkeitı. - Nobd. - Bdv. vgl. lon $1 ; 3 ; 5$.

Chron. Nürnb. 2, 10 Anm. 7 (nobd., 1422): den с₹и liebung, die ire hewser den wepnern gelihen heten. EBD. 3, 275, Anm. 2 (1440/4): vier tents, die man unserm herren künig bie oben bielt, mit weyn, obs, confect und liechten kost haben mitsampt der liebung, die man den geben hat, die des müe gehabt haben. EвD. 393, 24: ded. 2 Ut. hlr. n. unnsern püteln zu liebung von irer mue wegen. Loose, Tuchers Haushaltb. 155, 6 (nürnb., 1517): gab ich meinen schleiffern [...] auß einer sundern gunst, darumb das sie 3 tag [...] geeißt haben, c₹u einer liebung 2 t6. SAcHs 21, 57, 9 (Nürnb. 1524): Darff man dann kain liebung nemen für müe unnd arbeyt, so man groß summa gelt außleicht, das der müe werd ist? - Chron. Nürnb. 1, 466, 20; Lexer, Tucher. Baumeisterb. 123, 13.

2. >über den jm. zustehenden Lohn, über das vertragsgemäße Leistungsentgelt hinausgehende Zuwendung, Geschenk, Trinkgeld ‘; vgl. liebe 3; 5. - Nobd./oobd. Bdv.: erung, gesuch 6, gewinnung 3, schankung, schenkung, aufwechsel, übermas; vgl. liebmas, liebnis 2. - Synt.: l. fordern / geben, jm. eine l. schaffen / tun, jm. l. schuldig sein; etw. zu l. nemen, jm. etw. zu(r) l. geben / lassen / schenken. KöвLER, Ref. Nürnberg 287, 12 (Nürnb. 1484): ob der Crist von solchem gelihen gelt dem Juden icht gesuch. wechsell. genynnung. oder liebung gebẽ bett. LeXer, Tucher. Baumeisterb. 244, 30 (nürnb., 1464/75): die [bausfrawe] im dann zu dem newen jar auch ettwas scbencket als ein bemde oder dergleichen zu liebung. ЕвD. 277, 18: als man den meistern bißher von irs meister rechten und dargeliben zengs wegen schenckung oder liebung thun hat mussen, ist ein rat doran komen und gepeut ernstlich, das man denselben meister soliche schenckung oder liebung binfur nit mer scbuldig sein noch geben soll. SACHS 14, 166, 22 (Nürnb. 1551): Der kauffherr [...] spricht: Fraw, nembt zu liebung diesen ring. WINTER, Nöst. Weist. 2, 1054, 2 (moobd., 1491): den lobn sollen geben die weinzirel und mein herrn nichts, dann si geben ein erung und ein liebung. Turmarr 4, 836, 3 (moobd., 1522/ 33): Kaiser Severus und sein sun [...] schuefen den kriegsleuten 
ein êrung und schankung oder liebung über den gemainen sold. - Rwв 8, 1315. - Vgl. ferner s. v. aufwechsel.

3. `Sold (für Militärdienste)<; Spezialisierung zu 1. - Nobd. - Bdv.: vgl. paga, ${ }^{3}$ page, sold, stipendium.

Pfeiffer, Frk.-bay. Landfr. 74, 13 (o/nobd., 1377): $107 \mathrm{lb} .19 \beta$ blr. fur kost und in zu liebung und von iren pferden. EвD. 174, 28 (1395): $186 \mathrm{lb}$. und $81 / 2 \beta \mathrm{blr}$. kosten die 40 mit spiessen [...], den zug, dez man ubereyn ward, den man widerpot, daz man in ze liebung gab. CHRON. NürNB. 1, 172, 26 (nobd., 1388): so hat man iedem spiez in di bant geschankt ze libing 10 guld. EBD. 4, 17, Anm. 8 (1430): 50 guld., die Peter Volkmar dem Caspar von Waldenfels bezalet, die man im zu liebung in der vorgenanten teyding versprochen het. EвD. 407, 8: 150 U6. $n$. demselben Tetzel zú liebung.

4.; 5.; 6, s. lieben 9; 10; 11.

liebvater, s. lieb (Adj.) 3.

liebwirdig, s. liebe 4.

\section{liebzälig, Adj.}

1. >beliebt, leutselig, gern gesehen`; vgl. lieb (Adj.) 3, lieben 5. - Rib. - Bdv.: antunlich, ergezlich; leutselig. - Wbg.: liebgezal, liebräligkeit >Beliebtheit, Ansehen, Autorität<.

KLetT, J. v. Soest 5, 837 (Hs. ' wmd., 1470/80?): nu wass er eerst so lybgetzal | geworden, so das alles, dass | von hoffgesynd da geghen wass, | betrubt worn. EвD. 11, 904: als Venus hot myt krafft regyrt, | so wurt das selbig kynt probyrt, | das ess sol syn gantz lyb getzal | und selbest lyben uber al. Мелјвоом, Pilgerf. träum. Mönch 9268 (rib., 1444): As ich [Giricheit] wille, machen ich mich liebgetzale Ind ergetzlich altzo male. CHRON. KöLN 2, 490, 3 (Köln 1499): der selve burger hatte einen son [...] und was ein modich koen man, liefzalich van alre mallich. BUCH WEINSB. 1, 176, 29 (rib., um 1560): globten im das in sin hant, dan er seir leibrellich bei innen war. EBD. 2, 25, 27 (1553): Er [...] hat die meisten gnaden an sich, dan er war andoinlich, wolberedt, meissich, narhaftich, leibzellich. EBD. 4, 26, 32 (1588): ibr oberster, [...], so die leste belegerung mit graff Carln Trucksess auch in der stat Bon gewesen, allen handel wiste, were der leibzellickeit und dapferkeit, das es nube nit geschein wurde bis zum utersten zu. EBD. 5, 128, 16 (1578): Er [...] dede jedem vil guts, darumb er leibzelliger was, aber da er gestorben, war ein rait drie oder veirhondert daler an im zu achters. EBD. 1, 107, 26.

2. >aus Wohltätigkeit, Barmherzigkeit gestiftet «; vgl. liebe 1; 5 .

Siegel u. a., Salzb. Taid. 136, 32 (smoobd., 17. Jh.): bierdurch [...] auch den recht armen, alten, kranken [...] personen das liebseelige almusen entzogen [...] wird (hierher oder eigene Bildung: liebselig?).

liebzäligkeit, s. liebrälig 1.

liebzorn, s. liebe 4. liechen, V.; unr., abl.; Form von mhd. lûchen >zupfen (LEXER 1, 1974).

>etw. (Hanf) zupfen

Bihlmeyer, Seuse 136, 14 (alem., 14. Jh.): das si mit den andren jungen swóstern waz us gegangen uf einen aker ire werk liechen. MüLler, Grafsch. Hohenb. 1, 109, 31 (schwäb., 1392/3): ainem boten, lüff gen Oeningen [...] mit ainem brief von liechens wegen $[. .] .5 \beta 2 \mathrm{~h}$. WintTERLIN, Würt. Ländl. Rechtsqu. 2, 21, 11 (schwäb., 1537): hanf und flachs, wa der geseet (were), ist man auch deß drittails warten und stellt das kloster den dritten liecher.

liecher, s. liechen.

liecht, das; -es/- $\emptyset,-e,-e r$.

1. >Leuchte, Lampe; Licht der Lampe<; als Metonymien: >Lichtbehälter`; (seltener:) $>$ Öl, Unschlitt (als Brennstoff)<; halem. ist liecht vereinzelt im Zusammenhang mit feuer, herdstat belegt; offen zu 2. - Beleghäufung für Rechts- und Wirtschaftstexte sowie berichtende Texte. - Phras.: das ewige liecht (oft im Orientierungsfeld mit jarzeit, selgeräte); jm. (z. B. dem teufel) ein liecht anstekken >auf jn. hereinfallen`; jn. binter das liecht füren; das liecht mit molden in den keller tragen >Unsinniges tun «. - Bdv.: ampel 1, kerze 1, laterne, leuchte 1, leuchter, luzerne; vgl. ampulle 2, glasfas, immerliecht, lampe. - Synt.: l. anblasen / machen / geben, ein l. anzünden / aushenken / halten sunterhalten< / tragen, für das fenster benken, an die laterne setzen; das $l$. (Subj.) ausgehen, wo brennen, ein zeichen sein; bei l. dreschen, wo sitzen, mit / ane l. wo gehen, auf (>an ‘) l. denken (z. B. bei Feuer), mit liechten leuchten / suchen; das l. des hohmeisters; das blosse / brennende / eiserne / gute / verdekte l.; die materie des liechtes; das grab, der himmel mit liechten, öl zu liechten. Wbg.: liecht (Adj.) 1, liechtarbeit >Tätigkeit bei Lampenlicht`, liechtekasten >Kasten zur Lampenaufbewahrung`, lichterform (wohl dasselbe wie liechtform; möglicherweise >Form zum Lichtergießen`, so Dwв 6, 883), liechterweibe, liechtfas (s. die Belege), liechtform >Lampengehäuse<, liechtfutter >Schutzgehäuse für eine Lampe<, liechtgarn >Lampen-, Kerzendocht $<$ (a. 1529), liechtgeld >Geld für die Beleuchtung` (in verschiedenen Zusammenhän- 
gen; a. 1467 f.; Rwв 8, 1294), liechtglocke (a. 1457), liechthocke > Verkäufer(in) von Unschlittlichtern< (a. 1472), liechtholz, wohl >harzhaltiges Holz für Kienspäne<, liechtkämmerer > für die Beleuchtung eines herzoglichen Haushalts Verantwortlicher (a. 1396), liechtkelter wohl kontrahiert aus liechtgehälter >Lichtbehälter`, liechtkoche ( $\mathrm{Zu}-$ ordnung des $\mathrm{Gw}$ unklar) wohl >Lichtbehälter`, liechtkolbe eine Art Laterne (bdv.: laterne), liechtleiber >Person, die Lampen für verbotene abendliche Zusammenkünfte (nachtspiel, vgl. liechtstube) ausleiht<, liechtöl, liechtpfennig >Abgabe zur Unterhaltung des Kirchenlichtes` (um 1500), liechtpriester (abwertend), liechtputze, liechtputzer (bdv.: liechtschere), liechtquerdel >Lampen-, Kerzendocht (Gw zu mhd. quërder; LEXER 2, 323), liechtrame >Kerzengehäuse`, liechtschein, liechtscherbe >Lampe in Tiegelform`, liechtschere, liechtschirm a) >Lichtschere, b) >Lichtkegel oder >Schutzschirm gegen Licht` (nicht entscheidbar), liechtschnupfe (Beleg s. v. abbreche 1), liechtschupos > kleines / mittleres Bauerngut (genaue Motivation des Bw unklar; a. 1353), liechtseil >Lampe; Lampenseil, -aufhängung`, liechtsorge >aufmerksame Beobachtung der Leuchtgeräteく (a. 1642), liechtstab >Fackel<, liechtstal (a. 1437), liechtstar >Leuchte`, liechtstein >Steinbehältnis für Licht`, liechttegel >kleines Handlicht` (zum Gw s. SchweIz. ID. 12, 1108), liechttiegel auch: >Untersatz für eine Kerze oder Öllamper, liechtweihen.

SCHÖPPER 81b (Dortm. 1550): Lucerna. Liechtstarr leuchter lucern latern leucbte. Heцm, H. v. Hesler. Apok. 1667 (nrddt., 14. Jh.): Der lucbter ist des liecbtes stadel, | Alz ist daz liecht des libes adel. JOACHiM, Marienb. Tresslerb. 63, 9 (preuß., 1399): 1 scot eyn lichtfuter zu beslon, do man licht uf deme wege inne furet $2 u$ der messen. EBD. 497, 15 (1408): unsers homeysters lichte, das vor dem sacrario of dem buse bornet. Ziesemer, Marienb. Ämterb. 20, 25 (preuß., 1440): 1 scheffel senff, item eyn lichtforme, item 5 tonnen essig. Toeppen, Ständetage Preußen 1, 344, 31 (preuß., 1420): das ir belffet czu dencken off licbte, wenn der kellermeister off desse czeit keyner lichte noch unsslyt nicht mer enhabet. BLÜMCKe, Hans. Gesandtsch. 151, 9 (nrddt., 1603/05): vor 2 Lichttputzen ... 8 Sch. EBD. 197, 2: vor Papier, Calendaria [...], lichtkochen, liebtputscher, lichtt 11/2 Thlr. LUTHER, WA 11, 456, 21 (1523): ßo haben wyr doch yhe die rechte lauttere leer des Euangelii als eynen bellen liechtstar. EBD. 32, 450, 23 (1532): So bald kan man ein gloslin finden, damit man dem Teuffel ein liecht anstecke. EвD. 49, 751, 38 (1545): es stoßet die predigt auff ein hauffen altar, tempel, leuchter unnd liechtpriester und Leviten. Ders., WA Bibel 7, 216, 26 (1522): vnter wilchen [geschlecht] y br scheynet, als eyn liechtstar, ynn der welt. BLÜMCKe, Hans. Gesandtsch. 197, 2 (nrddt., 1603/05): Vor Papier, Calendaria oder almanach, lichtkochen, lichtputscher, lichtt 11/2 Thlr. Chron. Magdeb. 2, 152, 12 (nrddt., Hs. 1601): Diese selbigen haben [...] alle fahnenkasten und Lichtekasten umbgeworfen, zerstrewet. SсHмітт, Ordo rerum 200, 25 (rib./nd., 2. Dr. 15. Jh.): Ligmen [...] tacht - lichtquerdel [...] zach ader dacht. Bergner, Urk. Kahla 93, 29 (thür., 1455): das ein iglicher gastgebe [...] schicke und mache in seinen stellen, darin man mit lichten pfleit zu gehin, gute iserne vordacte lichten. EBD. 105, 27 (1474): So sollen [...] die meister der zweier handwerke zwei kerzen mit zweien lichten halden, got dem almechtigen und seiner wirdigen mutter Marien zu lobe. VOC. INC. TEUT. p, iv (Speyer um 1483/4): Liechtscherm Extinctoriũ. liechtscher Emũnctoriũ Cãdelariũ. Lichtschirm wl. rundel Antipira. EBD.: Liechtstab Facula. SснміDт, Frankf. Zunfturk. 1, 322, 38 (hess., 1478): so [bei fuer] sulde eyn iglicher, [...], eyn liecht in eyner luchten ußhencken. Lemmer, Amman/Sachs. Ständeb. 80, 6 (Frankf./M. 1568): Schón Liechtkolben ich auch bereit / | Bey Nacht / qu Gastung vnd Hochzeit. v. Tscharner, Md. Marco Polo 4, 10 (osächs., 2. H. 14. Jh.): das ole ist uneze, doch ist is gut czu burnen [...] und gibt vil provincien licht des nachtis. SKÁlA, Egerer Urgichtenb. 153, 12 (nwböhm., 1575): Zu nachts mit lichten geleicht haben sie [Krebse] sich deßdo biß fangen laßen. THiele, Chron. Stolle 505, 25 (thür., 3. Dr. 15. Jh.): dy luthe in oren busern sossen by lechte dy gancze nacht. WEISE. Jugend-Lust 112, 20 (Leipzig 1684): hat der Schiffer uns mit falschen Erzehlunge hinter das Licht geführet? WENDEHORST, UB Marienkap. Würzb. 53, 9 (nobd., 1390): daz ich [... bescheide mit disem brief [...], zu lob und zu eren dem almechtigen got, [...] an unser frawen capellen [...] ein ewig lyecht von gutem lutern lynól. HAMPE, Ged. v. Hausrat 1, 2, 9 (nürnb., n. 1480): Leüchter liecht scher vnd licht digel. Chron. Nürnb. 5, 583, 6 (nobd., E. 15./A. 16. Jh.): man [...] machet einen weibkessel pei dem sagrer, pei des Deichßler liechtkelterlein. Rupprich, Dürer 1, 162, 49 (nobd., 1520): hab 4 stüber geben für kesselbraun und ein lichschärlein. BaumanN, Bauernkr. Rotenb. 5, 11 (nobd., n. 1525): demnach der cerimonien, des weichwassers, salsweyhen, liecht-, palm-, wurzweyhen und vil anders an vilen orten abgieng. EBD. 12, 18: umb das er das weybwasser, wury, palm, sals, liechterweyhen und ander cerimonien abgestellt hatt. Vetter, Pred. Taulers 83, 1 (els., E. 14. Jh.): uf dem berge wesenlich so wübs die materie des liebtes, dis oley. KuRRELMEyer, Dt. Bibel 3, 304, 9 (Straßb. 1466): manche .vij. liechtuaz [Var. 1470-1475': lichtram; 1475²-1490: latern; LuTHER 1545, 2. Mose 25, 37: Lampen] oder luczernen: und setz. sy auff das kertzstal. HAMPE, Ged. v. Hausrat 4, 6, 13 (Straßb. um 1514): Ein Liechterform 
mit allen ierm plunder. DAsYPODIUs 373r (Straßb. 1536): Liechtarbeit der bauren. Rusticance lucubrationes. SCHAER, Pyr.-Thisbe-Sp. II, 99, 362 (Basel 1616): [Wie sie] Woll ein Lucern und Liecht zugleich | Fürs fenster bencken zum warzeich. Bremer, Voc. opt. 12041 (halem., 1. H. 14. Jh.): Lampas liehtuas [...] amppel [...] liechthus [...] Lampas [...] Grece generaliter dicitur incendium Latine. Sed in proposito [...] est vas vitreum olei pro materia luminis contentivum. Et proprie lampas vt vult Hugwicio est vas uel flamma in vertice lucens. EBD. 23029 (1. H. 14. Jh.): Antipira furschirm [...] liechtschirm [...] est instrumentum defensorium, quod locatur inter ignem et lumen ad oculos hominum, ne radii visuales per excellenciam luminis disgregentur. EBD. 23033 (1328ff.): Crucibulum tegel [...] liechtschein [...] liechtstain [...] liechtscherb [...] est vasculum unctuose materie luminis contentivum. RENNEFAHRT, Recht Laupen 202, 38 (halem., 1558): das in der kilchori Ferrenbalm, [...], sind 26 herdstet, da fur und liecht sind. MüLlER, Alte Landsch. St. Gallen 24, 36 (halem., M. 16. Jh.): es sol ouch niemandtr. [...], das feur und liechter bi und in den hüsern, scheuren, spichern, städlen noch sust onbewart tragen. JellineK, Friedr. v. Schwaben 1230 (schwäb., Hs. 1478): Er graif nach dem fúrzúg sein | Unnd zundt an ain liechlin. CHRON. Augsi. 1, 50 Anm. 1 (schwäb., zu 1376): $6 \beta$ d. umb unslit und liechtstain do man uf dem bus waz. und man den Zollner hauptot. EBD. 2, 23, 34 (Hs. 16. Jh.): ob im hett man gemacht ain himl mit vil liechten, da prunnen wol fünfbundert kertzen. Haszler, Kiechels Reisen 314, 10 (schwäb., n. 1589): dann es zu nacht zimlich lüecht ist von den lampn. GeHring, Würt. Ländl. Rechtsqu. 3, 348, 19 (schwäb., 1580): soll auch niemandts bey der nacht über die gassen [...] mit blaussem liecht gon. WINTTERLIN, Würt. Ländl. Rechtsqu. 2, 520, 27 (schwäb., 1601, Hs. 1788): In den lichtkërzen solle keine unordnung mit ärgerlichem gespräch, [...] gestattet [...] werden. Steinberger u. a., Urk. Hochst. Eichst. 119 (noobd., 1314): Mit demselben gelte wil min vorgenanter herre, der bischof, ein ewigez libt stiften. Thiel u.a., Urk. Münchsm. 162, 14 (moobd., 1343): daz daz vorgenant gotzhaus [...] nemen sol zimmerboltz, genug, liebtholt:, prigkboltz, [...], vnd zainholt\%. Auer, Stadtr. München Anh. [7], 97, 32 (moobd., n. 1347): Man sol under den chramen [...] chein fuir haben weder pei nabt noch bei tag ân liebtstein. DirR, Münchner Stadtr. 420, 23 (moobd., um 1365): Swer wagenläwt oder saemer herberget, der sol in mit unsliecht läwchten und mit chainem andern liecht. Fuchs, Kart. Aggsbach 165, 5 (moobd., 1399): ein ewig nachtlicht mit liechtóll vór sand Dorothee alter. FICHTNER, Füetrer. Trojanerkr. 5, 6 (moobd., 1473/8): das ain vakkel auss ir hertzen ginge, | die pran von liechten flammen. WinTER, Nöst. Weist. 1, 826, 14 (moobd., 1515): alles nachtspil [...], und wer daran begriffen würd, [...], der ist umb 72 s, desgleichen der würfl- und liechtleicher ist iedlicher um 72 ঐ. Qu. Brassó 5, 494, 24 (siebenb., 1614): 4 Flaschen, 4 Salzbixen, 4 Lichtert, 4 lichtscheren. - Ziesemer, Gr. Ämterb. 347, 21; PeIL, Rollenhagen. Froschm. 550, 1377; Fastnachtsp. 1216, 1; 1218, 27; Chron. Nürnb. 2, 29, 10; Thiele, Minner. II, 13, 383; Kurrelmeyer, a. a. O. 3, 304, 11 Var.; KoppITz, Trojanerkr. 22845; UB OB DER EnNs 10, 240, 25; Vogel, Urk. Heiliggeistsp. 1, 299, 37; Rennefahrt, Recht Laupen 24, 22; Ders., Statut. Saanen 152, 8; Boner, Urk. Brugg 369, 3; Jörg, Salat. Reformationschr. 767, 11; Chron. Augsb. 1, 61, 29; Hauber, UB Heiligkr. 2, 287, 20; GeHring, a. a. O. 3, 118, 6; Uhlirz, Qu. Wien 2, 1, 1333, 2; 2, 3, 5436, 12; Brunner, Rechtsqu. Krems u. Stein 134, 40; Moscouia C iiiv, 29; Bauer, Haller. Hieronymus-Br. 115, 13; ZingerLe, Inventare 158, 1, 30; Matzel u. a., Spmal. dt. Wortschatz. 1989, S. 192; Bremer, a. a. O. 23031; Schmitt, a. a. O. 199, 23; VOC. INC. Teut. p iv; West, Dasypodius. 1989, S. 335; MaAler 272v; Rwb 8, 1294; SChweiz. ID. 5, 1124; 7, 1304; 8, 1043; 11, 22; 12, 1107; PfäLz. Wв. 4, 97; 971; BüCher, Berufe Frankf. 1914, 78. - Vgl. ferner s. v. abbreche 1, ablöschen 2, aufbören 2, ausgehen 27; auswelben, baumöl 1 .

2. >Kerze ‘; meist angesprochen als Wirtschaftsgut, darunter als Abgabe, Stiftung in kirchlichen Zusammenhängen; in Vergleichen in bildhaften Bezug zur Liebe Gottes gesetzt. - Gewisse Beleghäufung für Rechts- und Wirtschaftsquellen. - Phras.: das liecht versäumen >das an der Abbrennstrecke von Kerzen o. ä. gemessene Zeitlimit überschreiten`. - Bdv.: keræ̌ 1; vgl. ${ }^{2}$ blas, golliecht, leuchte 1, tartis. - Synt.: das / ein l. anbrennen / ausrichten / brauchen / butzen / entründen / stelen; die selmesse mit l. singen; das grosse / kleine l.; stapel / schok liecht(e); die enthaltung der liechte. Wbg.: liechtbotzen >abgebrannter Kerzendocht< (a. 1635), liechtbutze (r) >Lichtschere<, liechtkerze (bdv.: vgl. liechtstuD), liechtmacher (auch mit Uml.; bdv.: kerzenmacher), wohl eher >Kerzen-` als >Lampenhersteller`(a. 1399 ff.), liechtmacherin (a. 1399 ff.), liechtmeister > für die Kerzen in der Kirche Zuständiger`, liechtstul.

SCHÖPPER 81b (Dortm. 1550): Lumen. Liecht kert\%: PeIL, Rollenhagen. Froschm. 559, 1671 (Magdeb. 1608): Wenn ers [Narr] Liecht putst / die Becher schwengt / | Vnd denn Bier oder Wein einschenckt. SCHAdE, Sat. u. Pasqu. 1, 162, 296 (о. O. 1587): Sie [Feuerschlucker] saufen aus hantfaßen und auß schuen, | Sie freßen glas zu einander, auch die Liecht. JOACHIM, Marienb. Tresslerb. 226, 25 (preuß., 1403): 8 sch. vor 2 stapil licht. BERGNER, Urk. Kahla 105, 23 (thür., 1474): wan die meister ein licht eins fingers lang ufstecken werden. EвD. 114, 14 (1492): Wert her aber uf eine nambaftige stunde zu komen vorbeischt und verseumpt das licht uf die selptige stunde von wachse eins virtel einer ellen lang gemacht ufgebrant [...] bußt vi \$. SKÁLA, 
Egerer Urgichtenb. 219, 1 (nwböhm., 1577): hab [...] gstoln, leinwandt, dan ein große bier Rindt fleiß [...], et乏lich licht, dan flachs, dan deller [... . haber er die schißel, licht, vnd etzlich deller thragen. Hertel, UB Magdeb. 3, 718, 43 (omd., 1503): $Z u$ des beygrafft die grosszen licht gebraucht, sal ein pfund wachs, und wu die cleinen licht genutzt ader angebrant, ein halb pfund wachs zu enthaldung derselbigen lichte gegeben werden. Asmussen, Buch d. 7 Grade 1683 (nobd., Hs. A. 15. Jh.): als ain libt / ain kerzen, | als enzunt in gaistlich berze | und in iren sinne | sein minne unser minne. Bremer, Voc. opt. 12046 (wschwäb., 15. Jh.): Candelabrum kerstal 1 kertastal [...] lichstull [...] Candelabrum [...] est instrumentum, super quod pontitur candela ardens. Dasypodius 141r (Straßb. 1536): Emunctorium, Ein abbrech / liechtbutzer. MaAler 247r (Zürich 1561): Das Kólble am liechtbutzen / in gestalt eines schwuñlins. Dict. Germ.-Gall.-Lat. 307, 41 (Genf 1636): Liechtbutze / $f$. Mouschettes, Emunctorium. UB OB DER ENNS 10, 53, 12 (moobd., 1381): so schol der pfarre ze Chirchdorf liechtmaister sehs stekchertzen zu dem jartag [...] leihen. - Ziesemer, Marienb. Ämterb. 96, 22; Bergner, a. a. O. 110, 19; Rennefahrt, Staat/Kirche Bern 76, 20; Hör, Urk. St. Veit 71, 30; Rechn. Kronstadt 2, 166, 25; Apherdianus 96; BüCHer, Berufe Frankf. 1914, 78; Ösт. Wв. 3, 683; - Vgl. ferner s. v. abbutzen 1.

3. >Fensteröffnung; Licht, das durch die Fensteröffnung fällt; Lichtrecht $<$; mit letzterem offen zu 5. - Obd.; gehäuft Rechtsund Wirtschaftsquellen. - Bdv.: fenster, gesicht 3; vgl. ${ }^{2}$ beie 1. - Synt.: l. haben, das l. verbauen, jm. das l. vermauern, unvermauert lassen; das l. auf jn., über die hofstat gehen; um l. klagen, etw. im l. verstängen / vernetzen; stürzel zu vier liechtern; das fenster mit einfallenden liechten.

Chron. Nürnb. 1, 375, 6 (nobd., 1420/41): da verkerten dy prediger sant Kathreyn closter den nunen ir regelen, und vermaurten sy in ire liebt und winden uberal und turren nimer flaisch essen. KöBlER, Ref. Nürnberg 401, 20 (Nürnb. 1484): von trupfen vnd liechten. Klaibung der nebenwende. Rennefahrt, Wirtsch. Bern 704, 23 (halem., 1557): Erstlich sol ein stürtzel zi 4 liechteren gelten -2 \&b. BAstian u. a., Regensb. UB 405, 11 (oobd., 1373): derselb schol noch enmag daz lyeht noch di venster dez êgenanten stainwerchs nicht verpawen. UHLIRZ, Qu. Wien 2, 3, 4324, 25 (moobd., 1470): dass die dreu venster, die aus der egenanten Hirsvoglin haus in des bemelten Hannsen Stettner bof geen, mit invallunden liechten gemacht, auch verneczt und verstengt werden sullen. - KöBlER, a. a. O. 417, 4; LeXER, Tucher. Baumeisterb. 280, 24; Auer, Stadtr. München Anh. [2], 23, 1, 4; 32, 23; 33, 28; 235, 47, 27; Welti, Urk. Rheinfelden 710, 255, 25; UB OB DER ENNS 9, 692, 11; Uhlirz, a. a. O. 2, 2, 2931, 20; 3066, 8; 2, 3, 4119, 32; Vogel, Urk. Heiliggeistsp. 1, 561, 3.
4. >Licht als Wesen, Seinsweise Gottes und gleichzeitig Gnadengabe an den Menschen; Ermöglichungsgrund religiöser Existenz des Menschen und damit zusammenhängender Qualitäten, u. a. religiöser Erkenntnisfähigkeit, Möglichkeit der Gottförmigkeit «. - Meist Texte der Sinnwelt ,Religion', oft der Mystik. - Bdv.: brunst 4, geist 3, glast 4, gnade 1, schein; vgl. ${ }^{1}$ lust 5. Ggs.: deusternis, finstere, finsternis. - Synt.: das l. seben / senden / annemen / erlangen / empfangen / haben, got der sele das l. einpflanzen, jm. ein l. aufstecken > gewähren〈; das l. (Subj.) gleichmachen / scheinen, jn. durchscheinen, im glauben entspringen, in der finsternis leuchten, geistlich sein, liecht und gnade einswesenlich sein; got, Cristus, der herre / vater das l. sein, Cristus das l. aller leuchten sein; $j$. des liechtes mangeln; im $l$. das leben sehen, jn. im l. erziehen, im l. gottes etw. erkennen, jn. mit dem l. füren, von l. finster werden, von dem l. zu der deusternis fallen, ob dem l. gnade sein; das l. gottes / Cristi, des evangeliums / glaubens / lebens, das l. der bekentnis / ewigkeit / glorie / gnade / warheit / welt; das ausleuchtende / einige / ewige / feurige / gnadenreiche / gotförmige / götliche / grundelose / belle / innerliche / klare / lautere / lebende / neue / übernatürliche / ungemischte / ungeschaffene / unsicbtige l.; der blike (>Blitz $<)$, der got / vater, die ausfliessung / ausgiessung / völle des liechtes; l. ane anfang / ende, das l. zur erkentnis. Wbg.: liechtbäre, liechtbärend 1, liechtbringerin (Bezug auf Maria), liechtig >lichtgestaltig<, liechtigkeit, liechtscheinend, liechtstram >Lichtstrahl<.

Strehlke, Nic. Jerosch. Chron. 4747 (preuß., um 1330/40): In den qîten ouch geschach $\mid$ [... $\mid$ daz der vurste lîchtbêre $\mid$ zu gote vil gewêre $|[. .]$.$| der marcgrêve von Mî̀in,$ | [...] | quam gevarn in Prûzinland. Luther, WA 10, 1, 1, 223, 12 ff. (1522): das wyr eben dasselb liecht, eben denselben gott, [...] [sehen] werden auff eyn ander weyßse. EBD. WA 10, 2, 469, 20 (1529): O herre fure uns mit deinem liecht. EBD. 37, 61, 33 (1533): was ist Sonn und Mond und alles liecht [dies $\mathrm{zu}$ 6] gegen dis licht? EBD. 49, 669, $24 \mathrm{f}$. (1545): Der berr des lichts geht auff, Christus, [...]. das das liecht inn die welt komen ist, und die Menschen haben die finsternis lieber denn das liecht. EBD. 54, 47, 1 (1543): Der HERR wird dein Ewiges Liecht sein. Ders. Hl. Schrifft. Joh. 8, 12 (Wittenb. 1545): Jhesus [...] sprach / Jch bin 
das Liecht der Welt. Alberus, Barf. Vorr. Alb. 8, 25 (Wittenb., 1542): wenn jnen schón das helle Liecht des Euangelij vnter die Augen scheinet. Pfeffere, Weigel. Ges. 4, 14 (Hamb. 1646): Allso ist Gott das wahre wesen Leben, vnd Licht obne Anfang vnd ende. EBD. 16, 6: Doher kombts das Creatur gutes vnd böses an ihr treget, das gutte von Gott, als Leben, Wesen, Licht, Geist. Ders., Weigel. Gn. S. 172, 24 (Magdeb. 1615): In deinem [Herr] Liecht sehe ich warlich das Liecht. Fischer, Brun v. Schoneb. 26, 23 (md., Hs. um 1400): der [Christ] sich wolt haissen stet | den flins, daraus uns schrät | unsers fewerleins liecht stram, | davon uns der anfank / kam. Chron. Köln 2, 8v, 40 (Köln 1499): geuallen van dem hoghen in dat nidder. van dem Liecht zo der duysternisse [Kontext: Sündenfall]. STEeR, W. v. Herrenb. Büchl. 473 (pfälz., 1436): Die licbtickeit auch jn dem füre ist nicht wesenclich das füre. EGGERs, Psalter 79, 12 (thür., 1378): by dir ist der born dez lebens, vñ in dime liechte sul wir seben daz liecht. Strauch, Par. anime int. 45, 21 (thür., 14. Jh.): di gnade ist an der sele alse lichtikeit ist an eime iclichin dinge. ie daz dinc der lichtikeit me an ume bait, io iz me geborit wirdit fon der erdin zu deme himmele. EBD. 127, 20: Got ist ein licht in ime selbir swebinde in einir stillin stille. dar ist daz einige licht, daz einige wesin sin selbis, daz. sich selbin forsteit und irkennit. daz forstentnisse des emigin lichtis daz. ist licht fon deme lichte, daz ist di persone des sonis. JosTes, Eckhart 68, 25 (14. Jh.): Daz ander liebt ist geistlich; dar, enspringt in dem gelauben. SERMON THAULERI 7va, 34 (Leipzig 1498): Das liecht leuchtet in der vinsterni $\beta$. Böнme, Morg.R. 13, 4 (Hs. 「schles., 1612ㄱ): Weisheit GOttes / die da ist das hauchen der Góttlichen Krafft und der glantz, des ewigen Liechts / gebe sich in des Lesers Seele. WAGnER, Erk. Ps.-J. v. Kastl 4, 25 (nürnb., 1. H. 15. Jh.): das war licht, das got ist. Gille u. a., M. Beheim 121b, 57 (nobd., 2. H. 15. Jh.): und [Kristus] hat sich selber mensch gemabt $|[\ldots]|$ Gemacht scheinber und libtig. Rieder, St. Georg. Pred. 160, 28 (Hs. rönalem.,

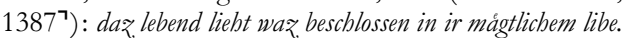
Vetter, Pred. Taulers 329, 23 (els., 1359): das liecht der gnaden. Das ist ein geschaffen liecht; das úber hebet die nature verre úber sich, [...]. Dar úber ist denne ein ungeschaffen liecht: das heisset man das liecht der glorien. Das ist ein gotlich liecht und das ist Got selber. Bihlmeyer, Seuse 17, 22 (alem., 14. Jh.): in der frólichen begirde gri̊t er die liechtbringerin des ewigen tages. EвD. 378, 5: Herr, dinú ogen sind ob der liehten sunnen glan₹, [...], dinú liebtberndú wengel gótlicher und menschlicher nature. Williams u. a., Els. Leg. Aurea 270, 10 (els., 1362): Dis ist der glast des der alle lieht hat geschaffen, der vns hat gelobet sin ewig lieht zu sendende. SCHмIDT, Rud. v. Biberach 162, 22 (whalem., 1345/60): di gotheit ist allein vbersubstancilich vnd het in ir selben dv volli alles liechtes vnd der guti. Eichler, Ruusbr. obd. Brul. 2, 1549 (els., E. 14. Jh.): zu der gnaden gottes - wan sv́ ist ein got geformig lieht, daz vns durch schinet und gelich machet, und sunder dis lieht, daz vns gelich machet, enmógen wir nút vereinigen viber naturlichen. RuH, Bonaventura 341, 12 (oschwab., 2. V. 15. Jh.): der wandlet nit in vinsternüsßen, me er wirt haben vnd erlangen das licht des lebens. WARNOCK,
Pred. Paulis 18, 17 (önalem., 1490/4): daz liecht (dies $\mathrm{zu} 5$ ) der natur wist den menschen och gott lieb ze haben eben als wol als daz liecht der gnaden. Aber doch so ist daz liecht der natur sich gar in vil weg widersetzen dem liecht der gnaden. EBD. 18, 126: [Plato, Aristotiles] manglotend des liechtes der göttlichen bekantnus. STEER, Schol. Gnadenl. 5, 117 (halem., 15. Jh.): Näch der mässe der gnäden wirt öch gemessen daz. natúrlich liecht der sele, wän genäde vnd vibernatúrlich liecht sint ainswesenlich ze nemen. Wyss, Luz. Ostersp. 9518 (Luzern 1545): Jesus, ein liecht der Ewigkeyt. HöVER, Bonaventura. Itin. B, 51 (moobd., 1450/60): der [Cristus] da ist ain wort und liechtscheinender glast des ewigen vater. Baptist-Hlawatsch, U. v. Pottenst. 61 (moobd., A. 15. Jh.): Der [Manes] leret, es wërn zwen götter und czpwen anefang, ainer ain got des liechtes, der ander ain got der vinster. - Helm, H. v. Hesler. Apok. 1792; Luther, WA 10, 1, 2, 384, 16; 14, 30, 4 ff.; 16, 234, 14; 35, 434, 17; Quint, Eckharts Pred. 1, 18, 7; 2, 116, 5; 325, 7; DERs., Eckharts Trakt. 428, 9; Sievers, Oxf. Benedictinerr. 1, 16; Steer, a. a. O. 142; Dubizmay, kurß zu Teutze 91, 5; Froning, Alsf. Passionssp. 1215; Strauch, a. a. O. 33, 15; Jostes, a. a. O. 13, 16 f.; 43, 37 f.; 101, 31; v. D. Broek, Suevus. Spieg. 66v, 12; Asmussen, Buch d. 7 Grade 959; Wagner, a. a. O. 7, 32; Gille u. a., a. a. O. 15, 4; Rupprich, Dürer 1, 171, 52; Bihlmeyer, a. a. O. 471, 27; Eichler, a. a. O. 1, 82; DERs., Ruusbr. steen 204; WARNOCK, a. a. O. 18, 13; Goldammer, Paracelsus 192, 17; Moscouia B iijr, 32; Bauer, Haller. Hieronymus-Br. 43, 34. - Vgl. ferner s. v. afterbussen, an₹ünden 1; 4; ausflus 3, ausgiessung 2 , icht 1 .

5. >Licht, Helligkeit des Tages, Tageslicht «; mehrfach im Ggs. zu finsternis und nacbt auf dem Hintergrund der Schöpfungsgeschichte (1. Mose 1, 4f.); auch >Tag` (s. u. Grimm, Weisth.; vgl. auch das Phrasem der liechte tag s. v. liecht, Adj., 3); hier anschließbar vereinzelte tropische Verwendungen, z. B. >natürliche Verhältnisse‘; >Tochter (in poetischer Überhöhung) r. Phras.: zwischen / unter den liechten in der Dämmerung〈; es wird liecbt $>$ der Tag bricht an (; sich selbst im liechte stehen >sich selbst schaden «; (erv) zu liechte bringen >fördern`; etw. an das liecht bringen $\mathrm{o}$. ä. (dazu bdv.: an den tag bringen); jn. / etw. bei liecht anseben / beschauen o. ä. >etw. bei Licht besehen<. Bdv.: vgl. glinster, tag 2, tagheitere. - Synt.: das l. erkennen / hassen / veraimmern >den Lichteinfall vom Nachbargrundstück her verbauen<, das l. nicht seben können, jm. das l. verbauen; das l. (Subj.) anbrechen / sterben, jn. an- 
scheinen; etw. bei l. [tun], das gerücht ans l. herfür treten; das l. des tages; das natüliche / rechte / stechende l.; glinster des liechtes. Wbg.: liechtbärend 2, liechtbrate >Schmaus zu Beginn des langen Abends`, liechten 1 >hell werden (vom Tag)<, 'liechtflieher, liechtflüchtig ' (s. u. jeweils MAALER), liechtgans >zum Michaelistag verzehrte Gans`, liechtgerade >so gerade, als ob man am anderen Ende das Tageslicht sehen könnte; völlig geradeく (a. 1582/ 5), liechtgerechtigkeit (a. 1546), liechtläuten >Glockenläuten zur Abend- und Morgendämmerung`, liechtmücke sowohl >Glühwürmchen` (dazu bdv: vgl. gleim 2) wie $>$ lichtsuchende Fliege<, liechtrecbt (a. 1483 f.), liechtscheu, liechtscheuend, liechtschnake (dazu bdv.: vgl. liechtmücke, nachtmückee).

SchÖpper 33a (Dortm. 1550): Notum facere. Offenbarn eroffnen verkinden kundt thun zu erkennen thun ansagen $q u$ wissen thun ans liecht bringen an den tag legen. LUTHER, WA 6, 321, 19 (1520): wer ubel handelt, der furcht das liecht. EBD. 33, 214, 15 (1531): unsere Rottengeister, wenn man sie bey dem liecht ansibet, leren nur allein von guten Wercken. EBD. 283, 10: was machet jr anders, denn das jr euch selbs im liechte stehet? EвD. 41, 421, 24 (1535): qui per oppera volunt bimel verdienen, Juden, Turcken cum suis rc. Si beym liecht, sinds buchstaben in corde. EBD. 50, 193, 194, 2 (1538): weil die liechtflüchtigen und tagschewende schelmen so jemerliche mühe haben, [...7. Volkmar 109 (Danzig 1596): Nitedula, eine licht muck / die des nachts leucht. Pfeffere, Weigel. Gn. S. 57, 25 (um 1571, Hs. 1615): Anfenckhlich schueff gott das licht, das ist die Engl, am ander Tage machte er das furmament. EBD. 90, 22: Manicher wirdt geboren zum Juristen, vnd wirdt ein Medicus. Jezt ist das liecht der Nattur verrukht. PeIL, Rollenhagen. Froschm. 513, 216 (Magdeb. 1608): Jhe ehr / vnd mehr es [Gerucht] sich außrecket / | [..] Vnd tritt obn schew herfur ans Liecht. EBD. 538, 990: JNsonderbeit las man zu Hauß / | Die Liechtschewende Fledermauß. Ingen, Zesen. Ros. 80, 17 (Hamb. 1646): weil auch stirbt das schone licht / | und die lange nacht einbricht. QuINT, Eckharts Pred. 1, 166, 7 (E. 13./A. 14. Jh.): der êrste umbelouf des himels dâ von ist ez tac. Dâ geschibet in einem nûu der sêle tac, und in irm natiurlîchen liebte, dâ alliu dinc inne sint, dâ ist ein ganzer tac. Loesch, Kölner Zunfturk. 1, 190, 4 (rib., 1293): geordenirt, dat die schorre mit lithe scheren solen van s. Andreas dage biz crissenath. CHRON. KöLN 2, 6v, 28 (Köln 1499): got sprach Dat licht werde. [...]. dat is eyn sunderlinge schijmberliche off clair wolck. die welche durch eyn vmblouff macht dach vin nacht. SkÁLA, Egerer Urgichtenb. 75, 9 (nwböhm., 1571): Die Khue Habe er [...] dem Michael Kieser Zwuschen den lichten gestolen. ERMISCH, Sächs. Bergr. 17, 24 (osächs., Hs. 15. Jh.): das derselbe buiwer
[... mit suwerre erbeit ercz irvolget yn eyme erben adir in lebenen [...] unde brenget das czu lichte. Fischer, Folz. Reimp. 46, 90 (Nürnb. 1479/80): die funft [fül >Trunkenheit], so man die lichtgans ist. Gilman, Agricola. Sprichw. 1, 554, 19 (Hagenau 1534): Eyn blinder kan den tag und das liecht nit seben. DAsYPODIUS 79v (Straßb. 1536): Lucifuga Ein liecht scheüch. EвD. 147v: Nitedula, ein liechtschnack, der zü nacht scheinet. Roloff, Brant. Tsp. 719 (Straßb. 1554): Ich gib dir das liecht [dochter] das ich ye gewan | Du solt sie zu einer gemahel han. JörG, Salat. Reformationschr. 251, 5 (halem., 1534/5): wurdend jnn underm liecht binweg füren. KopPITz, Trojanerkr. 5821 (Hs. ' noschweiz., 15. Jh. $\left.{ }^{\top}\right)$ : Der künsche belffenrich tegen | An ainem liechtberenden tage| Tett uff daz veld widersage. Grimm, Weisth. 6, 375, 45 (halem., 1555?): Es solle keiner befügt sein, ein neües gebeüw [...] aufzuführen, [...] wan andurch dem nachbaur [...] das liecht und prospect verbauet würd. EBD. 6, 134, 42 (oobd., 1402/70): wenn er dann di selb nuz und gewer übersizt ân alle ansprach zwo vinster und dreu liecht, da wider schol [...] weder prief urkund noch weisung nichz belfen [vgl. GRIMM, Rechtsalterth. 190, 557]. MAALER 272v (Zürich 1561): Liechtflieher / Liechtflüchtig / Der das liecht fleücht vnnd hasset. Lucifugus. EBD.: Liechtmugk. (die) Ein besondere mugk in Jtalia / scheynt zu nacht im fliegen zu $d^{\prime}$ zeyt der ernd. Cicindela. Liechtmugke. oder nachtmugk / die zu nacht in das liecht fleügt. Lampyris. Barack, Zim. Chron. 2, 416, 23 (schwäb., M. 16. Jh.): als die büchsenschützen daselbst gewonn sein, iren liechtbraten, das ist, so das järlich schiessen ain ende, zu herbstzeiten zu baben. BRÉvART, K. v. Megenberg. Sphaera 50, 25 (noobd., 1347/50): wir sprechen des morgens, so ez libt, ez sei tak. Spiller, Füetrer. Bay. Chron. 210, 29 (moobd., 1478/81): Ich muest mein not ye auch hierinn zu liecht pringen. Bischoff u. a., Steir. u. kärnt. Taid. 423, 6 (m/ soobd., 1562, Hs. 1735): das kein burger bei nächtlicher weil des abents nach liechtleuten biß auf morgen auch vor liechtleuten mit keinem liecht noch feier in kein fuetergeheuß kommen. РАтоскA, Salzwesen. 1987, 108 (oobd., 1582/5): durch ainen schenen liecht geraden Schurff gefahren. - OpITz. Poeterey 45, 20; Gille u. a., M. Beheim 288, 333; Fuchs, Murner. 4 Ketzer 241; Chron. Augsb. 1, 226, $27 ; 8,411,1$; Baptist-Hlawatsch, U. v. Pottenst. 326; McClean, Havich 3874; Fichtner, Füetrer. Trojanerkr. 485, 5; Volkmar 408; Dict. Germ.-Gall.-Lat. 307, 8; SAchs 13, S. 335; Rwв 8, 1290; 1294 f. (bezüglich des Rechtes auf Lichteinfall). - Vgl. ferner s. v. anbrechen 1, anbeben 1, anscheinen 1, argwönig 2, austilgen 4 , bedecken 1 .

6. >Licht, Helligkeit, die von Himmelskörpern ausgeht<. - Phras.: das angehende / neue / junge / zunemende liecht >Neumond <; das alte liecht $>$ Vollmond $<$. Bdv.: schein; vgl. glanz 1, stral. - Synt.: das l. sehen, l. von der sonne empfangen, der mond sein l. haben, die sonne das l. benemen; die liechter am himmel leiden >gehen ;; nach dem neuen l. pfropfen, der stern mit l. 
durch die wolken brehen; das l. des mondes, der sonne, der himmel; das klare l.; motus der liechter. Wbg.: liechten 2 >etw. (den Himmel) erleuchten<, liechtstriemel $>$ Lichtstrahl .

PeIL, Rollenhagen. Froschm. 682, 5516 (Magdeb. 1608): Wenn sie [Krebse] auch jrgend im finstern gehen / | Weder Sonn / noch des Mons licht sehen. Dubizmay, kurß zu Teutze 32, 17 (hess., 1463): Lobt in [heren] Sünne und monde lobt in alle sterne vnd licht. ScHмiDT, Frankf. Zunfturk. 1, 77, 30 (hess., 1586): sollen die balbirer uff die täg, uff welche nach gewönlicher außrechnung der gelehrten im liecht und zaichen nit gut ist, ader zu lassen, keine laßbinden außhencken. Sснмiтt, Ordo rerum 11, 30 (omd., 1466): Radius [...] liech streym [...] licht strimel [...] sicut rady solaris. Jungbluth, J. v. Saaz. Ackermann 14, 26 (Hs. ' ${ }^{\circ}$ omd., 1465`): Als wenig du kanst der sunnen ir licht, [...] oder dem wasser sein nesse benemen, als wenig magstu uns unser macht berauben! ERMisch u. a., Haush. Vorw. 91, 9 (osächs., 1570/7): setze sie [Huhn] mit dem angehenden neuen licht uber, so brüttet sie einen monat aus. EBD. 255, 34: säe 3 tage vor oder nach dem neuen licht waizen. WYss, Luz. Ostersp. 3, 79, 28 (Luzern 1597): Die liechter lydent allso schlimm | Am Himmel. Dict. Germ.-Gall.-Lat. 307, 24 (Genf 1636): Jung (liecht) n. Nouuelle lune. EвD. 26: Alt (liecht) n. Vieille lune, Vetus luna. EBD. 28: zunemmendt (liecht) $n$. Le croissant de la lune. Luna crescens. STEER, Schol. Gnadenl. 1, 11 (noschweiz., 15. Jh.): als der luft liecht enpfachet von der sunnen, also wirt die sele erlúcht von gótlicher gnäd. BRÉvART, K. v. Megenberg. Sphaera 59, 4 (noobd., 1347/50): Seit nu der mone kain aigen lieht hat. Pfeiffer, K. v. Megenberg. B. d. Nat. 62, 10 (oobd., 1349/50): daz er [morgenstern] ze mettenzeit gar mit klârem liebt durch die wolken her prehet. SPechtLer, Mönch v. Salzb. 26, 12 (oobd., 3. Dr. 14. Jh.): Iedoch du mit gestirn | unerzalt künikleich zirn, | in liechtes monen schein | liechten tuest himmel dein. - Bechstein, M. v. Beheim. Evang. Mk. 13, 24; Ermisch u. a., a. a. O. 100, 20; M. CuniTia. Ur. Prop. 155, 35; Eichler, Ruusbr. obd. Brul. 2, 1800. - Vgl. ferner s. v. adler 1, angelstern, auge 8, impfen 1.

7. >Sehkraft (der Augen)<; ütr.: >Erkenntnisfähigkeit und -tätigkeit des Menschen〈; dazu metonymisch: `Erkenntnis als Resultat der Erkenntnistätigkeit $\iota$, jeweils als Helligkeit, Licht gesehen, nur vereinzelt als innerweltliche, innernatürliche Größe gedacht, häufiger unter religiöse Aspekte gestellt. - Bdv.: vgl. augenstern, augsehe, begreiflichbeit 1, begreifung 3, begriffenheit, gemerke 3, gesehe, gesicht 4, mächtigkeit 4. - Ggs.: blindheit 2, finsternis; vgl. hindernis 4. - Synt.: das l. (der augen) verlieren, der vernunft ein l. setzen; das l. (Subj.) im menschen brennen; jn. des liech- tes (der augen) berauben; das l. des gemütes, der erkentnis / sele / natur / vernünftigkeit / warbeit; das falsche / innerliche / natürliche l.; die böhe des liechtes.

Luther, WA 10, 1, 2, 224, 19 (1522): Darnach [nach dem kreuchen ... ins Euangelion] kumpt ein ander liecht, ein ander entpfinden, eyn ander sehen. EBD. 17, 2, 102, 10 (1525): das liecht lebet und leuchtet ynn aller menschen vernunfft. PEIL, Rollenhagen. Froschm. 304, 1340 (Magdeb. 1608): wie die Sonn hilfft dem Gesicht / I So ist die Kunst der Seelen liecht. EBD. 407, 4559: wie die Sonn erleucht die Sternen $/|[. .]$.$| So seţt MENS der Vernunfft sein$ Liecht / | Darnach sie die Abmessung richt. Pfefferer, Weigel. Gn. S. 170, 11 (Magdeb. 1615): Also brennen drey Liechter im menschen / also seyn drey Doctrina im menschen / also in den dreyen ist der mensch perfect, vnd dieweil das ist / daß die untersten zimo / eine Finsternuß seyn gegen der dritten obersten / so seynd sie doch Liechter der Welt / in denen der mensch naturlich Liechter halben wandeln soll. MIEDER, Lehmann. Flor. 722, 22 (Lübeck 1639): wer der Vnwarbeit gewohnet / der kan der Warheit Liecht nicht als bald vollkómmlich sehen. STEER, W. v. Herrenb. Büchl. 123 (pfälz., 1436): die wirckend crafft der verstentnis. Die selb crafft glichet Aristotiles zu eynem liechte. $N u$ ist küntlich, das das liecht der sonnen schyne gibt allem dem, das jm tage gesehen wirt: dauon auch alle erkentnis der vernonfftikeit erspringt und sinen gründlichen anfangk hat von dem jnnerlichen liechte der sele, das warelichen leret und die warbeit öffenet. PEREz, Dietzin 1 316, 11 (Frankf. 1626): Die Dámpff vnd Nebell / so von dem Wein vber sich steygen verfinstern nicht allein Gesicht / sondern zugleich auch das Liecht des Gewuibts. Strauch, Par. anime int. 64, 6 (thür., 14. Jh.): daz licht der sonnen ist cleine wider deme lichte der fornuftikeit. BECHstein, M. v. Beheim. Evang. Mt. 6, 23 (osächs., 1343): ob daz lîcht daz in dir ist, di vinsternisse sint, wie grôz werden danne di vinsternisse selbir? WAGNeR, Erk. Ps.-J. v. Kastl 4, 69 (nürnb., 1. H. 15. Jh.): so der mensch also von im selbs schaidet, so kan er kein liecht der eygen erkentnuße in im baben. Harsdoerffer. Trichter 3, 925, 17 (Nürnb. 1653): Das Liecht hat die Deutung der Warbeit und werden auch vorneme Leute mit demselben verglichen / welche die Warbeit erkennen und andre erleuchten. VetTer, Pred. Taulers 406, 3 (els., E. 14. Jh.): das die heiden und die juden in iren wisen iren dingen vil rehter tint danne wir, noch irme naturlichen lichte, die doch alzumole in der blintheit sint. GOLDAMMER, Paracelsus 2, 423, 13 (1532/4): daß ich [...] das liecht der natur und beider, göttlicher und natürlicher, philosophia volkombene erkantnus hab. SAPPLER, H. Kaufringer 9, 68 (schwäb., Hs. 1464): si sprutzt in under die augen sein, das im vergieng des liechtes schein. EsCHENLOHER. Medicus 35, 21 (Augsb. 1678): ward er alsobald [...] deß Liechts seiner beyden Augen beraubt. Bauer, Zist.-Pred. Haller 45, 98 (tir., 1466): Der da nicht hat ain raines hercz, [...], der hat auch verlorn das liecht seiner angen. - STRAUCH, a. a. O. 138, 8; zu DohNA u. a., Staupitz/Scheurl 17; Vetter, a. a. O. 250, 5. - Vgl. ferner s. v. astronomei. 
8. >abends auf der Gasse gesungenes Lied, Gassenhauer`; zur möglichen Motivation s. den 2. Beleg! Die Schreibung lychter neben liecht läßt wie die Semantik ein eigenes, in den Wörterbüchern nicht gebuchtes Wort unklarer Zeichengestalt vermuten. Bdv.: vgl. gassenhauer, tanbäuser.

Turmair 1, 542, 12 (Augsb. 1517): poema lyricum: de variis rebus leviusculis [...]: ,ode, gesang, liecht, lychter, gassenhawer, die man auf der lauten schlecht'. EBD. 4, 255, 14 (oobd., 1522/33): das man auch von denen, die unrecht und pöse stuk, tuen, [...], lieder macht, dieselbigen [...] bei der nacht offenlich auf den gassen vor den heusern säng, so man das liecht aufkent het, darumb man dan auch solche gesäng, liechter' und ,liechtl' nent.

\section{9., s. liechtmesse.}

liecht, Adj., meist auf die Bezugsgrößen und -personen von liecht (das) bezogen, daneben zur Kennzeichnung einer offenen Menge anderer Bezugsgegebenheiten.

1., s. liecht (das) 1.

2. >aus, in göttlichem Licht strahlend; religiös verklärt, erleuchtet‘; vgl. liecht (das) 4. - Texte der Sinnwelt ,Religion / Didaxec. - Bdv.: vgl. gotbildig, gotförmig 1, klar 7, verklärt. - Synt.: das kreuz l. scheinen; der liechte brautgebe (ütr.), der liechte engel / schein (Gottes, der Engel), der liechte > visionäre s schlaf, die liechte >verzückter anstarrung, die liechten beiligen; das liechte wort. Wbg.: liechtglänzend (Beleg s. v. aufsteigen 3).

Asmussen, Buch d. 7 Grade 1104 (nobd., Hs. A. 15. Jh.): Dir ist zu hoh mein [got] libter schein. EBD. 1949: Wer kan gesagen den libten schein, | mit dem die engel geklaidet sein. Bihlmeyer, Seuse 188, 7 (alem., 14. Jh.): in einem lichten schlafe waz im vor gar bescheidenlich, wie [...]. - LuTHER, WA 41, 720, 7; Froning, Alsf. Passionssp. 7930; Williams u.a., Els. Leg. Aurea 7, 13; Bauer, Haller. Hieronymus-Br. 61, 3. - Vgl. ferner s. v. anstarren, aufsaz 3, aufsteigen 3.

3. >hell (von den Lichtverhältnissen des Tages)<; zu liecht (das) 5. - Phras.: der liechte (im Ggs. zu: finstere) tag >Tag (im Ggs. zu Nacht)<. - Bdv.: ${ }^{1}$ bel 3.

Luther, WA 6, 38, 30 (1520): ßo sol gott blenden [...], die seyn heyliges liechtes wort zur finsterniß machen. Grosse, Schwabensp. 213a, 33 (Hs. rnd./md., um 1410?): dat solen se tün offenbare vnde bi di lichten tage vor tïre vor offener straze. Quint, Eckharts Pred. 1, 133, 5 (E. 13./A.
14. Jh.): Daz sie [engel] got bekennent, als er aleine in im selben wesen ist, daz ist der liebte mittac. LOESCH, Kölner Zunfturk. 1, 99, 31 (rib., 1397): en solen si [meister] aventz, neit mit kerzen wirken noch morgens dan mit dem lichten, schoenen dage. Gilman, Agricola. Sprichw. 1, 175, 32 (Hagenau 1534): Liechter tag / liechte augen. Hiemit entschuldigen sich die Finantzer / welche den leutten [...] ein nase machen / [...] / Sie haben es gesehen / es sei liechter tag / warumb hat er die augen nicht auffgethon. BARACK, Zim. Chron. 2, 636, 37 (schwäb., M. 16. Jh.): der pfaff ist etlich stund also an der stangen offenlichen, helles, liechtes tags gehangen. Munz, Füetrer. Persibein 56, 7 (moobd., 1478/84): ich läg euch pey piß an den liechten morgen. SIEGel u. a., Salzb. Taid. 7, 15 (smoobd., 17. Jh.): so soll er [...] die pfand nemmen und bebalten, nemblichen essende pfand zwo finster und drei liecht, schreinpfand vierzëhen tag. - Schützeichel, Mrhein. Passionssp. 324; Chron. Köln 1, 968; Vetter, Pred. Taulers 172, 13; Klein, Oswald 101, 2; Spiller, Füetrer. Bay. Chron. 64, 1; Bischoff u. a., Steir. u. kärnt. Taid. 213, 4. - Vgl. ferner s. v. anzeigen 3.

4. >hell, leuchtend/; vom Licht der Himmelskörper, Wettererscheinungen, metaphorisch von religiös überhöhten, verklärten Personen gesagt; vgl. liecht (das) 6. - Ggs.: dinster, dunkel. - Synt.: der blik >Blitz< liecht sein; der liechte himmel / (morgen)stern, die liechte kläre / scheibe / sonne, die liechten beiligen, das liechte gewolken.

LuTHER, WA 22, 388, 1 (1544): ein Erbteil der Heiligen im Liecht oder der Liechten heiligen, Das sind die rechtschaffenen Heiligen. EBD. 34, 2, 126, 29 (1531): Ein heiliger Marterer [...] wird [...] gen Himel faren als ein heller, liechter Herrlicher Stern. STRAUCH, Par. anime int. 130, 33 (thür., 14. Jh.): alse einen grozin blic, der licht ist und aber dinster. Gille u. a., M. Beheim 3, 157 (nobd., 2. H. 15. Jh.): Ob ir wolt wissen, wo das licht gewolken kem: I man maint, got lies die sunnen daraus werden. EBD. 82, 144: Ainn stern, den sie mit libter cler $\mid$ auss vil zaichen sahen. BIHLMEYER, Seuse 469, 12 (alem., 14. Jh.): der liebte bimel machet die anbeis kriechen und den geswinden hirtz. loffen. Martin, $\mathrm{H}$. v. Sachsenh. Tempel 80 (schwäb., 1453): Maria, liechter morgenstern, | Durchglest min bercz das dunckel! - DüNNHAUPT, Werder. Gottfr. v. Bullj. 22, 28; Gleinser, Anna v. Diesb. Arzneib. 1989, S. 183; Chron. Augsb. 7, 356, 12. - Vgl. ferner s. v. ${ }^{2}$ auf 5, aufdringen 2, bedekkung 2, bedeutnis 5 , beginnen 6 .

5. > einleuchtend, erkennbar<; vgl. liecht (das) 7. - Bdv.: vgl. ansichtig 1, augenscheinlich 2, angensichtig, ${ }^{5}$ bar 2, ${ }^{1}$ bärlich 2, gesichtig 1, greiflich 2.

Dubizmay, kurß zu Teutze 11, 5 (hess., 1463): sein gebott ist licht und | erleuchtet die augen. JosTES, Eckhart 16, 32 (14. Jh.): Was ist ein uaflur? dar. ist ein beheglicheit seines 
willen mit eim lihten underscheid. SPILLER, Füetrer. Bay. Chron. 130, 3 (moobd., 1478/81): umb des willen will ich nämlich nachvolgen dem liechteren und vallen lassen das tunckler. - Bihlmeyer, Seuse 468, 27.

6. von Gegenständen verschiedenster Art, seltener auch von Personen gesagt, die tropisch oder bildlich mit Helligkeit, Licht als ihren idealen Eigenschaften assoziert werden können; im einzelnen z.B. a) >strahlend, hell (von den Augen)<; >rot leuchtend (von den Wangen) $<$; auch auf Personen, ihre Kräfte und Eigenschaften sowie auf die Erscheinungsweise von Personen, darunter des Teufels, bezogen, dann auch: >gleißend<; insgesampt dicht, teils formelhaft belegt. - Bdv.: fein, ${ }^{1}$ bel 4, klar (Adj.), scheinig, schön. Ggs.: dunkel. - Synt.: kräfte l. werden; der liechte glanz / schein / teufel, die liechte brünne / farbe, das liechte har, die liechten augen; etw. l. gemal (sein). Wbg.: liechtbrinnend >hellglänzend (von den Augen) <, liechtgrau, liechtheiliger, liechtpriester (beide Bildungen auch zu liecht, das, 4 stellbar. - b) >glänzend (von Waffen, Rüstungsteilen)<; schwach belegt. - c) > hell, licht (von Naturgegebenheiten verschiedener Art)<. - Bdv.: ${ }^{1}$ hel (Adj.) 3; 4, schöne. - Synt.: die natur l. sein; der liechte sommer, der liechte schein des meien, die liechte aue / wolke. - d) >farbenprächtig, bunt (von Blumen)<. - Synt.: der liechte glan₹, die liechte farbe, die liechten blumen. - e) >hell, leuchtend, strahlend (von Mineralien, Edelsteinen) $<$; dicht und teils formelhaft belegt. - Bdv.: fein, klar (Adj.) 5, weinfar. - Synt.: nitrum, der stein l. sein; der liechte rubein / stein / jochant. - f) >durchsichtig, klar (z. B. von der Brille, der Luft)<. g) >hoch aufragend, zum Himmel ragend (von Galgen) <. - h) ferner z. B. von Büchern, Häusern, Feuer, Tuchen, Metallen, auch von Personen gesagt. - Wbg.: liechtbraun, liechtrot.

$\mathrm{Zu}$ a): Luther, WA 24, 86, 7 (1527): die weisse farbe des Teuffels, denn er ist ein beller, liechter Teuffel, der [...]. Karnein, Salm. u. Morolf 111, 4 (srhfrk., Hs. um 1470): du [franwe] fellest uff die erde fur dot, I dine vil lichte farwe | die ist dannoch unverwandelt. KoppITz, Trojanerkr. 18421 (Hs. ' noschweiz., 15. Jh. '): Da₹, waren ritter liechtt gemal | Von crippel, wirtzall, zendal. BRANDSTETTER, Wigoleis 221, 24 (Augsb. 1493): fraw Laneyt [...] rauffet auß jr lyechtes har vnd schry mit klaeglicher stimme. PFEIfFer, K. v. Megenberg. B. d. Nat. 158, 9 (oobd., 1349/50): wenne ez. [aichorn] gar liehtgrâw ist, sô ist ez, vêch. EBD. 185, 7: sein [vogel] augen seint liehtprinnend in dem haupt. EBD. 280, 18: wan si [sternslang] hât auf irm ruk liebtgemâlt augen sam die stern. Hohmann, H. v. Langenstein. Quästio 195, 217 (moobd., 1. H. 15. Jh.): dye [chrefft] mit encziger ïebung wüerden liecht vnd scheinig. KLEIN, Oswald 98, 26 (oobd., 1430): Vil zarter, engelischer weib, | durchleuchtig schön, mit liechtem glan₹. | besessen haben meinen lib. SPILLER, Füetrer. Bay. Chron. 94, 19 (moobd., 1478/81): Er merckt an ir, das ire liechte augen stät voll träher waren. TuRMAIR 5, 151, 25 (moobd., 1522/33): [Er] het ain runden kopf, grosse liechtgrâbe augen nach der Teutschen art gehabt. Jungbluth, J. v. Saaz. Ackermann 20, 26; Klein, a. a. O. 21, 59. - Vgl. ferner s. v. amis 1, anblik 1, angesicht 2, augapfel. - Zu b): Karnein, Salm. u. Morolf 389, 2 (srhfrk., Hs. um 1470): ich wil dich mit dem liechten stabel wol bewarn. EBD. 491, 3: er sprach: ,nu bindent uff die belme liecht'. Koppitz, Trojanerkr. 2165 (Hs. ' noschweiz., 15. Jh. $\left.{ }^{\urcorner}\right): \operatorname{Der}$ [name] zü dem schwertt licht gemal | Hörtt. - Weber, Füetrer. Poyt. 290, 4. - Zu c): BECHSTEIN, M. v. Beheim. Evang. Mt. 17, 5 (osächs., 1343): dô her noch mit ime redete, sebt ein lîcht wolkin beschetewite si. Thiele, Minner. II, 14, 107 (Hs. 'wobd., 15. Jh. ') : wer denn hät gesamelt in $\mid$ inder liechten summer zitt. KLEIN, Oswald 102, 2 (oobd., 1427 ?): Sich manger freut das lange jar $\mid$ gen des liechten maien schein. - LutHeR, WA 10, 1, 2, 298. - Vgl. ferner s. v. ${ }^{1}$ aue 4. - Zu d): KLein, Oswald 2, 15 (oobd., 1421): plümlin auf der haid, | den geit er [herre] farb und liecbten glan \%. - Vgl. ferner s. v. ${ }^{1}$ anger 1, ausreuten 1, baum 1. - Zu e): Belkin u. a., Rösslin. Kreutterb. 166, 10 (Frankf. 1535): Nitrum ist zu erwelen der do liecht ist / weiß oder rot. KLEIN, Oswald 13, 20 (oobd., 1416?): recht als der liecht rubein | an pein pringt schein durchsichtig vein. - BELKIN u. a., a. a. O. 100, 7; 13; Kummer, Erlauer Sp. 3, 137. - Vgl. ferner s. v. balas, jochant. - Zu f): Lemmer, Amman/Sachs. Ständeb. 57, 2 (Frankf./M. 1568): Jch mach gut Brillen / klar vnd liecht. Pfeiffer, K. v. Megenberg. B. d. Nat. 207, 22 (oobd., 1349/50): ain iegleich tier fräut sich des liebten lautern luftes mêr denn des trüeben. - Zu g): Luther, WA 32, 84, 22 (1530): bencke sie [gute werck] viel eher an den liechten galgen unter die stinckenden diebe. v. Keller, Ayrer. Dramen 765, 25 (Nürnb. 1610/8): Ey, du Narr, pack dich an liechtn Galgn! - SACHS 14, 294, 28; Chron. Augsb. 2, 317, 16; Dienl, Dreytw. Essl. Chron. 30, 30. - Zu h): Ingen, Zesen. Ros. 94, 8 (Hamb. 1646): die du mit eignen fingern / | den kleinen berzens=zwingern / I ins lichte buch der angen schreibest ein. Dubizmay, kurß zu Teutze 37, 18 (hess., 1463): Dv [Maria] bist des hohen kunges thure und die lichte pfortte des lichten goldes. KARNEIN, Salm. u. Morolf 8, 3 (srhfrk., Hs. um 1470): Ein felle drug die kunigin, $|[\ldots]|$ sie was von edelm gestein unmassen liecht. Lauchert, Merswin 12, 11 (els., 1352/70): vnd 
wart gefüret in ein gar ziu mole schónes liebtes minnencliches bvs. VetTer, Pred. Taulers 369, 3 (els., 1359): als von vil kolen ein gantz, gros für wirt und slecht ein lichte flamme uf. Golius 406 (Straßb. 1579): Flammens, liechtrot. BAstian, Runtingerb. 2, 49, 27 (oobd., 1383): Und sol chauffen 5 samat, ein liehtgrin, ein liehtpraun, 1 satplaben. Dict. Germ.-Gall.-Lat. 307, 36; Bauer u. a., Kunstk. Rud. 244. - Vgl. ferner s. v. abbellen, auslugen 2.

liechtarbeit, s. liecht (das) 1.

liechtbäre, s. liecht (das) 4.

liechtbärend 1;2, s. liecht (das) 4; 5 .

liechtblau, Adj.

> hellblau<; vgl. liecht (Adj.) 6. - Bdv.: vgl. bimmelblau.

LipPert, UB Lübben 2, 231a, 13 (osächs., 1525): ein elle lichtblaw vor 7 ar. Pfeiffer, K. v. Megenberg. B. d. Nat. 457, 21 (oobd., 1349/50): der stain ist himelvar, wan er ist liehtplâ. - Henisch 409; Matzel u. a., Spmal. dt. Wortschatz. 1989, S. 191; Ösт. Wв. 3, 324.

liechtbotzen, s. liecht (das) 2.

liechtbrate, s. liecht (das) 5 .

liechtbraun, s. liecht (Adj.) 6h.

liechtbrehend, part. Adj.; Gw zu brehen. > hell strahlend, leuchtend <, in beiden Belegen innerhalb des Preises einer Frau (auch: Marias); vgl. liecht (das) 4; 6.

Jungbluth, J. v. Saaz. Ackermann 5, 6 (Hs. 'omd., 1465 $\left.{ }^{\urcorner}\right)$: Nicht mer geet auf mein lichtbrehender morgenstern. Pfeiffer, K. v. Megenberg. B. d. Nat. 412, 30 (oobd., 1349/50): si [unser fraw] ist ain liehtprehenden veltpluom, wan si stêt an der strẫ der gnâden.

liechtbringerin, s. liecht (das) 4.

liechtbrinnend, s. liecht (Adj.) 6a.

liechtbutze(r), s. liecht (das) 2.

liechtekasten, s. liecht (das) 1.

liechten, V.

1.; 2., s. liecht 5; 6.

3. >jn. belehren, jm. klare Einsicht geben<; vgl. liecht (das) 7. - Bdv.: vgl. beleren, ${ }^{2}$ leren 2.

Plant u. a., Main. Naturl. 294rb, 6 (ohalem., Hs. E. 14. Jh.): wonde aber niemã mich liebte noch nibt verstat so wil ich ein bizeichen setzin.

liechterform, s. liecht (das) 1.

liechterloh, (auch -lohen), Adv.

>lichterloh, in hellen Flammen`, vgl. liecht (das) $1 ; 2$, lohe.
Helm, H. v. Hesler. Apok. 359 (nrddt., 14. Jh.): Unse alde vater Moyses $\mid$ Den pusch sach brinnen liechterluon. LuTHER, WA 49, 486, 31, (1544): Das sehen wir, sie ligen schon im feuer, brinnen liechter lohe, wie Sodom. Harms u. a., Alberus. Fabeln 157, 17 (Frankf./M. 1550): das wie strob | Ein gantze Stadt brennt liechter loh.

liechterweihe, s. liecht (das) 1.

liechteweihe, s. liechtweihe.

liechtfar(be), vereinzelt: liechtgefar, Adj.

>strahlend, leuchtend, glänzend, hell (von religiösen und weltlichen Bezugsgegebenheiten und -personen zur Kennzeichnung von deren besonderer Qualität, vereinzelt zur Kennzeichnung heller Farbtöne); vgl. liecht (das) 4; 5; 6; 7, liecht (Adj.) 3; 4; 6. - Synt.: der gagates, die wängel l. sein, $j$ s. grund / leichnam l. werden, js. antlit l. rot sein; l. als ein jazint.

FISCHER, Brun v. Schoneb. 5590 (md., Hs. um 1400): di lilie wiz von kuscheit, | lichtvar an werken so man seit [von Maria gesagt). Bintmeyer, Seuse 224, 17 (alem., 14. Jh.): $\min$ [der ewigen Weisheit] ogen sint so klar, [...], minú wengel so liebtvar und so rösenröt. VETTER, Pred. Taulers 376, 10 (els., 1359): von dem blicke [des gotvarwen liecbte(s)] so werdent die menschen als durch lücbtet und wirt ir grunt als liechtvar. KoppITz, Trojanerkr. 3374 (Hs. 'noschweiz., 15. Jh. $\left.{ }^{\urcorner}\right)$: Daz ain so liecbtt farwer mund |Sol ainem valschen werden gegeben! Евр. 11916: yltt er dar | Do er der herren waz gewar, | Rich und liechtt gevar, | Worden. PÄPKE, Marienl. Wernher 5831 (halem., v. 1382): Ain wenig brait und liecbvar, $\mid$ Als ain jacintte schóne gar. PFEIFFER, K. v. Megenberg. B. d. Nat. 447, 12 (oobd., 1349/ 50): Gagates [...] ist zpairlai: swarz, und liebtvar. iedoch ist der liebtvar zwairlai: ainer weiæ, der ander gel. - ВӧнмЕ, Morg.R. 157, 11; Koppitz, a. a. O. 2034; Rieder, Gottesfr. 77, 80; Dict. Germ.-Gall.-Lat. 307, 43. - Vgl. ferner s. v. ange 8 .

liechtfas, s. liecht (das) 1.

liechtflieher, liechtflüchtig, s. liecht (das) 5.

liechtform, liechtfutter, s. liecht (das) 1.

liechtgans, s. liecht (das) 5 .

liechtgarn, s. liecht (das) 1.

liechtgefar, s. liechtfar(be).

liechtgehen, s. liechtstube. 
liechtgeld, s. liecht (das) 1.

liechtgerade, liechtgerechtigkeit, s. liecht (das) 5 .

liechtglänzend, s. liecht (Adj.) 2 .

liechtglocke, s. liecht (das) 1.

liechtgrau a; b, s. liecht (Adj.) 6a.

liechtgrün, Adj.

>hellgrün (von unterschiedlichen Bezugsgrößen)<; vgl. liecht (Adj.) 6 .

BELKIN u. a., Rösslin. Kreutterb. 118, 9 (Frankf. 1535): Der steyn Chrisolitus ist an der farb dünn und liecht grinn. Ott-Voigtländer, Rezeptar 206v, 15 (Hs. 'nalem., um 14007): dunket / jn [menschen] die spaichel gesalcren, und ist lieht grün / vnder den oggen. - Vgl. ferner s. v. liecht (Adj.) 6h.

liechtheiliger, s. liecht (Adj.) 6a.

liechthocke, liechtholz, s. liecht (das) 1.

liechtig, liechtigkeit, s. liecht (das) 4.

liechtkämmerer, liechtkelter, s. liecht (das) 1.

liechtkerze, s. liecht (das) 2.

liechtkoche, liechtkolbe, liechtleiher, s. liecht (das) 1.

liechtloch, das; -/-er + Uml.

enger Schacht, der von der Erdoberfläche auf einen Grubenbau der Wetterlösung halber niedergebracht wird; vgl. am ehesten liecht (das) 5. - Omd./ Östliches Inseldt.; bergbauliche Texte. - Synt.: ein l. fertigen / machen / sinken; aus dem l. einen durchschlag machen; das wetterische l.

ERmisch, Sächs. Bergr. 27, 1 (osächs., Hs. 15. Jh.): Hebet er syne wassirseyge uff adir fertiget syne lichtlócher. WUTKE, Schles. Bergb., Cod. Sil. 20, 248, 27 (schles., 1528): Sobald aber die gewerken, denen ihr ertz ausgehauen wurde, aus ibrem erbschacht oder lichtlöchern in ihrem massen zu den rauborttern ein ausgezimerten durchschlag machen, die andern gewerken bergmennisch beschreyen und beleuchten, alsdann sollen die gewerken, so geraubt haben, bey der peen funfzig mark mit ihrer arbeit ablassen. WeIzsëckeR, Graupn. Bergb. 223, 27 (osächs., 1541): wo den gewerken von der Grossen Klufft zu fuderung des stolns und wettersch lichtlocher zu machen ader zu sinken nottortig, sollen si diselben uff ir gelt und uncosten fertigen. - WutKe, a. a. O. 20, 218, 21; 21, 67, 19; Pilrainen, Igl. Bergr., S. 76; Veith, Bwb. 326; JeLINEK, Mhd. Wb. 473. - Vgl. ferner s. v. ansitzen 4. liechtmacher, liechtmacherin, liechtmeister, s. liecht (das) 2.

liechtmesabend, s. liechtmesse.

liechtmesse, seltener: liechtmisse, die; $-\emptyset /-$.

>Mariä Lichtmeß (2. Febr.)<; ein kirchliches Fest (mit Weihung der Kerzen) und gleichzeitig Rechts- und Zinstermin; zu den zugehörigen Glaubensvorstellungen vgl. die Belege; vgl. liecht 2. - Bdv.: hochzeit, unser lieben frauen tag, liecht (das) 9 (Kopfbildung); vgl. liechtweibe. - Wbg.: liechtmesabend >Tag und Abend vor Mariä Lichtmeß<, liechtmestag (a. $1473 \mathrm{ff}$. ), liechtmestrunk (a. 1654), liechttag (Klammerform).

Grosse, Schwabensp. 188a, 25 (Hs. ' nd./md., um 14107): Wil ein beren sinen zins man Von deme gude Wisen, Dat sal her tù lichtmissen tìn. Thielen, Gr. Zinsb. Dt. Ord. 26, 12 (preuß., 1437/8): Der czins uff lichtmesse. Item Ronisdorff bat 54 buben czinshafftig. LUTHER. WA 10, 1, 1, 442, 8 (1522): Was die ding sind, die sie [Jesu Eltern] [...] volnbracht, wirt das Euangelium auff unßer frawen liechttag geben. EвD. 34, 1, 145, 14 (1531): Wyr beben beute an zw feyren das fest von der Reynigung Mariae odder unser lieben frauen lichtmeß. Евр. 41, 478, 8 (1535): die zeit unser frawen liecht mes, das hiessen sie stad Rom reinigen. FISCHER, Brun v. Schoneb. 11, 34 (md., Hs. um 1400): dein lichtmess ist die selben vart, | so bilf uns, keusche muter zart, | das leib und seel sein wol bewart | sneweiss nach margariten art. Stambaugh, Milichius. Zaubert. 16, 30 (Frankf./ M. 1563): Auff den tag der Liechtmeß hat man Liecbter gevweihet. Thiele, Chron. Stolle 519, 24 (thür., 3. Dr. 15. Jh.): uff montagk noch unser lieben frowen tagk purificacionis zu dutcr. lichtmesse genant. LeXER, Tucher. Baumeisterb. 21, 4 (nürnb., 1464/75): Der febrer bat 28 tag. [...] unser frawen liecht. Binlmeyer, Seuse 29, 8 (alem., 14. Jh.): An unser frowen tag zu der lichtmiss bereit er [Seuse] vorbin drie tag mit gebete ein kerzen der himelschen kindbeterin, und dú kerz. was gewunden mit drin strangen also: [...]. Williams u. a., Els. Leg. Aurea 184, 17 (els., 1362): Uon der liebtemessen, purificacio. Djse hochzit het drie namen. Sú beisset candelaria, daz ist die liebtemesse, wenne die menschen zu dirre bochzit bürnende kerczen tragent. MOSCHEROsCH. Ges. Phil. v. Sittew. 52, 11 (Straßb. 1650): so gayl und rammelig als die Katzen umb Liechtmeß immer sein mögen. Müller, Stadtr. Ravensb. 115, 29 (oschwäb., 1356): in der zit, als man uss den torgeln gat untz, an die licbtmisse, so sol man ainem grübenraiter [...] fünf phenn(in)g ze taglon geben. Henisch 213 (Augsb. 1616): Der ist ein gebertzter Mann / [...] / der ein Wolff nicht furcht umb Liechtmeß / vnd einen Bawren in der Faßnacht. Turmair 5, 402, 8 (moobd., 1522/33): und starb [...] des ersten tags im hornung, am liechtmeßabent. Pirrainen, Stadtr. Sillein 
131b, 29/30 (sslow. inseldt., 1378): VIl eyn herre weysen seynen czins man von seym gut dazyn nicht an geborn ist daz sol er ym kundigen czu licht messe. - Grosse, a. a. O. 139a, 41; Gille u.a., M. Beheim 82, 497; Dinklage, Frk. Bauernweist. 69, 23; SACHs 17, 20, 25; Chron. AugsB. 1, 130, 24; 9, 162, 11; Hör, Urk. St. Veit 110, 28; 188, 23; Drescher, Hartlieb. Caes. 110, 3; Bretholz, Liechtenst. Herrsch. 248, 34; Rwв 8, 1295 (bezüglich der Abgabetermine), Schwerz. ID. 4, 448; 14, 1210 Öst. Wв. 1, 42; 4, 315; Matzel u. a., Spmal. dt. Wortschatz. 1989, 191 f. - Vgl. ferner s. v. andeuten 2, ausdingen 1.

liechtmestag, liechtmestrunk, liechtmisse, s. liechtmesse.

liechtmücke, s. liecht (das) 5.

liechtöl, liechtpfennig, s. liecht (das) 1.

liechtpriester, s. liecht, Adj., 6a.

liechtputze, liechtputzer, liechtquerdel, liechtrame, s. liecht (das) 1.

liechtrecht, s. liecht (das) 5 .

liechtreich, Adj. - 14. Jh.; Texte der Mystik.

1. >begnadend; gottgegeben, lichterfüllt, strahlend «; vgl. liecht (das) 4. - Synt.: die rose l. werden; den geist l. bekëstigen, das gut (ütr.) sich l. in den geist ergiessen; der lichtreiche glanz. I schein, die liechtreiche gnade / ingiessung.

JosTES, Eckhart 47, 17 (14. Jh.): wurcht di lichtrich ingizzung dez obersten guts an dem geist? Daz merchent, da(z) daz oberst gut sich lichtrichlich ergeuzet in den geist, da erhebent er den geist uber sein naturlich wonstat, da er lichtrichlich bekostiget wirt. Bihlmeyer, Seuse 64, 28 (alem., 14. Jh.): Der ros [...] ward als schön und liebtrich, daz er [Seuse] den ogen grossen lust brachte. STRAUCH, Schürebrand 17, 30 (els., E. 14. Jh.): und üch enpfenglich machent des heilgen geistes inflüsse und aller liebtrichen göttelichen gnoden. - BIHLMEYER, a. a. O. 190, 18; SсHмiDT, Rud. v. Biberach 163, 18.

$>$ lichtvoll, erleuchtet, visionär unterscheidend, erkennbar ‘; vgl. liecht (das) 7. - Synt.: der liechtreiche unterscheid, die liechtreiche bekantnis / klarbeit / vernünftigkeit.

LAUCHERT, Merswin 29, 10 (els., 1352/70): daz ir alle keinen gerehten geworen liebtrichen vernúnftigen vnderscheit in vich habende sint. STRAUCH, Schürebrand 25, 10 (els., E. 14. Jh.): obe in so vil underscheides und lichtricher bekantnisse von gotte gegeben ist. EBD. 40, 25: wanne er in der gnoden und in der geschrift vil liebtriches underscheides wuste. - BIHLmeyer, Seuse 194, 25; Strauch, a. a. O. 46, 2. - Vgl. ferner s. v. aufschwingen 2.

liechtrot, s. liecht (Adj.) 6h.

liechtscheinend, s. liecht (das) 4. liechtschein, liechtscherbe, liechtschere, s. liecht (das) 1.

liechtscheu, liechtscheuend, s. liecbt (das) 5 .

liechtschirm, s. liecht (das) 1.

liechtschnake, s. liecht (das) 5.

liechtschnupfe, liechtschupos, liechtseil, liechtsorge, liechtstab, liechtstal, liechtstar, lichtstein, s. liecht (das) 1.

liechtstok, der; $-s /$ auch $-\emptyset+\mathrm{Uml}$.

$>$ Leuchter nicht näher bestimmbarer Art; Lampengehäuse; Kerzenhalter<; vgl. liecht (das) 1; 2. - Bdv.: kerzenstok, leuchter, luzerne. - Wbg.: liechtstokplappert $>$ Münze mit einem liechtstok als Prägung< (a. 1416 f.).

ENDERs, Eberlin 2, 53, 14 (Grimma 1522): das ewangelium] tzeygt in [prediger] als ein stadt auff eynem berg, vnd als ein lycht auff eynem lycht stock. Alberus $\mathrm{Kk} \mathrm{IVr}$ (Frankf. 1540): Candelabrum, leuchter / oder liechtstock. Major, Haussradt A iijv, 7 (Basel 1569): Jch müßst han liechtstóck vnd tágel | Mistwagen vnd schlegel. KurreLMEYer, Dt. Bibel 6, 115, 3 (Straßb. 1466): gold zi der mancherhant maß eins iegklichen liechtstocks [Var. 1483-1518: leuchters] und seiner lutzern: zi gleicherweys zu den silbrin liecht stocken vnd iren lutzernen. - SсHмIтт, Ordo rerum 200, 26; MaAler 272v; Schweiz. ID. 10, 1736.

liechtstokplappert, s. liecbtstok.

liechtstram, s. liecht (das) 4.

liechtstriemel, s. liecht (das) 6.

liechtstube, die.

>beleuchtete Stube als soziale Einrichtung〈; metonymisch: >Raum für Abendzusammenkünfte mit sitten- und ordnungsstörender Tendenz‘; vgl. liecht (das) 1. Wobd. - Wbg.: liechtgehen (wohl Klammerform), liechtstubete.

JöRG, Salat. Reformationschr. 237, 14 (halem., 1534/ 5): darunder die secter nit mússig giengend [...] tichend stätz umbar wie der tüfel [...] und praktiziern jr sach zu gang bringen mogen / mit predyen / uff der gassen / jn urtenen, jn liechtstuben / by versamlungen der wyber [...7. MAALER 273 (Zürich 1561): Liechtstubeten (die) Conuenticulum. WINTTERLIN, Würt. Ländl. Rechtsqu. 2, 661, 7 (schwäb., 1587): die liechtstuben sollen fürobin abgeschafft werden, doch wa etwan arme weiber uß ermanglung der liechter [...] zusamengohn wolten, solle in einem flecken ein hauß [...] erwöhlt und zwen erbare männer darzu verordnet werden, die vleissige ach- 
tung darauf geben sollen, das in solchen liechtstuben gute ordnung [...] gehalten. GeHring, Würt. Ländl. Rechtsqu. 3, 521, 13 (schwäb., 1599): soll das liechtgehen [...] bei ebenmässiger straf $10 \beta$ \& abgethon sein. - WINTTERLIN, a. a. O. 1, 554, 8; MaAler 95r; Schweiz. ID. 10, 1138 (mit ausführlicher Behandlung).

liechtstubete, s. liechtstube.

liechtstul, s. liecht (das) 2.

liechttag, s. liechtmesse.

liechttegel, liechttiegel, s. liecht (das) 1. der.

liechttrage (vereinzelt), liechttrager,

1. >Lichtbringer, Träger eines Lichtspenders (generell)<; meist ütr. auf Christus (als Lichtbringer) sowie auf den gefallenen Engel Lucifer. - Texte der Sinnwelt ,Religion`. - Bdv.: vgl. lucifer 1.

Froning, Alsf. Passionssp. 154 (ohess., $1501 \mathrm{ff}$.): geheysßen was ich Lucifer, |ich was in dem obersten thron eyn licht-treiger! Rieder, St. Georg. Pred. 79, 10 (Hs. 「önalem., 1387?): da von spricht sant Bernhart: ,Lucifer, liecht trager, wárist du ain fúr trager gesin, so wárist du nie gevallen!" Kurrelmeyer, Dt. Bibel 2, 442, 4 (Straßb. 1466): biß das der tag erscheint vnd der liecht trager [Var. 14752-1518: morgenstern; so auch LUTHER 1545, 2. Petr. 1, 19] wirt geborn in eúern bertzen. MAALER 273 (Zürich 1561): Liechtrager / Der das liecht bringt. Luciferus. JAKsCHE, Gundacker 18 (oobd., Hs. 1. H. 14. Jh.): den [engel] nant er [Got] do Lucifer, | daz sprichet tiusche, ain liecht trager. Reithmeier, B. v. Chiemsee 24, 9 (München 1528): Vierder staffel ist accolitus, ain liechttrager, der gwalt hat zum altar liecht anzezünden. - Helm, H. v. Hesler. Apok. 10885; Palmer, Tondolus 835; Mayer, Folz. Meisterl. 29, 72; Dict. Germ.-Gall.-Lat. 307, 12.

>Lampe; Lampenseilı. - Bdv.: vgl. liechtseil.

BREMER, Voc. opt. 12050 (halem., 1328 ff.): Lucanar [...] liehtseil [...] liehttrag [...] liehttrager [...] est lampas ardens in aere; uel [lacunar] est instrumentum pendens in alto, cui multe candele ardentes affigentur. Et dicitur [lacunar] a lux et aer, quia lucem prebet aeri. - Vgl. ferner s. v. liecht (das) 1 .

liechttrager, s. liechttrage.

liechtung, die.

>Unterhaltung des ewigen Lichtes`; vgl. liecht (das) 1. - SchweIz. ID. 10, 1056 (a. 1567).

\section{liechtweihe, liechteweihe, die.}

$>$ Mariä Lichtmeß<; vgl. liecht (das) 2. Bdv.: vgl. liechtmesse.
StrehlKe, Nic. Jerosch. Chron. 529, 19498 (preuß., um 1330/40): und unsir vrounin tag irschein, | den man nennit lichtwie. ERmisch, Freib. Stadtr. 73, 27 (osächs., 1335): di munce zu Vribere sal man nicbt dicker [...] vernuwen anme slage unde an den pfenningen wen zu einem male in dem iare, unde daz sal sin $2 u$ unser vrowen tage lichtwie. Feudel, Evangelistar 133, 5; Ermisch, a. a. O. 253, 21; Thiele, Chron. Stolle 241, 30; Jelinek, Mhd. Wb. 473.

liechtweihen, s. liecht (das) 1.

liechtzaun, der.

ein Zaun nicht näher bestimmbarer Art; möglicherweise >durchsichtiger Zaun<; dann anschließbar an liecht (Adj.) $6 \mathrm{f}$.

SACHS 7, 202, 26 (Nürnb. 1536): [ich] kam für ein runden liecht-zaun, | Der umbfieng des keysers thiergarten. BAUMANN, Bauernkr. Oberschw. 18, 9 (schwäb., v. 1542): da waß ain ygel in den liechtraun gemacht, das niemet möcht zu dem zaun komen. Turmair 5, 18, 20 (moobd., 1522/ 33): schluegen alda ir veldgeleger, machten berumb ainen liecbtzaun, wurfen ainen graben auf, macbten ain beschüt berumb.

${ }^{1}$ lied, das; -/-Ø, -e, -en, -er; zu mhd. lit >Glied`(Lexer 1, 1938); vgl. gelied.

1. >Gelenk, Bindeglied, Band; Scharnier «, sowohl auf den Körper wie auf Sachgegenstände bezogen. - Bdv.: gelenk 1; vgl. 1 angel 3, ${ }^{2}$ gleich 1, grendel 1. - Wbg.: liedeband, liedfüge, liedgesucht, liedgesüchte (zum Gw s. gesüchte 2), liedsucht, liedsüchtig (dazu bdv.: gichtbrüchig, lamsüchtig. Ggs.: gesund, Adj., 1).

Ziesemer, Proph. Cranc Dan. 10, 16 (preuß., M. 14. Jh.): Herre min, an dinem gesicbte sint slaf wurden alle mine lidebant [Luther 1545: gelencke]. Kurrelmeyer, Dt. Bibel 1, 16, 14 (Straßb. 1466): die do hetten die teuffel und die monsucbtigen und die litsucbtigen [Var. $1475^{2}-1518$ : lamsuchtigen; Froschauer 1530: die der schlag hat getroffen; LuTHER 1545, Mt. 4, 24: Gichtbrüchigen] vnd er [jhesus] gesunt sy. BREMER, Voc. opt. 1200 (schwäb., 1441): Conpago lidfugi [...] gelid [...] Conpago [...] est colligacio membrorum per neruos. EBD. 43096 (wobd. / oobd., M. 14. Jh.ff.): Artetica lidsubt [...] lidgesucht [...] lidgesúcht [...] Artetica [...] est dolor iuncturarum. Et dicitur ab artus et teneo, vnde artetica quasi artus tenens, id est iuncturas uel concatenaciones; iuncture enim quasi cum kathena simul tenentur. Et bic morbus a laycis dicitur gutta. Et sunt tres species arthetice, scilicet sciatica podagra et cyrogra. CHRON. AugsB. 8, 156 Anm. 2 (schwäb., zu): vom stuel oder sitz, hab er ain lid, ain eisnes pendlen, bei 3 neglin lang. NIEWÖHNER, Teichner 106, 22 (Hs. ' ${ }^{\top}$ moobd., 1360/70 ${ }^{7}$ ): also ist von zwayer lit $>$ hat es zwei Gründe < | daz der mensch unczúchtig wart, | von gewonhait und von art (hierher?). ZINGERLE, Inventare 112, 2, 39 (tir., 1489): vier eysene lyder, do man 
türn da mit anhenngt. - Broszinski, Minner. Chir. Parva $79 \mathrm{r}, 18$.

2. >Teil eines Ganzen<; speziell: > einzelner Punkt, Artikel, Hauptteil einer Lehre, eines Textes<; >Einzelteil einer Goldschmiedearbeit<. - Bdv.: vgl. artikel 2. Wbg: liedegan₹ 1 >vollständig, unbeschädigt

Strehlke, Nic. Jerosch. Chron. 235 (preuß., um 1330/40): der sîn rede in stucke schicht, | ê er von der materien icht $\mid$ endehaftis spreche, | unde nicht vorbreche | der lidir ordenunge. KochendörfFer, Tilo v. Kulm 1145 (preuß., 1331): Vél lyd der geloube hat, | [...]: | Wil man, [...], | An sehn di do han gestift $\mid$ Den gelouben, so iz. der lied $\mid$ Di man sal balden bi der wied, | Nwer czwelve gar uber al. EвD. 1233: Di benanten virczen lid, $|[\ldots]|$ Czüt man uz dem gelouben. In den nächstfolgenden beiden Belegen explikativ für den Inhalt des Adjektivs bzw. Genitivs: KopPITZ, Trojanerkr. 3832 (Hs. ' noschweiz., 15. Jh. ') : Mitt vil kunstenlichen liden | Ward daz werk, gefügett. EBD. 7618: Wol dem edlen gold schmide! | Der siner hochen künste lide | Also zartlich gerüren kan. Vock u. a., Urk. Nördl. 2190 (schwäb., 1442): offen ledegantzen unverserten videmusbrieve.

3. >Teil des menschlichen oder (seltener) des tierischen Körpers, Glied, Organ`; im einzelnen bezogen auf die Extremitäten, die Finger, Zehen, Mund und Zunge, Genitalien, die Vorhaut, das Augenlid; ütr. auch: >Instrument (der Hölle) ২. - Phras.: leib und liede verdingen; etw. aus seinen lieden trekken >etw. mit der eigenen Händearbeit erwirtschaften «. - Bdv.: vgl. artikel 1, gelenk 1, gewerbe 9, lanke 1. - Synt.: kein l. geleichen >rühren< mögen, jm. die liede beschneiden / dekken / berauben / erschlagen, jm. eines der liede verderben, die lieder süchtig machen; die liede(r) geordnet / gesund sein, sterben, aus den gleichen kommen, von zären gefar werden, jm. schwaren, $j$ s. lied erquemen > erzittern ‘; an den lieden leiden, $j n$. an einem l. entlieden, die speise in die lieder gehen, brest in die liede kommen; der füsse lieder (gen. explicativus), das l. der augen, des fingers / hauptes / leibes; die blossen / jungen / müden / schwachen / zarten liede(r); die schwindung der liede; nägel an den liedern. Wbg.: liedbrechen (a.1385), lieddrïse >Auswuchs am Körper, Oberbein o. ä.ı (dazu bdv.: misgewächs), liedganz, > unversehrt<, liedhandschub >Eisenhandschuh mit Fingergeschübe`, lied- schärte >Verstümmelung der Glieder< (14. Jh.), liedeschärtig >gliederlahm (ütr.), krank «; ütr.: >verletzt, schwach`, liedtief (dazu bdv.: beinschrot, liedeschärtig, meisselwund; Beleg s. v. letzig).

Strehlke, Nic. Jerosch. Chron. 7858 (preuß., um 1330/40): swâ er [Swantepolk] indirt horchte | der dûtschin brûdre namin, | al sîne lit irquâmin. KOCHENDÖRFFER, Tilo v. Kulm 5206 (preuß., 1331): Merke wes du dich verczist | Und welches hobtes lit du siest! Grosse, Schwabensp. 47a, 35 (Hs. ' $\mathrm{nd} . / \mathrm{md}$., um 1410?): de vinfte sibbe stet an dem ersten lede des vengers. EBD. 156a, 4: Swer daz deme anderen met gewalt düt oder ime süs der lide eyn vorderuet, daz sal man ime Widere tün. QuINT, Eckharts Pred. 1, 313, 1 (E. 13./A. 14. Jh.): Nîu, stant in der porte'. Swer dâ stât, des lide sint geordent. Er wil sprechen, daz daz oberste teil der sêle sol stân îfgeribtet staticlîche. Stackmann u. a., Frauenlob 5, 10, 2 (Hs. rmd. auf nd. Grundl., v. M. 14. Jh. $\left.{ }^{7}\right)$ : Grif, herze, zu und hilf den sinnen ein lob smiden, | daz. allen liden | der kunst si wol gelenke. EвD. 5, 52, 15 (Hs. ' nobd., 3. V. 15. Jh. '): Ist disen zwein gelücke mit, | daz ist der belle ein erbesit, | ir erge lit, | ir höchster schrit. Мејјвоом, Pilgerf. träum. Mönch 2494 (rib., 1444): barmhertzicheit, I De sent Mertijn sich untcleyden dede | Dem armen zo decken syne bloisse lede. Chron. Köln 1, 2618 (rib., Hs. 1. H. 15. Jh.): dat sy vro woren aldamede $\mid$ dat sy verdingden liff ind lede. EBD. 5913: want luzzel volx myt muden ledin | hait selden her wail gestredin. EBD. 2, 291, 21 (Köln 1499): want be [Joseph7 die moder ind dat kint besorgen moist ind dat wis sinen leden strecken ein redeliche zit lank. HüBNER, Buch Daniel 3856 (omd., Hs. 14./A. 15. Jh.): Und weinte daz die lider | Wurden von zeren gavar. THiele, Chron. Stolle 275, 6 (thür., 3. Dr. 15. Jh.): als sy zu einem male dy gefangen wisten, ab sy noch ledegancz, weren. Pyritz, Minneburg 2129 (nobd., Hs. um 1400): Sie [werlt] ist an truwen gar lidschertig; | Sie ist spottig und hochfertig. EBD. 4680: Daz. min fraw, [...], | Ist gewest gein mir so stichel | [...], | Daz mir worden ist lideschertig | Min freude, daz ich mus alten Und leides mer drivalten. VetTer, Pred. Taulers 295, 18 (els., 1359): und get die spise denne durch die adern in alle die lider. Binlmeyer, Seuse 354, 18 (alem., 14. Jh.): eins ist in dem andern, und kein lid mag leben, daz usgeschidet ist. Adrian, Saelden Hort 1645 (alem., Hss. E. 14./ 15. Jh.): nach der e besniden |im [kint] sinú jungen murmen lider. Sudhoff, Paracelsus 7, 407, 26 (1529): so wachsen bieraus die moderschwammen, letdrusen, darumb seben sie dem gleich aus des knasp sie gewachsen sein. PFeIfFer, K. v. Megenberg. B. d. Nat. 490, 7 (oobd., 1349/50): die habent bundeshaupt und scharpf krumm negel an den lidern. ZINGERLE, Inventare 88, 2, 13 (tir., 1490): zway par lidhantschuech. - Helm, H. v. Hesler. Apok. 15258; Loesch, Kölner Zunfturk. 1, 9, 12; Меijвоом, а. а. O. 5120; 6253; Chron. Köln 1, 4772; Hübner, a. a. O. 4396; 5772; Pyritz, a. a. O. 4346; HaAge, Hesel. Arzneib. 16r, 10; Bihlmeyer, a. a. O. 316, 29; VetTer, a. a. O. 
159, 19; PÄPke, Marienl. Wernher 6042; 6976; Welti, Stadtr. Bern 235, 5; Pfeiffer, a. a. O. 477, 2; Klein, Oswald 38, 16; Rwв 8, 1318; Schweiz. ID. 5, 336; 8, 1307. - Vgl. ferner s. v. ${ }^{1}$ ader 4, aderschlag 1, amacht 2, augbrawe.

4. >Abhängiger (z. B. des Teufels); Kind (Gottes); Mitglied einer Gemeinschaft $<$.

Helm, H. v. Hesler. Apok. 2701 (nrddt., 14. Jh.): Der sal der tubel manic leit $\mid$ Tuen mit den argen sinen liden. EBD. 10810: Wen sie sint die Gotes lide, | Die sich nach dem vridesamen | Criste cristenen bie namen. Fischer, Brun v. Schoneb. 36, 93 (md., Hs. um 1400): das bimlisch paradeis $\mid$ mit hochem preis ler steigen deine lid. - Vgl. ferner s. v. ausscheiden 2 .

5. >Glied, Stufe in der Erbfolge «. - Bdv.: vgl. grad 4.

Grosse, Schwabensp. 47a, 31 (Hs. ' nd./md., um $\left.1410^{\urcorner}\right)$: Alsus is der vater vnde muter dat hoübet. $\int$ de kint, de ane zweyinge van vater unde van muter geboren sin, daz. sint rechte swestere. An den bouet sich der erste sibbe. de stent ouch von rechte an dem neisten lide bi deme houede, dar de arm an de schülderen stozent. daz lede heyzet de acsel. Jst aber zwevnge an den kinderen, so ne mogen se an deme lede nicht gesten vnde schriket an en ander let [stark bildhaft]. CHRON. KöLN 2, 621, 28 (Köln 1499): den ewigen guedigen got enmoegen wir alle sicherlich niet genoich [...] geloven mit allen den genen die uns zogehoeren bis in dat zehende lit.

6. >Teil (wohl ein Viertel oder die Hälfte) des geschlachteten Rindes «. Wbg.: liedfleisch (Beleg s. v. bandelfleisch).

LoEsCH, Kölner Zunfturk. 11, 124, 9 (rib., 13481407): so soillen de specksnider dat heuft in deme reichten lede afsniden zo reichte durch, ind dat sin geboer specks daan blive. Schnyder, Qu. Zürcher Wirtsch. 499, 38 (halem., 1428/9): als Bertschi Riem und Andres Holtzhalb iet wedra iij lid fleisch von einem rind verkouft hand. Boos, UB Aarau 339, 9 (halem., 2. H. 16. Jh.): Sy [mexger] sond ein lid rindfleisch an den bank bencken und nit mer und denselbigen lid howen und ein fierteil vom kopf darzi, und nit von dem lid wider in das schinthusz bencken.

7. >Verkaufstisch für Fleisch ‘; assoziativ mit ${ }^{3}$ lied verbindbar als $>$ Deckel der Ladenbank<. - Bdv.: vgl. band 11, beilbank, haubank, intumbank, judenbank, kamer 2, laden (der) 3.

Helbig, Qu. Wirtsch. 1, 46, 29 (md., 1487): also das er solch pfinnigk fleisch vornn auff die liedt lege und ein weiss tuch sichtiglich darunder. EBD. 2, 98, 4 (1357/8): ist recht, daz keyne lyt mêr sullin sin an den eckin wen eyn. WINTER, Nöst. Weist. 2, 730, 22 (moobd., 1430 / 1625): ob ain armes mensch zue im [fleischbacker] kämb und begehret ain pfenwert fleisch, so ist ers schuldig ime zue geben wie er dan daß ungevehrlich vor im auf der lidt hat geschroten.
${ }^{2}$ lied, das; es/-e oder -er.

1. >Lied in einem sehr generellen Sinne<, meist auf die Einheit von Text und Weise, teils eher auf den Text, teils eher auf die Melodie bezogen, ohne daß die Ganzheit von beidem in Frage stünde; unter ästhetischem Aspekt die ganze Spannweite vom Spottlied, Gassenhauer über z. B. das Liebeslied, den Heldengesang bis hin zum Kunstlied, zum geistlichen Lied umfassend und dementsprechend z. B. den gesungenen Alltagsschwank, das Liebeslied ebenso einbeziehend wie (im 17. Jh.) die Ode; auch inhaltlich auf ein außerordentlich weites Spektrum bezogen: Alltagssachverhalte, Nachrichten, große Ereignisse, Geschichtliches, Personen, Geistliches, Weltliches. Texte der weiteren Sinnwelt ,Religion / Didaxe', auch Chroniken. - Phras.: j. ist seines liedes >j. macht sich einen passenden Vers auf etw.<; ein lied auf einer seite fiedeln; $j$ s. lied singen (auch: pfeifen) o. ä. >sich js. Willen fügen ‘; jn. ein liedlein singen lernen o. ä. 〉jn. zur Vernunft (des Mächtigeren) bringen`; das alte lied singen; ein lied von etw. singen können (vgl. den Beleg Gilman), ein lied zu hoch anfangen und nicht aussingen >zu weit gehen, zu hoch greifen, es aber nicht ausführen`; dem tauben ein lied singen >Vergebliches tun<; ein liedlein von jm. singen >jn. verspotten`; lieder auf $j$ s. beutel machen $>$ jm. etw. zu Gefallen und zum eigenen Vorteil tun<; ein lied von etw. machen / singen >etw. sehr ernst nehmen, hoch aufhängen $1 ; 2\urcorner$, jeweils mehrfach, neue zeitung, ode, spruch, wiedersang; vgl. ${ }^{1}$ leich I, 1, leis, psalm, weise. - Synt.: ein l. begeren / erdichten / erfinden / pfeifen / singen (>häufig ‘), von etw. machen, von $j$ s. fromkeit singen, zu js. lob erzälen; das l. (Subj.) erklingen, den unmut vertreiben; willen zu unkeuschen liedern baben; dem l. eine weise geben; etw. in einem l. vermären; das l. auf jn. [z. B. auf ein weib], das l. mit [einer Anzahl] gesetzen, zu eren der kunst; das arme / bäurische / daketylische / deutsche / freundliche / geistliche / gemeine / gute / burische / lästerliche / neue / schandbare / schandliche / traurige / tro- 
chäische / tröstliche / unkeusche l.; der anfang des liedes. Wbg.: liedeln (um 1650), liederdichter, liedsprecher wohl > Ansager, Moderator bei Spielen oder >j., der Lobesverse vorträgt (s. u. Voc. Teut.-Lat.; vgl. Dwв 6, 995).

Schade, Sat. u. Pasqu. I 56, 75 (o. O. 1542): Die [stet] wolt er der maßen treiben und zwingen, | Daß sie im müsten seins gefallens ein liedlin singen. TOEPPEN, Ständetage PreuBen 3, 579, 32 (preuß., 1453): unde sungen vor em eyn nuwe schentlich lyth, das sie off uns allen unde den ganczen roth gemacht und geticht han. JOACHIM, Marienb. Tresslerb. 366, 23 (preuß., 1405): 1/2 m. dem lytsprechir. item $1 \mathrm{~m}$. den spelluthen. Luther, WA 2, 63, 5 (1519): Das man tzu schendlichen, untzuchtigen und unkeuschen worten, lyedern, bistoryen [...] willen gehabt hat. ЕвD. 10, 3, 153, 7 (1522): Unnd [Paulus] bilte yetzund seine sünde so verächtig, das er gleych ain lied da von macht und sang davon. EвD. 22, 145, 4 (1544): solche Geister [...] breitens weiter aus, singen ein liedlin von jm, lachen sein noch dazu in die faust hinein. EBD. 25, 487, 6 (1528): Sancti pseudoprophetae utcunque babent verbum dei, das einer seins lieds ist, quod plus diligit pecuniam. EBD. 28, 629, 25 (1529): und Moses sich nicht schemet, das er jmerdar ein Lied auff einer seiten [bezogen auf das erste Gebot] fiddelt, das ist: einerley treibt. EвD. 32, 275, 22 f. (1545): Man kan eyn gut lid nicht zuvil singen. EBD. 33, 581, 15 (1531): es hilfft nicht und ist dem Tauben ein Lied gesungen. ЕвD. 624, 29 (1531): Sie [Juden] gedachten: das Lied ist zu hoch angefangen, er wirds nicht binaus singen. EвD. 49, 416, 29 (1544): was Moses, Christus predigen, hilfft nichts, sie singen das alte liedlin lam, lam. EBD. 687, 37 (1545): das wir sollen clug sein, viel von uns haltten, andere verachten und das liedlin singen: Ich bins, Ich bins. Chron. Köln 3, 948, 130 (Köln 1483, Hs. 18. Jh.): dat was den goeden leit $\mid$ und kuntens gekeren niet $\mid$ und moisten singen ir liet. Wyss, Limb. Chron. 36, 25 (mfrk., 3. Dr. 14. Jh., Hs. 2. H. 16. Jh.): in der selben zit sang man ein nuwe lit in Duschen landen, daz was gar gemeine zu pifen unde zu trompen unde zu aller freude. EBD. 49, 5: in disem selben jare vurwandelten sich dictamina unde gedichte in Duschen lidern. Want man bit her lider lange gesongen hat mit funf oder ses gesetzen, da machent di meister nu lider di beißent widersenge, mit dren gesetzen. SснміDт, Frankf. Zunfturk. 1, 173, 24 (hess., Ende 16. Jh.): neue zeittungen, gesange, lieder und spruch, die seien geistlich oder weltlich. THIELE, Chron. Stolle 415, 22 (thür., 3. Dr. 15. Jh.): Hencze gutiar uns diez, lidelin sangk, $\mid$ sine winter cleydere die sint ome krangk. WeISE. Jugend-Lust 82, 8 (Leipzig 1684): ibr lieben herren; Auf euren Beutel mache ich keine Lieder. OpItz. Poeterey 15, 26 (Breslau 1624): der alten Cimber oder Dánen ebenmáßiger gebrauch / die von ibren Helden schone und geistreiche Lieder ertichtet haben. Voc. Teut.LAT. o iiijr (Nürnb. 1482): Herolt. heroldus. oder liedsprecher. poeta boliuus. SACHS 23, 108, 20 (Nürnb. 1556): Aller art ist der lieder sum. | Ains tails schriftlich zu gotes glori; | Ein grose sum weltlich histori,| Schuelkunst, fabel und poetrey, | Zwcht-ler aus der philosopherey, | Hofflich und abge- stolen renck, | Guet lecherlich possen und schwenck. BIHLMEYER, Seuse 26, 5 (alem., 14. Jh.): so gand die jungling dez. nabtes us [...] und bitent dez, gemeiten, daz ist, sú singend lieder und sprechent schónú gediht. Spanier, Murner. Narrenb. 15, 42 (Straßb. 1512): Sy wólten ein schlechten prelaten | Mit listen vnd mit spitzem fundt, | der ir liedlin singen kundt. EBD. 19, 43 (Straßb. 1512): Er müß syn [bischoff] lied on weren pfyffen. N Nachs bischoffs dantz syn reyen fieren. Gilman, Agricola. Sprichw. 1, 314, 5 (Hagenau 1534): der kan vil davon singen und sagen / daß ich wolt wol ein lidlein da von singen. EBD. 2, 79, 14 ([Augsb.] 1548): Das brot ich esse / das lied ich singe. Moscherosch. Ges. Phil. v. Sittew. 14, 31 (Straßb. 1650): Niemand aber ist der uns daselbsten grösser Dienst leiste als die Poeten, Lieder-Dichter, mit liegen und senften. MaAler 273 (Zürich 1561): Heilsams vnd frólichs Lied / das einem den unmüt / trawwren vnd sorg vertreybt / dz einen frólich und mütig machet. [...] Wist unnd unflatig Lied. Wyss, Luz. Ostersp. 3455 (Luzern 1575): sy [Krieger des Herodes] ryttend mitt grosser schar! Nemend ir der schönen fröwlin war | Vnd biessend ich ein liedlin singen. SAPPLER, H. Kaufringer 32, 116 (schwäb., Hs. 1464): Der ailft trunk mich von keften schied. | baide weis und auch lied | koment an ir alte statt. Baumann, Bauernkr. Oberschw. 61, 15 (schwäb., v. 1542): die briesterschaft zu schmechen und schenden mit schantlichen liedern zu singen auf der gassen. EBD. 79, 36: kamen sy mit gwalt in die stat, must ain frumer rat, auch etlich frum redlich menner auß der gemaint ir liedlin singen. BARACK, Zim. Chron. 2, 230, 21 (schwäb., M. 16. Jh.): so herr Hainrich [...] die zeit erlept baben sollt; wurt er schenk Eberbarten [...] ain anders liedlin haben singen lernen. EBD. 3, 306, 31: da baben die spaivögel ein liedt von gemacht, das thuet den Rotweilern zorn. Sснмітт, Ordo rerum 267, 16 (oobd., 1466): Gannio [...] sprecher [...] lietsprecher [...] klaffer. - Luther, a. a. O. 35, 473, 23; SCHEIN, NA 6 Xb, 12; Schmidt, a. a. O. 1, 152, 6; DüNNHAuPT, Werder. Gottfr. v. Bullj. 19, 22; OpITz, a. a. O. 11, 13; 44, 11; Gille u.a., a. a. O. 69, 205; 82, 99; 116, 165; Mayer, Folz. Meisterl. 90, 76; Ingen, Zesen. Ged. 388, 29; Bihlmeyer, a. a. O. 139, 28; 140, 3, ReNNeFAHRT, Staat/Kirche Bern 828, 10; Barack, a. a. O. 1, 300, 25; Hohmann, H. v. Langenstein. Quästio 207, 45; Klein, Oswald 41, 41; Kummer, Erlauer Sp. 4, 317; Bremer, Voc. opt. 35055; Dict. Germ.-Gall.Lat. 307; Schles. Wв. 2, 812. - Vgl. ferner s. v. anzäugen 1, auserreuten, ausmessen 7, papist, bäurisch 2.

$>$ Angelegenheit, Sache«; nur schwach und ansatzweise phrasematisch belegt.

Luther, WA 49, 330, 26 (1544): Sie [Heiden] aber nemen mich mit freuden an, bey ibn wil Ich sein, wie gefelt euch das lied? - EBD. 492, 32.

${ }^{3}$ lied, das; -/auch er; zu ahd. blit, mhd. lit >Deckel< (LeXer 1, 1939; SchweIz. ID. 3, 1088; Dwв 6, 982).

>Deckel auf Hohlkörpern (Gefäßen, Truhen u. ä.)<. - Bdv.: deckel 1, sturz; vgl. 
decke 1, laden (der) 4. - Synt.: das l. anhängen / zutun, von dem geschir stossen; das l. (Subj.) zustossen; den brunnen mit einem l. abdecken; das l. über die bütte; das überdekte / versperte l.; der kopf / laden, die schachtel / stande mit einem l., blumen auf dem l. Wbg.: liednagel >Nagel für einen Sargdeckel

Ziesemer, Gr. Ämterb. 2, 38 (preuß., 1374): 2 boe obirgolte koppe mit leden. Franz u. a., Qu. hess. Ref. Bd. 2, 2, 311, 23 (hess., 1539): hat man den goldschmieden befohlen, den sarck aufzubrechen, welche die liednägel abgezwengt. Lichtenstein, Lindener. Rastb. 55 (о. O. 1558): er machets so grob mit ir, daß das lid oder deckel an dem todtenbaum zu schlig. SCHNYder, Qu. Zürcher Wirtsch. 637, 10 (halem., 1455): ze machen diß werck, [...], mit namen einen kopff mit lid und mit füß und ein blimen uff dem lid. MaAler 248r (Zürich 1561): kast mit einem gewólbten deckel oder lid. LÖFfler, Columella/Österreicher 2, 303, 3 (schwäb., 1491): in den náchten zimpt es sich das lid zu zethind, das das tôn nit darin fall. - JоACHIM, Marienb. Tresslerb. 63, 3; Ziesemer, a. a. O. 567, 7; Намpe, Ged. v. Hausrat 4, 5, 3; LuginbüHL, Brennwalds Schweizer Chron. 1, 89; 26; Müller, Grafsch. Hohenb. 2, 29, 24; Barack, Zim. Chron. 2, 79, 24; Bauer u. a., Kunstk. Rud. 333; 544; 776.

liedbrechen, lieddrüse, s. ${ }^{1}$ lied 3.

liedeband, liedeganz, s. ${ }^{1}$ lied 1.

liedeln, s. ${ }^{2}$ lied 1.

liedemas, s. liedmas.

liederdichter, s. ${ }^{2}$ lied 1.

liedere, die.

>Lachsweibchen`, Bezeichnung, die vom Herbstmonat an gegeben wird (so SchweIz. ID. 3, 1093).

WeLti, Stadtr. Bern 135, 16 (halem., 1431): das nach aller heiligen tag hin weder vsser noch inder vischer keinen visch, die da lidernen oder lachss beissent, vachen [...] sóllent. Schmidt, Hist. Wb. Elsaß 227.

\section{liederlich, Adj.}

1. >nachlässig, leichtfertig (von der Haltung und den Handlungen des Menschen); seiner sozialen Verantwortung und Verpflichtung nicht nachkommend; durch Nachlässigkeit unfähig; schuldig, verantwortungslos`; auch: >gewöhnlichen Standes, gering «. - Bdv.: s. u. Schöpper, ferner: entbunden, faul, beillos 2; 3, las 2, leichtfertig 3; 4, nichtswürdig, träge, versäumlich, vertüig, un- nütze, weich. Ggs.: aufrecht 2; 3. - Synt.: j. (in seinem werk) l. sein; l. handeln / lernen, etw. l. verprassen, etw. (ein ampt) l. ausrichten, jn. l. vereren / verfüren, etw. l. wenden >leichtfertig behandeln<, sich l. mit jm. einlassen, l. einen krieg anfangen, sich e.S. l. verwegen; der liederliche haushalter / meister, das liederliche gebändze / haushalten, die liederliche tat / tochter, liederliche leute. Wbg.: liederliche (die), liederlichkeit.

Schöpper 30a (Dortm. 1550): Negligens. Hinlässig lässig seumig vnachtsam verachtig faarlessig liederlich verachtloß sorgloß ablessig $\uparrow$ seumling seumiger. EвD. 56a: Inutile. Unnutz. liederlich vnfürderlich nichtsóllig. LUTHER, WA 32, 527, 38 (1530/32): geschibet jn ja recht, die sich so liderlich verfuren lassen. Loesch, Kölner Zunfturk. 11, 46, 13 (rib., 1500): dardurch mench minsch in verschinener zijt des lidderlichen gebentz ind versummnis der wonden halven verwarloeist ind afliffich worden ist. SCHWARTZENBACH 28r (Frankf. 1564): Faul. [...]. Hinlessig. Liederlich. Ablessig. Nachlessig. Seumig. Vmfrutig. Vnwacker. LeXer, Tucher. Baumeisterb. 278, 33 (nürnb., 1464/75): Als dan liderlich meister bie aufgenummen sein auf dem hantwerck der decker piß her, die den morter nit gewist haben zu bereiten. FisCHER, Folz. Reimp. 18, 179 (Nürnb. um 1520): Wie er zu vil lyderlich sey | Mit vil dingen, und sagst darpey $\mid$ Der frawen fur, ir sey der man $\mid Z u$ hert. Kurrelmeyer, Dt. Bibel 8, 49, 19 Var. (Straßb. 1466): Der do ist linde vnd entpunden [Var. 14752 - 1518: liederlich; Froschauer 1530: faul, traag; EcK 1537: waich; LutHer 1545, Spr. 18, 9: lass] in seim werck: der ist ein brider des des seine werck seint verwust. Moscherosch. Ges. Phil. v. Sittew. 21, 26 (Straßb. 1650): werden also die arme Teuffel eben schlecht gehalten vnd liederlichen verehret, da wir doch so bungerig nicht sind, daß wir alles das so man vns zuwünschet annehmen wirden. HaAs u. a., Erasmus/Jud. Klag 26, 19 (Zürich 1521): Der nienar vff ein uffsehen hat dann uff gemeinen nutz / facht nit liederlich ein krieg an. MAaler 273r (Zürich 1561): Liederlich unnd nit aufrácht seyn in seinem ampt. [...]. Er ist eines Liederlichen vnnd schmáchlichen tods vmbkommen. EBD. 341r: Sag nit das es meiner liederliigkeit schuld seye. RENNEFAHRT, Zivilr. Bern 432, 11 (halem., 1564): von der unnutzen liederlichen lütten wegen, di ir güt mit merckten, tuschen, gält uffbrächen, entlenen, zeren, kouffen, verkouffen, verbürgen, versetzen, [...] verthünd. CHron. Augsi. 5, 388, 14 (schwäb., um 1530): es hett sich das auch niemandt liederlicher understeen [...]. Rот 358 (Augsb. 1571): Vanitet, Eytelkeyt / lug [...] / liederlichkeyt. Barack, Zim. Chron. 1, 423, 32 (schwäb., M. 16. Jh.): in dem er kain mangel oder etwas befunden, darin er fürnemlich, dann allain umb sein saumnus und liederligkait in aignen sachen, zu strafen gewesen. - v. D. Broek, Suevus. Spieg. 182 r, 41; Chron. Nürnb. 2, 371, 6; Goedeke, Fischart Flöh Haz 3456; Qu. SchweIz. Gesch. 1, 165, 3; BACHMANN, Haimonsk. 82, 3; Grossmann, Unrest. Öst. Chron. 157, 7; 192, 14; Dict. Germ.-Gall.-Lat. 307, 
24; Rwв 8, 1316; Schmidt, Hist. Wb. Elsaß 223; SCHweIz. ID. 3, 1100. - Vgl. ferner s. v. ${ }^{1}$ aufbrechen 13, befogtung, begschierer.

2. >schnell, rasch; unüberlegt, übereilt, voreilig‘; offen zu 1 und 3. - Bdv.: schnel, unbesonnen; vgl. bald 1 , leicht 5 , leichtfertig 2 ; 3, leichtiglich 3 .

SACHS 18, 353, 17 (Nürnb. 1565): Das alls kurtze zeit thut bestehn, | Gleich eim schlaff liderlich vergehn. ЕвD. 20, 299, 6 (Nürnb. 1563): Daß sie [lieb] nit liderlich abfellt, Weil lieb sein lieb helt trew und vest. LINDQvist, K. v. Helmsd. 2808 (halem., Hs. um 1435): Er [lïp] dring da durch än allen wank $\mid$ Als liederlich als ain gedank. Turmair 1, 198, 27 (moobd., 1529): er [Papst] ist je von bimel und hell mit seiner gewalt so liederlich verstossen worden, über welche er lange zeit die schlüssel so gewaltiglich gehabt hat, im sein schwert, den ban, [...], so liederlichen [dies zu 1] zucken lassen. EBD. 4, 12, 22 (moobd., 1522/33): wie unbestendig [...] der ruem, [...] seie, wie es gar schnel und liederlich zergê. EBD. 725, 30: Der angelt mit ainem gulden angel, der unbesunnen und liederlich sich in krieg begibt. Chron. Magdeb. 2, 200, 10; Bachmann, Haimonsk. 207, 15. - Vgl. ferner s. v. anæäugen 1.

3. เmühelos, leicht, ohne Schwierigkeiten, auf leichte Weiser. - Bdv.: leichtlich 3, ringlich; vgl. gering 4, leicht 3. - Synt.: l. davon kommen, ledig werden, etw. glauben, l. einen anfang machen, l. zu behaben >zu erhalten sein, l. vogtbar werden (von Gütern), eine stat l. nemen, sich l. vergleichen, sich l. abweisen / bereden / überwinden lassen, etw. (ein übel) l. zerrinnen lassen, l. bedenken, das [...].

Luther, WA 41, 585, 19 (1536); so wir sehen, wie sich die arme leute so liderlich lassen dabin reissen vom Wort. Jungbluth, J. v. Saaz. Ackermann 20, 30 (Hs. romd., 1465?): was scheuzlich ist, das ist leidenlich [Var. schwäb., um 1500: liederlich] zu haben. LugINBüHL, Brennwalds Schweizer Chron. 2, 153, 18 (halem., 1508/16): si [Zürcher; ...] hatend ein güt sach fersumt; den si dise stat abermals liederlich hetind mogen in nemen. UNGER, Richtes Stig 3, 12 (1474): durch sölich eigenschaft [libeigen sein] wer die gut liederlich vogtper. Mollwo, Rotes Buch Ulm 234, 7 (schwäb., 1418): als unser lite [...] untz, ber ringlich und liederlich in unser statt Ulme kommen sind. Müller, Welthandelsbr. 130, 12 (schwäb., 1506): So du dan waist, wie vil reali auf ain onz am gewicht, so magst liederlich machen, wie $d u$ ain allain nemen oder koufen solt. ReITHMEIER, B. v. Chiemsee 33, 7 (München 1528): Jme [Cristo] mit lieb, geduld vnd mitsamkait begegnen, auch dir das vbel der straff vnnd zeitlicher widerwaertigkeit liederlich zerynnen lassen, auf das du [...] selig werden moegst. - ToEppen, Ständetage Preußen 5, 620, 33; Luther, WA 30, 2, 701, 5; Schade, Sat. u. Pasqu. 3, 45, 37; Franz u. a., Qu. hess. Ref. 2, 342, 4; Chron. Nürnb. 5, 611, 3; SAChs 14,
315, 4; 20, 290, 28; BaCHMAnN, Haimonsk. 41, 6; Brandstetter, Wigoleis 201, 28; Reithmeier, a. a. O. 7, 9; Dict. Germ.-Gall.-Lat. 307, 29. - Vgl. ferner s. v. abweisen 5 , behelfen 4 .

4. >freiwillig, aus eigenem Antrieb; leutselig, gelassen ‘. - Bdv.: s. u. Höver; vgl. gern 4, gewillig, gunstwillig, gutwillig 2, los (Adj.) 11.

Höver, Bonaventura. Itin. B, 392 (moobd., 1450/60): die aller hochst ausgiessung mag nicht gesein, sy sey denn [...] selbstendigkleich naturleich, willig, freymutig, liederleich. TuRMAIR 5, 336, 20 (moobd., 1522/33): Hainrich, [...] der Lew, ist ein ernstlicher weiser fürst gewesen [...], ein milter liederlicher fürst mit allen leuten.

5. >geringfügigく. - Bdv.: vgl. geringfügig 1 , klein 9, kleinfüge 1, leicht 8. - Synt.: von liederlichen sachen, aus liederlichen ursachen, aus liederlichem wortgezänk, um liederliche dinge, um liederlicher ursachen willen.

Oоrschот, Spee/Schmidt. Caut. Crim. 279 a, 7 (Frankf./M. 1649): daß man meines erachtens offt auß gar liederlichen Ursachen [...] die gefangene auff die Folter erkennet. Koller, Ref. Siegmunds 309, 24 (Hs. ' Augsb., um $\left.1440^{\urcorner}\right)$: Wir sechen dick wol, das von liderlichen sachen offt groß krig auffsten. Müller, Alte Landsch. St. Gallen 236, 28 (halem., 1525): wenn also ein biderman umb liderlich ding verclagt, das dann der vernunft und dem rechten gemäs ime der vercleger anzögt und gestellt werde. - LUTHER, WA 49, 612, 32 (1544); Оonschot, a. a. O. 341a, 23.

6. > etwa, ungefährı.

Peil, Rollenhagen. Froschm. 5917 (Magdeb. 1608): Liederlich vmb den halben werth | Wie ein alt abgetrieben Pferd.

7. >reizend, anmutig (von Personen, Handlungen) «. - Bdv.: vgl. geschöne, gnadenreich 5, lustsam 1.

KLEIN, Oswald 18, 106 (oobd., 1416): In urtail, rat vil weiser hat geschätzet mich, | dem ich gevallen han mit schallen liederlich. EвD. 57, 18 (um 1402?): Weiplicher weib mensch nie gesach, I so liederlich an tadels punt. EBD. 91, 19 (1409?): so ich betracht und acht, | das mich liederlichen umbfacht | ermlin macht.

8. in formaler und inhaltlicher Anlehnung an luderlich: > mittels eines Köders

SACHS 13, 35, 8 (Nürnb. 1556): er muß sterben von meiner hand; | Ich wil im wol ein luder steln, | Das ich in liderlich wil feln.

liederliche, liederlichkeit, s. liederlich 1.

liedern, ledern (letzteres vereinzelt), V.

1. > (eine Tierhaut) beizen, gerben, (Tierhäute) zu Leder verarbeiten ten 1 , gerben $5,{ }^{1}$ lohen. 
Helbig, Qu. Wirtsch. 1, 86, 7 (md., 1459): von einem wolffe zu liedern drei pfennig. Jtem von einem hundert konelyn zu liedern ader zu gerben, [...7, iij groschen. SCHMIDT, Frankf. Zunfturk. 1, 279, 31 (hess., 1377): sal keyner, der kurssenerhantwerg zu Franck.fort tribet, binfure hondesbude oder felle gerben, lydern, [...] oder verarbeiden. BOBERTAG, Eulensp. 90, 13 (Straßb. 1515): Ulenspiegel nam die bereiten fel, [...] Und nimpt die trucknen fel und die geliddert waren. MülLER, Welthandelsbr. 177, 38 (schwäb., 1506): Man bringt auch aus Kathalonia vill weisser glidert fell gen Jenua. BARACK, Zim. Chron. 2, 548, 18 (schwäb., M. 16. Jh.): het er sein gewonlich klaidt an, war ain geliderte eselhut. - Loose, Tuchers Haushaltb. 79, 33; 156, 3; RaABE, Wortsch. Murner 2, 437. - Vgl. ferner s. v. bärenhaut 1 .

2. > hart und steif wie Leder werden<; ütr. (im Part. Prät.): sausgebildet, gelehrt, in einer Sache beschlagen

Spanier, Murner. Narrenb. 6, 118 (Straßb. 1512): Offt und dick sindt meyster worden, | Die nit also gelidert waren, | Das sy doch künten eyns erfaren, | Ob logica noch rechter lere | Gredt mülleryn geschwiger were. NyHOLM, Füetrer. Gralepen 702, 1b (moobd., 1473/8): So das in in dem munde dy zung begund zue ladern.

liedern (Adj.), s. ledern (Adj.).

liedeschärtig, s. ${ }^{1}$ lied 3.

liedfleisch, s. ${ }^{1}$ lied 6.

liedfüge, s. ${ }^{1}$ lied 1.

liedganz, s. ${ }^{1}$ lied 3.

liedgesucht, liedgesüchte, s. ${ }^{1}$ lied 1.

liedhandschuh, s. ${ }^{1}$ lied 3.

liedlich, s. liedlon 1.

liedlon, der, auch das; $-s /-$; zur rechtlichen Rolle des Liedlohns s. Rwв 8, 1298 ff.; zur Etymologie: Dwв 6, 994.

1. >Lohn für Gesinde, Dienstboten, Tagelöhner , in den Belegen überwiegend als Zahlungspflicht des Gesindeherren (Auftraggebers o. ä.) bzw. als Forderung, Recht des Dienstleistenden angesprochen, damit Gegenstand von Rechtsregelungen, in denen die Zahlungspflicht bzw. das Recht auf Zahlung, die Rangfolge des Liedlohns innerhalb anderer Pflichten / Rechte und die Art der Dienstleistung einen erhöhten Stellenwert einnehmen. - Keine omd. und nrddt. Belege; gehäuft Rechts- und Wirt- schaftstexte, vereinzelt didaktische Texte. Phras.: es brent und brät jm. um den liedlon jim. brennt der (unbezahlte) Liedlohn auf der Seeler. - Bdv.: besoldung, dienstlon, geld 1 , 2; 4, hofzins, trinkegeld 2. - Synt.: den l. fordern / verwirken, mit dem eide behaben (vom Berechtigten gesagt), den l. verhalten / verzieben / vorbehalten / anstehen lassen (vom Zahlungspflichtigen gesagt), jm. (den) l. ausrichten / geben / gelten, den l. an jn. fordern, l. zu $j m$. haben >zu fordern haben`, den l. ungefordert gestehen lassen; der l. ein jar bestehen, spännig erscheinen; jn. des liedlons berauben / bezalen; um den l. kommen, um l. zu recht fürbieten, pfand um l. geben, jm. um l. dienen, jn. um l. beklagen / pfänden (vom Berechtigten), jn. um l. ausrichten / vergnügen / bezalen (vom Pflichtigen), aufjn. um l. klagen, jm. etw. zu l. geben, sich durch l. verschulden, etw. für l. achten, jm. etw. für den l. geben sbillig bezahlen lassen<; der l. der knechte / dienstmeide / taglöner; der billiche / gebürende / schnöde / (un)verdiente l.; die bezalung / quittanz des liedlons; die klage um den l. Wbg.: liedlich > entsprechend liedlonBedingungen (als Klammerbildung auffassbar)<, liedlöner (dies reich belegt im Rwв 8, 1303).

STruck, Joh. Pfannstiel 155, 29 (mosfrk.,1542): Usgyfft vor das gesinde: Pauels Hen der schmit lidlone 9 fl. SCHмiDT, Frankf. Zunfturk. 1, 87, 17 (hess., 1594): im fall er [knecht] auch vor seiner gedingten zeit one erhebliche ubrsachen aus seinem dienst tretten wurde, soll er nit allein seinen verdienten liedtlohn dardurch verwurckt haben. BRINKMANN, Bad. Weist. 268, 34 (rhfrk., 1613): daß diejenige, so [...] den vogtsjunkern, [...] in billichem lidlichem lohn ₹u arbeiten schuldig, [...] gezwungen werden soll. Dinklage, Frk. Bauernweist. 113, 4 (nobd., 15. Jh.): umb lidlon mag ein iglicher einen phenden auf der gassen. Euling, Kl. mhd. Erz. 931, 22 (nobd., E. 15. Jh.): sag aller welt das groste leyt, | dï alle menschen müssen dulden | die sich durch lidlon also verschulden [sschuldig werden ‘]. FRANCK, Klagbr. 222, 19 ('wohl Nürnb. ${ }^{\top}$ 1529): Ja sind einer solichen vnuerschampten stirn / daß sie auch den zehend von dem lidlon der armen dienstmaid knecht und taglóner durffen fordern. GEIER, Stadtr. Überl. 344, 1 (nalem., 1532): wann miner herrn schulden, die bußzinser oder lidlöner vorhanden sind, so söllen deß ersten meine herrn umb ibr schulden, ald, so die nit weren, der bußzinser oder nach im der lidlöner sölliche gütter alle verganten lassen. KöBLER, Stattr. Fryburg 84, 31 (Basel 1520): so zi besorgen wer das uß des schuldners güt nit bezalüg geschebē mócht / so sol von allererst $v \beta$ dem selben sinem gemeinen verlassne 
güt / dz gefrónt würdt / [...] / sin libfall vnd begrebde / [...] / darnach die so im in solcher kranckheit gedient hetten / irs lidlons bezalt / [...] werden. Fuchs, Murner. Geuchmat 3767 (Basel 1519): Was ich genummen hab von dir, | für mynen bydlon gabstu es mir. ARGOVIA 4, 327, 13 (halem., 1550): Welicher sich laszt beclagen vmb zergelt, gelichen gelt und lidlon, dem soll [...] geboten werden, den cleger über ein twärnacht auszurichten, vnd welicher also vm lidlon beclagt wird, der verfallt vns [...] zuo buosz, drei pfund haller. EBD. 9, 73, 7 (1495): was ein schmid mit siner hand gedienet, das ist Lidlon; und wo er einem Isen git, das ist ein kouf, ûßgenommen das einer zum pflug brûcht, das ist Lidlon. EвD. 90, 16 (1572): Wellicher zu Einem Lidlon hätti, der mag's inzüchen $\hat{u} f$ einen Tag, ist es nit verjaret. B̈̈CHTOLD, H. Salat 81, 1 (halem., 1551): Ich wil es dir aber setzen vff din sel, da müs es dir brünnen vnd bratten $\mathrm{vm}$ sin lidlon, den er den gantz. nacht hett vssgeschribben, das er dich vergelti. MAALER 270v (Zürich 1561): Lidlon (der) Salarium, Merces, Pramium, Manupretium. EBD. 277r: Lydlon (das) Das man mit der hand gewunnen hat. Aes manuarium, Manuarium pretium. Hauber, UB Heiligkr. 2, 364, 17 (schwäb., 1484): von lidlons wegen, da soll sich dhainer vor gericht umb laussen beclagen [...7. Item es soll dhainer dhain unnutz pfand umb lidlon geben. Reu, Süddt. Kat. 1, 787, 42 (Augsb. 1543/4): sein gut vnnütz, verschwenden, vergeülen, verbanckethieren, den lidlon vorbehalten. Rössler, Stadtr. Brünn 393, $25 \mathrm{ff}$. (mähr. inseldt., 1. H. 14. Jh.): Von hofczins und von lidlon und von trinchgelt. Wer c₹u chlagen hat auf den andern um bofczins und um lidlon oder um trinckgelt, der mag drei chlag tuen, [...] doch schol man mercken, daz niemant lidlon mag behaben, er sei dan ein gedingter diner oder arbeiter eines herren und sei sein tegleich gesind und esse tegleich sein prot. - KREBS, Prot. Spey. Domkap. 1, 1956, 1; 3656, 1; Cirullies, Rechtsterm. Anh. 1981, 246; KöBler, Ref. Nürnberg 175, 7; Ders. Stattr. Fryburg 80, 6; Spanier, Murner. Schelmenz. 14, 32; SACHs 4, 220, 23; Sudhoff, Paracelsus 8, 34, 41; Geier, a. a. O. 343, 33; Graf-Fuchs, Ämter Interl./Unterseen 371, 12; Welti, Stadtr. Bern 98, 18 ff.; Rennefahrt, Wirtsch. Bern 614, 13; BoNER, Urk. Zofingen 566, 21; Adomatis u. a., J. Murer. Spieg. 1389; Argovia 4, 351, 35; 9, 117, 20; WintTERLiN, Würt. Ländl. Rechtsqu. 2, 784, 40; Chron. Augsi. 6, 59, 8; Wutzel, Rechtsqu. Eferding 86, 7; Siegel u. a., Salzb. Taid. 215, 29; Rössler, a. a. O. 390, 38; Dict. Germ.-Gall.-Lat. 307, 32; Schweiz. Id. 3, 1288; Schmidt, Hist. Wb. Elsaß 222; Vorarlb. Wв. 2,279 .

2. >Lohn der Bergarbeiter`, vereinzelt auch $>$ Lohn von Arbeiter(inne)n in Handwerksbetrieben`. - Omd.; Rechts- und Wirtschaftstexte.

ERmisch, Freib. Stadtr. 277, 31 (osächs., 1350/79): Welch man uf deme hantwerke von wollenslegern ader von spynnerynnen vor den meistern umme ir lon, daz se vordyent haben, wirt beclayt, demselben manne sullen dy meistere sin werg niderlegen alzo lange, biæ. [...]. Ders., Sächs. Bergr. (osächs.,
1479): also, das die sachen, die [...] zсu bergkerecht gehoren, als umb sammekost unnd vordints lidlon, [...] uff das slunigst geendt werden. WeIzsäcker, Graupn. Bergb. 229, 10 (osächs., 1542): dodurch der steiger sampt den arbeitern geursacht, umb iehren vordienten lidlon das recht dar₹u an₹ustellen. Wutke, Schles. Bergb., Cod. Sil. 20, 253, 19 (schles., 1528): dass ein ieglicher getreuer arbeiter seines verdinten lidlons [...] von seiner arbeit bekomen mag. EBD. 261, 6: So mer dann ein [...] verbot auf ein gut geschehen und vor gericht kumen, soll zuvoran gericht werden verdint lidlon. - LÖsCHER, Erzgeb. Bergr. 60, 32; WeIzsäcKer, a. a. O. 142, 30.

liedlöner, s. liedlon 1.

liedmag, liedmage, der; -/auch - $\emptyset$ (für -mag), -mage, -magen.

>Blutsverwandter<; auch: >Erblinie<; vgl. ${ }^{1}$ lied 6. - Alem.; Rechts- und Wirtschaftstexte. - Bdv.: (blut)freund; vgl. agnat, cognat, gesippe (der), leibsfreund, mag 1. - Synt.: der l. erben, ein gut kaufen; dem l. etw. bieten / leihen, dem l. etw. zustehen; der náchste l.; blutfreunde an der l. Wbg.: liedmagschaft.

Weltr, Stadtr. Bern 55, 31 (halem., A. 14. Jh.): So denne die muter stirbet, so erbent die nechsten lidmage. RENNEFAHRT, Staat/Kirche Bern 836, 8 (halem., 1546): so vil den grad der lidmagschaft betrifft, darin sich die trostung erstrecken soll, das die bemelt trostung sich nit wyter erstrecken sólle, dann biß in das dritt glid dero, so einandern zu erben [...] hand. Ders. Statut. Saanen 22, 1 (halem., 1397/ 98): denselben sóllent und mógent die nechsten frunde, [...], und lidmag an der linien der sibschaft, [...] erben. ЕвD. 214, 3 (halem., 1598): so soll sich des abgestorbnen git in zwo linien erben, der halbteil vater balben den nöchsten, der ander balbteil müter balben den nöchsten blutfründen an der (hier natürliches Geschlecht oder Kopfbildung für liedmagschaft) lyd mag und lynien der sipschaft. - GRAF-Fuchs, Ämter Interl./Unterseen 88, 16; Rennefahrt, Zivilr. Bern 154, 33; Sснміdт, Hist. Wb. Elsaß 222.

\section{liedmage, liedmagschaft, s. liedmag.}

liedmas, (vereinzelt:) liedemas, das, auch die; -/-en, -er, $\emptyset,-e+\mathrm{Uml}$.

1. `Gliedmaß, Körperteil<; zu ${ }^{1}$ lied 4; teils im Sinne der Beziehung von ,Haupt und Gliedern'; vgl. dazu ${ }^{1}$ lied 5. - Bdv.: vgl. auswendigkeit 1, ${ }^{1}$ bein 1, glied 1; 2, gliedmas (das) 1. - Synt.: keine l. haben, die l. greifen / sehen / regen, an sich nemen; die liedmas des leibes / leichnams, unter einem haupte; gleiche / rechte liedmas(sen); kundschaft der liedmassen.

Toeppen, Ständetage Preußen 5, 71, 27 (preuß., 1463): die [...] undir einem herrn geleiche rethe weren und undir eynem 
boupte geleiche lithmoszen. JosTEs, Eckhart 46, 23 (14. Jh.): di sel wurchet in einen influz in alle die leidmazzen des leibes an chuntschaft der lidmazzen. STRAuch, Par. anime int. 83, 6 (thür., 14. Jh.): wan daz kint inphangin wirt in der mudir libe, so bait ez lidemese. SCHÖNBACH, Adt. Pred. 8, 36 (osächs., 1. H. 14. Jh.): swer so geloubet an Christum der nützet in und horet im zu undist sin lidemaz. worden. MAYER, Folz. Meisterl. 19, 55 (nobd., v. 1496): Wo nit die kerefft $\mid$ Der sel all lidmas regten. KLEIN, Oswald 63, 37 (oobd., 1416): doch möcht ich es gefügen bas, | das ich die lidmass [von der gürtel umbevangk bis auf den füss] griff und säch. - RedLICH, Jül.-Berg. Kirchenp. 1, 270, 36; Mayer, a. a. O. 18, 140.

2. >Ebenmaß des Körpers und seiner Glieder^; speziell: >Gesichtszüge; Handlinien〈; vereinzelt: >Gleichgewicht generell<; zu ${ }^{1}$ lied 4.

Volkmar 329 (Danzig 1596): Lineamentum, die strich / oder liedmaß am antlitz / vñ hendẽ. Tiemann, E. v. Nassau-S. Kgn. Sibille 118, 22 (rhfrk., um 1435): Des keysers dochter [...] hat gar eynen schonen lyp vnd gude gelider in rechter lydemasse. KOLLER, Ref. Siegmunds 52, 7 (Hs. um 1475): das es nit mer woll gen mag, man hab dan ein rechte ordenung deß geistlichen und weltlichen standes, wann dye stend ploß on alle lidmaße. EвD. 173, 21 (Hs. rAugsb., um $\left.1400^{\urcorner}\right)$: an allen sachen, an den got gemaint sol werden und in zugehort; daran hat nymant kain rechte lidmaß mer, gots und seins rechtens ist gar vergessen (hierher?). V. ANsheLm. Berner Chron. 2, 152, 21 (halem., n. 1529): einen geraden körpel, also nach aller lidmauss ganz zuisammen getragen wurdend. Spechtler, Mönch v. Salzb. 1, 64 (oobd., 3. Dr. 14. Jh.): Keuschlichem leib | gab recht lidmass die modelscheib [auf Maria bezogen]. - Wackernell, H. v. Montfort 5, 50; Baumann-Zwirner, Augsb. Volksb. 1991, 208

liedmässig, Adj.

$>$ körperlich (wohl)geformt, gerade, schön`; zu liedmas 2. - Bdv.: ${ }^{2}$ gerade (Adj.) 2. - Wbg.: liedmässigkeit.

Ziesemer, Proph. Cranc Ez. 17, 3 (preuß., M. 14. Jh.): eyn groz adylar grosir vlogele, langir ledemasikeit [Worms. PROPH. / Froschauer 1530: schwingfádern; Еск 1537: schwaif der glider; LUTHER 1545: fittichen] vol veddirn. GILle u. a., M. Beheim 267, 76 (nobd., 2. H. 15. Jh.): zwai bain, lid messig ach | sein sy gerichtet nach der sichel. Евр. 268, 77: zwai rechte lid messige peine | und die sein als zwen pölcz gerichtet schaun. BARACK, Zim. Chron. 1, 338, 2 (schwäb., M. 16. Jh.): Derselbig herr Wörnher wardt von menigclichem als der gredest, lidmessigest herr [...], geachtet, desshalben er fast angesehen [...] ward.

\section{liedmässigkeit, s. liedmässig.}

liedmüte, der; Gw zu mhd. miete, müete ‘Lohn`(LeXER 1, 2134).
>Liedlohn, Gesindelohn^. - Bdv.: vgl. liedlon.

Winter, Nöst. Weist. 3, 818, 30 (moobd., v. 1483, Hs. 1643): wan ainer gegen dem andern spricht umb lidmüet, die mueß er bestetten mit seinem rechten.

liednagel, s. ${ }^{3}$ lied.

liedschärte, s. ${ }^{1}$ lied 3.

liedsucht, liedsüchtig, s. ${ }^{1}$ lied 1.

liedtief, s. ${ }^{1}$ lied 3.

liedweich, Adj.

'weich, biegsam (von Sachen); schlapp, matt, entkräftet (von Personen) <; dazu ütr.: >wankelmütig‘; vgl. ${ }^{1}$ lied 1 ; 4. - Bdv.: vgl. biegenlich, biegig, geleichnig, geschmeidig, gleichig, lenke. - Wbg.: liedweiche.

SCHÖPper 52a (Dortm. 1550): Imbecillis. Schwach unstarck, machtloß vnuermigig blód lidweich 1 luck vnuäst weich. HeLm, H. v. Hesler. Apok. 11030 (nrddt., 14. Jh.): Wen wir lide weichen $\mid$ Nach den worten uns bekarten $\mid$ Die jen alden uns vor larten. JörG, Salat. Reformationschr. 368, 11 (halem., 1534/5): das diß geschrifften allein für die ober / und erberkeytt / alls die bochverstendigen / und nit für die lidweychen gmit des unverstands / oder wyplich gscblecht / alls durch truck usgan noch kon wirdt. MAALER 270v (Zürich 1561): Lidweich / Weich / Zách als weyden. EвD. 277r: Lydweich / Zách / Das sich gern laßst zesamẽ legen oder biegen. [...] Lydweiche (die) Lentitia. Barack, Zim. Chron. 2, 140, 35 (schwäb., M. 16. Jh.): Do waren die pauren gegenwürtig, deren ieder ain lidwaichen tremmel in der bandt. - ЕвD. 3, 611, 42; SсHÖPper, a. a. O. 1146; SChweIz. ID. 15, 205.

liedweiche, s. liedweich.

liefergeld, s. liefern 1.

lieferherre $1 ; 2$, s. liefern $1 ; 3$.

lieferkauf, s. liefern 1.

liefern, V., über mnl. Vermittlung aus afrz. livrer >liefern< (RosenQvist, Frz. Einfluß. 1943, 385).

1. >etw. (sehr unterschiedliche Bezugsgrößen) liefern, abliefern, überstellen, aushändigen «. - Rechts- und Wirtschaftstexte, Chroniken. - Bdv.: überantworten, übergeben, zustellen; vgl. abdienen 2, abfüren 7, abschütten 6 , antworten 8, auserlegen, beigeben, stellen 1. Ggs.: verhalten. - Synt.: etw. (z. B. abholz./ geld / strob / silberwerk / steuer / zin / bü- 
ner / kälber / lämmer, die bete, einen topf, ein pfand / register / ros, eine ansprache / tafel) l. Wbg.: liefergeld >abgeliefertes Geld<, lieferberre 1 >Lieferant (von Rohstoffen)<, lieferkauf $>$ Lieferung (von Holz) aufgrund eines Kaufvertrags

Dat Nuwe Boych 432, 29 (rib., 1396): beuall, dat be dat gehauen gelt weder in die Rentkamer brengen vnd leueren sculde. LAMPRECHT, Dt. Wirtschaftsl. 476, 36 (mosfrk., 1548): doch das solche zuordnungh des nebenfursters lenger nicht, dan dis liferkouif volzogen, werhen. CHRON. MAINZ 1, 152, 33 (rhfrk., 15. Jh.): wir wolten sie [registere] ungern verhalten, sondern ine die gerne [...] geliebert haben. ЕвD. 1, 159, 8: also wart dem rade solich ansprache gelebert von den 20 of mandag. KöBlER, Ref. Wormbs 79, 7 (Worms 1499): das ich dẽ. N. verkaufft vñ er vmb mich kaufft hat ein Huß. Roß. Fuderwyn $\beta$ ec. das ich ym zu syner gewaltsam [...] gelibert oder vberantwort habe. SСHмIDT, Frankf. Zunfturk. 1, 246, 31 (hess., 1511): wurde aber eyner gemacht wergk, von eynem goltsmit [...] kauffen, so sal der liebererberre das probegelt betzalen. CHRON. NüRNB. 4, 170, 12 (nobd., 15. Jh.): und ieglich person, [...7, söllen von hundert milion lifer gelts [...] begabt werden. Merk, Stadtr. Neuenb. 121, 37 (nalem., 1616): das abholz und fleckling, so wider zue brauchen, in gewohnliche werkheuser zue liferen. MüLLER, Welthandelsbr. (schwäb., 234, 28): so bedingt einer wol, daß im $\beta$ der verkaufer ins scheff libert an die ort, (so) er haben will. Chron. Augsb. 8, 419, 9 (schwäb., zu 1556): daß sie dem kriegsvolck [...] alle wochen 140 klaster [holtz] lüffern. - Helbig, Qu. Wirtsch. 4, 94, 31; Kollnig, Weist. Schriesh. 125, 10; 16; KüTHER, UB Frauensee 372, 11; 14; Chron. Augsb. 5, 29, Anm. 4; Henisch 1460; Dict. Germ.-Gall.-Lat. 307, 42; Rwb 8, 1320; Pfälz. Wв. 4, 983. - Vgl. ferner s. v. achtmas, ausserman, pastor, befreien 2 .

2. >jn. ausliefern, überstellen; jn. (von Christus gesagt) dahingeben «. - Bdv.: vgl. anfeilen 3, anhändigen, antworten 9, aufantworten 1 , ausantworten 1, ausbetägen, ausfolgen 2, ausbingeben, ausliefern, dargeben 2, hingeben 2, lassen 8. - Synt.: jn. (z. B. die tochter, den son, das haus >die Familie, Dynastie<, den mistätigen man, den mistäter / herren) l., jn. jm. (z. B. dem nachrichter / tode) (an / in das gericht) l., sich jm. in die hand l.; j. der diebin geliefert sein.

Chron. Magdeb. 2, 97, 20 (nrddt., 1564): weil die Graffen von Mansfelt lautt deß ufgerichten vortrags daß hauß Gaterschleben nicht lieffern konten. Мєіјвоом, Pilgerf. träum. Mönch 8229 (rib., 1444): Ich wil dich balde leveren dẽ dode. EBD. 9002: Overmitz sij byn ich diesen alden dievynnen, [...] Gelevert. Buch Weinsb. 4, 202, 36 (rib.,1594): das mer gab, man sult den Sommer an das recht liebern. So wart er dem greven geliebert. Franz u. a., Qu. hess. Ref. 4, 104,
6 (hess., 1463): Es ist auch nicht ware, das sie sagen, wen man sie proscribert, man wolle sie nicht toden, aber andern wolle man sie libern, die sollens tun. CHRON. NürnB. 4, 170, 7 (nobd., 15. Jh.): ist dir das zu willen, so wöllen wir die tochter und das gelt [Zeugma] in unsern casten lifern von unser stat Alkeyro. zU DoHnA u. a., Staupitz/Scheurl 237 (Nürnb. 1517): got, der seins eignen suns nit verschont, sunder den für uns all gelifert. v. BIRKEN. Erzh. Österreich 71, 17 (Nürnb. 1668): der / ohne gegeben geleit [...] sich also seinem offenbaren Feind in die hand liefern dorfte. Kehrein, Kath. Gesangb. 1, 202, 43; PFÄLz. Wв. 4, 983. - Vgl. ferner s. v. ausleiten 1.

3. >(vor allem: Truppen) stellen, unterhalten, beköstigen «. - Bdv.: besolden 1 , balten 14 ; 18 ; vgl. aufenthalten 5 , aufhalten 8 . Wbg. lieferherre 2.

ENDERs, Eberlin 3, 35, 17 (Erfurt 1523): die [Stat] hat ein hauffen teuffels kriegsknecht, welche der teuffel do halt vnd lyfert. Neubauer, Kriegsb. Seldeneck 103, 23 (nobd., 2. H. 15. Jh.): so man den hauptman jn der beutt nennt, so jst domit gemeint der, deß der rutt vnd die sach jst vnd der den zeuck vff desmols geliffertt hott. BerNoulli, Basler Chron. 6, 446, 3 (alem., 1503): sind min herren von Bassel gezogen gon Beltzentz mit 500 man, und hend die selben 500 man geliffet in irem kosten [...]. Und ist min schwnoger Petter Offenburg und Fridrich zür Linden boblit gesin, und lifferher Willem Zegler. - Rwв 8, 1323; Vorarlb. Wв. 2, 283.

4. als Funktionalverbgefüge: >jm. eine Schlacht liefern

PeIL, Rollenhagen. Froschm. 570, 2007 (Magdeb. 1608): Da du erst gabst den falschen rath | Euch lieffern ein blutig Feldschlacht. MaAler 273 (Zürich 1561): Eim ein schlacht Liferen. Componere se alicui. - EschenloHer. Medicus 50, 16.

5. >jm. Erleichterung schaffen « (s. dazu lieferung 3). - Schmidt, Hist. Wb. Elsaß 221 (a. 1467).

\section{lieferung, die; $-\emptyset / ぬ$.}

1. >Belieferung von jm., Unterhalt, Versorgung, Verköstigung, die aus finanziellen Zuwendungen, Lebensmitteln, Kleidern, Ausrüstungsgegenständen u. a. besteht und (seltener:) an Einzelpersonen und Personengruppen, (häufiger:) an Truppen gegeben wird/; als Metonymie: >die konkrete Lieferung`(nomen acti); vgl. liefern 1; 3. Mittleres und späteres Frnhd.; Wirtschaftstexte, Chroniken. - Bdv. (bzw. zum Sachfeld gehörig): aufenthalt 2, enthalt, futer(ung), bilfe, proviant, rüstung, sold, treide, victali, zerung. 
- Synt.: l. finden / haben, js. l. gebrauchen, jm. l. geben / verschaffen; l. (Subj.) etw. gestehen >kosten ‘; die keranken mit l. versorgen, jm. etw. an l. abgeben; l. zum krieg; die gute l.; wein zu l. Wbg.: lieferungsbrief > schriftliche Anweisung auf Beköstigung` (a. 1522).

SchÖPper 1096 (Dortm. 1550): đ Commeatus. Fursorg vorrat 9 futerung liferung proviand victaly. HERBORN u. a., Rechn. Jülich 65, 11 (rib./snfrk., 1398/9): Dit is vort uyssgeve (n) [...] van ]...] lev(er)unge(n) en(d) vort van tzeruncge(n) ind coste (n), die ich ind vort mijns lieve(n) h(er)n vrunde [...] v(er)tzert hain. UB Zug 1848, 15 (halem., 1503): das sÿ och der selben unßer bottschafft zue lon geben sollen alle tag ein halben Rinschen guldin [...] darzu lüfferung, futter unnd mal, nagel und ÿßen. MaAler 75r (Zürich 1561): Bottenlon (der) alle lyferung und kosten oder zeergált [...]. Legatiuum. Schwarz, Awürt. Lagerb. 1, 156, 3 (schwäb., 1524): Dargegen soll die herschaft inen und den pferden liferung geben. Rотн, E. v. Wildenberg 137, 23 (moobd., v. 1493): das er überschlahen lies, was 10000 man gereissig zwei oder drei jar gestünden mit sold und lifrung zu taglichem kerig. Turmair 4, 174, 6 (moobd., 1522/33): verpurgen ir traid und ander groß hab und guet, mainten, die Teutschen würden kain liferung finden. - ENDERs, Eberlin 3, 9, 22; 150, 7; Meisen, Wierstr. Hist. Nuys 1288; Baumann, Bauernkr. Rotenb. 38, 20; 311, 14; V. Anshelm. Berner Chron. 4, 3, 26; 15, 3; 252, 23; JöRG, Salat. Reformationschr. 272, 1; 497, 10; TURMaIr 4, 257, 10; 323, $31 ; 387,3$

2. >Auflassung (eines Grundstückes)<; vgl. am ehesten liefern 1. - Bdv.: vgl. auflassung, aufreichung.

LoERsch, Weist. Boppard 299, 32 (mosfrk., 1592): ob nicht ein vogt lieberung thu mit halm und mund? Sprechen die scheffen: Ja.

3. > Erleichterung, Linderung (von Schmerzen, Krankheit, Bedrängung)<; als Metonymie an 1 anschließbar, dennoch semantisch fraglich; s. u. den Kommentar zu Schmidt; vgl. liefern 5. - Bdv.: labe 2, ergezlichkeit, erlösung.

Fuchs, Murner. 4 Ketzer 2050 (「wohl Straßb. ` 1509): So weisß ich noch ein wasser mer, | Das jm ein lüfrung kïnne machen. Michels, Murner. Badenf. 33, 13 (Straßb. 1514): Künd ich im nit ein liffrung geben, | Das schwitzen brecht in vmb sein leben. - SCHMIDT, Hist. Wb. Elsaß 221 (hier in der Schreibung lib-, was etymologische Umdeutung in Richtung auf lat. liber-nahelegt).

lieferungsbrief, s. lieferung 1.

liegbet, s. ${ }^{1}$ liegen 2.

liegeinung, die; $\mathrm{zu}^{2}$ liegen.

$>$ Buße für die Nichteinhaltung einer Auflage<; metonymisch: > Recht auf Einzug der
Bußer. - Bdv.: schelteinung. - Synt.: die l. haben, jm. die l. nachlassen; die unrechte $l$.

Brinkmann, Bad. Weist. 64, 13 (rhfrk., 1555): ein unrecht liegeinung [Var. 1661: liegeneinung], und so einer die offen tag versaumpt, ist funf to. heller. - Rwв 8, 1498.

liegelachen, das; zur Erklärung des Bw s. leiblachen, leichlachen, leilach 3.

>Bettuch<; zum Bw vgl. 'liegen 2; volksetymologische Umdeutung von leib-, leichlaken oder von leilach 3 (s. jeweils dort) möglich, aber nicht wahrscheinlich; Gw zu lakeen.

Struck, Marienst. Wetzlar 570, 14 (hess., n. 1400): 3 fl. vor dy czepei guden lygelachen unde anders alde ligelachen, banttwelen.

${ }^{1}$ liegen, V., unr. abl. - Nähesprachliches Verb mit schwer gliederbarem Bedeutungsspektrum; hohe Phrasemaffinität; zu speziell rechtsrelevanten Verwendungen s. RwB 8, 1325 ff., auch die zugehörige Kompositenreihe.

1. >wo liegen (von Sachen, wobei stehen als Gegensatz assoziiert werden kann); sich in der Fläche (wie z. B. Hagel) ausdehnen`; in mehrfacher Hinsicht spezialisiert, z. B. von Waren: >wo lagern, gelagert werden<; von Arzneimitteln: >stehen, ruhen, sich absetzen〈; von Verbandszeug: >aufgelegt, angebracht sein/; von Briefen: >wo aufbewahrt, hinterlegt werden «. - Ggs.: stehen 6. - Synt.: garben / gras / bolz / stro / hagel / karten / späne / steine / stricke, die tafel / kanne, der brief [wo; mit Ortsangabe] l., die karten auf dem tisch, die garben auf dem acker, die kandel in der lade l.; verschoben: der berg fol schne l.; liegendes hol:

PEIL, Rollenhagen. Froschm. 631, 3890 (Magdeb. 1608): Als Berg / Thal / Feld / vol schne noch lag. MEIJвоом, Pilgerf. träum. Mönch 7035 (rib., 1444): Laegen in myme wege stricke ind korden. KeIL, Peter v. Ulm 96 (nobd., 1453/4): guten essig; den scbütt doran daz er dorüber gee. Das laß ligen acht tag wol bedeckt. SACHS 17, 86, 28 (Nürnb. 1553): Mein Eulenspiegl, wie steht dein sach? [...] Eulenspiegel spricht: Mein sach stebt nit, sondern sie leit. BARACK, Teufels Netz 6840 (Bodenseegeb., 1. H. 15. Jh.): Ie naher es [strow] dem für lit, | Ie vester es die wermi git. Cirurgia H. Brunschwig 33 $3^{\text {vb }}, 6$ (Straßb. [1497]): das dï die wunden allen tag binden magst / nit dester minder das gebend belib ligen. GiLman, Agricola. Sprichw. 1, 228, 22 
(Hagenau 1534): Were eyn steyn nicht alleyn erbeben kan / der sol yhn auch selbander ligen lassen. CHRON. AugsB. 9, 308, 18 (schwäb., ): der [spruchbrief] ward der zunft geben mit aines rates großem insigel, der noch binder einer zunft leit. Bastian, Runtingerb. 2, 19, 16 (oobd., 1390): derselb leibtingbrif sol dacz ainem biderman ligen. ЕвD. 2, 56, 23 $(1383 / 84)$ : noch ligent da 2 to zigelfarber seyden $d R$ an 4 lot. Bauer u. a., Kunstk. Rud. 626 (oobd., 1607/11): 1 klein täfelin [...] ligt in einem fütterlin von lindenholt». ZINGERLE, Inventare 41, 1, 19 (vorarlb. / tir. 1478): i grosser schrein, darvnter lig smidezeng vi czangen. EвD. 71a, 20 (1484): ob mer guts in dem palhawss lëg, mag ain zollner auffgeben. Bischoff u. a., Steir. u. kärnt. Taid. 368, 18 (m/soobd., 17. Jh.): soll der forstner niemant kein [...] stebenten stamb boly, alleweil wüntfähl und ligunts vorbanden, außzaigen. Qu. BRAssó 4, 284, 35 (siebenb., 1664): welcher [Hagel] fast einen halben Tag bleibet liehen auf der Erd. - Hajek, Guite spise 14; M. Cunitia. Ur. Prop. 149, 25; v. Keller, Ayrer. Dramen 2447, 11; Siegel u. a., Salzb. Taid. 71, 1. - Vgl. ferner s. v. abschaben 1, abwenden 6, ächterkandel 2, äheren, aufhauen 3, ausrinnen 2.

2. >wo liegen (von Personen, auch von Tieren) ; je nach Ursache und Zweck des Liegens im einzelnen z. B.: >tot liegen<; >liegen, um zu ruhen oder zu schlafen<; >faul herumliegen`; >im Kindbett liegen`. Phras.: am rücken liegen >tief gefallen sein<; bei jm. liegen >mit jm. schlafen`; eines kindes liegen. - Synt.: schlafend l., an / auf / in dem bette, zu bette, an dem todbette, in der bütte / krippe, im grabe / stalle / kote, in anmacht, in seiner rube l. (>tot seins), bei dem schweher (im Grab) l., auf der wiese, auf dem rücken, vor jm., für dem tor, unter jm., unter / vor js. füssen, unter der stiege, unter dem altar, bei jm. (häufiger bei einer Frau, z. B. bei dem weibe, der frauen / nonne / magd, als bei einem Manne, z. B. bei dem bruder) l. Wbg.: liegbet >ausgestrecktes Bett zum Liegen〈, ${ }^{1}$ lieger >Lager, Bettstelle . PeIL, Rollenhagen. Froschm. 549, 1363 (Magdeb. 1608): Allein der Hund lag fur dem Thor. Grosse, Schwabensp. Grosse, Schwabensp. 149a, 6 (Hs. rnd./md., um 1410 $\left.{ }^{7}\right)$ : LJt ein man an deme totbette. EBD. 175a, 15: Begriffet eyn man eyne maget vf eime velde, [...] vnde legit der man bi ir, man sol in toten. CHRON. KöLN 1, 1005 (rib., Hs. 1. H. 15. Jh.): Sint saich bie sy aichter dem velde liegen | beide man, ors da neder. Tiemann, E. v. Nassau-S. Kgn. Sibille 160, 16 (rhfrk., um 1435): das niergen ein bube ist / er habe mit ir in den gräben gelegen. THür. CHRON. 2r, 9 (Mühlh. 1599): [Noab] ward Truncken / vnd lag in seiner Hütten. OpITz. Poeterey 32, 2 (Breslau 1624): Wann ich nach langer zeit schon lieg in meiner rbue. Loose, Tuchers Haushaltb. 174, 5 (nürnb., 1487): ein stund vor tagß, da ist mein weib eins kind gelegen. RuppricH, Dürer 1, 158, 133 (nobd., 1520): hab herr Niclaus Ziegler geschenckt ein toden liegenden Christum. EвD. 171, 129 (1521): Das sind die erschlagnen, unter dem altar gottes ligent. v. Keller, Ayrer. Dramen 2800, 9 (Nürnb. 1610/8): Schläfft jhr Mann, so bleibt sie nicht lign. EвD. 2803, 10 : Sonst liegen stets die Weiber vnder (hier ütr.: > unterlegen sein $<$. EвD. 2885, 31: Jahnn ligt vnder der Franen. EвD. 3093, 25: was machst du bie? | Ich dacht, du legst im bett. Gilman, Agricola. Sprichw. 1, 81, 24 (Hagenau 1534): Wer da ligt / der ligt / dem bilffet niemand widder auff. EBD. 79, 15: Da ligt es / sagt ihene gute magdt / da empfiel ybr das kind am tantze. EBD. 187, 26: Simonides fand ligen am mere eyn todten leichnam. EBD. 552, 2: lang ligen / und nicht wollen wider aufstehen / ist schande. EBD. 2, 239, 19 ([Augsb.] 1548): zwólff klaine Ferckeleyne|Die lagen unter ainer kalten stiegen. RIEDER, St. Georg. Pred. 7, 24 (Hs. $\left\ulcorner^{\circ}\right.$ önalem., 1387`): daz ain priester bi ainer gewibten nunnen wár gelegen. Wyss, Luz. Ostersp. 9505 (Luzern 1545): doselbst wend wir inne [jesus] bin tregen. | wann nie kein mensch dorinn [grab] ist glägen. MAALER 273r (Zürich 1561): Ligen / Rüwen. [...]. Auff dem angsicht Ligen. [...]. Die kindtbetterin Ligt binder dem vmbhang. [...]. Jch Lig im bett biß umb die viere. [...7. Im graaß Ligen. EBD. 273v: Liger / Etwas gespreits ze ligen / es seye dem menschen oder dem vych. HAIL. ALTVÅTER 107v, 1 (schwäb., E. 14. Jh.): do er als vnbeweglich lag. Morrall, Mandev. Reiseb. 157, 9 (schwäb., E. 14. Jh.): er lit nun vierstund des järes by sinen wiben. Heydn. maister 30v, 23 (Augsb. 1490): Die schweĩ ligê lieber jm kot dẽn i eĩ klarẽ wasser. BAUER, Geiler. Pred. 76, 23 (Augsb. 1508): lig vor seinen füssen und laß dich davon niemand abweißen / als lang untz das er dich begnadet. EBD. 316, 9: als ainem esel / dem liebt futer und hew und im stal zu ligen. Hauber, UB Heiligkr. 2, 484, 19 (schwäb., 1553): ain und zwainzig ligbeth, dreüundvierzig deckbeth. BArack, Zim. Chron. 1, 588, 36 (schwäb., M. 16. Jh.): Man sollt dich nit lan im landt, | Das du so gar am rugken leist. Henisch 341 (Augsb. 1616): Ligbeth / culcitra [...7. Ligbeth mit stro oder rohr. PRIMISSER, Suchenwirt 5, 525 (oobd., 2. H. 14. Jh.): Dem fúrstein junch und tugenthaft, | Sein schone fraw wêr gelegen, | Di het ein chind. SpILler, Füetrer. Bay. Chron. 21, 20 (moobd., 1478/ 81): so muest im Valerianus under seinen füessen ligen zu schmach. EBD. 47, 7: Im starb die edel heilig fürstin Nicostra und ligt bei irem sweber. EBD. 152, 11: unkeusche frawen; die gerte das zu ainem grafen, das er bei ir läg. BAUER u. a., Kunstk. Rud. 1805 (oobd., 1607/11): ist wie einer im bett schlaffendt ligt. - STEDTFELD, Roger-Glosse 85; Kehrein, Kath. Gesangb. 1, 83, 2; v. Keller, a. a. O. 2479, 11; 3320, 4; KoppITz, Trojanerkr. 3; ВАCHmann, Morgant 174, 25; Eschenloher. Medicus 85, 19; Chron. Augsb. 4, 177 [Var. zu Z. 23]; 8, 223, 14; SAppler, H. Kaufringer 10, 5; Klein, Oswald 58, 7; Bauer u. a., a. a. O. 2805. - Vgl. ferner s. v. äbich 3, absünen 2, abwesen, abrälen 1; ak 2, amacht 1, ${ }^{1}$ an 2, angesiegen 4, aussegnen 3, pagalette, bauerköter, begeben 6 .

3. >topographisch, katasteramtlich innerhalb, auf etw. liegen, das flächenhaft, als 
Linie o. ä. gedacht ist, wo situiert sein, sich befinden (von Fluren, Liegenschaften, Landflächen, Städten)<. - Rechts- und Wirtschaftstexte, berichtende Texte. - Synt.: der berg, das erbe / gut / land / vorwerk an / auf / bei / in / gegen / oberbalb / unter [einem topographischen Bezugspunkt] $l$.

Grosse, Schwabensp. 230a, 35 (Hs. ' nd./md., um $\left.1410^{\urcorner}\right)$: unde lat daz, gut nicht in dem gerichte, da der man beclaget wert, Man sol iz varbaz, halten. LAMPreCHT, Dt. Wirtschaftsl. 3, 509, 20 (mosfrk., 1301): die erbe, die da ligent, die geben alle zehen. Ralegh. America 12, 39 (Frankf. 1599): Diese Landtschafft Amapaia [...] ligt an dem Oronoke. Küther, UB Frauensee 233, 2 (thür., 1463): gerechtigkeit, [...] uff der wiesen zcu dem Rode bii der Roner wiesen gelegen. THür. Chron. 25v, 15 (Mühlh. 1599): Dann erst da jtzt die Stadt liegt ein gantz. Sóhrichter Eschenwalt. v. Tscharner, Md. Marco Polo 28, 17 (osächs., 2. H. 14. Jh.): Das lant das do umme lyt in mancher tage reyse. JAHR, H. v. Mügeln 901 (omd., Hs. 1463): in des landes mittel lak | ein burk. MAALER 273r (Zürich 1561): Es Ligt ein váld darzwischend. Dreckmann, $\mathrm{H}$. Mair. Troja 37, 19 (oschwäb., 1393): ain wazzer [...] tailt die stat in zwen tail, daran legend mülin on zal. HAIL. ALTVÁTER 81r, 17 (schwäb., E. 14. Jh.): die lant die in dem mere vnd in den ynsellen lägend. Grossmann, Unrest. Öst. Chron. 163, 28 (oobd., 3. Dr. 15. Jh.): Und wardt die ganntr gegent, darum gelegen, verderbt. MoscouIA C IIIv, 17 (Wien 1557): Drobobusch so oberbalb Smolensco ligt. Ralegh. America 1, 23; 11, 8; 12, 15; Bindewald, Texte schles. Kanzl. 2, 12; 74, 7; Chron. Augsb. 5, 303, 14; Rauwolf. Raiß 7, 16; Roth, E. v. Wildenberg 62, 12; Memminger Chron. Chr. 51, 5; Spiller, Füetrer. Bay. Chron. 115, 41; Schöpper 41a; Vorarlb. Wв. 2, 284. - Vgl. ferner s. v. abend 5, abgang 3, abschus 1, ${ }^{1}$ als $15,{ }^{2}$ anger $1,{ }^{1}$ aue 1 , auströlen, befangen 5 , beieinander 1 .

4. >sich innerhalb, in einem Raum oder einem räumlich gedachten Gebilde befinden; wo eingebunden, gefangen, situiert sein (von Personen und Sachen)<; der Ort der Situierung wird durch eine Raumangabe (meist Präpositionalangabe) zum Ausdruck gebracht. - Synt.: j. in eisen, in der falle, in dornen, im turm, in der kirche / belle, in einem ofen, in js. schos, an js. arm (iin js. Armen ২), im gestank / schlam l., [von Sachen:] schiffe [wo] l., das erz in der grube, die befe in einem fas, die därme im bauche, das land unter einer rauben luft $l$.

Dat nuwe Boych 425, 22 (rib., 1396): erbere lude, die in tornen laigen. Tiemann, E. v. Nassau-S. Kgn. Sibille 146, 26 (rhfrk., um 1435): Die frouwe ist zu mal schone /
Sy sol noch bint an myme arme lygen. OORschot, Spee/ Schmidt. Caut. Crim. 225b, 32 (Frankf./M. 1649): sie in jhrens schlam und gestanck. / darin sie offtmabls liegen zu trósten. Luther, WA 10, 1, 1 (1522): Es ligt das ertr. noch halb ynn der gruben. OpITz. Poeterey 14, 31 (Breslau 1624): das vnser Land vnter so einer rawen vnd vngeschlachten Lufft liege. v. Keller, Ayrer. Dramen 3006, 25 (Nürnb. 1610/8): die Frau, die all Tag | In der Kirchen führt solche klag | Vnd fast darinn ligt Tag und Nacht. LauATER. Gespånste 65r, 16 (Zürich 1578): Sy werdend in der hell ligẽ wie die schaaff. SAPPLER, H. Kaufringer 26, 61 (schwäb., Hs. 1472): der sollt ee bundert jar pflegen | in ainem feurin ofen ze ligen. Chron. Augsi. 8, 164, Anm. 2 (schwäb., zu 1562): so hat ain e. rat erkannt, daß er ain monat lang in Eisen ligen soll. - Gille u. a., M. Beheim 82, 166; Bachmann, Morgant 172, 3; Chron. Augsb. 2, 110, 6; Sappler, a. a. O. 19, 69; Dirr, Münchner Stadtr. 405, 11; Klein, Oswald 4, 54; MaAler 273r. - Vgl. ferner s. v. bauch 6 .

5. >sich wo aufhalten`; je nach Art des Aufenthaltes: >wo wohnen, siedeln; wo übernachten; wo hausen, verharren, herumhängen` (mit dieser letzteren Nuance eng an 2 anschließbar); > wo zu Besuch weilen r. - Bdv.: vgl. aufbleiben, aufhalten 10, bedonen, bleiben 1, gebauen 1, gesein 2, leben 5, pernoctieren. - Synt.: auch absolut; $j$. zu hause, auf dem hause / gute, an dem lande, in klöstern l., j. zu [einer Stadt, z. B. zu Wien] l., j. [eine Zeitlang, z. B. über nacht, drei tage] wo l., (oft:) bei / zu jm. l., j. gegen orient l. Wbg.: lieggrube >Spelunker (dazu bdv.: höle, laggrube), lieglingen $>$ liegend $<$.

Holland, H. J. v. Braunschw. V. e. Weibe 264, 24 (Wolfenb. 1593): Wenn ich nicht wanderte, und stets zu Hause ligen wolte, würde ich nicht viel erwerben. CHRON. KöLN 2, 283, 4 (Köln 1499): dat voulk dat am Rin up beiden siten liggen is. VOC. INC. TEUT. $\mathrm{p}$ iv (Speyer um 1483/4): Liggrub. Spelunca. WunderLich, Fierrabr. 125, 11 (Simmern 1533): Keyser Karle bleib da wol drei oder vier tag liegen. Kurz, Waldis. Esopus 4, 41, 16 (Frankf. 1557): So leistu bie, bist faul und treg. v. Tscharner, Md. Marco Polo 4, 29 (osächs., 2. H. 14. Jh.): In Mosil ist der patriarcha allir cristin di do legin kegin orient. ЕRMISCH, Freib. Stadtr. 49, 18 (osächs., 1335): Di wile muг der gast ligen unde warten. Rupprich, Dürer 1, 150, 89 (nobd., 1520): do logen wir übernacht und verzehrten 3 weispfenning. Chron. Augsi. 7, 208, 4 (schwäb., zu 1550): daß der Hanns [...] bei dem Jacob Meuttin gelegen. EBD. 219, 25 (1550): ist seiner trabanten ainer [...] auf in gefallen, dann in sonst die Spänier erstochen hetten also liglingen. МемміNGER Chron. Chr. 24, 26 (Ulm 1660): Es lag einer bier / hieß der Zeller / war sechs Tag lang hier mit vier Pferdten. Pfeiffer, K. v. Megenberg. B. d. Nat. 322, 12 (oobd., 
1349/50): daz ez. [volck] der veigen nibt az, dô er in dem land lag. - Ralegh. America 15, 3; Dinklage, Frk. Bauernweist. 40, 31; Chron. NÜRnb. 2, 78, 12; 81, 23; HaAs u. a., Erasmus/Jud. Klag 7v, 20; Chron. Augsb. 6, 24, 15; Bastian, Runtingerb. 2, 199, 25; Sснмгтт, Ordo rerum 34, 15. - Vgl. ferner s. v. alefan z 1, aufbieter, bald 3 .

6. >wo lagern, zu Felde liegen`; auch: >wo stationiert sein<; als Synekdoche: >unter jm. dienen`. - Gehäuft berichtende Texte. - Phras.: zu felde liegen. - Bdv.: vgl. legen 18, legern 2, losieren 2. - Synt.: vor der stat, um [eine Stadt], zu [einer Stadt], in den grenzen, in den schanzen, auf dem lande l., unter (z. B. unter einem hauptman) / über jm., auf / wieder jn. [z. B. wieder die ketzer] $l$.

PeIL, Rollenhagen. Froschm. 501, 25 (Magdeb. 1608): Also all Thier zu Felde liegen / | Jedoch jhr Geschlecht nicht bekriegen. v. LiLiencron, Dür. Chron. Rothe 782, 70 (thür., 1421): der lag uf die zeit yn Merern ouch uf die ketzer. Thür. Chron. 3r, 5 (Mühlh. 1599): eine grosse Stadt [...] Troia / vor der lagen die Griechen wol Zehen Jahr. CHRON. NüRNB. 1, 435, 3 (nobd., 1407): der [...7 in auch sust vast schaden getan hat und auch noch auf in c₹u velde ligt. MAALER 165r (Zürich 1561): Er ist vnder dem hauptmann Haßdrubale Gelagen / er hat vnder jm krieget. SPILLER, Füetrer. Bay. Chron. 133, 18 (moobd., 1478/81): Wo er nu zи nacht lag, da stack.t man ainen puntschuech auf. GroTHAUsMANN, Stadtb. Karpfen 18, 1 (mslow. inseldt., 1546): Satzany Peter, [...] im dorf zu BoZok, gelegen, schiessen gehört vom BöZok. - WYss, Limb. Chron. 92, 13; DAT NUwE Boych 428, 13; Franck, Decl. 350, 23; Chron. Strassb. 53, 26; V. Anshelm. Berner Chron. 4, 102, 24; Dreckmann, H. Mair. Troja 12, 14; Chron. Augsb. 6, 46, 23; 9, 88, 24; Qu. Brassó 5, 228, 28 ; 4, 263, 45; MaAler 273r. - Vgl. ferner s. v. ${ }^{1}$ an 3, panier 2.

7. >sich in einem Zustand befinden, der durch das Adv. (dies seltener) / präd. Attr. charakterisiert wird (von Personen und Sachen)<, insofern auch: >sein «; als Ellipse hier anschließbar: liegen >ruhen, unbebaut, nicht in Betrieb sein< (von Wirtschaftseinheiten, s. u. Löscher). - Synt.: j. alleine / einzig / begraben / elend / ernieder / erschlagen / erstorben / gebunden / gefangen / gewund / beftig / kalt / krank / nackend / tot (mehrfach) / ungearbeitet / ungebauen / unversonnen / verstolen / verwundet l., etw. ernieder / gefangen / unverkauft / verborgen / wüst / zerstrekt l.; ütr.: nabe l. (zeitlich).
Alberus, Barf. 355, 2 (Wittenb., 1542): fand er einen menschen liegen nackend / verwund. TiemanN, E. v. Nassau-S. Kgn. Sibille 130, 3 (rhfrk., um 1435): das phert das by yme doit lag. Froning, Alsf. Passionssp. 2473 (ohess., $1501 \mathrm{ff}$.): vor disser hochzyt, | die hie ßo nabe lyt. Cirullies, Rechtsterm. Anh. 1981, 246 (1366): und ritte ir, die [einen Geldbetrag] zu belegin uf einer wesin, daz sie faste legin. BergmanN, Ambr. Liederb. 79, 2 (Frankf. 1582): mein leide, | das mir mein junges hertr. gefangen leit. VAlLI, Baldemann 332 (rhfrk./nobd., um 1350): $Z u$ hant sie unfursunnen lag, Ir varwe gar verwandilt. $\mathrm{B} \mathrm{ECH}-$ Stein, M. v. Beheim. Evang. Mt. 8, 6 (osächs., 1343): min kint liget in dem hûse gichtec. JungBluth, J. v. Saaz. Ackermann 17, 28 (Hs. ' ${ }^{\circ}$ omd., 1465`): Mer knecht dann berren sach ich tot ligen. v. D. LEE, M. v. Weida. Spigell 17, 34 (omd., 1487): das offt Jn süssem honige, gar starcke gifft vorborgen leÿtt. LÖsCHER, Erzgeb. Bergr. 92, 10 (omd., um 1559): Da aber ein erbstoln lege, so mag der aufnemer uberfarne genge [...] an sich bringen. WUTKE, Schles. Bergb., Cod. Sil. 21, 29, 1r (schles., 1533): So ein perg oder gruben zwo wochen nocheinander [...] ungearbeit ligen bleiben. KeIL, Peter v. Ulm 155 (nobd., 1453/4): du must den fuß vor hen wol einrichten in ein laden, daz er gleich lig. v. Keller, Ayrer. Dramen 3005, 20 (Nürnb., 1610/8): jhr müst aber allein ligen | vnd habt kein, der euch wermen kan. VetTer, Pred. Taulers 157, 25 (els., 1359): die menschen die [...] mussig ire edelen krefte lossent ligen. EBD. 347, 9: Von disem inwendigen adel der in dem grunde lit verborgen. KöBLER, Stattr. Fryburg 203, 13 (Basel 1520): Welche büser iar vnd tag ongebuwen ligend. FUCHs, Murner. Geuchmat 1979 (Basel 1519): Es kumpt ein zyt / das solche wyb, $|[\ldots]|$ Werdendt kalt vnd eintrig lygen. RoLofF, Brant. Tsp. 293 (Straßb. 1554): du werst ermort / | Oder du legst ann ketten gefangen. Goldammer, Paracelsus 2, 255, 13 (um 1534): meint ir nit, sie [tochter] lig auch in dem spital krank? MAALER 273r (Zürich 1566): Gespreit vnd zerstreck.t Ligen. [...7. Weyn die lang Ligen vnnd bleyben mogend / vnnd noch gut sind. SAPPLER, H. Kaufringer 3, 697 (schwäb., Hs. 1464): trew und frumkait leit ernider. Bauer, Geiler. Pred. 104, 33 (Augsb. 1508): ir vernunfft / lond sy allzeit mússig ligen. HeNisch 133 (Augsb. 1616): Betrigliche asch / wann in der aschen noch beisse glut verborgen ligt. SpechtLer, Mönch v. Salzb. 11, 69 (oobd., 3. Dr. 14. Jh.): perg, anger, haide weit $\mid$ gar lustlich leit bedecket mit laub und gras. EBD. 17, 19: wenn in ir [mueter] verslossen lag | aller engel ostertag. KLEIN, Oswald 26, 58 (oobd., 1427): man barg mich vor der sunne schein, für springen lag ich zwainzig tag verholen. Munz, Füetrer. Persibein 148, 2 (moobd., 1478/84): alls die cbüniginne der clag lag dort erstorben. - CHRON. KöLN 1, 1078; MEIBOOM, Pilgerf. träum. Mönch 10715; Froning, Alsf. Passionssp. 6071; Jungbluth, a. a. O. 4, 5; Bell, G. Hager 158, 3; SAPpler, a. a. O. 3, 353; 4, 278; HAIL. Altvâter 73r, 16; 80v, 11; Chron. Augsb. 1, 351, 1; 7, 490, 8; Gilman, Agricola. Sprichw. 1, 552, 19; KLeIN, a. a. O. 1, 49; 33, 3; Bastian, Runtingerb. 2, 138, 17; FichtNer, Füetrer. Trojanerkr. 10, 4; Turmair 4, 41, 
29. - Vgl. ferner s. v. abschrecken 1, 1 anger 3, anstossen 9, irgend 1.

8. >sich in einem Zustand befinden, der durch einen präpositionalen Ausdruck näher charakterisiert wird (von Personen und Sachen) ২; im einzelnen z. B.: > sich befinden «; >mit etw. befaßt sein «; >in etw. verstrickt liegen ‘; in bestimmter Weise geartet sein (von Sachen) r. - Phras.: in der asche liegen >verloren gehen, am Boden liegens. Synt.: $j$. in arbeiten / pen / sünden / todesnöten / unkeuschbeit / zank, in einer not / sucht, in dem kindbette, in der asche, im fegefener / gebet / kampf, am sold, an der schwäche, an den äriten, auf dem anker, auf den knien, beim wein, zu krieg l., [von Sachen:] der bau in guten eren, das feld im aufbau, in der brache, das gut in aböde, die bube zum zehenten, der stand des lebens in sïssigkeit, der sin in der lateine, die sonne am morgen $l$.

Helm, H. v. Hesler. Apok. 5152 (nrddt., 14. Jh.): [Der] diesen durch langen tac $\mid$ In sinen arbeiten lac. LuTHER, WA 33, 615, 13 (1531): wen der heilige geist kommen wirdt und ihr in der aschen liegen werdet. EBD. 50, 313, 14 (1538): ligt also jr gesetze [...] jnn der aschen. Alberus, Barf. 264, 4 (Wittenb., 1542): Er bat auch / das er nicht lang im Feigfewr lege. Ralegh. America iijv, 12 (Frankf. 1599): nicht weit von dem Hafen / in welchem wir auff dem Ancker lagen. EвD. 8, 24: Wie er nun in Todtsnóten lag. v. LiLIENCRON, Dür. Chron. Rothe 708, 3 (thür., 1421): das eyne grosse geselleschaft uss Lampartin quam, die dor ynne am solde gelegen hatte. vaN INGEN, Zesen. Ged. 387, 31 (Breslau 1641): Horatius und andere ligen schon vor tausend Jahren in der Asche. Reichmann, Dietrich. Schrr. 72, 19 (Nürnb. 1548): Derbalb solten wir alle augenblick auff unsern knyen ligen. v. Keller, Ayrer. Dramen 2799, 5 (Nürnb., 1610/8): mein Frau und jr Mann | Ligen also in zanck vnd strauß. EBD. 3320, 13: Mein frau ist glegen in der nacbt Vnd, mit Züchten Zu Rebden, Ein Son gebracht. Gilman, Agricola. Sprichw. 1, 86, 25 (Hagenau 1534): daß eyn Einsidel inn eynem walde an seinem gebet gelegen ist. MAALER 273r (Zürich 1561): Mit eim zekerieg Ligen. SAPPLER, H. Kaufringer 13, 92 (schwäb., Hs. 1464): der herr lag in jamers dol. Eschenloher. Medicus 75, 14 (Augsb. 1678): daß ehegemelte seine Hausfraw Fastenzeit in der kindbeth gelegen. Baptist-Hlawatsch, U. v. Pottenst. Vorr. 98 (moobd., A. 15. Jh.): Darczu mag sich [...] aigne dewtsch nach der latein als die lawtet und nach dem text liget weder geschikechen noch gefügen. WeBER, Füetrer. Poyt. 182, 7 (moobd., 1478/84): vmb das er an der swech dy lenng nicht lege. Bischoff u. a., Steir. u. kärnt. Taid. 269, 39 (m/soobd., 15. Jh.): die bueben die zum zehent ligent. BAuer, Imitatio Haller 68, 14 (tir., 1466): der stant unsers lebens das leit nicht in der suessikechait vnd trostung der welt. Grothausmann, Stadtb. Karpfen 50, 17 (mslow. inseldt., 1525): Wen daśśselb feld, darin śie lait, in der Prach ligt. - Luther, WA 41, 649, 33; 49, 31, 34; Peil, Rollenhagen. Froschm. 515, 256; Quint, Eckharts Pred. 558, 4; Karnein, Salm. u. Morolf 84, 2; Kurz, Waldis. Esopus 2, 21, 81; BACHMANn u. a., Volksb. 111, 31; Welti, Stadtr. Bern 246, 21. - Vgl. ferner s. v. aböde, absagen 1, alraunpulver, anbau 1, arzet 1, aussaufen, bau 8, beflecken 2, angefär 3 .

9. >auf etw. lasten (von finanziellen Auflagen), auf js. Schultern liegen, jn. bedrükken, belasten (z. B. von Strafe); jm. zur Last fallen (z. B. einquartierte Soldaten); jm. im Nacken liegen «. - Bdv.: vgl. ${ }^{1}$ aufliegen 1.

Cirullies, Rechtsterm. Anh. 1981, 246 (1358): sal sin frawwen herfarn, waz uffe (= worauf) daz pant gelehen habe. Schönbach, Adt. Pred. 8, 23 (osächs., 1. H. 14. Jh.): ein swar joch liget üfe Adames kint. Mathesius, Passionale 34v, 4 (Leipzig 1587): Die straffe liegt auff jhm. Chron. Nürnb. 1, 146, 28 (nobd., 1388): da₹, dor₹̈̈ der krieg furbaz, mer wirt uff uns ligen, dann uff vil andern steten. EBD. 2, 37, 22 (nobd., 1421): des man vor als vast auf uns gelegen und für uns bin gecrogen ist. STAMMLER, Berner Weltger. 284 (ohalem., 1465): es ist an der zit! | Das jungste gericht vff vns lit! SAPPLER, H. Kaufringer 21, 11 (schwäb., Hs. 1472): so das alter auf im ligt. Uhlirz, Qu. Wien 2, 3, 4034, 7 (moobd., 1462): 48 s dn., die emaln gelegen gewesen sind auf Hannsen Planer, des ledrer, haus. Winter, Nöst. Weist. 3, 30, 39 (moobd., 1555): wer selbs pfant geit mit guetlichem willen, dieselben ligen auf vierzehen tag. Grothausmann, Stadtb. Karpfen 97, 18 (mslow. inseldt., 1610): gegen entrichtung gemeiner Stad drauf liegunten Lośung, Anśchnit vnd anderer Bürgerlicher auf lagen. - Rwв 8, 1327; Schweiz. ID. 3, 1205; MaAleR 473 r.

10. >aufhören, zu Ende gehen, sich legen . - Bdv.: ${ }^{2}$ anen 5, aubören 1, auskommen 13, ausleschen 3, nachlassen.

Helm, H. v. Hesler. Apok. 2262 (nrddt., 14. Jh.): Die rede lige stille. Jungbluth, J. v. Saaz. Ackermann 5, 7 (Hs. ' omd., 1465`): Nicht mer geet auf mein lichtbrehender morgenstern, gelegen ist sein schimmern. GuTH, Gr. Alex. 4049 (Hs. 'oobd., E. 14. Jh. `): Dar nach der wind began | Liegen.

11. >auf etw. bestehen, hartnäckig auf etw. beharren, an etw. festhalten<. - Bdv.: vgl. anhalten 4, ${ }^{1}$ anliegen 7 , ansteben 12, beharren 2; 3. Ggs.: vgl. ablassen 1.

Froning, Alsf. Passionssp. 5221 (ohess., 1501 ff.): wie lyt der Judde uff dem unglauben alßo hertlich. TOBLER, Schilling. Bern. Chron. 2, 152, 4 (whalem., 1484): Die botten von Frankenreich [...] lagen daruf, man solt dem kúng sechstu- 
sent manne umb sold senden. MAALER 273r (Zürich 1561): Gnauw auff einem wort Ligen / vnd darauff außhinfaren. Chron. Augsi. 5, 197, 9 (schwäb., 1523/7): so waren die 2 auch ewangelisch, und lagen alle 3 auff dem gotzwort. FISCHER, Eunuchus d. Terenz 118, 14 (Ulm 1486): Pitias [...] lag allain auff der sach die nach allen zaichen ainer geswechten junckfrawen zebeweisen gnig were. - SCHWEIz. ID. 3, 1205 .

12. >in etw. / jm. begründet sein; an etw. / jm. liegen, von etw. abhängen`; vereinzelt: >auf etw. hinauslaufen`. - Gehäuft Texte der Sinnwelt ,Religion / Didaxe'. Phras.: daran liegt macht >davon hängt viel $\mathrm{ab}<$.

Luther, WA 10, 1, 2, 393 (1526): Es ligt alles an eynem güten außleger. EвD. 33, 176, 38 (1531): Ehr redet von ihm selbest, do ligt auch macht dran. Pfefferl, Weigel. Ges. 38, 8 (Hamb. 1646): Es Liget sehr viel daran das man woll wiße und verstehe waß da heise Gesetze vnd Evangelium. Quint, Eckharts Pred. 2, 145, 4 (E. 13./A. 14. Jh.): götlichiu salicheit liget an drin dingen. Frantzen u. a., Kölner Schwankb. 1, 15 (Köln um 1490): Dat doe myr kont, wae an dyrss leyt. Schützeichel, Mrhein. Passionssp. 130 (mrhein., um 1335): daz am brode aleine nith | lige des menschen leben. EвD. 988: so bede sine martel nit irlost | die menscheit von der hellen not, | dar an lit aller der werlete drost. Gille u.a., M. Beheim 59, 20 (nobd., 2. H. 15. Jh.): mein trost, mein gir $\mid$ mein hofnung an dir leit. Reichmann, Dietrich. Schrr. 229, 8 (Nürnb. 1548): wie du disen trost [..] mercken mógest / deñ an dem ligt es alles. Schмidt, Rud. v. Biberach 5, 4 (whalem., 1345/ 60): daz beste, das in Cristo sin mag, an dem vnser zitlichvi und ewige selikeit lit. LindQvist, K. v. Helmsd. 1254 (halem., Hs. um 1435): alle úbung dar an lit $\mid$ Das in werd umb ir gottlich leben $\mid$ Hie lon in diser zÿt gegeben. $\mathrm{BACH}-$ MANN, Morgant 230, 5 (halem., 1530): man hat wol gsächen, an wemm es gelegen geweßt ist. Wyss, Luz. Ostersp. 6520 (Luzern 1545): an diser sach nit wenig lyt. MAALER 273r (Zürich 1561): Daran Ligt der gantz handel / das ist drr hafft. HAIL. ALTVÅTER 102r, 14 (schwäb., E. 14. Jh.): das lit nit allain dar an das er weltlich güt vnd er versmahe. SAPPler, H. Kaufringer 18, 8 (schwäb., Hs. 1472): ich waiß auf erd nichtz lebendig. | daran als vil faigkait lig | als an alten übeln weiben. EBD. 26, 100 (Hs. 1472): ain mensch, der nicht leidens pfligt, | waiß nicht, was nutz an leiden ligt. Turmair 4, 305, 25 (moobd., 1522/ 33): es leit alles an der üebung und an dem bedacht. - QUINT, Eckharts Pred. 2, 31, 2; 145, 7; 201, 16; 294, 7; 330, 2; 371, 3; Palm, Veter Buoch 41, 8; Froning, Alsf. Passionssp. 2864; Kurz, Waldis. Esopus 3, 7, 36; Bauer, Geiler. Pred. 103, 13; Vetter, Pred. Taulers 82, 28; Banz, Christus u. d. minn. Seele 1104; 1782; 2063; Lindevist, K. v. Helmsd. 1990; Wyss, a. a. O. 3164; 4985; Dreckmann, H. Mair. Troja 18, 7; PrIMISSER, a. a. O. 3, 85; 37, 7; 46, 141; Spechtler, Mönch v. Salzb. 1, 201. - Vgl. ferner s. v. afterwort.
13. phras. (alphabetisch geordnet nach dem mit liegen verbundenen Substantiv unter Beachtung onomasiologischer $\mathrm{Zu}$ sammenhänge, danach Ausdrücke ohne Subst., so auch in den Belegen): lange im armbrust liegen >lange zögern`; unter der bank liegen (von Sachen) >keinerlei Beachtung finden ‘; auf einer harten bank liegen >Mühen in Kauf nehmen<; auf der bärenhaut liegen; (mit jm.) unter der / einer decke liegen > (mit jm.) unter einer Decke stecken`; das / dies ding liegt jm. uneben / nicht eben; unter dem gaul liegen >den kürzeren ziehen<; an eigen und erbe liegen (Bezeichnung ,zur Verpfändung des gesamten Vermögens des Ehemannes für das eingebrachte Gut der Frau“, sо Rwв 8, 1328); jm. auf dem hals liegen retw. am Halse haben; jm. zur Last gelegt werden`; mit jm. zu har liegen $>$ mit jm. in der Wolle, im Streit liegen`; da liegt der hase im pfeffer; hier liegt der knote; da / hier liegts >darauf kommt es an〈; an dem jüngsten liegen >im Sterben liegen ‘; auf dem lezten liegen >alles bis zum letzten Pfennig eintreiben ‘; im kat (alternativ: im unrat, im wust) liegen; an dem lezten ker liegen >im Sterben liegen «; im luder liegen >dem Suff verfallen sein, in Unzucht leben`; auf der mutter liegen > der Mutter die Füße unter den Tisch strecken ‘; jm. in oren liegen; im recht liegen >sich in einem Rechtsstreit befinden〈; jm. liegt die rede $>$ jm. verschlägt es die Sprache`; im Rhein liegen >unter-, draufgehen`; $j m$. auf dem rücken liegen $>$ jn. unter dauernder Kontrolle halten <; jm. im sinne liegen; etw. liegt am tag; recht an dem ziel liegen >nahe dran sein «; es liegt darauf >darauf kommt es an<; fest auf jm. (im Beleg: auf den mäusen) liegen >jm. hart zusetzen<; es / etw. liegt jm. hart / (wunder)herte / wunderschwere >jm. geht es schwer, j. hat es schwer, jn. trifft es hart, es steht schlecht um jn. / etw.<; sich etw. herte / schwer liegen lassen > sich etw. zu Herzen gehen lassen, etw. schwer nehmen<; wie liegt es >wie kommt das?<; wie liegt es um etw. >wie verhält es sich mit etw.?‘; (also) mit jm. gelegen sein >(so) um jn. stehen, bestellt sein<; etw. ist jm. nicht gelegen (o. ä.) > etw. gefällt jm. gar 
nicht<; an einem mer gelegen sein denne an dem anderen sauf den einen kommt es mehr an als auf den anderen '; es ist viel an etw. gelegen >es kommt stark auf etw. an`; jm. liegt nichts / viel an etw. (z. B. an js. geschwäz / zorn) >etw. kümmert jn. nicht<; etw. liegen bleiben > unbebaut bleiben`; ob oder unten liegen >siegen oder untergehen ‘; mit jm. unten und ob liegen $>$ mit jm. kollaborieren, dauernd $\mathrm{zu}-$ sammen sein

Henisch 120 (Augsb. 1616): Man soll nicht lang im armbrust ligen / sondern ein ding dapffer angreiffen. LUTHER, WA 32, 516, 15 (1532): das sie der tollen vernunfft folget und lesset Gottes wort unter der banck ligen. ЕвD. 48, 31, 10 (1541): Wird im [Buch, der Bibel] gut, so ligts vnter der Banck. Henisch 182 (Augsb. 1616): Dem man sanfft gebettet hat / der stehet nit geren auff / vnd gehet auff ein barten banck ligen. Dict. Germ.-Gall.-Lat. 308, 9 (Genf 1636): Auff der Beerenbaut [liegen] Faullentzen. LuTHER, WA 33, 335, 27 (1531): weil jr Schelcke seid, als die Bischofe sind, ligt mit jnen unter einer decke. v. BIRKEN. Erzh. Österreich 65, 18 (Nürnb. 1668): Der Biscboff [...] lage beimlich auch mit unter der decke. KopprTz, Trojanerkr. 9046 (Hs. ' noschweiz., 15. Jh. $\left.{ }^{\top}\right)$ : Were dir dï mere kunde, | Du mittest, kind, dissen stritt: $\mid$ Won dir daz ding nitt eben litt. EвD. 19746: Dis dinge litt mir uneben, Dar umbe ich balde sterben sol. Lemmer, Amman/Sachs. Ständeb. 14, 8 (Frankf./M. 1568): Scblecht aber zuletzt ungluck $z u \mid$ Daß mein Parthey ligt vnterm gaul. LuTHER, WA 30, 2, 702, 7 (1530): Das die sach nicht allein uns auff dem hals ligt. Евр. 32, 34, 17 (1530): die febrlickeit, so uns vom Babst und Turcken auff dem bals ligt. ReichmanN, Dietrich. Schrr. 145, 34 (Nürnb. 1548): Dem Mann ligt ein grosse sorg auff dem balß / das er weyb vand kind nehren soll. Dietrich. Summaria 30v, 34 (Nürnb. 1578): trowet er jnen [den Pharisäern] grewlich / das alles vnschuldig blut auff jrem hals lige / vnnd musse an jnen gerochen werden. Qu. Brassó 4, 22, 27. Luther, WA 32, 112, 30 (1530): und wir also stets mit y $\mathrm{hm}$ [Teuffel] zu har liegen und uns mit yhm scblagen mussen. CHron. Augsb. 6, 33, 5 (schwäb., zu 1524): man wollt nit das gottes wort verbieten, aber da leit der has im pfeffer. Got leßs sein wort nit untergeen. LUTHER, WA 30, 3, 349, 21 (1531): das ist die frage, Hie ligt der knote. EBD. 48, 49, 2 (1541): HIe ligts, da stickts. EBD. 48, 114, 6 (1542/43): da ligts, das er wil das Himelreich geben, So wil die Welt das Erdreich haben. EвD. 49, 99, 11 (1540): fides ist medium, das zusamen kopelt me et Christum. Da ligts. Spiller, Füetrer. Bay. Chron. 170, 3 (moobd., 1478/81): Als der kranck was und an dem jüngsten lag, [...7. Sappler, H. Kaufringer 16, 766 (schwäb., Hs. 1464): dem lauget auch der tiefel ser, | da er lag an dem lesten ker. MaAler 273r (Zürich 1561): Die tugend Ligt im kaat [...]. Jm wisst unnd vnradt Ligen. SCHÖPPER 24b (Dortm. 1550): Potare. Sauffen schwelgen im sauß gehen im luder ligen. LUTHER, WA 47, 671, 23 (1539): non solt tag und nacht im luder ligen und der hurerey nacblauffen. Rupprich, Dürer 49, 55 (nobd., 1506): Rett mit dem puben, [...7, daz er ler und redlich balt, [...7, und nit ob der muter lich. SACHS 16, 218, 27 (Nürnb. 1562): jm mit fleiß in obren lagen | Nicbts von außwending [...] ding zu sagen. BARACK, Zim. Chron. 2, 408, 32 (schwäb., M. 16. Jh.): an seinem letzsten ende im die red also gelegen, das er nit [...7. BECKERS, Bauernpr. 61, 46 (Köln 1515/18): die nu beschermer syn|Woilden dat yr gemeyn leich in dem ryn. LuTHER, WA 22, 313, 4 (1544): wo er nicht selbs jmer jnen auff dem ricken ligt und treibet. KleIN, Oswald 73, 18 (oobd., v, 1408?): mein Nickel klïg, du leist mir in dem sinne. - Reichmann, Dietrich. Schrr. 74, 13. FrancK, Decl. 337, 31 Nürnb. 1531): ligt es nit am tag, das die vnstet tóricht lieb unsinnig ist? Guth, Gr. Alex. 1382 (Hs. 'oobd., E. 14. Jh.?): Eq leit rebt an dem zil | Daz, wir Dario sein her | Slaben wend. LutHer, WA 32, 204, 9 (1530): Es leyt drauff. PeIL, Rollenhagen. Froschm. 687, 5684 (Magdeb. 1608): Als sie aber jbnen zusaben / | Das sie fest auff den Meusen lagen. HaAge, Hesel. Arzneib. 14v, 13 (Hs. ${ }^{r_{n}}$ noobd./md., E. 15. Jh. $\left.{ }^{\top}\right)$ : Truckt es sich dieff bin ein und get wider auf als ein taick, so leit es hirt umb daz leben. Chron. Nürnb. 1, 156, 16 (nobd., 1388): daz er gar ubel mug und im gar hart lige. LeMmer, Brant. Narrensch. 124a, 395 (Basel 1494): Dem lydt syn dott am hertsten an. Chron. Augsb. 5, 44, 8 (schwäb., 1523/7): Die statt hett das kaisers volck inn, den lag es hert, dan sie betten mangel an speis. Adrian, Saelden Hort 3313 (alem., Hss. E. 14./15. Jh.): swel sich versument inder jugent | und sich mit ir [minne] verwirrent $\mid$ und in daz alter herrent, $\mid$ den lit es wunder sware. EBD. 10932: ez lit mir wunder berte $\mid d a z . d u$ mir ie erwurbe $\mid$ kint und dú muter sturbe. RoLOFF, Brant. Tsp. 698 (Straßb. 1554): wir bringen dir newe mehr|Die wóllest dir nit lassen ligen schwer. EвD. 1512: Herr nit laß es dir ligen hert. Haltaus, Liederb. Hätzlerin 2, 14, 424 (schwäb., 1471): ich sprach: wie ligt das? BANZ, Christus u. d. minn. Seele 1162 (alem., 1. H. 15. Jh.): lieber herr, wie lit es hierumb, $\mid$ Das du genn mir bist so tumb. KuRz, Waldis. Esopus 1, 98, 12 (Frankf. 1557): Mit freuel nimpst du mich entgegen, | Weils jetzt also mit mir gelegen. Meisen u. a., J. Eck 40, 17 (Ingolst. 1526): dann das wir allain solten bóren [...7, wil uns nit gelegen sein. MAALER $165 \mathrm{r}$ (Zürich 1561): Es ist mir yetz nit Gelagen mit dir zebalgen. [‥]. Es ist vil daran Gelágẽ welcherley weinráben es seye. [...]. EBD. 165r: Es ist mir vil daran Gelágen / vil zegewünnen. Franz u. a., Qu. hess. Ref. 2, 450, 23 (hess., 1546): es solt ein vergleicbung sein etc., so doch an einem mebr gelehen den an dem andern, wie man sagt, das man mehr legt an einen hengst den an einen esel. KNAPE, Messerschmidt. Bris. 13, 20 (Frankf./M. 1559): es lege jhm gar nichts daran / das er zpen Ritter vor jbm baben solt. Bergmann, Ambr. Liederb. 149, 17 (Frankf. 1582): an seinem geschwetz mir gar nicbts leid. BaChMANN, Haimonsk. 242, 11 (halem., 1530): got verflüeche mich, wenn mir neyswaz an sinem zornn ligge! ERMisch, Sächs. Bergr. 180, 20 (osächs., 1509): ap gleych ein tzech tzwuschen den quatembern lygen blybe. Bachmann u.a., Volksb. 146, 2; Chron. Augsb. 2, 191, 26; 5, 10, 4; Rwв 8, 1329; Schweiz. ID. 3, 1205. 
14. mit besonderer Häufigkeit belegt das polyseme Phrasem etw. / (seltener:) jn. liegen lassen >mit etw. / (seltener:) jm. nicht so verfahren, wie es der moralischen, wirtschaftlichen, gesetzlichen Verpflichtung, der Erwartung nach geschehen sollte, im einzelnen z. B. >etw. unbebaut lassen<; >etw. nicht verkaufen ‘; >etw. unerwähnt, unerörtert, auf sich beruhen lassen, übergehen«; >jn. verlassen, im Stich lassen〈; >jn. vergessen $\iota$ - Hierzu wiederum phras.: jm. bleibt das bier / der wein liegen $>j n$. überkommt eine so schwere Prüfung, dass er das Bier [...] verschmäht ‘; alles stehen und liegen lassen; es bleibt liegend > es bleibt beim Alten`.

Luther, WA 51, 662, 488 (um 1535): Was nicht dein ist, das las ligen. Stambaugh, Friederich. Saufft. 48, 15 (Frankf./O. 1557): Ob aber dennoch Gott eim ein solch Crentze aufflegte / das im das Bier / oder der Wein blieb ligen / so trage er solch Creutze mit gedult. Мегјвоом, Pilgerf. träum. Mönch 3642 (rib., 1444): Des ich up dese zijt wille geswygen | Ind umb des mynsten verdros wil laissen lijgen [>unerörtert lassen «]. THIELE, Chron. Stolle 378, 16 (thür., 3. Dr. 15. Jh.): unnd liessen allis stehe unnd lien, was sie hatten. v. D. LeE, M. v. Weida. Spigell 20, 15 (omd., 1487): lestu haw $\beta$, vnd hoff, wejppp vnd kinder lïgen, vnd lewfst Jm lande Jrre. EBD. 84, 11 (omd., 1487): gleich ap sÿ [eldern] nüe wern bye gewest vnd gleich den bunden ligen lassen. Wutke, Schles. Bergb., Cod. Sil. 21, 103, 31 (schles., 1546): Es soll auch Maz. Hirsch keinesweges one vorwissen und rat der herren gewercken diener orter belegen aber liegen lassen. CHron. Nürnb. 1, 32, 4 (nobd., 1407): di gefangen macht kayser Karel her nach zu Wirczburk ledig. alzo belaib ez ligend. VetTer, Pred. Taulers 107, 22 (els., E. 14. Jh.): Das sprechent die beiligen daz der mensche lieber sterben solte, aber das lasse ich nu liegen. JellineK, Friedr. v. Schwaben 4487 (schwäb., Hs. 1478): Schweig und laß das ligen. Chron. Augsi. 6, 34, 21 (schwäb., zu 1527): also hat der Jorg Vetter [...] die sach nit wollen lassen ligen. Memminger Chron. Chr. 38, 21 (Ulm 1660): Blieb also dieses Gut ligen Jahr vnd Tag. SpILLer, Füetrer. Bay. Chron. 52, 17 (moobd., 1478/81): Umb das las₹ ichs zu disem mal ligen [>gehe nicht darauf ein $]$. Вischoff u. a., Steir. u. kärnt. Taid. 485, 33 (m/soobd., 1593): Wenn ainer [...] unrecht zehent ligen lesst [>auf dem Felde zurück läßt und dadurch gegen die Abgabepflicht verstößt 1 , begriffen wierdt, der ist das geladne fueder sambt zug und zäg [...] dem zehentherren verfallen. Winter, Nöst. Weist. 3, 863, 8 (moobd. 1523): so sol er phluegsrederweit ainen acker ligen lassen. Pirrainen, Stadtr. Kremnitz 99 (mslow. inseldt., 16. Jh.): die Ienigen aber, So die Thurn bepholben sein, sollen alles steen vnnd ligen lassen. - Cirullies, Rechtsterm. Anh. 1981, 246; Schnurrer, Urk. Dinkelsb. 5,
1811; VORARLb. WB. 2, 284. - Vgl. ferner s. v. abschiessen 2, auffaren 11.

${ }^{2}$ liegen, V., unr. abl.; Trennung von den Brechungsformen von lügen und von den Einheiten des zugehörigen Wortbildungsfeldes nicht immer sicher möglich, s. d.

1. >jn. belügen, jm. die Unwahrheit sagen «. - Bdv.: vgl. ${ }^{2}$ geliegen.

Helm, H. v. Hesler. Apok. 6087 (nrddt., 14. Jh.): Da der lugenwise | Dieb der menscheite louc. LuTHER, WA 32, 91, 22 (1530): das wort kan uns nicht liegen. Fischer, Brun v. Schoneb. 10260 (md., Hs. um 1400): wen uns die werlt sus luget $\mid$ und in erem dinste betruget. GILMAN, Agricola. Sprichw. 1, 415, 23 (Hagenau 1534): Lengt yemandt / und ob ers schon vor den leuten zudecken kan / so kan er doch Gott nit liegen. - LuTHER, WA 48, 100, 9; Lemmer, Schernb. Frau Jutte 1315.

2. >täuschen, einen falschen Eindruck vermitteln<; offen zu 3. - Bdv.: vgl. ableiten 2, bestopfen 4, betriegen 1, bezücken 1, 1 gleichen 6, ${ }^{1}$ leichen 1 , letzen $1,{ }^{2}$ täuschen. - Wbg.: liegerei >Heuchelei, Betrugく.

Quint, Eckharts Pred. 1, 215, 14 (E. 13./A. 14. Jh.): Vil dinges glichet sich dem golde; er. linget und enist nibt golt. Gille u. a., M. Beheim 347, 34 (nobd., 2. H. 15. Jh.): dein munt sol mir mit liegen $\mid$ mein herc. nit mer petriegen. PÄPKE, Marienl. Wernher 4126 (halem., v. 1382): Er [got] ist war und wir gelogen ( $>$ falsch, verlogen<, auch zu 3 stellbar). Turmair 1, 212, 24 (moobd., 1529): Man tue das blutvergiessen, verräterei und liegerei ab. Sein das nit fein cbristen oder verstockt blind leut.

3. >lügen, über etw. / jn. die Unwahrheit sagen «; oft im Orientierungsfeld mit (be)triegen, afterreden, morden, rauben, stelen, verraten usw., zum Teil formelhaft. - Phras.: ane liegen Verwahrformel; um einen han liegen >um die Wette lügen`; den baum aus der erde liegen; in den eigenen beutel liegen; liegen, das sich die balken biegen. - Synt.: oft absolut; jn. (vor dem rechten) liegen heissen (>des Lügens bezichtigen ‘; mehrfach belegt; vgl. dazu RwB 8, 1500) / heischen; an der zal, auf die stat, auf / um jn. l., in seinen hals, in sein maul, durch seinen schlund, wieder die warheit, sonder not, wie ein schelm l.; fälschlich / frevelig / beimlich l.; subst.: ane liegen sprechen (verwahrformelhaft), den mund nicht mit liegen finden, sich des liegens massen. Wbg.: liegenheissen >Bezichti- 
gung e. P., dass sie lügt〈, liegenstrafe >Strafe für die Lügenbezichtigung<, liegezunge >Verleumdung` (a. 1460).

Luther, WA 16, 466, 20 (1525): das wir nicht schweren, fluchen, liegen, triegen, zaubern sollen. EBD. 30, 2, 442, 21 (1530): Dem Bapst kan ich nicht gleuben, denn er beisst sich selbs ynn sein maul liegen. EBD. 32, 193, 20: wer mit Menschen umgehet, der sol wissen, daß er mit denen umbgehet, die liegen und triegen. EBD. 35, 428, 2 (1524): Du sollt [...] | nicht liegen auff den nebsten deyn. FISCHER, Brun v. Schoneb. 4101 (md., Hs. um 1400): daz ist war und nicht gelogen. Quint, Eckharts Pred. 2, 454, 12 (E. 13./A. 14. Jh.): [want alles] was so valsch wider der warheit, das sie swigen vnd wolten nicht liegen. Reduch, Qu. Ratingen 51, 32 (snfrk./rib., 14. Jh.): scheltwort, vuystslege, liegenheyßßen dyefe moerder off verreder geschoulden dat an lyff off an ere trefft. Меijвоом, Pilgerf. träum. Mönch 9589 (rib., 1444): verstant wale, I Ich en liegen dir neit an deser tzale. Loesch, Kölner Zunfturk. 4, 54, 9 (rib., M. 15. Jh.): sower den anderen ernstlichen heischt liegen, die gilt 3. s. zo waisse. Frantzen u. a., Kölner Schwankb. 3, 898 (Köln um 1490): Du vule schalk, du luchst. ENDERs, Eberlin 3, 78, 23 (о. O. 1524): mügt ir mercken, das euer erste regul nit das Euangelion ist, als der anfang lygt. GRIMm, Weisth. 6, 395, 2 (rhfrk., 1378): Auch werden in diesem gericht [...] gestraft alle liegenstraf, fauststreich [...]. Jungbluth, J. v. Saaz. Ackermann 28, 15 (Hs. ' omd., 1465ㄱ): Triegen, listen [Var. E. 15. Jh.: liegen] smeichen, [...] lachen, weinen kan sie wol in einem angenblick. CHRON. NürnB. 3, 174, 20 (nobd., 1488): Es sei ein ursach zu liegen, daß die menschen peichten dem priester in sein ore. SACHS 5, 68, 31 (Nürnb. 1557): Du thust liegen, triegen und rauben. VETTER, Pred. Taulers 385, 22 (els., 1359): Got und der prophete enliegent nút. Bolte, Pauli. Schimpf u. Ernst 1, 236 (Straßb. 1522): Sprichwort: Wer gern lingt, der stilt auch gern. EBD. 1, 367: Du ligst uff mich, ich bin nit ein sollich Frau. Kurz, Murner. Luth. Narr 1115 (Straßb. 1522): Es ist ein sundere kunst zu liegen, | Das es geheb sei und nit rin: | Liegen hat ein besundern sin. Gilman, Agricola. Sprichw. 1, 503, 11 (Hagenau 1534): Er leugt wenn er das maul auff thut. EBD. 2, 93 ([Augsb.] 1548): Er leügt das sich die Balcken biegen. EBD. 2, 95: Er leüget in seinen aignen Beütel. EвD.: Leug dich nicht zu tod. Roloff, Brant. Tsp. 1668 (Straßb.] 1548): Wie kündten wir wider die warbeit liegen. GEIER, Stadtr. Überl. 370, 1 (nalem., 1532): Haist ain gewachsen mensch das ander liegen, ist peen zehen schilling pfennig. P̈̈pke, Marienl. Wernher 6677 (halem., v. 1382): Ich will die warbait hie von Got | Sprechen än liegen. Bachmann, Morgant 218, 34 (halem., 1530): Ruolland sprach zuo im: „Du lǔgst durch din schlund; wann ich bin kein dieb". V. Anshelm. Berner Chron. 4, 134, 1 (halem., n. 1529): ein teil luog, es wáre alles wol gefridet. $\mathrm{BACH}-$ MANN, Haimonsk. 124, 34 (halem., 1530): als man spricht gemeinlichen guot bluot nŭt lieggen. RODER, Stadtr. Villingen 193, 27 (önalem., 1582): So einer oder eine das redte, so liege er, oder si [...] wie ein schelm, dieb. Dict.
Germ.-Gall.-Lat. 307, 33 (Genf 1636): Liegen / sagen was nicht wahr ist. EвD. 39: Baum auß der Erden [liegen] vber alle massen triegen. HEYdn. MAISTER 8r, 24 (Augsb. 1490): Du sollt nit liegen. Chron. Augsb. 2, 307, 11 (schwäb., Hs. 16. Jh.): darumb han ich auf die erwürdigen stat pöslich gelogen. EBD. 4, 124, 19 (v. 1536): man hat köglet, scholder gehapt und um ain hannen gelogen. FISCHER, Eunuchus d. Terenz 125, 19 (Ulm 1486): Du bedarffst dem nit glauben. wann all knecht liegen gern. Turmair 1, 227, 37 (moobd. 1529): das die fürsten, hern und stett, so den Luther balten, ain jeglichen bleiben lassen, liegen, treigen, verraten, würgen, plöcken oder stöcken. WACKERNELL, H. v. Montfort 2, 62 (soobd. A. 15. Jh.): liegen, triegen fruo und spât, | das heissent si geschibikeit. BAuER, Haller. Hieronymus-Br. 28, 34 (tir., 1464): wenn si wider eüch rëden alles ïbel vnd eüch liegen haissen durch des menschen sunes willen. - Helm, H. v. Hesler. Apok. 5700; Мельвоом, a. a. O. 8370; Chron. Köln 1, 4488; 6208; Schade, Sat. u. Pasqu. 1, 4, 115; Brinkmann, Bad. Weist. 10, 15; Froning, Alsf. Passionssp. 411; Luther, WA 30, 3, 435; 32, 113, 3; v. D. Broek, Suevus. Spieg. 148v, 43; Asmussen, Buch d. 7 Grade 2012; Hoffmann, Würzb. Polizeisätze 157, 13; zu Dohna u.a., Staupitz/Scheurl 134; GILman, a. a. O. 2, 45; 93, 24; WYss, Luz. Ostersp. 10198; Enders, Eberlin 3, 41, 7; Österley, Steinhöwels Äsop 357; Primisser, Suchenwirt 19, 55; 22, 59; 30, 234; Turmair 4, 60, 2; WiessNER, Wortsch. Wittenw. Ring. 1970, 119; MaAler 273; Rwв 8, 1499 f. - Vgl. ferner s. v. afterreden, anzicht 1, arm (Adj.) 4, arzet 1 .

liegend, lexikalisiertes part. Adj. $\mathrm{zu}{ }^{1}$ liegen; in 1 subst.: liegendes, das liegende.

1. >unterhalb eines nicht senkrechten Erzganges liegende, gleichsam den Boden des Ganges bildende erzführende Gebirgsmasse<; vgl. am ehesten ${ }^{1}$ liegen 1. - Omd. und östl. Inseldt.; bergbaubezügliche Texte. Sснмітт, Fachprosa 37, 8 (osächs., um 1500): daz gangbafftig kupferertz. wirt besser und guldiger erfunden, nach dem der gang in seinem hangend und ligend mit einem edeleren vmd artigeren tzechstein voruast wirt. LÖsCHER, Erzgeb. Bergr. 85, 16 (omd., um 1559): Wo der aufnebmer seine fundtgrube und maßen in hangendts und ligents [...] nehmen will. EBD. 106, 12: Die beweisungen sollen von der fundtgruben [...] anzufahen und also bis an das strittige orte mit kendlichen hangendts und liegents sambt dem salbandt des gangs ins gesteine gebracht werden. Wutke, Schles. Bergb., Cod. Sil. 21, 6, 12 (schles., 1592): aufm Goltberge eine fundtgruebe [...] sampt einer wher in hangendes und ligendes. EBD. 24, 30 (1533): dass ein iede fundgrube im harten bergwerge haben soll dem gange nach zwoundviertzig lochtern, [...] in hangendes und liegendes sieben lochtern. - WeizsëCKer, Graupn. Bergb. 33, 28; 72, 7; Wutke, a. a. O. 20, 69, 5; 149, 6; Piirainen, Igl. Bergr. 31, 2, 20; S. 75; Paul, Wb. 
Bergmannspr. 1987, S. 211 - Vgl. ferner s. v. aufsetzen 27, ausschroten 5 .

2. >liegend im Unterschied zu beweglich, farend (von Gütern, Immobilien)<; vgl. ${ }^{1}$ liegen 3. - Rechts- und Wirtschaftstexte. Bdv.: grundfest, unbeweglich. Ggs.: farend. Synt.: das pfand l. sein; der liegende boden, die liegende babe, das liegende eigen / erbe / gut / pfand.

Perez, Dietzin 1 134, 1 (Frankf. 1626): dieses sind also die beweglichst vnd liegende Güter / die ich von meiner Mutter ererbt. KöBlen, Stattr. Fryburg 67, 10 (Basel 1520): So sich erscheint / das einer varendt hab / iar vñ tag / vnd ligende guter zehen iar lang [...] onangesprochen / ingehabt / der hat nach unser Statt puch / kuntschafft gnig / zu dem eigentumb. EBD. 77, 19: Wañ aber das pfand ligend ist / vnd der schultherr nit wyter gedult haben wolt / So sol dem schuldner [...] verkündt werden / [...]. EBD. 94, 15: Wer ouch das einer ein ligend gut / buß / acker / matten / boff 3c. vmb ein ierlich ewig gült verkouffte / das mag ouch wol sin / doch mit maß vñ bescheidenheit. Bremer, Voc. opt. 13017 (wobd., 15. Jh.): Fundus ligendeigen [...] grunt [...] duo significat: vno modo est infirma pars vasis rem liquidam continentis [...]. 2. modo (fundus) est ager, de quo sumitur fructus, uel area, super quam edificium construitur. GRAFFuchs, Ämter Interl./Unterseen 114, 33 (halem., 1404): wa vonbin ein vater oder ein muter ane elich gemechide stirbet [...], da nement und erbent die erren kinde an den ligenden gütren vorus ir libdinge. SсHів, H. Stockar 256, 45 (halem., 1520/9): so hatt sy yetzt angezeigte busarme zu iren rechten ... erben gesetst ... alles ires vbrigen gutz, ligennden vnnd varenden an goldt, barschaft, silber. GEHRING, Würt. Ländl. Rechtsqu. 3, 703, 58 (schwäb., 1585): wie es mit außziechung der fahrenden hab und güetter diß fabls gehalten, ebenmeßigerweiß soll es auch mit den ligenden güettern gebalten werden. Grothausmann, Stadtb. Karpfen 78, 27 (mslow. inseldt., 1605): nach śeinem todt aber, sollen solche ligende güeter vnd gründ alle, [...], Gemainer Statt KörPen Zufallen. - Dinklage, Frk. Bauernweist. 02, 19; KöвLER, a. a. O. 83, 28; 97, 12; RenNEFAHRT, Recht Laupen 32, 7; Rwв 8, 1330.

liegenheissen, s. ${ }^{2}$ liegen 3.

liegenschaft, die.

>Immobilie, liegendes Gut‘; vgl. ${ }^{1}$ liegen 3. - Rwв 8, 1332 (а. 1322).

liegenstrafe, s. ${ }^{2}$ liegen 3.

${ }^{1}$ lieger, s. ${ }^{1}$ liegen 2.

${ }^{2}$ lieger, der; $-/-\emptyset$.

>Lügner`, starke Tendenz zu 〉Betrüger`; vgl. ${ }^{2}$ liegen 3. - Bdv.: balräter, bieger, schalk, trieger (vgl. auch die Aufzählungen der Belege). - Wbg.: liegerin.

Jostes, Eckhart 65, 25 (14. Jh.): lieb on bute die ist [... ein fugerin der sunde und ist lag des teufels [...] und ist ein liegerin in der frag. Gille u.a., M. Beheim 115, 137 (nobd., 2. H. 15. Jh.): ripel, raier, läter, rüffian, | verreter, freibeit, puben, | Lieger und schelk verruchte | sein nun der frawen kamer knebt. Adrian, Saelden Hort 2719 (alem., Hss. E. 14./15. Jh.): wes hastu dich genomen an, | balretter, lieger, arger wiht! Pfeiffer, K. v. Megenberg. B. d. Nat. 45, 6 (oobd., 1349/50): der ist ain lieger, ain listiger und ain tôr. EвD. 600, 21: sneider: liger, schuster: trieger. Chron. Augsb. 2, 118, 5; Barack, Teufels Netz 13357; Voc. INC. TEUT. p iv. - Vgl. ferner s. v. äffer 2.

liegerei, s. ${ }^{2}$ liegen 2.

liegerhaft, Adj.

>krank, bettlägerig ‘; vgl. ${ }^{1}$ liegen $2 .-\mathrm{Bdv}$ : mat; vgl. betfest, betriese, betriesig, betsiech, langliegerig. Ggs.: gesund (Adj.) 1.

SACHS 4, 77, 34 (Nürnb. 1554): Zu eynem kind er wider wird, $|[\ldots]|$ Wirt inn kranckheit gantz ligerhafft. WINTER, Nöst. Weist. 1, 721, 16 (moobd., 16. Jh.): Ob auch ainer wuerd geschlagen anderstwo und kem her und wuerd do ligerbaft und starb, so [...].

\section{liegerin, s. ${ }^{2}$ lieger.}

liegering, liegerling, der; $-/-\emptyset$ oder $-e$. - Wobd.

1. >balkenähnliche, klotz-, scheitartige Unterlage für verschiedene Zwecke (zum Lagern von Holz, Dielen, Fässern) <; zu ${ }^{1}$ liegen 1. - Bdv. (bzw. sachzugehörig): afterschlag 1, klafterstecke, kloz 1, scheit, stumpf.

GeHring, Würt. Ländl. Rechtsqu. 3, 119, 33 (schwäb., 1554): es soll auch ain jeder, der [...] holz herein zue fibren schuldig ist, minder nit dann ain guet claffter, [...] aufladen, kein scheit, ligering [...] nit ligen lassen. EBD. 790, 33 (1607): sollen die claffter der lange nach und nit in der runde gestelt und die ligerling, darauf man die claufter setzt, nit uber ein spann hoch [...] gemacht werden. - SCHMIDT, Hist. Wb. Elsaß 223; SCHWeiz. ID. 3, 1216; Vorarlb. WB. 2, 285. - Vgl. ferner s. v. afterschlag.

2. >bettlägerige Person, Kranke(r)<; vgl. ${ }^{1}$ liegen 2. - Bdv.: vgl. ${ }^{2}$ betler, betlieger.

RiEDER, Gottesfr. 211, 31 (els., 1401/2): welre dúrftige [...] nút zumole ein ligering sige und ein zu swerer úberlast des gesindes [...], den soll [...].

3. >Fußhöhle<; als Metonymie: >Fußeinlager.

Voc. Teut.-Lat. ee iijr (Nürnb. 1482): Sole. subtel. $i$. solea. od' fußhol. od' ligering vnderschubel. fußstapff.

liegerling, s. liegering. 
liegerstat, die.

>Bett, Ruhe-, Schlafstätte<; zu ${ }^{1}$ liegen 2, vgl. stat (die) 1; 2. - Bdv.: vgl. ${ }^{2}$ betstat, bette 1 , gelieger, leger 1 , legerstat.

OоRschot, Spee. Trvtz-N. 62, 18 (wmd., 1634): Auß Rosen mir bereitet $\mid$ Gar weich die Ligerstat. SACHS 7, 443, 10 (Nürnb. 1557): Do [unter der stiege] ward es im [vater] gnaw gemessen, | Mit liegerstat, trincken und essn. Ders. 20, 151 (1560): Heiß warten ir mit allem fleiß, | Mit ligerstatt, getranck und speiß! - SACHS 13, 226, 4; 20, 75, 18.

liegezunge, s. ${ }^{2}$ liegen 3.

\section{lieggrube, lieglingen, s. ${ }^{1}$ liegen 5 .}

${ }^{1}$ liene, die; Etymologie? - Zu den Bezeichnungen für ,Muttersau' in den rezenten dt. Mundarten s. Dwa 4; 7, 3.

>erwachsenes weibliches Wildschwein, Wildsau .

LAMPrEcht, Dt. Wirtschaftsl. 472, 21 (mosfrk., 1465): wer ein liehe fahet, der ist schuldig ein zaume saw unt sechszig s. Graf-Fuchs, Ämter Interl./Unterseen 521, 19 (halem., 1657): von einer lienen, wie vil sy joch dero hat, [...] 3 d. Grimm, Weisth. 1, 386, 36 (wobd., 1456): da sollent sie von einem beren das höpt unnd von einem hawenden schwin vnd einer lienen öch das höpt geben. - EвD. 1, 384, 49; Dalby, Lex. MHG Hunt. 1965, 136; Vilmar 242.

2liene, die.

eine Pflanze, wohl >Waldreber. - Wbg.: lienenblume (a. 1542).

Otт-Voigtländer, Rezeptar 204r, 4 (Hs. ' nalem., um $\left.1400^{7}\right)$ : Dem das gesúbt in der stirnnen ald in den oggen ist, das nem linea. - MARZelL 1, 1047 (mit Überlegungen zur Etymologie); SchweIz. ID. 5. 82.

${ }^{3}$ liene, Genus und etymologische Zuordnung unklar, möglicherweise $\mathrm{zu}$ mhd. line >Geländer, Balken`(LeXer 1, 1926).

>Balken; Geländer, Lehne; Umgrenzung des Hauses, der Burg«?

Golius 7, 53 (Straßb. 1579): Paxillus, die Lien. NIEwÖHNER, Teichner 15, 33 (Hs. 'moobd., 1360/70? ): anders keiner do gewan $\mid$ wann daz man in warff durich die lienen. - SchweIz. ID. 3, 1286.

lienenblume, s. ${ }^{2}$ liene.

lieren, V.; onomatopoetische Spontanbildung mit Bezug auf liri, den Ton der Lerche.

> singen, trillieren $<$.

KLEIN, Oswald 50, 34 (oobd., v. 1408?): ir lierent, rierent I gracket und wacket. lierisch, Adj., teils subst.; nach der belg. Stadt Lier.

>von lierscher Machart, Herkunft ; subst.: >Tuch aus Lier<.

Sattler, Handelsrechn. Dt. Orden 430, 19 (preuß.,1391/9): 8 ellen van eyme swartzen lirschen. LEMMER, Amman/Sachs. Ständeb. 60, 4 (Frankf./M. 1568): Hereyn /wer Thuch zu scbáren hab / | es sey [...] | Mechlisch /Lundisch / Lyrisch / Stamet. Loose, Tuchers Haushaltb. 59, 5 (nürnb., 1507): adi 15 november czalt ich dem Peter Obermair durch mein sun fur 2 eln lirisch tuch [...], thut alles 23 tb. Spanier, Murner. Schelmenz. 45, 19 (Straßb. 1512/3): an dem rock [...] | Sammet / dammast / und von syden, | vnd laßt ir lindisch / lirisch schnyden, | Also bierisch und so frech. - SATTLER, a. a. O. 126, 23; Ziesemer, Gr. Ämterb. 84, 20.

liesch, das; zu mhd. liesche eine Grasart (Lexer 1, 1913; Dwb 6, 1019).

$>$ Riedgras, Sumpfgras vgl. binze 1, ${ }^{\text {gras, }}$ ror(kolb), schilf. - Wbg.: liestisch >gertenschlank (wie eine Binse)<.

Voc. Ex Quo C 481 (nrhfrk., 1411): Cirpus [...] semden / risch uel schelp [...] uel pinss [...] uel liße. Sснів, Н. Stockar 156, 17 (halem., 1520/9): Uff dye zitt hain ich keufft 3 liesch und kostend mich $3 \mathrm{gl} \mathrm{mit} \mathrm{alem.} \mathrm{ReNNEFAHRT,}$ Recht Laupen 193, 27 (halem., 1695): Mosgarten, so nur lischen tragt $1 / 2$ [mad]. Fischer, Eunuchus d. Terenz FISCHER, Eunuchus d. Terenz 59, 5 (Ulm 1486): sie [muttern] vertziehen in [Töchtern] die spys, und ob sie wol gut von natur ist, mit irem fleiß machen sis liestisch allso bat mans lieb. EвD. 59, 23: Mit irer [mutter] getzwingnuß [den leib in zwingen] machen sie [jungkfrawen] liestisch. Wann liesth sind die urlangen bints, nit knopffet und nabend in gleicher grósse und schmal. - SснмiDt, Hist. Wb. Elsaß 223; Schwerz. ID. 3, 1459; Ссншёв. Wв. 3, 1243.

lieschke, Genus und Etymologie? ein Ort ohne Gemeindeverfassung.

Toeppen, Ständetage Preußen 5, 589, 16 (preuß., 1516): auffm lande soll keyn kruger in lieschken oder dorffern maltz zur mule furen, er sall zuvorn vom amptman ein zeichen nemen.

liese, die; Lemmaansatz wegen der (wohl auch reimbedingten) Schreibung (ij für mhd. $-\hat{\imath}$, nhd. -ei) unsicher; möglicherweise zu nhd. Liese «Pustelく (Dwв 6, 1019), entsprechend mnl. liese (VERWIJS/Verdam 4, 568) oder mnl. leisse >Schnur` (Verwijs/ Verdam 4, 342), wie der Herausgeber der u.a. Quelle mit der Erläuterung >Schnur` anzunehmen scheint (semantisch nicht 
überzeugend); vgl. auch VILMAR 250: hier als Name der Krätze angegeben.

>Schramme, Kratzer`.

Meijвоом, Pilgerf. träum. Mönch 4098 (rib., 1444): Want id [pantzier] is van sulchẽ machsel ind prijse | Dat eme alle andere wapen neit eyne lijse | Noch eyne male mochten krencken.

liespfund, das; zum Wort: Dwв 6, 1020; Rwв 8, 333 (hier Herleitung aus: livisches pfund).

eine Gewichtseinheit, Zwanzigstel eines Schiffspfundes; das Maß war in den Niederlanden und im Ostseeraum gebräuchlich. - Конrт u.a., Alte Maße [...]. 1987, 164.

JoAchim, Marienb. Tresslerb. 84, 28 (preuß., 1399): 1 schok, flicken fleisch, dy wegen 10 pfundt und 4 lispfundt, das pfundt vor 11 firdung. Sattler, Handelsrechn. Dt. Orden 12, 34 (preuß., 1404): Cristan Klofhamer tenetur 25 schiff it und $1 / 2$ lis to Ungerisch ysen. ZIESEMER, Marienb. Konventsb. (preuß., 1412): $6 \mathrm{~m} .6$ d vor 4 bastard, dy wugen 4 schiffpfunt und 2 lispfunt. Toeppen, Ständetage Preußen 1, 374, 22 (preuß., 1420 ): Der herren von Thorun pfunde was czu leichte ... 11/2 lis Qto. - Ziesemer, a. a. O. 10, 14.

liessen, V.; zu mhd. liezen `losen` (LEXER 1, 1914).

>jm. eine Behandlung nach seinem Verhalten zuteil werden lassen

StackmanN u. a., Frauenlob 5, 26, 9 ( ${ }^{\circ}$ md. auf nd. Grundl., v. M. 14. Jh. $\left.{ }^{\top}\right)$ : Ba₹ möchte man die bösen von den bederben scharn: | die mislich varn, | den muz man mislich lieżen.

liestag, der.

>besonderer Tag, an dem die Wettervorhersage für das kommende Jahr vorgenommen wurde(?)<.

Beckers, Bauernpr. 54, 12 (Köln 1515/18): Die vj. daich na dê beilgen dreyen koeningen synt wyder lies daich vĩ besteten.

liestisch, s. liesch.

liftigen, V.; etymologische Zuordnung unklar.

>etw. (Weinreben) anheben, frei legen

LÖFFLER, Columella/Österreicher 2, 309, 19 (schwäb., 1491): So vil grósser die bitz. ist, so vil zimpt es sich dicker den win gefuret, erkelt und geliftiget werden.

liga, die, auch der; aus franz. lique >union offensive, défensiver (JonEs, French Borrowings 399, Belege 1579; 1616).
>Bündnis «. - Bdv.: (ver)bündnis; vgl. bund 4, einung, geselschaft 9, ${ }^{1}$ lege.

Barack, Zim. Chron. 1, 305, 37 (schwäb., M. 16. Jh.): so kammen die von Geroltzeck, mit dem schloss und der statt Sulz auch in disen liga. EBD. 3, 480, 33: Wie nun graf Gottridt Christof sein prebenda us dem stift bekamme, do gab es ain rechten ligam, [...], und es stande domals nit übel uf dem stift.

\section{lila, Genus?}

>Tuch aus Lille, nach der Machart von Lilleく.

MüLlER, Welthandelsbr. 261, 37 (schwäb., 1514/5): alle flemisch tuech, [...]. So muessen solliche tuech bie balten die sort Lila, Menin, Ycken, Thun, Armentini fina marcka, liüster, ain yedes 30 covido.

lilie, die; -/-n. - Vgl. auch gilge.

1. >Lilie $<$; in den Belegen begegnet lilie vielfach im Orientierungsfeld mit rose / veiel / zeitlose und im Gegensatz zu distel, dorn, nessel; starke Häufung tropischer und bildhafter Verwendungen; lilie steht dann im Vergleich mit der Brustwarze der Frau, als Symbol für keuschheit, sänftmütigkeit, als Tropus für Christus und (häufiger) Maria. - Gehäuft Texte der Sinnwelt ,Religion / Didaxe'. - Phras.: nicht zwischen rosen und lilien bängen >nicht nur angenehm sein «. Synt.: lilien ansehen / nemen (als Schmuck), die brust die lilien schauen lassen; die lilie / lilien [wo, z. B. in der aue] wachsen / reifen / blühen / welken, für den dornen geschönt sein, bel gleich den sternen sein, der welt gemeine sein, einen langen stengel haben; Maria / Christus die / eine l. sein; etw. schöner den lilien (>als Lilien`) sein; sänftmütigkeit der l. gleich sein, der l. überadeln wollen >die Lilie übertreffen wollen`; aus $l i$ lien öl machen, etw. mit lilien bestreuen / durchflorieren / zieren; die l. des ackers, der keuschbeit (mehrfach) / reinigkeit, die l. >Krone der arzenei; die blaue / gelbe / (schne)weisse / reine / feine / schöne / unbeflekte / minnigliche l.; die l. am wasser, aus der aue, im garten, sonder missefal, unter den dornen; die weisheit der lilien. Wbg.: lilienblank >weiß wie eine Lilies, lilienblat, lilienblume, lilienblut $\left(\mathrm{Gw} \mathrm{zu}^{2}\right.$ blut $)$, lilienbusch $>$ lilienbewachsener Ort , lilienfarbe, lilienfeld fiktiver Namensbestandteil eines romhöri- 
gen Königs, liliengarte, liliengerte (für Maria), lilienöl >öliger Auszug der Weißen Lilie`, liliensaft, liliental (für Maria).

Luther, WA 24, 401, 11 (1527): Das Euangelium [... benget nicht zwischen rosen und lilien. INGEN, Zesen. Ros. 79, 1 (Hamb. 1646): ihre brust ließ obne sorgen / | obne neid und binterhalt / | die schnee=weissen liljen schauen | auf den fol=beblubmten auen. EBD. 80, 29: [die Begierde] izt unter dem schatten der lieblichen augenlider / bald zwischen den zwe anmubtigen lust=bügeln / des liljen=blanken brust=feldes. EBD. 84, 27: Bald nahm sie lilien / bald rosen / und fugte sie an ihren weissen hals und ibre robte lippen. Fischer, Brun v. Schoneb. 319 (md., Hs. um 1400): du ein lilia uz. den ouwen, | du eine vroune obir alle vrouwen. EBD. 4858: durch waz. her si lilige heze, | vil note ich daz vorgeze, | ich sage iz. mannen und vrouwen: | liligen wachsen in den ouwen, | sint alle der werlt gemeine. Dubizmay, kurß zu Teutze 72, 19 (hess., 1463): Also liligen vnder den thornen also ist mein freundin vnder den Iungenfrawen. Neumann, Rothe. Keuschh. 2359 (thür., 1. H. 15. Jh.): einen langen stengel die lilie had, $|[\ldots]$.$| . ess bedudit das die kuscheit sal lange \mid$ in dem menschen weren. EBD. 2367: das lilien safft beilet zu hand | di wonden di da sint gebrand. HüBNER, Buch Daniel 2013 (omd., Hs. 14./A. 15. Jh.): Lylien sten in vuchte | In drierhande luchte (Minneallegorie). Bechstein, M. v. Beheim. Evang. Mt. 6, 28 (osächs., 1343): Seht an die lilien des acheris wî si wahsin, und si inerbeiten nicht. GILlE u. a., M. Beheim 110, 147 (nobd., 2. H. 15. Jh.): nach | des kaisers pstetikaite | Dez, von dem lilgen velde | werden sich die Jermani gseln $\mid$ und auss irn fursten ainn erweln. EBD. 245, 21: Der rauben disteln auff dem stil | und der nessel ist es ze vil, | ab ir art uber adeln wil | der lilgen, veigel, rasen, | Negel plümlein, zeitlasen. MaYer, Folz. Meisterl. 34, 627 (nobd., v. 1496): Do leg wir gancz am schadenn, | Ob nit dein [mutter Gottes] gut | Senfftmutiglich an neiget, | Das unns zu werd geeiget | Die lilgen plut. EBD. 84, 47 (1517/ 8): Den zorn hastw in güt gekert | [...] | Und uns funden die lilgengert, $|[\ldots]|$ Ee dw gabst form gestalt und pild $\mid$ Dem sün Gotts. EвD. 87, 31: Dw reine gert von Jesse stam, | In deinen lilgen garten clam |Jesus. KeHreIN, Kath. Gesangb. 1, 372, 63 (Nürnb. 1631): Dein [HERR] Edler gruch schmeck.t wunderlich, | Erbreite dich, O Lili schón. DAsyPODIUs 1145 (Straßb. 1536): Lilietum, Ein lilien busch da vil lilien wachßen. Bremer, Voc. opt. 50189 (wobd., 15. Jh.): Lilium lilgenplum. Rieder, St. Georg. Pred. 43, 1 (Hs. 「önalem., 1387`): ich engesach noch vernam nie, da man rosen phlantzat, daz da lylien wuichsent. aber uff dirre wurze der minne da wabsent alle edel blimen. EICHLER, Ruusbr. obd. Brul. 1, 734 (els., E. 14. Jh.): Reinekeit des libes gelichet men der wisheit der lilien und der luterkeit der engele. ЕвD. 2, 835: [Cristus] was die vnbefleckete lylie vnd die gemeine felt blime, do alle güte menschen das honig der ewigen süßekeit an ladent. Thiele, Minner. II, 14, 49 (Hs.

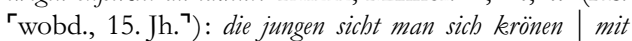
schappeln und mit rösen, | gemaht uß zittlösen, | mit lilyen durch florieret. Sudhoff, Paracelsus 14, 394, 13 (1568):
Darumb die materia tincturae das größt perlin [...] ist, das nach [...] aller menschen betrachtung auf erden sein mag. und das ist die lili der arznei und alchimei, welche die philosophi so beftig und streng gesucht haben. STRAUCH, Schürebrand 50, 19 (els., E. 14. Jh.): dar gegene dise materie glichet alse eine grobe rube tistele gegen allen den edeln liligen und rosen, die ie gewuibsent. WarNOCK, Pred. Paulis 2, 171 (önalem., 1490/4): Also die miniklich lily under den dornen ufgat, also ist min frúndin under den tochtren (Cant. 2, 2). EBD. 17, 53: die senftmütikait ist gelich ainem plimen oder edlen lylya. Rот 325 (Augsb. 1571): Lilgn, vom wort Lilium, ein Lilien oder blim / weiß / blaw / gelb lilgen / lilium conuallium. Pfeiffer, K. v. Megenberg. B. d. Nat. 59, 15 (oobd., 1349/50): Alsô tuot unser frawe, diu gibt [...] den pü̈ezern violisch varb, den martern rôter rôsen varb, den junkfrawen lilienvarb. ЕвD. 406, 16: liligenöl ist guot für der vergiftigen tier piz. EвD. 421, 22: des krautes pleter sint gestalt sam diu liligenpleter. Spechtler, Mönch v. Salzb. 7, 29 (oobd., 3. Dr. 14. Jh.): (du) [Maria] veldpluem, demüetig tal, | (du) lilge sunder misseval, | Christus aus dir plümleichen plüet. EвD. 8, 28: du demüetigs, reiches lilgental. Weber, Füetrer. Poyt. 190, 5 (moobd., 1478/84): Wie das raine weib | mit klarbait sunst thüet scheinen, | noch verre pas ist meiner frawen leib | für si geschönt alls lilien für den doren. - KEHREIN, a. a. O. 1, 106, 3; 2, 39, 31; 397, 10; FISCHER, a. a. O. 4804; 5589; Dubizmay, a. a. O. 63, 16; ERMISCH u. a., Haush. Vorw. 96, 24; GILle u. a., a. a. O. 110, 169; 343, 12; Sachs 21, 67, 10; Bihlmeyer, Seuse 440, 4; Rohland, Schäden, S. 466; Matthaei, Minner. I, 8, 464; Rieder, a. a. O. 75, 34; WARNOCK, a. a. O. 2, 81; 3, 221; PÄPKE, Marienl. Wernher 5834; 14404; EIs u. a., G. v. Lebenstein 43, 10 ; KeIL, Peter v. Ulm 54; Harsdoerffer. Trichter 3, 326, 5. - Vgl. ferner s. v. ${ }^{1}$ ban 5 , begreifen 2 .

2. >Lilie als Herrschaftszeichen, als Schmuckgegenstand, als Bild oder Gravur auf Gebrauchsgegenständen`. - Rechtsund Wirtschaftstexte, berichtende Texte. Synt.: die stole lilien haben, das wetter die lilien herabschlagen; die l. vergüldet, in einer fane sein, [wo] eingegraben [sein], perlen haben; der adler in der l. >Krone Frankreichs « nisten; die l. an / von gold / gestein, von steinen; die blaue / grosse / güldene / kostbärliche / vergüldete l.; der schild mit lilien, das humeral mit einer l., das wappen mit lilien. Wbg.: liliengefelde $>$ Schildfeld mit Lilien<, lilienzweig.

Ziesemer, Gr. Ämterb. 10, 17 (preuß., 1404): ein silberyn kopchin uff dem lede eine lylie gegraben. LutHER, WA 46, 730, 26 (1537/8): das aus seinem [Christi] Munde ein Schwert auff einer seiten gebe und ein liliensweig auf der andern seiten. PeIL, Rollenhagen. Froschm. 608, 3242 (Magdeb. 1608): Zur Lincken Frantrósiche Knecht / | Drey Lilgen waren im Febnlein / | [... $\mid$ Zwey ding prangen frólich 
berein | Die Lilg am Wassr / der Mann beym Wein. Loesch, Kölner Zunfturk. 11, 250, 8 (rib., 2. H. 15. Jh.): twe blawwe lelien, de sijn vergult end ellic beft 4 grote perlen. STRUCK, Marienst. Wetzlar 1158, 70 (hess., 1495): Trwa alt stolen, eyn gulden, $d y^{e}$ ander hait lilien etc. Helbig, Qu. Wirtsch. 2, 29, 14 (md., Hs. M. 16. Jh.): bej gefangenn Matz jm hosennlatz gefundenn 7 guldenn ringe [...] 1 lilien gefelde - solches der [...] richter $2 m$ sich jn vorwarung genomenn. STOLTziUs, Chym. Lustg. 87, 3 (Frankf./M. 1624): Sonn und Mon stehen hier zugleich / | Beide furren zween Lilien zweig. CHrON. Nürnb. 1, 60, 8 (nobd., 1407): unser wapen mit den dreyn lilgen burden unsern vordern verlichen von kunk Cunrat. EвD. 5, 680, 9 (nobd., E. 15./A. 16. Jh.): [das weter] slug die liligen und die plumen neben herab und durch daz gewelb. WENDEHORST, UB Marienkap. Würzb. 484, 31 (nobd., 1527): das humeral mit drey grossen silberen vergulten buchstaben und einer lilgen. Uhurz, Qu. Wien 2, 3, 4942, 19 (moobd., 1482): ain halbs vergulcz fraungesperr, [...], vir lilign von praunn staindlein. Grossmann, Unrest. Öst. Chron. 39, 26 (oobd., 3. Dr. 15. Jh.): Da was gerichtet ein altar, [...], in der mitten ein liligen an goldt und an gestain. LEIDINGER, V. Arnpeck 543, 20 (moobd., v. 1495): ain kostperliche liligen von golt und edlem gestain. Turmair 1, 199, 2 (moobd., 1529): er [künig in Frankreich] soll der Teutschen müessig stên [...] und der adler werd in die lilgen nisten. Ziesemer, a. a. O. 237, 14. - Vgl. ferner s. v. pater noster 2.

lilienblank, lilienblat, lilienblume, lilienblut, lilienbusch, lilienfarbe, lilienfeld, liliengarte, s. lilie 1.

liliengefelde, s. lilie 2.

liliengerte, lilienöl, liliensaft, liliental, s. lilie 1.

lilienwasser, das.

ygebranntes Wasser der Weißen Lilie (als Arzneimittel gegen Ausschläge o. ä. gebraucht) $<$; zu lilie 1.

Ermisch u. a., Haush. Vorw. 78, 20 (osächs., 1570/7): die maasen mit dem warmen oel geschmieret, auf den morgen mit warmen rosenwasser, weiß lilienwasser. HaAge, Hesel. Arzneib. 18v, 20 (Hs. ' noobd./md., E. 15. Jh. ') : nym lilgen basser und lans begrich und zbifel, und back daz durch ein ander. Eis u. a., G. v. Lebenstein 40, 9 (oobd., 1. V. 15. Jh.): Von dem weissen lilgen wasser Item das wasser hat die tugent, wer es in die augen sprengt, so vertreibt es al rot.

lilienweis, Adj.

>weiß wie eine Lilie`, oft auf die Schönheit einer Frau bezogen; zu lilie 1.

Luther, WA 7, 674, 23 (1521): Es sollte myr ewr [Emser] lilien weysse keuscheyt nit sagen, was meschen lere byrinnen setzet. Gille u. a., M. Beheim 337, 27 (nobd., 2. H.
15. Jh.): ir lilgen weisse wengel, | ir rosen varber mund | Tet mir grass fröude kunt. STACKMANN u.a., Frauenlob 14, 7, 9 (Hs. 'nobd., 3. V. 15. Jh. ${ }^{7}$ ): Baz dem munde zeme ein liljenwizez ja $\mid$ dann ein nein von jamer bla. - KEHREIN, Kath. Gesangb. 2, 448, 3; PÄpke, Marienl. Wernher 5785; Kummer, Erlauer Sp. 4, 124.

\section{lilienwurz, die.}

>Wurzelstock von Schwertlilienarten`; er diente der Herstellung von Heilsalben; zu lilie 1. - Bdv.: illesirke.

KeIL, Peter v. Ulm 64 (nobd., 1453/4): Die salbe heist vngentum Agrippa etc, Dye mach also: Nym bundes-kürbis j lb vnd weiß wein und rebenwurtzel ij lb [...], plaw liligenwurtzel vj lot vnd attich-wurtz vnd fech-distel-wurts. [im Folgendem genaue Herstellungsanleitung]. OTт-VoIGTLÄNDER, Rezeptar 209r, 6 (Hs. 'nalem., um 1400): Der das harn nit behaben mag, der nem bjlien wurczen vnd sied die in wasser. - RoHLAND, Schäden, S. 466.

lilienzweig, s. lilie 2.

limbisch, s. ${ }^{1}$ limbus.

${ }^{1}$ limbus, der; lat. Flexion; etymologische Zuordnung unklar.

eine Art gottgegebenen Urstoffs, der der männlichen Person eingegeben ist. - Wbg. : limbisch.

Sudhoff, Paracelsus 14, 161, 34 (1529/32): der mensch hat żo art an im, die limbisch und die sêlisch; das ist also ein exempel. es würd ein kint geboren auf die stunt und die minuten rc. [...]. nun iest folgt aus dem, dem kint ein nativitet, die wird also sein. EBD. 568, 6 (1591): aber Adam hat den besten teil von der essentia limbi aeterni gegen der Eva gerechnet. derwegen auch Eva den Adam so liebet, das heißt sie liebet das so hoch das in Adam der limbus aeternus ist. dan Adam liebet auch den spiritual cagastrischen limbum und stomachum cagastri matricis. EBD. 15: und dieweil Adam noch ein stück hat vom limbo aeterno, so hat Eva auch ein beger, lust und senen nach dem limbo aeterno, welcher in Adamo ist. EBD. 569, 40: nun ist in der frauen der limbus nicht aber der geist des limbi ist in ir. EBD. 572, 5: und wil beschreiben physicè den materialischen limbum, den got in die matrix microcosmi eingepflanzet hat. EBD. 689, 17 (hierher?): aber anima und matrix maioris mundi die ist inficirt worden und das ist dem irdischen limbo geschehen, dem baum so ir corallen heißt.

${ }^{2}$ limbus, der; aus lat. Limbus >Streifen` (GeOrges 2, 698).

> Vorhölle, zwischen Himmel und Hölle/ Fegefeuer liegender Ort für die Gerechten des Alten Testamentes $<$. Zur Sache: LeX. D. Mal. 5, 1990; LThK 6, 1986, $1057 \mathrm{f}$.

Adrian, Saelden Hort 3476 (alem., Hss. E. 14./ 15. Jh.): do hie lag tot $\mid$ uf erde sin lichame, | sin sel kunt 
Adame | und in die mit im wan behaft | inlymbo, liebe botschaft. EBD. 3929: von dem crutz zu dem grab, | von dem grab dort hin ab $\mid$ von linbun, da du [Got] lostest $\mid$ die rehten.

limel, der; zu mhd. limbel >Schuhfleckı, dies aus lat. limbulus (LeXer 1, 1922).

>Streifen, Flicken, Lappen (in der Regel von Leder oder Tuch)<. - Bdv.: blez 1, flek; vgl. ${ }^{1}$ hader, ${ }^{1}$ lappe, ${ }^{2}$ luder, lumpe 1, plak 2. Synt.: einen l. aufsetzen, jm. [eine Anzahl] l. geben; ein tuch mit l. setzen, den schuh mit limeln vernähen. Wbg.: limelleder, limeln >flicken (a. 1494).

Gille u.a., M. Beheim 1216, 220 (nobd., 2. H. 15. Jh.): da wir in [hern] sehn in rehter sip, | nicht als die berter in der krip | in swacher tüchlin limel. GRIMm, Weisth. 1, 675, 35 (els., o. J.): [der huber] sol im [meier] geben zwen bökhen schue [...] und dem ochsner [...] vir limmel. KuRRELMEYER, Dt. Bibel 4, 280, 1 (Straßb. 1466): gar alte geschúch die von rechtem alter mit lymelen [Var. $1475^{2}-1518$ : liderin flecken; LutHer 1545, Jos. 9, 5: geflickte Schuch] wurden verneet. RENNEFAHRT, Wirtsch. Bern 568, 2 (halem., 1487): von acht tuchen, mit einem limmet (!) gesetzt $8 \beta$. - Schmidt, Hist. Wb. Elsaß 224.

\section{limelleder, limeln, s. limel.}

\section{limit, Genus?}

$>$ Grenze (im räumlichen Sinne) <. - Bdv.: vgl. anstos 6, antrete 2, anwand 5, banscheide, bemärkung, bimark 1, confin 1, gemarke 2, grenze 1, letze 1, term 1, termin 2.

Hilliger, Urb. St. Pantaleon 525, 16 (rib., 1650): baben ibro hochw. [...] die grenzen und limiten des zehents [...] begangen und auf neu mit ibren vöhren [...] beschrieben. - AuBIN, Weist. Hülchrath 144, 23.

limitation, s. limitieren 2.

limitieren, V.; aus lat. limitāre >abgrenzen`(GEORGES 2, 660).

1. >etw. festsetzen; etw. limitieren; etw. auf etw. herabsetzen «. - Bdv.: vgl. abmessen 1, 1 abwägen 3, anschlagen 23 , ansetzen 8 , benennen 3, besetzen 13, statuieren.

KÖBLER, Ref. Franckenfort 36, 13 (Mainz 1509): So ordenen wir das solche erkantnissen gezogen / vnd limitirt sollen werden vff hundert gülden. Rot 325 (Augsb. 1571): Limitirn, Durchweg machen / vnter machen / außsylen / sich etwo bin enden / grentzen. Bischoff u. a., Steir. u. kärnt. Taid. 469, 29 (m/soobd., Hs. 17. Jh.): jedoch voriger inhalt allein von zechen ducaten limitiert auf vier ducaten die peen. - Rwв 8, 1335.
2. >sich auf etw. / jn. beziehen`. - Wbg.: limitation.

Rot 325 (Augsb. 1571): Limitirn [...7. Jtem sich auff etwas ziehen / referirn. Als so wir sprechen / Er Limitirt sich auff disen oder jenen. ЕвD.: Limitation, Anziehung / referirung, so da gleich zengnuß weiß geschicht. Jtem vntermachung / außweisung.

limme, s. limmen.

limmen, V., unr., abl.

>brüllen, schreien, bellen (von Raubtieren, vom Tod); klagen, laut jammern (vom leidenden Menschen)<. - Älteres Frnhd.; Verstexte. - Bdv.: vgl. brüllen, greinen 2, lören 1, ludemen, ${ }^{2}$ lügen 1 , plärren 1 ; 2. - Synt.: der ${ }^{1}$ bär / lewe / tot l., j. wunderlichen, in jamer / not, als ein hund l., js. zorn l. Wbg. : limme >lautes Klagen, Wehgeschrei<.

Strehlke, Nic. Jerosch. Chron. 15569 (preuß., um 1330/40): und begonde gâbin | wunderlichen limmende | und widir ubirswimmende $\mid$ ze bulfe siner rotin. EBD. 19252: der vorrêter an sî schrê $\mid$ mit vil jâmirlichbir vlêe $\mid$ in clegelichem limme. Reissenberger, Väterb. 2944 (Hs. rmd., 14. Jh. $\left.{ }^{7}\right)$ : Als ein tobende bunt er lam. STACKMANN u. a., Frauenlob 5, 34, 3 (Hs. 'md. auf nd. Grundl., v. M. 14. Jh. '): Ein hane sol keren, ein bunt sol bellen, kerren ein swin $\mid$ nach dünken min. $\mid$ so sol ein lewe limmen, $\mid$ und der ber sol brimmen. Henschel u. a., Heidin 609 (nobd., um 1300): Er fur limmende als ein ber. Pyritz, Minneburg 5071 (nobd., Hs. um 1400): ez. tut mir not $\mid$ Daz, ich so in jamer lymme, | Wann mich bat in starkem grymme | In sinen clowen ein mechtig ar. ЕвD. 5103: da von ich dol | Sulich not, dar inne ich lymme. Primisser, Suchenwirt 12, 30 (oobd., 2. H. 14. Jh.): Her tot, wie grimmicbleich ir limt, | Daz ir uns niemant lazzet bie, | Der milt und manbait ie pegie. EBD. 15, 202: den [lew] man vraidicbleich | Sach inn dem scbilde chlimmen | Und gen den veinden limmen. StrehlKe, a. a. O. 18157; Pyritz, a. a. O. 320 ; HoltzmanN, Gr. Wolfdietrich 1002, 2; 1128, 3; Gereke, Seifrits Alex. 5537; Primisser, a. a. O. 13, 113.

limone, die.

>Zitrone «. - Obd.

FIscher, Folz. Reimp. 45, 217 (Nürnb. 1482): altag nissen des sames citranguli oder der limonen pey aim halben quintin mit sülchen wassern. Morrall, Mandev. Reiseb. 107, 21 (schwäb., E. 14. Jh.): daz sie ir hend und ir fü $\beta$ wáschent mit ainem safft von ainem ópffel, der haisset bymon. EвD. 123, 8: wenn sie in den sów wóllent, so nement sie den safft von lymonen und von krúttern die sie hond, und bestrichent sich da mit. BAUER u. a., Kunstk. Rud. 1084 (oobd., 1607/11): Vasetti langlicht rund wie ein limoni. - Loose, Tuchers Haushaltb. 99, 8; Fischer, Folz. Reimp. 45, 
209; Rupprich, Dürer 1, 176, 189; Müller, Welthandelsbr. 187, 17; Gleinser, Anna v. Diesb. Arzneib. 1989, S. 184.

limpfen, V.; s. glimpf, auch nd. limpf >Ehre`(RwB 8, 1335).

setw. zweckentsprechend einrichten, justieren〈; im einzelnen z. B.: >etw. verdünnen . - Bdv.: vgl. ${ }^{2}$ justieren. - Wbg.: limpfieren (dies möglicherweise aus mlat. limphar) $>$ (mit Saft o. ä.) verdünnen`(DuCANGE 5, 114), limpfig (dazu bdv.: behende 2; 6, zügig), limpflich >gefällig; gut zu handhaben`, limpfige (die).

Toeppen, Ständetage Preußen 5, 120, 6 (preuß., 1464): dergleichen, offt ze en ok wes vorgheven, dat dem orden nicht vellich edder limplick were, dat scholden se ene to unwillen nicht leggen. Sснмітт, Ordo rerum 641, 4 (omd., 1452): Abilitare [...] bequeme machen [...] limphem [...] fügen machen schicken. Scholz, Lanfrank. Chir. Parva 232v, 12 (md./ oobd., 1446/8): so las in / ein linden wein nuczen, der do gelimpfirt sey ader mit waßer gemischt / sey. BIHLMEYER, Seuse 25, 1 (alem., 14. Jh.): Die spise, dú im nit linpfig was bot er zu tunken gen dem minnewunden herzen. KOLLER, Ref. Siegmunds 120, 42 (wobd., um 1448/52): an [... fasten, beden, zu kore gan, mag man wol nach nottorffege lymplichen erlychten, nit gantz abnemen. MAALER 273v (Zürich 1561): Limpffig / zügig / Das sich laßt hantzlen. Tener. Limpffige (die) Teneritas. Bоот, Cassiodor. Hist. Eccl. 20, 17 (moobd., um 1385): daz liecht waz als der glancz der stram und gaz darin ein suezzes trankech, und lympht es mit chaltem wazzer. - Toeppen, a. a. O. 5, 122, 29; Voc. Ex QUO A 20; Golius 373.

\section{limpfieren, limpfig, limpfige, limpf-} lich, s. limpfen.

lind, das; $-\emptyset /-$; entsprechend mnl. lint >Band`(Verwijs/Verdam 4, 663; s. auch LEXER 1, 1928); bei van VEen / van DER Sijs 1997, 514 wird auch Herkunft aus lat. linteum >Leinwand $<$ erwogen.

>Band, Leiste; Rindenbast $\iota$. - Bdv.: vgl. ${ }^{1}$ band 3, bendel, gebende 1, ${ }^{3}$ leiste 1. - Wbg.: ${ }^{2}$ linden >aus Bast $<$, lindzeichen $>$ Narbe $<$.

Scholz-BABisch, Klev. Rheinzollw. 402, 23 (rib./wfäl. 1587): allerhant lyndt den daller koepgeltr 1 alb. BuCH WeINsB. 3, 341, 14 (rib., 1586): das ist die hoede mit sidin, flawiln, lint zu foedern. JAHR, H. v. Mügeln 1338 (omd., Hs. 1463): sust dri personen gottes sint $\mid$ verstrikt in eines wesens lint. Chron. KöLN 3, 844, 3 (Köln 1499): [Karl] wart dairna vunden ind mirklich bekant an etzlichen lintzeichen, die hei an sich hadde. Voc. Ex QUO S 1145 (wmd., 2. H. 15. Jh.): Suberinus besten [...] linden, est aliquid de subere factum. EBD. S 1143 (15. Jh.): Suber bast oder lint / bast [...] dicitur arbuscula uel eius cortex. Mon. BoIcA, NF. 2, 1, 41, 23 (nobd., 1464): Wer ein lend abrysz [...], der verfiel von ir iglicher 30 schilling haller. НАMPE, Ged. v. Hausrat 1, 6, 6 (nürnb., n. 1480): Hawen vnd schaüffel ein waschstock | lendel [Diminutivum; hierher?] schaff züber ein waschdrock. - SchweIz. ID. 3, 1344 s. v. Lint.

lind, Adj., in adv. Gebrauch mehrfach lindiglich (auch -lichen).

1. dient in Antonymie zu hart 1 der Kennzeichnung sinnlich wahrnehmbarer Bezugsgrößen und diese voraussetzender Handlungen als weniger stark ausgeprägt, dadurch vielfach als sanft, angenehm empfunden; im einzelnen: a) > weich im taktilen Sinne «, je nach Bezugsgröße z. B. >streichbar (von Arzneien) «; >fluktuierend (von Geschwüren)く; 〉biegsam (von Metallen)<; $>$ locker (von der Erde) <; > weich (vom Stuhlgang)<. - Bdv.: biegig, sanft, weich. - Synt.: etw. (z. B. die lunge, das erdreich / würmlein) l. sein, j. (z. B. das weib) vom fleische l. sein, etw. (z. B. der schnee) l. werden, l. am leibe werden, etw. (z. B. das apostem) l. machen; l. liegen/ ruben, etw. lindiglich angreifen, jn. lindiglichen mit dem fuchsschwanz bestreichen; der linde wachs / weastein, die linde hand / baut / luft, das linde bet / tuch, linde bürsten / federn / hare / kleider / kolen / seiten. Wbg.: ${ }^{2}$ linde 1a >Weichheit`(Ggs.: bärte 1, schärfe), lindfüssig, lindgewirkt, lindig, lindlecbt, lindkästen eine Kastanienart, lindmütig 1. - b) >weich, sanft im akustischen Sinne <; als Ütr. zu a) auffaßbar; je nach Bezugsgröße z. B. >angenehm, sanft (von einer Melodie) <; > weich (von Lauten) $<$. - Bdv.: weich. Ggs.: rau, grob 2; 3, scharf, stark. - c) >angenehm schmeckend (z. B. vom Wein) «; ’gut, verweichlichend (vom Essen) $<$.

$\mathrm{Zu}$ a) Oorschot, Spee. Trvtz-N. 120, 14 (wmd., 1634): Dem Schöpffer sie zun Ehren |Jn lindgewircktem Flachs |Vnzablbar Fewr ernehren. KeIL, Peter v. Ulm 29 (nobd., 1453/4): pflaster, das do weichet vnd zeittiget vnd lind machet die apposten. EBD. 187: so thu die puluer auch dor ein [...] vnd am end mach es lind mit frawen-milch. Mayer, Folz. Meisterl. 55, 42 (nobd., v. 1496): Rein bockez plut list | Der stein der allen herten wider ist, | Dem gipt er lind. Rupprich, Dürer 1, 159, 16 (nobd., 1520): Jch hab 2 stüber für ein linden wecsstein geben. EBD. 3, 197, 
157 (1512/3): dÿ weiber sind lind fon fleisch. MatтHaEI, Minner. 1, 1, 1333 (Hs. ' nalem., 1459`): ringe liebe und linde kolen | sol niemand gern wit holen, | es entzint sich bald und lischt gern. Rieder, St. Georg. Pred. 316, 12 (Hs. 「önalem., 1387`): dez. [blime] natur ist also herte, swie lindeklich man in an grifet, so stichet er doch da wider. LiNDQvist, K. v. Helmsd. 1215 (halem., Hs. um 1435): wúrmlin. | So man das rirt, so ist das lind | Und hat doch als gar geschwind $\mid$ Durch boret des herten holtzes grat. BROszINSKi, Minner. Chir. Parva 82v, 2 (halem., 2. H. 15. Jh.): nach dem so ein glid / zesamengebeilt wirt und das man es lindmütig vnd gleichig mach. MAALER 273v (Zürich 1561): Linde / Weiche. (die) Mollities, Molitia. [...]. Lindfussig / Der lind / Weich oder zart füß hat. Mollipes. [...] Lindkestinen / Die vom braaten oder sieden lind werdend. Castanea molles. Lindlächt. Molliculus. EBD. 294v: Einem ein sanfften oder linden Stülgang machen. LÖFFLER, Columella/Österreicher 2, 7, 2 (schwäb., 1491): [ocbsen] ain dicken und kurtzen lib, [...] und in der begriffung und anrierung des libs aller lindost. Rauwolf. Raiß 35, 9 ([Lauingen] 1582): Ducaten / welche von gütem pur lauterem Gold gar lind und bigig seind. Turmair 1, 176, 18 (moobd., 1529): die Teutschen, sein nur fresser und weinsaufer, müessen alweg ir huern mitfiuern und die hauptleut ir linde pett am ars baben. EBD. 485, 30: schmirn macht lind heutt. - PeIL, Rollenhagen. Froschm. 358, 3083; Lemmer, Amman/ Sachs. Ständeb. 58, 6; KeIL, Peter v. Ulm 84; WeITZ, Albich v. Prag 162, 15; 171, 30; Gille u. a., M. Beheim 345, 43; Sachs 16, 480, 32; Bihlmeyer, Seuse 155, 17; Williams u. a., Els. Leg. Aurea 543, 16; Cirurgia H. Brunschwig 22va, 39; PÄpke, Marienl. Wernher 5929; Wiessner, Wittenw. Ring 9611; Sappler, H. Kaufringer 21, 76; Chron. Augsb. 5, 11, 4; Pfeiffer, K. v. Megenberg. B. d. Nat. 29, 25; Bauer u. a., Kunstk. Rud. 156; Bauer, Haller. Hieronymus-Br. 27, 3. - Vgl. ferner s. v. begreifen 19, behende 6, igel 2. - Zu b) Lappenberg, Fleming. Ged. 327, 29 (1631): Dieses Liedes linde weisen | sollen deine Zukunft preisen. OpIтz. Poeterey 29, 6 (Breslau 1624): soll man sie [buchstaben] doch sonsten mitt einander so wisßen zue vermengen / das nicht die rede dadurch gar zue raw oder zue linde werde. HarsDOERFFer, Trichter 1, 128, 14 (Nürnb. 1650): wann nemlich das Harte mit Hartem / das Linde [...] mit weichen Buchstaben geschrieben wird / als: dapfer / drucken / nicht tapfer / trucken. MüLlER, Quellenschrr./Kolr. 74 (Basel 1530): b. vnd d. ly $\beta$ lind. KOHLER, Ickelsamer. Gram. 40, 1 (wohl 'Augsb. 1. Dr. 16. Jh. `): kain stym kan zu gleich, mitainander geben den linden und berten laut. TurmaIr 4, 20, 12 (moobd., 1522/33): die Niderlender und Saxen nemen in [buechstaben B] für ain lind f. - JAHR, H. v. Mügeln 108, 455; KoHLER, Ickelsamer. Gram. 39, 29; Moscouia B, 35. - Zu c) Scholz, Lanfrank. Chir. Parva 232, 12 (md./oobd., 1446/8): so las in / ein linden wein nuczen. Kurrelmeyer, Dt. Bibel 8, 64, 6 (Straßb. 1466): Nicht sich an den wein so er schmoret: so sein varb leucht in dem glaße. Er get in lindiglich [EсK 1537: senft; LuTHER 1545, Spr. 23, 31: glat] | vnd becket zum iungsten als der slange. Dierauer, Chron. Zürich 247, 26 (halem., 15. Jh.): mocht ouch der win an reben nit lind werden. PRIMISSER, Suchenwirt 31, 83 (oobd., 2. H. 14. Jh.): Si hat in liep und tzúbt in tzart, | Lindiu chostel, guwten wein.

2. ssanft, milde, nachsichtig, freundlich, rücksichtsvoll<; vom Menschen, seinen Qualitäten und Handlungen sowie oft von Gott gesagt; vielfach in religiösen Kontexten. - Bdv.: freundlich, gütig 2, leutselig, milde, sanft, sänftmütig, sitsam, süsse, weich. Ggs.: hart 7, sauer, scharf. - Synt.: etw. (z. B. die minne) / j. (z. B. Jesus / got, der geistliche mensch) l. sein, das gemüte, der stat l. werden; das herz l. machen; l. antworten / sprechen, sich l. beweisen / erzeigen, jn. l. brennen / strafen / segnen, l. mit jm. umgeben; das linde herz. / wort, die linde antwort / mitlung / predig; l. in wort und werken, l. gegen jm. (z. B. gegen dem nächsten, gegen den feinden). Wbg.: lindentrit ssanftmütiger Mensch (a. 1650), lindmütig 2.

Strehlke, Nic. Jerosch. Chron. 16546 (preuß., um 1330/40): ₹u Colmin in dem lande was | ein lantcomentûwer, $|[\ldots]|$ den undirtânin swinde $\mid$ und kegn den vîndin linde. LuTHER, WA 10, 1, 2, 365, 38 (1526): also macht auch die süsse linde predig des Euangelions, das ich ein fein linndes hert? gegen Got und dem nechsten. ЕвD. 37, 380, 8 (1534): das Cbristus so freundlich, veterlich, sanfft und linde mit uns umbgehe. Schwartzendach 51v (Frankf. 1564): Gútig. Gutwillig / Milt. Sánfft. Lindt. Sanfftmuitig. Freundtlich. Jungbluth, J. v. Saaz. Ackermann 15, 2 (Hs. romd., 1465?): Süße und sauer, linde und hert, gütig und scharpf pflegt ir euch zu beweisen den, die ir meint $2 u$ betriegen. Chron. Nürnb. 5, 667, 19 (nobd., E. 15./A. 16. Jh.): da prent man den Veit Stoß durch ped packen und man het nie keinen so lind geprent. SACHS 14, 137, 1 (Nürnb. 1550): Forthin wil ich leutselig sein, | In wort und wercken lindt und sidtsam. RIEDer, St. Georg. Pred. 32, 6 (Hs.

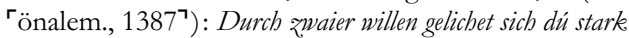
minne sidin saile. daz ain ist daz si starke ist; daz ander daz. si lind ist. EвD. 245, 9: wider den zorn sol man ban senfti und lindú wort. SсHмiDT, Rud. v. Biberach 185, 17 (whalem., 1345/60): daz gemite zer flusset, so es von andacht lind wird. JörG, Salat. Reformationschr. 22012 (halem., 1534/5): Antwurtend die botten / [...] Mit vil linden worten und früntlichemm abreden. V. ANshelm. Berner Chron. 4, 246, 26 (halem., n. 1529): do ich nútset anders vermocbt, gedacht inen iedoch lindmuietiklich ze widerstreben. GILMAN, Agricola. Sprichw. 2, 44, 12 ([Augsb.] 1548): Ain linde antwort stillet den zorn / aber ain bart wort richtet grimm an. - Chron. Magdeb. 2, 44, 14; Kehrein, Kath. Gesangb. 1, 172, 29; Pyritz, Minneburg 1466; 4886; Gille u. a., M. Beheim 120, 206; Reichmann, Dietrich. Schrr. 78, 13; SACHs 16, 480, 30; 18, 445, 5; 20, 
215, 3; Rieder, a. a. O. 44, 29; 280, 1 ; 316, 9; JörG, a. a. O. 596, 6; WIESSNER, Wittenw. Ring 1172; 3654; Lindevist, K. v. Helmsd. 1222; Sappler, H. Kaufringer 24, 51; Schöpper 75a; SCHLes. WB. 2, 814.

3. >leicht; leichtfertig, leichtsinnig‘. Bdv.: vgl. leichtiglich 4, leichtfertig 3, leichtsinnig 2.

Rosenthal. Bedencken 16, 7 (Köln 1653): etliche ibre Glaubens Lebren also gekrumbt [...] / daß sie denen / welchen sie sich begerten zu zugesellen / etwas linder vorkommen / als sie sonsten wahrẽ. OPEL, Spittendorf 154, 23 (osächs., um 1480): inrede, das die vorsteher linde eyde theten. NiEwöHNER, Teichner 96, 1 (moobd., 1360/70): Maniger wigt dw hell so lind $\mid$ sam ez sey ein milich chind. - OpeL, a. a. O. 113, 19; Bell, G. Hager 407, 2, 13; SchweIz. ID. 3, 1344 s. v. Lint.

${ }^{1}$ linde, die.

1. >Linde $<$, in den Belegen meist angesprochen als Gerichtsstätte, als topographischer Orientierungspunkt (mit ansatzweisem Übergang zum FIN), als Treffpunkt für Gelegenheiten unterschiedlicher Art, als wirtschaftsrelevante Gegebenheit, als Gegenstand poetischer Fiktion. - Phras.: reuter unter der linde halten sehen $>$ Unwahrscheinliches sehen . - Synt.: die l. (ab)hauen; einen bagstein an der l. haben, etw. (z. B. die pacht, den zins) an die l. bringen, das pferd an die l. heften, vor die l. treten, unter die l. gehen, unter der l. balten, etw. vorlesen, (zu gericht) sitzen / richten, den gerichtsplaz, eine gastung haben, sich zu der l. niederlassen, der berg bin zur l. gelegen sein; die breite / weite / gute l.; zu [+ ON] unter der l. Wbg.: 'linden, lindig' > aus Lindenholz<, lindenast (Beleg s. v. behangen 3), lindenbast, lindenbaum, lindenblut, lindenblutwasser >Destillat von Lindenblüten`, lindenbret, lindenholz, lindenkern >Fruchtkern der Linde` (Beleg s. v. baringenkorn), lindenkole >verkohltes Lindenholz $\iota$, lindenkrebe >umfriedeter Bezirk unter der Linder (zum Gw s. Sснwёв. Wв. 4, 719), lindenlaub > Goldverzierung in Form eines Lindenblättchens`, lindenrinde, lindensaft (dazu phras.: etw. mit lindensaft schmieren >etwas schönreden, schönfärben `); lindenseil >Seil aus dem Bast der Linder (nicht klar abgrenzbar von attr. Verwendung: das lindene seil), lindentolde $>$ Wipfel der Linde<; auch als Kosewort für die Geliebte gebraucht, lindenwasser, lindschälen >das Abschälen der Rinde zu gewerblichen Zwecken .

Ziesemer, Gr. Ämterb. 652, 31 (preuß., 1414): 12 leste lyndener kolen, item 7 schog buchsensteyne. LuTHER, WA 23, 510, 17 (1527): ein zeichen, das fride sey, gleich als wenn wir reuter sehen unter der linden halten, [...], Denn unter der linden pflegen wir zu trincken, tantzen. KLETT, J. v. Soest 5, 1270 (Hs. 'wmd., 1470/807): eyn bubschen bron, [...] |[... by eynem bubschen lynden bom. Aubin, Weist. Hülchrath 176, 35 (rib., 1515): sy [naebern] bekennen selves, dat sy ire pecht und zeins allwege [...] zo Fritzhem an die linde brengen seullen. Hilliger, Urb. St. Pantaleon 509, 36 (rib., 1624): Das laufstreffen des gewals, der gleichen das lobe und lintschelen [...] sollen [...] abgeschaft sein. LAMPrEcHT, Dt. Wirtschaftsl. 309, 7 (mosfrk., 1427): unter die linden, da sie dan ihre gerichtsplatz, haben. BELKIN u. a., Rösslin. Kreutterb. 54, 3 (Frankf. 1535): Christall polir mit gebrannten Christall und linden boltz. Lemmer, Amman/ Sachs. Ständeb. 16, 3 (Frankf./M. 1568): Ich entwuirff auff ein Linden Bret $\mid$ Bildnuß von Menschen oder Thier. KeIL, Peter v. Ulm 106 (nobd., 1453/4): Ein gut salb für den krampff. So nym linden-koln vnd sto $\beta$ die clein. EBD. 224: Wer sich verprent hab, der nem linden-rinden und sied die gar wol in wasser. Mon. BoICA, NF. 2, 1, 42, 6 (nobd., 1464): Wer ein lynten oder ein salhen abhybe, die sein klein oder grosz, der verfelt von eyner iglichen $10 \mathrm{lb}$. haller. MAYER, Folz. Meisterl. 102, 15 (nobd., v. 1496): Do fant ich pey einer linde | Gar eine schone binde. Dasypodius 176r (Straßb. 1536): Ein Lindenbaum. [... Item Linden bast / oder zarte linden rinden / darauß man seyl machet / vnnd vor zeitten zu kronen gebrauchet hat. EBD. 373v: Linden. Tiliaceus, a, um. [...] Lindenleüblin. Bracteola. Lemmer, Brant. Narrensch. 104, 54 (Basel 1494): Ich bin gar offt gerennet an |Wile ich diß schiff gezymberet han | Ich soll es doch eyn wenig färben | Und nit mit eychen rynden gårben | Sunder mit lynden safft ouch schmyerē. SudHOFF, Paracelsus 5, 374, 11 (1527/8): lindenblutwasser ist gut für den brant. SCHNYDER, Qu. Zürcher Wirtsch. 140, 11 (halem., 1367): eins pfuntz, wert linden seyl iij s. EвD. 176, 24 (1379): Eines pfuntz wert lindiner seilen $j$ to . LeIsi, Thurg. UB 7, 580, 27 (halem., 1387): den berg gelegen bin uff zer Linden, der järklich giltz und gelten sol ain mut kernen Willer messes. Boos, UB Aarau 161, 43 (halem., 1388): das ich [...] offenlich ze gericht sazz. ze Ernlispach in dem dorfe vor dem kilchoff under der linden. Welti, Stadtr. Bern 445, 21 (halem., 15. Jh.): Dis sint die dingstet der lantgerichten, als si von alter har sint komen [...] ze Niderdorf vnder der linden. [...] ze Ecikofen vnder der linden im dorf. WIESSNER, Wittenw. Ring 1860 (ohalem., 1400/08): Got grüess dich, lindentolde (hier als Kosewort gebraucht). EBD. 8850: Schreunend die unholden so $\mid$ Gen der lindentolden do. KopPITZ, Trojanerkr. 21434 (Hs. ' noschweiz., 15. Jh. ${ }^{7}$ ): Schwang der tegen mere $\mid$ Für die bruste nebend sich $\mid$ Ainem linden blatte gelich. MAALER 273v (Zürich 1561): Linden (die) Lindenbaum. [...]. Lindenbast / das ist die zart rinden 
zwüschend dem holtz vnd ausser rinden an den linden / darauff die alten schribend. Dict. Germ.-Gall.-Lat. 308, 5 (Genf 1636):Lindenblit / f. Fleur de tillet. Baumann, Bauernkr. Oberschw. 10, 21 (schwäb., v. 1542): daselbst ain grosse gastung gehapt mit dem adel, [...] under den linden. Wintterlin, Würt. Ländl. Rechtsqu. 2, 468, 16 (schwäb., 1522): soll er [gläubiger] das essendig pfand, [...], und(er) den lindenkreben [...] treiben und [...] feilbieten. Brandstetter, Wigoleis 206, 43 (Augsb. 1493): Hie beld herr Wigoleys zuo roß bey dem gekroeneten küng vnder einer linden vnd gibt jm der künig ein blat oder plued von der linden. - Chron. Köln 1, 1866; Thiele, Minner. II, 5, 87; Loose, Tuchers Haushaltb. 145, 1; LeIsI, a. a. O. 714, 25; Koppitz, a. a. O. 1362; Sudhoff, a. a. O. 6, 110, 18; Brandstetter, Wigoleis 195, 23/4; Pfeiffer, K. v. Megenberg. B. d. Nat. 350, 4; Weber, Füetrer. Poyt. 199, 4; Bauer u. a., Kunstk. Rud. 626; Voc. INC. Teut. p iv; Dasypodius 176r; 379v; Dict. Germ.Gall.-Lat. 308, 11; Harsdoerffer. Trichter 3, 227, 4; Rwв 8, 1336; Schweiz. ID. 3, 1319 f. - Vgl. ferner s. v. ${ }^{1} \mathrm{ab} 4,{ }^{1}$ bal 2 , ${ }^{1}$ bast 1 .

2. \Samen, Fruchtkern der Linde .

Buch Weinsb. 2, 182, 8 /rib., 1568): vur milch, botter, erzen, ullich, kirsn, wolber, negel, lint, summa 5 mr. 4 alb. $10 \mathrm{~h}$.

2linde, die.

1., s. lind (Adj.) 1 a.

2. >Milde; Sanftmut, Gutmütigkeit`; zu lind (Adj.) 2. - Bdv.: vgl. gelindigkeit, ${ }^{1}$ glimpf 5 , güte 2 , gütigkeit 1 .

Pyritz, Minneburg 2560 (nobd., Hs. um 1400): Nie gevangener wart gederret $\mid$ In gevenkniße so swinde $\mid$ Als ich. an alle linde | Man mich gar dick ultert. ADRIAN, Saelden Hort 5033 (alem., Hss. E. 14./15. Jh.): wan úser wider sach | gat süchent um und umb, | die slibti und die krumb, | die berte und och die linde, | wen er hie verslinde. LÖFfLER, Columella/Österreicher 2, 66, 19 (schwäb., 1491): was darnach uff denen empfangen ist, bătt behalten die mütterlichen lindi und die vátterlichen und ánischen farb (Bezug auf Schafe).

${ }^{3}$ linde, Genus und Etymologie? Möglicherweise Schreibung von lunde.

>Welle, Woge «. - Bdv.: ${ }^{2}$ bulge, welle; vgl. lunde 1, unde.

Sснмітт, Ordo rerum 27, 47 (rhfrk., 1414): Fluctus [...] builge [...] eyn bulge nel eyn lynnde [...] well.

\section{linden, $\mathrm{V}$.}

1. >etw. weich machen; (refl:) in seiner Schärfe zurückgehen (vom Wetter)<; vgl. lind 1a. - Bdv.: weichen; vgl. aberweichen, aufweichen, abschälen 1, 2 auserweichen, lindern 1.
Ggs.: bärten 1, stärken 1; 5. - Synt.: den schaden / stabel / stulgang, das eisen / glied, die blater l.; das wetter sich l. Wbg.: lindung >Linderung< (eines geschwers 1).

KeIL, Peter v. Ulm 29 (nobd., 1453/4): oder nym smaltz. oder öl. So hastu ein lindung zu den geschwerungen. EBD. 37: es [...] zihet den kreps vnd pöß geschwer zu samen vnd ist gut wo zu linden schaden sint. NiewöHneR, Teichner 378, 17

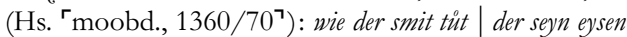
im vewr lindet. Fichtner, Füetrer. Trojanerkr. 237, 1 (moobd., 1473/8): Darnach das wetter linden sich ward. - Sudhoff, Paracelsus 2, 242, 13; Keil, a. a. O. 82; Gleinser, Anna v. Diesb. Arzneib. 1989, 184.

2. >etw. mildern, mindern, besänftigen<; $\mathrm{zu}$ lind (Adj.) 2. - Bdv.: sänftmütigen; vgl. begütigen, besänftigen, ${ }^{1}$ gelinden, gutwillen, leschen 2, stillen 9, 10, 11 .

Voc. INC. TeUT. p i v (Speyer um 1483/4): Lindē vō senftmutige Lenire $i$ mollire. KLEIN, Oswald 106, 26 (oobd., 1411 ?): ob sich ain klain ir widerstreb | bedächt gïtlich zu linden.

linden (Adj.), lindenast, lindenbast, lindenbaum, lindenblat, lindenblut, lindenblutwasser, lindenbret, lindenholz, lindenkern, lindenkole, lindenkrebe, lindenlaub, lindenrinde, lindensaft, lindenseil, lindentolde, s. ${ }^{1}$ linde 1.

lindentrit, s. lind 2.

lindenwasser, s. ${ }^{1}$ linde 1.

lindern, V.

1. `etw. (Gegenständliches, z. B. Eiterbeulen) weich machen «; vgl. linden (V.) 1. Bdv.: vgl. aberweichen, aufweichen, ${ }^{2}$ auserweichen, linden (V.) 1.

Broszinski, Minner. Chir. Parva 73r, 20 (halem., 2. H. 15. Jh.): Ein cur der bösen gescbweren [...] ist uff dry form: Das erst ist, das man die materi lindri. - BRoszINSKI, a. a. O. 72, 6; SChweIz. ID. 3, 1318.

2. setw. lindern, reduzieren, mäßigen, mindern, ermäßigen〈; refl.: >sich erbarmen (von Gott)<. - Bdv.: mässigen, mildern, weichen; vgl. ablassen 10, belinden, tempern 2. Ggs.: schärfen. - Synt.: die beschwerung / kirchenzucht / strafe / strengheit / ungnade, die hitze / hofnung, das trauren l., jm. die furcht, das gesez / recht / urteil l.; got sich l.; der lindernde umstand. Wbg.: linderung (dazu bdv.: begnadung 5, milderung. Ggs.: schwerung). 
Luther, WA 2, 245, 34 (1519): das ich lindern will, was mich dunck.t zu nabe sein. EBD. 10, 3, 256, 25 (1522): uff das er wissz die gestrengheit zi másszigen und linderen. EвD. 47, 83, 32 (1538): Du wirst linderung fbulen, das dein hertz zu frueden sej. Ingen, Zesen. Ros. 73, 31 (Hamb. 1646): daß ich mich lange zeit stálte / als wan ich noch keine linderung meiner schmerzen füblete. KeHrein, Kath. Gesangb. 3, 210, 2 (Köln 1582): All dein vngnad hastu gelindert Herr. Oonsснот, Spee/Schmidt. Caut. Crim. 323b, 19 (Frankf./M. 1649): dz alß ein Pfarrherr einem Gefangenen verschraubter weise linderung der Straffe versprochen. EBD. 261a, 2: werden sie [...] uns also die forcht der Gefahr / [...] / entweder gar benehmen / oder doch guten Theil lindern. Mayer, Folz. Meisterl. 52, 346 (nobd., um 1480): Ob in Got wollt die pet versagen, | Sich nit lan lindern. GrotHAUSMANN, Stadtb. Karpfen 35, 19 (mslow. inseldt., 1605): ist ibme wegen beśchehener üffter fürbitte dises vrtl. gelindert. - LutHer, WA 32, 315, 10 / 18; 27, 363, 2; $41,126,2 ; 41,474,18 ; 45,693,7 ; 47,65,9 ; 50,10$, 30; Kehrein, a. a. O. 3, 24, 2; zu Dohna u. a., Staupitz/Scheurl 239; SAchs 15, 222, 5; Broszinski, Chir. Parva 78v; Dict. Germ.-Gall.-Lat. 308, 15; RohLAND, Schäden 467; Rwb 8, 1337.

linderung, s. lindern 2. 1 a.

\section{lindfüssig, lindgewirkt, lindig, s. lind}

\section{lindigkeit, die.}

1. > Weichheit greifbarer Bezugsgegenstände`; vgl. lind 1a. - Ggs.: hertigkeit 1.

Hohmann, H. v. Langenstein. Untersch. 55, 34 (moobd., 1. H. 15. Jh.): ander geist [...] sint: die schon der sichtigen ding, die pegyrd der hörunden ding, die gevalnuss der smechunden ding, die süssichait der chostunden ding und die lindichait der greiffunden ding. BRETT-EvANS, Bonaventuras Leg. S. Francisci 67, 8 (önalem., v. 1478): So er [H1. Franziscus] etwen enffant lindikeit der keleider [...], so knupfte er inwendick ein seil dor an, daz er dester herter wurde. - Jelinek, Mhd. Wb. 474.

2. 'Sanftmut, Milde als dem Menschen eigene natürliche und vernünftige $\mathrm{Hal}-$ tung`; bei LUTHER in Gegensatz zur christlich begründeten lindigkeit gestellt; vgl. lind 2. - Texte der Sinnwelt, Religion / Didaxe'. - Bdv.: ergęlichkeit, liebe 4, mithellung, sänftigkeit, sanftmut, sänftmütigkeit, schlichtigkeit, süssigkeit, zartheit, zärtligkeit.

LuTHER, WA 10, 1, 2, 180, 14 (1522): Alßo ist die vernunfftige und naturliche lindigkeyt linde gegen die reychen, grossen, [...]; darumb ist sie falsch [...] gegen gott. EBD. 51, 103, 33 (1545): $N u$ leret Er, wie sie [Bezug auf Oberkeit] sich sollen gegen leutten haltten, 'Ewre lindigkeit' i.e. gutigkeit, freundtligkeit, opponitur rigori. PfefferL, Weigel. Gn.
S. 178, 6 (Magdeb. 1615): hitzige Ketzermeister [...] wollen den Glauben mit Schmáhworten verfechten / welcher doch in der Sanftmuth vnnd Lindigkeit stehen wil. KeHreIN, Kath. Gesangb. 1, 286, 4 (Nürnb. 1631): In Hitz bist du ein Lindigkeit, | Den weynenden ein Ergetzligkeit. KurreLMEYer, Dt. Bibel 4, 223, 14 (Straßb. 1466): Das iung weip [...] daz do nit mag eingen auf der erde [...] vmb die michel lindigkeit [LuTHER 1545, 5. Mose 28, 56: zertligkeit] vnd vmb die zartheit: die neidt iren man. HoHmann, H. v. Langenstein. Untersch. 65, 65 (moobd., 1. H. 15. Jh.): so ist ze lauffen zü den züaygüng des hailigen gaists, die da sint lindichait, süssichait vnd sittichait, mithállung, missichhait vnd lieb. - Luther, WA 10, 1, 2, 180, 18; Schmitt, Ordo rerum 498, 251; Volkmar 319.

lindiglich, s. lind.

lindkästen, lindlecht, s. lind 1a.

lindmütig 1, 2, s. lind $1 \mathrm{a} ; 2$.

lindschälen, s. ${ }^{1}$ linde 1.

lindung, s. linden 1.

lindzeichen, s. lind (das) 1.

lineal, das; $-/$ auch $-\emptyset$.

'Lineal (zum Ziehen von Linien); Senkblei, Lot; Winkel (im Bauhandwerk)<.

SCHÖPPER 88a (Dortm. 1550): Amußis. Linial richtscheid bleywag senckel schnur winckelmåß. Rот 325 (Augsb. 1571): Linial. Ein instrument das zum Liniren gebraucht wirt / Regel oder richtscheit. BAuER u.a., Kunstk. Rud. 2213 (oobd., 1607/11): futter, darin silberne außgetailte lineal und wincklmaß. - EBD. 2288; BREMER, Voc. opt. 23023; Voc. InC. TEUT. p iv; Dasypodius 373v.

lineament, s. linieren.

linge, Genus? zu lingen?

eine Schiffsart.

Aubin, Weist. Hülchrath 267, 2 (rib., Hs. A. 17. Jh.): wird erkant vur recht [...], dat die vehren van Monheim sullen vuiren mine gnedige herrn vam doimbcapitel [...] und sullen kommen zu Cöllen mit schiffungen und lingen.

lingen, V.; zu mhd. lingen >vorwärts gehen ( Lexer 1,1926). - Wobd.

1. >sich beeilen, Eile haben`; zur Geographie des Wortes in den rezenten Mundarten s. Dwa 2 (dazu: P. Seidensticker, in: ZfMaf. 24, 1956, 160 ff.). - Phras.: las dir lingen (o. ä.) >beeil Dichく. - Bdv.: belaufen 1, eilen, jagen 5 , hasten, schnellen.

Barack, Teufels Netz 1155, 8 (Bodenseegeb., 1. H. 15. Jh.): Sond si [Fährleute] denn ain erber man verdingen, 
| Der im muos laussen bald lingen, | Das er in selb vierd tüge füren, | Daran kan er in denn wol über rüren. KoppITz, Trojanerkr. 6964 (Hs. ' noschweiz., 15. Jh. '): Uss den liechtten ringen | Liesse er im balde lingen. B̈̈CHTOLD, N. Manuel. Zugabe H. R. Manuel 312, 205 (Zürich 1548): Louf hurtig, buib, und lass dir lingen, | Du müst uns ring und weggen bringen! Adomatis u. a., J. Murer. Nab. 978 (Mühlh. 1556): gond ir bed und land üch lingen | In thurn und thind den Naboth bringen. - Wyss, Luz. Ostersp. 8956; B̈̈снтоld, a. a. O. Todt 7, 2; Krankh. 227, 5; Schmidt, Hist. Wb. Elsaß 224; BaumannZwirner, Augsb. Volksb. 1991, 263.

2. >gelingen «. - Wbg.: lingerung > Vorteil (a. 1446), lingig >erfolgverheißend`, lingsam >geschicktく(16. Jh.).

Barack, Teufels Netz 11412 (Bodenseegeb., 1. H. 15. Jh.): Wer ins aber verdingot hett, | So war es geschehen an stett, | Und tat in ains tags bas lingen | Denn si in drin möchtint ufbringen. HAUBER, UB Heiligkr. 2, 18426 (schwäb., 1443): dar um haben wir in der gütlichi zu lingingem end gesprochen, das [...]. - SснміDт, Hist. Wb. Elsaß 224; SCHweIz. ID. 3, 1338.

lingerung, lingig, lingsam, s. lingen 2.

linie, die; $-n /-n$.

1. 'gerade oder gleichmäßig (etwa als Kreis) gezogene Linie auf Papier, in Zeichnungen, auf Geräten u. ä.; Richtung; Richtschnur; Zeile (z. B. die Verszeile); Stufer. Phras.: die äquinoktialische linie. - Bdv.: kreis, richtmas, ris, streich, strich, stufe, zeile, zug. Ggs.: beuge 1. - Synt.: eine l. machen / ziehen / färben / teilen; [eine Anzahl] linien (Akk.) aufsteigen (auf der Sonnenuhr); von der l. rücken, sich über eine l. ziehen, an einer l. [wohin] stehen; linien der liebe; die durchgehende / enge / gerade / gleiche / gleichteilende / punktierte / rechte l., angänge einer linien. Wbg.: linienrecht.

Mendthal, Geom. Culm. 22, 11 (Hs. 'preuß., Anf. 15. Jh. $\left.{ }^{\top}\right)$ : so sal sy [Ducze mile] doch noch der gemynen mose haben yn dy lenge 180 seyl linienrycbte uscrumessen. LUTHER, WA 41, 127, 6 (1535): sein wort [...] gehet fein stracks bindurch und machet reine gerade linien on alle benge. QUINT, Eckharts Pred. 1, 273, 2 (E. 13./A. 14. Jh.): Her umbe ist daz aleine ein gereht mensche, der alliu geschaffeniu dinc vernibtet hât und an einer glìchen linien âne aller. ûlluogen in daz énige wort geribtet stât. JAHR, H. v. Mügeln 36 (omd., Hs. 1463): du [got] linge, zirkel, winkelmaß: | nach dir sich alles wesen mißt: $\mid$ ouch von dem zentrum fürt din list $\mid$ die lingen zu dem ummesweif. EBD. 1605: ich [tugent] bin ein ling und ouch ein maß | die rechtem werke gibet saß. $\mathrm{M}$. Cunitia. Ur. Prop. 167, 12 (Öls 1650): Sinus recti seind grade Linien inner dem quadranten. Rupprich, Dürer 2, 47, 32 (nobd., um 1500): so teill der engsten linien eine jn der mit von ein ander. Kurrelmeyer, Dt. Bibel 5, 436, 11 (Straßb. 1466): Willtu das der schatt aufsteig ${ }^{*} \chi^{*}$ linien [Luther 1545, 2. Kön. 20, 9: stuffen]. Plant u.a., Main. Naturl. 295r (a), 11 (ohalem., Hs. E. 14. Jh.): ez. ist nvwen ein angenge d' zite als ein püctel einer linien anegenge ist. MAaler 273v (Zürich 1561): Linien (die) Ein rissz etwar auf. [...] Gerade durchgende oder gleychteilende Linien. [...] Linile oder zileten. Versiculus. KOHLER, Ickelsamer. Gram. 48, 7 (wohl ${ }^{\circ}$ Augsb. 1. Dr. 16. Jh. ${ }^{\urcorner}$): ain frag zeichen, [...] ist auch nach der stym art vnnd gleichnus geformiert also $. P . d z$ ain lini oder virgula über sich schnipt, wie sich die stym in ainer frag am ende erhebt. Rот 325 (Augsb. 1571): Lini vom wort Linea, Strich / kreiß / riß / zug / zeil. Fischer, Eunuchus d. Terenz 113, 13 (Ulm 1486): Es sind fünff linien der liebi. sehen. reden. lieplich straichen. kíssen. - Bömer, Pilgerf. träum. Mönch 2385; JAhr, a. a. O. 398; Ries, Rechenb. A 2r, 21; A 3r, 17; A 5v, 17; Lippert, UB Lübben 2, Eltg. 55, 37; M. Cunitia. a. a. O. 153, 1a; Rupprich, a. a. O. 2, 412, 117; SACHS 15, 221, 10; Vogel, Pract. Alg. Ratisb. 160, 5; Voc. Ex quo L 308; Hulsius L iijv; Dict. Germ.-Gall.LAT. 308, 20. - Vgl. ferner s. v. achselböhe, ${ }^{1}$ anglich, atlantisch, ausbiegen, ${ }^{2}$ base.

2. \Schnur, Seil<; auch: `Schnur, mit der eine Grenze, Umfriedung gezogen wird/, damit: >Grenzeく. - Bdv.: vgl. ${ }^{1}$ band 6, bindfaden, corde, drat, ${ }^{1}$ Kabel, leine, ${ }^{1}$ litze, schnur, strik. - Wbg.: linienschnur >Meßschnur des Zimmermanns $<$.

Chron. Magdeb. 2, 18, 17 (nrddt., 1565/6): ein geukkler, flohe ufm marckt offentlich uf einer linien von Moritz Almans Hause kegen dem Roland über. Thiele, Minner. II, 12, 86 (Hs. ' 'ndalem./sfrk., 1470/90?): wie du dich sassen | solt mit dinen lynien snüren, | unnd kundest den pfiler massen, | das er stee fry vor fulen unnd vor dorren [bezogen auf die Grundlegung eines Balkenfundamentes]. HuLsius P ijr (Nürnb. 1596): Schnur / oder linien der Zimmerleut. - Rwв 8, 1339.

3. >genealogische Linie, Generationenfolge einer Sippe; Verwandtschaftsgrad (als Stufe einer Linie gedacht<; ütr. auch für >Krankheitsfolger gebraucht. - Phras.: die absteigende / aufsteigende linie. - Bdv.: freundschaft, geschlecht 10, sipschaft, ${ }^{1}$ stam 3; vgl. liedmag 1. - Synt.: die l. an jm. >mit jm. enden; einer limien sein; $j$ s. (z. B. des heilands) aus einer l. gewarten, in einer linien sein / stehen, die krankheit nach dreien linien entspringen, etw. von seiner l. ererben, von einer (z. B. absteigenden) l. geboren sein ; die l. des blutes, der magenschaft / sipschaft, der krankheiten; die dritte / vierte / 
gerade / rechte / vorfarende / nachkommende / talsteigende / weibliche / zwerche l.; das ende, der buchstabe, die kinder einer linien; erben in einer l. Luther, WA 20, 551, 30 (1526): Aus dieser linien musten sie des Heylands gewarten. Gerhard, Hist. alde e 1047 (omd., um 1340): Entsprozen van Eleasar|Sint dize phaffen alle gar, $\mid$ Di in dizer lineen sten. KöвLER, Ref. Wormbs 209, 14/15 (Worms 1499): Natürlich oder gesipt erben [...] sind dreyerley wyse zurechen nemlich in abstygèder linien. oder in vffstygender linien. oder in d'z. zwerch und neben syten oder linien. In abstygender Linien sind kinder / Enckel Vrenckel vin also für zurechen. DeRs. Ref. Franckenfort 38, 12 (Mainz 1509): wóllē wir / das vatter oder müter vnd andere von vffstygender Linien / so kinder in absteigender linien haben / und Testament machen wóllen / das sie die selben kindere [...] zu erben machen. Ders. Stattr. Fryburg 148, 25 (Basel 1520): das deß einen eegemechd kinder von irer linien richer / dañ die kinder von dem andern eegmechd [...] erfunden [werden]. SudHoff, Paracelsus 7, 446, 9 (1529): Nun aber ein ander ursprung von den platern ist also, das noch ein lingen der kerankheiten ist, die nit nach der ordnung der sperma, [...], get, als die lingen der drei ersten. wan die krankheiten alle entspringen nach dreien lingen, das ist aus dreien ursprungen. SPILleR, Füetrer. Bay. Chron. 157, 28 (moobd., 1478/81): Diser künig liess Conradium, an dem auch diese liny endet. - Steer / Vogel, Rechtssumme 8, 2006, 660; Rennefahrt, Zivilr. Bern 795, 10; КӧвLER, Ref. Wormbs 218, 13; DERs. Ref. Nürnberg 192, 18; Spiller, a a. O. 50, 14; 65, 5; Winter, Nöst. Weist. 1, 740, 28; Rwв 8, 1331. - Vgl. ferner s. v. absteigend, auftreiben 14 , partei 3 .

4. vereinzelt mit oder als gen. explicativus, z. B. der unvernünste linie >Unvernunft<. JAHr, H. v. Mügeln 56 (omd., Hs. 1463): löse, got, die sinne min | von strenger unvernünste lin. EBD. 956: die [blume] kury, die lank, die lenger was, | als sie Naturen linge maß. EвD. 2351: der zirkel nach der lingen seil | sich teilet in zwelf gliche teil.

linienrecht, s. linie 1.

linienschnur, s. linie 2.

linier, s. linieren.

linieren, liniieren (o. ä.), V.

\Linien, Streifen ziehen, liniieren ‘. Wbg.: lineament, linier >Lineal, Richtscheit< (dazu bdv.: lineal).

v. Tscharner, Md. Marco Polo 70, 19 (osächs., 2. H. 14. Jh.): si [Inder] han eyn kleyne houbt und di corperige gelynygirt wys und rot. LoOse, Tuchers Haushaltb. 115, 22 (nürnb., 1514): mee dem Wagner davon [papier] einz̨upintten und czu lingiern 5 Go. MaAler 165v (Zürich 1561): Pergament mit bley Geliniert. EвD. 273v: Linier (der) Ein recht scheytle. Norma, Libella, Amussis. Linieren.
Lineam ducere. - Rot 325 (Augsb. 1571): Lineament, strich oder kraiß / Jtem glidmaß des angesichts. [...]. Linir. Ein Büchbinderischer werckzeng / damit sie die Búcher linirn / das ist die langen strich zwischen den modlen machen.

\section{liniieren, s. linieren.}

linimentum, das; aus lat. linimentum `Schmiere`(GEORGES 2, 667).

\Schmiere, Salber. - Bdv.: vgl. defensiv, gesälbe, ${ }^{1}$ lauge 4 , salbe.

Broszinski, Minner. Chir. Parva 72v, 21 (halem., 2. H. 15. Jh.): Ein linimentum vnd ist für die scrobulis: Rp. cinerum serpentis ein vntz, mellis vnd acceti [...]. EBD. 81v: Cura der wunden, die apostem habent. [...]. Rp. granatorum der süssen, súds in süssem win, bis [...], mach daruss ein linimentum.

\section{link, Adj.}

1. >links, im menschlichen Orientierungsraum nach der Sehrichtung linker Hand «. - Phras.: zur linken hand >links, nach links «; 'sich zur rechten und linken lenken, weder zur rechten noch zur linken ausweichen $>$ nicht von etw. abweichen'?; mit dem linken bein aufstehen o. ä. - Bdv.: tenk, sinister; vgl. lenk, linster, lirk, lurz 1. Ggs.: recht. - Synt.: l. essen / schreiben; in got weder rechtes noch linkes sein; die böcke zur linken stellen, sich zur linken lenken; der linke arm / fus / schatten / zan, die linke hand (dicht belegt; mehrfach mit Konnotation des Unheilvollen, Bösen o. ä.; vgl. 2), das linke ange; subst.: die linke >die linke Hand «. Wbg.: linkheit, linkicht, linkisch 1, linkitus >Linkshänder` (latinisierend), linkseitig.

Luther. Hl. Schrifft. Mt. 25, 33 (Wittenb. 1545): vnd [des Menschen son] wird die Schafe zu seiner Rechten stellen / und die Böcke zur Lincken. PfefferL, Weigel. Ges. 18, 5 (Hamb. 1646): böse aber ist sie [Creatur] vnd ein baum des Todes, wen sie sich lencket zur rechten vnd lincken, mit annehmung eigens willens. KLETT, J. v. Soest 11, 1939 (Hs. rwmd., 1470/80?): lynyg recht geweltiglich | streich er t₹n also meisterlich. Voc. Ex QuO S 702 (15. Jh): Sinister [... lincke [...] linckeit. VOC. INC. TEUT. P iv (Speyer um 1483/4): Linck wl. tenck Sinister. Diefenbach, Mlat.hd.-böhm. Wb. 250: (o. O. 1470): Sinister linkesch. Allg. Schau-BüHne 5r, 26 (Frankf. 1699): fand ein Kunst Hebraisch zu schreiben von der Rechten zur linken Handt. KüтHer, UB Frauensee 369, 9 (thür., 1526): gehor alle das, was zur leincken handtt gelegen, den fursten von Sachsenn. Jungbluth, J. v. Saaz. Ackermann 16, 22 (Hs. ${ }^{\circ}$ omd., 1465?): Der selbe man furet ein hauen in seiner 
rechten hant und ein schaufel in seiner linken hant. $\mathrm{BECH}-$ stein, M. v. Beheim. Evang. Mt. 6, 3 (osächs., 1343): wan du gibes dine almûsen, dine linke [MeNTEL 1466: winster] hant insal nicht wizzen waz. dîn rechte hant tüt. Вӧнме, Morg.R. 14, 4 (Hs. 「schles., 1612`): ein solcher wird nirgend anders bin als zur lincken hand des Richters unter die stinckende bellische Bócke gestellet. KEHREIN, Kath. Gesangb. 1, 5, 3 (Nürnb. 1613): Gedenck wie du wirst Aufferstehn, | [...] | Zur rechten oder lincken Hand, | Heilig oder der Hóllen brandt. Otт-Voigtländer, Rezeptar 205r, 5 (Hs. ' nalem., um 14007): Wem die nase blutet: ist es rechtend=halb, so verbind jm die zehen an dem linggen / fü $\beta$. DAsypodius 210r (Straßb. 1536): Scaua, Ein lingiduß / lincker der die lingke hand für die rechte brauchet [bei MAALER $273 \mathrm{v}$ semantisch anders: Lingituß / der beid bẽd gleychfertig braucht]. PÄPKE, Marienl. Wernher 9981 (halem., v. 1382): Do wart zem erst sin lingú hant | Mit ainem nagel uf gespant. Plant u. a., Main. Naturl. 297r (b), 2 (ohalem., Hs. E. 14. Jh.): vf der lingen siten sol man dè slaf vollebringen. Sснмітт, Ordo rerum 729, 20 (salem., 2. Dr. 15. Jh.): Sinistrorsum [...] lingsitich. MaAler 273v (Zürich 1561): Man kan sich vor dem streich deß der Lingk. ist nit búten. BARACK, Zim. Chron. 1, 313, 11 (schwäb., M. 16. Jh.): liess im auch, so oft es [pferdt] mit dem linken fuss heraustratt, wider in den stal ziehen. EsCHENLOHER. Medicus 77, 10 (Augsb. 1678): daß [...] die Medici und Chirurgi den Verlurst deß lincken Augs besorgt. AndreaE. Ber. Nachtmal 26r, 21 ([Augsb.] 1557): Damit wir [...] nicht zu weyt von der Warhait auff die Rechten oder lingken seyten gehen. EBD. 61v, 21: dieweil in Got nichts leiblichs / und demnach weder rechtes noch linckes ist. BRÉVART, K. v. Megenberg. Sphaera 46, 16 (noobd., 1347/50): wundert euch, daz die linken schaten der welde nibt gend. - LUTHER, WA 47, 42, 31; Bechstein, a. a. O. 20, 21; Feudel, Evangelistar 21, 9; Rupprich, Dürer 2, 409, 36; VETter, Pred. Taulers 206, 35; Cirurgia H. Brunschwig 17ra, 15; 25va, 16; Haszler, Kiechels Reisen 308, 4; Bauer u. a., Kunstk. Rud. 98; Bremer, Voc. opt. 1165; Dict. Germ.-Gall.-Lat. 308, 37/39; BaumannZwirner, Augsb. Volksb. 1991, 117; Rwв 8, 1340 (hier bezüglich des Orientierungswertes bei Rechtsritualen wie dem angang 8, dem kummer behandelt und belegt). - Vgl. ferner s. v. aufdringen 4, 1 ausweichen 2.

2. dient der Kennzeichnung von Personen, Haltungen, Handlungen, Gegenständen, die von einer situationsspezifisch unterstellten idealen Qualität abweichen oder ihr entgegengesetzt sind; im einzelnen z. B.: > falsch (von einer Lehre; ütr. auch von menschlichen Haltungen) 〈; 〉(Gott) entgegengerichtet ‘; >verdächtig, unecht (von Haltungen) <; > hinterhältig $<$; >mißtrauisch $<$; >verräterisch (ütr. von Wunden)<; >böseく. Phras.: (nicht) link im backen sein >(nicht) schlagferig sein. - Bdv.: lez 2. - Wbg.: linkisch 2, linkwürmisch >verschnörkelt .

Kochendörffer, Tilo v. Kulm 945 (preuß., 1331): 'Keczczerye, du bist linc, | Und di bimelische dinc | Dyn vernunft nicht kan verstan. EвD. 3562 (preuß., 1331): Uns ouch sullen wesen link | Der werlde lob, ir prysen. GERHARD, Hist. alde e 5472 (omd., um 1340): di secte nam ursprinc | Ken gote allerdinge linc. StrehlKe, Nic. Jerosch. Chron. 13055 (preuß., um 1330/40): In vorevils turste | trettinde dî linkin pfat, | dî ettiswen sin vatir trat. EBD. 17450: daz. ein mechtig edelinc. | ein Sudounit, an witzin linc | Lûdewige dâ betrûbete. LuTHER, WA 1, 692, 10 (1518): wann die sichtliche und leibliche reich oder guter seind genent die linck. hand gotes. EBD. 10, 1, 2, 14, 18 (1522): ynn der scbrifft bedeutt die lincke seytte widderwertigkeytt [...]. Die rechte seytte bedeutt die gluckselickeytt. EBD. 205, 21 : Die lincke seytte ist der leypliche verstand. VOC. Ex QUO, S 702 (15. Jh.): Sinister [...] lincke [...] ling [...] dicitur malus, nociuus peruersus vel linckiß. Kopp, Volks- u. Gesellschaftsl. 93, 4 (Hs. 'pfälz., M. 16. Jh. $\left.{ }^{\urcorner}\right)$: er war nit schon empfangen, | man sach in gar linkh ahn. HüBNER, Buch Daniel 2936 (omd., Hs. 14./A. 15. Jh.): Meitliche zucht ist da linc| Gepruvet bi dem ballen [>Tanzen`. EBD. 5316: $Z u$ hant wart im da irkant $\mid$ [...] wie disse dinc $\mid$ Weren gemachet vil linc $\mid$ Wider sines Gotis e. Cirurgia H. Brunschwig 17va, 32 (Straßb. [1497]): è cleine plut rū $\beta$ ist do eine die but biß vff $d \bar{z}$ fleisch v'wüt ist vin on masen oder linck zeichen geheilt mag werdè. Lemmer, Brant. Narrensch. 69, 30 (Basel 1494): Er [nydischer man] spricht $\chi_{u} u$ dir / fründt ys $\beta$ / vnd trinck | Doch ist syn hertz an dir gantz linck. SCHORER, SprachVerd. 27, 2 (1643): mit linckwurmischen Zügen gezierte Striche vnd Buchstaben. Wyss, Luz. Ostersp. vor 9151 (Luzern 1545): Der linngg schacher ret zum Saluattor. KochendörfFEr, a. a. O. 1917; Luther, WA 16, 224, 31; SCHWEIz. ID. 3, 1340.

linkheit, linkicht, s. link 1.

linkisch $1 ; 2$, s. link 1; 2.

linkitus, linkseitig, s. link 1.

linkwürmisch, s. link 2.

lins, Adj., etymologisch zu leise (ohne Diphthongierung und nasaliert; zu letzterem: FRnHD. GR. \ L 62, 4).

>unauffällig; sanft, angemessen; behutsam; gesittet $<$. - Wobd.

Barack, Teufels Netz 8383 (Bodenseegeb., 1. H. 15. Jh.): Das besliessend die zwen [keller und koch] vil lins. LÖFfler, Columella/Österreicher 1, 155, 6 (schwäb., 1491): dar nach ain [...] wecklin zwuschen die rind und den stammen nit minder dann dry finger lin $\beta$ und senfft in lassen, das du die butt nit letzest. MAALER 273v (Zürich 1561): Lins / Gütig / Milt. Mitis. Mit Linsem vnd wolgesetrtem 
schritt gon / [...] Vast büpschlich vnnd Linß gon / Wie ein dieb gon. [...]. Linß / sittlich / Nider. [...]. Linß und nider reden. [...] Mit niderer stiz̃ reden.

linse, die; $-\emptyset$ oder $-n /-n$.

1. \Linse (als Frucht)<; vereinzelt auch als Pflanze; mehrfach zur Kennzeichnung der Nichtigkeit e. S. gebraucht. - Phras.: nicht einer linsen wert sein; etw. um ein linsenmus verkaufen o. ä.; lieber mit got fisch als mit Esau linsen essen. - Synt.: linsen klein stossen / essen / verkaufen; linsen zu dem kleinen zehent gehören, kleiner dan keine l., leichter zu lassen sein als eine l.; ausser linsen mel machen, jm. an linsen schaden zufügen; der mut / scheffel, das luder linsen; die speise der l. Wbg.: linsebolle (16. Jh.; Gw zu bolle 2), linsenacker, linsengericht, linsenbülse, linsenkorn, linsenmäuchel (a. 1646; Gw zu mhd. mouch eine Art Brot; LEXER 1, 2210), linsenmus, linsensame, linsenwal.

Luther, WA 45, 389, 22 (1537): daß er [Esaw] dyse guter nicht helt, Sondern gering, verachts, verkeuffts umb ein Rot linßen muß. Quint, Eckharts Pred. 2, 305, 11 (E. 13./A. 14. Jh.): dem menschen waren alliu dinc als lîhte ze lâzenne als ein erweiz oder ein linse oder als nibt. BRINKMANN, Bad. Weist. 206, 11 (rhfrk., 1603): Zu jezgemeltem kleinen zehenden gehört rüben und kraut, obs, linsen und erbsen, sommergerst, beidenkorn, hirsen, flachs, lemmer. Österley, Kirchhof. Wendunmuth 1, 248, 27 (Frankf. 1563): Es mögen wol linsen heissen und haben den nammen mit irer wirckung, antwort der Baier, denn sie sind jo so leins von mir geschlichen, daß ich es im schlaff nit bin innen worden [hier Anspielung auf den Bauchwind]. GERHARD, Hist. alde e 1036 (omd., um 1340): Di erstekeit siner geburt | Er verkoufte uberal| um ein lutzel linsenwal [Hs.: -val; Konjektur zu -wal; s. Lexer 3, 646; möglich wäre auch -mal >Gericht]. THür. CHRON. 42v, 9 (Mühlh. 1599): der hatte eine weisse Tauben / welche er gewehnet / das sie ihm Korn vnnd Linsen auß den Obren laß. Gille u. a., M. Beheim 187, 91 (nobd., 2. H. 15. Jh.): Sand Augustinus sprichet: ,ich | ess lieber visch mit got messlich, I dann mit dem Esau linse'. Hirschmann, RogerGlosse 225 vb, 5 (omd., Hs. 15. Jh.): Ploster: / Nj̈m fenum grecom. linsen somen, / dÿ wurczeln von wilden papeln. Chron. Strassb. 254, 3 (els., A. 15. Jh.): Hienoch geschach, das Jacop bette ein lynsin mis gekochet. KurreLMEYer, Dt. Bibel 3, 123, 21 (Straßb. 1466): Vnd verkauft die ersten geburt: | vnd alsust enpfieng er [jacob] das prot und die speys der linsen [Luther 1545, 1. Mose 25, 34: Linsengericht]. Dasypodius 336 (Straßb. 1536): Folliculus lentium, Lynsen bülße. SCHNyder, Qu. Zürcher Wirtsch. 649, 13 (halem., undat.): ungelt ₹ü den toren $u \beta$. Item linsi, erws. bonen, birs, gersten und kernen, von ye dem mütt iiij denar. Dict. Germ.-Gall.-Lat. 308, 23 (Genf 1636): Linsenacker [...] Ager lenticularis. EBD. 25: Lensenmuß / Linsensupp [...] lus lenticulare. Barack, Zim. Chron. 3, 70, 4 (schwäb., M. 16. Jh.): „Ach des volien böswichts pfaffen! er sit doch nit ainer linsen wert". BRÉVART, K. v. Megenberg. Sphaera 12, 26 (noobd., 1347/50): Wanne e die natur eitel lid, e prech ain erein hafen von geprechen ains linsenkornes. Munz, Füetrer. Persibein 198, 7 (moobd., 1478/84): ergreiff ich dich zw meiner henndt, ich zerprich ich clainer dann ye ward kain linsen. BRETHOLZ, Liechtenst. Herrsch. 262, 30 (smähr. inseldt., 1414): babent mein herrn daselbs gancz traidczehent [...]; ze veld von traid, waic₹, chörn, gersten, habern, hanif, lins, chrawt, etc.; ze darff huner. - Ziesemer, Gr. Ämterb. 460, 9; ОттVoigtländer, Rezeptar 209r, 17; Rohland, Schäden 467; Schnyder, a. a. O. 928, 35; Leisi, Thurg. UB 5, 272, 18; 6, 487, 27; Gehring, Würt. Ländl. Rechtsqu. 3, 50, 24; Deinhardt, Ross Artzney 210; Bremer, Voc. opt. 13142; MaAler 273v; Pfälz. WB. 4, 1000; SCHWEIZ. ID. 4, 1173.

2. >Entenflott, kleine Wasserlinse .

Hirschmann, Roger-Glosse 214ra, 1 (omd., Hs. 15. Jh.): tranck: / Nj̈m [...] dÿ linsin dÿ off dem wasser swimmen, vnde wegebreit.

linsenacker, linsengericht, linsenhülse, linsenkorn, linsenmäuchel, linsenmus, linsensame, linsenwal, s. linse 1.

linster, Adj.; Schreibform zu link (mit Konsonantenerleichterung).

$>$ links`, die Seite kennzeichnend, die für Verdammung steht. - Bdv.: vgl. link 1.

Helm, H. v. Hesler. Apok. 10893 (nrddt., 14. Jh.): Her entzuge sich zu der linstern | Hende kegen der vinstern | Helle. EвD. 21988: Daz sie luchten in dem vinstern |Zur zeswen und zur linstern | Hende. - EBD. 7188.

lintwurm, der, $-/-e$, auch $-\emptyset$ (+Uml.).

ein drachenartig gedachtes, mit dem crokodil, der schosschlange und anderen großen, als gefährliche Ungeheuer geachteten Tieren (auch Vögeln) in eine Reihe gestelltes Wesen. - Bdv.: ${ }^{2}$ aspe, basilisk, kröte, natter, schlange, trache, unke, wurm. - Synt.: der l. umsichtig sein, menschen fressen.

Voc. Ex QUO I 10 (15. Jh.): Laculus est serpens, eyn lintworm. - est quidam serpens volatilis. DiefenBACH, Mlat. hd.-böhm. Wb. 5 (о. O. um 1570): Boas, boa est magnus draco [...] lyntworm. EвD. 147: Iaculus ein lynt wurm. LUTHER, WA 19, 604, 32 (1526): Hie hat der Lawe eine maus gefangen und lest sich duencken, er habe den Lindwurm uberwunden. ЕвD. 27, 386, 25 (1528): also hat man uns Sanct Georgen mit dem Lindwurm auch fürgemablet. PEIL, 
Rollenhagen. Froschm. 221, 5445 (Magdeb. 1608): Als er [Hertrog] mit seinem schwert vnd hand IJhm [Lewe] den lindwurm halff vberwinnen. Gille u.a., M. Beheim 451, 282 (nobd., 2. H. 15. Jh.): sie haben aller würm gestalt | greiffen, traken, lint wurme | und sust von allen ungeczivern, I da haben sie zu allen zeiten ain gesturme gar ungeheür. BREMER, Voc. opt. 46029 (wobd., 1. V. 15. Jh.f.): Cocodrillus [...] lindwrm [...] nix [...] haim [...] grill. Sudhoff, Paracelsus 14, 504, 40 (um 1570): die aller grausamsten und erschröcklichsten würm, als nemlich die schoßsschlang, [...], auch die lintwürm und crocodili, so die toten menschen auch gar fressen. - Gille u. a. a. a. O. 301, 61; 354, 47; SACHS 23, 53, 4; Sudhoff, a. a. O. 12, 524, 6; Bremer, a. a. O. 46012; Sснмітт, Ordo rerum 303, 24, 2; 328, 17; Voc. INC. TEUT. p iv. - Vgl. ferner s. v. begreifen 4.

\section{lintwürmlin, das.}

einem Vogelei entschlüpftes kleines, möglicherweis dem lintwurm ähnlich gedachtes, auch als Schmuckstück nachgebildetes Tier; gegenüber lintwurm lexikalisiertes Diminutivum; zur assoziativen Verknüpfung beider Ausdrücke vgl. gril und heim s. v. lintwurm (Beleg BREMER).

Gereke, Seifrits Alex. 523 (oobd., Hs. 1466): do cham aus der schal gechrochen | ain chlaines lindwurmelein; | ein chran was auf dem haubt sein. - Matzel u. a., Spmal. dt. Wortschatz. 1989, 193.

linze, die? - In den Wörterbüchern nicht belegt; aus mlat. linca >linx femininar (Du CANge 5, 144c); möglicherweise deformierte Schreibung von ${ }^{1}$ lun₹e.

>weiblicher Luchs`; ütr. (semantisch kaum motivierbar): > faule Frau<.

Österley, Kirchhof. Wendunmuth 1, 139, 38 (Frankf. 1602): antwortet die jung und faule lintze, er [doctor] solt den teuffel also artzneyen. Jungbluth, J. v. Saaz. Ackermann 24, 21 (Hs. 「omd., 1465ㄱ): So schönes mensche gesahestu nie, und bestu eines linzen augen [...], dir würde darüber grauen [Var.: ain lintz̃ñ augñ, des tieres lincetten (linteschen, linggen) augen; offensichtlich auch in frnhd. Zeit schwer zuzuordnen].

\section{lipf, s. lupfen 1.}

lipfel, das; Schreibform von leibbefil(d)e (s. d.).

$>$ Leichenbegängnis, Beerdigung $<$ - Bdv.: vgl. beerdigung, begängnis 3, begräbde, begräbnis 1, bestattung 1, leibfal 1, ${ }^{1}$ leiche 2 , leichenbegängnis, todfal.
Bolte, Pauli. Schimpf u. Ernst 1, 121, 29 (Straßb. 1522): und werffen mich in die Tiber und begon mir kein Lüpffel. EBD. 273, 11: da der Vatter gestarb, und nach dem Lipfel nam der Sun den Sack mit dem Gelt.

lipläp, s. lippen.

${ }^{1}$ lippe, die; $-/-n$; nahezu ausschließlich als pl. tantum belegt.

>Lippes. - Nrddt. / md. / nobd.; gehäuft poetische Texte. - Bdv.: lefze 1; vgl. befze, glef. - Synt.: die lippen anschauen/ behüten / entschliessen / auftun / regen / spalten, mit dem finger rüren; die lippen (Subj.) leuchten, beschlossen / ein türschlos sein, etw. reden; etw. (Subj., z. B. der türkis) für (১im Vergleich zu<) den lippen verbleichen, jn. mit den lippen anbeten / eren, sich mit den lippen zu etw. nähern, mit den lippen etw. berüren, unter den lippen vergiftnis tragen; des mundes lippen (explikativ); die röte der lippen; kancer an den lippen.

INGEN, Zesen. Ros. 73, 23 (Hamb. 1646): sie machte meine ráchte wunde viel túfer / als sie mit ibren lippen die meinigen berurete. Strehlke, Nic. Jerosch. Chron. 309 (preuß., um 1330/40): dávon ouch umbesnittin |nâch hovellichin sittin $\mid$ mînes mundis lippen sîn. LuTHER, WA 1, 218, 7 (1517): O gott, erloße meine seel von den triglichen lippen (hier tropisch), das ist, falschen leeren. Ders. $\mathrm{Hl}$. Schrifft. Ps. 34, 14 (Wittenb. 1545): Bebüte deine Zunge fur bösem / Vnd deine Lippen [MENTEL 1466: lespen; 1483-1490: lebßen; Mentel 1475¹ / Dietenberger 1534 / Еск 1537: leftren] / das sie nicht falsch reden. Fischer, Brun v. Schoneb. (md., Hs. um 1400): undir iren lippen [...] | tragen si bose vergiftenisse. ЕвD. 2948: di lippen sint [...] | ein ture sloz vor di zene. | di wil di lippen beslozzen sint, | so enkumpt dar zu noch wort noch wint. HüBNER, Buch Daniel 6679 (omd., Hs. 14./A. 15. Jh.): dar nach her zu mir neic | Eines glich des menschen kint $\mid$ Rurnde mines mundis spint, | Entsliezende die lippen. Bechstein, M. v. Beheim. Evang. Mt. 15, 8 (osächs., 1343): Diz volc êret mich mit den lippen [Mentel 1470: lespen; Froschauer 1530: lafftzen], abir ir herze ist verre von mir. OpIтz. Poeterey 41, 31 (Breslau 1624): für den Lippen muß ein Turrkiß auch verbleichen. IngEN, Zesen. Ged. 384, 16 (Breslau 1641): Es ist auch wol schóne und anmutbig die Rothe der Lippen. Bell, G. Hager 140, 3, 14 (nobd., 1595): thu auf die lüpen mein, | Das mein mund ver künd fein / allein | Deinen Rum. - Luther, WA 9, 127,$7 ; 41,183,20 ; 46,164,20$; INGEN, a. a. O. 72, 34; Ders., Zesen. Ged. 385, 13, Dünnhaupt, Werder. Gottfr. v. Bullj. 24, 2; Fischer, a. a. O. 244; StedtFELD, Roger-Glosse, S. 86; SCHÖPPER 35a.

$$
\begin{aligned}
& { }^{2} \text { lippe, s. }{ }^{2} \text { lüppe. } \\
& \text { lippel, s. }{ }^{1} \text { lippen. }
\end{aligned}
$$


${ }^{1}$ lippen, V.; zu ${ }^{1}$ lippe.

>schwatzen, wirres, dummes, törichtes Zeug von sich geben`. - Bdv.: ergillen; vgl. abkiefeln 2, blempern, schwatzen, tattern. Wbg.: lipläp, lippel, lippenlap, lippern >aufmukken, bellen (vom Hund) ; ütr.: >lecken, züngeln (von Flammen)

Luther. Hl. Schrifft. 2. Mose 11, 7 (Wittenb. 1535): bey allen kindern Jsrael sol nicht ein hund mit seiner zungen lippern [EBD. 1545: mucken; Mentel 1466: ergillen). DERs., WA 41, 251, 8 (1535): Zum munde er aus bat die flammen gelippert, quia in sacra scriptura dicuntur flamma, Ignis lingua, Omnes se mutuo inspexerunt, das inen ex ore lippern feurige zungen. Pyritz, Minneburg 559 (nobd., Hs. um 1400): So betuten auch die bunde | Brufer, claffer, die uz irem munde| Manig rede lippen lappen. FASTNACHTSP. 259, 11 (nürnb., v. 1486) Du Geigenkloß und App und Tapp | Und du Narrentotschz und her Lippenlapp, | Her Schlauraff. EвD. 586, 1 (15. Jh.): Es gehört keinem solchen lüllaffen an, | Der weder lippen noch laffen kan. EBD. 587: Du kanst vor frauen weder lippen noch sagen. Lemmer, Brant. Narrensch. 64, 21 (Basel 1494): Wann jnn [fraww] sunst wol ist mit geschwátz. Vnd lyplep / schnádern / tag vnd nacht. KLEIN, Oswald 27, 3 (oobd., 1420/ 1): Ich hab gehört [... $\mid$ mit ainem sprichwort dick ain toren triegen: | simm, Lippel wër ain güte ganns. EвD. 70, 19 (v. 1408?): Pfeiff auff, Hainzel, Lippel, snäggel! | frisch, frow, fri!

\section{${ }^{2}$ lippen V., zu ${ }^{2} l u ̈ p p e$.}

>jn. kastrieren<. - Bdv.: vgl. luppen 4.

Voc. Ex quo, C 226 (15. Jh.): Castrare geylen, [...] lippen, .i. testiculos ab aliquo prinare.

\section{lippenlap, lippern, s. lippen.}

\section{liquidation, die.}

>Vollzug, Ausführung, Abwicklung eines Rechtsanspruchs`. - Wbg.: liquidierung (a. 1501).

Hilliger, Urb. St. Pantaleon 534, 7 (rib., 1655): hat gemeltes capitulum dem heren licentiato Fabens 4 jahr seines als dahmals syndici restierendes gehalts [...] bis zur liquidation gemelter forderung eingehalten. LAUFs, Reichskammergo. 221, 6 (Mainz 1555): sachen der liquidation, execution und volnziebung der ortheyl. - Rwв 8, 1341.

liquidierung, s. liquidation.

liquiricie, die; zur etymologischen Einordnung s. KLuge/S., Et. Wb. 2002, 554 s. v. lakritze.

>Süßholz; süßholzhaltige Speise ‘.

J. W. von Cube. Hortus 120, 21 (Mainz 1485): thu die kyrsen $v \beta$ und syede eynen dranck [...] mit syropel von rosen von upoln vnd von sußholtz, genannt liquiricia. MENGE, Laufenb. Reg. 4697 (Hs. ' nalem., um 1470`): Das man sin zunge mit saltze berybe $|[\ldots]|$ Vnd ein wenig liquiricye. Bremer, Voc. opt. 49042 (alem., 1. H. 15. Jh.): Liquiricia $[$... $]$ süßholtr.

lirk, Adj.; zu mhd. lërc, lirc, lurc (LeXer 1, 1883).

>links, linksseitig‘; dient mehrfach der abwertenden Charakterisierung des Falschen, nicht Idealen. - Bdv.: vgl. link 1; 2. - Ggs. : recht.

Stackmann u.a., Frauenlob 5, 34, 11 (Hs. rmd. auf nd. Grundl., M. 14. Jh. $\left.{ }^{\urcorner}\right)$: sar dem sarewirken | zimt eben, der knecht zu dienste pflegen, beidenthalb der lirken (zum Verständnis s. den Apparat, Bd. 2, S. 752). LAUCHERT, Merswin 5, 11 (els., 1352/70): so sol aber mine lirke hant mine krancke böse nature bezeichen. wenne sie ist den lirken vngerehten weg lange zit irrende gangen. Williams u. a., Els. Leg. Aurea 728, 29 (els., 1362): Do erschein ime nebent sinre lirken siten eine swarze schette. Chron. STRAssb. 391, 24 (els., A. 15. Jh.): [Cosdroe] satte ₹ü der rehten hant das crüze und zu der lirken siten einen han. - Williams u. a., a. a. O. 452, 13; 763, 27; SснміDт, Hist. Wb. Elsaß 225.

\section{lirken, V.}

$>$ stottern<. - Bdv.: vgl. anstossen 8, lallen 2, lurken, stackeln, stottern.

Stackmann u. a., Frauenlob 5, 13, 12 (Hs. rnobd., 3. V. 15. Jh. $\left.{ }^{\urcorner}\right): e z[l o b]$ böbet, lenget, breitet sich, sin nennen niendert lirket.

lis, Genus nicht belegt; lat. Flexion; aus lat. lìs > gerichtliche Streitigkeit` (GEORGEs 2, 674).

$>$ Rechtsstreit, zweiseitiger, durch Klagbitte und Gegenbitte als Verfahrensgrundlage gebildeter Rechtsakt «. - Wbg.: litigant (a. 1548), litigieren > einen Rechtshandel führen<, litisconsorte >Streitgenosse im Prozess< (a. 1543), litisdenun₹iation >Streitverkündung (a. 1622), litiskontestation (a. $1521 \mathrm{f}$.).

LAufs, Reichskammergo. 223, 24 (Mainz 1555): Da aber eynem procurator litem zu contestiren, zu concludiren oder etwas anders [...] zu handlen mit urtheil ufferlegt, der sol solchs [...] zuthun schüldig sein. KuRz, Waldis. Esopus 4, 1, 61 (Frankf. 1557): Den Curtisanen wol bekant, | Die vmb Prebenden litigirn, $\mid Z u$ Roma in Rota Agirn. KöBler, Ref. Nürnberg 100, 13 (Nürnb. 1484): vnd [wo] der spruch verneynt oder veriawort. vnnd dardurch also lis contestiert wer worden. EBD. 100, 26: wo aber der Rechtlich krieg angefengt. vñ obbegriffen meynũg lis bestetigt [...] wer worden. - LAUFs, a. а. O. 251, 4; Rwв 8, 1345. 
liske, die. - Zur Einordnung: Toeppen, Altpreuß. Mon. IV, 511; Trautmann, Altpreuß. Sprachdenkm. 371.

ein Wirtschaftsgebäude im Ordensbereich. - Wbg.: liskenmole.

Thielen, Gr. Zinsb. Dt. Ord. 4, 19 (preuß., 1414/22): Thusund buben, dy czinsen mit mólen, kecremen und lisken $802 \mathrm{~m}$. Ziesemer, Gr. Ämterb. 222, 30 (preuß., 1422): 2 [gerethe] czu der huwsmolen, 1 czu der lyskenmole. - THIELEN, a. a. O. 73, 22.

liskenmole, s. liske.

lismen, V.; zu mhd. lismen >stricken<, nhd. laut KLuge/S., Et. Wb. 2002, 578 ,offenbar zu lesen“. - Zur Wortgeographie von ,stricken' in den rezenten Mundarten s. DwA 19, 11.

>stricken «. - Bdv.: vgl. stricken. - Synt.: bändschube, einen rok l.; der gelismete rok / strumpf.

Barack, Teufels Netz 1250, Var. B (Bodenseegeb., 1. H. 15. Jh.): Hendschuoch glisman [Var.: lisenen] und siden spinnen | Land sich ouch nit ungern minnen. Päpke, Marienl. Wernher 5507 (halem., v. 1382): Maria machte dem kindelin | Gar listeklichen ain róklin, [...] Ich wan es gelisemet ware. - Schmidt, Hist. Wb. Elsaß 225; Schweiz. ID. 3, 1424 (keine Angabe zur Etymologie).

lisnen, V.; etymologische Zuordnung unklar.

>lispeln<. - Schmidt, Hist. Wb. Elsaß 225 (a. 1517).

lispeln, lispelung, s. lispen.

lispen, $\mathrm{V}$.

>lispeln; stammeln«. - Bdv.: s. u. ScHÖpPER. - Wbg.: lispeln, lispelung, lispler.

SCHÖPper 366 (Dortm. 1550): Balbutire. Lallen schwapeln stamlen statzgen statzen lureken lurgen 9 lispeln lischben mit der zungen stossen. LutHer, WA 16, 23, 26 (1524): Moses habe [...] in die Kolen gebissen, daber hab Moses hernach gelispelt. ЕвD. 49, 100, 36 (1537): [die hohe Maiestet] schewet sich nicht mit jnen [den armen, ...] kindlicher weise zu lispeln und zu lallen. EBD. 49, 282, 33 (1543): wenn Er [Engel] also mit uns reden mocht, wurden wir todt bleybenn. Darumb lest Er sich herab und lisbelt mit unser sprach. Diefenbach, Mlat.-hd.-böhm. Wb. 52 (o. O. um 1470): Blesus lispinder. Cirurgia H. Brunschwig 15ra, 29 (Straßb. [1497]): werdē im aber die fordern zen [...] v/ gehowe daz ist das er lispen würt das ist ein grose wüd. KuRz, Murner. Luth. Narr 2809 (Straßb. 1522): Sie hören hinder den offen wißblen, | Was zwen gickenbeintzen lißblen. V.
Anshelm. Berner Chron. 4, 340, 19 (halem., n. 1529): ward understanden, im sine zungen abzehowen, so wit, dass er [...] lang ungeredt bleib, biss er [...] wider zi lischbender red kam. Dict. Germ.-Gall.-Lat. 308, 30 (Genf 1636): Lispelung [...] Balbutitio. Henisch 187/8 (Augsb. 1616): barbari, sind im anfang genant worden / diejenige so nicht wol haben reden kóñen / als die statzger / lispler / stamler. Pfeiffer, K. v. Megenberg. B. d. Nat. 15, 1 (oobd., 1349/50): als geschibt an den kinden, die in ir kinthait zärtlent, die lispent gern wenn si gewachsent. Rотн, E. v. Wildenberg 69, 2 (moobd., v. 1493): der liess ainen sone, was genant Balbus, der listbet. - Voc. Ex QUO, B 169; Voc. INC. TEUT. p iv; VOLKMAR 64.

lispler, s. lispen.

lisse, Genus und etymologische Zuordnung?

>Fischlaich $\prec$ - SchmidT, Hist. Wb. Elsaß 225 (a. 1449).

list, der; -(e)s/-e, vereinzelt die.

1. >gestaltende Fähigkeit, Fertigkeit, Geschicklichkeit vor allem in den Handwerken (Schmiedehandwerk, Weberei, Baukunst), auch in der Kriegskunst, Rhetorik und Alchemie<; oft mit Bezug auf religiöse Glaubensinhalte, dann: >Schöpfungsgeheimnis, -kraft Gottes «. - Älteres und mittleres Frnhd.; Verstexte der Sinnwelt ,Religion / Didaxe'. - Bdv.: kunst; vgl. arbeit 8, behendigkeit 2. - Synt.: l. schenken, einen l. betrachten / finden / suchen, liste können, jm. einen l. geben; mit listen fechten, etw. mit list(en) bauen / machen / malen / überklügen / weben / wirken, von der $l$. des beiligen geistes empfangen werden, mit l. geworcht sein, auf den l. lausen; des beiligen geistes / schmiedes / friedes, der naturen l.; der gütliche / kluge / reiche / spähe l.

Mone, Adt. Schausp. 1, 96 (Hs. 'omd., 13917): Jhesu Crist, $\mid$ der von dez, heilgen geistes list $\mid$ ufferden by ${ }^{\mathrm{e}}$ onphangen wart. JAHR, H. v. Mügeln 1436 (omd., Hs. 1463): ich wil beslijen nu, $\mid$ das tugnde flu $\beta$ von gotte ist $\mid$ und nicht $u \beta$ der Naturen list. v. Groote, Muskatblut 16, 37 (nobd., 1. H. 15. Jh.): Maria meit gotlicher list $\mid z \ddot{u}$ dir han ich den glauben | daz du docbter vnd muter bist. STACKMANN u. a., Frauenlob 3, 8 (Hs. ' nobd., 3. V. 15. Jh. ${ }^{7}$ ): In ir schoz. bant sie [zwar] die bimel alle, | siben planeten dienten ir mit schalle, | er barg sich schone unter ir brust, $\mid$ daz, was des alten listes. Bihlmeyer, Seuse 552, 2 (alem., 14. Jh.): din [du, geminter] munt, der da waz ein schül aller tugent und künsten, uss dem er schankte alle kunst und liste. PÄPKE, Marienl. Wernher 969 (halem., v. 1382): Ir [Maria] ober brawen, 
sere kling, | Als sú mit listen warind gar $\mid$ Gemalet an ain bilde dar. Martin, H. v. Sachsenh. Tempel 314 (schwäb., 1453): Als ers zuosamen fuegt, | Mitt listen überkluegt, | In hohen künsten fry. EBD. 635: Als dort der tempel wart | Gebüwen hoch und zart, | Mit lysten manigvald. PRIMISSER, Suchenwirt 24, 119 (oobd., 2. H. 14. Jh.): Dar ob mit reichen listen $\mid$ Ein token chlar von perlein vein. Spechtler, Mönch v. Salzb. 39, 55 (oobd., 3. Dr. 14. Jh.): sunder istikleicher christ | mit seinem leichnam pure | lauset ganz auf spahen list | in kurzer klausure. WEBER, Füetrer. Poyt. 261, 6 (moobd., 1478/84): Poytislier alls mit lissten vacht, | kunnd im aus seinen schlegen schwär wol sprinngen. - Helm, H. v. Hesler. Apok. 9219; Fischer, Brun v. Schoneb. 2739; Мельвоом, Pilgerf. träum. Mönch 6383; Meisen, Wierstr. Hist. Nuys 2238; Jahr, a. a. O. 1994 Mone, a. a. O. 1, 2270; LindQvist, K. v. Helmsd. 1897; KLeIN, Oswald 12, 44. - Vgl. ferner s. v. alchimist, ametist, antwerk 1, balsam 2, illuminist.

2. >Weisheit Gottes; Erkenntnis, kluge Einsicht, Aufmerksamkeit des Menschenґ. - Älteres und mittleres Frnhd.; Texte der Sinnwelt ,Religion / Didaxe“. - Bdv.: sin, vernunft, weisheit. - Synt.: die l. verbergen, jm. l. verleihen, seine l. an got keren; der l. (Subj.) etw. bekennen / erdenken; dem l. etw. verborgen sein; der l. von >über got; des menschen / herzen l.; der geistliche / bohe / irdische l. - Wbg.: listenreich.

Helm, H. v. Hesler. Apok. 4535 (nrddt., 14. Jh.): Dar bi kere dine list $\mid$ An den Got der immer ist. EBD. 5288: Got in des wil gunnen $\mid$ Daz sie von geistlicher list $\mid$ Wizzen mugen waz, Got ist. Thiele, Minner. II, 24, 1 (Hs. ' $\mathrm{md}$./ rhein., 1. V. 15. Jh. $\left.{ }^{7}\right)$ : So bo of ouch soe listen rych $\mid$ de winde in heyl dier gelych. Mone, Adt. Schausp. 1, 710 (Hs. 'omd., 1391ำ): der suße (vater) Jhesu Crist, | daz her uns mit siner list $\mid$ behutet (uns) vor der ewigen not. PYRITZ, Minneburg 4890 (nobd., Hs. um 1400): Daz er [Got] verrer schoner ist, | Dann kan derdenken keins menschen list. Gille u. a., M. Beheim 76, 2 (nobd., 2. H. 15. Jh.): Du parmbercziger Jesu Crist, | verleich mir weishait, syn und list. Koller, Ref. Siegmunds 59, 35 (Hs. ' ${ }^{\circ}$ Basel, 1440? ${ }^{\urcorner}$): das du [vatter] die list an geistlichen boubtern verborgen hast [...], und hast es geoffenet den cleinen. BretT-Evans, Bonaventuras Leg. S. Francisci 57, 65, 33 (önalem., v. 1478): das er nùt dar hette gesetzet noch sin selbes listen, wen das er die [Ordensregel] alle hette gedin schriben, als jm von gott was erofnet. ÖsterLey, Steinhöwels Äsop 358 (Ulm 1474/82): List. Was der mensch nit mit krefften überkomen mag, das tuo er listig mit syner vernunfft. - HeLm, a. a. O. 1711; JAhr, H. v. Mügeln 14; 841; PYrITZ, a. a. O. 4822; KLeIN, Oswald 1, 5.

3. >Gewitztheit, Einfallsreichtum als Haltung; einzelner gewitzter Einfall, Plan, geschickte Maßnahme zur Bewältigung ei- ner Aufgabe, zur Ausführung eines Planes; List, Trick, witzige Maßnahme zum Teil moralisch bedenklicher Art zur Erreichung eines Zieles; gewitzte Einzeltat (bezogen auf Bereiche wie Erotik, Possen, militärische Zwänge u.a.)<; einerseits an 1, andererseits an 4 anschließbar; im Unterschied zu 1 und 2 keine religiösen Konnotationen. - Phras.: listles mit jm. spielen >jn. austricksen<; list gegen list brauchen; ane / sonder list (Verwahrformel). - Bdv.: anschlag 4; 5, grif 3, kunst, posse 2, rank, schelmenstük; vgl. beberzenheit 2, herzhaftigkeit 2. - Synt.: eine(n) l. erdenken / finden / können / wissen; j. der liste vol sein; l. (Subj.) kraft, kein ende haben, nicht verlangen, nicht zu etw. helfen; sich eines listes besinnen / brauchen; sich mit listen lösen / weren, mit listen nach etw. ringen, jm. mit listen einen possen reissen, die stat mit listen gewinnen, etw. mit l. erforschen, jn. mit listen [wohin] bringen, etw. aus l. ersinnen, jm. durch l. das leben fristen; der frauen / weiber (mehrfach) / menschen, des weines l. ।Wirkung ‘; der geschwinde / grosse / kluge / neue / spähe l. Wbg.: listweise >schlau (um 1476).

Holland, H. J. v. Braunschw. V. e. Weibe 285, 9 (Wolfenb. 1593): So wisset jhr doch selber wol, das der Weiber list kein ende habe. MiedER, Lehmann. Flor. 481, 4 (Lübeck 1639): List vnd Gluck haben grosse Krafft. FiscHer, Brun v. Schoneb. 435 (md., Hs. um 1400): noch schreib ein meister sundir list: | [...]. v. Tscharner, Md. Marco Polo 42, 6 (osächs., 2. H. 14. Jh.): do was keyn der Tartirn der do kunde vindin di list wy man di stat gewunne. SACHS 17, 13, 8 (Nürnb. 1552): Sie wird deß listles mit ihm [juncker] spielen. Dasypodius 3730 (Straßb. 1536): List gegen list brauchen. Vulpinari cũ uulpe. UkenA, St. Jörg 138 (oschwäb./tir., 1486/n. 1520): Wir miessen erdencken ainen list $\mid$ Das wir fristen vnser leben. SAPPLER, H. Kaufringer 7, 395 (schwäb., Hs. 1464): Mit der red ich das nun preis, $\mid$ das die frawen sind so weis $\mid$ und der cluogen list als vol. Pfeiffer, K. v. Megenberg. B. d. Nat. 248, 5 (oobd., 1349/50): daz man in [walvisch] mit kains menschen künsten noch listen gevâhen mag. KLEIN, Oswald 117, 46 (oobd., n. 1438): Also bört ir des weines list, | daran ich nicht vil loben mag, wie gut er ist. SPILLER, Füetrer. Bay. Chron. 116, 18 (moobd., 1478/81): striten sy paid sunder waffen nur mit stainen, wann Rueland muest sich nur mit listen und wencken weren. FICHTNER, Füetrer. Trojanerkr.

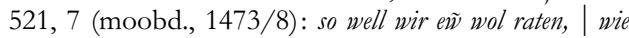
ir Troya dy statt gewinnet mit listen. - QuiNT, Eckharts Pred. 2, 555, 2; Lichtenstein, Lindener. Rastb. 7; Opitz. Poeterey 42, 26; Goedeke, P. Gengenb. 128, 
421; Sachs 17, 101, 21; v. Keller, Ayrer. Dramen 1825, 4; Karnein, Salm. u. Morolf 241, 3; Bihlmeyer, Seuse 137, 8; Moscherosch. Ges. Phil. v. Sittew. 16, 21; Adomatis u. a., J. Murer. Bab. 205; Barack, Zim. Chron. 4, 230, 10; Weber, Füetrer. Poyt. 37, 7; 254, 7; Turmair 4, 455, 4; Maaler 274r; Dict. Germ.Gall.-Lat. 308, 32; Baumann-Zwirner, Augsb. Volksb. 1991, 376. - Vgl. ferner s. v. aufenthalten 7.

4. >Arglist, Tücke, Hinterhältigkeit als Haltung wie auch aus der Haltung hervorgehende heimtückische Handlung`; speziell (dicht belegt): >Bosheit, Betrug, Täuschung (generell sowie als Verführungskunst und Handlungsweise des Teufels $\iota)$. - Phras.: ane (alle) list (Verwahrformel). - Bdv.: s. u. SCHÖPPER, ferner: anschlag 5, beschis, bosheit 2, falschbeit, praktik 3, spiegelfechtung. Ggs.: recht. - Synt.: einen l. auskünden / brauchen / (er)suchen / finden, l. zu triegen haben, js. (z. B. des teufels) l. überwinden; der l. (Subj.) jn. (!) nicht helfen, l. js. rüstung sein; die könige vol l. sein; mit l. [wol] wandeln, mit list(en) abgerürt / gerïstet sein, etw. mit l. an sich bringen, vieh mit listen inhalten, sich mit listen beraten, jn. mit list(en) fangen / bintergehen / betriegen, jm. etw. mit list abdingen (z. B. das gesinde) / abspannen (z. B. das pferd, den garten) / ablausen / abstreichen / entfremden, jm. mit listen beiwonen, nach dem erbe stehen, jn. durch l. umbringen, etw. für einen l. halten; der l. des hellehundes / satans / teufels / feindes / fuchses; der bose / betriegliche / falsche / schädliche / teuflische l.; der rat mit l., der drache mit seinen listen, die obrigkeit mit listen. Wbg.: listen >listig handeln<, listige (die).

StrehlKe, Nic. Jerosch. Chron. 18909 (preuß., um 1330/40): [der tûvil] irsûchete manche list | $\hat{u}$ r tûvilischim sinne, $\mid$ wî er an dem beginne $\mid$ den gotishelt vorirrete. LUTHER, WA 35, 456, 5 (1528/29): gros macht und viel list | sein [feind] grausam ruistung ist. SCHÖPPER 166 (Dortm. 1550): Fallacia, seu dolus. List betrug auffsatz trug argduck binderlist listigkeit túcke trügnuß ránck triegerey geferd behendigkeit gescheidigkeit feinantz feinantzerey mouendeln bintergang. PEIL, Rollenhagen. Froschm. 548, 1311 (Magdeb. 1608): Der Han sagt / füer des Fuchses list / Auff dem Balcken mein Schlaffstedt ist. Stambaugh, Milichius. Zaubert. 12, 6 (Frankf./M. 1563): Unter den listen und beymlichen practikken damit er [Teuffel] den menschen nachstellet / ist die Zauberey nit die geringste. JAHR, H. v. Mügeln 1910 (omd., Hs. 1463): daran der mensche [...] | [... gefallen ist $\mid$ in marter von tüfels list. JungBluth, J. v. Saaz. Ackermann 28, 15 (Hs. ' ${ }^{\circ}$ d., 1465ㄱ): triegen, listen, smeichen, spinnen [...] kan si [weib] wol in einem augenblick. KeHREIN, Kath. Gesangb. 1, 11, 4 (Bautzen 1567): Das vns der bóß kein schad zufug, | Nach vns mit seinem list betrig. VETTER, Pred. Taulers 371, 14 (els., 1359): Die kunst was do er [Christus] des túfels list úberwant mit dem bitterlichesten schentlichesten tode. Lemmer, Brant. Narrensch. 33, 78 (Basel 1494): Die weltt steckt voll beschysß vnd lyst. Schade, Sat. u. Pasqu. 3, 12, 28 (orhein., 1520): wann ein wolf ein geiß ankïmbt, so brucht er solichen list, daß ers nit glich anfelt mit bißen, sunder [...]. RoLofF, Brant. Tsp. 1443 (Straßb. 1554): Darumb ist war das bóße rabt mit list | Dem Rabtgeb aller schandlichs ist. Wyss, Luz. Ostersp. 5061 (Luzern 1574): Vnd süchtend aber einen list, | Damitt sy Todtend Jesum Christ. Barack, Zim. Chron. 3, 205, 19 (schwäb., M. 16. Jh.): Der allmechtig verleibe uns allen sein gnad, das wir seine [Satan] listige und strick beharrlichen entgeen mögen! EsCHENLOHER. Medicus 32, 24 (Augsb. 1678): sonder bielten es [Gesicbt] nur für ein Spiegelfechtung / List / und Betrug. WACKeRNELL, Adt. Passionssp. St 1, 126, 4, Var. B (tir., v. 1496): Die weill der pöse trügner | Mit seinem list und falscher ler | Thut in unsern gepiete wandln. - LUTHER, WA 1, 167, 34; 30, 1, 360, 10; Меiјвоом, Pilgerf. träum. Mönch 11791; Henschel u.a., Heidin 893; Gille u.a., M. Beheim 52a, 12; Franck, Klagbr. 221, 36; Goldammer, Paracelsus 2, 135, 25; P̈̈PKE, Marienl. Wernher 10637; WYss, a. a. O. 3539; 3, 93, 249; BARAck, a. a. O. 2, 526, 28; Bischoff u. a., Steir. u. kärnt. Taid. 266, 37; Rwв 8, 1342. - Vgl. ferner s. v. ${ }^{3}$ aber 5, ablausen 2, abrüren 4, abstreichen 3, abtäuschen, afterrede 1, anmassen 1, anneiden, anricbten 16, anschlag 5, auge 10, auskiunden 1, behauren 3

5. mit Adjektivattribut oder (explikativem) Genitivattribut in der Weise gebraucht, daß das Adj. bzw. der Genitivausdruck die Bedeutung von list stark, teils bis zur Aufhebung (vgl. die Phraseme) überlagert; je nach Beleg an 1-4 anschließbar und insofern auch dort einordenbar. - Gehäuft Verstexte des älteren Frnhd. - Phras.: ${ }^{\top}$ sunder falsche list, ane arge(n) list o. ä. ${ }^{\urcorner}$, jeweils Verwahrformeln im Sinne von statsächlich, ohne Zweifel<; mit solcher list >auf solche Weise<; keine list >nichts, keine Möglichkeitく. - Synt.: der arge / böse / falsche / schwache l.; der Juden l., des irtumes / sünders / todes l., der schlangen $l$.

Helm, H. v. Hesler. Apok. 11375 (nrddt., 14. Jh.): volle sete; Die teilet sich mit sulcher list $\mid$ Daz der niemant hat gebrist. EвD. 13606: irretumes list her brichet $\mid$ Und scheidet u₹ die warbeit $|[\ldots]|$ Von der irdachten lugene. ЕвD. 17597: und sach dar bi keine list $\mid$ Der menscheite zu einer gnist. Fischer, Brun v. Schoneb. 6073 (md., Hs. um 1400): daz si [Maria] al sundir valsche list $\mid$ obir der engele kore gehoet ist. VALLI, Baldemann 367 (rhfrk./nobd., um 
1350): Und lost uns do uz bandin, | Do her was uff erstandin, Do uns in braht der slangen list. Gerhard, Hist. alde e 2707 (omd., um 1340): Din minster vinger ist | Turer, grozer, an arge list, | Wan dines vater rukke was.

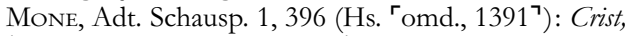
| der hat uns mit sines todes list | uns alle erlost. EBD. 674: der [Jhesu Crist] hat von der Juden list $\mid$ dy marter geleden durch unser schulde. Rennefahrt, Stadtr. Bern 227, 25 (halem., 1384): [verziechen wir ins] aller buntnusche, eytgnosschaft, geselleschaft, aller ander ufsetzen, arger listen, funden und usziechung. LindQvist, K. v. Helmsd. 2365 (halem., Hs. um 1435): Der wilend durch des sunders list | Begraben bie uff erden ist. KLEIN, Oswald 9, 62 (oobd., 1421?): ich wolt uns raten, | möcht wir aus disen swachen listen waten. - DAT NUWE BOYCH 442, 1; JAHR, H. v. Mügeln 2404.

liste, die; im 16. Jh. aus dem Ital. rückentlehntes endogen dt. Wort.

>Liste, Verzeichnis`. - Schulz / BASLeR 2, 1942, 31 (а. 1597); Rwв 8, 1343.

listen, s. list 4.

listenreich, s. list 2.

listig, Adj., als Adv. meist -lich o. ä.

1. >mit Gestaltungskraft ausgestattet; kunstvoll<; vgl. list 1. - Bdv.: vgl. artig 4, artlich 1, behende 6, statlich 1 .

Jungbluth, J. v. Saaz. Ackermann 10, 12 (Hs. 'omd., $\left.1465^{\urcorner}\right)$: alle irdische creatüre, wie künstig, wie listig, wie stark. sie sein [...] müßen zu nichte werden. PÄPкE, Marienl. Wernher 5500 (halem., v. 1382): Maria macbte dem kindelin | Gar listeklichen ain róklin.

2. >klug, ein-, umsichtig, weise ; vgl. list 2. - Bdv.: bescheide 1, klug 4, könnend, sinnig, weise.

Luther, WA 9, 151, 16 (1518): dartzu horet gar ein scharff gesichte und listige Vornunfft, ab trusundern eins van dem andern. EBD. 10, 3, 275, 27 (1522): wie der haußhalter da listig ist auf sein tìn, also sóllen wir auch sein das ewig leben zigewinnen. Quint, Eckharts Pred. 2, 553, 3 (E. 13./A. 14. Jh.): Ein meister sprichet: der listic ware und wol dâ mite künde, der ordente wazzer über wîn, alsô daz des wines kraft möbte dar inne gewürken. KOLLER, Ref. Siegmunds 69, 7 (Hs. ' ${ }^{\circ}$ Basel, um 1440?): zytlich bose gewin hant den loff; wer sich darinne übet, den schótzet man für wise und listig und wol kiunent. Sснмгтт, Ordo rerum 501, 10 (oobd., 2. H. 15. Jh.): Jndustris Jndustriosus lisstig sinnig weys pescheid.

3. sgewitzt, einfallsreich, versiert, wendig, klug, schlau, gerissen (von Menschen, menschlichen Handlungen, auch menschenähnlich gedachten Wesen sowie von
Tieren gesagt)<, bezogen auf die gesamte Bandbreite zwischen 2 und 4; vgl. list 3. Bdv.: aufgeblasen, aufgescbraubt, aufsetzig 2; 3 , ausgestochen, böse 6, gemeit 1, klug 4, auch 5, schalkhaftig, spitzig, spirfündig, vielköonnend; vgl. gescheibe, tausendfündig. - Synt.: j. l. sein; jm. l. zugehen, jn. l. [wohin] bringen, etw. (z. B. zweitracht) l. anrichten; etw. (z. B. ein schlos) listigerweise einnemen; der listige fuchs / leie / mensch / Ulisses / spruch, die listige katze / tat, das listige tier / weib / wort, listige fünde.

Luther, WA 6, 247, 14 (1520): dan der schalckhaftige Adam gar listig ist y hm selb urlaub zusuchen. EBD. 34, 2, 394, 9 (1531): 'Bose geister', das ist: nicht allein klug, spitzig und listig, hoch und weit uber menschen vernunfft und weisheit, Sondern auch gifftig, bose und bitter. HüBNER, Buch Daniel 7475 (omd., Hs. 14./A. 15. Jh.): Wand ieclicher in stillen | Gedachte gantz. volbringen |Al sines herzen ringen | Listlich an der vil guten [Susanna]. CHRON. Nürnb. 3, 172, 11 (nobd., 1488): man bielt in [Huß] auf der schuel fuir einen listigen [Z. 19: gelerten; Var.: lustigen] und kleprigen laien. MAALER 274 (Zürich 1561): Listig vnd geschwind vneinigkeit vnd zuytracht anzerichten. [...] Listige oder spitzfündige that. [...] Listiger außgestochner mensch. Listiger fuch $\beta$. SAPPLER, H. Kaufringer 10, 103 (schwäb., Hs. 1464): seit von natur den weisen man |Aristotilem gewan | ain weib listig und gemait. Chron. Augsb. 2, 309, 26 (schwäb., Hs. 16. Jh.): der Ochsenfueß [...] ist alle sein tag ain vilkeünnet man gewesen, schalkhaftig und listig. - v. KELLER, Amadis 7, 33; Oorschot, Spee/Schmidt. Caut. Crim. 323b, 6; Gajek, Seidelius. Tych. 10, 15; Karnein, Salm. u. Morolf 224, 2; Sснӧnbach, Adt. Pred. 26, 35; Rauwolf. Raiß 4, 28; Pfeiffer, K. v. Megenberg. B. d. Nat. 151, 26; Grossmann, Unrest. Öst. Chron. 234, 5; Dict. Germ.-Gall.-Lat. 308, 36; Rwb 8, 1344. Vgl. ferner s. v. anschlagen 16, aufblasen 3, aufsetzig 3, ausschläufen 3, arm (Adj.) 1, begreifen 8, jägermeisterin.

4. strügerisch, verführerisch (von Sachen; relativ selten belegt); heimtückisch, hinterlistig, arglistig, betrügerisch (von Menschen, Handlungen, Haltungen); vom rechten religiösen Weg abführend, teuflisch, verführerisch (vom Teufel o. ä.)<; zu list 4. - Bdv.: abgünstig, ängstlich 1, arg (Adj.) 4, aufsetzig 3, betriegig, betrieglich 1; 2, böse 6, böslich, falsch (mehrfach), füchsisch, ränkisch, schädlich, schalkhaftig, schandlich, verfiurisch, vergiftend, verschlagen, wolfisch. Ggs.: alber, schlecht. - Synt.: der teufel, die schlange / etw. (z. B. die welt, die dinge der welt) l. sein, $j$. (die Juden) listiglich karg sein; $j$. (der satan) listiglich zu Eva 
schleichen, der teufel mit jm. listiglich umgehen, etw. l. überkommen, jn. l. betriegen / verfüren / veruntreuen, in das verderben bringen, jm. etw. listiglich ablausen; der listige anlauf / geist / mord / satan / tausendkünstler / wicht [= schlange], die listige anweige / bosheit / bure / lüge / schalkheit / trogenheit / versuchung / zunge.

SCHÖPper 166 (Dortm. 1550): Dolosus. Listig arg / trieghafft betrieglich betriegig tückisch fücbsisch auffsetzig ränckisch. LUTHER, WA 32, 174, 9 (1530): Diaboli sind nicht alle gleich, Einer ist listiger, schalkhaftiger, bóser denn der ander. EBD. 53, 247, 11 (1542): denn wir sind gegen solche Listige und Unergruendliche [...] Kluegheit [...] Albere Scheflin. LemMER, Amman/Sachs. Ständeb. 111, 5 (Frankf./M. 1568): Wie ich [Geltnarr] nur groß Gelt vnd Reichthumb / | Vnverschempt listig vberkumb. OоRSсHOт, Spee/Schmidt. Caut. Crim. 392a, 17 (Frankf./M. 1649): der Teuffel sey ein sebr listiger tausent Kinstler. zu DoHNA u. a., Staupitz/ Scheurl 213 (Nürnb. 1517): unser red auf die schlangen, die listiger ist dann alle andre tier. WAGNer, Erk. Ps.-J. v. Kastl 7, 6 (nürnb., 1. H. 15. Jh.): das er [mensch] sich von allen dingen diser werlt abcribe [...], wann sie sein schedlich, falsch, listig, betrigent, vergiftent. ÖsterLey, Steinhöwels Äsop 39, 35 (Ulm 1474/82): Welcher under üch allen den andern listenclichen understat ze veruntrïwen, desselben but sol mit sölichem lon [öffentlichem Auspeitschen] geziret [...] werden. Chron. AugsB. 7, 422, 31 (schwäb., zu 1564): [er] hat genanten Beckenstain schandtlich, beßlich und listigclich umb all sein bab [...] in das eusserst verderben gebracht. Hohmann, H. v. Langenstein. Quästio 193, 192 (moobd., 1. H. 15. Jh.): das der mensch müg erchennen [...] dy listig trogenhait des pösen geist. Grossmann, Unrest. Öst. Chron. 181, 13 (oobd., 3. Dr. 15. Jh.): gegen dem ennd [ward er] durch valsch verreter [...] listiglich betrogen. Helm, H. v. Hesler. Apok. 14066; Ders., H. v. Hesler. Nicod. 5123; Luther, WA 1, 218, 7; 6, 14, 5; AlbeRus, Barf. Vorr. Alb., 5, 28; SснӧNBACh, Adt. Pred. 6, 42; Mayer, Folz. Meisterl. 12, 112; v. Keller, Ayrer. Dramen 2799, 23; WAGNER, a. a. O. 7, 61; GoldAMMER, Paracelsus 3, 279, 5; BANZ, Christus u. d. minn. Seele 1131; Pfeiffer, K. v. Megenberg. B. d. Nat. 45, 6; Hohmann, a. a. O. 179, 4; 179, 21; 205, 5; 213, 128; SpechtLer, Mönch v. Salzb. 10, 83; 25, 21; Baptist-Hlawatsch, U. v. Pottenst. 1513; Maaler 274r. - Vgl. ferner s. v. abgünstig, ablausen 2, anfecbten 4, anlauf 4.

listige, s. list 4.

listigkeit, die.

1. \Kunstfertigkeit; handwerkliches Können; Kriegstüchtigkeit‘; vgl. listig 1. - Bdv.: geschiklichkeit 1; 3, kunst; vgl. list 1 .
Luther, WA 21, 21, 17 (1528): Es ist auch noch nie nicht solch pflantzen gewest und solche listickeit und geschicklickeit die narung zu suchen. DAsYPODIUs 364r (Straßb. 1536): Kunst / listigkeyt. Ars, Techna. Brandstetter, Wigoleis 192, 18 (Augsb. 1493): durch wz krafft oder listigkeit jch [Gabon] überwunden bin weyß jch nicht. Aber soelt mir mein schwertt gancz beliben sein. jch woelt eüch vnd all eüer listigkeyt [...] überwunden haben. EBD. 218, 9: karyos vnd marin belfen wenig mit jrer stoercke vnd listikeit. mit dem sy das land befridt [...] haben. - DAsYPODIUs $13 \mathrm{r}$.

2. >Klugheit, Weisheit (wie sie dem Menschen, in nichtchristlichen Glaubensvorstellungen auch z. B. der Schlange, zugeschrieben und im Vergleich mit Gottes Erkenntnis-Allmacht abwertend beurteilt wird)<; vgl. listig 2. - Bdv.: fürwiæ, vernunft, weisheit.

Perez, Dietzin 1 43, 25 (Frankf. 1626): Von [...] Aristotele dichten die Alten / als neme er seine Schrifften auß dem Herzen einer Schlangen: Dieweil sie das Symbolum oder Zeichen der Weißheit / Listigkeit vnnd der Vernunfft ist. SACHS 18, 288, 18 (Nürnb. 1562): Wenn sollichs in anfechten thut, | So dencket er, das thut fleisch und blut, | Daß sein fürwitz. und listigkeit $\mid$ Bey gott sey ein lautre thorheit. BAUER, Imitatio Haller 77, 13 (tir., 1466): Du solt kechain getrauen baben in dein kechunst noch in kechaines menschen listikechait, aber du solt getrauen in die genaden gottes. EBD. 101, 29: Du würt die willig gehorsam vil mer erböcht denn alle menschleiche listikechait. - SACHS 17, 482, 4.

3. Klugheit, Schlauheit, Gewitztheit, Geschicklichkeit, schlauer Einfall<; vgl. listig 3 - Bdv.: geschwinde (zu geschwind 1), klugheit 3, subtiligkeit. Ggs.: simpelheit.

LutHer, WA 30, 1, 25, 14 (1528): scbleicht er [Teufel] einem nach mit listigkeit. PeIL, Rollenhagen. Froschm. 297, 1119 (Magdeb. 1608): als Reinick Fuchs ankam / | [...] | Gab er nach seiner listigkeit / | Dem Kónige solchen bescheid. Chron. Köln 2, 9v, 36 (Köln 1499): He [...] verkeirde also die eyrste simpelheit in kloicheit vnd listicheit. Mon. BoicA, NF. 2, 1, 248, 30 (nobd., 1464): lehen, das sein vatter der herrschaft mit listikeyt abgetragen hat in sein hwbe. MAaler 274r (Zürich 1561): Die Listigkeit / Geschwinde / oder subtyligkeit ze dißputieren. PFafF, Tristrant 121, 4 (Augsb. 1498): er [...] hat mit listigkeit und grosser gebeisse ab geworffen all meins herren lantsessen. SEEMülLER, Chron. 95 Herrsch. 10, 11 (oobd., Hs. 1. H. 15. Jh.): Er [Cham] was gesprëch und überwant si mit listichait, das si für gotes zoren buben an ze pawen den turen.

4. >Arglist, Tücke (des Menschen)<, schwach belegt; >Hinterhalt, Heimtücke, Verführungskunst des Teufels` mit der Folge: >Versuchung (für den Menschen) ; dicht belegt; vgl. listig 4. - Texte der Sinn- 
welt ,Religion / Didaxe'. - Bdv.: s. u. SCHÖpper s. v. list 4, ferner: anweige, betriegnis $1 ; 2$, bosheit 1, falschbeit, trogenheit, versuchung. - Synt.: die l. erkennen / verbergen / versuchen >ausprobieren<, etw. l. bedeuten; l. (Subj.) sein [>darin bestehen $]$, das $[. .] ;$.$j s. l. entgehen; die$ l. des feindes / bösen geistes / teufels (dicht belegt), der bulerin.

zu Dohna u. a., Staupitz/Scheurl 217 (Nürnb. 1517): Darumb ist die erst kunst, des teufels betrignus und listikeit zu entgeen, zemung der begirlikeit, alle dingk zu wissen. KurRelmeYer, Dt. Bibel 1, 168, 14 (Straßb. 1466): Do er west ir [phariseer] listikeit [LuTHER 1545, Mr. 12, 15: heucheley] er sprach zu in. Was versucht ir mich? WARNOCK, Pred. Paulis 7, 291 (önalem., 1490/4): und betút die menschen, die also allain daz. wulli, daz, gütt und ainfaltig, lassent sechen, aber ir boshait und listikait verbergent sú. Hohmann, H. v. Langenstein. Quästio 193, 180 (moobd., 1. H. 15. Jh.): Dye sechst listichait vnd versüchung ist, mit der er [Teufel] dem menschen rätt dye sünd vnder der gestalt einer tugent. EBD. 193, 192: das der mensch müg erchennen dy falschen listichait [...] des pösen geist. KLEIN, Oswald 26, 49 (oobd., 1427): mein listikait hett in der fürst $\mid$ die oren vol erschellet. - KeHrein, Kath. Gesangb. 3, 34, 3; v. D. LeE, M. v. Weida. Spigell 79, 5; WARNOcK, a. a. O. 7, 286; HoHmAnN, a. a. O. 183, 27; 187, 71; 187, 89; Bauer, Haller. Hieronymus-Br. 107, 28; MaAler 46r. - Vgl. ferner s. v. immer 2, inbitzig 1.

listweise, s. list 3 .

litargirum, das; s. DU CANGE 123b.

ein Pulver für verschiedene Zwecke; >Bleiglätte, Bleioxid (PbO)<. - Fachtexte. Bdv.: bleiweis, glätte 2 .

Follan, Ortolf. Arzneib. 147, 6 (rib., 1398): nym [...] litargiri twey lot de gummi saltu seden in eynen vyrteyl starkez. etekes [angewandt gegen alle fistulen vnde alle drose, unde beylet de wunden]. BELKiN u. a., Rösslin. Kreutterb. 150, 9 (Frankf. 1535): Nim ól von nüssen / mische darunder das puluer Litargirium, schmier die reudigkeyt damit. EBD. 15: Wer da het das rot / der nem essig / vnnd mische darunder des pulvers Litargiri [...] mach drauß ein cristier. KeIL, Peter v. Ulm 165 (nobd., 1453/4): ein gut graw pflaster. Nim weiß wachs, hart₹, [...], grünspan [...], holwurť, weyrauch, [... litargirum, das ist glet. - Broszinski, Minner. Chir. Parva 70v, 11; 78, 24; StedtFeld, Roger-Glosse, S. 86.

litauisch, Adj.

$>$ litauisch<; im einzelnen: >der diet, Litauer' zugehörig‘; >in ,Litauen' üblich, gebräuchlich «. - Wbg.: litisch.

Strehlke, Nic. Jerosch. Chron. 19690 (preuß., um 1330/40): darûffe dâ von gestin | was vil Littowschir rotin | von andrin gegenôtin. EBD. 19697: Dâ bettin der Littouwschin dît | dî brûdre vil irslagin. EBD. 25838: [Darnâch] sprengte ein her littouschir dît $\mid z u$ Wilow in daz gebit. ZIESEMER, Gr. Ämterb. 71, 37 (preuß., 1510): 2 bolczsschlietten, $i$ littawisch schlietten. EBD. 202, 24: 1 balben kornscheffel, 3 littausche holtzwegen. Moscowia C 3r, 40 (Wien 1557): So liessen die Mosconiter aufblasen / und griffen die Littischen an.

litierige, die; aus frz. litière.

> von zwei Pferden an Doppelstangen getragene Sänfter (so SchweIz. ID. 3, 1500). Rennefahrt, Staat/Kirche Bern 737, 29 (halem., 1634): das an Sonn- und Festtagen in Bern anstößige fahren mit gutschen, berlinen, chaisen ind littiergen und andere üppigkeiten mehr.

litigant, litigieren, s. lis.

litisch, s. litauisch.

litisconsorte, litiscontestation, litisdenunziation, s. lis.

litter, ${ }^{2}$ letter, der, auch die; -/auch -en.

>Buchstabe; Drucktype«; metonymisch dazu: >Literalsinn, eigentliche Bedeutung des Geschriebenen`; ‘Schriftsatz, Brief‘. Bdv.: vgl. abkerung 3, buchstabe. - Wbg.: letterwechsel > Anagramm<, litterat, litteratur.

ENDERs, Eberlin 3, 162, 28 ([Eilenb.] 1524): auch gebrauchen die Trucker bóß papyr, bóße litera. CHrON. KÖLN 2, 10r, 7 (Köln 1499): Abraã hait vondẽ die kaldeeschen litterẽ. EвD. 2, 388, 3 (Köln 1499): he [konink] machte so vil cloistere as sin litteren in dem a b. BUCH WeINSB. 1, 277, 35 (rib., um 1560): wan der eirste boichstaif an jeder taifel ist roit und wan die allereirste litter an jederer und allen taifeln beiein pracht werden, so begriffen sei sinen nammen. ЕвD. 2, 193, 4 (1569): darvur mit guldin litteren diss vers stunde geschriben. EBD. 2, 312, 22 (1575): dar der eirst, der mittelste ungeferlich und leste litter einen besondern sensum und sinne machten (Akrostichon). Gropper. Gegenw. 18v, 1096 (Köln 1556): obe die wort der Schrifft nach jrem rechten unnd eigentlichen siñ / wie sie nach der Litter stehen vnd lauten / oder aber Methaphorischer [...] weiß zuuerstehen sein. MATthaei, Minner. I, 8, 398 (Hs. 15. Jh.): ,wilt du versten myn litter", sprach fraw Marze, ,ich schriben dir". FRANZ u. a., Qu. hess. Ref. 2, 67, 33 (hess., 1528): das zu einer jeden pfarrkirchen [...] ein neu testament in grober litter z.u sein fast not [...] sein solt. Helm, Maccabäer 6984 (omd./ nrddt., Hs. A. 15. Jh.): Ptolomeus wart ouch vugen, | daz. er besande die rittre | des landes, daz wurben littre. Rот 325 (Augsb. 1571): Literat. Ein Gelehrter / der etwas inn den freyen kinsten versteht vnd kan. Ein Gschrifftglerter / kunstreicher. EвD.: Literatur. Gschrifft / kunst der gschrifft / gschrifftgelerte weiß vnd kunst. - Rwв 8, 1239.

litterat, litteratur, s. litter. 